\title{
Coming of Age in Byzantium
}




\section{Millennium-Studien}

zu Kultur und Geschichte

des ersten Jahrtausends $\mathbf{n}$. Chr.

\section{Millennium Studies}

in the culture and history

of the first millennium C.E.

Herausgegeben von / Edited by

Wolfram Brandes, Alexander Demandt, Helmut Krasser, Hartmut Leppin, Peter von Möllendorff, Karla Pollmann

\section{Volume 69}




\section{Coming of Age in Byzantium}

Adolescence and Society

Edited by

Despoina Ariantzi

\section{DE GRUYTER}


Veröffentlicht mit Unterstützung des Austrian Science Fund (FWF): Projekt P22773

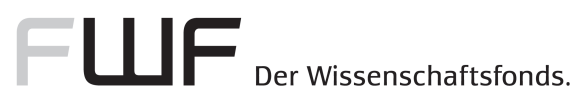

\section{(cc) BY-NC-ND}

This work is licensed under a Creative Commons Attribution-NonCommercial-No-Derivatives 4.0 License. For details go to https://creativecommons.org/licenses/by-nc-nd/4.0/.

ISBN 978-3-11-057646-7

e-ISBN (PDF) 978-3-11-057649-8

e-ISBN (EPUB) 978-3-11-057660-3

ISSN 1862-1139

\section{Library of Congress Cataloging-in-Publication Data}

A CIP catalog record for this book has been applied for at the Library of Congress.

\section{Bibliographic information published by the Deutsche Nationalbibliothek}

The Deutsche Nationalbibliothek lists this publication in the Deutsche Nationalbibliografie; detailed bibliographic data are available on the Internet at http://dnb.dnb.de.

(C) 2018 Despoina Ariantzi, published by Walter de Gruyter GmbH, Berlin/Boston The book is published with open access at www.degruyter.com.

Printing and binding: $\mathrm{CPI}$ books $\mathrm{GmbH}$, Leck

@ Printed on acid-free paper

Printed in Germany

www.degruyter.com 


\section{Acknowledgments}

This volume evolved from an international symposium on the subject "Coming of Age - Adolescence and Society in Medieval Byzantium," held in Vienna on February 3-4, 2014. The subject of this symposium was closely related to the research project "Coming of Age, Leaving the Nest: Models of Adolescence in Byzantium (Sixth-Eleventh centuries) hosted at the Department of Byzantine and Modern Greek Studies at the University of Vienna and funded by a three-year research grant (2011-2014) of the Austrian Science Fund (FWF): Project-Nr. P22773.

I would like to express my warmest gratitude to all of the contributors for participating in the conference and presenting their findings in this volume.

My special thanks go to the project leader Prof. Johannes Koder for his cooperation and his unwavering support in many matters. Last but not least, I thank the series editors, Wolfram Brandes, Alexander Demandt, Helmut Krasser, Hartmut Leppin, Peter von Möllendorff und Karla Pollmann, for accepting the book, the anonymous readers for their positive evaluation, and the Austrian Science Fund (FWF) for the financial support.

Despoina Ariantzi

Vienna, June 2017 



\section{Contents}

Despoina Ariantzi

Introduction: Approaches to Byzantine Adolescence $\left(6^{\text {th }}-11^{\text {th }}\right.$ centuries $)-1$

Béatrice Caseau

Too Young to Be Accountable: Is 15 Years Old a Threshold in Byzantium? 19

Günter Prinzing

Adoleszenten in der kirchlichen Rechtsprechung der Byzantiner im Zeitraum

13. -15 . Jahrhundert -29

Alice-Mary Talbot

The Adolescent Monastic in Middle and Late Byzantium — 83

Tonia Kiousopoulou

Adolescence in the Late Byzantine Society $\left(14^{\text {th }}-15^{\text {th }}\right.$ centuries $)-99$

Petra Melichar

Adolescent Behavior in Byzantine Sources? Some Observations on Young

Byzantine Women Pursuing their Goals 105

Despoina Ariantzi

Soziale Identitätsbildung im Jugendalter in Byzanz — 117

Leslie Brubaker

Images of Byzantine Adolescents — 141

Cecily Hennessy

Representations and Roles of Adolescence with a Focus on Apocryphal

Imagery - 175

Catia Galatariotou

The Byzantine Adolescent: Real or Imaginary? — 203

Ulrike Sirsch

Erwachsenwerden oder Erwachsensein? Ausgewählte Aspekte zu Jugend, „Emerging Adulthood“ und jungem Erwachsenenalteraus Sicht der

Entwicklungspsychologie -215 
Thomas Pratsch

Jugendliche und Heilung -237

Hans-Werner Goetz

Adolescentia in abendländischen Quellen des frühen Mittelalters zwischen Kindheit und Erwachsensein? Ein begriffsgeschichtlicher Zugang — 251

List of Contributors -295

Index -297 


\title{
Abbreviations
}

\author{
AASS Acta Sanctorum \\ AnBoll Analecta Bollandiana \\ AnnalesESC Annales Economies, Sociétés, Civilisations

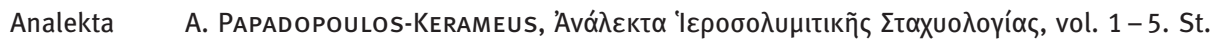 \\ Petersburg 1891-1898 \\ B. Basilicorum Libri LX, ed. H. J. SCHeLtema-N. VAN DER WAL. Gröningen 1962. \\ BBA Berliner Byzantinistische Arbeiten \\ BBS Berliner Byzantinistische Studien \\ BF Byzantinische Forschungen \\ BH Bibliotheca Hagiographica Graeca \\ BMFD Byzantine Monastic Foundation Documents \\ BMGS Byzantine and Modern Greek Studies \\ BSI Byzantinoslavica \\ BV Byzantina Vindobonensia \\ Byz Byzantion \\ BZ Byzantinische Zeitschrift \\ BHG Bibliotheca Hagiographica Graeca \\ C. Corpus luris Civilis, Codex Justinianus, vol. 2, ed. P. KRUEger. Berlin 1963. \\ CCCM Corpus Christianorum. Continuatio Mediaevalis \\ CSEL Corpus Scriptorum Ecclesiasticorum Latinorum \\ CCSG Corpus Christianorum, Series Graeca \\ CCSL Corpus Christianorum. Series Latina \\ CFHB Corpus Fontium Historiae Byzantinae \\ CSHB Corpus Scriptorum Historiae Byzantinae \\ CTh Codex Theodosianus, vol. 1-2 in 3 Teilen, ed. Th. Mommsen-P.M. MeYer. Berlin 1905 \\ (reprint Princeton 1952) \\ D. Digesta, Corpus luris Civilis, vol 1, ed. Th. MOMmSEN-P. KRüger. Berlin 1872 (reprint \\ 1973) \\ DOC 1 A. Bellinger, Catalogue of the Byzantine Coins in the Dumbarton Oaks Collection and \\ in the Whittemore Collection, 1: Anastasius to Maurice, 491-602. Washington DC \\ 1966 \\ DOC 2 P. Grierson, Catalogue of the Byzantine Coins in the Dumbarton Oaks Collection and \\ in the Whittemore Collection 2. Washington DC 1968 \\ DOC 3 P. Grierson, Catalogue of the Byzantine Coins in the Dumbarton Oaks Collection and \\ in the Whittemore Collection 3. Washington DC 1973 \\ DOC 4 M. Hendy, Catalogue of the Byzantine coins in the Dumbarton Oaks Collection and in \\ the Whittemore Collection 4. Washington DC 1999 \\ DOP Dumbarton Oaks Papers

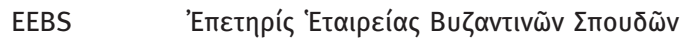

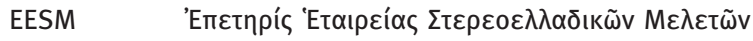 \\ FM Fontes Minores \\ GOrThR The Greek Orthodox Theological Review \\ GCS Die griechischen christlichen Schriftsteller \\ GRBS Greek Roman Byzantine Studies \\ IRAIK Izvestija Russkago arheologičeskago instituta v Konstantinopole \\ JECS Journal of Early Christian Studies \\ JGR Jus Graecoromanum, ed. I. Zepos-P. Zepos, vol. 1-8. Athen 1931 (reprint Aalen 1962)
}


JÖB Jahrbuch der Österreichischen Byzantinistik

Lampe $\quad$ G. W. H. LAMPE, A Patristic Greek Lexicon. Oxford 1961

LBG Lexikon zur byzantinischen Gräzität, ed. E. TRAPP [et al.]. Wien 1994-2017

LexMa Lexikon des Mittelalters

LS H. G. LIDDELL - R. SCOTT, A Greek-English Lexicon. With a Revised Supplement. Oxford ${ }^{9} 1996$

LThK Lexikon für Theologie und Kirche

MGH Monumenta Germaniae Historica

OCP Orientalia Christiana Periodica

ODB The Oxford Dictionary of Byzantium

PG Patrologia Graeca

PL Patrologia Latina

PLP Prosopographisches Lexikon der Palaiologenzeit

PLRE 3 J. R. MARTINDALE, ed., The prosopography of the later Roman empire 3, AD 527-641. Cambridge 1992

PmbZ Prosopographie der mittelbyzantinischen Zeit

RAC Reallexikon für Antike und Christentum

RE Rauly's Real-Encyclopaedie der classischen Altertumswissenschaft

REB Revue des Études Byzantines

ROC Revue de l'Orient Chrétien

SC Sources Chrétiennes

StP Studia Patristica

SubHag Subsidia Hagiographica

SynaxCP Synaxarium ecclesiae Constantinopolitanae. Propylaeum ad Acta sanctorum Novembris, hrsg. H. Delehaye. Brussels 1902

TM Travaux et Mémoires

VV Vizantijskij Vremennik 


\section{Introduction: Approaches to Byzantine Adolescence $\left(6^{\text {th }}-11^{\text {th }}\right.$ centuries)}

The idea of adolescence as a distinct stage of biological and psychological development clearly differing from childhood and adulthood can be observed in all human societies and goes far back to antiquity. ${ }^{1}$ Yet the question how the changes in the personality of adolescents are perceived and which possibilities and limits determine their behavioral patterns depends upon the cultural, social, and economic conditions $^{2}$ of a given society and its historical context.

The topic of "youth" was discovered as a study area as early as the beginning of the twentieth century, but only slightly explored. On the basis of diary entries, the behavior of adolescents was analyzed and categorized into certain typifications. Between the 1920s and 1960s, a number of studies treated questions of developmental psychology and pedagogy, primarily addressing educators. Since the 1970s, research on adolescence - especially in the USA - has further developed as a result of advances in biology, sociology, psychology, anthropology, and medicine. Thus it became clear that adolescence is not only a socio-historical construction, but also a phenomenon of multidisciplinary interest.

In the Roman-Byzantine legal tradition, majority generally began between the age of 20 and 25. According to the Byzantine understanding, the stage of adolescence preceding legal age covered a period starting at the age of twelve years for girls and fourteen for boys. ${ }^{3}$ This gives us a first approach from the viewpoint of family law defining the relations of young people with their relatives, their social milieu, and the state authorities. The public sphere clearly discerns an intermediate stage between childhood and full legal capacity. But what does this mean for the role of adolescents in Byzantine society as a whole? How and to what degree is this intermediate status reflected in the narratives and statements provided by various genres of Byzantine literary traditions? The "Coming of Age" project aimed to explore the presuppositions, parameters, and particularities determining the phases of transition from childhood to adulthood, in which the material, emotional, and spiritual release from the parents and the incremental emancipation of the individual were accom-

\footnotetext{
1 E. Eyben, Restless Youth in Ancient Rome. London - New York 1993; M. KLEIJwEgt, Ancient Youth. The Ambiguity of Youth and the Absence of Adolescence in Greco-Roman Society (Dutch Monographs on Ancient History and Archaeology 7). Amsterdam 1991; Тн. Widemann, Adults and Children in the Roman Empire. London 1989.

2 K. HuRRELmann, Lebensphase Jugend. Eine Einführung in die sozialwissenschaftliche Jugendforschung. München ${ }^{9} 2007,19$.

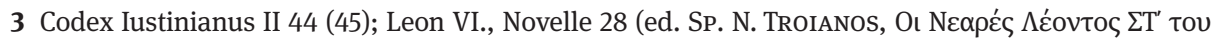

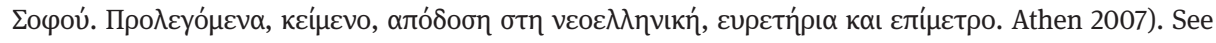
for the legal age limit and concerning terminology.
} 
plished. Current psychological and socio-anthropological research dedicated to life stages interpret this phase as a definitive or at least as an important step towards maturity and personality development, which in the most recent studies has become increasingly ascertainable and describable also as a historical phenomenon. ${ }^{4}$ As chronological limits we have chosen the period between the sixth and the eleventh centuries, two watersheds in the development of the Byzantine state and society, marking the end of Late Antiquity and the emergence of new attitudes towards human individuality. Our corpus of primary sources consists mainly of hagiographic, legal, and medical texts, while we glean additional information from theological treatises, historiography, and letter collections. There are no texts offering an extensive treatment of adolescence and its characteristics. In this sense we cannot say that adolescence was a central concern, a subject of investigation, or at least a topic of sophisticated debates among Byzantines. Nonetheless, there are recurring patterns of thought, plenty of stereotypes, moral suggestions, legal concepts, medical descriptions, and, in few cases, even statements expressing individual experiences of adolescence. This set of ideas in its entirety reflects collective attitudes and prevailing modes of perception in Byzantine society. By investigating this material we cannot claim to reach the social reality of the period under discussion or to discover traits of diachronic development in the centuries-long continuities of generations of young people. In early medieval contexts this is something that can be rarely achieved anyway, even in regions and subject areas where the available source material is much richer than in Byzantium. What we can actually do is to try to establish a comprehensive typology of concepts and perceptive patterns related to adolescence and to work on methods and tools for a verifiable description and interpretation of these phenomena. To this end, the project has developed a framework of investigation which combines a careful analysis of the texts based on methods of source criticism with modern theories of psychology and social sciences. This is not to say that theoretical approaches developed on the basis of data provided by twentieth-century realities would always be applicable to early medieval social contexts. What matters is to detect overlapping zones between the modern terminology and the semantic fields offered by our Byzantine sources. Reading them against the background of modern experiences helps us better understand and decipher the cultural code encapsulated in the rhetoric modes of expressions of Byzantine texts. Thus, we have chosen the process of the release of youths from parental care, which according to modern theories forms one of the key moments of adolescence, as the thematic core issue of the project. According to youth psychology it is during this process

4 M. Bucholtz, Youth and Cultural Practice. Annual Review Anthropology 31 (2002) 525-552; E. ERIKson, Jugend und Krise. Die Psychodynamik im sozialen Wandel. Stuttgart 1988. HurrelmanN, Jugendforschung; A. FlAMMER - F. AlSAKER, Entwicklungspsychologie der Adoleszenz. Die Erschließung innerer und äußerer Welten im Jugendalter. Bern - Göttingen - Toronto - Seattle 2002; G. Mietzel, Wege in die Entwicklungspsychologie: Kindheit und Jugend. Weinheim ${ }^{4} 2002$. See also in this volume the paper of C. GALATARIOTOU and U. SIRsch (with further literature). 
that decisive steps towards self-actualization and the assumption of (personal) selfresponsibility are often completed and decisions about the direction of one's personal future are made. ${ }^{5}$ It also contains a key social component, as it enables the proliferation of the younger generation, which is exposed to the tension between acceptance of and resistance to the older generation and thus is able to spark conflicts and to initiate social changes. This definition certainly provides us with a much broader spectrum than the scarce statements of Byzantine legal treatises. It makes us think about the possibilities to interpret the descriptions, terms, and allusions of Byzantine texts with respect to the psychological and social context in which the literary conventions and perceptive patterns of Byzantine authors have to be embedded. In other words, the thought world of Byzantine authors has to be contextualized within the framework of Byzantine family relations and the social network of young people.

Generally speaking, the field of Byzantine Studies has so far only insufficiently probed the set of questions proposed here. To date there has been no systematic examination of adolescence in Byzantium. Our knowledge of this subject is based almost exclusively on research devoted to the more general phenomenon of childhood. Even this subject was until recently only in its beginnings with respect to Byzantium and, in the last three to four decades, has produced more precise results, admittedly restricted to specialised areas. Évelyne Patlagean was among the first Byzantinists involved in the study of children and youths. ${ }^{6}$ In her view, coming of age is not an institution of mass transition applied to a group of a specific age, but a personal passage of varied duration and complexity depending upon social conditions. The different forms of this passage determined the place of the youth of both genders. Using legal terms of the thirteenth and fourteenth centuries, she has demonstrated that coming of age occurred in stages and varied according to gender. Patlagean made an important advance in the study of youth with her investigation of the role of young men in the public life of the cities of the early Byzantine era. Within the military and socio-political currents of the early Byzantine period the age group of young men played a double role: on the one hand, they took up an active military role and,

\footnotetext{
5 A. B. Hollingshead, Elmtown's Youth. New York 1958; A. Flitner, Soziologische Jugendforschung. Darstellung und Kritik aus Pädagogischer Sicht (Anthropologie und Erziehung 7). Heidelberg 1963; E. H. ERIKSON, Identität und Lebenszyklus. Frankfurt 1979; IDEM, Jugend und Krise. Stuttgart 1980; G. R. ADAms, Psychosocial development during adolescence (Advances in adolescent development 8). Newbury Park, California 1996.

6 E. Patlagean, L'enfant et son avenir dans la famille byzantine, Annales de démographie historique, 1973, 85-93 [= Structure sociale, famille, chrétienté à Byzance $I V^{e}-X I^{e}$ siècle (Variorum Reprints CS),

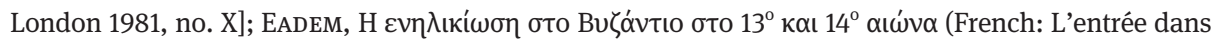

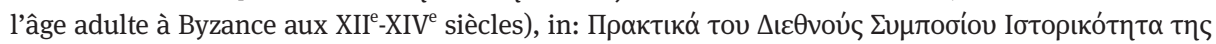

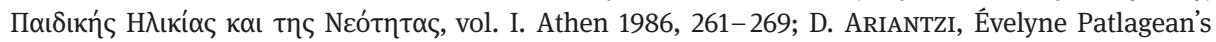
Ideas on Childhood: A Presentation of her Views and their Importance for Future Research, in: Les Réseaux Familiaux. Antiquité tardive et Moyen Âge in memoriam A. Laiou et É. Patlagean, ed. B. Caseau (Collège de France - CNRS, Centre de recherche d'histoire et civilisation de Byzance. Monographies 37). Paris 2012, 77-92.
} 
on the other, they acted as rioters or individuals who displayed violent or extreme behavior. Hence, she made clear that "young men" constitute a separate social group with special characteristics and a corresponding social role, which differs from both that of children and of adults. "Young men" are presented as wavering between the maturity that characterizes adults by taking on social responsibility and the adolescent immaturity that drives them to acts of violence and disturbance of the social order. ${ }^{7}$

In her study of young and old people in late Byzantine Epirus, Tonia Kiousopou$\mathrm{lou}^{8}$ investigates the relevant decisions of the ecclesiastical law court of Naupaktos under Archbishop John Apokaukos. She arrives at the conclusion that the changes during adolescence were certainly marked by biological characteristics, but they were not related to specific social events. Cecily Hennessy ${ }^{9}$ provides a brief outline of different aspects of childhood and youth in Byzantine society. Another major development in the study of childhood and youth occurred in 2006 when Dumbarton Oaks organized a symposium on the topic of "Children and Childhood in Byzantium" ${ }^{10}$ the proceedings of which were published in 2009. The volume brought about an important broadening of the thematic scope and new methodological approaches. My own $\mathrm{PhD}$ thesis published in 2012 focuses on the various stages of a child's life from birth to a possible early death. Since in some respects its effects can be observed beyond this stage, adolescence is also included as a transitional phase from childhood to adulthood. ${ }^{11}$

All in all, there is still much to do in order to reach a more comprehensive and more systematic understanding of this subject area. In the context of my current project, I have applied the methodological principles outlined above to an investigation of the terminology and semantic fields of Byzantine legal, medical, and hagiographical texts. ${ }^{12}$ As in other areas of the Byzantine intellectual and mental world, one must pay attention to the parallel use of terms from antique written records and the adaptive and innovative tendencies of medieval linguistic usage as well as the resulting shifts in meaning.

The Byzantine terms of adolescence are formed to a great extent by Roman legal practices and Byzantine medical doctrines on human ages. Certain semantic similar-

7 Patlagean, Les “jeunes” dans les villes byzantines: émeutiers et miliciens, in: Le charivari, eds. Le Goff - J.Cl. Schmitt. Paris 1981, 123-129; ARIANTZI, Childhood 81-82.

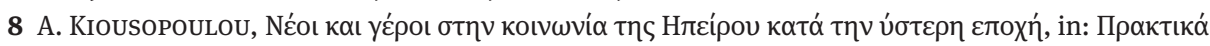

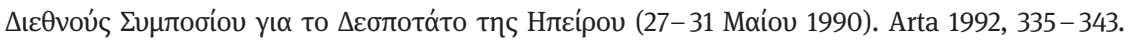

9 C. Hennessy, Young People in Byzantium, in: Companion to Byzantium, ed. L. James. Chichester 2010, 81-92.

10 Becoming Byzantine: Children and Childhood in Byzantium, ed. A. Papaconstantinou - A.-M. Talbot (Dumbarton Oaks Byzantine Symposia and Colloquia). Washington D.C. 2009.

11 D. ARIANTzI, Kindheit in Byzanz. Emotionale, geistige und materielle Entwicklung im familiären Umfeld vom 6. bis zum 11. Jahrhundert (Millennium Studien 36). Berlin - Boston 2012.

12 D. ARIANTZI, Terminologische und sozialhistorische Untersuchungen zur Adoleszenz in Byzanz (6.-11. Jahrhundert). Teil I. Theorien, Konzepte, narrative Quellen. JÖB 63 (2013) 1-31. 
ities with modern theories occur in the concepts of phases and sequences of adolescence: early, middle and late adolescence. ${ }^{13}$ As expected, the Byzantines were given the periodization models available to them by ancient Greek authors. The rich treasure of Greek composite nouns and adjectives drawing on temporal prepositions offer manifold possibilities for subtle differentiations in the description of these phases. A comparison of the available material leads us to the conclusion that there is a high degree of uniformity among the various literary genres regarding the use of prevailing terms and expressions. In most cases they maintain their classical meaning, but there are clear differences in the frequency of occurrences in the individual text categories. As regards the spectrum of variety, it can be observed that historiographical texts have a stronger proclivity to terminological reduction and reiteration than hagiographical texts as regards ages in general and adolescence in particular. This may have to do with the linguistic registers used by individual authors, but is mainly due to the fact that historians rarely refer to young people or their social and political role while in saints' lives the youth in a holy man's biography frequently appears as a decisive formative period for his religious zeal, his love for the monastic way of life, and his exemplary moral qualities as a future saint. ${ }^{14}$

Terms denoting various stages of adolescence are usually framed by nouns and adjectives referring to the qualities and behavioral patterns of young people. In this respect we always have to consider the binary oppositions on the basis of which the authors of hagiographical texts juxtapose future holy men and "normal" youngsters. These contrastive presentations yield a catalogue of positive and negative qualities of adolescents as well as a number of characteristics which were regarded typical for young people. The relevant semantic fields refer to physical qualities, the outward appearance, mental conditions, and forms of social interaction. Thus we discern groups of expressions defining norms in the thought world and perception of the authors. The written sources name in particular readiness and helpfulness or enthusiasm as positive characteristics, whereas immaturity, irresponsibility, unreliability, easy suggestibility, uncontrolled emotional reactions and love of pleasure rate among the markedly negative characteristics. An interesting aspect is the question to what extent establishments of vice and places of ill repute (theater, brothel, and tavern) ${ }^{15}$ are linked to persons of youthful age. Moreover, it becomes clear that external appearance (hairstyle, clothing) and patterns of social behavior serve to differentiate youths in general and to describe the resulting tensions with the older generation or the authorities. Thus we come to the intersection between the qualities of adolescents and their functions as social actors on various levels of urban societies.

Adolescence was normally marked in the texts by signs of psychical and psychosexual development. The beginning and the end of adolescence do not always corre-

13 ARIANTZI, Adoleszenz 9-14.

14 ARIANTZI, Adoleszenz 28-31.

15 See in this volume ARIANTZI, Soziale Identitätsbildung 128-134. 
spond to particular years of life or to specific terms. In order to make them clear, the authors either refer to biological characteristics, ${ }^{16}$ such as the appearance of facial hair for young boys (three phases), ${ }^{17}$ physical strength, and beauty, or dwell on different ways of a young person's leaving parental care, such as the parents', guardians', or relatives' decision to marry their child, or to send him to a monastery, to Constantinople, or another urban center for higher education or a professional career in the secular or religious spheres. ${ }^{18}$

Significant and often rapid physical changes are associated with psychological change and are reflected in social interactions. The end of adolescence is individually determined by social characteristics, such as by achieving a relatively autonomous life style. Criteria such as the completion of education or vocational training and the social and material independence from the parents or from the family environment appear to be crucial. Such criteria are differentiated in different cultures and historical epochs and lead to a variable average duration of adolescence. ${ }^{19}$

In Byzantium, as in other pre-modern cultures, ${ }^{20}$ the transition into adult life was associated with certain initiation rites (rites de passage), ${ }^{21}$ which were partly symbolic in character. Since Christianity shaped the formation of human life and society in Byzantium in a special way, religious rites had developed for baptism, naming, engagement, marriage and the entry into the adult age. ${ }^{22}$ On the latter, we find in the Euchologion prayers to gender-specific initiation rites such as the cutting of a

16 ARIANTZI, Adoleszenz 14-23.

17 ARIANTZI, Adoleszenz 14-15: The bearded growth ('וou $\lambda$ os) is above all a clear indication for the age determination of a young person. Three phases are reported that correspond to the three stages of

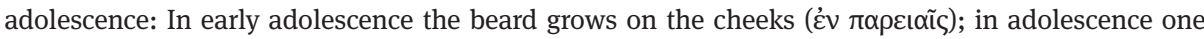

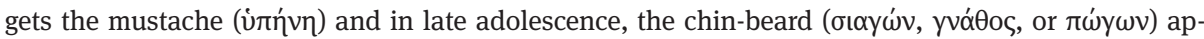
pears as evidence of complete adolescence and entry into the male age.

18 ARIANTZI, Kindheit 231-298.

19 Flammer - Alsaker, Entwicklungspsychologie der Adoleszenz 21. Cf. Hurrelmann, Jugendforschung 19; Mietzel, Entwicklungspsychologie Chapter 10, 351; ARIANTZI, Adoleszenz 8.

20 R. GARLAND, The Greek Way of Life from Conception to Old Age. London 1990, 185-187; KLEIJWEgt, Ancient Youth, 44, 90-101; Cf. G. Cole, The Social Function of Rituals of Maturation. ZPE 55 (1984) 233/244. E. EYBEN, Jugend, in: RAC 19, 388-442, hier 399; IDEM, Die Einteilung des menschlichen Lebens im römischen Altertum. Rheinisches Museum 116 (1973) 150 -190; IDEM, Restless Youth in Ancient Rome 113, 128-129, 142-143, 151, 182, IDEM, Jugend, 388-442, hier 409-410. Cf. A. B. SCHWARz, Die justinianische Reform des Pubertätsbeginns und die Beilegung juristischer Kontroversen. Zeitschrift der Savigny-Stiftung für Rechtsgeschichte: Romanistische Abteilung 69 (1952) 345-387. A. VAN GenneP, Les Rites de Passage. Paris 1909, 93-163.

21 S. Wilson, The Magical Universe: Everyday Ritual and Magic in Pre-Modern Europe. London 2000, especially chapter 7-10.

22 J. BAUn, Coming of Age in Byzantium: Agency and Authority in Rites of Passage from Infancy to Adulthood, in: Authority in Byzantium, ed. P. Armstrong (Publications of the Centre for Hellenic Studies, King’s College London 14). London 2013, 113-135. Cf. GenneP, Rites de Passage 93-163. 
young man's chin beard ${ }^{23}$ and the high-haired hairstyle or veil of the head of a young woman. ${ }^{24}$ They signal to the outside world the end of adolescence and the beginning of adult life or the new social life of a man or a woman.

The anatomical, physiological and hormonal changes also have a serious impact on psychological and social levels. ${ }^{25}$ The almost adult body and the new cognitive abilities usually prompted the young people to deal constructively with the important development task of detachment from the parents in order to prepare themselves for adult life. In most cases detachment is associated with the attainment of emotional independence from the parents, in which the orientation of attitudes and actions is no longer primarily directed to the parents, but to members of their own generation, as the psychoanalytic tradition postulates. Psychology speaks of two types of contacts with peers: belonging to peer groups and intimate emotional connections, namely friendship, sexual relationship, or marriage. In this way, the young person gains an increased degree of autonomy and self-responsibility, which allows his gradual integration into society, the so-called secondary socialization. ${ }^{26}$

Another field of investigation is the question of gender-specific differences between young men and women as far as these were perceived and described in narrative and normative sources. In this context one has also to take into account the social and economic status of given families mentioned in the sources. Striking changes of place, social environment, and career perspectives were restricted to young men of aristocratic families related to the imperial court and the political, ecclesiastical, and administrative elite of Constantinople. These are also the cases we mostly hear of when referring to typical Byzantine biographies. People of lower social strata appear almost exclusively in hagiographical contexts. Their life planning is characterized by a high degree of stability and immobility within the familial and social environment they belong to. Boys and girls continue to play their roles in domestic work, in agriculture, stock breeding or the parent's workshops until they were ready to found their own family through betrothal and marriage. These descriptions are widely typified and model-oriented, hardly leaving any room for individualities or particularities of specific urban spaces or social environments. ${ }^{27}$

The childhood of girls in aristocratic circles was not devoid of education and it prepared them for married life, except in the case of young nuns. The span of time

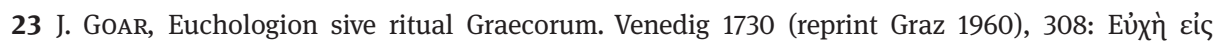

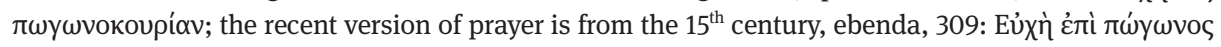

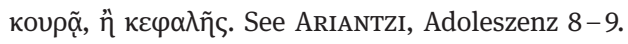

24 Anna Komnene speaks of a veil that covers the face: Alexias II 5, 8 (ed. D. REInsCH - A. KambyLis,

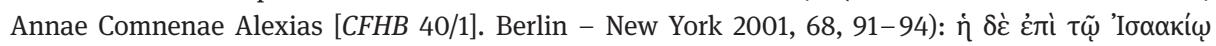

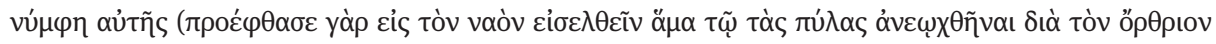

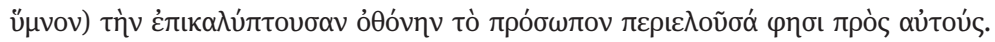

25 HuRRELManN, Jugendforschung 26.

26 ARIANTZI, Soziale Identitätsbildung 117-140.

27 ARIANTZI, Soziale Identitätsbildung 118-119. 
between betrothal and marriage was spent at home. Byzantine law retained the age limits for marriage that were in force under Roman law, twelve for girls and fourteen for boys, ${ }^{28}$ and the Church adopted the same limits. ${ }^{29}$ Betrothal was permitted from the seventh year of age for both sexes. ${ }^{30}$ Nevertheless, marriage remained a family matter, not an individual one. ${ }^{31}$ Early marriages were particularly common among the aristocracy. The practice of early marriage applies to both genders, but mainly to young girls, who had no career opportunities. Young girls entered adult life, by marrying or by becoming nuns. There was no transitional period between childhood and adulthood for young girls. They sometimes refused to marry and secretly left the parental house to enter a monastery: this is a very common topos in hagiography, not only for young girls but also for young men. ${ }^{32}$ Female adolescents had little opportunity for an off-home group formation. As a field of activity, it was almost exclusively the domestic sphere that was assigned to them, because, according to the norms of behavior in Byzantine society, girls were to be protected from men. ${ }^{33}$ Thus their only possibility to cultivate friendships was with neighbors or relatives. ${ }^{34}$ Their upbringing was not aimed at careers or public offices, which, in addition to a good education, would have required good social contacts, but to become good wives and mothers. ${ }^{35}$

For most boys of the urban elite and aristocracy, however, there existed yet another transitional stage of socialization and education before coming of age, a training that took place outside the paternal household. Boys had two paths toward coming of age, one via the desire for learning based on the Greco-Roman tradition and the other through feats of valor in battle and on the hunt. ${ }^{36}$ During adolescence, parents sent their boys, or they went of their own accord, to urban centers to continue their studies or to get training in the military arts (hunting, the use of weapons). ${ }^{37}$ The sources (hagiography and historiography) clearly show a social differentiation

28 Codex Justinianus (Corpus Iuris Civilis), ed. P. Krueger, vol. II, Berlin 1963, V, iv, 24, A. 530; L. Burgmann, Ecloga. Das Gesetzbuch Leons III. und Konstantinos V. (Forschungen zur Byzantinischen Rechtsgeschichte 10). Frankfurt am Main 1983, II, 1; Procheiros Nomos, in: Jus Graecoromanum II, ed. P. - et I. ZEPos. Athens 1931 (reprint Aalen 1962), 107-228, here IV, 3; Eisagoge, in: Jus Graecoromanum II, ed. P. et I. ZEPos. Athens 1931 (reprint Aalen 1962), 229-368, here XI, 2. Cf. PATLAGEAN, L'enfant 86.

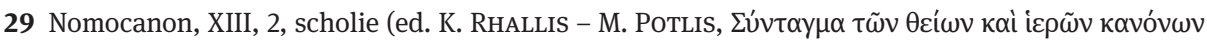

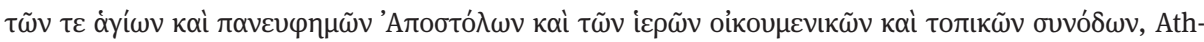
ens 1852-1859, vol. I, 288) Cf. PATlageAn, L'enfant 86.

30 Ecl., I, 1; Proch., I, 7; Eisag., XIV, 11; Jus Graecoromanum I: Imperatorum Leges Novellae, ed. P.-et I. ZEPos, Athens 1931(reprint Aalen 1962), 305-309, here Nov. 24.

31 Cf. Patlagean, L'enfant 90.

32 Numerous cases by ARIANTZI, Kindheit 253-271; See also in this volume the article of P. MELICHAR, Some Observations on Young Byzantine Women 115-125.

33 ARIANTZI, Kindheit 165-167; EAdEM, Adoleszenz 18.

34 ARIANTZI, Soziale Identitätsbildung 121.

35 ARIANTZI, Kindheit 139.

36 ARIANTZI, Soziale Identitätsbildung 123-128.

37 ARIANTZI, Kindheit 231-245. 
in various sports activities. Hunting and riding were part of the training of youths of the social upper class of urban and rural origin, while running, jumping, wrestling, and fighting were evidently accessible to young people of all social strata, because this did not require expensive equipment, such as horses and weapons. Texts from the aristocratic milieu of the Comnenian dynasty and hagiographical texts of earlier centuries clearly indicate a common horizon of experience in which physical exercise and attainment of physical abilities are understood as an important instrument and as a framework for the socialization processes of adolescents. The nature of the activity was determined by economic conditions and the associated value propositions in a layer-specific manner and accordingly had a high signal effect both externally to other groups as well as internally in the sense of consolidating collective social identities and internal coherence. Certain forms of hunting and exercises in the field of warfare emphasized, above all, military ideals, which were accepted as norms and identification features of young aristocrats. On the other hand, there were less complex physical activities for youthful groups from lower social strata, which fulfilled similar functions of self-assurance. ${ }^{38}$

Another important issue in studying Byzantine adolescence is the procedure of leaving parental care. Basically we can single out three substantial motives for leaving parental or family care: The first one was related to obtaining general or professional education. Both higher education and professional careers in secular or religious spheres were restricted to Constantinople or other urban centres. Children and adolescents were sent to uncles or relatives with prominent positions, who would be able to facilitate the children's career advancement. ${ }^{39}$ The reasons that led children and young boys to choose a specific profession are diverse. They had to do, for instance, with the social position of the family, the expectations of senior family members, the continuation of a family tradition, the improvement of the family's social and economic situation through the child's career, and the child's own desires. In some cases children rejected the career that their parents or relatives had chosen for them and followed their own paths (Symeon the New Theologian, ${ }^{40}$ Athanasios in the life of Niketas of Medikion). ${ }^{41}$ To understand why a particular secular (military or bureaucracy) or ecclesiastical profession was selected, one has to take into consideration various factors, such as financial security and the influence of precedents. It is noteworthy that some fathers belonging to the lower social strata sought their son's professional advancement, because they coveted the economic benefits and so-

38 ARIANTZI, Soziale Identitätsbildung 125-128.

39 ARIANTZI, Kindheit 240-243.

40 Life of Symeon the New Theologian (BGH 1692) 10 (ed. I. HAUSHERR - G. HorN, Vie de Syméon le Nouveau Théologien [949-1022] par Nicétas Stéphanos [Orientalia Christiana 12]. Roma 1928, 18); Cf. ARIANTZI, Kindheit 240-241.

41 Life of Niketas of Medikion (BGH 1341-1342a) 11 (AASS Apr. I [3d Ed.], Appendix, XVIII-XXVII, here XXIB-C). Cf. ARIANTZI, Kindheit 243-244. 
cial recognition that this would entail not only for the child but also for themselves and their entire family. ${ }^{42}$

The second reason for leaving the nest was due to engagement or marriage. The father or parents made the choice of future partners according to a broad range of criteria, such as physical beauty, virtuous lifestyle, piety, or the future partner's social status, wealth, and descent. ${ }^{43}$ The examples of hagiographical texts illustrate some of the decision-making processes concerning engagement and marriages with households and family units. As is to be expected, the predominant person was the father in his capacity as the head of the household. After his death, his place was taken by the mother, provided she did not marry again, and thereafter by the grandparents or other relatives or guardians. Apart from current clichés concerning the bride's physical and mental virtues, the examples reflect the parents' strategy of establishing relationships through engagement and marriages with other families enjoying a similar social and material status. The texts point to the parents' attempts to enhance their position and to strengthen their influence within the social group they belonged to, while marriages to men or women of inferior social standing would have caused severe criticism, as is shown in the Life of Neilos the Younger $^{44}$ and the miracle stories of Paul of Monembasia..$^{45}$ In case a family was not able to provide a girl with a dowry because of financial constraints, people were forced to choose a husband from lower social strata. ${ }^{46}$ In this way, hagiographical texts provide intriguing insights into mechanisms of hierarchical thinking and social consciousness determining mentalities and courses of action in the process of establishing inter-familiar ties of marriage. Another recurring topic in saints' lives is the opposition between conformity with the norms and expectations posed by a person's familiar environment vs. the rejection of those norms in favour of an alternative way of life as provided by monastic ideals and the concept of living in the service of God. In this framework children and youths complied with their parents' decision to marry, contracting a union out of respect and obedience, yet later they abandoned their families and entered a monastery. ${ }^{47}$

42 ARIANTZI, Kindheit 241-242; 243-245 (as in the case of Athanasios, in the life of Niketas of Medikion and Constantine, son of the Metrios); 249-250; EADEM, Childhood 88.

43 ARIANTZI, Kindheit 276-279; EADEM, Childhood 88.

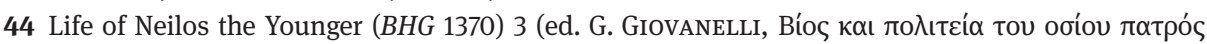

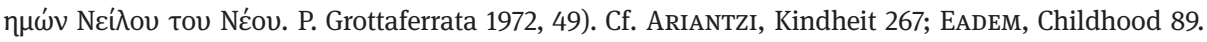

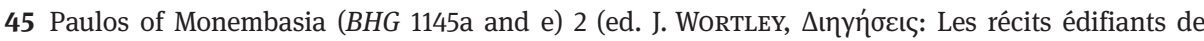
Paul, évêque de Monembasie et d'autres auteurs [Sources d'histoire médiévale]. Paris 1987, 98). Cf. ARIANTZI, Kindheit 268; EADEM, Childhood 89.

46 ARIANTZI, Kindheit 269-270 (as in the case of one father in the life of Nicholas of Myra, Encomium Methodii [BGH 1352z], ed. G. ANRICH. Hagios Nikolaos. Der Heilige Nikolaos in der griechischen Kirche. Texte und Untersuchungen. Vol. 1. Leipzig - Berlin 1913, 153-182, here 158).

47 ARIANTZI, Kindheit 263-264, 297 (Life of Euthymios the Younger, Life of Neilos the Younger). 
Entering a monastery ${ }^{48}$ is, according to the available source material, the third main motive for leaving the nest in Byzantine family life. The diachronic development of regulations concerning the age of admission to monasteries can be examined on the basis of legal sources: the 18th Canon of Basil the Great, the 6th novel of Emperor Leo VI, monastic foundation documents, and other monastic rules. From a typological viewpoint, the following factors were taken into consideration for the decision in question: the desire of the parents ${ }^{49}$ or of the children themselves ${ }^{50}$ (and the clash that the decision to enter a monastery provoked between the two sides (as in the cases of Symeon the New Theologian and of Athanasios in the life of Niketas of Medikion, who abandoned their careers to follow the monastic vocation and came into conflict with their fathers); ${ }^{51}$ the influence of relatives, such as brothers and uncles, who were already monks and served as models for the children (Euthymios, Theodore Stoudites, Peter of Atroa, Peter of Argos, etc.); ${ }^{52}$ and the particular circumstances of life, for example being an orphan in the case of Paul of Latros, ${ }^{53}$ the widowhood of the mother, as in the case of Theodosia of Constantinople and Symeon, brother of David of Mytilene, ${ }^{54}$ and the absence of parental care, as in the case of Sabas. ${ }^{55}$ Vows taken by the parents ${ }^{56}$ (as in the case of Theodora of Kaisaris and Theodora of Thessaloniki, who gave up her six-year-old daughter) and financial problems, such as the inability or unwillingness of parents to provide a dowry, were considerations of particular importance. ${ }^{57}$ Due to the abundance of hagiographical material, we can expect an over-emphasis in written sources on the model of a pious child's or youth's choice of the monastic life, and thus the descriptions do not necessarily match social reality. The children's alleged wish to enter a monastery can be explained by the fact that the hagiographical genre inevitably depicts monasticism as an ideal form of life. Just as in the case of any career, family connections and social networks played a role in the decision to enter a monastery, in general,

48 Cf. R. GREEnfIELD, children in Byzantine Monasteries: Innocent Hearts or Vessels in the Harbor of the Devil?, in: Becoming Byzantine: Children and Childhood in Byzantium, eds. A. Papaconstantinou - A.-M. Talbot. Washington, D.C. 2009, 253-282; See also in this volume the article of Alice-Mary Talbot, The Adolescent Monastic in Middle and Late Byzantium.

49 ARIANTZI, Kindheit 282-284: as in the case of Stephan the Younger, Nicholas the Studite, Andrew of Crete, Phantinos the Younger, etc.; EADEM, Childhood 80.

50 ARIANTZI, Kindheit 287-297: as in the case Theodore of Sykeon, Alypios Stylites, Anthony Kauleas, etc.; EADEM, Childhood 80.

51 ARIANTZI, Kindheit 149-150, 194, 226, 232-233 (for Symeon the New Theologian) und 131-132, 160, 235-236 (for Athanasios in the life of Nicetas of Medikion).

52 ARIAnTzI, Kindheit 279 (Kyriakos); 291 (Theodore Stoudite); 281 (Peter of Atroa); 290 (Peter of Argos).

53 ARIANTZI, Kindheit 286-287. EADEM, Childhood 90.

54 ARIANTZI, Kindheit 284-285. EADEM, Childhood 90.

55 ARIANTZI, Kindheit 286. EADEM, Childhood 90.

56 ARIANTZI, Kindheit 271-272 (Theodora of Kaisaris); 272-273 (Theodora of Thessalonike); EADEM, Childhood 90.

57 ARIANTZI, Kindheit 270-271. 
and in the choice of a specific monastery. Brothers ${ }^{58}$ and uncles, ${ }^{59}$ who were monks or held high positions in a monastic hierarchy, introduced their brothers and nephews to the monastic lifestyle and could help them make a carrier within the monastery. Hagiography offers a number of examples for such a course of action. ${ }^{60}$ It was common for family members to seek company in the same monastery, as in the case of Peter of Argos or John Psichaites, being also united by another kind of kinship, the spiritual one. ${ }^{61}$

To sum up, modern social and psychological theories, a broad spectrum of terms and semantic fields gleaned from the primary sources, as well as a set of collective perceptions, behavioral patterns, and external appearances resulting from the terminological analysis constitute the main axes of investigation which the Coming of Age project is following. In this way it contributes, on the one hand, to a better understanding of adolescence within the broader context of Byzantine society and its particularities and, on the other, to a more accurate definition of adolescence in various genres of Byzantine literature. The anonymous mass of young people and the fictitious characters of holy men belong to common social reference systems, which have to be expounded by putting literary descriptions and perceivable social realities in relation to each other.

This volume aims to examine Byzantine adolescence from an interdisciplinary viewpoint with the collaboration of Byzantinists, medievalists, art historians, and psychologists. The contributions gathered therein treat seven subtopics that correspond to crucial questions in the current research on adolescence: (1) the legal status of adolescents; (2) the reasons for leaving parental care as a mechanism of transition from childhood to adolescence; (3) the socialisation and gradual integration into adult society; (4) adolescents in Byzantine art; (5) psychological aspects of adolescence from medieval to modern times; (6) illnesses of adolescents; (7) adolescents in the western medieval world. There is no strict chronological frame so that the discussion extends over different periods between the fourth and the fifteenth century.

Béatrice Caseau concerns herself with the threshold between childhood and adulthood, which can be defined as the time when a person becomes fully accountable for his or her actions. At what age were young people considered mature enough to make up their own mind on life-altering decisions? Caseau approaches this question by focusing on decision-making in the area of property management, the monastic profession, and marriage. Byzantines had the notion that fifteen years of age marked a threshold. Decisions taken by the young were considered more solid

58 On the role of brother cf. ARIANTZI, Kindheit 206-215; EADEM, Childhood 91.

59 On the role of uncle cf. ARIantzi, Kindheit 219-226; EADEm, Childhood 91.

60 ARIANTZI, Kindheit 221 (the uncle of Ignatios of Bathyrrhyax, Basileios), 221, 291 (Platon of Sakkoudion and his nephew Theodore the Stoudite).

61 ARIANTZI, Kindheit 207, 290, 295 (Peter of Argos); 289-290 (John Psichaites); EADEM, Childhood 91. 
and informed after than before this age. The period between ten and fifteen was thus perhaps an equivalent of what we call teenage years, when the young are past early childhood but not yet completely trustworthy to take important decisions, even if it was not formally recognized as a particular period of life the way it is now. Parents could consecrate one of their children to a virginal life or marry him or her off while the child had no say in this matter. A girl could consent to a wedding at twelve but she was not to be asked to consent to a monastic life at that young age. Girls were not required to possess intellectual or emotional maturity in order to get married. The decision was taken for them, but we can say that girls who were married at a very early age were in fact deprived of their adolescence. They had to cross from childhood to adulthood without transition. These early weddings were particularly numerous among the aristocracy or members of the imperial house. Boys, on the other hand, had more time to adjust to the changes linked to puberty, and, for a few years, they could expect some leniency towards misbehavior.

Günter Prinzing's study is based on a total of 49 cases collected from files relating to adolescents of both sexes. The material is extracted from three source groups comprising documents related to the administration of justice by ecclesiastical authorities in Epiros (first half of the thirteenth century), on the one hand, and in Constantinople (fourteenth/fifteenth century), on the other hand. The Epirotic collections contain documents issued by Bishop John Apokaukos of Naupaktos between 1200 1232 and documents issued by Archbishop Demetrios Chomatenos of Achrida/Ohrid between 1216-1236. The Constantinopolitan corpus consists of documents issued by the patriarchs in the years 1315-1402, which came down to us in two surviving manuscripts of the register of the Patriarchate of Constantinople (PRK) preserved in the Austrian National Library at Vienna. As it is difficult to make a direct comparison between the three source groups, the value of this study mainly lies in a careful examination of the thematic focus of the relevant cases. It turns out that in court cases adolescents were primarily confronted with problems related to inheritance and property law and to a lesser degree with problems related to matrimonial and family law. It should be emphasized that orphans and half-orphans were in an especially precarious position. They were frequently facing attempts on the part of their stepparents or relatives to prevent them from taking possession of the landed property that legally belonged to them. Some conflicts, in which adolescents were involved, however, arose in connection with the career or discipline of clerics. Only from the region of Epiros, finally, we know of a few cases, in which adolescents fell victim to homicide.

Analyzing the procedures of leaving the family allows us insights into the expectations of parents and their social environment regarding the future of the new generation, especially with respect to changing attitudes resulting from broader politi- 
cal, social, and economic developments. ${ }^{62}$ Of special interest in this context are gender-specific expectations. Leaving parental care in order to enter a monastery was one of three substantial possibilities for young people. Alice-Mary Talbot offers an overview of monastic adolescents in middle and late Byzantium. Because of the relative lack of evidence for youthful nuns, she focuses, although not exclusively, on male adolescents. There were significant variations in the experience of young monks, depending on the monastery where they lived. A limited number of monastic foundation documents, saints' lives, and Athonite acts might give the initial impression that monastic adolescents were forbidden in Byzantium. The rules are clear for a number of major monasteries and holy mountains, "no beardless youth under the age of 20 or 26 was granted access". In reality the situation was much more complicated. Although there were misgivings about the admission of youthful postulants, each monastery had its own rules on the subject and a number of monastic typika permitted teenagers to become novices. Hagiographical texts also provide examples of teenagers entering monasteries. Talbot examines various aspects of this phenomenon, such as opposition to monastic adolescents, variations in age of monastic tonsure, monastic life of novices and youthful monks, education of novices and young monks, servile duties of young monks and novices, and abusive treatment. The author concludes that the novitiate was often an arduous experience for prospective monastics, a training period designed to weed out those who were physically or mentally unable to endure the harsh challenges of cenobitic life, and to prepare those who were well suited to a monastic vocation for a life of spiritually satisfying prayer and service to the community.

Tonia Kiousopoulou examines the notion of adolescence in late Byzantine society. The study of saints' lives in comparison with more secular texts, such as the invective of John Argyropoulos against Katablattas or Mazaris' Journey to Hades, shows that adolescence was marked by biological characteristics and carnal exercise in both rural areas and cities. In contrast to earlier periods, late Byzantine texts give only few details about the saint as a youth. Apparently, biographers wished to construct a saintly person who was familiar to his readership. Therefore, the saint, before devoting himself to asceticism, had to be an almost normal person by the standards of his time. While the saint of the middle period often was exposed to carnal desires, which in any case defined him as an adolescent and youth, the saint of the Palaiologan period struggled with the social dictates of marriage, which he often rejected by running away from the family home. Leaving parental care was a momentous passage to a new phase of live. In the late Byzantine period, however, it did not have the characteristics of a rupture. In rural societies, a rupture with one's family was neither possible nor desirable on account of the need to preserve the relationships of production in the countryside. The difference between the countryside and the cities lies in

62 See more about these three substantial possibilities for leaving parental care or family group ARIANTZI, Childhood 87-91. 
the fact that in a city like Thessaloniki the passage to youth via adolescence was much more marked, primarily through education in letters or the military arts. Urban life permitted or enforced rites of passage and socialization.

Petra Melichar deals with seven stories of Byzantine adolescent women and their behavior and interprets them in accordance with the general findings of modern psychology. Observing the actions of the young girls gathered from chronicles, hagiographic texts, letters and a eulogy, the author inquires into the kinds of wishes these women had, the methods and behavioral patterns they employed in order to achieve them, the difficulties they faced, and the role of their families in their eventual success. This study presents different social and familial expectations regarding young girls and the conflicts that arose if adolescent women made their own decisions against their parents' will. Unlike young men, whom the sources typically depict as running after court offices or eager to prove their military skills, adolescent women focused primarily on relationships of various kinds. Young Byzantine women had the possibility to choose between marriage and becoming a nun, as is especially highlighted by hagiographic texts. When the time came for the parents to give the daughter in marriage, she refused, wishing instead to maintain her virginity and purity so as to become a bride of God. In this case some girls secretly left their family und entered a monastery. Hagiographical sources also provide evidence for romantic relationships and the dramatic passion of adolescent love by depicting the love for a man as a work of the devil. Melichar concludes that being aware of a person's age allows the reader to understand him or her better and may also lead to new interpretations of the sources.

Despoina Ariantzi treats ways of gradual integration into society and social identity formation of adolescents. Her study focuses exclusively on male adolescents, because female juveniles had little opportunity for contacts with the public sphere. As field of activity, they were almost exclusively assigned the domestic sphere because, according to the norms of behavior in Byzantine society, girls were to be protected from men. A crucial aspect of the integration of adolescents into society was the contact with other peers; in other words, the belonging to a particular group of people of the same age and socio-economical background outside the family. It should be stressed that such peers could form a separate social group with specific physiological and intellectual characteristics that gave them a socio-political function. Hagiographic sources reveal different forms of group identity and feelings of belonging to specific, status-related systems of values. Common sport and leisure activities and the commitment to group-specific norms make it possible to discern peer groups that show an articulated level of independence with respect to society as a whole. The holy man remains largely isolated and often appears as their moral counterpart so that they were often described as being at odds with common Christian and monastic ideals.

Two contributions concern themselves with the visual representations of adolescents in Byzantine art. Leslie Brubaker compares Byzantine written and visual images of youth, examining the significance of the similarities and differences between 
how adolescence was communicated in words and in images, and how and why male and female were differentiated in both. The author shows us that the Byzantine painters could and did clearly distinguish between various stages of the life course trajectory, sometimes quite intentionally, but they did not always do so. The presence or absence of a beard is a key distinguishing mark between adolescent and adult males in Byzantine imagery, but it is also a sign of status. Similarly, the presence or absence of head covering can distinguish adolescent and adult females in Byzantine representations, but head covering too is a sign of status. The youths in their early teens are distinguished from both younger children and adults by their height (with children shorter and adults taller than the youths portrayed) and their slight builds. As visualized in middle and late Byzantium, growing up, for women, was not a gradual process, but an instant transformation effected by marriage. Indeed, after Iconoclasm, young (pre-married) females look remarkably the same. The only distinction between young women, unmarried or old women is the color of their hair.

Cecily Hennessy explores how adolescents are shown in imagery derived from apocryphal writings. To set this in context, she discusses approaches to interpreting adolescence and looks at some of the complexities of defining adolescence, at a brief survey of legal issues, and at areas of responsibility; then she turns to pictorial representation and reviews how adolescence is depicted in contexts other than apocryphal ones, both biblical and historical. Finally, she focuses on a selection of imagery related to the Virgin, Christ, and their family from apocryphal stories not included in the canonical texts. The author concludes that representations of youth, of girls and boys in the period between childhood and adulthood, show subtle gradations between boy and man, girl and woman, and appear to have been both customary and appreciated. In the imagery of Christ's family, his brothers and his mother, various portrayals also emphasize childhood and adolescence. In apocryphal iconography, the origin of the imagery is still not clear.

As regards the psychological aspect of adolescence, Catia Galatariotou seeks to answer the question of whether there was a Byzantine conceptualization of adolescence as a separate and distinct phase of the life cycle. Galatariotou presents insights, reports, and theories on adolescence from the fields of cultural anthropology and especially of psychoanalysis. Her main question is whether any of it is of relevance to our search for the Byzantine adolescent. Byzantine sources themselves seem to neglect adolescence. Galatariotou believes that we do encounter in our sources traces of an unmistakably adolescent psychic world, in the twelfth century at least. The locus of this psycho-cultural registration is the fictional literature of twelfth-century Constantinople and the reduction of epic poetry that preceded it (the novel of Digenes Akrites und the love story of Drosilla and Charikles). The surprise here is how astonishingly closely the Byzantine twelfth-century (psychic) description of adolescence agrees with the psychoanalytic description of western twentieth-century adolescence. Byzantine societies do not seem to have acknowledged the transition from "tender child" to adolescent through explicit puberty rituals or initiation rites of the type anthropologists describe. However, the novels contain ref- 
erences to markers or entry-points from boyhood to manhood, like riding and especially hunting skills, with emphasis on socialization, friendship, and induction in a peer group.

With the consideration to follow the development of the age-stage adolescence from Byzantium to the modern age, in order to recognize similarities or continuities and innovations, this volume included the contribution by Ulrike Sirsch, who refers to a "specific feature" regarding the expansion of youth, a new development phase called "Emerging Adulthood". This is an independent development phase for young people between 18 and 25 or 29 years, which can be distinguished from adolescence and the adult age. In recent decades, social changes have increasingly caused young people to postpone social transitions, which are defined in the sociological literature as the beginning of the adult age, such as the completion of school, the parental home, the beginning of professional activities - the productive area of culture and society - and marriage and birth of the first child - as transitions into the reproductive sphere. Opinions about the future meaning of the phenomenon are diverging. Some specialists believe that "emerging adulthood" will expand even further as a result of globalization. Critics see "emerging adulthood", however, limited to more educated young people in western industrialized societies.

Thomas Pratsch examines illnesses and healing of adolescents through incubation. The Christian and Byzantine tradition continues the ancient rite of incubation in a temple after asking the pagan god or goddess for cure from disease or illness with only a few modifications. The place of incubation is now usually a church or another place of worship and the person who is asked for a cure is now a Christian holy man or woman. In the literary genre of saints' lives, we find many a description of such incubations and in most cases the following healings. This literary tradition starts in the fourth to fifth centuries and goes on to at least the eleventh century. According to this tradition there was a wide range of diseases that could be cured by incubation in a church and invocation of a holy man or woman. Sometimes this therapy may have been successful, in other cases its effects were questionable from a scientific point of view. In any way these spiritual healing methods stood in competition with the medical doctors and worldly physicians. Apart from religious belief, the quarrel was also about money, as health care was a financial problem already in the Middle Ages. Pratsch comes to the conclusion that youngsters and young adults in Byzantium basically had the choice between the healing methods of traditional medical doctors and of the clergy. There are a lot of examples showing that they could use both healing methods successively or in turns.

Hans-Werner Goetz provides a careful terminological analysis of early medieval western sources. By examining the medieval notion of adolescentia, he focuses on aspects of frequency, age and years of life, characteristic features, behavior, as well as duties and activities. His survey confirms and further defines the theory of ages and its symbolic interpretations, though in the age between 12 and 20 (instead of 14 and 28). Physically, it is the age of growing strength and beginning fertility, but adolescence is mainly perceived as a difficult, unstable, but nevertheless decisive pe- 
riod of transition, in which the person is still 'unmoulded' and can be easily enticed. The result of puberty is shame as well as lust. The adolescent can be cheeky, but his faults are still forgivable: he needs supervision as well as leniency. Adolescence is the age not only of marriage and copulation but also of moral and vocational formation, preparing a character's development towards good or - frequently - towards evil. At the same time, duties and activities grow through education and, among lay people, through training in hunting and warfare. The (noble) adolescent takes up services at court or chooses an ecclesiastical career, and often assumes functions as count, margrave, duke, king, or emperor, thus being fully integrated into social and political life. However, the perception of adolescents remains ambiguous: the adolescent sways between depraved behavior and a virtuous sense of responsibility. The western notion of adolescence evinces a number of similarities with Byzantium, where the terminology is much more differentiated and the legal definition of agestages is more precise. In both East and West, there are tensions between the volatility of age and the demands on the adolescents so that they were admonished accordingly. 
Béatrice Caseau

\section{Too Young to Be Accountable: Is 15 Years Old a Threshold in Byzantium?}

In our contemporary societies, we tend to define the teenage years as a period of transition between childhood and adulthood, marked by physical transformation and the need to adjust to adult responsibilities. We also tend to see this period as a time of crisis, intergenerational conflicts and sometimes rowdiness. ${ }^{1}$ Was there a transitional period for young Byzantines as well? Some historians such as Mark Kleijwegt deny the existence of adolescence, as we understand it nowadays, in the ancient world. ${ }^{2}$ In texts that divide the life stages, from the Roman, late antique and mediaeval period, the concept certainly exists. Ambrose, Augustine, and Isidore of Seville all mention adulescentia as a period of life starting after childhood (pueritia), after the end of the 14th year. ${ }^{3}$ In Byzantine sources, even if the vocabulary is rather

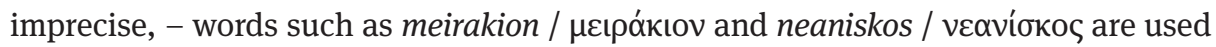
for children from 6 to 14 and for teenagers from 14 to 18 or 20, as Günter Prinzing noted in an article published in 2009. ${ }^{4}$ Since that article, Despoina Ariantzi has listed all the words used to talk about teenagers in Saints Lives and she concludes that the Byzantines may have distinguished two phases in the teenage years, the first one being centered on the appearance of a beard in young boys. ${ }^{5}$ Clearly, the notion of a period of life starting with puberty existed, even if there was no clear agreement on when that period ended. ${ }^{6}$ In Roman law, puberty was legally defined at 12 years for girls and 14 years for boys. The legal majority was fixed at 25 by Roman

1 M. Breviglieri - V. Cicchelli, Adolescences méditerranéennes: l'espace public à petits pas. Paris 2007; V. CicchelLI - C. MARTin, Young Adults in France: Becoming Adult in the Context of Increased Autonomy and Dependency, in: Youth and Family: Intergenerational Tensions and Transfers. Journal of Comparative Family Studies 35-4 (2004) 615-626.

2 M. KLEIJwegt, Ancient Youth: the Ambiguity of Youth and the Absence of Adolescence in GraecoRoman Society. Amsterdam 1991, XII: "we shall likewise come to understand that adolescence was defined in a different way in preindustrial society.”..."adolescence, as we know it in modern society, was not a universal phenomenon in the past."

3 J.-P. NERAUdAU, La jeunesse dans la littérature et les institutions de la Rome républicaine. Paris 1979; E. Eyben, Restless Youth in Ancient Rome. London 1993.

4 G. Prinzing, Legal Status of children and the Stages of Childhood in Byzantium, in: Becoming Byzantine. Children and Childhood in Byzantium, eds. A. Papaconstantinou - A.-M. Talbot. Washington, D.C. 2009, 15-34, here 19.

5 D. ARIANTzI, Terminologische und sozialhistorische Untersuchungen zur Adoleszenz in Byzanz (6.-11. Jahrhundert). Teil I. Theorien, Konzepte, narrative Quellen. JÖB 63 (2013) 1-31.

6 C. Jounnno, "Le Roman d'Alexandre ou l'enfance d'un héros", Enfants et enfances dans les mythologies. Paris 1995, 269: l'enfance est loin d'être délimitée de façon claire et précise dans le monde grec antique et médiéval. [..] En fonction de la périodisation adoptée, l'âge enfantin dure jusqu'à quatorze, quinze, dix-huit ans...”

Ә OpenAccess. (c) 2018 Béatrice Caseau, published by De Gruyter. (c))BY-NC-ND This work is licensed under the Creative Commons Attribution-NonCommercial-NoDerivatives 4.0 License. 
law and adopted by Byzantine law as well. Can we say that the period in between was an equivalent to what we call the teenage years? If we can agree about the mentioning of this stage of life in medical literature and in legal texts, there is a debate, however, among social historians about how the transition from childhood to adulthood was managed in Byzantine youth. Was it the same for boys and girls? Was it the same for all social classes? Was this long period of eleven to thirteen years in fact shortened by different methods?

By distrusting the young, and imposing a majority rather late in life, the Romans had created, at least for boys, a period of life where the young were not considered as adults and responsible citizens. In childhood studies, the establishment of married life, paid work and legal responsibilities are often considered to be signs of adulthood. These are criteria that we can adopt for contemporary societies, but do they work for the Byzantine world?

One way to make progress in understanding how the Byzantines viewed the transition between childhood and adulthood is to study decision-making. At what age were young people considered mature enough to make up their own mind on life-altering decisions? Byzantine society raised the question of the accountability of young people and this paper discusses the ages considered to be a threshold between childhood and adulthood, seen as a time when a person becomes fully accountable for his or her actions. In this paper, the focus will be on decision-making in the area of property management, the monastic profession and marriage. The sources on such a topic are mainly legal, such as canon or civil law. Examples taken from historical narratives and saints' Lives can complete and nuance the image.

\section{Threshold for an adult decision}

\section{From 25 to 20 or 18}

Majority was reached at the age of 25 . Roman law had decided to protect young people from their inexperience and lack of maturity. ${ }^{7}$ Constantine reinforced this protection and allowed young people older than 25 to sue their tutors for mismanagement. ${ }^{8}$ Yet, the same emperor decided that majority could also be reached before the age of 25, if one petitioned the emperor. This decision has been preserved in the Theodosian code (2.17):

If any youths possessed of honorable character should wish to govern more advantageously by their own management their paternal savings or their ancestral patrimonies, whether urban or rural, and should begin to need imperial aid, thus finally they may venture to impetrate this benefit of legal age, when the age of completed youth, the twentieth year, has begun to open for them the door for the entrance upon most stable young manhood, provided that after the ben-

7 J.-P. NERAUdAu, Etre enfant à Rome. Paris 1984.

8 Codex Theodosianus 2.16, transl. Cl. PHARR. 
efit of legal age has been impetrated, the aforesaid youths who make a formal allegation of this special grant of imperial favour shall not only themselves prove that they have attained the number of years prescribed by law, but they shall also call as witnesses men endowed with the rank of honourable or an equal or greater dignity, who shall testify to their moral conduct and the integrity of their character by the evidence of an honourable life. [...] We sanction also that since the age of women outstrips that of men by two years because of an earlier puberty, the interval of time shall be observed also in this case, and they can obtain the rights of legal age when they have passed their eighteenth year, provided that they are recommended by honourable character, intelligence of mind, and an established good reputation. [...] On the aforesaid age, since it is midway between fully complete youth and most robust young manhood. We impose the designation of the age of stability (firmata aetas). Therefore the first age is childhood, the following youth, this age of stability is the third, the fourth is legal age, and the fifth, old age." The emperor cautions these young persons "who have obtained this special grant of imperial favour" to "continue to be exceptionally careful in selling their landed estates.

The age of 18 for a young lady and 20 for a young man are therefore the thresholds marking the entrance into an age of stability, which means the ability to take responsible adult decisions. This imperial favour is not meant for everyone, and distrust of youthful folly is still present in the background of the text. Constantine requires witnesses of good conduct for young men and good reputation for young women.

\section{From $18 / 20$ to 17}

Two centuries later, Justinian granted the possibility of writing a will to those of an age inferior to that decreed in Roman law. A novel written by Justinian decides to let young masters write a will and dispose of their properties, including the manumission of their slaves, and alludes to the former prohibition of this in Roman times. The Lex Aelia Sentia in $4 \mathrm{AD}$ had limited the performance of manumission to masters younger than the age of 20. Justinian's code also includes this age restriction, which also invalidates the freedom granted by a testator younger than 20 . The Institutes, however, decides that since a person can write a will, even if they have not reached the age of 17 , as long as they have reached puberty, it is acceptable for them to free their slaves. ${ }^{9}$ Here is the text of the Institutes:

Thus the lex Aelia Sentia having prescribed a certain mode of manumission for owners under twenty, it followed that though a person fourteen years of age could make a will, and therein institute an heir and leave legacies, yet he could not confer liberty on a slave until he had completed his twentieth year. But it seemed an intolerable hardship that a man who had the power of disposing freely of all his property by will should not be allowed to give his freedom to a single slave: wherefore we allow him to deal in his last will as he pleases with his slaves as with the rest of his property, and even to give them their liberty if he will. But liberty being a boon beyond price, for which very reason the power of manumission was denied by the older law to owners under twenty years of age, we have as it were selected a middle course, and permitted persons under twenty years of age to manumit their slaves by will, but not until they have

9 Institutiones I 6, 7, transl. J. B. MoYLE. Oxford 1911. http://amesfoundation.law.harvard.edu/digital/ CJCiv/JInst.pdf; I wish to thank A. Schminck for discussing the point with me. 
completed their seventeenth and entered on their eighteenth year. For when ancient custom allowed persons of this age to plead on behalf of others, why should not their judgment be deemed sound enough to enable them to use discretion in giving freedom to their own slaves?

Two points are interesting here: first, the age to write a will is 14 , but although puberty has been reached, the period between 14 to 17 is not recognized as a time to completely trust a young person with managing properties. From this text, we understand that at 18 one is considered old enough to be sensible in the economic sphere.

Thus 17 is yet another threshold of adult responsible behaviour, an age after which a young man is able to take the right decisions. The age of 17 is also mentioned in Roman sources for new soldiers. For Thomas Wiedemann, in the Roman world "the crucial division between child and adult was at 17: the age at which a male could learn to fight. The ceremony of putting on the adult toga at about 16 was the formal preliminary to full citizenship. It was followed by a year's preparation for service, tirocinium". ${ }^{10}$

\section{From 17 to 15}

Two centuries later, in 741, an interesting text, included in the Ecloga, considers that orphans who were influenced by others and imprudently got themselves engaged, should be able to break up their engagement without penalty, unless they have reached their fifteenth birthday. ${ }^{11}$ This shows that the Byzantines had some notion that before 15, young people are not completely adults and that sexual maturity (granted at 12 and 14) is not a threshold for the ability to take wise decisions.

These three examples reveal that between the early Roman Empire and the $8^{\text {th }}$ century, the threshold for adult behavior and responsible decisions had been lowered by ten years.

The age of 15 appears in a number of Byzantine texts, where more than sexual maturity matters. For young boys, 15 years seems to be a threshold. Günter Prinzing cites a text, which defines an ephebos as someone over 15 years old. ${ }^{12}$ The physical transformation which accompanies sexual maturity, in particular the appearance of a beard in young men, is described in the medical literature, and more generally in literary documents, to describe someone's age. Some monasteries, such as those at Mount Athos, refuse unbearded young men in their midst.

Naturally, girls started this period earlier than boys, officially at 12, although there are discussions about the actual age of puberty during the Middles ages. This might have been later than 12, around 13 to 14 years of age, depending on how poorly fed young girls were. For girls too, puberty was not always considered

10 Th. Wiedemann, Adults and Children in the Roman Empire. London 1989, 114.

11 Ecloga, I, 4 (ed. L. Burgmann, Ecloga: das Gesetzbuch Leons III. und Konstantinos V. [Forschungen zur Byzantinischen Rechtsgeschichte 10]. Frankfurt am Main 1983, 170).

12 PRINZING, op. cit. 23. 
the threshold of intellectual maturity. There is another text, which shows that girls were considered more responsible after 15 than before 15. It concerns monastic vows.

\section{Basil and the monastic profession}

Monastic life supposed an ascetic lifestyle, including celibacy for one's entire life, and fasting, but also the rejection of worldly values such as ambition, career seeking, and wealth accumulation. Because monastic life eventually became established as a holy lifestyle, following many of the New Testament's recommendations, parents started offering their children to monastic institutions or consecrating one child to a virginal life at home. In homilies addressed to families, Eusebius of Emesa encouraged parents to dedicate a child to a virginal and ascetic life. Not only will the child reap the rewards reserved to the saints in heaven, but his or her parents will benefit from it too. ${ }^{13}$ Ambrose congratulates a father who has consecrated to God one of his daughters, explaining to him the great advantage of having a child who will never leave home. ${ }^{14}$ In Christian families of the Late antique period, it was not rare to see one of the girls consecrated to God and living in the family home. ${ }^{15}$ In the $D e$ inani gloria, John Chrysostom recommends that parents take good care of leading children towards a pious life, by teaching them the value of virginity and detachment towards wealth and glory as soon as possible. ${ }^{16}$ In the Adversus oppugnatores vitae monasticae, John Chrysostom hopes to see Christian families prepare their children for an ascetic life at home, when they are little, and then, when they have reached 10 years of age, entrust the child to monks or nuns for a Christian pious education. ${ }^{17}$ Although John Chrysostom hoped that children raised in a monastery would want to remain there permanently, he recognised that it was not a realistic goal and admitted their return into society: "Raise an athlete for Christ. I am not telling you to refuse his marrying or to send him into the desert or to prepare him to live the life of the monks. No I am not saying that. I want that and I pray that all embrace it, but since it seems to be a burden, I will not constrain you. ${ }^{18}$ " In these texts, parents decided to consecrate one of their children to a virginal life or to marry them with the child having no say in the matter. Parents could also give a child to a monastery, not only for the pur-

13 Eusebius of Emesa, Homily VII, 24: asks parents to help their consecrated daughters fulfill their promise to God. D. AmAND de MEndiEtA, La virginité chez Eusèbe d'Emèse et l'ascétisme familial dans la première moitié du IV siècle. Revue d'histoire ecclésiastique 50 (1955) 777-820, at 804. 14 Ambrose, De institutione virginis 1.

15 B. CASEAU, Stratégies parentales concernant les enfants au sein de la famille: le choix de la virginité consacrée, in: Parenté et stratégies familiales dans l'Antiquité tardive (III ${ }^{\mathrm{e}}-\mathrm{VI}^{\mathrm{e}}$ siècle), eds. Ch. Badel - Ch. Settipani. Paris 2012, 247-264.

16 John Chrysostom, De inani gloria 17 (ed. and transl. A. M. MALINGREy. Paris 1972, 100).

17 John Chrysostom, Adversus oppugnatores vitae monasticae, III, cc. 11, 18. PG 47, c. 376*.

18 John Chrysostom, De inani Gloria 17 (102-104 MALINGREY). 
pose of a pious education, but as a monk or a nun, to fulfill a vow made to God. ${ }^{19}$ Fathers had the upper hand when it came to decisions for their children, but mothers could also give a child to monks or nuns. The Life of saint Theodore of Sykeon mentions a mother healed by the saint who gives her son, already old enough to teach, to the monastery. Rich women who transformed their houses into nunneries, often invited family members and servants to join them in a life of prayer and asceticism. ${ }^{20}$ Jerome's letters provide us with examples of such a behaviour among aristocratic women in Rome. When a widow transformed her house into a nunnery, if she had young daughters, these too would live an ascetic life, whether or not they had an inclination for it. Susan Ashbrook Harvey has studied Syriac saints Lives praising mothers and daughters living together in such a life. ${ }^{21}$ Other family members could act as a guardian of a child and decide on its future. Saint Anthony's sister was deprived of her inheritance and entrusted to nuns by her brother. Nothing in the Life written by saint Athanasius indicates that it was her choice. ${ }^{22}$ While it was very difficult for minors to contest the choice of spouse made for them by their father, discussions started as to the binding power of a parent's power to impose a celibate life on a child. Could the child decide or not to break a parent's vow of virginity upon reaching adulthood? If so, what would be the appropriate age to do so?

On this question, we have the opinion of Basil of Caesarea in the so-called Long Rules or Regulae fusius tractatae (also called the great Asceticon), which Basil wrote after 370 , at the end of his life, when he was a bishop. ${ }^{23}$ He points out that entering monastic life is an important decision, which cannot be taken by a person too young to fully comprehend what it entails. He suggests that if children, especially orphans, can be welcomed inside a monastery, this living arrangement should not be the equivalent of a formal monastic profession and that they should be able to leave, once they are adults. He recommends that monastics do not accept vows from persons too young to understand the nature of their decision. For girls, he suggests that it is important to wait until they have reached their sixteenth or seventeenth birthday to give them the veil. We can deduce from this that Basil considers that at such an age, a person is a responsible adult who should then stay in the monastic orders once committed, and be punished otherwise. He sets the punishment of nuns,

19 M. DE Jong, In Samuel's Image. Child Oblation in the Early Medieval West. Leiden 1986; V. VuoLANTO, Children and asceticism. Strategies of continuity in the late fourth and early fifth centuries, in: Hoping for continuity: childhood, education and death in Antiquity and the Middle Ages, ed. K. Mustakallio et alii. Rome 2005, 119-132.

20 P. LAUREnce, Jérôme et le nouveau modèle féminin. La conversion à la “vie parfaite”. Paris 1997. 21 S. AshbrooK HARvey, Sacred Bonding: Mothers and daughters in Early Syriac Hagiography. Journal of Early Christian Studies 4/1 (1996) 27-56; S. BROCK - S. ASHBRooK HARVEY, Holy Women of the Syrian Orient. Berkeley 1987.

22 Athanasius of Alexandria, Life of Antony 2, 4-5 and 3, 1, ed. and French translation by G. J. M. BARTELINK, Paris 1994, 134-136.

23 The manuscript tradition of these texts is very complex: J. GRIBOMONT, Histoire du texte des Ascétiques de saint Basile (Bibliothèque du Muséon 32). Louvain 1953. 
who fail to keep their vows, to the age when they took such vows. In the Long Rules, he writes:

it is not proper to consider children's words entirely final in such matters, but she who is above sixteen or seventeen years, and is mistress of her faculties, who has been examined carefully and has remained constant and has persisted in her petitions for admittance, should then be enrolled among the virgins, and we should ratify the profession of said virgin, and inexorably punish her violation of it. ${ }^{24}$

Basil writes this canon because parents have started pressuring children to adopt the life of consecrated virgins or have given children to monasteries with the same effect, without considering the inclination of the child towards a celibate and ascetic life. If some bishops recommend checking with the child before deciding on a consecration to God, most admit the freedom of parents to decide the future of their children. Basil, however, considered the risk of a fall for these unwilling or half-willing young virgins. A number of them refused to accept their parent's decision, and this caused scandal in their communities. Eusebius of Emesa mentions unfaithful virgins, who fell into temptation or decided to change lifestyle and marry. ${ }^{25}$ Basil may have been aware of the problem in his own family, if the letter of reproach he wrote to a fallen virgin, was in fact addressed to one of his sisters. ${ }^{26} \mathrm{He}$ was obviously grieved and called her an adulterer and a prostitute. She probably lived an ascetic life in her parental house, with Macrina, her eldest sister, but without feeling it was her choice in life. Basil's advice concerning the proper age to let a young girl take the veil and make her vows stems from such examples, that Basil wanted to avoid in future. He considered that the salvation of the individual person was at stake. The decision of Basil to postpone the monastic profession to 16 or 17 is extremely interesting for our purpose. He obviously considered that this type of decision required an intellectual and emotional maturity that reaching puberty alone would not ensure. He created a space of a few years, which we can equate to our understanding of adolescence. A girl could consent to a wedding at 12 but should not be asked to consent to a monastic life at that young an age.

\section{Boys and girls: marriage and adult life}

Basil did not challenge parental authority for weddings, and admitted that a girl would have to obey her father if he decided to marry her at puberty or soon after. He probably believed that married life was less of a challenge than monastic life. Intellectual or emotional maturity was not required of a young girl for marriage, the

24 Basil of Cesarea, Regulae fusius tractatae XV 4. PG 31, c.956.

25 Eusebius of Emesa, Homily, VII.

26 Basil of Caesarea, ep. 46; P. Maraval, Encore les frères et sœurs de Grégoire de Nysse. Revue d'Histoire et de Philosophie Religieuses 60 (1980) 161-166. 
decision was taken for her, but we can say that girls who were married at a very early age were in fact deprived of their adolescence. They had to cross from childhood to adulthood without transition, especially when confronted with the task of managing servants, for middle and upper class women, as well as giving birth and parenting soon after the wedding. ${ }^{27}$

These early weddings were particularly numerous among the aristocracy. In the imperial family, some girls were married even before puberty, such as Fl. Maxima Fausta, the daughter of the emperor Maximianus, who married her to Constantine in 307 when she was around 9 years old. The first daughter of Fausta and Constantine, Constantia was also married to a cousin when she was around 9 years old. It needs to be said that these princesses were part of political alliances, and these marriages may not have been consummated. Constantia's first living child was born when she was around 19. Still, these young brides were expected to behave like adults. Robert Etienne has shown that, in the imperial family from the time of the Julio-Claudian dynasty to the 4th century, the majority of women were married between 10 and 15 years of age, which left very little time to adjust after puberty. ${ }^{28}$ Was it the same for more ordinary families? ${ }^{29}$ Based on the family of Ausonius, Robert Etienne had shown that the median age for girls to be married was 15 in the 4th century. ${ }^{30}$ The study of 246 Christian epitaphs from Italy, mostly dated to the 4 th and 5 th century, reveal that girls were married between 12 and 16, while boys were married later, between 18 to 25 years. ${ }^{31}$ Being a bride at 12 was not uncommon. Augustine's mother offered such a young bride to his son. ${ }^{32}$

So, we need to separate the case of boys and that of girls. Young girls of the upper class were thrown into adult life, by being married at an early age. For these young girls, in pagan times, there was a ceremony marking the transition from childhood to married life, where a young girl would offer her dolls and toys to a deity. In Greece, they offered dolls to Artemis at Brauron. Venus was often chosen

27 Same conclusion by Patlagean for the end of the Middle Ages, see É. PATLAGEAN, L'entrée dans l'âge adulte à Byzance aux XIII ${ }^{\mathrm{e}}-\mathrm{XIV}^{\mathrm{e}}$ siècles, in: Historicité de l'enfance et de la jeunesse. Athens 1986, 267.

28 R. Etienne, La démographie des familles impériales et sénatoriales au IV siècle après J.C”, in: Transformations et conflits au IVe siècle ap. J.-C., colloque organisé par la Fédération Internationale des Etudes Classiques (Bordeaux, 7-12 septembre 1970). Bonn 1978, 133-168.

29 B. SHAW - R. SALLER have pushed back to the late teens the age of first marriage for girls, except those belonging to the elite. More on the topic in: M. HARLOW - R. LAURENCE, Growing up and Growing Old in Ancient Rome. A Life Course Approach. London 2002.

30 R. Etienne, La démographie de la famille d'Ausone, in: Etudes et chroniques de démographie historique. Paris 1964, 15-25.

31 É. Patlagean, L'enfant et son avenir dans la famille byzantine (IVème-XIIème siècles). Annales de démographie historique (1973) 85-93 at 90 (= EADEM, Structure sociale, famille, chrétienté à Byzance, $\mathrm{IV}^{\mathrm{e}}-\mathrm{XI}^{\mathrm{e}}$ siècle [Variorum Reprints] London 1981).

32 B. D SHAw, With whom I Lived: Measuring Roman Marriage. Ancient Society 32 (2002)195 - 242; Id., The age of Roman girls at marriage: some reconsiderations. JRSt 77 (1987) 30 - 46; IDEM, The family in Late Antiquity: The experience of Augustine. Past and Present 115 (1987) 3-51. 
for such a rite of passage. ${ }^{33}$ This ritual shows what was expected of young brides: a very quick, even brutal transition between childhood and adulthood. Boys, on the other hand, had more time to adjust to the changes linked to puberty, and they could expect, for a few years, some leniency towards misbehaviour. Libanius and John Chrysostom both mention young men attending school whose fathers are almost proud because they misbehaved sexually, through lack of self-control. Both authors on each side of the religious divide agree that fathers, should guide their sons in a stricter manner. The fact that some fathers looked with indulgence at their sons' poor moral judgment irritated Libanios ${ }^{34}$ and scandalised Chrysostom. ${ }^{35}$ Students' rowdiness appears in different sources, from saints' Lives to legal texts in the Late Antique period and is considered a fact of life. It occasionally led to violent clashes. In these sources, we may catch a glimpse of boisterous groups of teenagers, golden boys belonging to the upper strata of society, studying, free of cares, and feasting when they were not arguing. Unlike the little slaves whose childhood was shortened by the work they were required to do, unlike peasants and workmen whose sons had to start working early too, and unlike young girls of their milieu who were married at an early age, these privileged boys could enjoy a few years of adolescence. In my opinion, this is where we have to look to find teenagers.

\section{Conclusion}

The threshold for adulthood is a movable one in Byzantine society. It is in fact different for boys and girls, it also depends on social level, the presence or absence of properties to manage, freedom or slavery. The ages proscribed to take life-altering decisions and be trusted as an adult are clearly later in the Roman period and earlier in the Byzantine world. Was life shorter in Byzantines times than it had been in Roman times? Was it necessary to take important steps earlier in life, for fear of not being able to do so later? Or should we consider that this evolution reveals a positive more trusting attitude towards young people? Did Christianity influence the idea that children or even teenagers can be wise and should be trusted to know their own mind at the age of 15? These are difficult questions. The evolution towards an earlier date for important decisions such as monastic life or marriage could also be interpreted as the increasing power of parents to decide the future lives of their children. Yet the striking point is the diversity of cases, and the flexibility of Byzantine society to accommodate different ways of managing life.

For example, the prudent advice given by Basil for monastic vows was copied in Byzantine canonical collections, but rarely followed. His idea that children could de-

33 B. Rawson, Children and Childhood in Roman Italy. Oxford 2003, 144-145.

34 Libanios, Or. 62, 24-25

35 A. PuEch, Saint Jean Chrysostome et les mœurs de son temps. Paris 1891; A.J. FESTUGIÈRE, Antioche païenne et chrétienne: Libanius, Chrysostome et les moines de Syrie. Paris 1959. 
cide for themselves did not agree with the general understanding that parents decided what was best for their children in view of what was best for the family, and family networks. ${ }^{36}$ In spite of well-known examples in saints' Lives of children choosing the monastic life against their father's wishes, such as Michael Maleinos, children were consistently married or sent to monasteries following their parents' wishes, and few could oppose their father's will. ${ }^{37}$ This is why Basil's recommendations were ignored by the bishops of the council in Trullo, convened in 692, who decided to admit young boys to the monastic profession at the age of ten. In fact, for marriages, they left an adult in charge of such a decision, and they placed bishops in charge of deciding on early vocations. At the same time, they also ruled that 10 was the age of consent for such life-altering decisions. Emperor Leo VI tried to make sense of the discrepancy between the opinions of Basil of Caesarea and the bishops of the council in Trullo. He explained that Basil chose the age of 16 because children could dispose of their properties, at that age. Yet, he did not want to disagree with an assembly of bishops, and decided that there should be no objection to letting a ten years old child enter the monastic life and receive tonsure or the veil. He refused to give preference to one or the other, and decided that anyone willing to take the religious habit may do so after the age of 10 or after the age of 16 . We can suppose that if a child agreed to take monastic vows at 10 , it is was with the supervision of an adult, while at 16 , the decision was his own. This imperial indecision is a good illustration of the flexibility of the Byzantines when it came to age. Nothing was really cast in stone in this area, as the numerous examples in Despoina's book also show. ${ }^{38}$

36 B. CaSeau, Les réseaux familiaux. Antiquité tardive et Moyen Âge. Paris 2012.

37 V. Vuolanto, Choosing Asceticism: Children and Parents, Vows and Conflicts, in: Children in Late Ancient Christianity, eds. C. Horn - R. Phenix. Tübingen 2009, 255-291.

38 D. ARIANTZI, Kindheit in Byzanz. Emotionale, geistige und materielle Entwicklung im familiären Umfeld vom 6. bis zum 11. Jahrhundert (Millennium-Studien 36). Berlin - Boston 2012. 


\title{
Adoleszenten in der kirchlichen Rechtsprechung der Byzantiner im Zeitraum 13. - 15. Jahrhundert
}

\begin{abstract}
Einleitung. - I. Zur kirchlichen Gerichtsbarkeit und Judikatur in der Berichtszeit. - I.1: Kirchliche Gerichtsbarkeit und Judikatur. - I.2 Quellen zur kirchlichen Rechtspraxis. - II. Quantitative, inhaltliche und formale Beobachtungen zu den ,Adoleszenten-Akten` der einschlägigen Quellenkomplexe. - II.1 Mengenangaben - Themenbereiche - Frauenanteil. - II.2 Der Halb- und Vollwaisen betreffende Aktenbestand. - II.3 Direkte bzw. indirekte Ego-Dokumente bzw. Aussagen in den Akten. - III. Kasuistik: III.1 Der „Gang in die Fremde“ verwaister Söhne, das „Verschwinden“ frisch verlobter oder verheirateter Männer und der Undank von Söhnen oder Ziehtöchtern. - III.2 Gewaltanwendung mit Todesfolge und sonstige Gewalt- oder Druck-Ausübung. - III.3 Ehe-/Familienrechtliche Probleme, auch mit Blick auf die klerikale Karriere $(\mathrm{E}+\mathrm{K})$ oder mönchische Lebensweise $(\mathrm{E}+\mathrm{ML})$. - III.4 Fragen im Zusammenhang mit Karriere und Disziplin. - III.5 Vermögens- und erbrechtliche Probleme. - IV. Gesellschaftliche Stellung der Adoleszenten. - V. Fazit. - Anhang A: Überblick zur Fall-Diversität bei den erb- u. vermögensrechtlichen Fällen aus den $P D$ und dem PRK. - Anhang B: Tabelle II: Von Adoleszenten handelnde Fälle aus kirchlichen Gerichtsakten 1204-1402.
\end{abstract}

\section{Einleitung}

Wie sahen die Lebensverhältnisse byzantinischer Adoleszenten ${ }^{1}$ beiderlei Geschlechts und unterschiedlicher sozialer Stellung im gegebenen Zeitraum, d.h. um 1200ca.1400, konkret aus? Das detailliert und sozialgeschichtlich fundiert herauszuarbeiten, dürfte, wenn nicht ganz unmöglich, so doch äußerst schwer sein, schon wegen der massiven Quellenverluste. Sinnvoller scheint es daher zu sein, nach konkreten oder typischen Problemen zu fragen, mit denen sich Adoleszenten im späten Byzanz konfrontiert sahen. Doch wie lässt sich hierüber etwas in Erfahrung bringen? Gibt es spezielle Quellen oder Quellenkomplexe, in denen man historisch belegten Personen dieser Altersgruppe und ihren Problemen näherkommen kann? Diese Frage hat bereits 1950 Paul Lemerle († 1989) im Zuge seiner Untersuchungen zur Judikatur in der Paläologenzeit in dem Beitrag zum synodalen Patriarchatsgericht konzis beantwortet: „Les textes les plus intéressants sont ceux où l'on voit le tribunal prendre les intérêts d'un mineur, $\dot{\alpha} \varphi \tilde{\eta} \lambda \iota \xi^{\prime} .^{2}$ Damit ist implizit schon angedeutet, dass es vor allem kirchliche Gerichte waren, die mit familienrechtlichen Fragen befasst waren, so dass von ihren

\footnotetext{
1 Vgl. für den Zeitraum 6.-11. Jh. den grundlegenden Artikel von D. ARIANTZI, Terminologische und sozialhistorische Untersuchungen zur Adoleszenz in Byzanz (6.-11. Jh). Teil I. Theorien, Konzepte, narrative Quellen. JÖB 63 (2013) 1-31.

2 P. LEMERLE, Recherches sur les institutions judiciaires à l'époque des Paléologues. II. Le tribunal du patriarcat ou tribunal synodal. AnBoll 68 (1950) 318-332 (NDr. in: DERS., Le monde de Byzance: Histoire et Institutions. London 1978, Art. XII), hier 322.
} 
Entscheidungen wichtige Aufschlüsse über die Lage von Adoleszenten zu erwarten wären. Warum aber waren es überhaupt kirchliche Gerichte, die sich mit dieser Materie befassten, und wie war es mit der Struktur und der „Zuständigkeit“ kirchlicher Gerichtsbarkeit im späten Byzanz bestellt? Der Klärung dieser Fragen gilt im Folgenden der Abschnitt I.1, während die auf uns gekommenen Quellen bzw. Quellenkomplexe mit kirchlichen Gerichtsakten aus dem Zeitraum 1204-1402 im Abschnitt I.2 beschrieben werden. Deren Auswertung führte zu einer Liste von 49 konkreten Fällen, die sich deshalb als einschlägig erweisen, weil

1. entweder zur Abfassungszeit der Quelle eine (oder mehrere) von ihr erwähnte(n) Person(en) den Adoleszenten zuzurechnen war(en) oder weil

2. von der Quelle ein Sachverhalt berichtet wird, der die bereits länger zurückliegende Adoleszenz-Zeit der in ihr erwähnten Person(en) beleuchtet.

Die in den erfassten Akten enthaltenen Daten sind mit Hilfe der Tabelle II im Anhang B leicht zu überblicken. Sie bildet daher die Grundlage dieses Beitrags.

Schon an dieser Stelle sei jedoch als eine Spezifik des herangezogenen Quellenmaterials hervorgehoben, dass man bei seiner Auswertung auch direkten bzw. indirekten Ego-Dokumenten oder -Aussagen von Adoleszenten begegnet. Als direkte EgoDokumente bzw. Aussagen gelten im Folgenden seitens eines Petenten/einer Petentin dem Gericht schriftlich vorgelegte Eingaben oder vom Gericht protokollierte Aussagen der betreffenden Petenten, die in den Akten unserer Quellen zitiert werden; als indirekte Ego-Aussagen indes gelten vom jeweiligen Aussteller der Akten erstellte Paraphrasen entsprechender Dokumente bzw. mündlicher Aussagen. Beide Arten von Ego-Aussagen, auch wenn sie zumeist nur biographische Fragmente darstellen, besitzen als Selbst-Aussage der fokussierten Jugendlichen naturgemäß einen erhöhten Quellenwert, könnten somit jeweils wichtige Aufschlüsse zur Person enthalten. ${ }^{3}$

3 Zur Bedeutung dieser Art Quellen vgl. W. SchulzE, Ego-Dokumente. Annäherung an den Menschen in der Geschichte? Vorüberlegungen für die Tagung „Ego-Dokumente“, in: Ders. (Hrsg.), Ego-Dokumente. Annäherung an den Menschen in der Geschichte, Berlin 1996, S. 11-30. Vgl. zu solchen Passagen D. SImon, Byzantinische Provinzialjustiz. BZ 79 (1986) 310 - 343 (bezieht sich ausschließlich auf Chomatenos-Akten), hier 322: ,...können wir nur in ganz wenigen Fällen uns einen Eindruck verschaffen, wie die Parteien ihre Sache vorgetragen haben, wie sie formulieren und welche Meinungen sie äußerten. Zwar gibt es einige Akten, in denen das Vorbringen der Bittsteller in direkter Rede protokolliert ist, aber es ist klar, dass die Authentizität dieser Äußerungen nicht größer ist, als die heutiger Gerichtsprotokolle, die gleichfalls nach den Relevanzkriterien des Entscheidungsstabes formuliert werden." Die byzantinistische Sekundärliteratur zu autobiographischen Texten hat sich mit den aus Texten der Rechtsprechung eruierbaren autobiographischen Passagen, die man als mehr oder weniger fragmentarische Ego-Dokumente bezeichnen könnte, bislang nur am Rande befasst, vgl. M. HINTERBERGER, Autobiograpische Traditionen in Byzanz. Wien 1999, hier außer 95, 256f. (zu autobiographischen Fragmenten in Testamenten u. Typika) besonders $376 \mathrm{f}$. den Hinweis auf das 1394 vor der Patriarchalsynode „abgelegte Schuldbekenntnis des ehemaligen lateinischen Patriarchen von Konstantinopel und Hochstaplers Paulos Tagaris“ (376), das der Synodalschreiber Akindynos Perdikes im „,ungefähren Wortlaut“, gestützt auf Notizen, wiederzugeben sich bemüht habe, so dass sein Text „nicht als wörtliche Äußerungen des Tagaris zu verstehen“ (377) sei, und die weiteren, paraphrasie- 
Aber zurück zur Tabelle II: Eine umfassende Auswertung der in ihr registrierten Fälle lässt sich im Rahmen dieses Beitrags nicht vornehmen, dazu wäre die Mitarbeit eines ausgewiesenen Rechtshistorikers notwendig. Hinzu kommt, dass sich manche Fragen, die man an die Quellen richten möchte, mangels einer hinreichend engmaschigen Quellenlage nur sehr bedingt, teils überhaupt nicht beantworten lassen. Mit dieser Einschränkung sollen von vornherein zu hohe Erwartungen an den vorliegenden Beitrag gedämpft werden.

\section{Zur kirchlichen Gerichtsbarkeit und Judikatur in der Berichtszeit}

\section{I.1 Kirchliche Gerichtsbarkeit und Judikatur}

Generell stehen uns zur byzantinischen Rechtspraxis, also der weltlichen und kirchlichen Rechtsprechung, viel weniger Quellen zur Verfügung als zu den Texten mit den rechtlichen Normen, d. h. vor allem zur Gesetzgebung. ${ }^{4}$ Immerhin aber liegen uns aus der spätbyzantinischen Epoche, welche die Zeit der Fragmentierung des Reiches durch den Lateinereinbruch 1204-1261 und die Palaiologenzeit 1261-1453 umfasst, drei Quellenkomplexe aus dem Bereich kirchlicher Rechtsprechung vor. In ihnen wird man für die hier zu diskutierende Thematik in größerem Maß fündig, wie sich noch zeigen wird.

Doch warum ist es gerade die kirchliche Gerichtsbarkeit, aus der das Quellenmaterial kommt? Der Grund hierfür liegt darin, dass die orthodoxe byzantinische Kirche $^{5}$ im Lauf der Zeit immer mehr Einfluss erlangt hatte auf die Regulierung und Gesetzgebung der Bereich Ehe-, Familien- und Erbrecht. ${ }^{6}$ Dieser Umstand führte dazu, dass sich eben auch die kirchliche Gerichtsbarkeit immer stärker auf diesen Bereich auszudehnen vermochte, ohne jedoch hierbei jemals die ausschließliche Zuständigkeit zu erlangen: $\mathrm{Zu}$ jeder Zeit war es dem Kaiser möglich, in ein Verfahren einzugreifen bzw. es an sich zu ziehen, so dass sich kirchliche Richter gegebenenfalls einem

renden Angaben hierzu, 377-381; vgl. zuletzt M. ANGoLD, Memoirs, Confessions and Apologies: The Last Chapters of Byzantine Autobiography. BMGS 37 (2013) 208-225, hier 210 (zu Tagaris), aber sonst ohne Bezug zur Rechtsprechung.

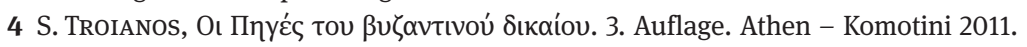

5 Vgl. zu ihrer Geschichte noch immer klar und konzis H.-G. BECK, Geschichte der orthodoxen Kirche im byzantinischen Reich. (Die Kirche in ihrer Geschichte Bd. 1, Lieferung D). Göttingen 1980, sowie ergänzend etwa P. Schreiner, Byzanz 565-1453 (Oldenbourg Grundriss der Geschichte, Bd. 22). München 2011, 86-101, doch wird darin die kirchliche Rechtsprechung nur kurz behandelt: 72.

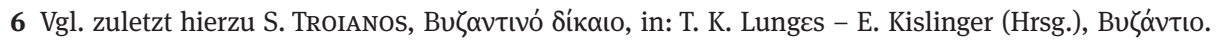

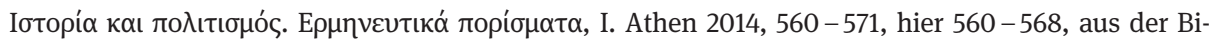
bliographie (ab S. 607) 609-612 (zum Familien- und Erbrecht u. 616f. (zur Rechtsprechung). 
kaiserlichen Gerichtsurteil beugen mussten, umgekehrt konnten sie auch, z. B. bei mangelnder Kompetenz, ihrerseits einen Fall delegieren, so auch an den Kaiser. ${ }^{7}$

Entsprechend der hierarchischen Struktur der Kirche sind bei der Rechtsprechung drei Ebenen zu unterscheiden: die der jeweils regional zuständigen Bischöfe, dann die Ebene der ihnen (den sog. [Suffragan-]Bischöfen) übergeordneten Metropoliten bzw. Erzbischöfe, und zuletzt die Ebene des Patriarchen von Konstantinopel, dem alle Metropoliten und Erzbischöfe des Reiches unterstanden. Nur die autokephalen Erzbischöfe der Erzbistümer „Bulgarien“ (mit Sitz in Achrida/Ohrid) und von Zypern (mit Sitz in Nikosia) nahmen hierbei lange Zeit eine Sonderstellung ein, insofern sie (mit ihren Suffraganen) vom Patriarchen formal unabhängig waren und nur dem Kaiser unterstanden. ${ }^{8}$ Somit war die kirchliche Hierarchie auf diesen zwei bzw. drei Ebenen mit Auseinandersetzungen über die Zulassung oder Gültigkeit von Verlobungen, Eheschließungen oder auch Ehescheidungs-Begehren einerseits bzw. über erbrechtliche, ehe-(und familien-)rechtliche oder vermögensrechtliche Auseinandersetzungen andererseits oftmals befasst. Dies geschah nicht selten auch aufgrund entsprechender Delegation durch eine weltliche Instanz. Nicht $\mathrm{zu}$ vergessen ist dabei, dass die kirchliche Judikatur natürlich in erster Linie für innerkirchliche, den Klerus betreffende Rechtsfälle zuständig war. ${ }^{9}$ Obgleich man davon ausgehen kann, dass sich die kirchliche Judikatur $\mathrm{zu}$ allen Epochen der byzantinischen Geschichte auch in schriftlicher Form niederschlug, hat sich davon aus dem Zeitraum vor 1204 nur ein relativ geringer Anteil erhalten, mehr indes aus spätbyzantinischer Zeit (13. - 15. Jh.). $\mathrm{Zu}$ verdanken ist dies dem Erhalt der erwähnten drei Quellenkomplexe. ${ }^{10}$

7 Vgl. Simon, Byz. Provinzialjustiz (wie Anm. 3) 314f., 317 f., 324-331.

8 Für Zypern gilt dies bis 1185 bzw. ab 1191 wohl ca. 1208-1260, vgl. A. BERGER - J. RICHARD, s.v. Zypern, in: LMA 9 (1998) Sp. 738-742; für Bulgarien/Achrida 1020 - ca. 1202 und von 1216 bis mindestens 1334, im Prinzip aber noch bis ca. 1386, dem Beginn der Osmanenherrschaft über Ohrid, letztlich sogar bis zur 1767 erfolgten Eingliederung des Erzbistums in die Jurisdiktion des Ökumenischen Patriarchats, vgl. G. PrInzING, s.v. Ohrid, LMA 6 (1993) 1376-1379 und zuletzt Ders., Emperor Manuel II and Patriarcharch Euthymios II on the Jurisdiction of the Church of Ohrid, in: M.-H. Blanchet - M.-H. Congourdeau - D. I. Mureşan (Hrsg.), Le Patriarcat oecuménique de Constantinople et Byzance hors frontières (1204-1586). Actes de la table ronde [...], Sofia 22-27 août 2011 (Dossiers byzantins 15). Paris 2014, 243-271, hier 247.

9 Vgl. zur kirchlichen Judikatur in Byzanz, also dem Gerichtswesen grundlegend H.-G. BECK, Kirche und theologische Literatur im byzantinischen Reich. München 1959, 74-78, für die 1. Hälfte d. 13. Jh.s (unter Einschluss auch der weltlichen Rechtsprechung) Simon, Byz. Provinzialjustiz (wie Anm. 3) 314 f., 317 f., 324-331, 333-340; DERs., Die Bußbescheide des Erzbischofs Chomatian von Ochrid. JÖB 37 (1987) 235-275, hier 236, 256, 258f., 260 und dort auch zur Komplexität des Ineinandergreifens von weltlicher und geistlicher Rechtsprechung (unter Einschluss der Frage der Effektivität) die Erwägungen

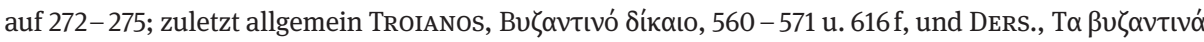

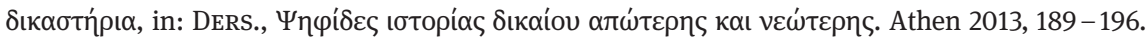

10 Vgl. zu diesen kanonistischen Quellenkomplexen im Überblick Sp. Troianos, Byzantine Canon Law from the Twelfth to the Fifteenth Centuries, in: W. Hartmann - K. Pennington (ed.), The History of Byzantine and Eastern Canon Law to 1500. Washington, D.C., 2012, S. $170-214$, hier 192-197, und

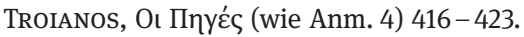




\section{I.2 Quellen zur kirchlichen Rechtspraxis}

Bei ihnen handelt es sich 1. um Synodal-Urteile oder -Bescheide bzw. briefliche Auskünfte des Metropoliten Ioannes Apokaukos von Naupaktos (um 1155 - 1234/35), ${ }^{11} 2$. um das Corpus der Ponemata diaphora (künftig: $P D$ ) des Erzbischofs Demetrios Chomatenos von Achrida/Ohrid (1216-ca.1236) ${ }^{12}$ und 3. um das als Original auf uns gekommene, in Wien aufbewahrte Patriarchats-Register von Konstantinopel (künftig: PRK) aus dem Zeitraum 1315-73 und 1379-1402, das in den Codices Vindobonenses hist. gr. 47 und 48 vorliegt. ${ }^{13}$ Während sich die beiden erstgenannten Quel-

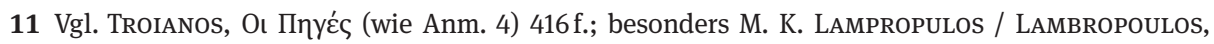

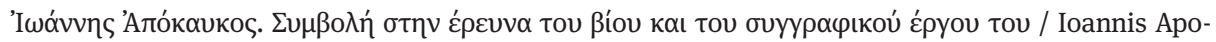
kaukos. A Contribution to the Study of his Life and Work. Athen 1988, hier 99-107 und 260 - 299. Hierin sind die Synodal-Urteile, Bescheide und Gutachten des Apokaukos in Kapitel 4, 260-299) regestenartig aufgelistet und durchnummeriert (im Folgenden und in den Tabellen: Lam Nr./\#). Die Apokaukos-

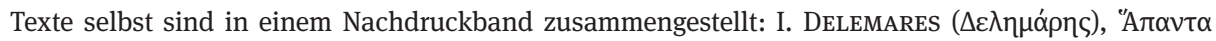

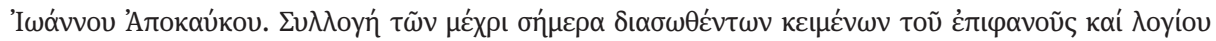

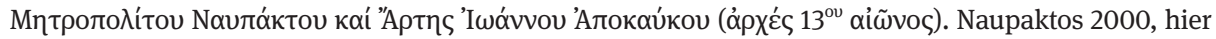
Kap. 5.2. (Texte von Synodalurkunden etc.): 349-457, Numerierung nach Lambropulos; vgl. auch die bibliographischen Hinweise zur Tabelle II. Zu seiner Rechtsprechung im Vergleich zum Patriarchatsregister und zu Chomatenos vgl. zuletzt E. PAPAGIANNI, Un témoin de la réalité juridique byzantine: la jurisprudence patriarcale au XIV siècle, FM 11 (2005) 213-227, hier $213 \mathrm{f}$.

12 Demetrii Chomateni, Ponemata diaphora, rec. G. PrinzIng (CFHB, 38). Berlin u. Boston 2002 (= Dem. Chom, Ponemata). Zu ihm u. seinem Werk: Simon, Byzanz. Provinzialjustiz (wie Anm. 3), zur

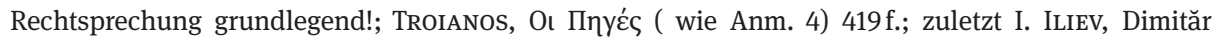

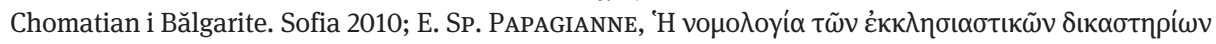

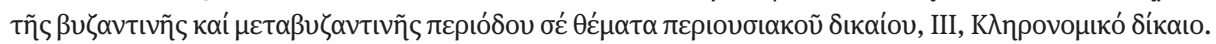
Athen - Komotini 2010, 279-281; G. PRINZING, Konvergenz und Divergenz zwischen dem Patriarchatsregister und den Ponemata Diaphora des Demetrios Chomatenos von Achrida/Ohrid. In: Ch. Gastgeber - E. Mitsiou - J. Preiser-Kapeller (Hrsg.), The Register of the Patriarchate of Constantinople. An Essential Source for the History and Church of Late Byzantium. Proceedings of the International Symposium. Vienna, $5^{\text {th }}-9^{\text {th }}$ May 2009. (ÖAW, phil-hist. Klasse, Denkschriften 457. Bd. - Veröffentli-

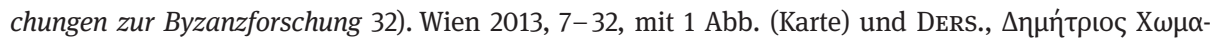

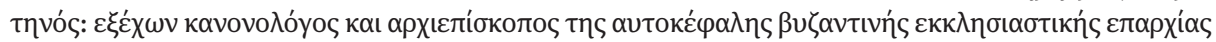

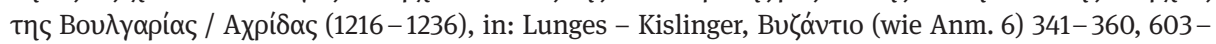
605.

13 Die alte Edition des Patriarchatregisters (Acta et diplomata graeca medii aevi sacra et profana, I-II, hrsg. v. F. MIKLOSICH - J. MÜLLER. Wien 1860 - 1862) [= MM, I/II] ist erfasst in den Patriarchatsregesten: J. DARrouzÈs, Les Regestes des actes du patriarcat de Constantinople, I, Les Actes des patriarches, Fasc. V. Les Regestes de 1310 à 1376. Paris 1977, hier ab Nr. 2030-Nr. 2680, und Fasc. VI, Les Regestes de 1377 à 1410. Paris 1979, Nr. 2682-3259, vgl. dazu auch den Index: Fasc. VII, Les Regestes de 1410 à 1453, suivis des Tables générales des Fascicules I-VII. Paris 1991, 81-195. In Neuedition liegen vor: Das Register des Patriarchats von Konstantinopel, 1. Teil. Edition und Übersetzung der Urkunden aus den Jahren 1315-1331, hrsg. v. H. Hunger - O. Kresten, unter Mitarbeit v. C. Cupane et alii (CFHB 19/1). Wien 1981 (= PRK I); PRK, 2. Teil: Edition und Übersetzung der Urkunden aus den Jahren 1337-1350, hrsg. H. Hunger - O. Kresten - E. Kislinger - C. Cupane, unter Mitarbeit v. W. Fink † et alii). (CFHB 19/2). Wien 1995 (= PRK II); PRK, 3. Teil: Edition und Übersetzung der Urkunden aus den Jahren 1350 -1363, hrsg.v. 
lenkomplexe vor allem auf Gebiete der Provinz in Westgriechenland, Epiros und den ionischen Inseln beziehen, konzentrieren sich die Fälle des 3. Quellenkomplexes überwiegend auf den Raum Konstantinopel und die noch bei Byzanz verbliebenen östlichen Regionen der Balkanhalbinsel. ${ }^{14}$

Vor der näheren Auswertung der drei Quellenkomplexe sei aber im Hinblick auf die Materie, mit der die kirchliche Rechtsprechung befasst war, noch auf einen weiteren Punkt hingewiesen: In bestimmten Fällen konnten, in der Regel wohl aufgrund einer Selbstanzeige, auch kriminelle Tatbestände bis hin zu Totschlag oder Mord, Gegenstand eines kirchlichen Verfahrens werden. Das geschah in der Form, dass die Kirche - nach entsprechender Untersuchung des Vergehens - den bußbereiten Delinquenten einen formalen Bußbescheid erteilte, der die epitimia (Bußstrafen) festlegte, mit denen die Kirche das Vergehen in Kombination mit den verschiedenen Stufen der Exkommunikation (bei Klerikern auch durch befristete oder dauerhafte Amtsenthebung) ahndete. ${ }^{15}$ Dadurch aber, dass sich Delinquenten, die sich als Petenten an das Synodalgericht des Erzbischofs von Achrida oder an den Patriarchen gewandt hatten, diesem Verfahren freiwillig unterwarfen und hiermit die ihnen auf-

J. Koder - M. Hinterberger - O. Kresten, unter Mitarbeit von A. Giannouli et alii (CFHB 19/3). Wien 2001 (= PRK III). Weitere Bände sind im Druck bzw. in Vorbereitung.

14 Vgl. zum Patriarchatsregister u.a. LEMERLe, Le tribunal (wie Anm. 2) 318-329; H. Hunger, Das Patriarchatsregister von Konstantinopel als Spiegel byzantinischer Verhältnisse im 14. Jahrhundert, (Erstpubl. 1978), NDr. in: DeRs., Epidosis. Gesammelte Schriften zur byzantinischen Geistes- und Kulturgeschichte. München 1989, Art. X, 117-136, bes. 130-135; R. MACRIDES, Dowry and Inheritance in the Late Period: some cases from the Patriarchal Register, in: D. Simon (Hrsg.), Eherecht und Familiengut in Antike und Mittelalter. München 1992, 89-98; PAPAGIANnI, Un témoin (wie Anm. 11); CH. GASTGEBER, Das Patriarchatsregister von Konstantinopel der Österreichischen Nationalbibliothek, Historicum. Zeitschrift für Geschichte (Frühjahr-Sommer 2007) 9-19; J. PREISER-KAPELLER, Die hauptstädtische Synode von Konstantinopel (Synodos endemusa). Zur Geschichte und Funktion einer zentralen Institution der (spät)byzantinischen Kirche, Historicum. Zeitschrift für Geschichte (FrühjahrSommer 2007) 20 -31; CH. R. Kraus, Ehe und Verlobung im Patriarchatsregister, Historicum (ebenda), 43 - 48; DERS., Kleriker im späten Byzanz. Anagnosten, Hypodiakone, Diakone und Priester 1261-1453. Wiesbaden 2007, 10 -12; E. GamillscheG, Hohe Politik und Alltägliches im Spiegel des Patriarchatsregisters von Konstantinopel, in: F. Daim - J. Drauschke (Hrsg), Byzanz - das Römerreich im Mittelalter. Teil 2,1: Schauplätze. Mainz 2010, 145 -152; E. Mitsiou, Interaktion zwischen Kaiser und Patriarch im Spiegel des Patriarchatsregisters von Konstantinopel, in: M. Grünbart - L. Rickelt - M. Vučetić (Hrsg.), Zwei Sonnen am Goldenen Horn? Kaiserliche und patriarchale Macht im byzantinischen Mittelalter, Akten der internationalen Tagung vom 3. Bis 5. November 2010. Münster 2011, 79-96, hier 82-88; Gastgeber - Mitsiou - Preiser-Kapeller (Hrsg.), The Register (wie Anm. 11).

$15 \mathrm{Zu}$ den Bußbescheiden (u. ihrer Entstehung) und vergleichbaren Dokumenten kirchlicher Rechtsprechung vgl. M.V. STRAZZERI, Drei Formulare aus dem Handbuch eines Provinzbistums. FM 3 (1979) 323-351, bes. 332 u. 336 - 338, (mit tabellarischem Überblick, S. 342-351); SIMON, Byz. Provinzialjustiz (wie Anm. 3) 316f., und vor allem DeRs., Die Bußbescheide (wie in Anm. 9), 236f. und passim. Nur kurz gehen E. Katerelos, Auflösung der Ehe bei Demetrios Chomatianos und Johannes Apokaukos. Ein Beitrag zur byzantinischen Rechtsgeschichte des 13. Jahrhunderts. Frankfurt/M. etc. 1992, 21f. und M. ANGoLD, Church and Society in Byzantium under the Comneni 1081-1261. Cambridge 1995, ${ }^{2} 2000,224$ (mit Bezug auf Apokaukos, doch nichts zu den Bußbescheiden des Chomatenos) auf sie ein. Zu den Bußstrafen allgemein A. P(APADAKIS), s.v. Epitimion, in: ODB 1, $723 \mathrm{f}$. 
erlegten Bußstrafen auf sich nahmen, erhielten sie anscheinend die Möglichkeit, sich einer weiteren Verfolgung durch weltliche Autoritäten zu entziehen. Denn das kirchliche Gericht konnte, wie dies besonders aus den Chomatenos-Akten hervorgeht, solchen Petenten $\mathrm{zu}$ ihrer Sicherheit ein entsprechendes Schreiben (einen sog. Kirchenbrief) ausstellen, tat dies aber nicht immer. ${ }^{16}$

Nicht wenige Texte der kirchlichen Rechtspraxis können uns somit wegen der in ihnen behandelten, öfter auf Konflikten beruhenden Sachverhalte (die eben zu ihrer Lösung den Gang zum kirchlichen Gericht als notwendig bzw. nützlich und/oder hilfreich erscheinen ließen), wertvolle Einblicke in die konkreten Lebensverhältnisse von Byzantinern beiderlei Geschlechts, diversen Alters und aus unterschiedlichen Schichten verschaffen. ${ }^{17}$ Denn wiederholt stößt man auf Sachverhaltsschilderungen, in denen auch von (rechtlich) Minderjährigen im Bereich der Altersspanne von 12 (bei Mädchen) /14 (bei Jungen) bis 25 Jahren, also von Adoleszenten, die Rede ist, zumal wenn entsprechende Altersangaben ausdrücklich mit angeführt werden. Fehlen aber solche numerischen Altersangaben, lässt sich oftmals der altersbezogenen Begrifflichkeit, wie z.B. den Adjektiven $\alpha \dot{\varphi} \tilde{\eta} \lambda ı \xi / \pi \alpha v \alpha \varphi \tilde{n} \lambda ı \xi$ („qualifiziert“ minderjährig/ ganz , aber pubes/mannbar, geschlechtsreif, heiratsfähig: im Alter zw. 12 [Mädchen]/

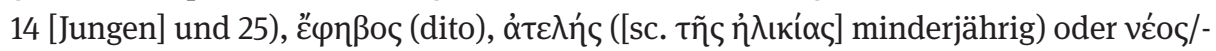

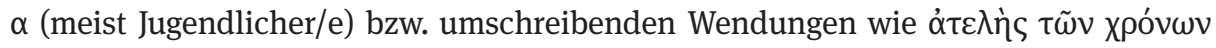
([noch] nicht volljährig, minderjährig) entnehmen, dass eine hiermit bezeichnete Person zu den jungen Leuten (Adoleszenten) zählte, somit kein(e) anēbos/anēlix = Jugendlicher/-e unter 12/14 Jahren mehr war, aber eben auch noch keine rechtlich volljährige Person. ${ }^{18}$ In dem Zusammenhang sei hier auch festgehalten, dass endgültig mit den einschlägigen Novellen von Kaiser Alexios I. Komnenos von 1084 und 1092 generell festgelegt wurde, dass unter Einsegnung vorgenommene Verlobungen und Ehen nur dann gültig seien, wenn die Partner mindestens das Alter von 12 Jahren (bei

16 Vgl. Simon, Byz. Provinzialjustiz (wie Anm. 3) 316f., und DERs., Die Bußbescheide (wie Anm. 9) hier 236, 256, 258f. u. 260. Zu den sog. Kirchenbriefen vgl. Dem. Chom., Ponemata (wie Anm. 12) 280*-283*. 17 Vgl. z. B. die einschlägigen Arbeiten von A. LAIOU, Contribution à l'étude de l'institution familiale en Épire au XIII ${ }^{\text {ème }}$ siècle, FM 6 (1984) 275-323; DIES., Marriage Prohibitions, Marriage Strategies, and the Dowry in Thirteenth-Century Byzantium, in: J. Beaucamp - D. Dagron (Hrsg.), La transmission du patrimoine: Byzance et l'aire méditerranéenne. Paris 1998, 129-160 (Ndr. Dies., Women, Family and Society in Byzantium, ed. by C. Morrisson - R. Dorin. Farnham - Burlington 2011, Art. II); A. KIO-

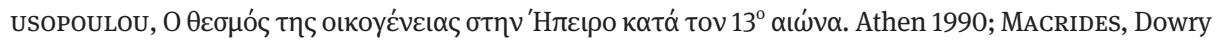
(wie in Anm. 13); Katerelos, Auflösung (wie Anm. 14) und Angold, Church (wie Anm. 15) 223-225 (zum Gericht des Apokaukos), 242-247 (zu dem des Chomatenos).

18 Vgl. C. Hennessy, Images of Children in Byzantium. Farnham - Burlington 2008, 10 f., G. PRINZING, Observations on the Legal Status of Children and the Stages of Childhood in Byzantium, in: A. Papaconstantinou - A.M. Talbot (Hrsg.), Becoming Byzantine. Children and Childhood in Byzantium. Washington, D.C., 2009, 15-34, hier 17-23 und ARIANTZI, Untersuchungen zur Adoleszenz 9-14. 
Mädchen) und 14 Jahren (bei Jungen) erreicht hatten. (Nur der Kaiser blieb befugt, hinsichtlich des Mindestalters eine Ausnahme zuzulassen). ${ }^{19}$

\section{Quantitative, inhaltliche und formale Beobachtungen zu den ,Adoleszenten-Akten' der einschlägigen Quellenkomplexe}

\section{II.1 Mengenangaben - Themenbereiche - Frauenanteil}

Die folgenden Abschnitte beruhen auf der Sichtung der einschlägigen Akten aus der Gesamtmenge besagter drei Quellenkomplexe. Es handelt sich insgesamt um 53 Akten zu 49 Fällen (Urteile, Bescheide, briefliche Auskünfte) und um weitere zwei Akten allgemein gehaltenen Inhalts, bestehend aus einem Aufruf des Patriarchen Kallistos I. und einer Selbstverpflichtung des Priesters K. Strongylos. Die 49 Fälle mit den dazugehörigen Akten finden sich übersichtlich aufgelistet (mit Hinweis auf die jeweilige Akten-Nr.) in der im Anhang B beigegebenen Tabelle II, wobei ihr Inhalt möglichst knapp referiert wird, mit Hinweisen auf die Zeitstellung, formale Aspekte und relevante Akteure, gelegentlich auch relevante Editionen / Übersetzungen / Literatur. Diese Tabelle II dient dem Leser grundsätzlich zur Orientierung, doch darüber hinaus auch dazu, die folgenden Angaben jeweils nachvollziehen bzw. überprüfen zu können.

Die verstreut überlieferten Schriften des Ioannes Apokaukos (s. oben Anm. 11) weisen insgesamt 45 Urteilsprotokolle, Gutachten oder sonstige Entscheidungen auf, von denen aber nur fünf Akten thematisch einschlägig sind. (N.b.: ein Asterisk [*] neben der spezifischen Akten-Nr. [hier und in den folgenden Aufzählungen wie auch in den Tabellen I und II] besagt, dass entweder die betroffene jugendliche Person weiblich ist oder dass zu den jeweils betroffenen Adoleszenten auch mindestens eine weibliche Person gehört). Bei Apokaukos handeln zwei Akten (Nrn. Lam 2* u. 10) von Tötungsdelikten (strafrechtlich gesehen), die zu einem Bußbescheid führten, und zweieinhalb Akten zu ehe-rechtlichen Fragen (Nrn. Lam 17^, 28 [halb] u. 37^), da eben Akte \# 28 auch zur Hälfte der Frage nach der Möglichkeit des weiteren Aufstiegs zum Priester, also der Karriere, gilt. ${ }^{20}$ Somit ist bei den Apokaukos-Akten der Bereich Karriere/Disziplin auch mit einer halben Akte (Lam \# 28 [halb]) vertreten. - Der Anteil

19 Vgl. F. DöLGER, Regesten der Kaiserurkunden des Oströmischen Reiches von 565-1453, 2. Teil: Regesten von 1025-1204. Zweite, erweiterte u. verbesserte Auflage, bearb. von P. WIRTH, Nr. 1116 und 1167, Katerelos, Auflösung (wie Anm. 15) 217-221 und zuletzt Prinzing, Observations (wie Anm. 18) $28 \mathrm{f}$.

20 Mit der hier soeben verwendeten und dann auch im Folgenden öfter vorkommenden Bezeichnung X [halb] bzw. Akte X,5 wird dem Umstand Rechnung getragen, dass eine Akte zwei verschiedenen Sachkategorien angehört. (Bei der Addition zählt die Akte also als X, 5). 
der Adoleszentinnen bei den Apokaukos-Akten beläuft sich auf drei jeweils namentlich genannte Frauen.

Aus den insgesamt 149 Akten umfassenden $P D$ des Chomatenos indes entfallen 123 Akten auf die Rechtsprechung; sie bestehen teils aus Gutachten bzw. Auskünften in Form von Rechtsbescheiden, teils aus Urteilen, zumeist Synodalurteilen. In 24 (oder 23) dieser Akten waren Adoleszenten involviert: Neun Akten betreffen Fälle zu ehe-

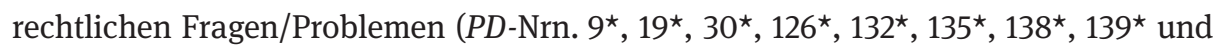
$\left.141^{\star}\right)$, wobei Nrn. $9^{\star}$ und $138^{\star}$ zugleich einen Bußbescheid ${ }^{21}$ darstellen. Die Mehrzahl aber, nämlich 14 (oder 13) Akten, beziehen sich auf erb- und vermögensrechtliche Fälle (PD-Nrn. 30 [+ 43 ident. Petent], 32, 60, 64*, 73, 81, 83, 84, 85, 90, 91 und 105 [bedingt auch 99]). Letzterer stellt hier einen bedingt mitgezählten Fall dar, weil die Jugendlichkeit des Petenten nicht genau erkennbar ist. ${ }^{22}$ Der dritte uns interessierende Bußbescheid aus den $P D$, nämlich \# 129 ${ }^{\star}$, ist innerhalb der $P D$ insofern ein Sonderfall, als er quasi ein Pendant zum (buß-)strafrechtlichen Apokaukos-Fall Lam-2 ist: Er diente der Ahndung eines indirekt verübten Tötungsdelikts (Verstümmelung mit Todesfolge), für das die schon aus (dem Bußbescheid) \# 9* bekannte Petentin Zoe als Auftraggeberin verantwortlich war (vgl. unten bei III.2). Wie gesagt, von den $P D$ sind insgesamt 24 (23?) +1 = 25 Akten für unser Thema einschlägig. Dabei sind Adoleszentinnen, wie zu erwarten, an allen neun eherechtlichen Fällen als Betroffene beteiligt, wobei sie (mit Ausnahme von \# 30) auch namentlich genannt werden. Bei den 14 Akten mit erb- und vermögensrechtlichen Fällen war nur in den Fall \# 64* möglicherweise eine (oder mehrere) Adoleszentin(nen) involviert (das genaue Alter der in der Akte erwähnten minderjährigen, inzwischen verheirateten Halbschwestern ist unbekannt). Das Gleiche gilt für den einzigen (buß-)strafrechtlichen Fall (\# 129*). Insgesamt waren also Adoleszentinnen in 11 der 24 einschlägigen $P D$-Akten involviert.

Bei den ca. 100 auf die Judikatur entfallenden Akten des $\boldsymbol{P R K}$, das insgesamt rund 700 Akten umfasst, ${ }^{23}$ wird man bei 24 Akten konkret fündig, ${ }^{24}$ bei zwei weiteren handelt es sich um die oben erwähnten allgemeinen Verfügungen [Nrn. 219 u. 240], die von nun an außer Betracht bleiben. Im Folgenden befassen wir uns also ausschließlich mit den 24 Akten des PRK zu konkreten Fällen (Nrn. 11, 14*, 89*, 92, 100* [+102* ident. Personen], 125*, 136, 236, 392, 442, 464* [hier Adoleszenz unsicher], 553/1 +2 [da 2 Regest-Nrn], 569^, 554, 571, 575, 584, 587, 610, 613, 646*, 678^). Adoleszentinnen waren nur in die Fälle von neun Akten (der 24) involviert, wobei sie in den meisten

$21 \mathrm{Zu}$ den Bußbescheiden vgl. oben Anm. 15.

22 Den komplexen Fall PD \# 59 habe ich a priori ausgeschlossen, weil er großenteils Vorgänge aus der Zeit vor 1204 betrifft; auch bleibt die rein theoretische, ohne jede konkrete Angabe erfolgte Erörterung eines sachenrechtlichen Problems [PD \# 69], zu dem der Bischof der Stadt Serbia eine Auskunft erbeten hatte, ausgeklammert (trotz Erwähnung von Adoleszenten: Dem. Chom. Ponemata (wie Anm. 12) 241, Z. 78).

23 Vgl. Lemerle, Le tribunal (wie Anm. 2) 319f., Hunger, Das Patriarchatsregister (wie Anm. 14) 117; Troianos, Oı Пெyćs (wie Anm. 4) 422.

24 Vgl. Lemerle, Le tribunal (wie Anm. 2) 321-323, bes. die Abschnitte 1., 2. und 4. 
Fällen ohne den Taufnamen erwähnt werden, vgl. Nrn. 11*, $14^{\star}, 89^{\star}, 92^{\star}, 464^{\star}, 569^{\star}$, $678^{\star}$ ), nur einmal bleibt die Adoleszentin ganz anonym (in \#125). - Thematisch verteilen sich beim $P R K$ die einschlägigen 24 Akten wie folgt: fünfeinhalb betreffen den

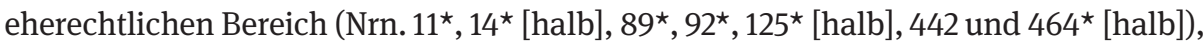
vierzehneinhalb beziehen sich auf erb- und vermögensrechtliche Auseinandersetzungen (Nrn. 14* [halb], 100* [+102*], 553/1+2, 569*, 554, 571, 575, 584, 587, 610, 613, 646 und 678) und vier auf Fragen der Karriere bzw. Disziplin (Nrn. 125*[halb], 136, 236, 392 und $464^{\star}$ [halb]). Auffällig ist, dass sich Adoleszenten-Akten aus diesem zuletzt genannten Themenbereich nur einmal (zur Hälfte) auch bei Apokaukos (Lam \# 28), doch nirgends in den $P D,{ }^{25}$ vor allem also im $P R K$ finden.

Zählt man nun die Einzelsummen aus den drei Quellenkomplexen zusammen, so beläuft sich die Summe auf $(5+24+24=) 53$ Akten, wovon $(3+11+9=) 23$ (ganz oder unter anderem) Adoleszentinnen betreffen.

Die folgende Tabelle I erleichtert die Übersicht über die Verteilung der Akten auf die Themenbereiche:

Tabelle I: Verteilung der ,Adoleszenten-Akten“ nach Themenbereichen

\begin{tabular}{|c|c|c|c|c|}
\hline $\begin{array}{l}\text { Bezugsbereich } \\
\text { der Fälle }\end{array}$ & $\begin{array}{l}\text { Eherecht / } \\
\text { Bußbescheide } \\
(=\mathrm{Bb})\end{array}$ & $\begin{array}{l}\text { Erb- und Ver- } \\
\text { mögensrecht }\end{array}$ & $\begin{array}{l}\text { Karriere u. Dis- } \\
\text { ziplin }\end{array}$ & $\begin{array}{l}\text { Strafrecht } \\
\text { (Tötungs- } \\
\text { Fälle) }\end{array}$ \\
\hline $\begin{array}{l}\text { Apokaukos } \\
\text { Lam }\end{array}$ & $17^{\star}, 28$ [halb], 37* & & 28 [halb] & $2^{*} /$ Bb., 10 \\
\hline $\begin{array}{l}\text { Chomatenos } \\
P D\end{array}$ & $\begin{array}{l}9 * / \text { Bb., 19*, 30*, } \\
126^{\star}, 132^{\star}, 135^{\star}, \\
138^{\star} \text { Bb., 139*u. } \\
141^{\star}\end{array}$ & $\begin{array}{l}29,42,32,60,64^{\star}, 73,81,83 \text {, } \\
84,85,90,91 \text { u. } 105 \text {; bedingt: } \\
99\end{array}$ & & $129^{*} / \mathrm{Bb}$. \\
\hline PRK & $\begin{array}{l}11^{\star}, 14^{\star} \text { [halb], } 89^{\star} \\
92^{\star}, 125^{\star} \text { [halb], } 442 \\
\text { u. } 464^{\star} \text { [halb] }\end{array}$ & $\begin{array}{l}14^{*}\left[\text { halb], } 100^{*}\left[+102^{\star}\right], 553\right. \\
(2 x), 569^{\star}, 554,571,575,584, \\
587,610,613,646 \text { u. } 678\end{array}$ & $\begin{array}{l}125^{\star}[\text { halb], } \\
136,236,392 \\
\text { und } 464^{*} \\
\text { [halb] }\end{array}$ & \\
\hline $\begin{array}{l}\text { Summe } \\
53\end{array}$ & $\begin{array}{l}2,5+9+5,5= \\
17\end{array}$ & $\begin{array}{l}14+14,5= \\
28,5\end{array}$ & $\begin{array}{l}0,5+4= \\
4,5\end{array}$ & 3 \\
\hline
\end{tabular}

25 Der Striktheit dieser Feststellung widerspricht möglicherweise PD \# 14: Darin geht es um die Frage, ob ein Mann Priester werden könne, der, noch unmündig, mit einem 8-jährigen Mädchen verlobt worden sei, aber nach dessen Tod, nunmehr dem Alter nach prosēbos / nahezu mannbar (also knapp 14-jährig [nicht aber, wie im Regest, $75^{\star}$ steht, „als Volljähriger“], eine andere Frau geheiratet habe. Auch wenn diese Akte nicht in die Tabelle II aufgenommen wurde, sollte man sie als Grenzfall betrachten und insofern berücksichtigen. 


\section{II.2 Der Halb- und Vollwaisen ${ }^{26}$ betreffende Aktenbestand}

Die Gesamt-Menge der 53 Adoleszenten-Akten lässt sich noch in bestimmte Teilmengen unterteilen. So gibt es, kaum überraschend, mehrere Akten, in denen verwaiste Adoleszenten, und zwar Halbwaisen (Hw) bzw. Vollwaisen (Vw), die Betroffenen sind oder als Petenten hervortreten: Dies sind bei den PD insgesamt 13 Akten (Nrn. 29 [mit Nr. 42]): Hw; 32: Vw; 64*: 5 Hw/davon 4 weibliche; 73: Vw; 81: Hw; 83: 4 Vw; ${ }^{27}$ 84: 2 Vw; 85: Hw; 90: 4 Vw; 91: Hw; 99: Hw [keine Altersangabe!]; 105: 2 Hw). Beim PRK aber sind es nur drei Akten (Nrn. 92*: Hw, weiblich; 125* Vw; und 236: Vw). Somit betreffen 17 Akten dieser beiden Quellenkomplexe Halb- oder Vollwaisen im jugendlichen Alter, wobei in nur zwei Akten von weiblichen (Halb-)Waisen die Rede ist. Alle Waisen-Akten aus den $P D$ betreffen den erb- und vermögensrechtlichen Bereich, während die drei Akten aus dem PRK sich auf den eherechtlichen Bereich (\# 92*; 125* [halb]) bzw. den Bereich Karriere/Disziplin (\# 125* [halb]; \# 236) verteilen.

\section{II.3 Direkte bzw. indirekte Ego-Dokumente bzw. -Aussagen in den Akten}

Hier sei kurz zusammengestellt, in welchen Akten sich solche Dokumente bzw. Aussagen im oben (S. 30) definierten Sinne finden.

Auf direkte Ego-Dokumente bzw. -Aussagen trifft man in den $P D$ fünfmal. Sie verteilen sich einerseits auf drei in schriftlicher Form dem Gericht vorgelegte Suppliken, die in den Nrn. 81 und 84 von den persönlich erschienenen Petenten vorgelegt wurden (und sich in der Akte zitiert finden), während im Fall \# 138* die Supplik wegen dienstlich bedingter Abwesenheit des Petenten von seinen beiden Vertretern verlesen wurde (s. unten) und in der Akte entsprechend zitiert wird; andererseits auf zwei mündlich vorgetragene und als solche protokollierte Aussagen in den Nrn. 90 und 99 desselben Aktenbestandes. Sie alle wurden von Halb- oder Vollwaisen vorgelegt oder vorgetragen, die sich zur Vorbereitung sicherlich auch nach Möglichkeit fremder Hilfe bedient haben: So kann man zumindest im Fall der Nrn. 81 und 84 mit Dieter Simon annehmen, dass „eine Schreibhilfe am Werk“ war. $^{28}$

26 Zur Lage der Waisen in Byzanz vgl. T. S. MiLleR, The Orphans of Byzantium. Child Welfare in the Christian Empire. Washington, D.C., 2003.

27 In Übereinstimmung mit dem Regest zu \# 83 meiner (ihm noch unbekannten) Ausgabe (S. 174) spricht auch MilleR, The Orphans (wie Anm. 26) 80 zu Recht davon, dass es sich hier um vier Brüder Lubroi handelt. Deshalb korrigiere ich hier nachträglich meine Ausgabe durch die Ergänzung des

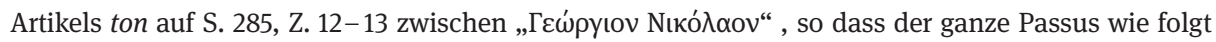

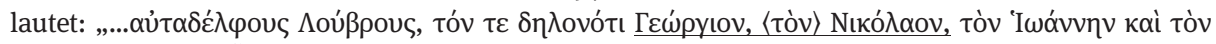

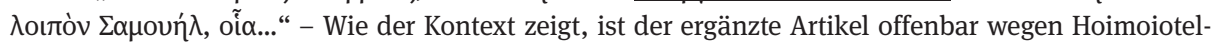
euton vor Nikolaon ausgefallen.

28 Simon, Byz. Provinzialjustiz (wie Anm. 3) 322. 
Eine Sonderrolle in formaler Hinsicht spielt indes die Supplik des halb verwaisten Melias aus Berroia (PD \# 29, S. 113, Z. 34-46), abgefasst als indirektes Ego-Dokument: Sie findet sich in einem Bitt-Brief des Chomatenos, den er um 1224 an den damaligen Dux von Berroia, den Sebastos Ioannes Plytos, gerichtet hatte, ${ }^{29}$ nicht ohne darin einleitend wortreich die Rolle des Dux als Beschützer der Hilfsbedürftigen und als Garanten des Gemeinwohls zu betonen. Chomatenos wollte auf diese Weise Plytos mit dem Gesuch des Melias vertraut machen, der darin schilderte, er werde nach dem Tod seiner Mutter von seinem Vater, der inzwischen mit einer Konkubine zusammenlebe und mit ihr auch Kinder habe, auf gröbste Weise vernachlässigt und müsse sogar um

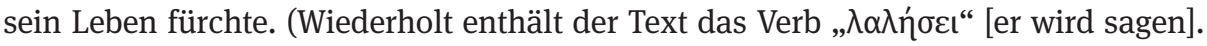
Dadurch verdeutlichte Chomatenos, dass er lediglich stellvertretend für Melias agiere als derjenige, der dem Dux den Wortlaut seines Beistandsgesuchs übermittelt). Ziel der Supplik des Melias war nun einerseits, den ihm zustehenden Vermögens-Anteil aus dem Erbe seiner Mutter (genauer: eines intestat verstorbenen Bruders seiner Mutter) zu erhalten, andererseits aber sicherzustellen, dass die Konkubine seines Vaters und ihre Kinder nicht mehr als ein Zwölftel des väterlichen Vermögens erhalten wird. Der ganze Vorgang lässt vermuten, dass Melias wohl der oberen Mittelschicht (evtl. auch der Oberschicht) entstammt. Der Name seines Vaters bleibt zwar ungenannt, aber sein Großvater mütterlicherseits hieß Theodosios Basilikos, und vielleicht hat auch er dessen Familiennamen getragen. ${ }^{30}$ So scheint Melias von der Kirche, d.h. von Chomatenos, auch in rechtlichere Hinsicht, voll unterstützt worden zu sein, da der Erzbischof sogar versucht hat, auch die weltliche Seite, den regional zuständigen Dux, zu mobilisieren. Wir wissen zwar nicht, ob sich Plytos tatsächlich so für Melias eingesetzt hat, wie von Chomatenos erhofft, vermutlich aber hat Plytos der Bitte entsprochen. So überrascht es auch nicht, dass sich Melias neun Jahre später (1230) nochmals Beistand von Chomatenos holte: Dies beweist die Akte PD \# 42, aus der hervorgeht, dass der nunmehr 33 Jahre alte Melias, der von Berroia aus in Achrida selbst vorstellig geworden war und auf sein dort vorgebrachtes Anliegen einen Synodalbescheid erhielt, mit dem seine Position in einer Streitsache mit einem Ortsfremden um Immobilien-Besitz bestärkt wurde.

Indirekten Ego-Aussagen indes begegnet man im selben Quellenkomplex (den PD) elfmal: in den Nrn. 9, 29, 32, 42, 73, 85, 91, 132*, 135*, 139^ und 141. Abgesehen von den zwei letztgenannten Akten, die eherechtlichen Fragen gelten, betreffen die übrigen fünf Akten erb- und vermögensrechtliche Probleme, wobei die indirekten EgoAussagen von Voll- oder Halbwaisen stammen.

In den PRK-Akten findet sich lediglich ein direktes Ego-Dokument, und zwar in \# 392 (aus dem Bereich Karriere/Disziplin): Dabei handelt es sich formal um eine

29 Vgl. hierzu G. Prinzing, Nochmals zu den adressierten Briefen des Demetrios Chomatenos, in: S. Kotzabassi - G. Mavromatis (Hrsg.), Realia Byzantina. Berlin - New York 2009, 223-245, hier 230 u. $240 \mathrm{f}$.

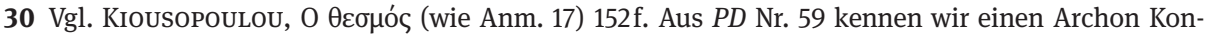
stantinos Basilikos aus dem Thema von Skopia/Skopje. 
Promissio, die im Rahmen unserer Untersuchung singulär ist. Hingegen gibt es ebenfalls, wie in den $P D$, sieben indirekte Ego-Aussagen, enthalten in den Akten Nrn. 100^, 125*, 464 [halb], 587, 610, 613 und 678*. Hiervon gelten fünf (Nrn. 100*, 587, 610,613 und $678^{\star}$ ) dem erb- und vermögensrechtlichen Bereich, zwei halbe Akten (\# $125^{\star}$, \# 464^) betreffen den eherechtlichen Bereich, und nur zwei Akten beziehen sich je zur Hälfte auf den Bereich Karriere/Disziplin (\#125^ [halb]; \# 464: halb]).

\section{Kasuistik}

Dieser Abschnitt besteht aus mehreren Teilabschnitten, von denen jeder einzelne einen bestimmten Sachverhalt bzw. eine Kombination von Sachverhalten hervorhebt, die für mehrere Fälle kennzeichnend sind. So gesehen, lässt dieser Abschnitt als Ganzes wesentliche Aspekte der inhaltlichen Vielfalt aller registrierten Fälle deutlich hervortreten, mit anderen Worten: Aus ihm ergibt sich ein Überblick über die Spezifik der von der kirchlichen Gerichtsbarkeit behandelten Fälle, in die Adoleszenten involviert waren, letztlich also ein Überblick über Probleme, mit denen besonders Adoleszenten mehr oder weniger häufig konfrontiert waren.

\section{III.1 Der „Gang in die Fremde“ verwaister Söhne, das „Verschwinden“ frisch verlobter oder verheirateter Männer und der Undank von Söhnen oder Ziehtöchtern}

Der (zeitweilige) Gang in die Fremde wird fast ausschließlich in den Waisen-Akten $P D$ Nrn. 85, 90, 91, 99 thematisiert. Bei all diesen Akten geht es um erb- und vermögensrechtliche Auseinandersetzungen, doch einen besonders anschaulichen Fall zum „Gang in die Fremde“ stellt hierbei \# 85 dar: Der Petent und Konvertit Manuel (als Jude hieß er zuvor Israel) gab an, er sei nach dem Tod seines Vaters von seinem Stiefvater Moses, der noch zu Lebzeiten seiner (bald darauf ebenfalls verstorbenen) Mutter seinen bei Kastoria gelegenen Weinberg aus dem Vatererbe widerrechtlich verkauft hatte, so vernachlässigt worden, dass er in die Fremde gegangen sei. Dort herumirrend habe er ein armseliges Leben als Bettler und Lohnarbeiter geführt, bis er schließlich eine Arbeit als Koch gefunden habe. ${ }^{31}$ Dies habe ihn später in die Lage versetzt, in die Heimat zurückzukehren. Dort habe er erfolgreich beim Bischof von Kastoria gegen seinen Stiefvater einen ersten Prozess anzustrengen vermocht. Ihm und anderen Halbund Vollwaisen, die in früher Jugend mehr oder weniger mit der Notwendigkeit konfrontiert wurden, in die Fremde zu gehen, um ihr Überleben zu sichern, weil man

31 Vgl. zur Akte zuletzt G. PRInZING, In Search of Diasporas in the Byzantine „Successor State“ of Epirus (c. 1210 - 1267), in: G. Christ (et alii), Union in Separation. Diasporic Groups and Identities in the Eastern Mediterranean (1100 - 1800). Rom 2015, 123-136, hier 133. 
sie z.B. ihres Erbes - nicht selten durch betrügerische Machenschaften - beraubt hatte, bot sich, wie auch durch diese Akte (und z. B. PD \# 91, aber auch durch die Nrn. 42, [59, s. zu ihr oben II.1, Anm. 22] und, nur indirekt, 84) belegt wird, eine Chance, ab dem 14. Lebensjahr innerhalb einer Frist von 30-Jahren ihr Erbe wieder einzuklagen. ${ }^{32}$ In $P D$ \# 90 war übrigens der Gegner des verwaisten Klägers Konstantas und seiner Brüder, ihr Onkel Makronikolaos, derjenige, von dem es heißt, er sei in seiner Jugend in die Fremde gegangen. ${ }^{33}$

In $P D$ \# 99 indes wird der „Weggang in die Fremde“ von dem Grammatikos Theodoros Syropulos gegen zwei seiner Söhne wie folgt instrumentalisiert: Syropulos, aus zweiter Ehe Vater von drei Söhnen und vier Töchtern, verhielt sich besonders seinen Söhnen Nikephoros und Ioseph gegenüber so feindlich, dass sie aus Not in die Fremde gingen. Dort auch verstarb Ioseph, wohingegen Nikephoros noch längere Zeit dort verblieb. (Der anonyme älteste der drei Söhne ging nicht nach auswärts, sondern zog es vor, für sich allein in einer eigenen Behausung in Armut zu leben). Daher hat der Vater gegen Lebensende die beiden weggezogenen Söhne unter dem Vorwand, sie seien noch zu Lebzeiten der Eltern in die Fremde gegangen, testamentarisch bis auf ein kleines Legat völlig enterbt, so dass das elterliche Erbe ihrer Schwester Theodora zufiel. Als nun Nikephoros bei dem Versuch, wieder in sein Erbe eingesetzt zu werden, in Achrida um Auskunft bat, ob der Vater ihn und seinen Bruder auf diese Weise habe rechtens enterben können, obwohl sie erst nach dem Tod ihrer Mutter in die Fremde gezogen seien, erhielt er einen für ihn günstigen Bescheid. Ihm wurde bestätigt, dass er Anrecht auf den Pflichtanteil habe. Zudem wird festgestellt, dass der Wegzug in die Fremde nicht zu den 15 bereits von Justinian I. festgelegten Gründen gehöre, die es Eltern gestatten, (undankbare) Kinder zu enterben. ${ }^{34}$

Wurde hier seitens des Vaters nur indirekt auf den Undank der Söhne angespielt, so konstatierte im Fall der Akte $P R K$ \# 14* (2. Hälfte 1315) die Tante des Kaisers (Andronikos II.) und Megale Papiaina, Eudokia Nestongonissa, ausdrücklich nicht nur „Undankbarkeit“, sondern auch noch Boshaftigkeit bei ihrer Adoptivtochter NN. Sie hatte diese als siebenjähriges Pflegekind zu sich genommen und „fast wie ein leibliches Kind geliebt ...“.35 Vor dem Patriarchats-Gericht führt sie aus, ihrer Adoptivtochter im Lauf der Zeit viele Dinge teils in Verwahrung, teils im Hinblick auf eine spätere legale Eheschließung für die Mitgift gegeben zu haben. ${ }^{36}$ Doch dann habe jene

32 Vgl. auch Simon, Byz. Provinzialjustiz (wie Anm. 3) 313 (lies aber in Anm. 16: Pitra Nr. 85 statt 84), und Prinzing, Observations (wie Anm. 18) 32 (mit Anm. 85) u. 34.

33 Ergänzend zu den Lit.-Angaben zum Regest (Dem.Chom., Ponemata [wie Anm. 12] 188*) vgl. auch LAIOU, Marriage Prohibitions (wie Anm. 17) 154 (case IV).

34 Vgl. Prinzing, Observations (wie Anm. 18) $30 \mathrm{f}$. (mit Anm. 77).

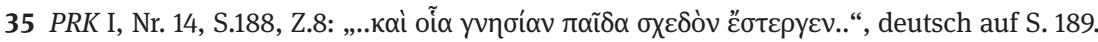

36 Anders als R. MACRIDES, Kinship by Arrangement: The Case of Adoption. DOP 44 (1990) 109-118 (repr. in: EADEM, Kinship and Justice in Byzantium. Aldershot 1999, Art. II), hier 116, meint PAPAGI-

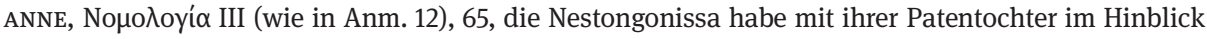
auf deren Mitgiftausstattung nichts vertraglich vereinbart. 
nicht nur ohne ihre Zustimmung geheiratet, sondern sie auch noch verhöhnt und sich ihr gegenüber unverschämt aufgeführt. Sie (Nestongonissa) wende sich daher wegen der Bosheit der NN mit der Forderung auf Rückerstattung an den Patriarchen, um die o. a. Dinge rückerstattet zu bekommen. Ihre Supplik wurde positiv beschieden, man gab ihr Recht, vorbehaltlich der Überprüfung des Falles vor Ort. Hiermit hat man den zuständigen Metropoliten von Philippoi sowie den Erzbischof von Drama beauftragt. Leider besitzen wir über diese örtliche Überprüfung keinerlei Unterlagen, wissen daher auch nichts über eine Reaktion der NN. Soweit ich sehe, ist diese Akte ihrem Inhalt nach singulär, ein vergleichbarer Vorgang sonst anscheinend nicht belegt.

Der Großteil dieser Fälle zeigt, dass es wohl oft prekäre familiäre Verhältnisse, insbesondere mangelnde Fürsorge von (Stief-)Vätern für jugendliche (Stief-)Söhne und/oder rücksichtslose Missachtung der Rechte minderjähriger Jugendlicher am elterlichen Erbe durch ältere Verwandte waren, welche die Jugendlichen in eine solche Not trieben, dass sie sich gezwungen sahen, ihren Lebensunterhalt zumindest eine Zeit lang in der Fremde zu suchen. Andererseits lässt der zuletzt genannte Fall erkennen, dass eben auch auf Seiten der Adoleszenten schuldhaftes Verhalten, Undank und mangelnder Respekt gegenüber Verwandten und Pflegeeltern nicht a priori auszuschließen sind.

Ein anderer, öfter thematisierter Grund für den Weggang oder das scheinbar spurlose (vielleicht auch fluchtartige) Verschwinden in die Fremde offenbart sich beim Studium von Ehescheidungsverfahren: die Entdeckung des (zumeist unter Mitwirkung der Eltern jung oder viel zu früh) verlobten bzw. verheirateten Mannes, dass er gar nicht mit der für ihn ausersehenen Frau zusammenleben könne, anders gesagt: die (im Nachhinein erkannte oder vorgeschobene) Unverträglichkeit (Inkompatibilität) der Partner. Ein hierdurch motiviertes Verschwinden bzw. eine Flucht dieser Art in die Ferne oder Fremde werden in unserem Dossier dreimal bei Scheidungsprozessen angesprochen (Apokaukos Lam \#17*, PD \# 126* und PRK\# 11*): Die Akte Lam \#17 stellt einen gutachtlichen Bescheid des Apokaukos dar, den er (wohl vor 1226) dem vor ihm erschienen Priester Michael Kaludes aus Leusista/Lapsista im Bistum Ioannina im Beisein des zuständigen Bischofs Neophytos, hochrangiger Weltlicher und anderer erteilte. Kaludes wollte wissen, ob seine Tochter Eirene eine neue legitime Ehe eingehen könne, nachdem sie als knapp 12-Jährige vor 3 1/2 Jahren mit dem sprunghaften, begabten und rührig dienstbereiten Konstantinos legal verheiratet worden sei, der aber daraufhin verschwunden sei. Apokaukos riet nun dem Bischof, den von glaubwürdigen Zeugen als Dieb und Betrüger bezeichneten Mann zunächst förmlich zu sich zu zitieren und bei Erscheinen zur Fortführung der Ehe zu überzeugen. Scheitere dies oder lasse der Mann sich weiterhin nicht blicken, so stünde einer Scheidung und dem Eingehen einer neuen Ehe nichts mehr im Wege. ${ }^{37}$

Die Akte PD \# 126 (aus dem Zeitraum ca. 1216-1224) handelt indes von einem weniger schwierigen Sachverhalt: Preabos war nach seiner zu früh erfolgten, daher

37 Vgl. die unten in Tab. II, Fall 3 angegebene Literatur. 
illegitimen Verlobung mit der 9-jährigen Stana auf acht Jahre in die Fremde unter Mitnahme der Arrha ${ }^{38}$ verschwunden, kehrte aber nach seiner Auffindung wieder zurück zu Stana, um dann, trotz Erneuerung der Verlobung mit ihr, schon bald erneut zu verschwinden. Petros aus Resine/Resen (am Prespa-See), der Vater der Braut, wandte sich daraufhin an den Erzbischof mit der Frage, was mit seiner inzwischen in voller Jugend stehenden Tochter nun zu geschehen habe. Preabos erhielt nach Prüfung und Bestätigung seiner Angaben über Stanas Alter den Bescheid, sie dürfe wegen der Nichtigkeit der früheren Verlobung eine neue Verbindung mit einem anderen Mann eingehen.

Besonders komplex ist schließlich der Fall PRK \# 11* (von 1315), der vom Scheidungsprozess des ehemaligen kaiserlichen Pagen Konstantinos Palates gegen die Partei seiner Verlobten NN, Tochter der N. Alamanina (die beide aus Melenikon stammten) handelt. Palates hatte eine ursprünglich mit ihrer Mutter bei Austausch von „Enkolpia“ (d.h. Brustkreuzen, Medaillons oder vergleichbarem, religiös bestimmten Schmuck) ${ }^{39}$ beschlossene Verlobung mit NN sofort gelöst, nachdem er sie erstmals traf und ihre körperlichen Mängel wahrnahm. Durch unlauteres Vorgehen ihrer Mutter wurde aber dann doch K.s Verlobung, ohne Mitwirkung seiner Eltern, bei Leistung der Arrha (?) besiegelt und ein Mitgiftvertrag geschlossen. Als K.P. dann (wie schon zuvor der frühere Verlobte der NN, Georgios Tarchaniotes) nach der Verlobung (als sog. Esōgambros) ${ }^{40}$ auch ins Elternhaus der NN aufgenommen wurde, entdeckte er ihre diversen körperliche Mängel: Daraufhin verweigerte er sich der Eheschließung und entfloh für 4-Jahre ins Ausland. Nach Rückkehr von dort, beantragte er aus anhaltend tiefster Abneigung und Hass gegen die NN die Scheidung. Diese wurde auch von der Synode verfügt, nicht zuletzt, weil 1. seitens der Eltern des K.P. keine Billigung seiner Verlobung bzw. Eheschließung vorlag, und 2. auch wegen seines Hasses auf die Braut sowie wegen der Machenschaften der Alamanina; beide könnten eine neue Ehe eingehen. Die Partei des K.P. solle zudem den Wert der von ihm behaltenen oder verbrauchten Mitgift-Anteile der Braut ersetzen. Für die Vollstreckung des Beschlusses solle der Metropolit von Melenikon sorgen.

Ganz anders gelagert ist indes der aus den Apokaukos-Akten bekannte Fall Lam \# 28[halb], der vom Scheidungsgesuch eines 18-jährigen Anagnosten handelt. Diesem war die Flucht aus der Ehe (mit einer viel älteren Frau) gelungen, die einzugehen ihn

38 Das ist ein Verlobungsgeschenk, vgl. M. Th. FöGEN, s.v. Arrha sponsalicia, in: $O D B$ 1, $185 \mathrm{f}$.

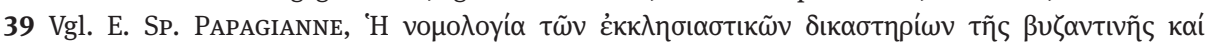

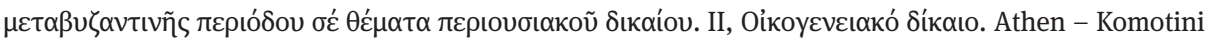
1997, 9, und KraUs, Ehe und Verlobung (wie Anm. 14) 45.

40 In der Akte taucht der Begriff allerdings nicht auf. Eine Esōnymphē (im Text ebenfalls nicht als solche bezeichnet) begegnet in der Akte PRK \# 89, vgl. unten bei III.2. - Zum Phänomen der Hausgemeinschaft mit einem/r Esōgambros/Esōnymphē vgl. D. Simon, Byzantinische Hausgemeinschaftsverträge, in: F. Baur - K. Larenz - F. Wieacker (Hrsg.), Beiträge zur europäischen Rechtsgeschichte und zum geltenden Zivilrecht. Festgabe für Johannes Sontis. München 1977,91-128. Der Begriff Esōgambros ist erstmals in der Peira (11. Jh.) belegt. 
eine Archontin, der er unterstand, gewaltsam gezwungen hatte. (Dazu gleich mehr in III.2).

\section{III.2 Gewaltanwendung mit Todesfolge und sonstige Gewalt- oder Druck-Ausübung}

Von Gewaltdelikten mit Todesfolge ist in zwei, vielleicht sogar in drei Akten (zwei aus den Apokaukos-Akten: Lam \# 2 und \# 10; eine aus den PD: \# 129) die Rede. Andere Formen von Gewalt kommen in den Akten Lam \# 28 [halb] (Missbrauch der Amtsautorität/-Gewalt), $P D$ \# 141* (elterliche Gewalt gegen die Tochter durch Züchtigung), und PRK \# 89 (häusliche Vergewaltigung durch den Ehemann) zur Sprache.

So betrifft Lam \# 2* einen Mord bzw. Totschlag im Affekt, den ein Basileios Kaliges aus Leukas, angestachelt von seiner Ehefrau, an seinem Sohn Konstantinos im Jahr 1217 beging, der ohne Zustimmung der Eltern die hübsche Pankalo geheiratet hatte. Da das junge Paar bei den Eltern noch wohnte, hatte sich die Abneigung der Schwiegereltern gegen die Braut so sehr gesteigert, dass es zu diesem Gewaltakt kam. Die Untat des Vaters Basileios Kaliges wurde mit auf 5 Jahre angelegten Bußstrafen geahndet.

Klar ist der Sachverhalt auch bei der Akte $P D \# 129$, dem 2. Bußbescheid für die bereits 1216 wegen (sukzessiver) Bigamie (siehe $P D$ \# 9) mit Bußstrafen belegte Zoe aus dem Thema Koloneia (im Bistum Diabolis/Devol). Diesmal war sie wiederum freiwillig vor dem Kirchengericht erschienen, um zerknirscht ihre Mitschuld am Tod eines des wiederholten Diebstahls überführten Bediensteten zu bekennen: Sie hatte die übrige Dienerschaft ihres Hause veranlasst, den Betreffenden zu ergreifen und ihm die Hände ohne Wundversorgung $\mathrm{zu}$ amputieren, so dass er verblutete und starb. Das Gericht verordnete ihr in Anbetracht ihrer weiblichen Schwäche sowie ihrer Reue ergänzende Bußstrafen auf drei weitere Jahre (zur bereits bestehenden zweijährigen). ${ }^{41}$

Der Fall Lam \# 10 indes, der sich auf Korfu zugetragen hatte und 1222 auf Betreiben des dortigen Metropoliten Georgios Bardanes (1219 - ca. 1240/42) der in Arta tagenden Metropolitansynode von Naupaktos zur Revision vorgelegt worden war, betraf einen Jugendlichen/neaniskos NN, der möglicherweise durch Stockschläge eines Priesters zu Tode gekommen war. Der Junge hatte sich einst zur Zeit einer Hungersnot im Wald abends am Wegesrand schlafengelegt, als der Priester NN vorbeikam, der sich verlaufen und in der Dunkelheit Angst bekommen hatte. In Erwartung, der Junge könne ihm den Weg zeigen, forderte er ihn lauthals auf, ihm den Weg zu zeigen. Der aber, vom Hunger erschöpft, reagierte abweisend, so dass ihn der Priester mit seinem

41 Vgl. dazu zuletzt G. PRINZING, Hausbedienstete oder -Sklaven in Byzanz zwischen tödlicher Repression und größter Hochachtung. Ein Streiflicht anhand von vier konkreten Fällen, in: S. Hanß - J. Schiel (Hrsg.), Mediterranean Slavery Revisited (500-1800). Neue Perspektiven auf mediterrane Sklaverei (500-1800), with editorial assistance by C. Schmid. Zürich 2014, 187-199, hier 191-194. 
Wanderstock zwei-dreimal traktierte. Zwar gelangte jeder von beiden nach Hause, doch der Junge starb einen Monat später. Nachdem der frühere Metropolit von Korfu, Basileios Pediadites (gest. 1217/18) (2) $^{2}$ den Priester für schuldig am Tod des Jungen gehalten und auf 11 Jahre vom Dienst suspendiert hatte und nun schon drei weitere Jahre vergangen waren, kamen in der Synodalsitzung zu Arta nun Zweifel am Urteil des Pediadites wegen unklarer Beweiserhebung auf, weil z.B. nicht bewiesen war, ob der Tod des Jungen absichtlich herbeigeführt worden war. Daher hat man das frühere Urteil des Pediadites kassiert. ${ }^{43}$

Ein Beispiel dafür, dass zumindest gelegentlich auch auf Adoleszenten durch einflussreiche Personen (oder Archonten) erheblicher Druck ausgeübt worden ist, damit z.B. eine ihnen irgendwie nahestehende oder von ihnen protegierte Person doch noch geehelicht wird, bietet die Apokaukos-Akte Lam \# 28 [halb]: Darin geht es, wie schon in III.1 angedeutet, in erster Linie um das Gesuch des 18-jährigen Anagnosten/Lektors Ioannes, von seiner viel älteren, „barbarisch“ sprechenden Frau Rusa, die aus der Belechatuia stammte, geschieden zu werden. Denn die Ehe war nicht nur bisher nicht vollzogen, sondern ihm auch aufgezwungen worden durch die Prōteuusa, d.h. die Archontin der (bergigen Region bzw. des Drungos) Malaina, ${ }^{44}$ kyra Theodora (Tochter des Indanu), der beide, Ioannes und Rusa, unterstellt waren: Da Ioannes sogar wegen seiner anfänglichen Weigerung, diese Ehe einzugehen, durch Anordnung der Proteuusa Theodora quasi in ,Beugehaft' genommen wurde, steht fest, dass diese Dame ihre Amtsgewalt rüde missbraucht hatte, um die ältliche, fast zahnlose Rusa, bei der es sich um anscheinend um eine Vlachin gehandelt hat, ${ }^{45}$ unter die Haube zu bringen. Der Fall hat einigen Wirbel verursacht, nämlich den Widerstand der örtlichen Kleriker hervorgerufen, da laut Akte „keiner von den Priestern des Ortes, nachdem sie von der Gewaltanwendung und der Unfreiwilligkeit des Lektors erfahren hatten, in der Kirche die Segnung dieser Ehe vollzog, sondern ein anderer aus einer anderen Provinz, der von der Urheberin dieser Ungerechtigkeit herbeigerufen worden war". ${ }^{46}$ Es war wohl die Verweigerungs-Haltung der örtlichen Priester, die den jungen Lektor zusätzlich darin bestärkte, nun auf seine Weise sich aus der Zwangsehe zu befreien. Jedenfalls hielt Apokaukos im Protokoll Folgendes fest: „Und sobald die zwangsweise

42 Vgl. Dem. Chom., Ponemata (wie Anm. 12) 70* und 106*.

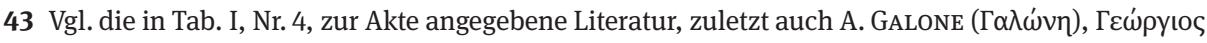

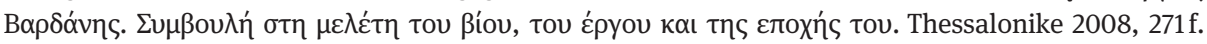
44 Vgl. hierzu G. PRINZING, Historisch-geographische Bemerkungen zu Carev dvor und Malaina. BSl 49 (1988) 213-221, hier $220 \mathrm{f}$.

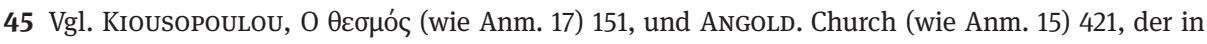
seiner überaus knappen Paraphrase der Akte Theodora als „lady of the manor“ bezeichnet und Rusa als „one of her aging Vlach servants“. Hier sei noch kurz darauf hingewiesen, dass Ioannes mit seinen 18 Jahren wohl vorzeitig zum Anagnosten gemacht worden ist, weil ein solcher an sich mindestens 20 Jahre alt sein sollte, vgl. BECK, Kirche (wie Anm. 9) 79 u. KRAUs, Kleriker (wie Anm. 14) 23 f. und 319.

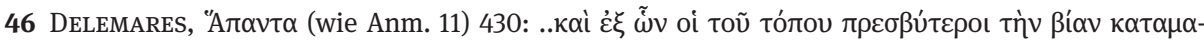

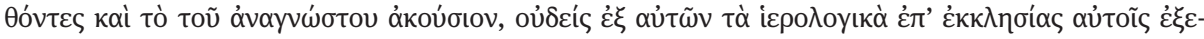

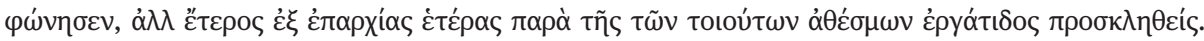


Segnung vollzogen war, sich nächtliche Finsternis über die Erde ausbreitete und man hoffte, dass Johannes mit seiner Ehefrau (und ihrem Alter nach: Großmutter) schlafen würde, ist er - ohne dass alle anderen etwas mitbekamen (wörtlich: verborgen vor den Augen aller), da sie mit Trommeln, Tänzen und Hochzeitsgesängen beschäftigt waren - gleichzeitig aus dem Brautgemach entflohen und hat von da an bis jetzt bald bei diesem, bald bei jenem in Dienst gestanden und die verblühte Braut ohne Bräutigam hinterlassen. Seinen Unterhalt verdient er mit seiner eigenen Hände Arbeit“. ${ }^{47}$ Apokaukos betonte in seinem Urteil die Nichtigkeit dieser Ehe, weil sie zwangsweise, ohne die unbedingt notwendige Zustimmung herbeigeführt wurde, verfügte daher die Scheidung. Auch die mit dem Antrag auf Scheidung verbundenen zwei Zusatz-Fragen des Ioannes, ob er wieder heiraten und auch Priester werden dürfe, bejahte Apokaukos (vgl. auch bei III.3).

Mit dem gerade behandelten Fall im Kern vergleichbar ist, wenn auch nicht in den äußeren Bedingungen, der oben erwähnte Fall aus PD \# 141: Auch in dieser Akte geht es um die Scheidung einer zwangsweise geschlossenen Ehe: der des mittellosen Handwerkers Theodoros Chloropodes und seiner Frau Eirene, die von ihm des unerträglichen Hasses und häufiger Aushäusigkeit (und damit Ehebruchs) angeklagt worden war. Es waren Eirenes Eltern, der gleichfalls arme Handwerker Michael Bodaniates und seine Frau, die ihrer Tochter, die eigentlich einen anderen Mann (den von ihren Eltern abgelehnten Georgios Cholaures) liebte, diese Ehe ganz gegen ihren Willen aufgezwungen hatten. Aus Abneigung und Hass gegen ihren Mann war sie, wie beim Prozess herauskam, kaum länger als zwei-drei Tage zu Hause, um sich umso länger auswärts aufzuhalten, so dass ihre Eltern sie öfter, auch unter Gewaltanwendung (sie griffen sogar zur Peitsche), zu ihrem Mann zurückgebracht haben. Eirene steigerte sich vor Gericht in Selbstmorddrohungen für den Fall, dass die Scheidung nicht vollzogen würde. Das Gericht schied die Ehe wegen Eirenes Ehebruch, Chloropodes durfte eine neue Ehe eingehen, Eirene indes wurde nur mit den für sie anzuwendenden Bußstrafen belegt, nicht aber mit materiellen Strafen, weil sie mit der Armut, in der sie ohnehin lebte, bereits gestraft genug sei. - Es ist anzunehmen, dass solche Fälle elterlicher Gewaltausübung zu dem Zweck, eine aus ihrer Sicht ungebührlich renitente Tochter daran zu hindern, aus der ihr verhassten, weil bereits aufgezwungen Ehe auszubrechen und sie ihrem Ehemann wieder gefügig zu machen, nicht gerade selten waren. Schließlich ist aber noch auf die Akte PRK \# 89 hinzuweisen, weil sie zeigt, wie gefährlich gerade für junge Mädchen (grundsätzlich aber für Kinder beiderlei Geschlechts) die Tatsache war, dass es bis in die spätbyzantinische

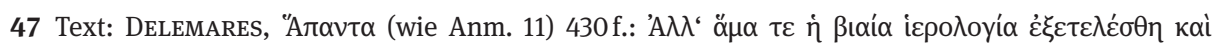

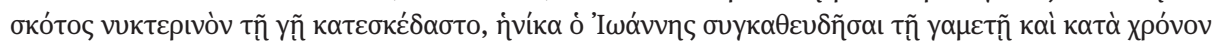

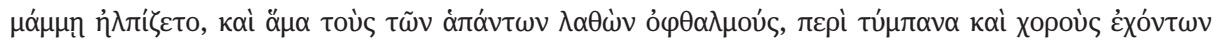

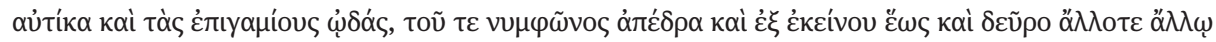

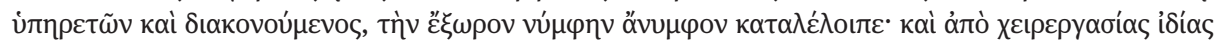

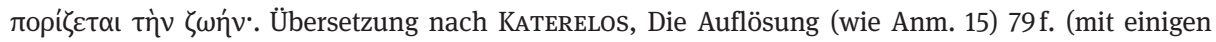
Änderungen). 
Zeit vorkam, dass Eltern ihre Kinder gern auch schon vor dem gesetzlichen Ehefähigkeitsalter von 12 (bei Mädchen) und 14 Jahren (bei Jungen) verheirateten. ${ }^{48}$ Denn in solchen Umständen konnte es sich eben auch, wie in diesem Fall, zutragen, dass der Bräutigam bzw. Ehemann versuchte, das 11-jährige Mädchen auch in seinen (oder seiner Eltern) Haushalt aufzunehmen (analog dem Brauch, dass Brauteltern den Bräutigam als sog. esōgambros bei sich wohnen ließen). Wenn sich der Vater der Braut, wie hier berichtet wird, darauf einließ und glaubte, mit Hilfe eines dem jungen Ehemann abgenommenen Versprechens es ausschließen zu können, dass dieser mit seiner bei ihm wohnenden Braut/Frau nicht sexuell verkehren würde, solange sie noch nicht ihr gesetzmäßiges Heiratsalters erreicht haben würde, der handelte (jedenfalls aus heutiger Sicht) letztlich fahrlässig: Aber auch als Byzantiner hätte er wohl damit rechnen können, dass sich der Bräutigam nicht daran halten würde. Jedenfalls trat genau das hier ein, obwohl die Eltern beider Seiten Kleriker waren, und zeitigte schlimme Folgen: Das junge Mädchen wurde dabei offenbar vergewaltigt und so schwer verletzt, dass es dauerhafte körperliche Schäden erlitt. Die Ehe wurde, weil zu früh geschlossen, geschieden, und der Schwiegervater der NN, der archōn tēs ekklēsias Konstantinos Argyros, dazu verurteilt, dem Brautvater nicht nur die Mitgift zu erstatten, sondern ihm auch das sog. Theōretrohypobolon, die voreheliche Gabe, bestehend aus theōretron und hypobolon, zu entrichten. ${ }^{49}$

\section{III.3 Eherechts-Problematik, auch mit Blick auf eine kirchliche Karriere $(E+K)$ oder mönchische Lebensweise $(E+M L)$}

Nach dem, was oben zur ,Zuständigkeit‘ der kirchlichen Gerichte und zur Zusammensetzung der Aktenmenge gesagt wurde, überrascht es nicht mehr, dass zu diesem Themenkomplex insgesamt (vgl. auch Tabelle I) relativ viele (17) Akten vorliegen: so

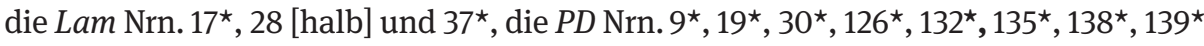
und $141^{\star}$, und die PRK Nrn. $11^{\star}, 14^{\star}$ [halb], 89*, 92*, 442 und $464^{\star}$ [halb]. Einige davon wurden schon zuvor näher behandelt: Lam \# 17* oben in III.1, \# 28 [halb] in III.2; PD \# $126^{\star}$ in III.1 und \# 141* in III.2; die PRK Nrn. \# 11* und \# 14*, in III.1, \# 89* indes in III.2.

Da die $P D \# 9$, 30* sowie $P R K$ \# 442 (zur Frage, ob eine dritte Ehe eingegangen werden darf), aber auch PD 19* oder \# 139* (Prozess des Stratioten Rados gegen seine 13-jährige Frau Slaba, die von ihrer Dienerin zum Ehebruch mit deren Bruder verführt wurde) wegen der Inhalts-Angaben in Tab. II nicht weiter kommentiert zu werden

48 Vgl. M. Th. FöGEN, Rechtsprechung mit Aristophanes. Rechtshistorisches Journal 1 (1982) 74-82, hier: 77: „In unzähligen Fällen dürfte der Verstoß gegen die Gesetze folgenlos geblieben sein. Denn hatte die frühe Ehe Bestand, so gab es niemanden, der Anstoß genommen hätte. Gerichtskundig wurde die Sache erst, wenn ein Partner, eine Familie oder auch beide an der Auflösung der Ehe ein Interesse hatten."

49 Vgl. M. Th. FöGEN, s.v. Theoretron, in: $O D B$ 3, 2068f., und ANGold, Church (wie Anm. 15) 417. 
brauchen, ${ }^{50}$ kann man sich hier auf die nähere Diskussion der \# $132^{\star}$ und \# $135^{\star}$ beschränken, um dann gesondert auf die PD \# 138 ( E+ML) und PRK Nrn. 446 u. 125 sowie die Apokaukos-Akte Lam \# 28 [halb] (E+K) einzugehen.

Den wohl bemerkenswertesten Fall dieser Gruppe von Akten stellt wieder eine Apokaukos-Akte dar: Lam \# 37. Der Metropolit hatte (wohl 1200/1232) über den Antrag des vor ihm erschienenen Ehepaares, des schmächtigen, 16-jährige Konstantinos Papaioannopulos, und seiner stattlichen, ja üppigen, reifen Frau Anna, auf Ehescheidung zu richten. Die Ehe war schon im Kindesalter zur Zeit der Unmündigkeit beider Partner geschlossenen und eingesegnet worden, doch später niemals vollzogen. Konstantinos war Anna augenscheinlich überhaupt nicht gewachsen, so dass Anna auch schon fremdging. Entsprechend dem Wunsch beider Partner, hat Apokaukos die Ehe geschieden wegen der unzulässig früh vorgenommenen Verlobung nicht aber wegen der subtil, mit Witz und Ironie geschilderten Gegensätzlichkeit bzw. Inkompatibilität des $\mathrm{Paares}^{51}$, die er voll erfasst und so treffend geschildert hat, dass ihm das Protokoll nachgerade $\mathrm{zu}$ einem literarisch-juristischen Kabinettsstück geriet. $^{52}$

Die Akten PD \# 132 und \# 135* werden hier kurz besprochen, weil sie inhaltlich singulär in unserem Dossier sind: \# 132 gilt dem in Achrida abgehaltenen Scheidungsprozess des Ehepaares Georgios Serbopulos und seiner Frau Sbina. Es war sie, die ihren Mann verklagte (1216/22), da er seit fünf Jahren seinen ehelichen Pflichten nicht nachgekommen war. „Ihr kam auch deshalb die Scheidung in den Sinn, sagt sie, weil sie befürchte, dass, wie es natürlich ist, die Jugend sie irgendwann in eine gesetzwidrige Verbindung treiben würde “. ${ }^{53} \mathrm{Ihr}$ Mann stellte seine Impotenz zunächst in Abrede, räumte diesen Sachverhalt aber ein, als Sbina auf ihre Jungfräulichkeit hin untersucht werden sollte, und meinte, er habe sich für sie sexuell nicht entflammen können. Das Urteil, das zeigt, dass ein Scheidungsbegehren sowohl von der Frau (wie auch vom Mann) gerichtlich vorgebracht werden konnte, entspricht dem Antrag Sbinas und verfügt die Scheidung. Sbina darf ihre Mitgift behalten und erneut heiraten, wobei ausdrücklich noch festgestellt wird, es sei unnötig, dass das die junge Frau die Impotenz (w. das Unmännliche) weiterhin mit Schweigen bedecke, da die (sc. für eine Scheidung aufgrund von Impotenz erforderliche) gesetzliche Dreijahresfrist schon auf fünf Jahre ausgedehnt worden sei. ${ }^{54}$

50 Dies gilt für die Akte \# $139^{\star}$ auch deswegen, weil sie erst kürzlich kommentiert wurde, vgl. PRINZING, Hausbedienstete (wie Anm. 41) 194-195.

51 Vgl. dazu besonders LAIou, Institution (wie Anm. 17) $308 \mathrm{f}$.

52 Unbedingt lesenswert ist die mit einem eingehenden Kommentar verbundene brillante Übersetzung dieses Textes durch Marie Theres Fögen, angefertigt anhand ihres Neudrucks der Erstedition durch S. Pétridès [1909]): FöGEN, Rechtsprechung (wie Anm. 48).

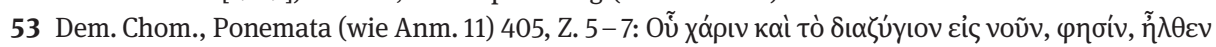

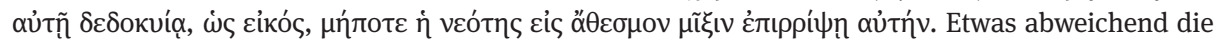
Übersetzung von KATERELOS, Die Auflösung (wie Anm. 15) 47.

54 Dem.Chom., Ponemata (wie Anm. 12) 405, Z. 24f. Vgl. Katerelos, Die Auflösung (wie Anm. 15) 47 und 123. 
In der Akte $P D \#$ 135* ist in der Streitsache des Ioannes, Sohn des Priestermönchs Barnabas, und einer gewissen Frau Dragoste die Rede. Beide waren vor Gericht erschienen (sie mit einem Säugling auf dem Arm): Er erklärte, beim stürmischen Drang der Jugend (akathektois hormais neotētos) mit Dragoste ein Konkubinat (kata pallakismon) gepflegt zu haben, jetzt aber sei er zur Wahrnehmung der Sünde gelangt und weise sie mit ihrem Wunsch auf dauerhaftes Zusammenleben ab. Sie bestätigte dies, glaubte aber nach fünfjährigem Zusammenleben sowie wegen des gemeinsamen Kindes (zwei weitere von ihm waren verstorben) ein Recht auf dauerndes Zusammenlebens mit ihm zu haben, und bat um die übliche Einsegnung. Chomatenos forderte Ioannes, Dragoste kirchlich zu ehelichen, doch dieser weigerte sich weiterhin. So verfügte der Erzbischof mit beiderseitigem Einverständnis, Dragoste solle für sich und das Kind die dem Ioannes gehörende Kuh und einen entsprechenden Anteil vom Saatfruchtertrag erhalten. Jeder könne dann nach Belieben das Seine tun.

Wie die Akte erkennen lässt, verstand Chomatenos in dieser Akte unter pallakismos ein längeres außer-eheliches Zusammenleben eines unverheirateten Mannes mit einer Frau. ${ }^{55}$ Festzuhalten ist, dass hiermit belegt wird, dass es innerhalb der byzantinischen Gesellschaft junge Leute wie dieses Paar gab, die längere Zeit ein Familienleben in einer an sich illegalen Verbindung führten, weil sie nicht regulär verheiratet waren. Zugleich wird deutlich, dass Frauen in einer solchen Beziehung auf jeden Fall die schwächere Position gegenüber dem Mann hatten, besonders auch dann, wenn sie den Versuch unternehmen wollten, diese Beziehung im Fall ihrer drohenden oder - wie hier - bereits eingetretenen Abkühlung in eine legale, kirchlich eingesegnete Ehe zu überführen, um für sich selbst mitsamt ihrem/n Kind(ern) in eine gesicherte Position zu gelangen. Wurde dies abgelehnt, wie in diesem Fall, konnte ein kirchlicher Richter mit Autorität wie Chomatenos ${ }^{56}$ immerhin den von beiden Seiten quasi als Urteil akzeptierten Vorschlag durchsetzen, dass Ioannes seiner bisherigen Gefährtin und ihrem Kindes seine Kuh überlässt und dazu einen entsprechenden Anteil am Ernteertrag der Saatfrucht. ${ }^{57}$ Das Alter von Ioannes und Dragoste zum Zeitpunkt ihres Scheidungsverfahrens ist unklar, sie könnten schon über 25 gewesen

55 Die Akte ist eine von insgesamt 11 Akten der $P D$, in denen Chomatenos die Begriffe pallakismos / pallakē verwendet, und zwar in drei Bedeutungsvarianten, vgl. dazu LAIou, Institution (wie in Anm.17), 284-300; dort auch allgemein (s. 284f. u. 296f.) zu dem in Byzanz seit dem 10. Jh. verbotenen Konkubinat, neben J. H(ERRIN) - A. K(AZHDAN), s.v. Concubinage, in: ODB 1, 493.

56 Vgl. zur Art seiner Autorität G. PRINZING, The Authority of the Church in Uneasy Times: The Example of Demetrios Chomatenos, Archbishop of Ohrid, in the State of Epiros 1216-1236, in: P. Armstrong (Hrsg.), Authority in Byzantium. London 2013, 137-150, bes. 142-150.

57 Vgl. zu dieser Akte Laiou, Institution (wie Anm. 17) 286, 293; DiEs., The Evolution of the Status of Women in Marriage and Family Law, in: Mother, Nun, Deaconess: Images of Women According to Eastern Canon Law (Kanon XVI). Egling 2000, 71- 85 (Ndr. DiEs., Women [wie in Anm. 17], Art. III), 76. Simon, Byz. Provinzialjustiz (wie Anm.3) 318 sieht die vorgesehenen Ab-Gaben des Ioannes an Dragoste als eine von ihr verlangte „Abfindung“ an, was aber nicht dem Text zu entnehmen ist, daher Vermutung bleibt. (N.b.: Lies dort am Ende des 1. Absatzes Nr. 135 statt 136). 
sein, aber der Hinweis auf gegenseitige Zuneigung in der Jugend rechtfertigt die Hereinnahme der Akte in die Tabelle II.

Nun zu den zwei thematischen Sonderfällen dieses Teilabschnitts, zu denen dreieinhalb Akten gehören, einerseits die zwei PRK Nrn. 464 u. 125 sowie - zur Hälfte - die Apokaukos Akte Lam \# 28 [halb], andererseits die PD \# 138.

Sonderfall 1: Eherechtsproblematik mit Blick auf die kirchliche Karriere (E+K): Der eine der beiden Hauptfälle zu diesem Thema ist enthalten in PRK \# 464. Der Synodalbescheid an den Metropoliten von Methymna (Lesbos) aus dem Jahr 1394 handelt vom Gesuch des Demetrios Asomatianos, der Geistlicher werden wollte: Seinem Bericht nach war er vor einiger Zeit einzig durch eine Übereinkunft mit der Tochter NN des Nikolaos Bambakas verlobt worden. Dies ging vonstatten ohne Verlobungsurkunde und Enkolpion. Nun aber wollte er diese Abmachung wegen der Minderjährigkeit (auch Unreife im Hinblick auf einen Heiratstermin) ${ }^{58}$ des Kindes des Nikolaos aufheben und mit einer anderen Frau eine legale Ehe eingehen. Er ersuchte nun die Synode um einen Synodalbeschluss, „dass er, wenn er in Bezug auf das Verübte unschuldig ist, in das Diakons-/Priesteramt befördert [oder: zum Diakon/Priester geweiht wird]“. ${ }^{59}$ Der Patriarch wies den Adressaten an, den gesamten Vorgang, genauestens zu überprüfen, auch anhand der Auskunft des Betroffenen, und schriftlich Bericht zu erstatten. Im Fall des Asomatianos erfährt man übrigens nichts über seine familiäre Stellung zum Zeitpunkt seiner Verlobung, so dass auch unbekannt bleibt,

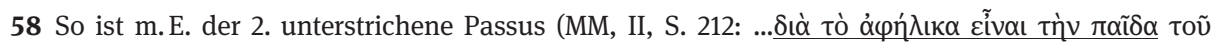

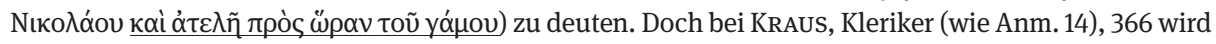
daraus: „Eine Eheschließung habe bis dato nicht stattgefunden.“ Kraus gelangt zu dieser abwegigen Deutung, weil er die erste unterstrichene Passage bei seiner Paraphrase der Akte völlig ausgeblendet und dann auch übersehen hat, dass sich der zweite unterstrichene Passus auf die minderjährige Tochter des Nikolaos bezieht.

59 MM II, 212, wo m.E. der Text verderbt ist und wie folgt lauten müsste (die Neuedition wird dann

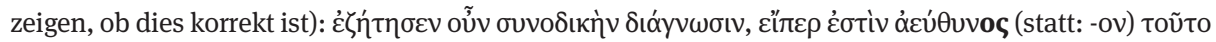

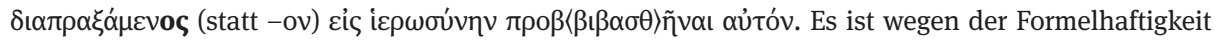
des unterstrichenen Ausdrucks die dortige Ergänzung vorzunehmen, vgl. dazu Ch. KRAUs, Xeıрoтovía,

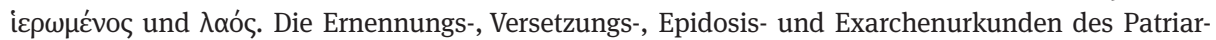
chatsregisters von Konstantinopel: Die Weihen, Einsetzungen und die kirchlichen Grupen, in: Gastgeber - Mitsiou - Preiser-Kapeller, The Register (wie Anm. 12) 139-151, mit Belegen auf 156, Anm. 79 (zu PRK II \# 136, S. 288, Z. 8), 157 Anm. 83 (zu PRK I \# 70, S. 418, Z. 28 f. ) und ebd. Anm. 88 (zu MM I \# 228, S. 489, Z.27 f.), sowie die von ihm übersehene Stelle PRK I, \# 125, S. 184, Z. 35. Der Begriff hierōsynē hat im PRK in der Regel die Bedeutung Priesteramt, Priesterwürde oder Weihegrad, vgl. C. Cupane - E. Schiffer (unter Mitarbeit v. E. Kislinger), Das Register des Patriarchats von Konstantinopel. Indices zu den Urkunden aus den Jahren 1315-1356. Wien 1995, s.v. 190. Gelegentlich aber kann er sich auf die Diakonsweihe beziehen (vgl. Kraus, Kleriker [wie Anm. 14] 365, Anm. 249, mit Hinweis auch auf den hier oben zitierten, damals noch ungedruckten Cheirotonie-Artikel), für die das Mindestalter $25 \mathrm{~J}$. traditionell vorgeschrieben war, vgl. ebd., 23f. Vgl. auch hier unten bei IV, PRK \# 392. Daher könnte im Fall des Asomatianos sehr gut der Diakonsweihegrad gemeint sein, so dass A. vielleicht auch noch Adoleszent war, als er sich an die Patriarchatssynode wandte. 
wer von seiner Seite damals die Verlobungsvereinbarung mit dem Brautvater Nikolaos Bambakas getroffen hat.

Anders war es im zweiten Hauptfall, dem der Akte PRK \# 125 vom Mai 1340: Der Petent Demetrios Eskammatismenos, ein Kleriker (wohl Anagnost oder Hypodikakon) aus Thessalonike, war als Vollwaise aufgewachsen, betreut von seinen beiden älteren Brüdern. Sie waren es, die vor langer Zeit mit dem Neffen NN des Michael Phortos ${ }^{60}$ lediglich vereinbart hatten, Xene, die Tochter des NN mit ihm, Eskammatismenos, zu verloben. Nach Umsetzung der Vereinbarung, habe er dann er erkannt, dass Xene gänzlich minderjährig (aphēlika pantelōs) sei, und die Vereinbarung umgehend für nichtig erklärt. Auch seitens der Ortskirche galt sie nach einer Besprechung als ungültig und gesetzwidrig, weil das Mädchen noch nicht das für die Eheschließung gesetzliche Mindestalter besaß. Einige Zeit danach heiratete er legal (war also mindestens 14jährig) eine (andere) Frau. Als er um die (sc. Diakons-/Priester-)Weihe

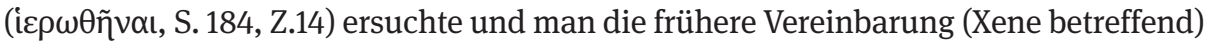
ansprach, wies der Metropolit von Thessalonike seine Beamten an, den Vorgang zu untersuchen (durch Befragung mehrerer Familienmitglieder und Verwandter der Xene bzw. des Portos). Daraus habe sich klar ergeben, dass sie bei Inkrafttreten der Vereinbarung „etwas über fünf Jahre alt“ (S.185) gewesen sei. - Die Patriarchatssynode war also doppelt instruiert, durch Eskammatismenos selbst und durch den schriftlich vorliegenden Bericht aus Thessalonike. Man entschied, dass die besagte Vereinbarung ungültig war, da sie gegen das (von Leon VI. und Alexios I.) für die Braut vorgeschriebene Mindestalter von 12 Jahren verstieß sowie die erforderliche kirchliche Einsegnung fehlte. Daher stünde der Weihe des Eskammatismenos zum Diakon/ Priester nichts im Wege. ${ }^{61}$ - Er musste nur mindestens 25 Jahre alt gewesen sein, wenn er, wie zu vermuten ist, zunächst wohl doch eher das Diakonat im Blick hatte als das Priesteramt, für das er mindestens 30 sein musste.

Vergleichbar war in beiden Fällen also die recht ähnliche Ausgangslage, die aus der jeweils auf völlig gesetzwidrige Weise durch Dritte eingefädelten und zustande gekommenen Verlobung mit einem altersmäßig noch gar nicht heiratsfähigen Mädchen bestand. Da dieser Umstand beiden Kandidaten bei ihrem jeweiligen Gesuch, die geistliche Laufbahn (letztlich zum Priesteramt) einzuschlagen und sich (neu) zu verheiraten, potentiell gefährlich werden konnte ${ }^{62}$ waren beide mehr oder weniger schnell darauf erpicht, diese gesetzwidrige Verlobung zu annullieren. Eskammati-

60 Phortos/ PLP 30020 (hier Phortes) war der Bruder eines Drungarios, wie aus der Akte im letzten Drittel hervorgeht.

61 Vgl. im Hinblick auf die Weihe im Text der Akte bes. S.184, Z. 14-15 und 35-36), dazu KRAUS, Kleriker (wie Anm. 14) 149f. und besonders oben Anm. 59.

62 Vgl. KraUs, Kleriker (wie Anm. 14) 25 (mit weiteren Nachweisen): „Kandidaten für die Weihe können im Prinzip verheiratet und unverheiratet sein. Eine Heirat nach dem Empfang der Hypodiakonsweihe ist nicht mehr möglich. [...] Kein Kleriker darf ein sukzessiver Bigamist (zweimal verheiratet) oder mit einer Witwe verheiratet sein. [...]. Anagnosten können ein zweites Mal heiraten, allerdings ist dann eine höhere Weihe unmöglich. Ehe und förmliche Verlobung werden hier gleich gewertet.“ 
smenos soll das nach Inkrafttreten der Verlobung umgehend bei der Ortskirche (Thessalonike) in Angriff genommen haben; Demetrios Asomatianos tat das erst relativ kurz vor seiner Eheschließung und der Bewerbung um das geistliche Amt direkt in Konstantinopel. Bei Eskammatismenos nahm auf sein Betreiben die für ihn zuständige Metropolitansynode eine erste Untersuchung seiner Verlobung vor und erklärte sie für gesetzwidrig, so dass er auch bald, diesmal ganz legal, sich verheiratete, um danach das geistliche Amt anzustreben. Patriarch und Synode sahen am Ende kein Problem in seiner früheren gesetzwidrigen, daher nichtigen, durch Absprache seitens Dritter vorgenommenen Verlobung, und ebenso dürfte auch in Thessalonike am Ende für Asomatianos entschieden worden sein. Was wir in beiden Fällen nicht genau wissen, ist das Alter beider Kandidaten. Ich vermute aber (oder möchte es nicht ausschließen), dass beide zur Zeit ihres Antrags an die Patriarchalsynode noch Adoleszenten waren (vielleicht kurz vor 25).

An dieser Stelle sei nochmals daran erinnert, dass, wie in III.2 ausgeführt, auch schon der von Apokaukos entschiedene Antrag des Anagnosten Ioannes (Lam \# 28 [halb]) den beiden eben behandelten Fällen darin glich, dass der Antrag des Petenten auf Ehescheidung mit der Bitte verbunden war, die Erlaubnis zu erhalten, eine neue, reguläre Ehe eingehen und Priester werden zu dürfen (den niederen Weihegrad des Anagnosten besaß er schon). Beides wurde zugelassen. ${ }^{63}$

Sonderfall 2: Eherechts-Problematik mit Blick auf mönchische Lebensweise (E +ML): Er liegt vor in die Akte PD \# 138, einem Bußbescheid des Chomatenos, wohl aus dem Jahr 1222. Er betrifft den Bruch des Gelübdes, das ein aristokratisches Ehepaar, der noch adoleszente Georgios Komnenos (analog auch seine ebenfalls adoleszente Frau Theodora) knapp acht Jahre zuvor abgelegt hatte, als er schwer erkrankt war, wegen irriger Prognose eines unfähigen Arztes den baldigen Tod vor Augen sah und ihm Verwandte zu dem Gelübde geraten hatten. Darin versprach er, den Mönchshabit acht Jahre nach seiner Genesung zu nehmen. Er genas aber und bat nun, kurz vor Ablauf der Frist - angesichts seiner Furcht vor dem Zorn Gottes bei Bruch seines Gelübdes, aber auch wegen der Befürchtung, gegen das Gelübde in seinem jugendlichen Alter zu verstoßen, zumal wenn er an seine junge und hübsche Frau denke um Besänftigung bzw. Linderung seiner doppelten Furcht. Beide Ehepartner erhielten wegen des Bruchs dieses Gelübdes, weil es nicht aus wirklich eigenem Antrieb geleistet worden war, diverse Bußstrafen auferlegt. Georgios trug übrigens nicht selbst sein Anliegen dem Gericht vor, sondern ließ sich durch Vertrauenspersonen, darunter der Bischof von Moglena, vertreten. - Dieser Fall, der einen Sachverhalt aus der religiösen Praxis bei lebensbedrohender Krankheit beleuchtet, ${ }^{64}$ ist in der Fallsammlung auch ohne Parallele.

63 Vgl. hierzu aus kanonistischer Sicht speziell Katerelos, Die Auflösung (wie Anm. 15) $155 \mathrm{f}$.

64 Zur Begründung der Zuordnung dieser Akte zu den Fällen eherechtlicher Problematik vgl. auch Dem.Chom., Ponemata (wie Anm. 12) 297*. 


\section{III.4 Fragen im Zusammenhang mit Karriere und Disziplin}

Die vier Akten zu diesem Themenbereich, die, wie kaum anders zu erwarten, sich alle in der Hauptsache auf Kleriker beziehen oder nur Geistliche betreffen, umfassen gemäß Tabelle I die Apokaukos-Akte Lam \# 28 [halb], sowie die PRK Nrn. 125 [halb], 136, 236, 392. Hiervon wurden die drei Halb-Akten bereits in III.3 unter Einschluss des Karriere-Aspekts behandelt, da dieser immer mit dem eherechtlichen Aspekt und der Frage nach der Möglichkeit einer zweiten Heirat verknüpft gewesen war. Von den noch verbleibenden Fällen wurde die Akte $P R K$ \# 392, in der es um die Anfrage des Subdiakons Manuel Sguropulos geht, schon in II.3 kurz angesprochen. Er hatte, wie aus der Akte, der von den Editoren (Miklosich/Müller) als Promissio bezeichneten urkundlichen Selbstverpflichtung vom 22. März 1387 hervorgeht, als 22-jähriger Subdiakon der Großen Kirche (der Hagia Sophia) den Patriarchen gebeten, ihn vorzeitig zum Diakon zu ernennen: Man entsprach seiner Bitte, ließ ihn aber sicherheitshalber auch urkundlich versprechen und unterschreiben, später auf Befragung hin dieses vorzeitige Alter stets korrekt anzugeben; anderenfalls verlöre er seine Stellung. Christof Kraus vermutet sicherlich zu Recht (m. E. auch im Hinblick auf die zentrale Stellung des Klerus an der Hagia Sophia), dass wohl ,außer dem Patriarchen selbst noch weitere Personen für die Entscheidung zum Weiheempfang zuständig“ waren und der Weihe zum Diakon ein von mehreren „Funktionsträgern“ vorgenommenes, im Ergebnis für den Patriarchen bindendes „Prüfungsverfahren“ vorausging, bei dem man sich auch über das Weihealter des Kandidaten erkundigte. ${ }^{65}$

Auch dem in PRK \# 136 (vom April 1342) als Petent an die Synode herangetretenen, offenbar gebildeten Laien (vielleicht auch Anagnosten?) und - wie aus der Anrede zu erschließen ist - Angehörigen der oberen Mittelschicht, ${ }^{66}$ kyr Michael Kabasilas, ging es nach der begründeten Ansicht von Kraus wohl um die Gewährung der Diakonsweihe (und nicht, wie es noch in der Übersetzung heißt, um die Beförderung ,in den Priesterstand“ [S. 289]). ${ }^{67}$ Für das genaue Alter Michaels fehlen entsprechende Angaben, aber er dürfte auf jeden Fall zur Zeit der Ausstellung der Akte bereits mindestens kurz vor 25 gewesen sein. Es steht fest, dass Kabasilas in der Zeit seiner Adoleszenz eine zunehmend wichtige Funktion im Haus seines Mentors einnahm: Dieser, der Metropolit und (vormalige, wegen des Verdachts auf Bestechung abgesetzte Katholikos Kritēs) Ioseph von Apros, hatte Kabasilas bereits als Kind in sein Haus aufgenommen, erzogen und ausgebildet: Schließlich heiratete er Iosephs Nichte und wurde dem Metropoliten auf „kaiserliche Verfügung hin untergeordnet“, ${ }^{68}$ so dass

65 KRAUS, Kleriker (wie Anm. 14) 325f.. (mit den Zitaten).

66 Ebenda, 328.

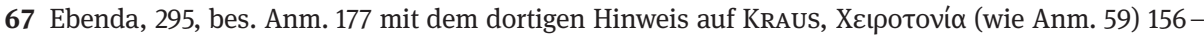
158, wo er seine These nochmals erhärtet hat, vgl. die oben Anm. 58 aufgelisteten Belege (unter Einschluss der Akte PRK \# 136).

68 Vgl. DöLgER, Regesten (wie Anm. 19). 4. Teil: Regesten von 1282-1341. München 1960, Nr. 2743 (vgl. auch PRK \# 136, S. 286). 
er in die Funktion eines Sekretärs und Dieners hineinwuchs. Als solcher war er befähigt, ihm „Urteile und Entscheidungen“ schriftlich auszuarbeiten (289). Kabasilas ist somit ein Beispiel dafür, dass und auf welche Weise in Byzanz, wie auch schon in mittelbyzantinischer Zeit, bereits im Kindesalter bis in die Adoleszenz hinein (im Normalfall) die Grundlage für eine spätere Karriere im Bereich der kirchlichen Hierarchie begründet werden konnte; es zeigt aber auch die Gefahren auf, die mit der jahrelangen, persönlichen Bindung an eine einflussreiche Person unter Umständen verbunden waren. Denn mit der Absetzung des Metropoliten und hohen Richters wegen des Vorwurfs der Bestechlichkeit, verlor Kabasilas Iosephs Rückhalt, so dass er sich nach einer neuen Stellung umsehen musste. Es gelang ihm, die erwünschte Diakonsstellung zu bekommen, obwohl es Stimmen gab, die ihn wegen seiner möglichen Verstrickung in Machenschaften seines Mentors und Dienstherrn von geistlichen Ämtern hatten fernhalten wollen.

Die dritte Akte, $P R K$ \# 236 enthält die im Jahr 1360 abgegebene Erklärung des Priesters Georgios Kallistos, der verstorbene Priester Manuel Kallistos (vielleicht ein Bruder oder enger Verwandter des Georgios?) habe nie den Knaben Alexios formell adoptiert. Das versichere er ausdrücklich vor dem Beichtvater des Manuel K. und Priestermönch Ioakeim. Diese Akte stellt im Vergleich mit der zuvor behandelten Akte eine Variante des Heranwachsens eines Jugendlichen in einem Klerikerhaushalt dar und wirft vor allem ein Schlaglicht auf das Leben des Vollwaisen Alexios aus WestAnatolien, den Manuel in seinen Haushalt mit aufgenommen hatte. Alexios lernte bei ihm „Lesen und Schreiben“ und fungierte auch jahrelang als sein Diener, bis der Einzug des Alexios zum Militär und entsprechenden Fronarbeiten drohte. Um dies zu verhindern, soll Manuel den Alexios als sein Kind ausgegeben haben. Darüber einmal in der Kirche durch Alexios befragt, was er (Manuel) denn (von ihm) wolle oder in Bezug auf ihn vorhabe, habe dieser (Manuel) geantwortet: Er schwöre bei der Theotokos, dass Alexios, wenn er sich nur richtig und ordentlich verhalte, von nun an sein Kind sei.- Einige Zeit später, sei Alexios der Trunksucht verfallen und in seinem Lebenswandel heruntergekommen. Darum habe Manuel ihn aus dem Haus gejagt, so dass Alexios nie wieder dessen Haus und Hof betreten habe, auch nicht nach Manuels Tod. Von einer Adoption zu dessen (Alexios') Gunsten habe er nie etwas vernommen, es habe sie nicht gegeben. ${ }^{69}$ (Außerdem bestätigten Georgios Kallistos und andere Priester, der Anagnost Georgios Euschemanos sei der Diakons-Weihe würdig).

Am Ende muss man sich im Hinblick auf Alexios fragen, wie glaubwürdig dieses Schlaglicht auf sein Leben ist. Aufgrund des letztlich negativen Tenors dieses merkwürdigen Dokuments, dessen Anlass sich nicht hinreichend genau klären lässt, aber doch wohl bezweckt, die (von unbekannten Dritten) behauptete Adoption, die man seit Leon VI. [Nov. 24] im Zuge einer kirchlichen Einsegnung legal vornehmen konn-

69 Vgl. zur möglichen Deutung dieser Aussagen und des ganzen Vorgangs KRAUs, Kleriker (wie Anm. 14) 324 Anm. 41. 
te, ${ }^{70}$ in Abrede zu stellen, könnte es durchaus sein, dass speziell die negative Entwicklung, die Alexios bald nach dem Gespräch genommen haben soll, eine aus welchen Gründen auch immer in Umlauf gesetzte Erfindung der Aussteller der Akte war. Da beide Hauptakteure, Manuel und Alexios tot waren (oder hatte man Alexios nur für tot erklärt?) und keine sonstigen Zeugnisse von ihnen überliefert sind, bleibt es unklar, was wirklich geschehen ist.

\section{III.5 Vermögens- und erbrechtliche Probleme}

Die oben im Abschnitt II.1 (vgl. auch Tabelle I) zur Quantität der einzelnen Aktengruppen gemachten Angaben zeigten bereits, dass in den $P D$ und im $P R K$ die Akten über Fälle zum Themenbereich der vermögens- und erbrechtlichen Fragen die jeweils größte Menge ausmachen: 14 in den $P D$ (Nrn. 29, 42, 32, 60, 64* , 73, 81, 83, 84, 85, 90, 91 u. 105 [bedingt 99]) und 14,5 im PRK (Nrn. 14* [halb], 100* [+ 102*], 553/1+2, 569*, 554, $571,575,584,587,610,613,646^{\star}$ und $\left.678^{\star}\right)$.

Da man sich aus den unterschiedlich knappen Inhaltsangaben in Tabelle II einen hinreichend genauen Überblick zu den Fragen verschaffen kann, aus welchen Gründen, in welchem Kontext und mit welchem Ergebnis Adoleszenten in die hier registrierten Verfahren involviert waren, soll hier im Prinzip auf eine breitere, ausführliche, teils auch vergleichende Darstellung bzw. Analyse der einzelnen Fälle aus diesem Segment verzichtet werden; zumal das in einigen Einzelfällen - wie auch bei den vorigen Teilabschnitten - auch schon an anderer Stelle geschehen ist: So wurden die PD Nrn. \# 29 u. 42 (zum Petenten Melias aus Berroia) in II.2 näher vorgestellt, in III.1 jedoch die PD Nrn. 85 und 99, sowie, wenn auch in geringerem Umfang, die Nrn. 91, 42, 84.

Verzichtet man also auf eine breitangelegte vergleichende Einzelanalyse all dieser Fälle und strukturiert sie stattdessen in einer Übersicht, wie sie im Anhang A (Überblick zur Fall - Diversität bei den erb- und vermögensrechtlichen Fällen) vorgenommen wurde, so erkennt man Folgendes: Alle PD Akten aus diesem Segment und nur wenige $P R K$ Akten finden sich in der Kategorie I.1: Sie umfasst die Fälle zu Streitsachen/Prozessen mit der Konstellation A(doleszent) gegen andere (oder umgekehrt). Bei den $P D$-Akten handelt es sich einerseits um vier Fälle, die zu Prozessen (Nrn. 73, 83 [Fall der 4 Waisen Lubroi], 91 u. 105) und entsprechenden Urteilen führten, andererseits um 10 Streitfälle, bei denen es sich um Bescheide/Stellungnahmen zu noch anhängigen bzw. bislang nicht endgültig entschiedenen oder noch immer schwelenden Streitfragen handelte. $\mathrm{Zu}$ den noch nicht endgültig entschiedenen Bescheiden zählen die der Akten Nrn. 29 und 42 (Melias betreffend), dann die der Nrn. 32, 60, 64* ${ }^{\star}, 81,84,85$ (Israel/Manuel betreffend), 90 und 99, von denen \# 81 besonders komplex ist und \# 90 zeigt, wie unendlich lang ein Verfahren sich hinziehen konnte,

70 Vgl. R. M(ACRIDES), s.v. Adoption, in: ODB 1, 22, und Miller, Orphans (wie Anm. 26) 171. 
wenn der an sich rechtlich unterlegene, aber mächtige Gegner (ein Onkel des Klägers) gar nicht daran dachte, sich irgendwann mit einer Niederlage abzufinden. ${ }^{71}$ In fast allen diesen Prozessen bzw. Streitfällen aus den $P D$ ging es Adoleszenten (oder den für sie Agierenden) um die Rückgewinnung des ihnen tatsächlich oder vermeintlich zustehenden, aber von Mitgliedern der engeren Familie (inkl. Brüdern oder Stiefvätern), Verwandten oder Dritten auf verschiedene Art und Weise scheinbar legal, gerichtlich oder auch gewaltsam abspenstig gemachten Grundbesitzes. Nur in einem Fall (\# 105) ${ }^{72}$ gab das Synodalgericht einem Petenten Recht gegen die Partei des Adoleszenten. So im Streit des Leon Kontos aus Bodina mit der Witwe Anna he tu Kometos des Anagnostes Ioannes Kukumes und ihrem Sohn, dem Adoleszenten Konstantinos: Anna hatte einst an Kontos als Vormund für ihre beiden Söhne zwei brachliegende Weinberge aus deren väterlichem Erbe verkauft, später aber hartnäckig versucht, diesen legal abgewickelten Verkauf rückgängig zu machen. Doch vergebens, sie unterlag in zwei Gerichtsverfahren in Berroia. Unbeeindruckt davon, besetzte sie kurzerhand die von ihr beanspruchten Grundstücke. Daher bat Kontos in Achrida um Auskunft, ob sie hierzu berechtigt sei. Der Synodalbescheid stellte klar, dass Anna samt ihren Söhnen keinerlei Anrecht mehr auf diese Grundstücke habe, da am Verkauf nichts zu beanstanden und Kontos der rechtmäßige Eigentümer sei. Aber schließlich schlug man Kontos in Achrida seitens des Gerichts (!) vor, in Erwägung zu ziehen, Anna mit folgendem Kompromiss zu beruhigen: Leon werde auf jeden Fall 1/3 der Fläche unangefochten behalten, während er 2/3 von ihr an Anna (und ihre Söhne) von sich aus übergeben würde: Dafür sollte sie ihm jedoch eine (nach amtlicher Schätzung) festgesetzte Summe zur Kompensation seiner Aufwendungen für die Melioration zahlen. So hat Annas Sturheit am Ende nicht dem Recht, sondern ihrem Egoismus vielleicht doch noch zu einem Teilerfolg verholfen.

Unter den oben angeführten Fällen aus dem $P R K$ finden sich indes kaum Prozesse (nur zwei) und nur eine Streitsache mit Bescheid (PRK \# 14* [halb]), wobei sich die beiden Prozesse, wie auch bei den $P D$ um Probleme mit (Erb-)Grundbesitz drehten. Ob dies auch bei \# $14^{\star}$ [halb] der Fall war, ist unklar, weil Immobilien nicht explizit genannt werden. Allein dieser Fall aber (der auch teils einem familienrechtlichen Aspekt gilt) ist nicht nur der einzige zu einer Streitsache mit Bescheid, sondern auch der einzige PRK Fall aus Kategorie I.1, der für die betroffene Adoleszentin (die anonyme Zieh- und Adoptivtochter der Megalē Papiaina Nestongonissa) negativ ausgeht. Demgegenüber endeten bei den $P D$ Akten die drei Prozesse ganz oder zumindest teilweise positiv, wie auch die meisten Streitsachen mit Bescheid positiv für die Adoleszenten ausgingen, nur zwei indes negativ (\# 60 [vgl. Anm. 72] und \# 105, vgl. aber oben!).

71 Vgl. die erhellenden Schlussfolgerungen von Simon, Byz. Provinzialjustiz (wie Anm. 3) $342 \mathrm{f}$. 72 Nur bedingt ähnlich zu \#105 ist der Fall PD \# 60, doch war hier der Adoleszent Salerinos aus der 4. Ehe seiner Mutter Kale, der Gegnerin des prozessierenden Kalupolos, nur indirekt involviert, also kein Kläger oder Mitkläger. 
Demgegenüber finden sich in der Kategorie I.2 ausschließlich solche PRK Akten, die in den beiden anderen Quellensammlungen nie aufgetaucht sind: Hierin geht es um Fälle (ab 1398), in denen die Interessenten den Patriarchen jeweils gebeten hatten, als Garant oder Kontrollinstanz zu fungieren, um damit sicherzustellen, dass bei vermögensrechtlich relevanten Verträgen, Immobiliengeschäften oder Teilungsregelungen ererbter Güter, in die (oder von denen) Adoleszenten mit ihrem Vermögen eingebunden bzw. betroffen waren, letztere nicht benachteiligt werden und die Vermögen von Adoleszenten/innen (hier: ihre Mitgift) geschützt bleiben. Die verschiedenen Vorgänge, die zu dem jeweiligen Gesuch führten, erstreckten sich auf a) Vereinbarungen im Zusammenhang mit einer Erbgüter- oder Vermögensteilung bzw. einem Verkauf (Nrn. 553[2x], 613, 646* und 678), b) den Schutz eines Testamentsvollstreckers vor späteren Regressforderungen des Adoleszenten bei Rückgabe eines Legats an ihn (Nrn. 575, 584), c) die Ermöglichung der Absicherung eines Darlehens an Dritte aus Grundeigentum eines/einer Adoleszenten/-in (Nrn. 569 und 571), und d) den Abschluss eins Vertrags mit Dritten für die Aufnahme einer Hypothek durch Adoleszenten auf eigene Immobilien zur Rückzahlung von Schulden (\# 610). In allen hier genannten Fällen kam der Patriarch dem Wunsch der Interessierten nach. Es ist anscheinend weder rechtshistorisch, noch historisch - politisch ein Zufall, dass gerade diese Art Akten aus dem Zeitraum (1398 - Ende 1401) stammen, als Konstantinopel durch die osmanische Blockade unter Sultan Bayezid I., die von 1394 bis 1402 dauerte, äußerst bedroht war. ${ }^{73}$

\section{Gesellschaftliche Stellung der Adoleszenten}

Die gesellschaftliche Stellung der Adoleszenten lässt sich mangels genauer Daten nur ungefähr abschätzen, nicht wirklich hinreichend präzise ermitteln, und zwar in der Regel nur anhand von Angaben der jeweiligen Akte zum jeweiligen Sachverhalt, manchmal auch noch vom Namen bzw. der Anrede her. Wie auch immer, es sei dennoch versucht, eine grobe Einteilung wie folgt vorzunehmen (die Nummerangabe erfolgt hier nach den Fällen, also der laufenden Nummer der Tabelle II):

[Abkürzungen: $\mathrm{B}=$ Braut, $\mathrm{Bm}=$ Bräutigam, $\mathrm{K}=$ Konkubine, $\mathrm{P}=$ männl. Partner, $\mathrm{vM}=$ vermögende Mittelschicht]

- $\quad$ Arme Unterschicht/Handwerker/Bettler: 2, 24/K, 27 Summe: 2, 5

- Bürger/Mittlere Schicht: 1, 3(B), 5, 9 (od.vM?), 10, 17, 19, 20 (od. vM?), 22, 23, 25, 31, 37, 38 Summe: $\mathbf{1 3 ,} 5$

- Speziell: Klerus-Familie u. ihr Umfeld: 3 (Bm), 4, 13, 14 (od. VM?), 21, 24/P, 30, 33, 34, 35, 36, 45, 46 Summe: 12

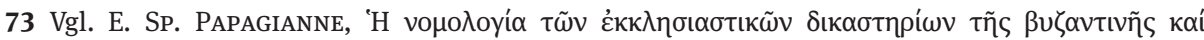

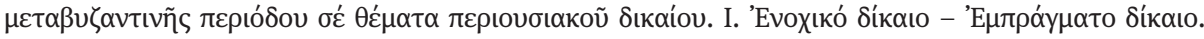
Athen 1992, $7 \mathrm{f}$. , und Papagianni, Un témoin (wie Anm. 11) $215 \mathrm{f}$. 
- Vermögende, obere Mittelschicht (= VM) 6a+6b, 8a+8b, 11, 12, 15, 16,18, 28, 39, 42, 44, 4 Summe: 14

- $\quad$ Oberschicht 7, 25, 29, 32, 40, 48, 49 Summe: 7

Die Verteilung spricht im Grunde für sich und bedarf kaum eines weiteren Kommentars. Die Abgrenzung (zwischen vermögenden oder weniger vermögenden Personen) mag teilweise anfechtbar sein, doch ist eine leichte Mehrzahl der Fälle bei den mehr oder weniger einfachen Bürgern (inklusive der Kleriker-Familien) zu verorten, wobei der Übergang zum vermögenden Bürgertum teilweise fließend ist. - Auf weitergehende Überlegungen sollte man angesichts der disparaten Quellenbasis für diese Zahlen verzichten, da sie nur sehr bedingt für statistische Zwecke verwertbar ist.

\section{Fazit}

Diesem Beitrag liegen insgesamt 53 erfasste Fälle aus den einschlägigen, auf Adoleszenten bzw. Adoleszentinnen sich beziehenden Akten dreier Quellenkomplexe zur kirchlichen Rechtsprechung im späten Byzanz zugrunde: Es handelt sich einerseits um Fälle aus den Akten des Metropoliten Ioannes Apokaukos von Naupaktos (5 Fälle) und des autokephalen Erzbischofs Demetrios Chomatenos von Achrida/Ohrid (24 Fälle aus den $P D$ ), andererseits auch um 24 Fälle aus den im $P R K$ registrierten Akten des Patriarchatsgerichts von Konstantinopel aus dem Zeitraum 1305-1402. Angesichts der ganz ungleichen Zeitspannen ihrer Entstehungszeiträume, aber auch aus anderen Gründen, die hier nicht diskutiert werden können, ist es evident, dass die Menge der Akten zu einschlägigen Fällen aus dem Machtbereich der Herrscher von Epiros statistisch kaum oder nur sehr bedingt mit der Menge entsprechender Fälle aus dem $P R K$ vergleichbar sind. Eher vergleichbar und auf jeden Fall aussagekräftig ist indes die jeweilige thematische Verteilung der Fälle. Wertet man (was angesichts der geringen Zahl der Fälle aus den Apokaukos-Akten zulässig ist) die Fälle aus Epiros als einen gemeinsamen Aktenkomplex und stellt sie den Fällen aus dem $P R K$ gegenüber, so schälen sich deutlich zwei große Themenkomplexe heraus, in denen es in der Alterskohorte (Mädchen/Frauen zwischen 12 und 25, bzw. Jungen/Männer zwischen 14 und 25) der Adoleszenten/-innen zu Problemen und Konflikten kam, die gerichtlich zu klären waren:

Hierbei handelt es sich zum einen um ehe- bzw. familienrechtliche Problemfälle, zum anderen um erb- und vermögensrechtliche Problemfälle.

Im epirotischen Quellenkomplex umfassen erstere 11,5 Fälle (von denen neun den $P D$ entstammen), ${ }^{74}$ während das $P R K$ 5,5 solche Fälle aufweist, zusammen 17 Fälle. Für den zweiten Themenkomplex ergeben sich aus der epirotischen Quellengruppe 14 Fälle und für das PRK 14,5, zusammen 28, 5 Fälle. Damit wird deutlich, dass es (freilich

74 Zur Erklärung des Zustandekommens von Halb-, bzw. 0,5 Werten vgl. oben Anm. 20. 
immer „nach Aktenlage“) in erster Linie erb- und vermögensrechtliche Probleme waren, mit denen sich Adoleszenten/-innen konfrontiert sahen, und in zweiter Linie ehe- bzw. familienrechtliche Probleme. Ein dritter, allerdings quantitativ viel weniger ins Gewicht fallender Themenkomplex bei den Fällen bilden Probleme im Zusammenhang mit Karriere und Disziplin von Klerikern, die allerdings im Raum Epiros fast gar nicht im Hinblick auf Adoleszenten/-innen belegt sind. Dafür ist in diesem Raum eine, auch wiederum verschwindend kleine Menge von drei Fällen namhaft zu machen, die sich auf Tötungsdelikte erstrecken, die man mit Bußbescheiden geahndet hat.

Mit den verschiedenen Problemkonstellationen, denen man bei den jeweiligen Fällen (unter Berücksichtigung der zum Teils sich verschränkenden Themenbereiche) begegnet, befasst sich das Kernstück dieses Beitrags, der umfangreiche Abschnitt III (Kasuistik) mit seinen insgesamt fünf Unterabschnitten.

In sozialer Hinsicht scheint es kein Zufall zu sein, dass Adoleszentinnen, speziell im Zusammenhang mit Fällen erwähnt werden, in denen es um Ehescheidung, teilweise auch im Zusammenhang mit der Karriere-/Disziplin von Klerikern geht; und dass - nach den 13 Fällen aus Epiros zu urteilen, die alle den PD entstammen und sich auf erb- und vermögensrechtliche Probleme beziehen - Voll- oder Halbwaisen unter den Adoleszenten eine besonders gefährdete Gruppe darstellen: Sie hatten oft damit zu kämpfen, dass Stiefeltern oder Verwandte sie, oft jahrelang, an der Inbesitznahme eines ihnen rechtlich zustehenden Grundeigentums zu hindern versuchten. Gerade auch aus der Gruppe der Halb- und Vollwaisen liegen uns besonders interessante Egodokumente vor, deren Bedeutung im Rahmen dieses Beitrags insgesamt in Abschnitt II.3 erörtert wird.

Aus dem PRK betreffen zwar auch drei Fälle die Personengruppe der Waisen / Halbwaisen, gehören aber thematisch zu den ehe-/familienrechtlichen Fällen und partiell auch zum Karriere-/Disziplinbereich. Im Hinblick auf die soziale Schichtung der Adoleszenten/-innen ergibt sich aus Abschnitt IV, dass die Mehrzahl der Fälle wohl im Bereich der einfacheren Mittelschicht (inkl. des Klerus) mit 13,5 Fällen (sowie 12 aus dem [Um]Feld von Klerikern) etwa doppelt so stark wie die vermögende Mittelschicht (14 Fälle) vertreten ist, während sich der Oberschicht sieben Fälle zuordnen lassen. 


\title{
Anhang A: Überblick zur Fall-Diversität bei den erb- und vermögensrechtlichen Fällen aus den $P D$ und dem PRK
}

\author{
Abkürzungen und Symbole \\ $A=$ Adoleszent(en) \\ $\mathrm{F}=$ Fürsorger \\ $A^{*}=$ Adoleszentin \\ OA. $=$ Ortsansässiger \\ Aem $=$ volljährig gewordener Adoleszent \\ $\mathrm{OF}=$ Ortsfremder \\ At $=$ adolesz. Tochter \\ Pkm. $=$ Priester $/$ Kleriker $/$ Mönche \\ Av. $=$ Anverwandte \\ $\mathrm{S}=$ Schwester \\ Azt $=$ adolesz. Ziehtochter \\ $\mathrm{Sg}=$ Sicherheitgarantie \\ B. $=$ Bruder $/$ Brüder \\ V. = Verwandte \\ D. $=$ Dritte \\ $Z M=$ Ziehmutter \\ $\mathrm{Eg}=$ Ehegatte \\ = gegen \\ E/Stief-E. $=$ Elternteil
}

I.1 Konstellation: Streitsache/Prozess A gegen andere (oder umgekehrt)

PD Nrn. 29: A > E. - 32: A V. - 42: A > OF. - 60: A > Aem. - 64: A > Stief-E. - 73: A

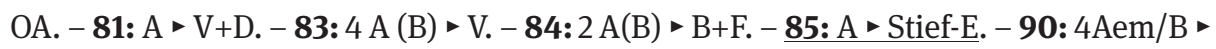
V. - 91: • OF. - 99: A+B - S. - 105: $D>A+E$

PRK Nrn. 14[halb]: ZM $>$ Azt. $-100^{\star}+102^{\star}: \mathrm{E} \bullet \mathrm{At}+[+\mathrm{Eg}] .-587^{\star}: \mathrm{A} \bullet \mathrm{V} / \mathrm{P}$

Erfolg oder Misserfolg des/der A bei Prozessen

A gewinnt: PD Nrn. 73 (weitgehend), 83, 91 (teilweise). - PRK Nrn. 100*+102* (At†+Eg), nur z.T. in $100^{\star}$; Revision in $102^{\star}$ verordnet. -587 (A)

bei Gutachten/Bescheiden

A bekommt Recht $P D$ Nrn. 29, 32, 42, 64* $, 81,84,85,90$, [99] ). - A ist im Nachteil: PD \# 60. - 105: D

- A + E (evtl.abgeändert). - PRK \# 14*[halb]: ZM • Azt

I.2 Konstellation: Zustimmung des Patriarchen (+ Synode) zur Gewährung einer Sicherheitsgarantie $(=\mathrm{Sg}$ ) oder dazu, als Kontrollinstanz zu fungieren bei Verträgen über Hypothekenaufnahme bzw. Immobiliengeschäfte, in die Adoleszenten involviert waren oder von denen sie tangiert wurden; somit

a) für eine Erbgüter-/Vermögens-Teilung oder auch für einen Verkauf (an Verwandte oder Fremde), um (Grund-/Haus)Eigentum bzw. die Mitgift eines/-r Adoleszentin/-en zu schützen

PRK Nrn. 553 (2x): A - D. - 554: Sg für V wegen A. - 613: Sg für V wegen A. - 646*: Sg (mit Regelungsvorschlag) für D wegen $A^{\star} / E g . ~-~ 6^{\star} 8^{\star}$ : Sg für A/Eg wegen $A^{\star} / E g$

b) für den Schutz der Testamentsvollstrecker vor späteren Regressforderungen des Adoleszenten bei Rückgabe eines Legats 
PRK Nrn. 575: Sg für D wegen Aem. - 584: Sg für V wegen A

c) für Erteilung der Altersdispens bzw. venia aetatis (syngnōmē hēlikias $=$ Sh) zur Ermöglichung der Absicherung eines Darlehens an Dritte aus Grundeigentum eines/-er Adoleszentin

PRK Nrn. 569^: Sh für A wegen A*/Eg. - 571: Sh für A wegen E

d) zu einem Vertrag mit Dritten für die Aufnahme einer Hypothek durch Adoleszenten auf ihre eigenen Immobilien (zur Rückzahlung von Schulden).

PRK Nr. 610: Sg für Pkm wegen A+A 


\section{Anhang B: Tabelle II}

Von Adoleszenten handelnde Fälle aus kirchlichen Gerichtsakten 1204-1402.

Anhand der Akten von Ioannes Apokaukos, Demetrios Chomatenos und dem

Patriarchat von Konstantinopel aus dem Zeitraum 1315-1402

\section{Hinweise und Abkürzungen:}

$\#=\mathrm{Nr}$.

Allg. = Allgemeine Aussagen oder Ermahnungen (aus der Zählung der

Fall-Akten herausgenommen)

Bf. $=$ Bischof

Bußb. = Bußbescheid

$\mathrm{D}=$ Datierung

Ebf. $=$ Erzbischof

EgoD $=$ Direktes Ego-Dokument

$$
\begin{aligned}
& \text { Ind.EgoD = Indirektes Ego-Dokument } \\
& \mathrm{K}=\text { Kommentar } \\
& \text { Mpt. = Metropolit } \\
& \text { PLP = Prosopographisches Lexikon der } \\
& \text { Palaiologenzeit } \\
& O=\text { Ort } \\
& \mathrm{U}=\text { Übersetzung } \\
& \text { Ur = Urkunde }
\end{aligned}
$$

Die gerichtlichen Apokaukos-Akten werden angeführt und nummeriert nach der Analyse bzw. dem jeweiligen Regest von K. Lampropulos (Lam \# = Lampropulos Nr.) und der Werkausgabe von Delemares, "Aп $\alpha v \alpha$ (Delem. SE \# = Delemares, Kap. 5.2 [Synodika Engrapha] Nr.), vgl. oben, Anm. 11. - Die Chomatenos-Akten werden nach der Ausgabe der Ponemata diaphora $(=P D)$, ed. Prinzing, nummeriert (\#) u. (teilweise in der letzten Spalte wg. spezieller Hinweise, nach §/Seite) angeführt, vgl. Anm.12. Die Patriarchats-Akten $(P R K)$ werden analog nummeriert bzw. angeführt nach der CFHB-Neuausgabe des Patriarchatsregisters von Konstantinopel, ed. H. Hunger, O. Kresten, J. Koder et alii, (= PRK \#; erschienen bislang Bd. 19/1-3, ed Wien 1981-) bzw. nach MM II, vgl. oben Anm. 12, ergänzend auch nach der Nr. der Patriarchatsregesten von J. Darrouzès (Darr. Reg.), vgl. wieder oben Anm. 13. Grundsätzlich wird für die Edition jeder Akte im Fließtext auf die Angabe in der 2. Spalte in dieser Tabelle verwiesen. Zur Bedeutung des Asterisk vgl. oben II.1 (S. 36).

Für weitere Abkürzungen bei Quellen- u. Literaturhinweisen vgl. die Bibliographie.

Bei der Sachverhaltsschilderung erscheinen nur die Namen der nachweislichen (oder mutmaßlichen) Adoleszenten im Fettdruck. Personennamen aus der Palaiologenzeit werden in der Regel zusammen mit ihrer PLP- Nummer angeführt. Ergänzend zu der in der Tabelle angeführte Literatur zu einzelnen Fällen verweise ich für Akten aus den $P D$ auch grundsätzlich auf die Lit.-Angaben zu den Regesten meiner Edition im Rahmen der Prolegomena. 
Tabelle II. Von Adoleszenten handelnde Fälle aus kirchlichen Gerichtsakten 1204-1402

Mpt. Ioannes Apokaukos

1

Akte: Lam \# 2*

Edition: Delem SE \# 2, 353 f-355

Datierung: 1217

Ort/Region: Leukas

Art d. Akte: Synodal-Urteil mit Bußb.

Sachverhalt: Basileios Kaliges u. seiner Frau missfiel die Braut Pankalo ihres Sohnes Konstantinos. Dieser hatte sie gegen den Willen seiner Eltern geheiratet. Der Konflikt zw. dem jungen Paar und den Eltern K., bei denen sie wohnten, eskalierte so sehr, dass Basileios Kaliges seinen Sohn im Affekt tötete.

Auskunft, Bescheid oder Urteil (samt Strafen oder Auflagen): Der Totschlag wird als Mord gewertet. Diverse Epitimia auf 5 Jahre

Quellen- u. Literatur-Hinweise: Lam., 262 f.; Strazzeri, Drei Formulare, 348f., Nr. XVI

Besondere Bemerkungen: Altersangaben zum Sohn u. seiner Braut fehlen.

Akte: Lam \# 10

Edition: Delem SE \# 10, 378-380

Datierung: März 1222

Ort/Region: Korfu

Art d. Akte: Synodalbescheid der Synode in Arta, ergangen im Beisein u. auf Antrag des Mtrp. von Korfu, G. Bardanes.

Sachverhalt: Tod des Jugendlichen/neaniskos NN: Ein Priester NN traf ihn nachts im Wald und zwang ihn mit Schlägen, ihm den Weg zu zeigen. Der Junge starb 28 Tage später. Pediadites, der Amtsvorgänger des Bardanes hatte den Priester auf 11 Jahre vom Amt suspendiert.

Auskunft, Bescheid oder Urteil (samt Strafen oder Auflagen): „Das Verfahren des Pediadites wird“, weil die Schuld des Priesters im letzten Verfahren nicht bewiesen wurde, „mißbilligt und der Priester von der Strafe befreit, wenn auch mit Zögern.“ (Strazzeri, Drei Formulare, S. 343.) Quellen- u. Literatur-Hinweise: Lam., 269 ff.; Strazzeri, Drei Formulare, 334, 342 f., Nr. III

Akte: Lam \# 17*

Edition: Delem SE \# 17, 396 f.

Datierung: vor 1226

Ort/Region: loannina

Art d. Akte: Protokoll eines Bescheids zur Möglichkeit einer Scheidung

Sachverhalt: Bescheid zum Anliegen des Priesters Michael Kaludes aus Leusista bei Ioannina, ob seine Tochter Eirene geschieden werden könne. Der Bescheid ergeht im Beisein des Bf.s Neophytos von loannina und hoher weltlicher Personen. Kaludes zufolge war seine Tochter Eirene vor $31 / 2$ Jahren, als sie gerade im gesetzlichen Heiratsalter war, mit dem begabten Konstantinos vermählt worden, doch verschwand dieser sogleich. Nach glaubwürdigen Zeugen war er ein Dieb u. Betrüger.

Auskunft, Bescheid oder Urteil (samt Strafen oder Auflagen): Der Bf. soll den Mann durch Ausruf (1 Monat lang) zu zwecks Ermahnung zu sich zitieren. Erscheint er auch dann nicht, kann der Priester Eirene wieder verheiraten. Bekennt aber ihr Mann den Fehler vor einem Bf. u. verschwindet nicht wieder, muss Eirene mit ihm wieder zusammen leben.

Quellen- u. Literatur-Hinweise: Lampropulos, 276 f.; Katerelos, \# 15, S.29, 56 f.(Ü), 134-136, 195, 197 -199; Laiou, Institution, S. 207 
Besondere Bemerkungen: Das Alter des Konstantinos ist unklar.

Akte: Lam \# 28

Edition: Delem SE \# 28, 429-431

Datierung: ZW. 1200 und 1232

Ort/Region: Malaina/Belechatuia

Art d. Akte: Bescheid

Sachverhalt: Scheidungsbegehren des 18-jährigen Anagnosten loannes NN von seiner viel älteren, barbarisch sprechenden (offenbar vlachischen) Frau Rusa aus der bergigen Belechatuia. Die Ehe wurde gegen den Willen des loannes auf Druck der Theodora, Prōteuusa der Region Malaina, geschlossen (Ioannes kam zeitweilig in Haft), aber nicht vollzogen. Frage: Darf er eine zweite Ehe eingehen und auch Priester werden?

Auskunft, Bescheid oder Urteil (samt Strafen oder Auflagen): Die Ehe ist, weil unter Zwang und ohne Konsens geschlossen, ungültig. zumal sie nicht vollzogen wurde. Daher darf loannes wieder heiraten und auch Priester werden.

Quellen- u. Literatur-Hinweise: Lampropulos, 289f.; Katerelos, \# 25, 32 f., K 155-156, 265; Ü 78-80; Laiou, Institution, 308f. ; Kiusopulu, 18, 23, 66, 151; Prinzing, Carev dvor; 220 f.

Akte: Lam \# 37*

Edition: Delem SE \# 37, $446 \mathrm{f}$.

Datierung: ZW. 1200 und 1232

Ort/Region: ?

Art d. Akte: Urteil des Mpt.

Sachverhalt: Scheidungsfall ungleichen Paares: Der 16-jährige Konstantinos Papaioannopulos und die reife Anna wurden (eti paidia ontes ... kai hēlikias en ateleia) im Kindesalter kirchlich verheiratet. Doch Konstantinos war seiner reifen, üppigen Frau nicht gewachsen, so dass sie Ehebruch beging.

Auskunft, Bescheid oder Urteil (samt Strafen oder Auflagen): Apokaukos lässt die Ehe scheiden, Anna darf erneut heiraten, aber nicht den, mit dem sie Ehebruch beging. Der Priester, der beide getraut hatte, wird suspendiert.

Quellen- u. Literatur-Hinweise: Lampropulos, 295f.; Katerelos,34, 89f., Ü + K. 167 -169; Laiou, Institution,308f.; Fögen, Rechtsprechung (Ü+K); Papadatou, Divorce, 272

\section{Ebf. Demetrios Chomatenos}

6a Akte: \# 9* (vgl. auch \# 129*, mit anderem Sachverhalt, aber selbe Delinquentin)

Edition: $P D$

Datierung: 1216 (1219)

Ort/Region: Koloneia

Art d. Akte: Synodalurteil, mit Bußb.

Sachverhalt: Bußbescheid für Zoe aus Koloneia. Sie ist zum vierten Mal verheiratet, zweimal in der Zeit der Unmündigkeit / anēbotēs (vor dem 12. J.); dann zweimal nach ihrer Reife (meta de... tēn ephēbon tricha). Zoe fragte, inwiefern sie sich durch ihre ,Polygamie‘ schuldig gemacht habe u. was sie tun müsse, um von der Kirche wieder angenommen zu werden.

Auskunft, Bescheid oder Urteil (samt Strafen oder Auflagen): Beide Früh-Ehen Zoes sind ungültig, wg. ihrer Unmündigkeit. Z. gilt daher nur als Bigamistin und darf, falls ihr Mann stirbt, nicht nochmals heiraten. Mit Hinweis auf ihre Jugend (neotēs hēlikias) erhält sie Bußstrafen, die bestehende Exkommunikation wird um ein Jahr verlängert.

Quellen- u. Literatur-Hinweise: Ind.EgoD.; Katerelos, 35,172-174; Laiou, Priests, 46 
Edition: $P D$

Datierung: vor 1225

Ort/Region: Ohrid

Art d. Akte: Urteil

Sachverhalt: Ehescheidungsprozess des Archonten Nikolaos Tzachenos, dem Beaufragten seiner Schwägerin Eirene, Witwe des loannes Lakapenos (oder: Lampenos), u. ihrer 12-jähr. Tochter Kale gegen Basileios Drugubilos, um den zwischen ihm und Eirenes verstorbenen Mann abgeschlossenen Verlobungsvertrag aufzulösen. Kale war als 5-Jährige verlobt und im Alter von 7 über den Vertrag informiert worden. Sie erklärte, eher Selbstmord zu begehen, als mit Basileios zusammenkommen zu wollen.

Auskunft, Bescheid oder Urteil (samt Strafen oder Auflagen): Der Vertrag wurde annulliert, weil Kale bei Abschluss noch unmündig war und es keine von ihr gab. Ein Streit beider Seiten über Umfang und Wert der Dinge, die Bas. wg. der Verlobung (mit eingebracht hatte (u.a. Textilien, +150 Nomismata trikephala) wurde mit einem Vergleich beigelegt. Wg. der Rüstung des B. sollte man das Kaisergericht befragen.

Quellen- u. Literatur-Hinweise: Katerelos, 35, 95 f.-97 (Ü), 171 f., 203, 230

8a $\quad$ Akte: \# $29(+\# 42$ = 8b)

Edition: $P D$

Datierung: Um 1222

Ort/Region: Berroia

Art d. Akte: Supplik des Ebf.s an den Sebastos Plytos

Sachverhalt: Chomatenos teilt dem Adressaten (Plytos) mit: Melias Basilikos, Halbwaise aus Berroia, wurde nach dem Tod seiner Mutter von seinem Vater total vernachlässigt, vor allem, weil er das M. zustehende Muttergut verbrauchte. Er bevorzugt nun die Kinder seiner Konkubine, die wohl sogar M.s Leben bedroht.- Plytos möge den Sachverhalt prüfen. Vgl. unten $\# 42=8 \mathrm{~b}$ !

Auskunft, Bescheid oder Urteil (samt Strafen oder Auflagen): Chomatenos: Das Anliegen des Melias ist berechtigt, denn: Väter sind verpflichtet, heiratswillige Kinder, die sich korrekt verhielten, von Rechts wegen zu unterstützen.

Quellen- u. Literatur-Hinweise: Ind. EgoD. 113, 34-46; Prinzing, Observations, 33, A.85, mit

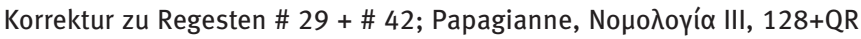

$9 \quad$ Akte: \# 30*

Edition: $P D$

Datierung: $1225-30$ ?

Ort/Region: ?

Art d. Akte: Responsum an einen Panhypersebastos: Plytos?

Sachverhalt: Anfrage: Ist die 3. Ehe erlaubt für eine 18-jährige NN, nach Verlust ihrer zwei vorigen Ehemänner?

Auskunft, Bescheid oder Urteil (samt Strafen oder Auflagen): Bescheid: Die 3. Ehe NNs wird erlaubt wegen der Jugendlichkeit der Betroffenen.

Quellen- u. Literatur-Hinweise: Katerelos, 263; Prinzing, Adress. Briefe, 230, 241 
Sachverhalt: Fall des verwaisten Jugendlichen/neaniskos Romanos aus Choropanion bei Prilapos, : Ein ihm gehörendes Erbgrundstück will sein Onkel Bladimeros, der uneheliche Sohn seines gleichnamigen Großvaters (mütterlicherseits), verkaufen. Romanos' Vater Nektanos war eine 2. Ehe eingegangen. Hat Bladimeros ein Anrecht auf sein (R.s) Grundstück?

Auskunft, Bescheid oder Urteil (samt Strafen oder Auflagen): Romanos kämpft, weil noch nicht 25, zu Recht um sein mütterliches Erbe. Bladimeros ist als unehelicher Sohn des Großvaters Bladimeros nicht $a b$ intestato erbberechtigt, darf das Grundstück daher auch nicht verkaufen.

Quellen- u. Literatur-Hinweise: IndEgoD. § 1, Z. 5 -16, zu mdl. Aussage. - Miller, Orphans, 304

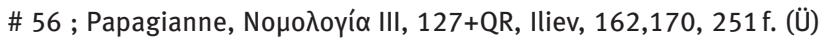

8b! Akte: \# 42

Edition: $P D$

Datierung: 1230

Ort/Region: Berroia

Art d. Akte: Synodal-Bescheid

Sachverhalt: Aktuell ist Melias (vgl. \# 29) 33 J. alt. Vor 9 Jahren erfolgte der Verkauf eines Grundstücks aus seinem mütterlichen Erbe, da war er 24, und wurde im folgenden August 1222 volljährig, also 25 .

Auskunft, Bescheid oder Urteil (samt Strafen oder Auflagen): Melias bekommt Recht; der Gegner kann keinen Anspruch geltend machen.

Quellen- u. Literatur-Hinweise: Ind.EgoD., § 2-3: mdl. - Katerelos, 225. Im PD-Regest lies $116^{\star}, 4$. Z. durchaus“ statt „nicht“.

11 Akte: \# 60

Edition: $P D$

Datierung: ab 1220

Ort/Region: Kerkyra

Art d. Akte: Synodal-Bescheid

Sachverhalt: Bescheid zum Prozess des Marinos Kalupolos aus Korfu um das Erbe (Grundstücke) seiner Frau (aus der Fam. Akronaras) gegen Kale, Witwe des verstorb. Vetters von Marinos' Schwiegervater Nikolaos Akronaras, Nikolakios. Kale hatte einen Sohn aus 4. (illegit.) Ehe (mit einem einflussreichen Ehemann), den 12-jähr.Theodoros Salerinos, zum Erben $a b$ intestato des Nikolakios bestimmt.

Auskunft, Bescheid oder Urteil (samt Strafen oder Auflagen): Bescheid: Theodoros Salerinos hat keinen Rechtsanspruch auf das Erbe. Recht bekommt also Kalupolos.

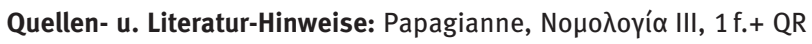

Besondere Bemerkungen: Kalupolos führt den Prozess seines verstorbenen Schwiegervaters Nikolaos Akronaras fort, im Auftrag seiner Frau.

Akte: \# 64*

Edition: $P D$

Datierung: nach 1219/20

Ort/Region: Berroia

Art d. Akte: Synodal-Bescheid

Sachverhalt: Bescheid zum Erbschaftsstreit zw. Ioannes Tantures (vertreten durch Stephanos Kappadokes) u. Michael, seinem Stiefsohn aus der 1. Ehe seiner Frau Kale; bei Kales 2.

Eheschließung (mit Tantures) war Michael 3-jährig, jetzt ist er 20 u. sollte (so der Stiefvater) nun heiraten. Er trennte sich aber von seinem Stiefvater und fordert sein Erbe (u. a. einen 6 modioi großen Weinberg) aus dem elterlichen Vermögen, hat aber 4 Halb-Schwestern (aus 
Kales 2. Ehe). Diese waren beim Tod ihrer Mutter Kale noch ateleis /minderjährig. Tantures hatte dann nach dem Tod Kales für alle ihre Kinder gesorgt (also für Michael u. seine vier Halbschwestern). Jetzt will er wissen, ob Michael, trotz der Trennung von ihm, nun zu Recht sein Erbe beansprucht.

Auskunft, Bescheid oder Urteil (samt Strafen oder Auflagen): Bescheid: Wegen der 2. Ehe der Kale, Michaels Mutter, erben nur Kinder aus ihrer 1. Ehe, also nur Michael, d. h. ihm steht auch der Grundbesitz an dem Weinberg aus der Mitgift für seine Mutter (bei ihrer ersten Ehe) zu.

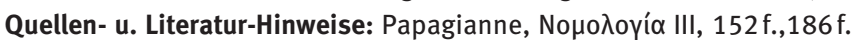

13 Akte: \# 73

Edition: $P D$

Datierung: zw. 1225 u. 1230

Ort/Region: Achrida

Art d. Akte: Urteil d. erzb. Gerichts

Sachverhalt: Prozess des loannes Teichotitzes (Sohn des gleichnamigen verstorbenen Priesters des Ebt. Ohrid) um ein Erbgrundstück (Weinberg in Brysis) gegen den Baumeister Athanasios. Der hatte es sich den Weinberg angeeignet, als lo. nach d. Tod des Vaters ganz minderjährig/panaphēlix war. Jetzt ist er aber volljährig/enēbēsas und 25 und erhebt Anspruch auf den Weinberg.

Auskunft, Bescheid oder Urteil (samt Strafen oder Auflagen): Der Kläger loannes jr. bekommt Recht, weil Athanasios sich auf unlautere Weise (durch Tausch mit Dritten u. Fälschung einer Verkaufsurkunde) das Grundstück in seinen Besitz gebracht hatte.

Quellen- u. Literatur-Hinweise: Ind. EgoD 252, 5-253,15+25-31. - Iliev,170, 270 (Ü) Besondere Bemerkungen: Der Beklagte zeigt die gefälschte Verkaufs-Urkunde.

$14 \quad$ Akte: \# 81

Edition: $P D$

Datierung: nach $1219 / 20$

Ort/Region: Berroia

Art d. Akte: Synodal-Bescheid

Sachverhalt: Bescheid für den jugendlichen. Manuel Sbenilos, Halbwaise, Sohn des vor 12 Jahren verstorbenen Diakons und Castrensis Leon Sbenilos. Er wehrte sich gegen diverse, gegen ihn vorgebrachte, aber strittige (teilweise auch schon anderswo vor Gericht verhandelte) Ansprüche seines Onkels Theodoros Sbenilos u. eines gewissen Panagiotes, in denen es zumeist um deren angebliche Eigentumsrechte an Immobilien ging, die als Gegenstand des vor 12 Jahren erstellten Testaments seines Vaters teilweise auch ihm (Manuel) zugesprochen worden waren. Ein Teilaspekt betraf auch die Ansprüche eines Dritten (anonymen) Mannes, wobei es um ein Wassermühlen-Grundstück und die Kostenerstattung für dessen rechtliche Absicherung ging. $M$. hatte dazu 5 Fragen zu Details der Erbschafts- bzw. Besitzprobleme. Auskunft, Bescheid oder Urteil (samt Strafen oder Auflagen): Manuel erhält in Bezug auf die 5 Fragen bei den ersten vier ganz, bei der fünften weitgehend Recht.

Quellen- u. Literatur-Hinweise: Schriftl. Dir. EgoD. (§ 1-6). Laiou, Mariage, 138-145 (Ü). -

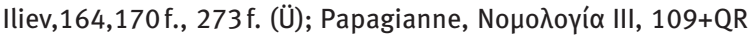

15 Akte: \# 83

Edition: $P D$

Datierung: um 1227

Ort/Region: Bela

Art d. Akte: Urteil 
Sachverhalt: Vier minderj. Waisenbrüder Lubroi (Georgios, Nikolaos, loannes, Samuel) prozessieren zum 2. Mal gegen den Verwandten loannes Ganadaios um ihr väterliches Erbe aus Immobilien. Denn dieser befolgte nicht das im 1. Prozess für sie günstig ausgefallene Urteil (beim Duxgericht), sondern wandte sich an den Kaiser, der eine Untersuchung durch den Ebf. anordnete. (Hinweis: Text-Ergänzung in § 2, Zeile 13, s. oben, Anm. 27.

Auskunft, Bescheid oder Urteil (samt Strafen oder Auflagen): In Ohrid unterliegt Ganadaios, wegen der (damaligen) Unmündigkeit /anēbotēs der Lubroi.

Quellen- u. Literatur-Hinweise: Miller, Orphans, 80 f. und 304, \# 59; Stefec, Regesten, Nrn. 52 und 53

Akte: \# 84

Edition: $P D$

Datierung: wohl nach 1225

Ort/Region: Berroia

Art d. Akte: Synodal-Bescheid

Sachverhalt: Bescheid für den volljährigen, aber noch vor dem Alter von $9 \mathrm{~J}$. verwaisten loannakios Achyraites aus Berroia. Er ist der drittgeborene von ursprünglich 4 Brüdern u. spricht auch für seinen älteren Bruder Leon. Bevor auch ihre Mutter starb (da war Leon 13, er [loannakios] 9 J. alt), setzte sie den ältesten Bruder, Konstantinos zum Testaments-Vollstrecker ein. Doch dieser und andere Fürsorger / Kuratoren für loannakios u. Leon handelten eigennützig $u$. betrügerisch, u. a. durch Fälschung einer Schenkungsurkunde (für Weinberge).

Auskunft, Bescheid oder Urteil (samt Strafen oder Auflagen): Angebliche, von den Adoleszenten gemachte, urkundl. festgehaltene, gefälschte Schenkungen sind, da von Minderjährigen stammend, nicht rechtskräftig! Daher sind loannakios und Leon gegen ihren Bruder Konstantinos im Recht: Ihnen steht die Restitution der ihnen zustehenden Weinberge samt Erträgen zu.

Quellen- u. Literatur-Hinweise: Wichtig § 1, 4-14: Prooimion. Das Dir.EgoD, § 2 -9, schriftl. Korrektur zum Regest 176*: Abs.1: lies in Z.3:“Brüder, Konstantinos, zum...“ und in Abs: 2, Z.1 - 2 lies: ,... damals 9 und 13 Jahre alt“. - Miller, Ophans, 79 f. u. 304 \# 61; Papagianne,

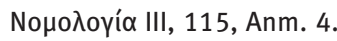

17 Akte: \# 85

Edition: $P D$

Datierung: ab Nov. 1227

Ort/Region: Raum Kastoria

Art d. Akte: Synodal-Bescheid

Sachverhalt: Bescheid für Manuel (jetzt 37), früher genannt Israel, Vollwaise, hatte beim Bf. v. Kastoria seinen Stiefvater Moses verklagt wg. Vernachlässigung: Denn dieser hatte mit Manuels (bald danach verstorbener) Mutter die Grundstücke des noch ganz minderjährigen / panaphēlix M.s verkauft u. andere Dinge verbraucht. M. ging in die Fremde, war Bettler u. Lohnarbeiter, dann Koch. Er erhob nach Rückkehr noch innerhalb der inm verbliebenen 7 Jahre der 30-jähr. Frist (14 J + 30=44) Klage, um seine Grundstücke bei Kastoria wieder zu erlangen. M. hatte zuerst bei dem klugen Bf. von Kastoria Recht bekommen (s. das Hypomnēma), nun bat er um ein Gutachten des Ohrider Synodal-Gerichts.

Auskunft, Bescheid oder Urteil (samt Strafen oder Auflagen): Manuel hat korrekt argumentiert, ebenso der Bf. von Kastoria.

Quellen- u. Literatur-Hinweise: Ind.EgoD.

Besondere Bemerkungen: Hinweis: Simon, Byz. Prov., 313 Anm.16 muss sich auf \# 85 beziehen, nicht 84. Wichtig das Prooimion; Miller, Orphans, 93 f. (u. 304, \# 62) ignoriert Israels Taufnamen; Laiou, Priests, 52 
Edition: $P D$

Datierung: $1225 / 30$ oder $1230 / 35$

Ort/Region: Kerkyra

Art d. Akte: Synodal-Bescheid

Sachverhalt: Gutachten f. den verwaisten Konstantas aus Korfu, der (mit seinen $\mathbf{3}$ Brüdern) in Ohrid um Rechtsbeistand bat: Die Eltern, erst der Vater, dann die Mutter (Erbin der Hälfte des elterlichen Grundbesitzes), starben früh. Sein Onkel (Bruder der Mutter) Makronikolaos, ging als Jugendlicher (ephēbos) in die Fremde; so sorgten die Großeltern (d. Mutter) für ihre 4 minderjähr. Enkel (tois aphēlixin), so auch für Konstantas, u. verwalteten das Muttergut der Kinder, die noch zu Lebzeiten der Großeltern volljährig wurden u. ihre Erbgrundstücke erhielten, wie auch der inzwischen zurückgekehrte $M$. Sie nutzten sie 22 Jahre. Aber M., mit dem Erbteil unzufrieden, riss Grundstücke aus dem Erbteil K.s u. seiner Brüder an sich. Ihren Protest ignorierte M. Daher riefen die Geschwister das Kaisergericht an. Dort sagte M., seine Schwester sei ausgesteuert worden $u$. habe keine Grundstücke geerbt. Er verlangte Einsicht in ihren Mitgiftvertrag, der aber unauffindbar war. K. u. die Brüder boten die Beeidung ihrer Aussage an, was ihr Onkel Kalos (väterlicherseits) übernehmen wollte. M. stimmte zu. Der Kaiser wies mit einem die Position der Brüder stärkenden Endurteil den Dux von Kerkyra an, Kalos den Eid in der Nikolaos-Kirche Korfus abzunehmen u. das Urteil zu vollstrecken. 2 Jahre später strengte M., den Eid bezweifelnd, einen 2. Prozess bei einem anderen Richter an, unterlag aber. Daher die Anfrage der Brüder.

Auskunft, Bescheid oder Urteil (samt Strafen oder Auflagen): Das Ohrider Synodalgericht stützte die Brüder: Makronikolaos hätte in den 22 Jahren seiner Anwesenheit binnen 10 Jahren klagen müssen. (Abwesende binnen 20 Jahren). Das für die Brüder positive kaiserliche Beweisurteil muss $M$. respektieren.

Quellen- u. Literatur-Hinweise: Dir.EgoD, mdl.: § 2-5. Die Petenten aber sind inzwischen volljährig, s. Hinweis auf die 22 Jahre Grundstücks-Nutzung ab ihrer Volljährigkeit! - Papa-

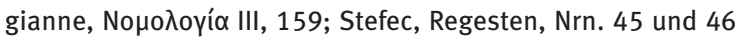

19 Akte: \# 91

Edition: $P D$

Datierung: ca. 1220

Ort/Region: Achrida

Art d. Akte: Urteil (ohne Synode)

Sachverhalt: Prozess des Georgios Kuritzes (Halbwaise) aus Ohrid gegen Silbestros aus Bidobiste um einen Weinberg aus G.s väterlichem Erbe. Diesen hatte G.s verwitwete Mutter, als er noch unmündig / anēbos war, aus Not an S. verkauft. G. ging in die Fremde und kehrte, nun älter als 14, zurück, war somit berechtigt, die Restitution von Eigentum zu fordern. Der Verkauf war (laut Urkunde) 1210 erfolgt..

Auskunft, Bescheid oder Urteil (samt Strafen oder Auflagen): Georgios hatte Erfolg: Seine Mutter durfte kein Vatergut verkaufen bei Unmündigkeit G.s (bis 14). G. erhob innerhalb der ab 14 geltenden 30-jähr. Frist die Klage. Kosten für den Kauf u. die Melioration werden S. mit 1/3 aus dem Ertrag erstattet.

Quellen- u. Literatur-Hinweise: Prooimion knapp (mit Bezug zu anēbotēs + Waisentum). IndEgoD.; Miller, Orphans, 252 u. 305, \# 63; Iliev, 164, 171, 279 (Ü) 
Art d. Akte: Synodalbescheid

Sachverhalt: Bescheid für Nikephoros Syropulos aus Berroia aus einer Fam. von 3 Söhnen und 4 Töchtern. Der seinen Söhnen einst feindlich gesinnte, verstorbene Vater hatte ihn u. den zweiten Bruder enterbt, wg. ihres Wegzugs in die Fremde zu seinen u. der Mutter Lebzeiten. (Der dritte Bruder aber, Nikephoros, sagt, sie seien erst nach dem Tod der Mutter in die Fremde gezogen, und fragt nach der Rechtmäßigkeit der Enterbung von (nach dem Tod der Mutter) in die Fremde gegangenen Söhnen.

Auskunft, Bescheid oder Urteil (samt Strafen oder Auflagen): Ausführungen zu den väterlichen Sorgepflichten. Hinweis auf die 15 gesetzlich festgelegten Gründe für Enterbung von Kindern durch die Eltern. Hierzu zählt aber nicht der Wegzug von Kindern. Nikephoros bekommt Recht. Quellen- u. Literatur-Hinweise: Dir.EgoD (mdl.): § 2

Besondere Bemerkungen: Nur bedingt einschlägig: Hinweise auf das Alter der Kinder fehlen. Aber thematisiert sind: Dank u. Undank von Kindern.

Edition: $P D$

Datierung: $1225-1230$

Ort/Region: Bodina und Berroia

Art d. Akte: Synodalbescheid

Sachverhalt: Bescheid für Leon Kontos von Bodina im (2.) Streit mit Anna he tu Komētos, Witwe des Anagnosten Ioannes Kukumes, aus Berroia. Er hatte einst ihr u. ihrem Sohn Konstantinos 2 Weinberge abgekauft. Doch sie besetzte die Grundstücke wieder, da sie die Verkaufs-Urkunde wg. d. Minderjährigkeit ihrer 2 Söhne K. und Leon, für ungültig hielt. Durfte nun Anna als Vormund ihrer 2 Söhne den Verkauf ihrer Grundstücke umstoßen unter Hinweis auf ihre andauernde Witwenschaft (keine 2.Ehe), ihre Notlage u. die Minderjährigkeit / aphēlikiōsis der hungernden Kinder? Es lagen schon zwei Gerichtsurteile vor.

Auskunft, Bescheid oder Urteil (samt Strafen oder Auflagen): Annas Klage wird abgewiesen und ein Kompromiss vorgeschlagen: 1/3 kann Leon K. unbedingt behalten. 2/3 solle er Anna, um sie zufrieden zu stellen, überlassen $u$. dafür den ihm gebührenden Preis erhalten wg. seiner Ausgaben für die Melioration: Leon K. hatte im guten Glauben gehandelt; Anna durfte ja als Vormund für ihre Kinder verkaufen, da sie auch keine 2. Ehe einging.

Quellen- u. Literatur-Hinweise: Mdl. Aussage des L. Kontos vor Gericht in § 1-3 der Akte (aber er ist kein Adoleszent; doch Konstantinos war dies und vielleicht auch sein Bruder). - Papa-

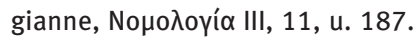

Akte: \# 126*

Edition: $P D$

Datierung: Wohl zw. „vor 1216 und $1224^{\text {“ }}$

Ort/Region: Rasine/Resen

Art d. Akte: Urteil d. erzb. Gerichts

Sachverhalt: Scheidungsprozess. zw. Stana, Tochter des Petros, und ihrem Mann, Preabos. Stana hatte man als 9-Jährige mit ihm kirchlich verlobt. Danach aber verschwand er 8 Jahre in der Fremde. Wieder aufgefunden, wurde er zurückgebracht u. sollte mit ihr zusammenleben, verschwand jedoch erneut. Wg. der zu früh erfolgten Verlobung war die Ehe ungültig. Stana hatte nun aber ihr Pubertätsalter überschritten. Ihr Vater fragt, was er mit ihr machen solle. Auskunft, Bescheid oder Urteil (samt Strafen oder Auflagen): Stana darf eine neue Ehe eingehen.

Quellen- u. Literatur-Hinweise: Katerelos, 99f. (Ü), 174-77 (K), wo K. feststellt, Stana sei 17jährig gewesen, falls Preabos sie sofort nach der Verlobung verließ; Iliev, 165, 172, 298 (Ü) 
Edition: $P D$

Datierung: 20.6.1219

Ort/Region: Koloneia

Art d. Akte: Bußb.

Sachverhalt: Zoe bekennt sich mitverantwortlich für die Verstümmelung (mit Todesfolge) eines diebischen Dieners durch ihr Personal.

Auskunft, Bescheid oder Urteil (samt Strafen oder Auflagen): Verhängung neuer Bußstrafen. Zuvor soll der Bf. von Diabolis den Fall prüfen.

Quellen- u. Literatur-Hinweise: Strazzeri, Drei Formulare, 336, 344 f., Nr. VII; Prinzing, Hausbedienstete, $191-194$.

23 Akte: \# 132*

Edition: $P D$

Datierung: wohl zw. 1216 u. 1222

Ort/Region: wohl Achrida/Ohrid

Art d. Akte: Urteil d. ebf. Gerichts.

Sachverhalt: Scheidungsprozess der Sbina, Tochter des verstorbenen Georgios Nestor, gegen ihren Mann Georgios Serbopulos. Sbina fühlt sich in ihrer Jugend / neotēs gefährdet durch die bereits 5-Jahre andauernde Impotenz d. Georgios. Dieser widersprach ihr zunächst. Als aber Sbina untersucht werden sollte, räumte er doch diesen Sachverhalt ein und meinte, er habe sich nicht mehr für Sbina sexuell erwärmen können.

Auskunft, Bescheid oder Urteil (samt Strafen oder Auflagen): Die Scheidung wird verfügt. Sbina behält ihre Mitgift und darf eine neue Heirat eingehen, Georg erhält sein Hochzeitsgeschenk zurück.

Quellen- u. Literatur-Hinweise: Ind. EgoD.; Katerelos, 47 (Ü), 123f. und 214f.; Iliev, 165,172 (Ü)

24 Akte: \# 135*

Edition: $P D$

Datierung: Ca. 1219-1222

Ort/Region: Achrida/Ohrid

Art d. Akte: Urteil d. erzb. Gerichts

Sachverhalt: Streitfall zwischen loannes (Sohn d. Hieromon. Barnabas) und „einer gewissen Frau“ Dragoste, die ihren Säugling auf dem Arm hielt. Er hatte in jugendlichem Drang (akathektois hormais neotētos) 5 Jahre mit ihr im Konkubinat zusammengelebt, erkannte jetzt aber seine Sünde und wies ihren Wunsch auf dauerhaftes Zusammenleben ab. D. gab dies zu und wollte auf keinen Fall die Trennung (das Kind sei von ihm, ebenso wie zwei weitere, aber verstorbene Kinder). D. glaubt, nach so langer Zeit ein Anrecht auf das Zusammenzuleben mit ihm zu haben, und beantragt die übliche Einsegnung. Ioannes lehnt dies gänzlich ab.

Auskunft, Bescheid oder Urteil (samt Strafen oder Auflagen): Die Trennung wird unter der von beiden Seiten akzeptierten Maßgabe verfügt, dass loannes seiner bisherigen Gefährtin seine Kuh u. einen Anteil am Ernteertag aus der Saatfrucht abtritt. Danach sind beide frei.

Quellen- u. Literatur-Hinweise: Ind. EgoD. Iliev, 166, 172, 302 f. (Ü)

Besondere Bemerkungen: $\mathrm{Ob}$ beide noch jugendlich waren, ist offen.

Art d. Akte: Bußb. des erzbf. Gerichts. 
Sachverhalt: Bußbescheid für den Dux von Skopje, Georgios Komnenos (ein eugenēs anēr, Z. 35f.) wg. Aufgabe seines Gelübdes, acht Jahre nach seiner Gesundung von einer Krankheit Mönch werden zu wollen. Das hatte er gelobt auf Anraten von Verwandten, als ihm ein unfähiger Arzt den baldigen Tod prognostiziert hatte. G. fürchtete angesichts des nahen Fristendes den Zorn Gottes beim Bruch des Gelübdes, aber auch, dass er wg. seiner Jugendlichkeit (tēn kat' eme...neotēta nicht in der Lage wäre, dieses einzuhalten, zumal im Hinblick auf die Jugend u. Schönheit seiner Frau Theodora.

Auskunft, Bescheid oder Urteil (samt Strafen oder Auflagen): Das Gericht verhängt diverse Bußstrafen...für beide, also auch für Theodora, die, wie während des Verfahrens bekannt wurde, ein ähnliches Versprechen (Nonne zu werden, falls er Mönch werde) abgelegt hatte. So werde sie ihrem Mann bei der Buße eine Stütze sein.

Quellen- u. Literatur-Hinweise: Ego.Dok. (vor Gericht in Vertretung verlesen, durch den Bischof von Moglena und eine weitere Vertrauensperson). Simon, Bußb., 237 f., 239; Simon, Byz. Prov., $316 \mathrm{f}, 332$

Edition: $P D$

Datierung: ca. nach 1222

Ort/Region: Mokros

Art d. Akte: Synodal-Urteil

Sachverhalt: Ehescheidung im Prozess von Slaba u. dem Stratioten Rados aus Mokros: Slaba, erst 13 alt, wurde von ihrer Bediensteten Kale zum Ehebruch mit deren Bruder verführt.

Auskunft, Bescheid oder Urteil (samt Strafen oder Auflagen): Freispruch Slabas wg. jugendl. Alters. Rados kann sich von ihr trennen oder bei ihr bleiben; er verweigert aber eine Sicherheitserklärung für sie, daher wird die Scheidung verfügt. Wolle aber Rados reumütig die Ehe fortsetzen, ginge das innerhalb von zwei Jahren.

Quellen- u. Literatur-Hinweise: Ind. Ego-Aussagen. - Katerelos, s. bes. $38 \mathrm{ff}(U ̈), 107 \mathrm{f}$. (gute Interpr.); Iliev, 166, 173, 305 f. (Ü); Prinzing, Hausbedienstete, $194 \mathrm{f}$.

27 Akte: \# 141*

Edition: $P D$

Datierung: nach 1222

Ort/Region: wohl Achrida/Ohrid

Art d. Akte: Urteil d. erzb. Gerichts

Sachverhalt: Scheidungsprozess des jugendlichen/neaniskos Theodoros Chloropodes, der von eigener Arbeit u. Bettelei lebte, gegen die ebenfalls jugendliche Eirene, Tochter des Michael Bodeniates, der ebenso wie Th.Chl. lebte. Chl. beantragt die Scheidung, weil seine Frau ihn hasse u. das Zusammenleben mit inm verweigere. E. bestätigt dies und droht mit Selbstmord, falls die Ehe nicht geschieden wird. Die Eltern sagen, sie hätten u. a. durch Gewalt, auch mit der Peitsche, versucht, die öfter tagelang dem Haus ihres Mannes entflohene E. zu inm zurückzubringen. Wichtig die Aussage der Maria, Schwester des Theodoros, dass E. einen Georgios Cholauros liebe und sogar wochen- und monatelang aushäusig war. Die Eltern wussten von E.s Neigung zu Cholauros, mochten ihn aber nicht: Darum hätten sie E. mit Chloropodes verbunden.

Auskunft, Bescheid oder Urteil (samt Strafen oder Auflagen): Der Ehebruch Eirenes ist erwiesen. Daher wird die Scheidung verfügt. Theodoros darf eine neue Ehe eingehen. Wg. der Armut wird aber, abgesehen von den kanonischen Strafen für Ehebruch, auf mögliche materielle Strafen etc. verzichtet für die Seite der Frau. Denn die Armut und ihre Folgen sind Strafe genug.

Quellen- u. Literatur-Hinweise: Katerelos, 39-41 (Ü), 109-111 (K)

Besondere Bemerkungen: Ind. EgoD. beider vor Gericht 
PRK

Akte: \# 11*

Edition u. Regest: PRK I; Dar. Reg. 2039

Datierung: 1315

Ort/Region: Region Melenikon

Art d. Akte: Synodal-Urteil

Sachverhalt: Scheidungsprozess des ehem. kaiserlichen Pagen, Konstantinos Palates/21561, gegen die Partei seiner Verlobten NN, Tochter der N. Alamanina/ 539 aus Melenikon, mit der (ohne Mitwirkung seiner Eltern) bei Leistung der Arrha ein Mitgiftvertrag geschlossen worden sei. Als K.P. (wie schon zuvor der frühere Verlobte der NN, Georgios Tarchaniotes) nach Verlobung und Aufnahme ins Elternhaus der Verlobten bei ihr diverse körperliche Mängel feststellte, verweigerte er sich der Eheschließung und entfloh für 4 Jahre ins Ausland. Nach Rückkehr von dort, beantragte er, da seine Abneigung gegen $\mathrm{NN}$ anhielt $\mathrm{u}$. sich noch zum Hass gesteigert hatte, die Scheidung.

Auskunft, Bescheid oder Urteil (samt Strafen oder Auflagen): Die Scheidung wird verfügt. Gründe: Der Hass zwischen dem Brautpaar, die Täuschung durch die Alamanina, keine Mitwirkung der Eltern des Konstantin Palates. Da man inn in die Hausgemeinschaft der Brauteltern aufgenommen habe, solle seine Partei den Wert des von ihm behaltenen und verbrauchten Mitgift-Anteils der Braut ersetzen. Der Mpt. von Melenikon solle dies vollstrecken.

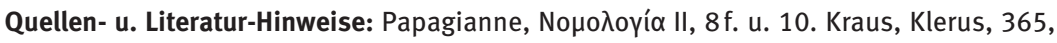
Anm. 249 (kein Hinweis auf Papagianne)

Besondere Bemerkungen: Altersangaben für Palates und die NN fehlen. Aber die mangelnde Mitwirkung seiner Eltern und die für NN agierende Mutter lassen auf die Minder jährigkeit beider schließen.

29 Akte: \# $14^{\star}$

Edition u. Regest: PRK I; Dar. Reg. 2043

Datierung: 2.H. 1315

Ort/Region: Region Drama

Art d. Akte: Synodal-Brief

Sachverhalt: Bescheid für die Tante des Kaisers, die Megalē Papiaina, Eudokia Nestongonissa/20197 im Hinblick auf ihre Forderung gegen die von ihr adoptierte Ziehtochter NN. Diese lebte seit ihrem 7. Lebensjahr bei hrer Ziehmutter, von der sie viele Dinge zur Verwahrung, aber auch für ihre Mitgift erhalten hatte. Da die NN aber nun ohne Zustimmung der E.N. geheiratet hatte und sich ihr gegenüber undankbar, bösartig und unverschämt verhielt, richtete E.N. an den Patr. die Forderung auf Unterstützung bei der Restitution all dieser Dinge.

Auskunft, Bescheid oder Urteil (samt Strafen oder Auflagen): Bescheid: Die Nestongonissa fordert zu Recht die Restitution. Der zuständige Mpt. von Philippopel soll mit dem Bf. von Drama am Ort den Sachverhalt überprüfen und das Urteil vollstrecken.

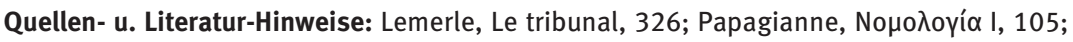

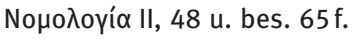

30 Akte: \# 89*

Edition u. Regest: PRK I; Dar. Reg. 2122

Datierung: Mai 1325

Ort/Region: Region Ainos

Art d. Akte: Synodal-Urteil 
Sachverhalt: Scheidungsverfahren zw. dem Sohn NN des Klerikers (archōn tēs ekklēsias) Konstantinos Argyros/ 1289 u. der 11-jährigen Tochter NN d. Klerikers Manklabites /16073 wg. Unmündigkeit / anēbotēs der Braut, zu früh vollzogenem Beischlaf u. Körperverletzung der Frau. Die Braut war in das Haus d. Bräutigams eingezogen, nachdem dessen Vater zugesichert hatte, dass sein Sohn solange nicht mit ihr verkehren werde, bis sie das gesetzliche Heiratsalter erreicht haben würde. Doch der Bräutigam verstieß mit Gewalt gegen die Abmachung: Die Braut wurde körperlich schwer verletzt u. erlitt dauernden Schaden.

Auskunft, Bescheid oder Urteil (samt Strafen oder Auflagen): Die Ehe wird geschieden mangels Ehemündigkeit der Frau u. wg ihrer erlittenen körperlichen Schäden mit Dauerfolgen. Der Vater des NN muss die Mitgift erstatten u. das theoretrohypobolon zahlen, beides an den Brautvater.

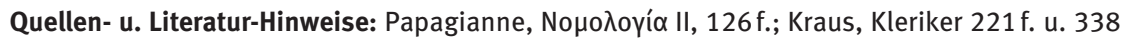
(vgl. auch das Regest im PRK) nimmt an, dass die Braut vergewaltigt wurde.

Edition u. Regest: PRK I; Dar. Reg. 2125

Datierung: Juni 1325

Ort/Region: Ennakosia (Thrakien)

Art d. Akte: Synodal-Urteil

Sachverhalt: Vor 5 Jahren war die damals 8-jährige Halbwaise, Tochter NN der Witwe Apostolina / 1202 aus Ennakosia/ Thrakien mit Theodoros Diakonites/ 5379 bei Leistung der Arrha und unter priesterlicher Einsegnung verlobt worden. Diakonites machte sich aber in den 5 Folgejahren zunehmend bei der Braut u. ihrer Mutter verhasst. Daher wollte die Apostolina die Verlobung außer Kraft setzen lassen und klagte auf deren Aufhebung, um die Eheschließung zu verhindern.

Auskunft, Bescheid oder Urteil (samt Strafen oder Auflagen): Das Gericht erklärte wg. der gesetzwidrigen Unmündigkeit/anēbotēs der bei ihrer Verlobung erst 8-jährigen Braut die Verlobung für gesetzwidrig. Die Scheidung beider wird verfügt, bei Zurückzahlung bestehen der Verpflichtungen. Der Priester wird vom Amt suspendiert.

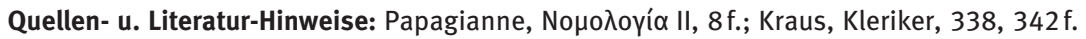

Besondere Bemerkungen: Das Alter des Theodoros ist unklar.

Akte: \# 100*

Edition u. Regest: PRK I; Dar. Reg. 2153 (+ \# 102*, Dar. Reg. 2156)

Datierung: Febr.1329 bzw. April 1330

Ort/Region: Adrianopel

Art d. Akte: Synodal-Urteile

Sachverhalt: Erbschaftsstreit zwischen der jetzigen Nonne Euphrosyne Petraleiphina/29013 u. ihrem Schwiegersohn, kyr Theodoros Branas/3170: Seine Frau Maria Branaina/3150, die Tochter der Petraleiphina, war im Alter von 22 Jahren kinderlos verstorben, hatte aber vor ihrem Tod mündlich u. vor Zeugen (darunter ihre Mutter) ihren Ehemann als ihren Erben eingesetzt. Aber 3 Monate später focht die Mutter das Testament als ungültig an, (weil ihre Tochter noch keine 25 J. alt war u. nur mündlich testiert hatte). Dagegen klagte dann Branas. - In \# 102 bestritt die Mutter die Sachverhaltsdarstellung des Branas, verwies auf eine Vergleichsurkunde mit ihm, die ihr die volle Verfügunggewalt einräumte, mit Ausnahme der Häuser in Adrianopel.

Auskunft, Bescheid oder Urteil (samt Strafen oder Auflagen): Branas erhielt zuerst 2/3 des Erbes zugesprochen, die Mutter 1/3; doch nach ihrem Hinweis auf die von ihr (wg. Verhinderung des Branas) besorgten spiritualia für ihre Tochter, bekam sie ein weiteres Drittel zugesprochen. Ihr Einwand, die Verfügung der Tochter sei wg. deren Minderjährigkeit ungültig, 
ist rechtlich unbegründet. - In \# 102 wird nach dem Widerspuch der Petraliphina die Aufteilung der Erbanteile beider Parteien revidiert u. die Überprüfung der Verhältnisse in Adrianopel angeordnet.

Quellen- u. Literatur-Hinweise: Ind. EgoD. der verstorbenen Maria Branaina in \# 100. Hier ist

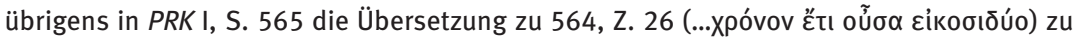
korrigieren: Lies: „da sie noch eine 22-Jährige sei“ statt „, und das bei ihren 22 Jahren !“;

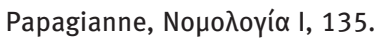

33 Akte: \# $125^{*}$

Edition u. Regest: PRK II; Dar. Reg. 2197

Datierung: Mai 1340

Ort/Region: Thessalonike

Art d. Akte: Synodal-Urteil

Sachverhalt: Demetrios Eskammatismenos/6143, Kleriker aus Thessalonike, zum zweiten Mal verheiratet, ersuchte um die Priesterweihe $u$. wollte daher wissen, ob sein wg. Min-

derjährigkeit / aphēlikiotēs der Braut (Xene) annullierter 1. Heiratsvertrag ein Hindernis für die Priesterweihe sei. Damals war E. als voll verwaister Knabe seinen zwei älteren Brüdern unterstellt gewesen. Die Untersuchung entlastete ihn.

Auskunft, Bescheid oder Urteil (samt Strafen oder Auflagen): Eskammatismenos kann zum Priester geweiht werden.

Quellen- u. Literatur-Hinweise: Ind. EgoD. Kraus, Kleriker,149 f., 323.

Besondere Bemerkungen: Eskammatismenos war zur Zeit des Antrags wohl Anagnost oder Diakon, s. ebd., 150.

34 Akte: \# 136

Edition u. Regest: PRK II; Darr. Reg. 2227

Datierung: 1342

Ort/Region: Region Apros

Art d. Akte: Synodal-Urteil

Sachverhalt: Michael Kabasilas/10101, fragt, ob er kirchliche Weihen empfangen dürfe. Von klein auf stand er in der Obhut des Mtrp. u. Katholikos Kritēs loseph von Apros/9027 u. hatte inm bei seinen Urteilen etc. assistiert. Nach dessen Amtsverzicht habe auch er verzichtet, wünschte nun aber Priester zu werden. Er wurde aber von einigen der Habgier und Bestechlichkeit bezichtigt, auch im Zusammenhang mit dem Amtsverzicht des Metropoliten. Dies war zu prüfen.

Auskunft, Bescheid oder Urteil (samt Strafen oder Auflagen): Die Synode sah keinen Anlass, Kabasilas den Zutritt zum Diakons- bzw. Priesteramt zu verwehren.

Quellen- u. Literatur-Hinweise: Eine indirekte Altersangabe zu Kabasilas ist im Mindestalter für Diakone (25) zu erblicken oder in dem für Priester: 30; Kraus, Kleriker, $295 \mathrm{f} ., 328$; Ders., Хعıротоvía, 156.

Allg 1 Akte: \# 219*

Edition u. Regest: PRK III; Darr. Reg. 2431

Datierung: 1360

Ort/Region: reichsweit

Art d. Akte: Rundschreiben

Sachverhalt: Allgemeine Ermahnung des Patriarchen Kallistos' I., dass man das Heiratsverbot für (unreife, nicht heiratsfähige: anēboi) Minderjährige einhalten sollte.

Besondere Bemerkungen: Allgemeiner Aufruf 
Akte: \# 236

Edition u. Regest: PRK III; Darr. Reg. 2421

Datierung: März 1360

Ort/Region: Umland von Konstantinopel (?)

Art d. Akte: Zu den Akten genommene Erklärung des G. Kallistos

Sachverhalt: Der Priester Georgios Kallistos /10488 bezeugt, der (verstorbene) Waisenknabe Alexios sei keineswegs urkundlich durch den (verst.) Priester Manuel Kallistos / 10493 adoptiert worden. Vielmehr habe er A. als Vollwaise aus Anatolien in sein Haus aufgenommen, wo er Lesen u. Schreiben lernte und eine Zeit lang sein Diener war. Um ihm vor Militär- u. vor Frondienst zu bewahren, gab er ihn als seinen Sohn aus. Als dieser sich aber der Trunksucht ergab, habe er Alexios für immer fortgejagt.

Auskunft, Bescheid oder Urteil (samt Strafen oder Auflagen): Zeugenunterschrift des Georgios Kallistos u. anderer. Zur Bestätigung, auch vor loakeim, dem Beichtvater des Priesters Manuel Kallistos, dass es zu keiner Adoption gekommen sei.

Quellen- u. Literatur-Hinweise: Kraus, Kleriker, 283, 321, 324f. mit Anm. 36-41, 401, 473

Besondere Bemerkungen: Vgl. PRK III \# 235, S. 359+ 361, die Bemerkungen zu Alexios (PLP 00), Georgios Kallistos u. Manuel Kallistos.

Allg 2 Akte: \# 240*, s. 390, 95 f. u. 392,97-109

Edition u. Regest: PRK III; Darr. Reg. 2402

Datierung: Dez. 1357

Ort/Region: wohl Konstantinopel

Art d. Akte: siehe unten

Sachverhalt: Annahmeerklärungen der dem Exarchen loannes Petriotes/23043 unterstehenden Kleriker, hier die des Priesters Konstantinos Strongylos/26954, der u. a. bekräftigt, weder Eheschließungen Minderjähriger/Unmündiger ( $a \bar{e} b \bar{b} n$ ) vornehmen, noch Beihilfe zu einer solchen leisten zu wollen.

Quellen- u. Literatur-Hinweise: Kraus, Kleriker, 303 (Anm. 240), 340, 403

Besondere Bemerkungen: Allgemeine Erklärung, Selbstverpflichtung

Akte: \# 392, $97-98$

Edition u. Regest: $M M$ II; Darr. Reg. 2817

Datierung: 1387, März

Ort/Region: wohl Konstantinopel

Art d. Akte: Promissio

Sachverhalt: Der 22-jährige Subdiakon Manuel Sguropulos/25027 hatte um Ernennung zum Diakon schon in diesem Alter (statt des Mindestalters von 25) gebeten.Versprach, immer sein korrektes Alter anzugeben.

Auskunft, Bescheid oder Urteil (samt Strafen oder Auflagen): Seiner Bitte wurde stattgegeben, unter der Bedingung, dass er dies angibt bei späteren kanonischen Erhebungen.

Quellen- u. Literatur-Hinweise: EgoD., Promissio. - Kraus,Kleriker, 321, 325f., 330; Kraus, Хعıротоví $\alpha, 157$

37 Akte: \# 442, 176

Edition u. Regest: MM II; Darr. Reg. 2933

Datierung: 1393, Okt.

Ort/Region: wohl Konstantinopel

Art d. Akte: Protokollnotiz (Semeioma) 
Sachverhalt: Der noch junge Konstantinos Rhamatas/24071 bittet darum, eine dritte Ehe eingehen zu dürfen.

Auskunft, Bescheid oder Urteil (samt Strafen oder Auflagen): Sie wird zugelassen wegen seiner Jugend / to néon. Doch die „andere“ (heteron) Tochter wird über den Wingert in Astrabiki, 2 Häuser, ein Warenlager u. 10 Exagia/ (Nomis.) chrysaphin verfügen.

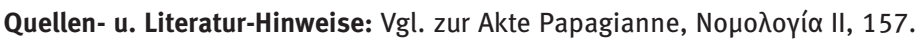

Besondere Bemerkungen: Diese „andere“ Tochter, ein paidion, war wohl 4-10 Jahre alt (vgl. Prinzing, Observations,17). Nach Darrouzès war sie aus 2. Ehe, wofür aber ein Beleg fehlt.

38 Akte: \# 464*, 212

Edition u. Regest: $M$ M II; Darr. Reg. 2962

Datierung: 1394, Mai

Ort/Region: Methymna

Art d. Akte: Synodal-Bescheid

Sachverhalt: Bescheid an den Mpt.von Methymna / Lesbos zum Gesuch des Demetrios Asomatianos/1601. Seiner Aussage nach wurde er vor einiger Zeit nur auf Basis einer Vereinbarung, ohne kirchliche Bindung, mit der Tochter NN des Nikolaos Bambakas / 2130 verlobt.

Diese Verlobung mit dem minderjährigen/aphēlix (noch nicht heiratsfähigen) Kind wolle er nun aufheben, um eine andere Frau legal zu heiraten. Er bat die Synode auch um einen Bescheid, ob „das Getane“ (= besagte Verlobung) ein Hindernis für den Erwerb eines Weihegrades sei. Auskunft, Bescheid oder Urteil (samt Strafen oder Auflagen): Der Adressat möge die Angelegenheit prüfen, worin der Vertrag bestand, und das Ergebnis schriftlich mitteilen.

Quellen- u. Literatur-Hinweise: Ind.EgoD. Vgl. Kraus, Kleriker, $365 \mathrm{f}$.

Besondere Bemerkungen: Das Alter des Asomatianos ist unklar.

39 Akte: \# 553, 354, 1-14), und nochmals: \# 553, 354-355

Edition u. Regest: MM II; (Darr. Reg. 3065), und Reg. 3108

Datierung: Febr. 1398 und Febr. 1400

Ort/Region: Konstantinopel

Art d. Akte: Synodal-Urteil

Sachverhalt: Beschluss für den minderjährigen (aphēlix/neos) lakobos Tarchaniotes/27481, der Immobilien an das benachbarte Kloster Myrelaion verkaufen wollte.

Auskunft, Bescheid oder Urteil (samt Strafen oder Auflagen): Der Verkauf soll unter Kontrolle erfolgen. Der anfangs (1398) zu niedrig festgesetzte Preis wird nach lakobos' wiederholten Beschwerden unter dem im J. 1400 v. Patr. eingesetzten Vormund Alexios lagupes/7819 erhöht: Das Kloster muss nun den ca. doppelten Preis zahlen.

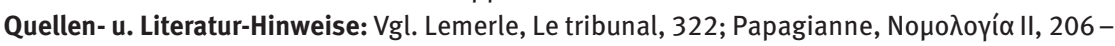
208

40 Akte: \# 569*, 382-383

Edition u. Regest: $M M$ II; Darr. Reg. 3069

Datierung: März 1399

Ort/Region: Konstantinopel

Art d. Akte: Praxis

Sachverhalt: Bestätigung eines Darlehensvertrags, mit Sicherung der Unantastbarkeit der Mitgift der Frau. Kyr Michael Palaiologos/ 21523 (er ist älter als 20 J., jedoch noch minderjährig/aphēlix) will an kyr Michael Magistros Pothos/23456 76 Hyperpyra als Darlehen geben. Das Eigentum des Michael Palaiologos ist in der Mitgift seiner Frau NN enthalten, die auch noch minderjährig ist. 
Auskunft, Bescheid oder Urteil (samt Strafen oder Auflagen): Die Synode gewährt Michael Palaiologos die Altersdispens (syngnōmē hēlikias). Aber er soll ein anderes seiner Grundstücke sicherheitshalber der Mitgift seiner Frau übertragen.

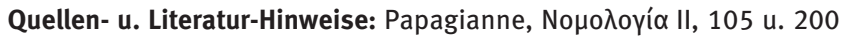

Besondere Bemerkungen: Vgl. auch unten Fall 49!

41 Akte: \# 554, 355-358

Edition u. Regest: $M M$ II; Darr. Reg. 3109

Datierung: Febr./ Mz. 1400

Ort/Region: wohl Konstantinopel

Art d. Akte: Synod.-Patr.-Bescheid

Sachverhalt: Bescheid zur Teilung der ererbten Güter der drei kaiserlichen oikeioi und Söhne des kyr Perrios Lampadenos / 14412, deren jüngster der minderjährige (aphēlix/neos) Ioannes Palaiologos/21482 war. Sie baten daher den Patriarchen, sicherheitshalber die komplizierte Sache zu beaufsichtigen. Dies geschah unter Beiziehung von Experten.

Auskunft, Bescheid oder Urteil (samt Strafen oder Auflagen): Der Patriarch verfügt, der Jüngste dürfe nicht benachteiligt werden. Er garantiert ihm die Bewahrung seines Anteils bis zu seiner (d. Patr.) endgültigen Entscheidung.

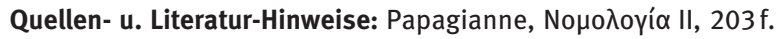

42 Akte: \# 571, $386 \mathrm{f}$.

Edition u. Regest: $M M$ II; Darr. Reg. 3129

Datierung: Mai 1400

Ort/Region: Konstantinopel

Art d. Akte: Synodal-Urteil

Sachverhalt: Kyr Demetrios Pegonites / 23254, hatte testamentarisch seiner Frau NN die Häuser in der enoria tōn Chalkoprateiōn, aber seinem Sohn Konstantinos Pegonites/23154 die Häuser in der geitonia tu Mustare vermacht; dieser benötigt als Minderjähriger/ aphēix noch die syngnōmē hēlikias/Altersdispens für evtl. eigene Verfügungen. In Geldnot borgte er sich 36 Hyp. von der Mutter, fuhr nach Sympolos (Cembalo/Krim), kehrte ganz verarmt zurück, ließ die ebenerdigen Häuser abreißen $u$. hatte das Gleiche auch vor mit dem mehrgeschossigen Haus. Besorgt um die Rückzahlung des Darlehens, wandte sich die Mutter an den Patriarchen, um den Hausverkauf zu ermöglichen. Der Patr. urteilte nach Anhörung beider Seiten. Auskunft, Bescheid oder Urteil (samt Strafen oder Auflagen): Der Patriarch erlaubt es Konstantinos mit der Altersdispens (s. Fall 40!), das Haus zu verkaufen. K.s Mutter tritt nun als Käuferin auf, bietet 85 Hyperp., nimmt aber nur 20 für sich und gibt den Rest ihrem verarmten Sohn. Zugleich wird sie damit Eigentümerin des Hauses.

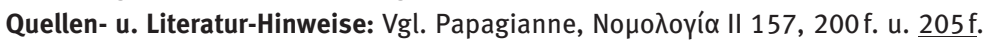
Besondere Bemerkungen: Erwähnt das urkundlich abgefasste Testament des Vaters Kyr Demetrios $P$.

Akte: \# 575, $390 \mathrm{f}$.

Edition u. Regest: MM II; Darr. Reg. 3133

Datierung: Mai 1400

Ort/Region: Konstantinopel

Art d. Akte: Patr.-Bescheid.

Sachverhalt: Bestätigt wird die reguläre Rückgabe eines Legats von 50 Hyp.an die Mutter des jugendl./ aphēlix loannes. Er war ein Neffe des Erblassers Demetrios Kydones/13876 und Sohn der Prōtomaistōrissa. Auf Wunsch der Testamentsvollstrecker (hier Manuel Gabalas) sollte der Patriarch die Rückgabe kontrollieren. 
Auskunft, Bescheid oder Urteil (samt Strafen oder Auflagen): Der Patr. bestätigt den Schutz der Testamentsvollstrecker vor späteren Reklamationen des erwachsen) gewordenen Kindes / paidiu (es war arm und mittellos) und seiner Mutter.

Akte: \# 584, 406 f. (nicht \# 583, 404 f., wie Darr. angibt)

Edition u. Regest: MM II; Darr. Reg. 3143

Datierung: Juni/Juli 1400

Ort/Region: Konstantinopel

Art d. Akte: Synodal-Urteil

Sachverhalt: Mehrmals schon hatte Andronikos Trichas / 29344 versucht, sein väterliches Erbe, das ein Vormund, sein Onkel Manuel Trichas für inn verwaltete, in seine Hand zu bekommen. Das aber hatte der Patriarch 2 Jahre lang verhindert wegen der Jugend des A. (der Patr.sah to neon tutu tēs hēlikias). Nun aber wird Manuel Trichas von seiner Aufgabe, Vormund für seinen Neffen (und dessen Brüder) zu sein, befreit. Dies geschah nach Rückgabe von 340 Hyperp.+ 8 kokkia und der aufgelisteten beweglichen Güter an eben den Neffen. Aber:

Auskunft, Bescheid oder Urteil (samt Strafen oder Auflagen): Andronikos Trichas darf nach der Aufteilung und Erhalt seines Anteils keinerlei Streit mit Manuel Trichas anfangen über die erhaltenen Gelder und Güter. Das wird garantiert. Kokkíon $=1$ keration $=1 / 24$ Nomisma, cf. Trapp LBG.

Quellen- u. Literatur-Hinweise: Trotz ähnl. Sachverhalts in Darr. Reg. 3069 u. 3129 hier keine

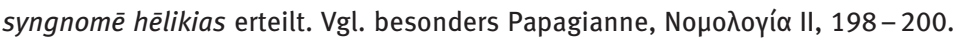

Akte: \# 587, $412 \mathrm{f}$.

Edition u. Regest: MM II; Darr. Reg. 3146

Datierung: Juli 1400

Ort/Region: Konstantinopel

Art d. Akte: Synodal-Urteil

Sachverhalt: Ioannes Chrysaphes/31079 klagt gegen den Priester Phokas/30226, seinen Schwager. Der verstorbene Blasios Tzakalos/27692 hatte noch vor seinem Tod dem Chrysaphes ein Legat von 40 Hyperp. vermacht, das der Priester Phokas für den minderjährigen / aphēlix Chrysaphes verwahren sollte. Phokas aber gab das Geld aus und ist daher zahlungsunfähig.

Auskunft, Bescheid oder Urteil (samt Strafen oder Auflagen): Der Patriarch gewährt eine 4Monatsfrist zur Regelung, danach kann Chrysaphes das Haus des Priesters an sich nehmen u., falls dies nicht genüge, auch von der Mitgift der Frau NN (u. Komplizin) des Ph. so viel, wie Chrysaphes zur Tilgung des Legats benötige.

Quellen- u. Literatur-Hinweise: Ind.EgoD. des Chrysaphes; Darrouzès schreibt versehentlich 50 statt 40 Hyperp.; Papagianni, Un témoin, 224

Akte: \# 610, $443 \mathrm{f}$.

Edition u. Regest: $M M$ II; Darr. Reg. 3170

Datierung: Okt. 1400

Ort/Region: Konstantinopel

Art d. Akte: Patr.-Brief mit Bescheid

Sachverhalt: Die minderjährigen Söhne, NN / 22348 u. Chrysoberges (er ist der ältere) des Priesters Pepagomenos/22352 möchten eine Hypothek von 50 Hyperpyra auf ihr Haus u. die Kirche des Hag. Theodoros Blachopulos aufnehmen, um sie auch den Gläubigern zu erstatten. Mit den Mönchen des mit diesen Gebäuden eng benachbarten Christos PhilanthropenosKlosters wollen sie darüber einen Vertrag schließen. Die Mönche aber ersuchen wegen der Minderjährigkeit/ aphēlikiotēs der 2 Brüder u. aus Furcht vor Belastungen den Patriarchen um 
Kontrolle des Vertrags. Die schon bestehende Hypothek (22 Hyperpyra) zahlen die Söhne dem Priestermönch Makarios/16221 (ident. mit 16225?) zurück.Da die Brüder die Stadt verlassen wollen, erklärt Chrysoberges dem Patriarchen u. den Mönchen, wie man den Rückkauf der Hypothek beim Tod eines der Brüder regelt.

Auskunft, Bescheid oder Urteil (samt Strafen oder Auflagen): Der Patr. stimmt dem Vertrag zu, damit den Brüdern geholfen sei. Die Rechte am Haus und der Theodoros-Kirche verbleiben bis zur Erfüllung des Vertrags bei dem Philanthropenos-Kloster.

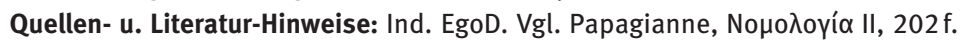

47 Akte: \# 613, $447 \mathrm{f}$.

Edition u. Regest: $M M$ II; Darr. Reg. 3173

Datierung: Nov. 1400

Ort/Region: Konstantinopel

Art d. Akte: Synod.-Patr.-Bescheid

Sachverhalt: Nikolaos Exotrochos/6085, minderjähriger/aphēlix Sohn des verstorbenen Exotrochos/6079, beklagt es, keine anderen Ressourcen als die Häuser zu haben, die aus dem elterlichen, mit seiner Schwester geteilten Erbe stammen. Er forderte, diese Häuser zu verkaufen. Der Patriarch hat anfangs Bedenken wegen N.s Minderjährigkeit.

Auskunft, Bescheid oder Urteil (samt Strafen oder Auflagen): Der Patr. stimmt schließlich doch zu: Ein Experte schätzt den Wert der Häuser auf 250 Hyperpyra, die nur N.s Kusine kyra Theodora Baropolitissa/ 2320 kaufen will, für 240 Hyperp. Dieses Geld, so der Patr., solle Martha, die Nonne u. Tante des Nikolaos E., verwahren u. für evtl. Ausgaben verwalten. Theodora solle unbesorgt sein.

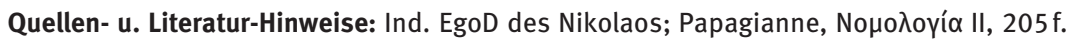

Akte: \# 646*, 492-494

Edition u. Regest: MM II; Darr. Reg. 3208

Datierung: Mai 1401

Ort/Region: Konstantinopel

Art d. Akte: Synodal.-Patr.-Bescheid

Sachverhalt: Der oikeios d. Kaisers (u. geistl. Sohn des Patr)., kyr Manuel Buzenos/3018, möchte aus wirtschaftl. Not ein Haus aus der Mitgift seiner minderjährigen/aphēlix Frau, kyra Theodora Buzene Philanthropene/29744, an den (von auswärts gekommenen) Argyropulos/ 1251 verkaufen, laut Vertrag für 270 Hyperpyra. Dafür bat er um Zustimmung. Der Patriarch billigt den Vertrag, schlug aber eine 8-Tages Frist für eine Versteigerung vor zur Hebung des Verkaufspreises. Argyropulos zieht zurück, keiner will ersteigern. Aber ein Archon, kyr Thomas Kallokyris /10640, zahlt die 270 Hyperp.

Auskunft, Bescheid oder Urteil (samt Strafen oder Auflagen): Die Synode teilt diese Summe, weil sie zur Schuldentilgung u. zur Unterhaltssicherung der Buzenos-Familie benötigt wird. Der Patriarch bestätigt die Gültigkeit des Vertrags u. erklärt, alles zur Wahrung der Interessen der minderjährigen Frau des Buzenos unternommen zu haben.

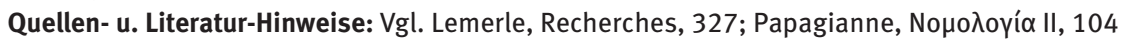
Besondere Bemerkungen: Aussteller der Verkaufsurkunde sind die Philanthropene (Theodoras Mutter), Buzenos sowie seine Frau.

49 Akte: \# 678*, $557 \mathrm{f}$.

Edition u. Regest: MM II; Darr. Reg. 3240

Datierung: Nov. 1401

Ort/Region: Konstantinopel

Art d. Akte: Synod./Patr.-Brief 
Sachverhalt: Kyris Michael Palaiologos/ 21523 bat um Erlaubnis für den Verkauf des Weinbergs (aus der Mitgift seiner minderjährigen/aphēlix Frau NN) nahe der Auferstehungskirche im Viertel Hag. Romanos. Die Käufer hätten Bedenken wg. der Minderjährigkeit seiner Frau. Er benötige dringendst Geld, sonst drohe der Ruin.

Auskunft, Bescheid oder Urteil (samt Strafen oder Auflagen): Der Patriarch will die Äquivalenz der Mitgift u. auch die freie Verfügbarkeit der Grundstücke durch den Mann sicherstellen. Drei kirchl. Archonten begeben sich zu Michaels Frau, lesen ihr d. Vertrag vor u. überprüfen Details. Sie erhält ersatzweise ein Feld u. einen Weinberg von 280 Hyp. Ihr Mann darf verkaufen wie geplant. Kein Anlass zur Beunruhigung.

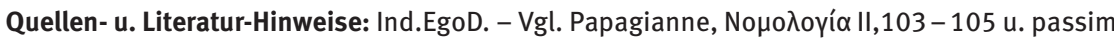
(s. Register).

Besondere Bemerkungen: Michael gilt im PLP (wohl zu Recht) noch als unmündig, vgl. auch oben Fall 40.

\section{Bibliographische Hinweise zur Tabelle}

Delemares, "Aп $\alpha v \tau \alpha$ : wie in Anm. 11

Fögen, Rechtsprechung: wie in Anm. 48

Iliev: wie in Anm. 12

Katerelos: wie in Anm. 15

Kiousopoulou: wie in Anm. 17

Kraus, Klerus: wie in Anm. 14

Kraus, Ehe und Verlobung: wie in Anm. 14

Kraus, Xeıрotovía: wie in Anm. 54

Laiou, Institution: wie in Anm. 17

Laiou, Mariage: A.LAIOU, Mariage, amour, et parenté à Byzance aux Xie-III ${ }^{\mathrm{e}}$ siècles. Paris 1992

Laiou, Priests: A. LAIOU, Priests and Bishops in the Countryside, Thirteenth to Fifteenth Century, in:

D. ANGelov (Hrsg.), Church and Sociey in Late Byzantium. Kalamazoo 2009, S. 43-57. (NDr. in

DIES., EconomicThought and Life in Byzantium, ed. by C. MorRISSON and R. Dorin. Farnham

u. Burlington, 2013, Art. VIII).

Lemerle, Le tribunal: wie in Anm. 2

Miller, Orphans: wie in Anm. 26

MM II: F. MıKLosich - J. MüLleR (Hrsg.), Acta et diplomata graeca medii aevi sacra et profana, II.

Wien 1862

Papadatou, Divorce: D. Papadatou, Divorce bx Mutual Consent and its Customary Application in

Byzantium, BSI 58 (1997) 269-273

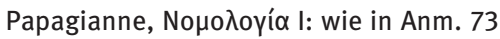

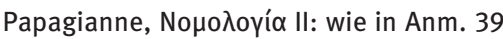

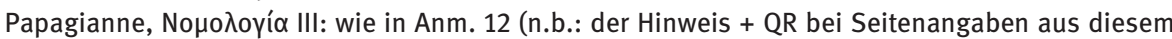

Werk bedeutet: vgl. auch das Quellen-Register des Bandes)

Papagianni, Un témoin: wie in Anm. 11

Prinzing, Hausbedienstete: wie in Anm. 4

Prinzing, Carev dvor: wie in Anm. 44

Prinzing, Adress. Briefe : wie in Anm. 29

Simon, Bußb.: wie in Anm. 9

Simon, Byz. Prov.: wie in Anm. 3

Stefec, Regesten = R. STEFEC, Die Regesten der Herrscher von Epeiros 1205-1318, RHM 57 (2015)

$15-120$ 


\section{Alice-Mary Talbot \\ The Adolescent Monastic in Middle and Late Byzantium}

The individualized nature of Byzantine monasteries is revealed in manifold ways, among them attitudes toward the inclusion of children and teenagers in monastic communities. As Richard Greenfield brilliantly demonstrated in a comprehensive study for the Dumbarton Oaks symposium of 2006 on "Children and childhood in Byzantium", monastic founders were strongly divided in opinion as to whether children and beardless youths should be permitted within the cloister walls. ${ }^{1}$

Perusal of only a select number of monastic foundation documents, saints' lives and Athonite acts might give the initial impression that in fact there could not be any adolescent monastics in Byzantium. The prohibitions are clear for a number of major monasteries and holy mountains: at Athos, ${ }^{2}$ Menoikeion $^{3}$ and the lavra of Stylos on Latros, ${ }^{4}$ no beardless youth under the age of 20 was granted access; at Kosmosoteira in Thrace no one younger than 26 could be considered a candidate for admission (and this was a modification of the original limit of $30 !){ }^{5}$ at the monastery of St. John on Patmos, at Phoberou in Bithynia, and Mar Saba in Palestine no beardless youths at all were admitted. ${ }^{6}$ At Eleousa in Macedonia admission of youths under 18 was strictly prohibited, but they were apparently allowed to become novices at

1 R. GREENFIELD, Children in Byzantine Monasteries: Innocent Hearts or Vessels in the Harbor of the Devil?, in: Becoming Byzantine: Children and Childhood in Byzantium, eds. A. Papaconstantinou A.-M. Talbot. Washington, D.C. 2009, 253-282.

2 The prohibition on beardless youths on Athos first appears in the typikon of John Tzimiskes (971-2), ch. 16 (J. P. Thomas - A. C. Hero, Byzantine Monastic Foundation Documents. Washington, D.C. 2000, 1:238, hereafter cited as BMFD; for Greek text see D. PAPACHRYssanthou, Actes du Prôtaton. Paris 1975, 209-215), and was reiterated by Constantine IX Monomachos in his typikon of 1045 ([15] Constantine IX, ch. 1, BMFD 1:285; PAPAChrysSAnThou, Actes du Prôtaton, 224-232). See also act no. 1 of 1089 of the Xenophontos monastery, stating that no beardless youth under the age of 20 will be admitted to the Holy Mountain (D. PAPACHRYssanthou, Actes de Xénophon. Paris 1986, 68, 70.36, 71.70, 74.174).

3 The rule for the monastery of Prodromos on Mt. Menoikeion ([58] Menoikeion, ch. 14, BMFD 4: 1601), prohibits boys and youths under 20 even to visit the monastery.

4 Laudatio of Paul the Younger of Latros, ch. 48, ed. H. Delehaye, in: T. WiEgand, Der Latmos. Berlin 1913, 153; (7) Latros Typikon, ch. 9, BMFD 1: 141: "I order that never any smooth-looking beardless fel-

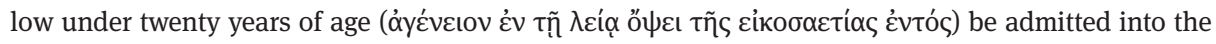
community of Lavra."

5 (29) Kosmosoteira, ch. 49-50, BMFD 2: 787, 822.

6 Patmos: (24) Christodoulos, ch. A10, BMFD 2: 583: "no[r] young men in their boyish prime, before their beard appears”; Phoberou: (30) Phoberos, ch. 58, BMFD 3: 939; Mar Saba: (42) Sabas, ch. 1, BMFD 4: 1314. Theodore of Edessa became a monk at Mar Saba precisely at age 20; see his vita, ch. VIII (ed. I. PomjalovskiJ, Žitie iže vo svjatych otca našego Feodora archiepiskopa Edesskogo. St. Petersburg 1892, 7).

Ә OpenAccess. (C) 2018 Alice-Mary Talbot, published by De Gruyter. (cc) BY-NC-ND This work is licensed under the Creative Commons Attribution-NonCommercial-NoDerivatives 4.0 License. 
a metochion, and then be tonsured at Eleousa once they reached the age of $18 .^{7}$ In Constantinople the 14-year old Symeon (the future New Theologian) was denied permission to become a novice at Stoudios until he was 20 on the grounds of his immaturity. $^{8}$

The repeated reissue of rules prohibiting beardless youths on Athos shows that their exclusion was not always rigorously observed, but the evidence of hagiography suggests that on the whole young teenagers were discouraged from coming to the Holy Mountain. Thus in the $14^{\text {th }} \mathrm{c}$. when the future Athanasios of Meteora first visited Athos as a youth, he was turned away as "beardless" (áyćveı $)$ ). He then went to Crete where his beard began to grow, and returned to Athos where he was tonsured at age 30. ${ }^{9}$ Likewise, in the same century, Germanos Maroules, while still a beardless student in Thessalonike, met John of Athos, his first spiritual mentor, and begged to join him on the Holy Mountain as his disciple. John, however, advised him to continue his studies, since his chin was still bare, and to come to Athos once fuzz appeared on his cheeks. ${ }^{10}$

\section{Opposition to monastic adolescents}

Because Greenfield has researched the subject in depth, I will only briefly summarize his findings on some of the reasons for opposition to the presence of young people, especially in male monasteries. As so vividly expressed in the typikon for the Phoberou monastery, a key factor was the fear of homosexual relations between older and younger monks, the latter described as "seething with fleshly passion". ${ }^{11}$ Unease with the presence of youth is well illustrated by a story from the $9^{\text {th }}$-c. vita of Anthony the Younger. Iakobos, who became Anthony's disciple, was first introduced to his

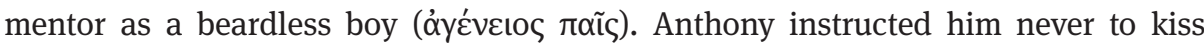
any monk who came to visit, whether an acquaintance or stranger. In fact he was admonished not even to engage in the exchange of the kiss of peace during the liturgy until his beard had grown to the length of a palm, that is, four fingers' breadth

7 (10) Eleousa, ch. 17, BMFD 1: 186.

8 R. GReenfield, The Life of Saint Symeon the New Theologian. Cambridge, Massachusetts 2013, 9-11, ch. 4.

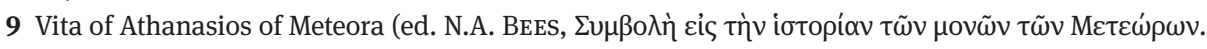

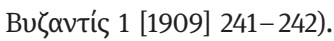

10 Vita of Germanos Maroules, ch. 7-8 (ed. P. Joannou. AnBoll 70 [1952] 60 -64). Note, however, that

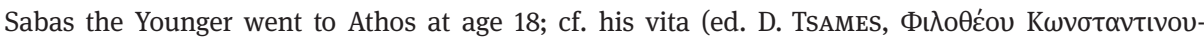

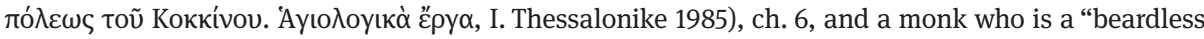
youth" is mentioned in ch. 16 of the vita of Niphon of Athos (ed. F. HALKIN, La vie de Saint Niphon, ermite au Mont Athos (XIVe s.). AnBoll 58 [1940] 23.24).

11 (30) Phoberos, ch. 58, BMFD 3: 940. This passage is borrowed from a much earlier abbot, Paul Helladikos, at the Elusa monastery in Idumaea (Palestine). 
or several inches. ${ }^{12}$ It is curious, however, that youths were deemed less of a sexual temptation once they had grown a beard, since at least one hagiographer admitted that the growth of facial hair also signified the onset of sexual awareness and desire; thus in the vita of Blasios of Amorion, the hagiographer refers to the moment when "down was about to cover his cheeks" as a time "when the swarm of passions insinuates itself into youth". ${ }^{13}$

Some founders also felt that teenagers would disrupt the tranquility of monastic life because of their tendency to noisy, boisterous behavior. Thus the rule for the monastery of Charsianeites did not permit any youths under the age of 16, "precisely on account of their tender years and their tendency to loose behavior and the scandal produced therefrom by the devil". ${ }^{14}$ The same document noted that the unstable and flighty temperament of teenagers was unsuited to the monastic regimen, and they should wait to become novices until they were more mature. ${ }^{15}$ Yet another reason for forbidding the admission of adolescent youths or eunuchs (both beardless, with smooth faces) was the fear that a woman might gain entry in disguise; this is the rationale of emperor Manuel II in his typikon for Athos of $1406 .^{16}$

\section{Variations in age of monastic tonsure}

Thus many monasteries prohibited adolescent novices, but this was by no means the rule everywhere, and in reality the situation was much more complicated. Although clearly there were misgivings about the admission of youthful postulants, following "the tradition of the fathers," 17 each monastery had its own rules on the subject which varied widely. In the $13^{\text {th }} \mathrm{c}$. Nikephoros Blemmydes was an outspoken advocate of the desirability of training future monks from a young age, when they were still malleable, calling them "innocent hearts"; he permitted novices as young as 12 at his monastery of Emathia near Ephesus. ${ }^{18}$ The nunnery of Neilos Damilas on Crete accepted postulants at age $13,{ }^{19}$ and Charsianeites and $\operatorname{Lips}^{20}$ in Constantinople

\footnotetext{
12 F. Halkin, Saint Antoine le Jeune et Pétronas le vainqueur des Arabes en 863. AnBoll 62 (1944) $222.13-18$.

13 Vita of Blasios of Amorion, ch. 7.72-74 (Acta Sanctorum, Nov. 4. Brussels 1925, 660).

14 (60) Charsianeites, ch. C2, BMFD 4: 1652.

15 (60) Charsianeites, ch. A3, BMFD 4: 1634.

16 (59) Manuel II, ch. 13, BMFD 4: 1621.

17 See, for example, Papachryssanthou, Actes de Xénophon, 71, 70.

18 See GreEnFIELD, Children in Byzantine Monasteries 254-256, and (36) Blemmydes, ch. 9, BMFD 3: 1202-3. He also prescribed a seven-year novitiate and tonsure at age 20.

19 (54) Damilas, ch. 5, BMFD 4: 1470.

20 Girls raised in the convent could be tonsured at 16, while those who came to the convent as teenagers could be tonsured at age 20, after a novitiate of three years; see (39) Lips, ch. 17-18, BMFD 3: $1270-71$.
} 
took in novices at ages 16 and 17 respectively. ${ }^{21}$ Biographical accounts of the careers of monks and nuns also attest to teenagers taking monastic vows: Antony Kauleas at 12 (before down began to grow on his cheeks), ${ }^{22}$ Luke of Steiris at $14,{ }^{23}$ the future patriarch Matthaios I at $15,{ }^{24}$ Eirene Choumnaina at $16,{ }^{25}$ and Sabas the Younger and Bakchos the Younger at $18 .^{26}$ The chart I have drawn up suggests that 18 was the most common age for admission as a novice (Appendix I).

Before moving on to the experiences of teenagers within the cloister, I should like to present evidence from hagiography on the question of how long adolescence lasted in Byzantium. For Byzantines the defining characteristic of male puberty was the growth of facial hair; in fact no other bodily alteration, such as deepening of the voice, is ever mentioned. There is very limited textual evidence for the age of beard growth, but the few passages that discuss this issue seem to indicate that it occurred later in the middle ages than in the contemporary world. From hagiographical texts we learn that facial hair first appeared on the faces of future saints at the following ages:

Theophanes the Confessor $-18^{27}$

Gregory of Dekapolis - $18^{28}$

Elias of Heliopolis - $20^{29}$

Luke of Steiris - ca. $21^{30}$

Less precise evidence informs us that Joseph the Hymnographer was still beardless at $15,{ }^{31}$ Maximos the Hutburner at $17,,^{32}$ Leontios of Jerusalem ca. $17-18,{ }^{33}$ and Bak-

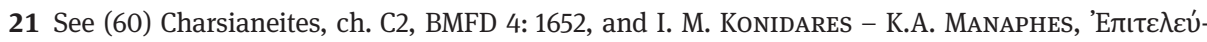

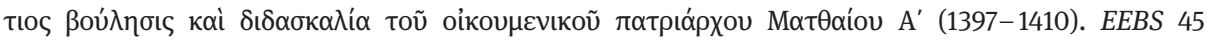
(1981-82) 497.

22 Vita of Antony Kauleas, ch. 4 and 6 (ed. P. L. M. LEONE, L'Encomium in patriarcham Antonium II Cauleam' del filosofo e retore Niceforo. Orpheus n.s. 10 [1989] 415, 90 and 416, 137-139).

23 C.L. Connor -W.R. Connor, The Life and Miracles of St. Luke [of Steiris]. Brookline, Massachusetts 1994, 140-141, ch. 86.

24 (60) Charsianeites, ch. A2-3, BMFD 4: 1633-1634.

25 A. C. Hero, Irene-Eulogia Choumnaina Palaiologina, Abbess of the Convent of Philanthropos Soter in Constantinople. BF 9 (1985) 121 and n. 9.

26 For Sabas the Younger ( $14^{\text {th }}$ c.), see n. 10 above; Vita of Bakchos the Younger, ch. 3 (ed. F. A. DE-

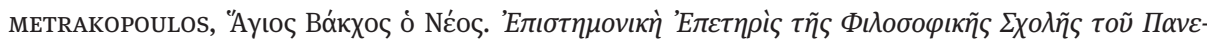

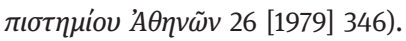

27 Vita of Theophanes by Methodios, ed. V.v. LATYŠEv, Mefodija patriarcha Konstantinopol'skogo Žitie prep. Feofana Ispovednika. St. Petersburg 1918, 6.3-6.

28 F. Dvornik, La vie de s. Grégoire le Décapolite et les Slaves macédoniens au IXe siècle. Paris 1926, 47, 11-48, 1.

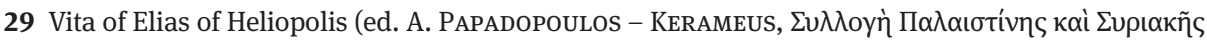
Ayıภoyías [= Pravoslavnij Palestinskij Sbornik 19.3 (1907) 47, 31-48, 1]).

30 Connor - Connor, The Life and Miracles of St. Luke, ch. 34, p. 54-55. The Connors postulate the age of 21 based on the chronology provided in ch. 86; see their note to ch. 34.3 on p. 159.

31 Vita of Joseph the Hymnographer, ch. 3 (ed. A. Papadopoulos -Kerameus, Monumenta graeca et latina ad historiam Photii patriarchae pertinentia, 2. St. Petersburg 1901, 3, 11-12). 
chos the Younger at $18 .{ }^{34}$ Moreover, the common prohibitions on admission to a monastery for beardless and smooth-faced youths under 20 implies that many young Byzantine men did not start to grow facial hair until their early 20s. Recent findings of a decline in the age of puberty for American and European teenagers in past centuries $^{35}$ suggests a need for caution in applying modern data to the medieval period; but this is a question well beyond the scope of this paper. In any case, I will include in my discussion information on Byzantine youths beyond their teenage years, into their early twenties.

\section{Desire for a monastic vocation during adolescence}

Longing for a monastic vocation was kindled in the hearts of many youths as young teenagers, but often they had to spend several more years at home or in school before gaining permission to enter a monastery. Such was the case of the future St. Athanasios of Athos, who came to Constantinople as an orphan to live with some distant relatives and pursue his education. Although he was living in an upper class household, he imposed upon himself a frugal, ascetic regimen. He refused to eat the white bread and fish that the servants prepared for him, but insisted on eating barley bread, and raw fruits and vegetables, and drank water instead of wine. He kept nocturnal vigils, splashing his face with ice-cold water in the effort to stay awake, and if he did succumb to drowsiness, he would sleep in a chair instead of a bed. Moreover he kept giving away his clothes to the needy. In the words of his hagiographer, "he was recognized as a monk even before he adopted the habit of the monastic way of life". 36

The future Symeon the Theologian also came to Constantinople as a child to live with relatives, in order to be educated and find greater career opportunities than in his native Paphlagonia. But when he turned fourteen, he decided to reject the secular world and enter monastic life. When he sought admission to the Stoudios monastery, however, Symeon Eulabes, his spiritual father, told him he was too young and to come back when he was more mature. So Symeon pursued the ascetic life on his

32 Vita of Maximos by Theophanes, ch. 3 (ed. F. HALKin, Deux vies de s. Maxime le Kausokalybite, ermite au Mont Athos [XIVe s.]. AnBoll 54 [1936] 69, 5).

33 See ch. 18 of the vita of Leontios (ed. D. Tsougarakis, The Life of Leontios, Patriarch of Jerusalem. Leiden 1993, 52-55). According to Tsougarakis's calculations, Leontios was born between 1110 and 1115 and arrived on Patmos as a beardless youth ca. 1130 -1132; thus, he could have been anywhere between 15 and 22, but was most likely in his late teens.

34 Vita of Bakchos the Younger, ch. 3 (346 Demetrakopoulos).

35 See, for example, E. WeIL, Where have all the sopranos gone? New York Times Magazine, November 10, 2013 (http://www.nytimes.com/2013/11/10/magazine/where-have-all-the-sopranos-gone.html? pagewanted=all\&_r=0).

36 Vita B of Athanasios of Athos, ch. 6 (ed. J. NoRET, Vitae duae antiquae sancti Athanasii Athonitae. Turnhout 1982, 131-132). 
own, praying and reading spiritual texts into the early hours of the night, or even cockcrow. On a visit home, he discovered a copy of the Spiritual Ladder of John Klimax in the family library, and read it assiduously along with holy scripture. He would shut himself up in the family chapel for hours in order to pray, sometimes holding his arms aloft for long periods of time. He also engaged in fasting and vigils, and visited tombs to meditate upon death. At one point he was granted an ecstatic out-of-body visionary experience. After six years of this self-imposed regimen, Symeon Eulabes finally permitted the youth to become a novice at Stoudios. ${ }^{37}$

The future patriarch Matthaios I decided to become a monk at age twelve, idolizing the men who devoted themselves to God and "led an angelic life upon earth." He became "obsessed with this [idea]," and his soul "burned like fire" with his passionate desire to abandon the world. His parents, however, were very upset at his plan, and sought to dissuade him, reminding him of the hardships of monastic life, which would be "difficult and scarcely tolerable for immature youths" like himself. ${ }^{38}$ He persevered, however, and three years later, when he was fifteen, his parents agreed to let him become a novice at the Charsianeites monastery. Even then the abbot Mark was reluctant to accept the youth, "on account of [his] tender years. For [immature youths] flit from one thing to another, and tire easily of the intensity of virtuous discipline and soon take their leave". ${ }^{39}$

\section{Monastic life for novices and youthful monks}

The period of the novitiate varied in Byzantine monasteries, ranging from six months to three years, with the longer term the norm for youthful postulants. Even the most enthusiastic teenager might be challenged by the discipline of a cenobitic monastery during the trial period. The novitiate was intentionally a tough period of probation, designed to separate youths with a true vocation from those of weaker character, to train flighty and unfocussed teenagers in routine, patience, and humility, and especially in obedience to their elders and the abbot. Not all postulants were accepted for tonsure, although it is impossible to determine what proportion of novices was successful. ${ }^{40}$ The typikon of Evergetis states that after an initial six-month trial period, if novices "appear to have grown tired, 〈and are) grumbling and being negligent about instructions," the abbot must decide whether or not to dismiss them. ${ }^{41}$ At the nun-

37 Greenfield, Life of Saint Symeon the New Theologian, ch. 4-11.

38 (60) Charsianeites, ch. A2, BMFD 4: 1633-4.

39 (60) Charsianeites, ch. A3, BMFD 4: 1634. For another description of a youth who began an ascetic life before taking monastic vows, see the vita of Maximos Kausokalybites by Theophanes, ch. 2 (67-69 HALKIN [as in n. 32]).

40 Thus, the typikon for the Black Mountain refers to the dismissal of a novice; see (77) Black Mountain, ch. 77, BMFD 1: 407.

41 (22) Evergetis, ch. 37, BMFD 2: 494-5. 
nery of Lips after a three-year novitiate teen-age novices were examined by all the nuns and either accepted or sent home. ${ }^{42}$ Hagiographic literature offers only tantalizing glimpses of young monastics who had second thoughts about their vocation, or succumbed to sexual temptation, like the girl who had abandoned a suitor in Cappadocia to take vows at the Constantinopolitan convent of Chrysobalanton. We are told that "the girl was unexpectedly attacked by a seething passion which maddened her with a frantic lust for her former suitor and did not allow her to control herself. Violently leaping, screaming, moaning, crying, and calling out his name in a loud voice," she threatened to commit suicide if she were not allowed to see her lover. ${ }^{43}$

Hagiography also provides illuminating glimpses into the experiences of more spiritually inclined young postulants. Paul of Latros went to extreme lengths to stay awake during night vigils: to keep from falling asleep, he used to tie large stones to his shoulders and walk around the monastery or on the mountainside. ${ }^{44}$ In the $12^{\text {th }}$ c. Leontios, the future patriarch of Jerusalem, was admitted to the Patmos monastery while still beardless, ${ }^{45}$ although such a practice had been prohibited by Christodoulos. Perhaps because of this breach of the rules, the abbot, Theoktistos, kept Leontios closely confined to his cell and instructed him not to associate with the other monks; he was to recite his prayers and chant the psalms in his cell, and apparently not attend church services. But Leontios imposed severe mortification upon himself as well: he engaged in self-flagellation (almost never attested in Byzantine monasticism), using a whip with nails embedded in it, and wept often and copiously. ${ }^{46} \mathrm{He}$ also used to sneak out of his cell during the night, go to the cemetery, and climb naked into a coffin that still held bones, as a "voluntary corpse" to contemplate death. ${ }^{47}$ Later on the youth was assigned to duties in the church; when from lack of sleep he would lean his head against the wall and doze off during services, the abbot would bang his head against the wall to wake him up. ${ }^{48}$

As for the young Symeon the Theologian there was no spare cell when he arrived at Stoudios, so he slept under the stairs in the cell of his mentor, Symeon Eulabes, in a tomb-like space into which he would squeeze his body with much difficulty. He engaged in fasts and vigils, and was assaulted at night by demons, especially those of fornication; as a result he suffered from sleepiness, lethargy and dizziness. ${ }^{49}$

42 (39) Lips, ch. 17, BMFD 3: 1270.

43 Vita of St. Irene, ch. 13 (ed. J.-O. Rosendvist, The Life of St. Irene, Abbess of Chrysobalanton.

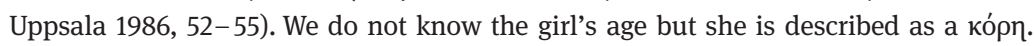

44 Vita of Paul of Latros, ed. H. Delehaye, in: T. Wiegand, Der Latmos. Berlin 1913, 108.

45 Vita of Leontios, Patriarch of Jerusalem, ch. 18 (ed. D. TsougARAKIs, The Life of Leontios, Patriarch of Jerusalem. Leiden 1993, 52-55). Leontios had previously taken monastic vows in Constantinople around the age of 15-17 (Ibidem 2), but was evidently treated as a novice on Patmos because he was still a beardless youth when he arrived there.

46 Ibidem ch. 19.

47 Ibidem ch. 20.

48 Ibidem ch. 21.

49 Greenfield, Life of Saint Symeon the New Theologian, ch. 13, 32-35. 
A frequent tactic of abbots was to deny to the most zealous novices permission to engage in strict asceticism, teaching them both obedience and humility. Thus on Mt. Kyminas Michael Maleinos told the newly arrived Athanasios that he must eat every three days rather than once a week as he preferred, and that he had to sleep on a mat on the floor instead of in a chair, as had been his habit as a layman. Michael's goal was to separate Athanasios "from his will," and to make him "a child of obedience". ${ }^{50}$ Likewise Symeon Eulabes, wanting to "eradicate" the younger Symeon's will, often made him eat and sleep, when he would have preferred to fast and keep vigil. ${ }^{51}$

\section{Education of novices and young monks}

The paucity of references to education and schools in typika and in hagiography demonstrates what John Thomas has called a less prominent "commitment to literacy and ... sense of educational mission ... in medieval Byzantine monasteries".52 There are in fact only a handful of references to the education of young monks and nuns in medieval Greek monastic foundation rules. Thus the typikon for the Bebaia Elpis convent in Constantinople refers to the role of the ekklesiarchissa (who among her many functions served as choir mistress) in teaching young nuns their letters and proper chanting; it also states that no lay girls were to be admitted for schooling, only girls who intended to take monastic vows. ${ }^{53}$ At the convent of Damilas on Crete, girls between the ages of 10 and 13 were to learn to read. ${ }^{54}$ Adolescent youths were sometimes relegated to a separate institution, often a metochion of the main monastery. Thus at the Bačkovo monastery, where boys who had not reached

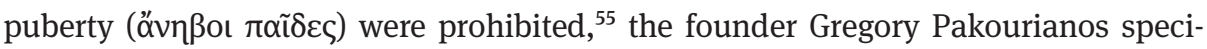
fied that six youths ( $\left.v \varepsilon о \gamma v \alpha \dot{\alpha} \pi \alpha ı \delta^{\prime} \alpha, \mu \varepsilon ı \rho \alpha ́ \kappa ı\right)$ ) were to be trained in Holy Scriptures in order to become priests, but should live separately at the nearby monastery of St. Nicholas. Once they grew beards, they could be ordained as lower clergy to serve at Bačkovo itself. ${ }^{56}$ This type of segregation of students from the monks is also mentioned in some of the very rare hagiographical allusions to monastic school-

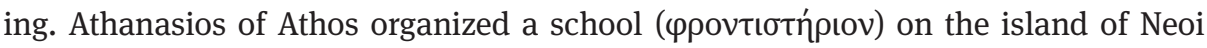
where younger monks at the Lavra could be educated, while apparently maintaining

50 Vita B of Athanasios of Athos, ch. 9 (ed. J. NoRET, Vitae duae antiquae sancti Athanasii Athonitae (CCSG 9). Turnhout 1982, 124-213, here 135-136).

51 GREENFIELD, Life of Saint Symeon the New Theologian 30-33, ch. 12.

52 BMFD 1: 28.

53 (57) Bebaia Elpis, ch. 53, 148, BMFD 4: 1538, 1564.

54 (54) Neilos Damilas, ch. 5, BMFD 4: 1470.

55 (23) Pakourianos, ch. 17, BMFD 2: 541.

56 (23) Pakourianos, ch. 31, BMFD 2: 550-51. 


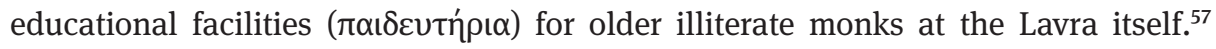
Nicholas of Stoudios went at age 10 to the Stoudios monastery to be mentored by his paternal uncle, the monk Theophanes, a familiar pattern. He was educated

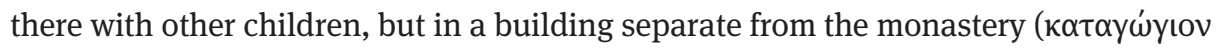

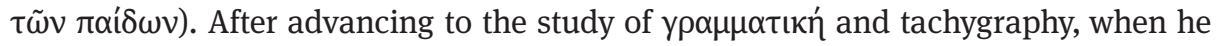
was presumably in his teens, he was tonsured once he started to grow a beard. ${ }^{58}$ The typikon for the Machairas monastery on Cyprus specifies that only bearded youths could be admitted as novices, and then trained in the holy scriptures; but even they were to be relegated to a "special cell". ${ }^{59}$ The experience of Makarios Makres seems relatively unusual: he had begun his studies in Thessalonike, but around 1400 was able to continue his training in the enkyklios paideia with a teacher named Armenopoulos at the Vatopedi monastery on Athos; this highly educated geron taught him rhetoric, logic, geometry, arithmetic and astronomy. Makres was in his late teens and early 20 s at the time. ${ }^{60}$

Such references to the pursuit of advanced studies, including the quadrivium, in a monastic environment are rare, and mostly from the late period of Byzantium. Ca. 1237 Nikephoros Blemmydes became hegoumenos at the monastery of Gregory Thaumatourgos in Ephesus, and at the request of the emperor John III Vatatzes established a school for five external lay students, among them George Akropolites. ${ }^{61}$ After the restoration of Byzantine rule to Constantinople in 1261, George of Cyprus probably offered instruction at the Akataleptos monastery, while Maximos Planoudes taught a wide range of advanced subjects at an unidentified imperial monastery. ${ }^{62}$ But their students were primarily destined to become civil servants or literati, not monks, and should be the subject of another paper.

\section{Servile duties of young monks and novices}

There is much evidence that novices and young monks were assigned menial and unpleasant duties, to teach them humility and to harden their bodies, although the typika are quite vague on the expected services. The Evergetis rule of the $11^{\text {th }}$ c., for example, states that novices "should be appointed tasks according to their abilities", ${ }^{63}$ while that of Mount St. Auxentios ( $13^{\text {th }}$ c.) says that they "must be exer-

57 Vita B of Athanasios of Athos, ch. 57, 45 (193, 179-180 NoREt [as in n. 50]).

58 Vita of Nicholas of Stoudios, $P G$ 105, 869C-872C.

59 (34) Machairas, ch. 115, BMFD 3: 1155.

60 Vita of Makarios Makres, ch. 14-16 and ch. 23-24 (ed. A. ARgYriou, Macaire Makres et la polémique contre l'Islam. Vatican 1986, 191 and 194-195).

61 See J. Munitiz, Nikephoros Blemmydes. A Partial Account. Leuven 1988, 20, 71-72.

62 C. Constantinides, Higher education in Byzantium in the thirteenth and early fourteenth centuries, 1204-ca. 1310. Nicosia 1982, $70-72$.

63 (22) Evergetis, ch. 37, BMFD 2: 494. 
cised in every service in the community" ${ }^{\prime 4}$, and the typikon for the Lips convent (ca. 1300) ordains that the novice nun should "be assigned to various duties, willingly switching from this duty to that one ... if she is so ordered by the superior". ${ }^{65}$ At St. Mamas the rule specified that young monks were to serve those advanced in age. ${ }^{66}$ Even at Phoberou, with its hostility toward beardless novices, an exception was made if there was an urgent need for attendants to care for elderly monks; in this case beardless youths were housed separately on a monastic estate, but during the day came to the monastery to minister to the elderly. ${ }^{67}$ The emphasis seems to have been on exposing the young monastic to various aspects of the type of work necessary to maintain a cenobitic community, and ensuring that the younger members assist the elderly and incapacitated ones. The abbot also welcomed the heavy labor that could be performed by strong and youthful monks and postulants.

As so often hagiography provides much more specific information than the typika. Thus, in the $9^{\text {th }}$ c. Euthymios the Younger, when he arrived at the monastery of Pissadinoi on Bithynian Mt. Olympos, probably in his early 20s, was first placed in charge of the pack animals, then worked in the kitchen as the cook's assistant, next as the cellarer's assistant, and finally as an ox team driver. ${ }^{68}$ In the following century Nikon "ho Metanoeite" was tonsured immediately after his arrival at the monastery of Chryse Petra in Pontos, and put to work carrying wood and water on his back up a steep mountainside, and serving meals in the refectory. Despite his hard labor he engaged in a harsh ascetic regimen, drinking only water and eating a piece of moldy barley bread once a week. After a two year trial he was relieved of his arduous duties, and permitted to devote himself to contemplation. ${ }^{69}$ Upon entering the Stoudios monastery as a novice, the future Symeon the New Theologian became essentially a servant to his mentor, Symeon Eulabes, carrying out menial responsibilities in his cell. ${ }^{70}$ When Symeon the Theologian himself became old, ill with dysentery and bedridden, he would accept care only from the young Nikephoros, who came to the monastery at age 14 as his servant. ${ }^{71}$ (It is not clear whether Nikephoros was officially a novice at this time, but he was tonsured after Symeon's death.) One of his duties was to use a mechanical device to help Symeon shift his position in bed to avoid getting bed sores. The youth slept on the floor in a corner of the holy man's cell. But because he slept so soundly, as teenagers often do, it was often difficult for Symeon to rouse him when he needed a bedpan or assistance

64 (37) Auxentios, ch. 12, BMFD 3: 1228.

65 (39) Lips, ch. 17, BMFD 3: 1270.

66 (32) Mamas, ch. 34, BMFD 3: 1018.

67 (30) Phoberos, ch. 58, BMFD 3: 939.

68 Vita of Euthymios the Younger, ch. 10 (ed. L. Petit, Vie et office de saint Euthyme le Jeune. Revue de l'Orient Chrétien 8 [1903] 176-177).

69 Vita of Saint Nikon, ch. 5 (ed. D. Sullivan, The Life of Saint Nikon. Brookline, Ma. 1987, 40 - 47). 70 GreENFIELD, Life of Saint Symeon the New Theologian, ch. 11, 28-31.

71 Ibidem ch. 116, 272-275. 
in going to the toilet. So Symeon advised him to sleep on top of a large chest close to his bed, so that he might be more easily roused. ${ }^{72}$ It is noteworthy that in choosing an adolescent boy as his attendant, who shared his room, Symeon was disregarding the admonition of Theodore, the founder of Stoudite monasticism, that adolescent disciples were to be banned from the abbot's cell. ${ }^{73}$

In the $14^{\text {th }} \mathrm{c}$. Romylos of Vidin moved early in his monastic career to a new monastery being built in the wilderness of Paroria by Gregory of Sinai. Since Romylos was so young and strong, Gregory assigned him the most arduous duties: hauling timber and stone from the mountainside to the construction site, and carrying water uphill from the river below. He also mixed lime and water for mortar, and worked in the kitchen and bakery. ${ }^{74}$ Next Romylos was charged with the care of a feeble but cantankerous elderly monk. The old man had a delicate stomach and a hankering for fresh fish, so Romylos wove fishnets with which to catch fish for the old man's dinner. In winter, when the water in the stream froze solid, Romylos had to break the ice up with a hammer, and wade in icy pools in order to catch the fish in a leather bag. ${ }^{75}$

The young disciples of solitaries, especially elderly ones, were indispensable attendants, who ministered to their every need, hauling water, cutting firewood, making fires, cooking, and fishing. They also ran errands, and walked long distances to monasteries to secure food supplies for their mentors. Luke of Steiris served a stylite at Zemena for ten years, carrying wood and water for him. He also did his cooking, mended nets and went fishing to catch his supper. ${ }^{76}$ The vita of Germanos Maroules, who spent his monastic career on Athos, gives a vivid picture of the control solitaries exerted over their youthful servants. The hagiographer, Philotheos Kokkinos, praises Germanos's obedience, noting that he would come immediately when summoned by his geron or elder; if he were copying a manuscript, he would stop in mid-stroke of forming a letter! ${ }^{77}$ Sometimes his geron would send him to Vatopedi, on the other side of the peninsula, a five-hour walk with a heavy load, involving a steep ascent and descent in both directions. Philotheos comments that he himself had made this hike without carrying a pack, and was panting and sweating so much he resolved never to try it again!

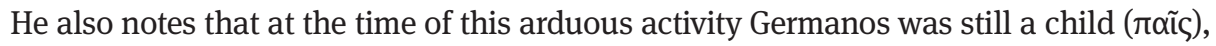

72 Ibidem ch. 125, $300-303$.

73 "You shall not have an adolescent disciple in your cell out of affection, but you shall be served by various brothers and by a person above suspicion"; see ch. 18 of the testament of Theodore, BMFD 1: 78.

74 Vita of Romylos of Vidin, ch. 4-5 (ed. F. HALKIn, Un ermite des Balkans au XIVe siècle. La vie grecque inédite de Saint Romylos. Byz 31 [1961] 111-117, here 119-121); Eng. tr. by M. BARTUSIS K. Ben NASSER - A. LAiou, Days and Deeds of a Hesychast Saint: A Translation of the Greek Life of Saint Romylos. Byzantine Studies/Etudes Byzantines 9 (1982) 24-47 at 29-30.

75 Ibidem ch. 6.

76 Connor - Connor, The Life and Miracles of St. Luke, ch. 35.

77 Vita of Germanos Maroules, ch. 9 (65 JoAnNou [as in n. 9]). 
who was not used to such strenuous exertion, since he came from a well-off family. ${ }^{78}$ Another holy man eulogized by Philotheos, Sabas the Younger, was praised for his ability to carry heavy loads when he served a harsh elder; on one occasion when he was sent to Esphigmenou to get provisions, he not only carried back his mentor's food but the load of an exhausted traveling companion as well. ${ }^{79}$

When the future Athanasios of Meteora first came to Athos, he served the geron Gregory, a strict taskmaster who treated him like a "beast of burden." Carrying a pad on his back like a mule's saddle, he had to walk long distances in all weather to fetch provisions; once when caught in a blizzard, he almost froze like ice; then wrapping clothes around his feet, he used them like snowshoes to complete his journey. ${ }^{80}$

\section{Abusive treatment}

Clearly these young novices and monks endured severe hardships and were in danger of both verbal and physical abuse by domineering older monks to whom they owed obedience. The future patriarch Matthaios I notes that he was sometimes assigned to "particularly difficult and unpleasant tasks" at Charsianeites. ${ }^{81}$ Once when the young Paul of Latros dozed off and yawned during services, his mentor, the abbot Peter, smacked him in the face, and he never dozed off again. ${ }^{82}$ Niketas Stethatos relates a shocking episode in his vita of Symeon the New Theologian. A bishop who was residing in a monastery had brought his young nephew to live with him and serve him, a common practice. On one occasion when he asked for a cup of wine mixed with water, he was infuriated because the boy did not make the concoction sufficiently hot. In a fit of anger he threw his staff at the boy, hitting him on the head and killing him..$^{83}$ This was an accident; other abuse was deliberate. The vita of Blasios of Amorion contains a lengthy story about a young kitchen worker, Euphrosynos (it is not clear whether or not he was a novice), who was ridiculed and beaten for having a garment soiled by soot. ${ }^{84}$ We learn that the young Maximos Kausokalybites was mocked by his geron, even though he was submissive and well trained in asceticism. ${ }^{85}$ Sabas the Younger had a particularly strict geron, harsh as the terrain he inhabited, who had alienated previous disciples by his severe treat-

78 Ibidem ch. 9, p. 66. Likewise Makarios Makres carried provisions for his geron, Harmenopoulos, using a packsaddle; sometimes he carried wheat on a daily basis; see anonymous enkomion of Makarios Makres, ch. 21-23 (193-194 ARgYRIou [as in n. 60]).

79 Vita of Sabas the Younger, ch. 12 (179-181 Tsames [as in n. 10]).

80 Vita of Athanasios of Meteora (243 BeEs [as in n. 9]).

81 (60) Charsianeites, ch. A3, BMFD 4: 1634.

82 Vita of Paul of Latros, ch. 16 (107 Delehaye).

83 Greenfield, Life of Saint Symeon the New Theologian, ch. 52, 114-117.

84 Vita of Blasios of Amorion, ch. 4-6 (as in n. 13).

85 Vita of Maximos by Theophanes, ch. 3 (69 HALKIN [as in n. 32]). 
ment of them. He also beat and abused Sabas, but the latter persevered and eventually won the respect and even affection of his mentor. ${ }^{86}$ Once when the aforementioned Romylos of Vidin spent too long catching fish for his geron, the irascible old man heaped ridicule on him and made him spend the night outside in the snow; he was found half dead the next morning, buried in a snow drift. ${ }^{87}$ When Romylos himself became a geron, he proved to be a severe taskmaster to his young charges. On one occasion when he sent his disciple Gregory to another elder to borrow a book, Romylos abused the youth harshly when he came back, because he had dared to read a chapter of the book during his return journey! ${ }^{88}$ On the other hand, when the disciple of another abusive geron was tempted to run away to escape his miserable existence, Romylos intervened on behalf of the maltreated youth and urged the elder to act with greater moderation. ${ }^{89}$

It is rare to find a more compassionate attitude toward novices, as in the rule of Makarios Choumnos, founder of a monastery in $14^{\text {th }}$-c. Thessalonike. He urged his monks to be patient and tolerant of inexperienced novices, even if they were surly. ${ }^{90}$ This attitude may derive from the Evergetis typikon which states that novices should not be punished as severely for disciplinary lapses as experienced monks. ${ }^{91}$ Choumnos also revealed a sensitivity to human nature when he recommended that the novice Theodoulos, from a peasant background, be permitted to spend time in the vineyard where he could feel at home and relax. ${ }^{92}$

\section{Conclusion}

I would conclude that as in so many aspects of monastic life there were significant variations in the experience of adolescent monastics, depending on the monastery where they lived. A number of institutions viewed beardless novices with suspicion and forbade their presence, others admitted them but tried to keep them separate from older monks, ${ }^{93}$ yet others welcomed, or even exploited, young postulants as a useful resource, essential for rigorous physical labor and assistance with the care of the elderly. For many starry-eyed youths, admission to a monastery was the fulfilment of a long-held desire, and they relished the opportunity to mortify their bodies while their spirits rejoiced in the routine of hymnody and prayer. The period of the

86 Vita of Sabas the Younger, ch. 8-9 (174-176 TsAmes [as in n. 10]).

87 Vita of Romylos, ch. 7 (123-124, 1-20 HALKIN [as in n. 74]).

88 Ibidem ch. 18.

89 Ibidem ch. 20.

90 (52) Choumnos, ch. B9, BMFD 4: 1448.

91 (22) Evergetis, ch. 4, BMFD 2: 474.

92 (52) Choumnos, ch. A17, BMFD 4: 1443.

93 Thus, Blemmydes, who favored the admission of very young teenagers as novices, stipulated that "they should be kept to themselves"; see (36) Blemmydes, ch. 9, BMFD 3:1202. 
novitiate was an arduous experience, however, and a resolute temperament and strong constitution were required to persevere and earn the privilege of tonsure. Hagiography, of course, focuses on the success stories; but I suspect that for every novice who thrived in the monastery and ended up as an abbot or saint, there were others, unheralded, who had a difficult transition to spiritual life; they may have left the monastery or been dismissed, or remained reluctantly and become grumpy and cantankerous senior monks. 
Age at Monastic Tonsure in Middle and Late Byzantium

6 Epiphanios of Maximina monastery (in Constantinople)

9 John of Kathara

12 Antony Kauleas

13 Phantinos the Younger

14 Luke of Steiris

15 Demetrianos of Chytroi

Joseph the Hymnographer

Matthaios I

Stephen the Sabaite

Stephen the Younger

Ca. 15-16 Ignatios the patriarch

Ca. 15-17 Leontios of Jerusalem

16

Eirene Choumnaina Palaiologina

Ca. 17 Nicholas the Stoudite

18 Bakchos the Younger

Euthymios the Younger

George of Mytilene

Gregory of Dekapolis

Makarios Makres

Michael Maleinos

Neophytos Enkleistos

Peter of Atroa

Sabas the Younger

19 Theophylaktos of Nikomedeia

20 Eustratios of Agauros

Hilarion of Dalmatos

Theodore of Edessa

20/21 Theophanes the Confessor

21 Theodore of Stoudios

22 Symeon of Lesbos

Age of Beard Growth in Byzantium

18 Theophanes the Confessor

Gregory of Dekapolis

20 Elias of Helioupolis

Ca. 21 Luke of Steiris

\section{Age when Still Beardless}

15 Joseph the Hymnographer

17 Maximos the Hutburner

ca. 17-18 Leontios of Jerusalem

18 Bakchos the Younger 



\section{Adolescence in the Late Byzantine Society $\left(14^{\text {th }}-15^{\text {th }}\right.$ centuries)}

It is well known that the late Byzantine Saints' Lives are not distinguished for their originality. Although many of them are not "old wine in new bottles", as Alice-Mary Talbot insightfully puts it, writing about new saints during the Palaeologan period is characterised by the basic rules of the genre. ${ }^{1}$ Even so, there is still evidence. Precisely, because they narrate the life of a person from birth until death, they have much to contribute to the subject that concerns us here, namely the notion of the adolescence in the Late Byzantine society, i.e. what adolescence meant in the Byzantine collective consciousness during the $14^{\text {th }}$ and $15^{\text {th }}$ centuries.

I am primarily interested in investigating how the authors of the Saints' Lives measure time, so that I can evaluate the information that they offer us on this subject. For the purpose of this paper, I have selected only a few Lives, which I consider representative: firstly, because they concern figures who lived during the Palaeologan period; secondly, because they concern figures with different qualities for which they were sanctified; and, thirdly, because their authors did not all have the same level of education. I have therefore examined the Lives of Patriarch Athanasios I, Athanasios of Meteora, Bishop Ioannis of Herakleia, Germanos of Athos, Romylos of Vidin, and Maximos of Kausokalyvia.

When reading these texts, we can immediately see that, just as during the middle period, ${ }^{2}$ so now the saint is constructed as a historical and therefore living person. He is located, that is, within a specific place and time. In other words, the author locates the saint within historical time, either determining the action in relation to himself (since the author often knew the saint and was his student), or by interweaving the action with events that the audience of the text apparently knew of. This is followed by the usual narrative motifs, relating the life of the saint in chronological order. The author puts the time of the saint's life into broader historical time, which is a neatly arranged time. I believe that the author articulates his text on

1 A.-M. TALвот, Old Wine in New Bottles: The Rewriting of Saints Lives in the Palaeologan Period, in: The Twilight of Byzantium, ed. S. Curcić - D. Mouriki. Princeton 1991, 15-26. For saints of the Palaeologan period see also A. LAIOU-THomadAKIS, Saints and Society in the Late Byzantine Empire, in: Charanis Studies. Essays in Honor of Peter Charanis, ed. A. Laiou - Thomadakis. New Brunswick N.J. 1980, 84-114; R. MACRIDES, Saints and Sainthood in the Early Palaeologan Period, in: The Byzantine Saint, ed. S. Hackel. London 1981, 67-87 and recently A.-M. TALBoT, Hagiography in Late Byzantium 1204-1453, in: The Ashgate Research Companion to Byzantine Hagiography I., ed. St. Efthymiadis. Farnham - Burlington 2011, 173-195.

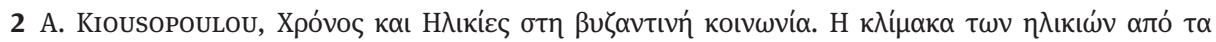

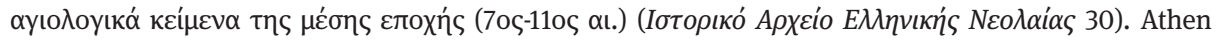
1997, 137-141.

Ә OpenAccess. (c) 2018 Tonia Kiousopoulou, published by De Gruyter. (cc) BY-NC-ND This work is licensed under the Creative Commons Attribution-NonCommercial-NoDerivatives 4.0 License. 
the basis of the concept of time, not simply because this is what the rules of the genre demand, but also as a narrative choice.

The narrative always begins with a reference to the family origins and childhood of the biographical person. ${ }^{3}$ There follows a series of topoi, such as his noble descent, the effort his parents made so he would learn the Holy Scripture, his unusual behaviour as a child and, of course, his inclination towards the divine.

The decision of the individuals under investigation to abandon the family home with the ultimate aim of becoming a monk marks their passage to the next phase of their lives, adolescence. During this period or, more precisely, towards its end, the saint withdraws from secular life, often preferring to become a hermit rather than getting married. However, the limits of adolescence, just as with those of childhood, do not always correspond to particular numbers. In order to make them clear, the biographers either select biological characteristics, such as the appearance of facial hair, or point to the parents' decision to marry their child, or they may even refer to ecclesiastical offices, which one is only eligible for after a certain age.

For example, Romylos of Vidin leaves his house in secret and goes to the Monastery of Hodegetria at Turnovo at an age, which is not specified but, nonetheless, coincides with the appropriate age for marriage according to his parents. ${ }^{4}$ Germanos also refuses to marry and leaves his family, ${ }^{5}$ as does Maximos who was ordained a monk at the age of seventeen ${ }^{6}$. Athanasios of Meteora, after his captivity, and having also indirectly had to face the dilemma of marriage or monastery, withdrew to a deserted place, "his facial hair having recently grown". ${ }^{7}$ Athanasios served barefoot in the refectory of Esphigmenou, before being accepted in the Monastery of Lazaros in

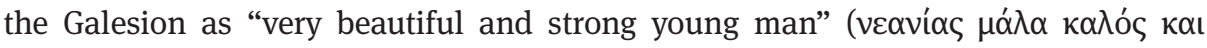

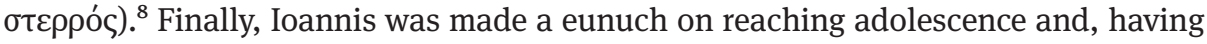
rejected the life of luxury of a eunuch in the imperial court, became a monk at the age of twenty. ${ }^{9}$ It is worth noting, however, that while the saint of the middle period

3 Kiousopoulou, Xpóvos 61-75; B. Chevalier-Caseau, Childhood in Byzantine Saints Lives, in: Becoming Byzantine. Children and Childhood in Byzantium, ed. A. PAPAConstantinou - A.-M. TALBOT). Washington D.C. 2009, 127-166. D. ARIANTZI, Kindheit in Byzanz. Emotionale, geistige und materielle Entwicklung im familiären Umfeld vom 6. bis zum 11. Jahrhundert (Millennium-Studien 36). Berlin-Boston 2012.

4 F. Halkin, Un érmite des Balkans au XIVe siècle. La vie grecque inédite de St. Romylos. Byz 31 (1961) $116-145$.

5 P. IoAnnou, Vie de S. Germain l'Hagiotite par son contemporain le Patriarche Philothée de Constantinople. AnBoll 70 (1952) 35-115.

6 F. Halkin, Deux vies de S. Maxime le Kausokalybe, érmite au Mont Athos (XIVe s.). AnBoll 54 (1936) 38-112, esp. 68-69.

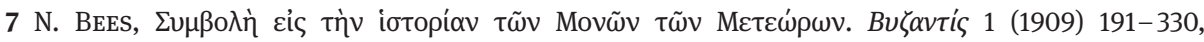
esp. $208-260$.

8 H. Delehaye, La Vie d'Athanase patriarche de Constantinople (1289-1293, 1303-1309), in: Mélanges d'Hagiographie grecque et latine. Bruxelles 1966, 125-149, esp. 131-132.

9 V. Laurent, La vie de Jean, Métropolite d'Héraclée du Pont par Nicéphore Grégoras. Apxeĩov Пóvтov 6 (1934) 4-66. 
often has to struggle with his carnal desires, which in any case define him as an adolescent and a youth, ${ }^{10}$ the saint of the period which we are studying here also has to struggle with the social dictates of marriage, which he rejects by running away from the family home.

In each case we can see that, until the saint begins his timeless path to perfection, time is measured on the basis of his age. The distinction between childhood and adolescence is thus clearly made, if not always numerically. Also, in contrast with the preceding period, few details are given about the life of the saint as a youth. Only the most educated of the authors, such as Nikephoros Gregoras, place importance on the role of education in the parents' plans for their child..$^{11}$

Given all this, the question that arises is what information the Lives offer us regarding the meaning of adolescence. The answer to this is linked to the question of what the biographer's aim is when specifically referring to the saint's childhood and adolescence.

I am of the opinion that the biographer wants to construct the saint as a person who must be familiar with the addressees of his text. For this reason, and until he begins his harsh asceticism, the saint must be almost a normal person by the standards of his time. In seeking to teach his public, the biographer was obliged to use elements that, in order for them to work, the audience could comprehend. As such, the notion of time, divided according to the phases of a human life, as used by the biographer, would have to be understandable and acceptable. I will not expand further on this argument, but I would like to point out that, because in pre-capitalist societies time is defined by its content and because it cannot be conceived abstractly, the biographer uses the phases of the different ages as his audience was familiar with this way of measuring time.

If we bear in mind, as I believe the case is, that the Lives sought to secure the cohesion of the monastic communities and to legitimize the hesychast values, then for their audience, who were the monks, adolescence involved the same dilemma as for the individuals about whom the biographies were written. For both, the saints and the monks, separation from their families was a momentous passage to the next phase of their lives. In late byzantine period, however, it did not have the characteristics of a rupture. ${ }^{12}$ In rural societies, a rupture with one's family was nei-

10 Kiousopoulou, Xpóvos 96-116; D. ArIANTZI, Terminologische und sozialhistorische Untersuchungen zur Adoleszenz in Byzanz (6.-11. Jahrhundert). Teil I. Theorien, Konzepte, narrative Quellen. JÖB 63 (2013) 1-31, here 14-23.

11 M. Hinterberger suggests that the Life of Ioannis, written by Nikephoros Gregoras, is more secular biography than a work of hagiography: M. HINTERBERGER, Les Vies des Saints du XIVe siècle en tant que biographie historique. L’oeuvre de Nicéphore Grégoras, in: Les Vies des Saints a Byzance. Genre littéraire ou biographie historique?, ed. P. Odorico - P. Agapitos. Paris 2004, 295-301.

12 É. PAtlageAn, L'entrée dans l'âge adulte à Byzance aux XIIIe-XIVe siècles, in: Historicité de l'enfance et de la jeunesse. Actes du colloque international Athènes 1-5 octobre 1984. Athen 1986, 261269. 
ther possible nor desirable, on account of the need to preserve the relationships of production in the countryside. Consequently, the passage to adolescence was undergone silently for the members of the family. This can more clearly be seen in the court decisions of Demetrius Chomatenos, Archbishop of Achris and Ioannis Apokaukos, Bishop of Naupaktos. Evelyne Patlagean has observed that, for demographic and economic reasons, rural society in practice did not consider being underage an obstacle to marriage, as we can indeed see in the many early marriages that took place and which Apokaukos called the "Naupakteian disease". ${ }^{13}$ The saint, however, was a person who was destined to acquire particular social power. A preparation stage was thus needed, which all monks were obliged to imitate.

Within a different environment, that of Thessaloniki in the early $15^{\text {th }}$ century, Katablattas, later to become a judge, was showing the first signs of his nefarious life. In the invective that Ioannis Argyropoulos wrote against him, ${ }^{14}$ Katablattas is said to have been of humble origins, in contrast with the author, who was from an aristocratic family. At an age when Argyropoulos was still continuing his education, Katablattas was making one mistake after another. He wandered around the streets of the city accompanying the mime artists on his untuned kithara. At some point he found himself serving in the Ottoman army at Prousa, from where he returned to Thessaloniki with many "barbaric", so Argyropoulos claims, experiences, including sexual experiences with men. After his return, Katablattas went to school and, indeed perhaps a little older than his classmates, initiated them into the act of sodomy, which was the destruction of the youth. He was expelled from the school and for a time led a life of immorality, although not specifically defined. Finally, he found himself - an illiterate - running a school and, as was to be expected, failing to discipline the youths he had undertaken to educate. Worst of all, he helped to fill Thessaloniki with all the evils that homosexuality entailed.

Katablattas' biography is obviously exaggerated and there is clearly no point in investigating how true it is. Nonetheless, it is certainly worth commenting on the narrative choices of the author. Argyropoulos seeks to morally shame his rival and, in this way, to undermine the potential for social mobility illustrated by Katablattas' career path. He thus selects to place the first indications of Katablattas' depravity in his adolescence, during which he consorted with theatre people, did not get an education, and acquired barbaric habits. The author thus echoes the collective understanding that in adolescence one is initiated into whatever will comprise the content of his adult life. By this measure, Katablattas is the opposite of a saint, who, at the same age, adolescence, manifests the signs of his moral integrity. It is indeed at this age that Katablattas, as a teacher, educates his students in singing, dancing and lewd acts.

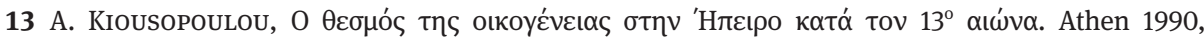
$27-30$.

14 P. CANivet - N. Oikonomides, [Jean Argyropoulos], La comédie de Katablattas: Invective byzantine du XVe siècle. Diptycha 3 (1982-1983) 5-97. 
If we place the saints and Katablattas within the same social context - more specifically, if we consider them as members of the same medieval society - then we can see that the biological changes, as they are manifested in the body and through sexual desire, that marks the passage from childhood to adolescence, are a feature of their parallel paths. Education among the higher social strata made this passage controllable, just as this passage was also made controllable by marriage. So, adolescence is the passage to a definitive age category, the category of youth, and its end does not therefore need to be defined. Indeed, Argyropoulos' insistence on describing Katablattas' behaviour at school leads me to think that sodomy may have been chose by him as a literary rite of passage, analogous to the process of hardening and physical asceticism through which the saints go. At almost the same time, in Constantinople, Mazaris criticize a person who refuses to bear a title of the Great Church and to sing the glorious hymns of the holy liturgy. He prefers to be found with a promiscuous crowd of intoxicated, depraved youths, singing, dancing and babbling away, with the incoherence of a madman. ${ }^{15}$

The difference between the countryside and the cities lies in the fact that in a city, such as Thessaloniki, the passage to youth via adolescence was much more expressed, primarily through education in letters or the military arts. The potential for it to be expressed existed in the cities, because the urban life permitted or enforced the rites of passage and the socialisation. In any case, what a text such as that of Argyropoulos or his near-contemporary Mazaris shows us is not that the vaguely defined "young men" sing and dance, but that the scholars of the $15^{\text {th }}$ century were willing to describe the secular version of adolescence, by individualizing it, even if only to condemn it. And their audience was willing to amuse themselves by listening to it. This was an innovation in relation to earlier periods.

15 Mazaris' Journey to Hades, or Interviews with Dead Men about Certain Officials of the Imperial Court, (ed. J. N BARRY - M. J. SHARE - A. SMithiEs - L.G. WeSternick). Buffalo 1975, 52-54. 



\section{Adolescent Behavior in Byzantine Sources? Some Observations on Young Byzantine Women Pursuing their Goals}

While searching the sources for information on adolescent women, I was intrigued by the goals they chose to pursue and the behavior they adopted in order to achieve them. Based on the presupposition that behavioral patterns accompanying each stage of human development remain constant throughout history, I focused on the aims and actions of seven ${ }^{1}$ Byzantine teenage women ${ }^{2}$ captured in the primary sources. I attempted to interpret them in the light of elementary findings of psychological research in order to see whether such treatment would render new readings of these passages and/or a better understanding of the female protagonists.

Unlike young men, whom the chronicles typically depict as running after court offices or eager to prove their military skills, the goals of Byzantine adolescent women seem to have been mostly connected with relationships. Among these the relationship with God played an important role. Embraced and promoted by the Byzantine society, family and culture, it often figures especially in the hagiographic texts.

The present study has been completed as part of the project Foreign Bride, Negotiator and a Pious Woman: Empresses in Late Byzantium (project nr. 14-08304P) funded by the Grant Agency of the Czech Republic (GAČR).

1 Byzantine sources provide very little information on young women who rarely influenced the public life of their society. As the passages often offered no clues as to whether the woman in question was a child, an adolescent or a young woman, a number of women could not be included in the present study.

2 The youth and childhood of the Byzantines has been discussed in various studies such as D. ARIANTZI, Kindheit in Byzanz. Emotionale, geistige und materielle Entwicklung im familiären Umfeld vom 6. bis zum 11. Jahrhundert (Millennium-Studien 36). Berlin-Boston 2012; EADEM, Terminologische und sozialhistorische Untersuchungen zur Adoleszenz in Byzanz (6.-11. Jahrhundert). Teil 1 Theorien, Konzepte Narrative Quellen. JÖB 63 (2013) 1-31. D. DE ABRAHAMsE, Images of Childhood in Early Byzantine Hagiography. The Journal of Psychohistory 6/4 (1979) 497-517. P. HatLIE, The Religious Lives of Children and Adolescents, in: Byzantine Christianity (People's History of Christianity 3), ed. D. Krueger. Minneapolis 2006, 182-200. C. Hennessy, Young People in Byzantium, in: Companion to Byzantium, ed. L. James. Chichester 2010, 81-92. R. GreEnfIELD, Children in Byzantine Monasteries: Innocent Hearts or Vessels in the Harbor of the Devil?, in: Becoming Byzantine: Children and Childhood in Byzantium, ed. A. Papaconstantinou - A.-M. Talbot. Washington, D.C. 2009, 253-282. A. PAPaconstantinou - A.-M. TAlbot (ed.), Becoming Byzantine: Children and Childhood in Byzantium. Washington, D.C. 2009. É. PATLAGEAN, L'entrée dans l'âge adult à Byzance aux XIIIe - XIVe siècles, in: Historicité de l'enfance et de la jeunesse: Actes du colloque international, Athèn 1-5 Octobre 1984. Athèn 1986, 261-270.

Ә OpenAccess. (C) 2018 Petra Melichar, published by De Gruyter. (cc))BY-NC-ND This work is licensed under the Creative Commons Attribution-NonCommercial-NoDerivatives 4.0 License. 
The Life of Matrona of Chios, ${ }^{3}$ a fourteenth-century saint, describes the young heroine's early yearning to become a bride of Christ:

(...) when the time came for her parents to give her in marriage she refused wishing instead to maintain her virginity and purity in order to become a beautiful and blameless bride of the immortal bridegroom Christ (...). She secretly left her family, forsaking her father, mother and sisters and came to the hills called Katabasis where she opened a stadium of ascetic fights (...). Her parents not knowing what had happened and where she was were overcome with grief and did not cease to search for her until they found her. When they discovered her whereabouts, they did not deem it right to leave her there alone but persuaded her to return to their house. A servant of God, she obeyed the blessed order of her parents and returned home. ${ }^{4}$

Though runaway saints were a relatively common sight in earlier Byzantine vitas, the above description does not exactly fit the established topos. For one thing, the fugitives usually did not return home. As the passage does not concur with an earlier depiction of the saint's youth, it seems that Matrona, at that time still the teenage Maria, ${ }^{5}$ left home after a fight with her parents over a less-than-desirable marriage and was later reconciled to them, due perhaps to the protracted discomfort on the Katabasis heights. Her behavior reflects not only the tendency to impulsive and radical $^{6}$ behavior and the desire to acquire her goal immediately ${ }^{7}$ but also the need for security and protection ${ }^{8}$ which finally brought her back home. Her ensuing life in the house of her parents offered excellent conditions to the young adolescent, who lived as a nun following the monastic regimen; she acquired the necessary freedom as well as the support and protection vital to this phase of her development. When Maria's parents died some years later, she was a mature adult well prepared to leave her home and launch a successful monastic career.

3 PLP, no. 17399.

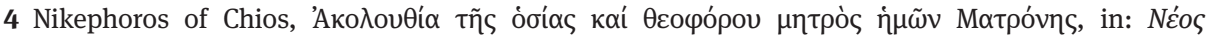

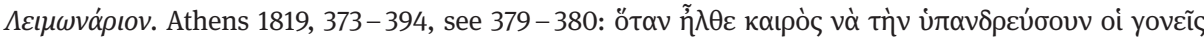

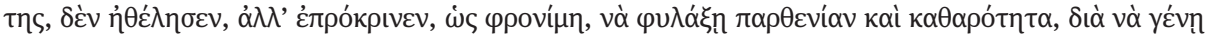

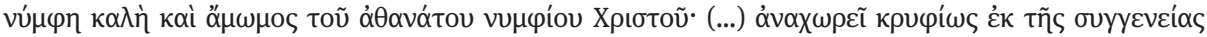

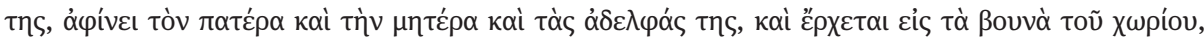

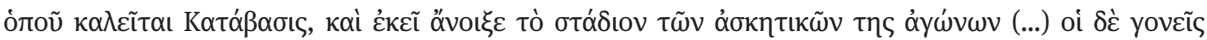

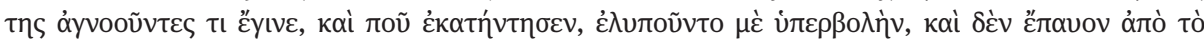

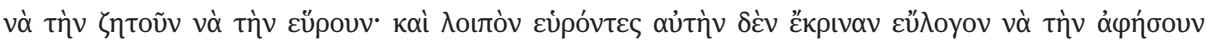

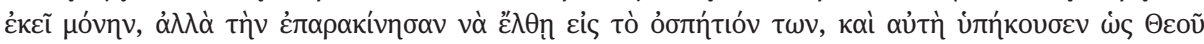

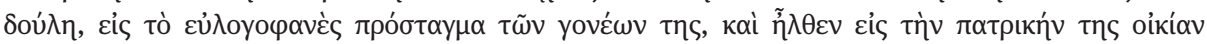
(...). Also see ARSENIJ, Nila, mitropolita Rodosskago, Četyre neizdannyja proizvedenija. Moscow 1891, 1-54, see $13 \mathrm{f}$.

5 The text notes that her flight took place at a time when her parents would 'give her in marriage' that is probably around or sometime after she reached her twelfth year, the canonical age for women to get married.

6 M. VÁGNEROVÁ, Vývojová psychologie, dětství a dospívání (Developmental psychology; childhood and adolescence). Prague 2012, 388, 390.

7 Ibidem 468.

8 VÁGNEROVÁ, Vývojová psychologie 387. 
While reflecting a teenage solution to a difficult situation, Matrona's story underlines the complicated position of young women within the Byzantine society. As the well-known stereotype implies, these adolescents stood under constant pressure to adjust to the social norm and assume a subordinate place within the family and community being frequently denied the right to choose their spouse. Whatever the circumstances of Matrona's adolescent life, the relationship with God and his saints, maintained through public and private worship offered teenage girls, who were often kept under strict supervision in the parental home, a personal space in which they could develop their self-image and self-confidence and loosen filial bonds; all crucial milestones of this developmental stage. Orthodox faith had apparently much to offer to girls who found themselves in Matrona's situation. God, depicted as a loving Father, could replace the angry earthly parents still very important to a teenage person, while a commitment to Christ freed young women from the dreaded prospect of an unwanted union and gave them an opportunity to decide their own fate.

As the sources indicate, the relationship with parents, though often complicated in their teenage years, was still crucial to Byzantine adolescent women. Even if they may have wished to acquire more freedom, losing the support and protection represented by this bond ${ }^{9}$ was another matter. At the beginning of the Vita of Mary-Marinos, the hagiographer includes a scene in which the widowed father of Mary announces his decision to enter a monastery. ${ }^{10}$ Mary, who refuses to be separated from him, is willing to brave the harshness of monastic life and follow him dressed as a boy.

In Mary's case, the physical changes occurring in adolescence play an important role in establishing that she was in her teenage years at this point in her life, a fact which the Vita does not explicitly mention. As children under the age of ten ${ }^{11}$ were not easily admitted to Byzantine monasteries and her father apparently considered her old enough to take care of herself, Mary could not have been a minor. The author makes this circumstance clear when he no longer calls her a child ( $\pi \alpha \mathrm{I} \delta$ íov) as in the

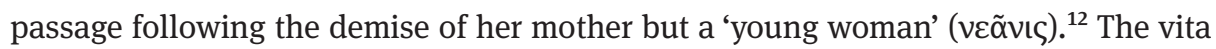

\footnotetext{
9 Ibidem 387.
}

10 Vita of Mary-Marinos 2 (ed. M. RICHARD, La vie Ancienne de Sainte Marie surnommé Marinos, in: Corona Gratiarum. Miscellanea patristica, historica et liturgica Eligio Dekkers O.S.B. XII Lustra complenti oblata, I. Brugge 1975, 83-94, here 9-11, 87f. [repr. M. RICHARD, Opera minora, III. Leuven, 1977]). See Translation: N. Constans, The Life of Saint Mary/Marinos, in: Holy Women of Byzantium: Ten Saints' Lived in English Translation, ed. A.-M. Talbot. Washington, D.C. 1996, 1-12, see 8. For further information and literature on Mary-Marinos see the footnotes in ibidem 1-5. For aspects of a child's life in Byzantine monasteries see GrEenfIELD, Children (as in n. 2).

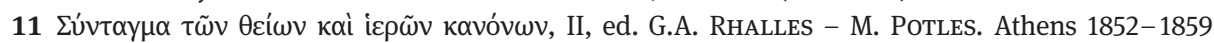
(repr. 1966), 398. Byzantine Monastic Foundation Documents: a complete translation of the surviving founders' typika and testaments, I-V (Dumbarton Oaks Studies, 35.), ed. A. Constantinides Hero - G. Constable - R. Allison - J. Thomas. Washington, D.C. 2001, III, $1202 \mathrm{f}$.

12 Vita of Mary-Marinos 2 (8-11, 87 RICHARD). 
further states that "after she lived for a few years in the monastery, some of the monks considered her to be a eunuch, for she was beardless and of delicate voice". ${ }^{13}$ Had Mary entered the monastic community as a grown woman, she would have been considered a eunuch immediately. The text, however, suggests that she came at an age when the transformation into manhood was expected and did not occur.

While Mary's wish to remain close to her father is naturally interpreted by the hagiographer as a sign of love, obedience and humility very proper in a future saint, considering Mary's behavior from the perspective of her age gives her story a new dimension. Instead of the faultless and perfect woman sketched by the author, it reveals a teenage girl insecure about assuming adult responsibilities and desiring stability, continued protection, and contact with her parent, all very natural feelings in an adolescent. ${ }^{14}$

Young Byzantine women who for various reasons entered monastic life often wished for a relationship with a spiritual father who would stimulate their spiritual growth, give them a sense of protection and advise them in their ascetic struggles. While late Byzantine nuns commonly received such care in the convents, as the contemporary typika (monastic rules) inform us, ${ }^{15}$ the written sources preserve little evidence on specifically teenage recipients of such instruction. In at least one case, however, such guidance was bestowed in writing, due to the preoccupation of the spiritual father. The pupil was Eulogia Choumnaina, ${ }^{16}$ daughter of the famous Nikephoros Choumnos, and her guide was Theoleptos, bishop of Philadelphia, a wellknown statesman and theologian. Early in her adolescence (spring 1303), the noble Eirene became the wife of John, third son of Andronikos II. Their marriage was ended prematurely by the prince's death four years later (1307). Soon after John's demise, Theoleptos cut the golden locks of the then sixteen-year-old basilissa who on this occasion took the monastic name Eulogia. As she refused even the comfort of her own family, it was Theoleptos who guided her through the first months of her untimely widowhood. At this time she became abbess of the female part of the

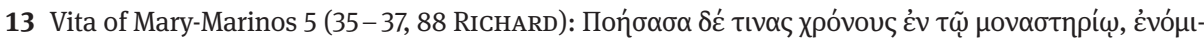

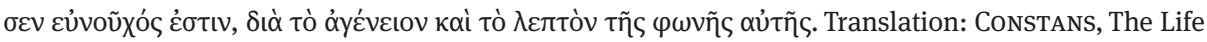
8.

14 Marie Delcourt, on the other hand, interprets Maria's change of clothes as self-mutilation and her desire to enter a male monastery as an expression of her longing to attain 'male' perfection. M. DELCoURT, Le complexe de Diane dans l'hagiographie chrétienne? Revue de l'histoire des religions 153 (1958) 1-33. EADEM, Female Saints in Masculine Clothing, in: Hermaphrodite: Myths and Rites of the Bisexual Figure in Classical Antiquity. London 1961, 84-102.

15 See H. Delehaye, Deux typica byzantins de l'époque des Paléologues (Mémoires de l'Académie de Belgique, Classe des lettres, ser. II, 13/4). Brussels 1920, 109, 5; 112-113, 11. A. Constantinides Hero, Byzantine Monastic Foundation Documents p. 1292, 5; 1269, VI/11-13; 1476, 15; 1479, 9. S. PÉTRIDES, Le typikon de Nil Damilas pour le monastère de femmes de Baeonia en Crète. IRAIK 15 (1911) 92-111, see here 103, 107.

16 For a list of sources and literature on Eirene see PLP, no. 30936. 
double monastery of Christ Philanthropos Soter, which she renovated and provided for from her own property. To Theoleptos fell the uneasy task of supervising the transformation of a pampered teenage princess into an ascetic leader of a monastic community, an example of virtue and source of guidance to her nuns. As his letters to her witness, it was not an easy assignment. His first preserved missive from spring 1307, which finds her already in the nunnery, contains the following recommendations:

Since therefore you lost the pleasure of the body and are walking in the land of mourners, take pains to make your rational soul suitable to God the Word, to attach yourself to Christ and to establish with Him the spiritual marriage. Give up the frequent visits at the palace, let go of the multitude of servants and run from the vanity of all flattery. ${ }^{17}$

While Eulogia's moods apparently remained volatile throughout her life, the survey of her behavior described above reflects the extremes well known to contemporary parents and psychologists: on one hand the refusal to see her family ${ }^{18}$ and the decision to become a nun, on the other an unwillingness to submit to the discipline and austerity of convent life, profound depression followed by the decision to give up the world one day and longing for her former home the next. The premature death of her husband, her inability to re-marry without losing her status and her marred ambition, which she apparently never internally resolved, offer a new avenue of understanding Eulogia's problems in her later life; her longing to leave the nunnery, her difficult adjustment to monastic rule, conflicts with her nuns and her desperate insistence on personal contact with her second spiritual guide, expressed in her own preserved letters. ${ }^{19}$

Her strong relationship with Theoleptos also deserves attention as it reflects the need to find a replacement of sorts for the parent, a person "who would impress [her], represent a natural authority [to her] and treat [her] as [an equal]". ${ }^{20}$ It seems that the bishop was able to create such a bond with his young charge, which then enabled him also to correct and guide her.

Though not frequently described in Byzantine chronicles, romantic relationships, endowed with the dramatic passion of adolescent love, form another group of goals

17 Theoleptos of Philadelphia, Letter 1 (ed. A. Constantinides Hero, The Life and Letters of Theo-

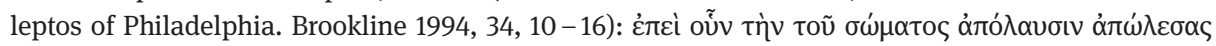

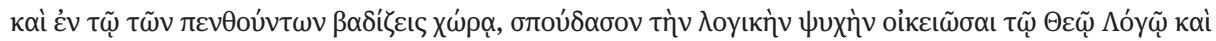

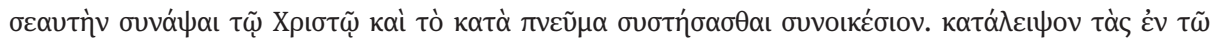

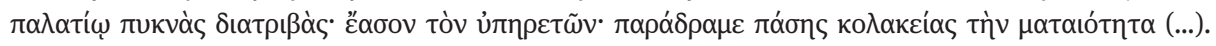
Translation 35.

18 VÁGNERovÁ, Vývojová psychologie 395. Conflicts with family and refusal to subordinate and show one's feelings to parents are common in adolescents.

19 A Woman's Quest for Spiritual Guidance: The Correspondence of Princess Irene Eulogia Choumnaina, ed. A. Constantinides Hero. Brookline, Massachusetts 1986.

20 VÁGNERovÁ, Vývojová psychologie 410 (Translation mine). 
pursued by Byzantine adolescent women. Theodora Palaiologina, ${ }^{21}$ niece of Michael Palaiologos (later Michael VIII), was engaged by Emperor Theodore II Laskaris to a man of rather modest origin named Balaneidiotes who, according to the sources, nevertheless won the heart of this exalted lady. After he had become well acquainted with his fiancée and her family, the emperor suddenly changed his mind and forced Theodora to marry another nobleman, Basileios Kaballarios. In the words of George Pachymeres "those ladies [Theodora and her mother, Martha Palaiologina ${ }^{22}$ ] who preferred the previous union and considered it a shame to change it, despised the [new] fiancé. According to the order of the emperor, the usual rituals none-the-less took place along with a splendid wedding”. ${ }^{23}$ In the following days the young groom did not consummate the union, a fact which he ascribed to the sorcery of his mother-in-law. Soon afterwards, however, Theodore II Laskaris died and the uncle of the unhappy bride, Michael, assumed his place. At the request of his sister, he allowed Theodora to separate from Kaballarios and marry Balaneidiotis, who on this occasion rose to the honor of grand stratopedarch. ${ }^{24}$

Though the sources do not explicitly state the precise age of the bride, we know that her mother, Martha Palaiologina, married in 1237 and her uncle, Michael VIII, came to power in 1258. Theodora is known to have had at least three siblings, of whom she may not have been the eldest. Thus, by the time of her engagement and first marriage, she would have been in the second half of her adolescence.

Theodora's willingness to brave the anger of the emperor and even endanger her family's fortunes seems an unusual behavior in a well-bred Byzantine lady but not in a teenage girl able to fall violently in love, as the novelists put it, without regard for the established system. ${ }^{25}$ It is not very likely, therefore, that the clever and subtle plan which resulted in the annulment of Theodora's marriage came from the princess's own head. In fact, as the groom blames his failure not on his bride but on his mother-in-law, it must have been this formidable lady herself who decided to aid Theodora, possibly in order to prevent some scandalous or desperate action. Such an image also fits within the framework of the mother-daughter relationship, often characterized, also in the adolescent phase, by a tendency to mutual support. ${ }^{26}$

What puzzled me as I reread the above story was the question of how to reconcile the princess, determined to take a serious risk, with the later grave and rather silent nun standing, at least from the perspective of the primary sources, in the shadow of her mother Martha and half-sister Nostongonissa as they took their place in the front lines of the Arsenite controversy, or her aunt Eirene and cousin Theodora Raoulaina, who very effectively opposed the Union of Lyons (1274). Could the psycholog-

21 For sources and literature see PLP, no. 27510.

22 Ibidem no. 21389.

23 George Pachymèrés I, 55. (ed. A. FAILLER, Relations historiques [CFHB 24/I-IV]. Paris 1984).

24 George Pachymèrés I, 55. (ed. A. FAILLER, Relations historiques [CFHB 24/I-IV]. Paris 1984). 25 On the willingness to risk common in adolescents see VÁGNERovÁ, Vývojová psychologie 372. 26 Ibidem 405. 
ical changes which temporarily occur in adolescence ${ }^{27}$ have been co-responsible for the above-described 'misconduct'?

The Life of St. Eirene of Chrysobalanton includes another case of youthful passion, which concerns a nun under the guidance of this formidable abbess. Her age is once more not specified, but the fact that she is called a 'girl,' kore, suggests that she was in her adolescent years. Of noble Cappadocian descent, she entered Eirene's ${ }^{28}$ convent and took the monastic vows. Her distant lover did not forget her, however, and with the aid of a sorcerer rekindled the girl's love for him:

(...) the girl was unexpectedly attacked by a seething passion which maddened her with a frantic lust for her former suitor and did not allow her to control herself. Violently leaping, screaming, moaning, crying, and calling out his name in a loud voice, she assured with fearful oaths that unless someone let her see him with her eyes and enjoy to excess his sight and conversation, she would hang herself. One could see her continually running to the gateway, urging her escape and with inarticulate screams and shameless gestures ordering the door-keeper to let her out. ${ }^{29}$

The abbess reacted promptly, ordering the sisters to pray, fast and make a thousand genuflections a day, and set a constant guard over the bewitched girl. Through several dreams, she then received instructions as to how to proceed and miraculously procured "two idols made of lead, one resembling the suitor, the other the sick nun, embracing each other and bound together with hairs and threads (...) inscribed on them [was] the name of the author of the evil and appellations of servant demons". ${ }^{30}$ Needless to say, once these objects were destroyed, the Cappadocian nun quickly recovered both her wits and her passion for the spiritual calling.

If we admit the possibility that the girl was not acting under a spell, we behold a tragic story of a young woman who apparently took her vows before she was completely certain of her heart or under the pressure of material circumstances. In her case, however, there was no way back as the sacred promises could not be undone.

27 The general characteristics of adolescence include also refusal to compromise, a tendency to radical solutions and a need to instantly achieve one's goals. See ibidem $385 \mathrm{f}$.

28 PmbZ I, Nr. 1452.

29 The Life of St. Irene Abbess of Chrysobalanton 13 (ed. O. RosEnQUisT, The Life of St. Irene Abbess of Chrysobalanton: A Critical Edition with Introduction, Notes and Indices. Uppsala 1986, 22, 52, 6,

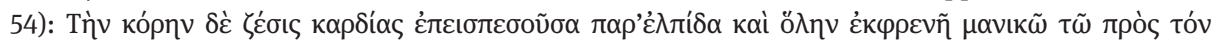

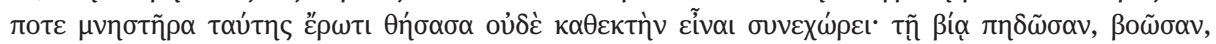

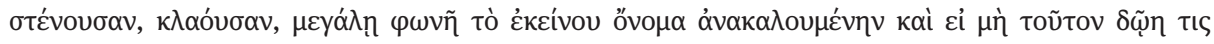

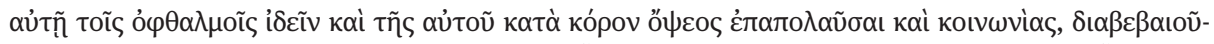

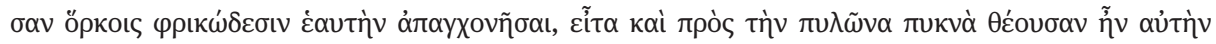

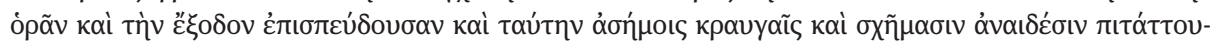

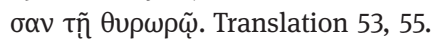

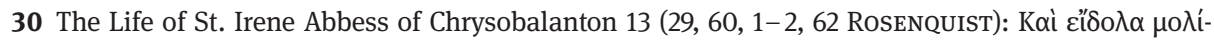

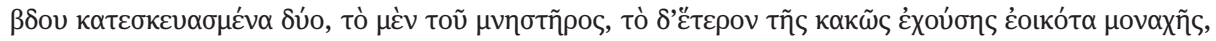

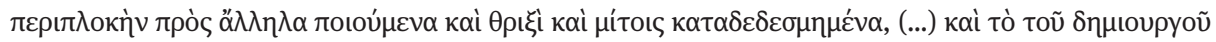

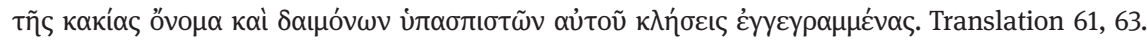


The girl's violent entreaties, screams and threats fit well the model of adolescent behavior. ${ }^{31}$ The young nun did not have a mother nearby to help her devise a stratagem and so she struggled loudly but in vain. While the Byzantines did have a sense of adolescence as an age of seeking and testing one's calling, ${ }^{32}$ the monastic founders nevertheless had the final word in deciding at what age the novices could take the veil. Once this ritual had taken place, the Orthodox Church considered the vows irreversible.

In problematic families and with girls of strong character, the need for relationship, essentially the need for love, may have transpired in a socially undesirable way. The sinful youth of St Mary of Egypt, as recounted by Sophronios, ${ }^{33}$ is a notorious example. In her confession to Father Zosimas, she admits that at the age of twelve she left her parents and went to Alexandria, where she led a debauched life for seventeen years, stressing that her reasons for immorality were not material. ${ }^{34}$ Though Mary's figure has been reshaped by Sophronios and her despicable youth creates the plot of the legend and counterpart to her later ascetic lifestyle, it does not describe a wholly improbable event. A young woman would run from home, like the above Mary-Matrona of Chios, most probably after a fight or misunderstanding with her parents. More problematic is the detail confessed to Father Zosimas, that she led a promiscuous life without material necessity, which would indicate a need to rebel against the values of her parents or a desire to receive love and recognition, which the latter failed to confer. Whether due to the miraculous intervention of the Theotokos on her own journey to maturity, Mary later became a holy woman and a famous ascetic - an extreme example of the more common transformation of a wild teenager into a balanced and responsible individual. ${ }^{35}$

In its description of adolescence, the World Health Organization depicts this phase as "movement toward social and economic independence," in which "development of identity and the acquisition of skills needed to carry out adult relation-

31 Strong emotional reactions are typical in adolescents: VÁGNEROVÁ, Vývojová psychologie $390 \mathrm{f}$. 32 The Council of Carthage for example only allowed women of 25 to take the monastic vows (Les canons des Pères Grecs [= Discipline générale antique], ed. P.-P. JoAnNou. Grottaferrata 1962, I/1, 175 -177). This wise precept was nevertheless later changed by the Council in Trullo (962) to seventeen (JoAnnou, Les canons I/1, 176/13-16).

33 There are three different versions of the vita: the first by John Moschos presents Mary as a pious nun in a monastery who left for the desert after a young man fell in love with her in order to avoid sin (see $P G, 87,3049$ ). Another story is presented by Cyril of Scythopolis, who introduces Mary as a singer at the Church of the Resurrection in Jerusalem who, on account of her great beauty, did not want to become a snare for men and therefore left for the desert (ed. E. ScHWARTz, Cyril of Scythopolis's Life of of Cyriacus. Leipzig 1939). The third version is that of Sophronios, used above, who depicts Mary as a repentant harlot.

34 For Mary's Vita (Sophronios version) see (BHG 1042) PG 87, 3697-3726, (leaving home, 3709D) for translation and further literature see M. KouLI (transl.), Life of St. Mary of Egypt, in: Holy Women 70-93, see 65-93 (as in n. 10); ODB 2, 1310.

35 VÁGNEROVÁ, Vývojová psychologie 394, 449. 
ships and roles"36 take place. While most, if not all, Byzantine women received instruction in domestic tasks and sometimes in the family trade, the eulogy of Michael Psellos $^{37}$ dedicated to his mother confirms that at least some of them also desired education. Following the description of her childhood and directly preceding the depiction of her marriage, the adolescent Theodote, according to her son, regretted that "she happened not to be a man by nature and that she was not allowed to study literature freely". ${ }^{38}$ Determined woman that she was, she nevertheless managed to overcome this handicap:

Evading the attention of her mother whenever she could, she picked up the basic principles of letters from someone and soon began through her own efforts to join them together and to form syllables and then sentences, without having any need for an instructor in the basics. ${ }^{39}$

Psellos's text offers a testimony not only to the scholarly interests of his mother but also to the difficulties faced by gifted Byzantine women of unprivileged background who desired knowledge as a means of personal growth..$^{40}$ On the other hand, it is encouraging to see that Theodote, and perhaps others, had the perseverance to teach themselves to read and write. If not absolutely exceptional, Theodote's story may indicate that basic education among the Byzantine female population was not as rare as the preserved sources would have us believe.

\section{Conclusion}

Despite the paucity of examples, the above sources indicate that Byzantine adolescent women focused primarily on relationships of various kinds. Upon inspection, the texts also reveal the tendencies and emotions typical of this age group, including willingness to risk, internal turmoil, egocentrism and the need to immediately achieve their goals.

36 World Health Organization on adolescent development: http://www.who.int/maternal_child_ adolescent/topics/adolescence/dev/en/\# (seen 12.8.2014).

37 Michael Psellos, Encomio per la madre (ed. U. CRIscuolo, Michele Psello: Autobiografia, Encomio per la madre, (Testo critico, introduzione, traduzione e commentario). Naples 1989. Tranl. A. KALDELLIS, Mothers and Sons, Fathers and Daughters: The Byzantine Family of Michael Psellos. Notre Dame 2006. Also see J. Gouillard, La femme de qualité dans les lettres de Thédore Stoudite. JÖB 32/ 2 (1982) 445- 452.

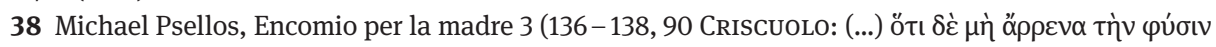

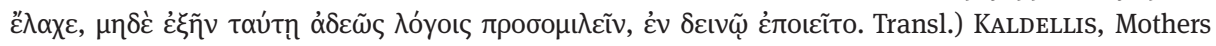
and Sons 55.

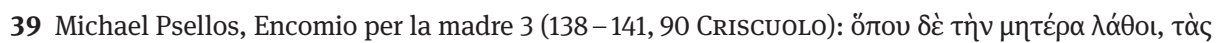

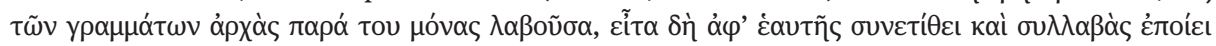

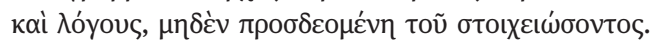

40 Modern research confirms the development of cognitive processes in adolescence, which frequently stimulate teenagers’ desire to learn. VÁGNERová, Vývojová psychologie 371-390. 
Regarding family, though adolescence in Byzantium was apparently likewise marked by parent-child conflicts and a tendency to explore new ideas rather than quickly accept adult authority, a stable family background had a crucial impact on teenagers' future choices and success in life. Its importance is apparent in the successes of Matrona of Chios, Theodora Tarchaneiotissa or Mary-Marinos, while its lack brought Mary of Egypt as well as the nameless nun from the monastery of Eirene of Chrysobalanton into serious difficulties.

The seven descriptions also reflect tensions related to the social expectations which were placed on young women. The approved or ideal desires clearly included serving God, obeying parents, maintaining chastity, remaining faithful to one's fiancé and keeping sacred vows. Conversely, they reveal the problems faced by teenaged females. Among these, the inability to choose partners, enter or leave the monastery, remain with parents, escape difficult circumstances, or acquire basic knowledge obviously ranked high on the list.

The idealized aims of Byzantine adolescent women interestingly stand in direct contrast to those of their male counterparts established by the recent study of Despoina Ariantzi. While men desired good looks, recognition and strength, ${ }^{41}$ young women were expected to completely negate such wishes by remaining hidden within their homes or monasteries, neglecting their beauty and cherishing no other ambition than piety. In pursuing their dreams which went beyond the approved limited sphere they almost always had to take irregular and often ingenious paths.

Surprisingly, several patterns connected with contemporary adolescent women do not appear in the above sources. One of them is the tendency to become a member of a peer group. ${ }^{42}$ The dearth of information about these groupings may certainly be ascribed in part to a lack of interest of the Byzantine authors. Nevertheless, their existence may have been limited by the expectation that the more prominent women would spend most of their time in their homes, while extensive help in the household may have kept their less fortunate peers from attending.

Missing in the above examples is also any note of the adolescent focus on apperance. It is, of course, not likely that young Byzantine women would have neglected their looks as both the preserved images of Byzantine women and archeological finds of jewelry indicate. Whether due to lack of financial means or lack of social significance, they apparently failed to make the result of their efforts provocative enough to capture the attention of contemporary authors who after all perhaps were not as opposed to female beauty as the lives of the Byzantine female saints would have us believe.

In applying developmental psychology on literary figures, which were not even based on real personalities, one must, of course exercise caution. While constructing

41 ARIANTZI, Adoleszenz 19-21 (as in n. 2). On gender-oriented needs also see VÁGNERovÁ, Vývojová psychologie 471-482.

42 For the peergroups in Byzantium see in this volume the paper of D. ARIANTZI, Soziale Identitätsbildung im Jugendalter. 
completely fictive characters, the authors may have more or less projected their own fears, ideas and expectations into the (female) figures in order to achieve their purposes, which most frequently entailed issuing a warning or encouragement to their audiences. Such 'figments of imagination' were not meant to resemble human beings - and they did not. On the other hand, with sources describing historical women (and men) who had to pass through the elementary processes of infancy, adolescence, adulthood and aging, the findings of modern psychologists may provide much insight. Though their individual experiences of these seasons may have differed to a certain degree, they always shaped their stories - and left a mark on the texts. 



\section{Soziale Identitätsbildung im Jugendalter in Byzanz}

Modernen soziopsychologischen Theorien zufolge haben die anatomischen, physiologischen und hormonalen Veränderungen auch auf die seelische und soziale Ebene gravierende Auswirkungen. ${ }^{1}$ Die fast schon erwachsene Gestalt und die neuen kognitiven Fähigkeiten veranlassen den Jugendlichen normalerweise, die wichtige Entwicklungsaufgabe ${ }^{2}$ der Ablösung ${ }^{3}$ von den Eltern konstruktiv zu bewältigen, um sich auf das Erwachsenenleben vorzubereiten. Meistens wird Ablösung mit dem Erreichen emotionaler Unabhängigkeit von den Eltern assoziiert, bei der sich die Orientierung von Einstellungen und Handlungen nicht mehr vorrangig nach den Eltern, sondern nach Angehörigen der eigenen Generation ausrichtet, wie es auch die psychoanalytische Tradition postuliert. Die Psychologie spricht über zwei Arten von Kontakten mit Gleichaltrigen: Zugehörigkeit zu Peer Gruppen ${ }^{4}$ (Gleichaltrigen) und Aufnahme von intimen emotionalen Bindungen, nämlich Freundschaft und sexueller Beziehung bzw.

\footnotetext{
Vorliegender Beitrag entstand im Rahmen des vom „Fonds zur Förderung der wissenschaftlichen Forschung (FWF)“ finanzierten Projektes „Entlassung aus elterlicher Obhut: Modelle der Adoleszenz in Byzanz vom 6. bis zum 11. Jahrhundert“ (Projekt-Nr. P22773, geleitet von J. Koder). Zu danken habe ich für konstruktive Bemerkungen Alexander Beihammer und für sprachliche Korrekturen Anneliese Paul.
}

1 K. HuRRELMANN, Lebensphase Jugend. Eine Einführung in die sozialwissenschaftliche Jugendforschung. München ${ }^{9} 2007,26$.

2 A. Flammer - F. AlSAKER, Entwicklungspsychologie der Adoleszenz. Die Erschließung innerer und äußerer Welten im Jugendalter. Bern - Göttingen - Toronto - Seattle 2002, 56. Der Terminus „Entwicklungsaufgaben“ (englisch: Development Task) ist durch den amerikanischen Pädagogen Robert Havighurst (Development Tasks and Education. New York 1948 [Nachdruck 1959]) etabliert worden. Er legte dar, dass „Entwicklungsaufgabe“ eine Aufgabe ist, die zu einem bestimmten Lebensabschnitt des Individuums entsteht, deren erfolgreiche Bewältigung zu dessen Glück und Erfolg bei späteren Aufgaben führt, während ein Misslingen Unglücklichsein, Missbilligung durch die Gesellschaft und Schwierigkeiten mit späteren Aufgaben nach sich zieht. Die Entwicklungsaufgaben einer bestimmten Gruppe haben ihren Ursprung in drei Quellen: 1. der körperlichen Entwicklung, 2. dem kulturellen Druck (den Erwartungen der Gesellschaft), 3. den individuellen Wünschen und Werten.

3 G. MietZEL, Wege in die Entwicklungspsychologie: Kindheit und Jugend. Weinheim ${ }^{4} 2002$, 362. Was unter dem Begriff „Ablösung“ zu verstehen ist, ist nicht eindeutig definiert, sondern variiert in Abhängigkeit vom theoretischen Hintergrund. Ablösung aus der Sicht der Jugendforschung beinhaltet die Entwicklung von Autonomie und emotionaler Unabhängigkeit durch verstärkte Kontakte mit den Peergroups, Auflehnung gegen den elterlichen Einfluss, Aufbau einer eigenen intimen Beziehung, Wahl eines Berufes, Auszug und Gründung eines eigenen Haushaltes, Partnerwahl und eventuell Heirat und materielle-finanzielle Selbstständigkeit.

4 Der Terminus Peergroup geht als Fachbegriff aus der Pädagogik, Soziologie und Sozialpädagogik zurück auf Charles H. Cooley (1864-1929), der das Konzept der Primärgruppen entwickelte. 
Ehe. ${ }^{5}$ Dadurch gewinnt der Jugendliche ein erhöhtes Maß an Selbstständigkeit und Eigenverantwortung, was seine schrittweise Integration in die Gesellschaft, die sogenannte sekundäre Sozialisation, ermöglicht. ${ }^{6}$ Diese vollzieht sich problemlos, wenn das allgemeine Normensystem einer Gesellschaft mit dem der Familie übereinstimmt. Denn das Individuum kann seinen vollen Mitgliedstatus innerhalb des sozialen Systems durch Verhaltensmuster erreichen, die es in der Familie erworben hat. Gesellschaften, in denen diese Bedingung erfüllt wird, werden als partikularistisch oder universalistisch bezeichnet. In diesen Gesellschaften gibt es oft Initiationsriten, ${ }^{7}$ die weniger im Lernen neuen Verhaltens bestehen als in der kognitiven und affektiven Zuteilung von Pflichten und Rechten. ${ }^{8}$ Bei solchen Gesellschaften verlief die Jugendsozialisation ohne große Probleme im Gegensatz zu postindustriellen Gesellschaften. Forscher ${ }^{9}$ bezeichnen diese als kontinuierliche Sozialisation, die durch fließende Übergänge, beispielsweise vom Spiel zur Arbeit und von Abhängigkeit zu Unabhängigkeit oder von Sorglosigkeit zu Selbstverantwortung gekennzeichnet war. In der vorindustriellen Gesellschaft hatten schon kleine Mädchen Pflichten bei der Herstellung von Mahlzeiten oder in der Betreuung von jüngeren Geschwistern; Knaben arbeiteten im Rahmen der Familie in der Landwirtschaft oder als Hirten, teilweise parallel mit „schulischer“ Ausbildung; nach dem Ende der Kindheit traten sie in die Berufslehre ein; ${ }^{10}$ sie zogen aus beruflichen Gründen oder nach der Heirat aus der

5 HURRELMANN, Jugendforschung Kap. 3.3. Intime emotionale Bindungen führen in erster Linie zur Sozialisierung und spielen eine wichtige Rolle bei der Entstehung von vertrauensvollen Beziehungen mit gemeinsamem Erleben von Gefühlen.

6 Mietzel, Entwicklungspsychologie $351 \mathrm{ff} .$, Kap. 10.

7 In Byzanz war wie in anderen vormodernen Kulturen der Eintritt in das Erwachsenenleben mit bestimmten Initialriten, die teilweise symbolischen Charakter hatten, verbunden. $\mathrm{Zu}$ praktizierten Initiationsriten, die den Jugendlichen in Byzanz den Eintritt in den neuen Lebensabschnitt erleichtern: D. ARIANTzI, Terminologische und sozialhistorische Untersuchungen zur Adoleszenz in Byzanz (6.11. Jahrhundert). Teil I. Theorien, Konzepte, narrative Quellen. JÖB 63 (2013) 1-31, hier 8-9. Zu Adoleszenz - Riten vgl. A. vAN GENNEP, Les Rites de Passage. Paris 1909, 93-163.

8 Flammer - AlsaKer, Entwicklungspsychologie der Adoleszenz 40 - 41: Die individuelle Existenz wird wesentlich durch Sozialisation gewährleistet, zunächst durch die Primärsozialisation in der Familie. Wenn das Kind zeugungsfähig und selbständig überlebensfähig wird, wird die sekundäre Sozialisation, d. h. die Integration in die Gesellschaft, fällig.

9 In einer anderen Form hatten Vertreter der Kulturrelativismus-These z. B. Ruth Benedict (R. BENEDICT, Kulturen primitiver Völker. Stuttgart 1949 [Originaltitel: Patterns of Culture. New York 1934]) und Margaret Mead (M. MEAD, Der Konflikt der Generationen. Jugend ohne Vorbild. Olten - Freiburg 1971 [Originaltitel: Coming of Age in Samoa. A Psychological Study of Primitive Youth for Western Civilisation. New York ${ }^{7} 1957$ ]) die Unterscheidung zwischen partikularistischen und universalistischen Gesellschaften vorausgedacht. Der Kulturrelativismus betonte die kulturelle Bedingtheit menschlichen Verhaltens. Benedict und Mead führten Untersuchungen an Cheyenne-Indianern, aber auch auf Samoa, Bali und Neuguinea durch und verwendeten für diese Gesellschaften den Begriff „primitive“ statt „partikularistische Gesellschaften“.

10 Zum Arbeitseinsatz von Kindern und Jugendlichen: D. ARIANTzI, Kindheit in Byzanz. Emotionale, geistige und materielle Entwicklung im familiären Umfeld vom 6. bis zum 11. Jahrhundert (Millennium Studien 36). Berlin - Boston 2012, 135-141; EADEM, Kinderarbeit in Byzanz im Rahmen der Familie auf 
elterlichen Wohnung aus; sie wurden sexuell aktiv; sie gründeten eine Familie und trugen dafür Verantwortung; sie verdienten Geld; sie wechselten Rollen, Pflichten und Rechte, Familie und Gesellschaft betreffend. Trotz der zweifelsohne großen Unterschiede zwischen mittelalterlichen und modernen Sozialstrukturen und der verschiedenen Formen sozialer Identifizierung und Selbstwahrnehmung begegnen einem in den byzantinischen Quellentexten Aussagen, die durchaus mit Phänomenen der sekundären Sozialisation korrespondieren und eine ganz ähnliche Problematik skizzieren, wie sie in heutigen sozialen Milieus zutage tritt.

Im Rahmen dieses Artikels möchte ich mich den Peergruppen in Byzanz vom 6. bis zum 12. Jahrhundert als einem Weg zur Sozialisierung, ihrer Typologie bzw. Identität und Funktion sowohl für die Adoleszenten als Individuen als auch für die jeweilige Gesellschaft widmen. Die Sozialisierung erfolgte durch das Aufbauen von Kontakten mit Menschen außerhalb des Familien- bzw. Verwandtenkreises, was das Individuum selbst ohne Hilfe oder Vermittlung der Familie schaffte.

Die Präsentation erfolgt auf der Basis hagiographischer und historiographischer Quellen. Heiligenviten und verwandte hagiographische Texte stellen die wichtigste Textgruppe dar, da sie für die Untersuchung des Alltagslebens einer großen Bandbreite der Bevölkerung besonders aufschlussreich sind und vielfältige Einblicke in diverse menschliche Verhaltensformen gewähren. ${ }^{11}$ Trotz der unschätzbaren Bedeutung dieser literarischen Gattung ${ }^{12}$ ist man bei der Untersuchung mit Schwierigkeiten konfrontiert.

Das Hauptproblem der Analyse hagiographischer Texte ${ }^{13}$ besteht darin, dass Aussagen über soziale Gruppen in der Regel einem idealisierten Heiligentyp, der durch die sogenannten Topoi ${ }^{14} \mathrm{zu}$ erkennen ist und die monastischen Ideale und

Grund der hagiographischen Quellen vom 6. bis zum 11. Jahrhundert, in: Laetae Segetes Iterum. Brno 2008, 405-413.

11 É. Patlagean, Ancienne hagiographie byzantine et histoire sociale. Annales. Economies, Sociétés, Civilisations 1 (1968) 106-126 (= Structure sociale, famille, chrétienté à Byzance IVe-XIe siècle. London 1981, Nr. XI); FR. HALKIn, L'hagiographie byzantine au service de l'histoire, in: 13th International Congress of Byzantine Studies. Oxford 1966. Main Papers, 345-354 (Nr. XI) (= Recherches et documents d'hagiographie byzantine [Subsidia Hagiographica 51]. Brüssel 1971, 260 - 269); G. DAGRON, L'ombre d'un doute: L'hagiographie en question, VIe-XIe siècle. DOP 46 (1992) 59-68; L. RYDÉN, New Forms of Hagiography: Heroes and Saints, in: 17th International Byzantine Congress. Major Papers. New Rochelle - New York 1986, 537-554.

12 Unter diesem Gesichtspunkt werden diese Texte vornehmlich als kulturelle, weniger als literarische Produkte ihrer Zeit betrachtet. Die Frage, ob Heiligenviten eine literarische Gattung oder historische Biographien sind, bildete das Thema eines internationalen Colloquiums in Paris im Jahr 2002. Les vies des saints à Byzance. Genre littéraire ou biographie historique? Actes du IIe colloque international philologique „EPMHNEIA“ Paris, 6-7-8 juin 2002 organisé par l'E.H.E.S.S et l'Université de Chypre sous la direction de Paolo Odorico et Panagiotis A. Agapitos (Dossiers Byzantine 4). Paris 2004.

$13 \mathrm{Zu}$ den Problemen der Analyse hagiographischer Texte für die Erforschung der Kindheit bzw. Adoleszenz vgl. ARIANTzI, Kindheit in Byzanz 8-11; EADEM, Adoleszenz in Byzanz 5-7.

14 Th. Pratsch, Der hagiographische Topos. Griechische Heiligenviten in mittelbyzantinischer Zeit (Millennium Studien 6). Berlin - New York 2005. 
Einstellungen der Umwelt des Autors widerspiegelt, vergleichend gegenübergestellt werden. Dementsprechend erscheinen Informationen über „normale“ Jugendliche zumeist in den Kontext einer Erzählung über die Adoleszenz eines Heiligen am Rande eingebettet und mit dessen idealtypischem Verhalten kontrastiert. Es kommt selten vor, dass die Autoren gesondert auf die Aktivität normaler Jugendlicher genauer eingehen und wenn, dann nur flüchtig und ohne Detailangaben. Im hagiographischen Schrifttum werden uns normale Adoleszenten präsentiert, die auf der Suche nach der eigenen Identität Schwankungen ${ }^{15}$ erleben und Erfahrungen sammeln auf Wegen, die von den Autoren aus ihrer religiösen Sicht abwertend beurteilt werden. Deren Hauptintention war es, die Diskrepanzen zwischen normalen und heiligen Jugendlichen aufzuzeigen. Aus diesem Grund hoben sie den außergewöhnlichen Lebensstil heiligmäßig lebender Jugendlicher, die mit der geistigen Reife von Erwachsenen (im Sinne des puer senex) ein klares Ziel vor Augen hatten, hervor. Der Heilige erscheint als beispielgebender Antitypus zum Gemeinplatz des kraftvollen, aber unbeherrschten, Leidenschaften und Vergnügungen ausgesetzten Jugendlichen. ${ }^{16}$ Da er der Protagonist der Erzählung ist, sollte seine Kindheit bzw. Adoleszenz und sein entsprechendes Verhalten die künftige Heiligkeit vorankündigen. Literarische Topoi bzw. direkte Vergleiche mit „normalen“ Jugendlichen, die als Nebendarsteller manchmal absichtlich übertrieben schlecht, schwach und unmoralisch dargestellt werden, dienen dazu, die Besonderheit des Heiligen zu unterstreichen. Durch diese narrativen Techniken bekommt man den Eindruck, dass zwei extreme Positionen einander gegenübergestellt werden. Dieser Umstand macht die Bewertung und Interpretation der Aussagen nicht leicht. Aus methodischer Sicht werde ich zunächst Beschreibungen normaler Jugendlicher aus dem Kontext herauslösen und in weiterer Folge versuchen, über den Kontrast mit den relevanten Verhaltensformen der Heiligen diejenigen Wahrnehmungsmuster und Deutungsformen herauszuarbeiten, die in der Lebenswelt der Autoren und ihres Publikums für die Sozialisierungsprozesse von Adoleszenten als charakteristisch angesehen werden können. Der Artikel wird sich geschlechtsspezi-

15 Adoleszenz wird unter anderem in der Psychologie und Anthropologie als Zeit der persönlichen Krisen bezeichnet. Über Schwankungen und Krisen (,identity crises“), die Jugendliche auf der Suche nach der eigenen Identität erleben, siehe M. Bucholtz, Youth and Cultural Practice. Annual Review Anthropology 31 (2002) 525-552; E. ERIKSON, Jugend und Krise. Die Psychodynamik im sozialen Wandel. Stuttgart 1988.

16 Über Aspekte der Kindheit und Jugend der Heiligen: ARIANTZI, Kindheit; B. ChEvallier-CASEAU, Childhood in Byzantine Saints' Lives, in: Children and Childhood in Byzantium, ed. A. Papaconstantinou - A.-M. Talbot (Dumbarton Oaks Byzantine Symposia and Colloquia). Washington, D.C. 2009, 127-166; A.-M. ТАLвот, The Death and Commemoration of Byzantine Children, in: Becoming Byzan-

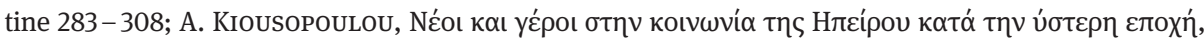

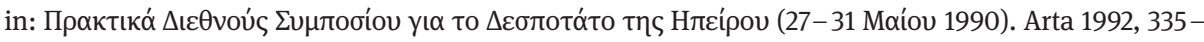

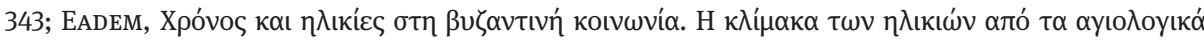

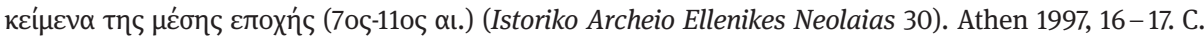
HenNESsy, Image of Children in Byzantium. Farnham u. a. 2008; EADEM, Young People in Byzantium, in: Companion to Byzantium, ed. L. James. Chichester 2010, 81-92. 
fisch auf männliche Jugendliche konzentrieren, da weibliche kaum Möglichkeiten zu einer außerhäuslichen Gruppenbildung hatten; ihnen wurde als Tätigkeitsfeld fast ausschließlich der häusliche Bereich zugewiesen, weil nach den Verhaltensnormen der byzantinischen Gesellschaft Mädchen vor Männerblicken geschützt werden sollten. So blieb ihnen nur die Möglichkeit, Freundschaften, z. B. mit Nachbarinnen, zu pflegen. Ihre Erziehung war nicht auf Karriere bzw. Bekleidung öffentlicher Ämter ausgerichtet, für die neben einer guten Ausbildung auch gute soziale Kontakte erforderlich gewesen wären, sondern darauf, gute Ehefrauen und Mütter zu werden. ${ }^{17}$

Moderne psychologische Studien zeigen, dass Gleichaltrige in der Gegenwart mehr Kontakte untereinander haben als etwa vor 150 Jahren oder zeitlich noch davor, was mit bestimmten geschichtlichen Veränderungen der jüngeren Zeit zusammenhängt. Die byzantinische wie jede vorindustrielle Gesellschaft ${ }^{18}$ schaffte Bedingungen, unter denen Kinder und Jugendliche mindestens ebenso häufig mit Erwachsenen wie mit Ihresgleichen zusammen waren, sowohl im privaten Bereich als auch bei der Arbeit. In den überwiegend landwirtschaftlichen ${ }^{19}$ Familien herrschte eine einheitliche Organisationsform für das Zusammenleben von Erwachsenen und Kindern bzw. Jugendlichen. Zwei oder drei Generationen wohnten unter einem Dach, wuchsen unter den gleichen Wirtschafts- bzw. Sozialbedingungen und Erziehungsidealen auf, hatten viele gleiche Aufgaben und Beschäftigungen im Tagesablauf und ähnlich strukturierte Sozialkontakte. So konnten Kinder bzw. Jugendliche von den Eltern und Großeltern als Vorbilder alles erfahren, was sie benötigten, um ihre späteren Aufgaben als Erwachsene zu erfüllen. ${ }^{20}$ Jugendliche beiderlei Geschlechts standen unter väterlicher Gewalt, ${ }^{21}$ männliche als Lehrlinge auch unter der Gewalt des Handwerksmeisters. Der Weg zum Erwachsenwerden war geschlechtsspezifisch bedingt unterschiedlich. Er führte für männliche Jugendliche über berufliche Ausbildung zur Selbständigkeit und materiellen Autonomie, für weibliche über Haushalt und Handarbeit zur EheschlieBung. 22

Jugendliche wurden in der Beziehung zu Gleichaltrigen nicht mit einer völlig neuen Aufgabe konfrontiert. Bereits in der Kindheit machten sie während der

\footnotetext{
17 Zur unterschiedlichen Erziehung und Bildung der Jungen und Mädchen ARIANTZI, Kindheit 125180.

18 Mietzel, Entwicklungspsychologie 361; HuRRELMAnN, Jugendforschung 20.

19 In Byzanz war die Mehrheit der Bevölkerung mit Landwirtschaft und Viehzucht beschäftigt. Ein kleiner Prozentsatz übte handwerkliche Berufe aus und ein anderer besaß kleine Betriebe. Siehe dazu: The Economic History of Byzantium. From the Seventh through the Fifteenth Century. Bd. 3, ed. A. Laiou. Washington D.C. 2002.

20 Vgl. zur gemeinsamen Arbeit der Generationen in Byzanz. Kinder und Jugendliche lernten dadurch vermutlich die Arbeit von den erfahrenen bzw. älteren Familienmitgliedern: ARIANTZI, Kindheit 135141; EADEM, Kinderarbeit in Byzanz 405-413.
}

21 Zur väterlichen Gewalt (patria potestas) in Byzanz: ARIANTZI, Kindheit 41-47.

22 ARIANTZI, Kindheit 6.1 und 6.2. 
„schulischen“ Ausbildung ${ }^{23}$ bzw. bei der Arbeit mit solchen Bekanntschaft und das prägte die Erwartungen, die sie in die gegenwärtige Situation setzten. Die Kontakte im Kindesalter wurden von den besorgten Müttern kontrolliert, die wegen des möglichen schlechten Einflusses der Gleichaltrigen auf das Verhalten ihrer Kinder strenge Maßnahmen zu deren Schutz trafen. Man denke etwa an diesbezügliche Aussagen der hagiographischen Texte. Die Mutter des Michael Psellos wollte von ihren Kindern nicht nur über den Lernstoff, sondern auch über ihre Kontakte in der Schule informiert werden; ${ }^{24}$ die Mutter des Nikephoros von Miletos begleitete ihren Sohn auf dem Schulweg, weil sie verhindern wollte, dass das Kind durch den Kontakt mit Altersgenossen schlechtem Einfluss ausgesetzt werde. ${ }^{25}$ Die Mutter des künftigen Patriarchen Tarasios versuchte ihren Sohn im Kindesalter dazu anzuhalten, nur mit guten und frommen Personen Umgang zu pflegen. ${ }^{26}$ Kinder aus bäuerlichen Familien, die als Hirten oder in der Landwirtschaft arbeiten mussten und daher keine Ausbildung

23 ARIANTZI, Kindheit 168-181: In Byzanz gab es kein staatliches Bildungssystem, sondern Bildung ging ausschließlich auf Privatinitiative zurück und hierin folgte man der antiken Tradition. Wenn in den Heiligenviten von Elementarschule die Rede ist, muss man an einen Raum in der Kirche, im Kloster oder seltener in einem öffentlichen Gebäude denken, in dem Kinder verschiedenen Alters von einem Lehrer einzeln oder in einer Gruppe unterrichtet wurden. Die Elementarbildung wird von den Autoren der Heiligenviten unter folgenden Begriffen zusammengefasst: Vorerziehung ( $\pi \rho \pi \alpha$ เ $\left.\delta \varepsilon^{\alpha} \alpha\right)$, einfache

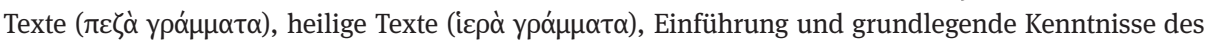

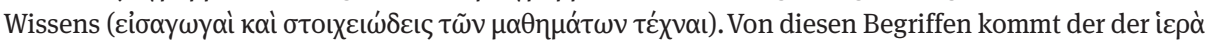
үра́ $\mu \alpha \alpha \alpha$ in den Heiligenviten besonders oft vor. Während der Elementarbildung lernten die Kinder anhand kirchlicher Texte Lesen, Schreiben, Silben, Worte und Sätze zu bilden und etwas Arithmetik.

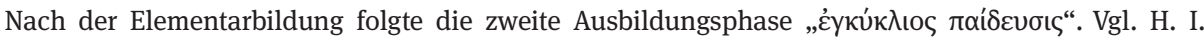
MARRou, Geschichte der Erziehung im klassischen Altertum. München ${ }^{7} 1977$; A. MARKopoulos, H

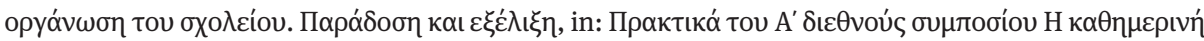

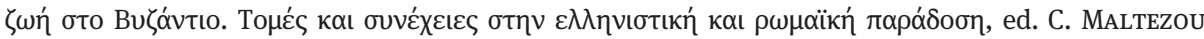
Athen 1989, 325 - 333, hier 327; N. Kalogeras, Byzantine Childhood Education and its Social Role from the Sixth Century until the End of Iconoclasm. Ph.D. Diss. History. University of Chicago 2000, $3 \mathrm{ff}$. 24 Michael Psellos, Encomio per la madre 10 (ed. U. CRIscuolo, Michele Psello Autobiografia Encomio per la madre. Testo critico, introduzione, traduzione e commentario. Napoli 1989, 105, 582-585):

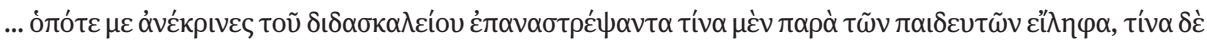

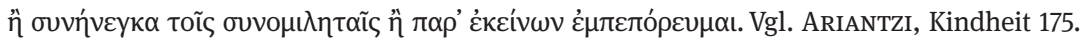

25 Vita des Nikephoros von Miletos (BHG 1338) 5 (ed. T. WiEgAND, Milet 3,1. Der Latmos. Berlin 1913,

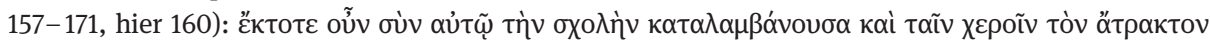

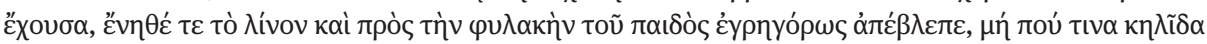

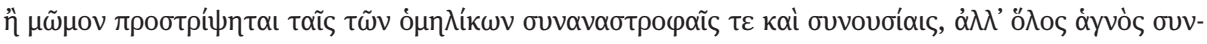

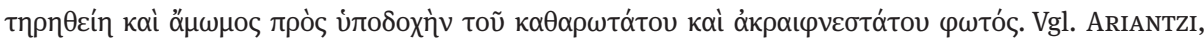
Kindheit 156.

26 Vita des Tarasios I. Patriarch (BHG 1698) 6 (ed. ST. EfthymiAdIS, The Life of the Patriarch Tarasios by Ignatios Diakon (BHG 1698). Introduction, Text, Translation and Commentary [Birmingham Byzantine and Ottoman Monographs 4]. Aldershot [u.a.] 1998, 74, 1-5-75, 6-7). Vgl. ARIANTzI, Kindheit

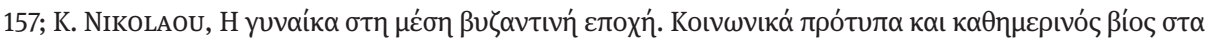

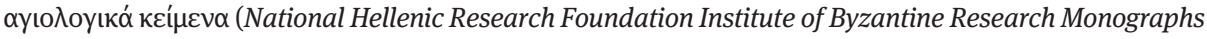
6). Athen 2005, 134. Zu Tarasios PmbZ I/4, Nr. 7235. 
genossen, bekamen Kontakt mit Gleichaltrigen bei der Arbeit, wo sie auch nebenbei Gelegenheit zum Spielen miteinander fanden. ${ }^{27}$

Dank dieser und ähnlicher Erfahrungen waren bei den Jugendlichen bereits Sozialkompetenzen entwickelt. Das Formenspektrum der Peergruppen in Byzanz reicht von spontan entstehenden Kleingruppen männlicher Jugendlicher bis zu „Jugendbanden“. Hagiographische Texte belegen, dass Peergruppen vor allem große Bedeutung für die Gestaltung der Freizeitaktivitäten hatten. Aus diesen können wir Einblicke in das Leben von Jugendlichen aus verschiedenen sozialen Schichten städtischen und ländlichen Milieus gewinnen. Die Quellen beschreiben Aktivitäten bzw. Interessen und Verhaltensformen der Peers, sodass drei verschiedene Typen von Gruppenidentität sichtbar werden:

In der ersten Kategorie manifestiert sich die Körperkraft und Vitalität von Jugendlichen in sportlichen Tätigkeiten ${ }^{28}$ wie Laufen und Springen, ${ }^{29}$ Ringen und Faustkampf, Jagd ${ }^{30}$ und Reiten. Die letzteren Sportarten bevorzugte seiner Vita zufolge Theophanes der Bekenner aus Konstantinopel, wobei er sich besonders im Umgang mit Tieren ${ }^{31}$ als mutig und geschickt erwies. Mit der Kraft der Jugendblüte geht die

27 ARIANTZI, Kindheit 135-141.

28 Über die genannten sportlichen bzw. kriegerischen Aktivitäten, die Lungenprobleme durch Überbelastung verursachen konnten, wird in den medizinischen Quellen berichtet. Siehe dazu: Stephanos von Athen, Kommentar zu den Aphorismen des Hippokrates V 10 (ed. L. G. WeSTERINK, Stephanus of Athens Commentary on Hippocrates'Aphorisms Section V-VI [CMG XI, 1, 3, 3]. Berlin 1995,

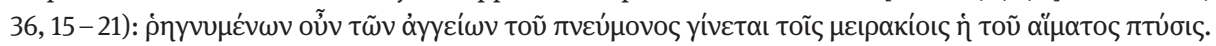

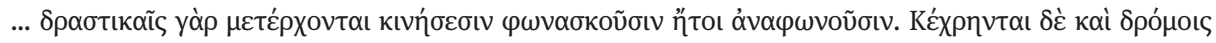

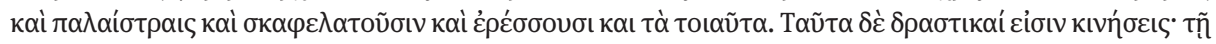

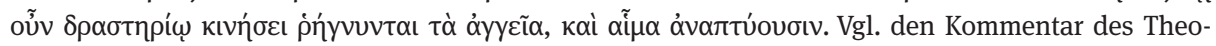
philos Protospatharios und Damaskios, die offenbar den Text des Stephanos von Athen gekannt und inhaltlich übernommen haben: Theophilos Protospatharios und Damaskios, Scholia in Hippocratis aphorismos 5. Teil, 9 (ed. F. R. Dietz, Apolloni, Citiensis, Stephani, Palladii, Theophili, Meletii, Damascii, Ioannis, aliorum Scholia in Hippocratem et Galenum, II. Königsberg 1834 [Nachdruck Ams-

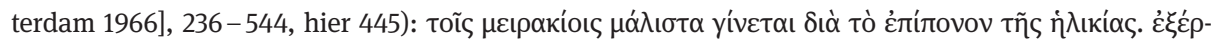

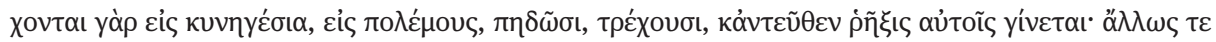

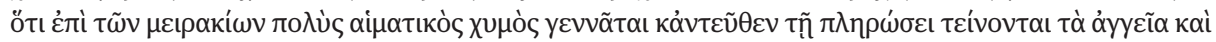

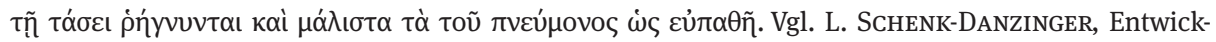
lungspsychologie (Schriften zur Lehrerbildung und Lehrerfortbildung 1). Wien $1988^{20}$, 276: Der modernen Psychologie zufolge besteht in der Zeit der Vorpubertät ein erhöhtes, mit großer körperlicher Leistungsfähigkeit verbundenes Bewegungsbedürfnis, das sehr oft bei sportlichen Wettbewerben ausgelebt wird.

29 Vita des Niketas von Medikion (BHG 1341-1342a) cap. 5 (AASS Apr. I, Appendix, XVIII-XXVII): ... $\dot{\omega} \varsigma$

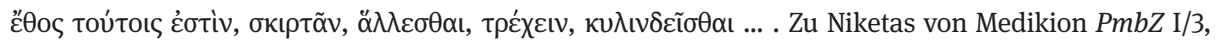
Nr. 5443.

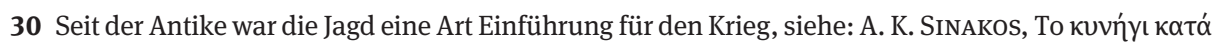

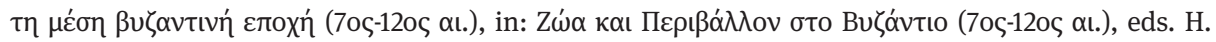
Anagnostakis - T. G. Kolias - E. Papadopoulou. Athen 2011, 71-83.

31 Zu Tieren in Byzanz: H. KRoLl, Tiere im Byzantinischen Reich. Archäozoologische Forschungen im Überblick (Monographien des Römisch-Germanischen Zentralmuseums 87). Mainz 2010, speziell zu 
Vorstellung wilder Leidenschaft einher, es wird aber in Hinblick auf die künftige Heiligmäßigkeit ausgleichend die Selbstbeherrschung des Theophanes betont. ${ }^{32}$ Auch Jugendliche aus der Vita des Nikon Metanoeite, die wahrscheinlich aus demselben sozialen Milieu wie der Heilige, nämlich aus begüterten Landbesitzerfamilien in Paphlagonien stammten, liebten Reiten und Laufen. ${ }^{33}$ Der ungeschickte Umgang mit Waffen bei der Jagd konnte allerdings zu Unfällen führen, wie der Fall des achtzehnjährigen Sohnes eines Illoustrios namens Johannes aus der Stadt Amorion in Phrygien zeigt. Als der Jugendliche (pais und neaniskos) 15 Jahre alt war, ging er mit seinem Vater auf die Jagd und wurde mit einem Speer verletzt. Drei Jahre lang war er unfähig zu gehen, ${ }^{34}$ bis sein Vater ihn zum Heiligen Theodoros von Sykeon brachte,

Jagdtieren 192-199: Den Tierknochenfunden nach zu urteilen, spielte die Jagd eine nur sehr geringe Rolle im Alltagsleben. Von Region zu Region sind andere Jagdtiere zu finden, weil ihre Existenz von klimatischen Umständen und Ernährungsmöglichkeiten abhängig war. Allgemein werden Hasen, Wildschweine, Rothirsch, Damhirsch und Reh, Hirsche anstelle verschiedener Gazellen- und Antilopenarten und Wildvögel erwähnt. EADEM, Animals in the Byzantine Empire. An Overview of the Archaeozoological Evidence. Archeologia Medievale 39 (2012) 93-121.

32 Vita des Theophanes des Bekenners (BHG 1787z), cap. 6 (ed. V. V. LATYŠEV, Mefodija patriarcha

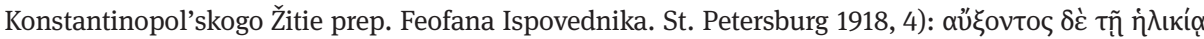

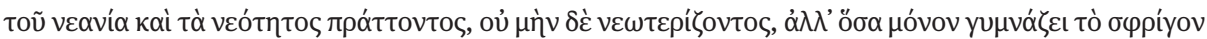

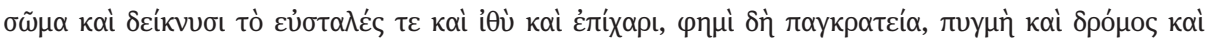

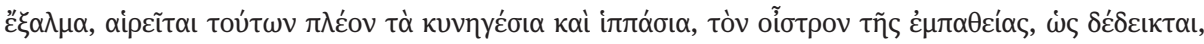

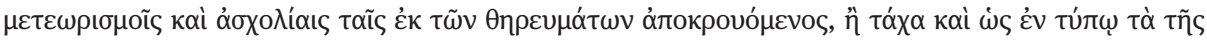

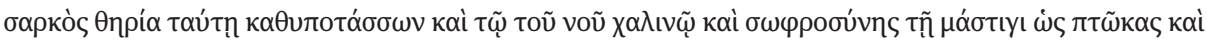

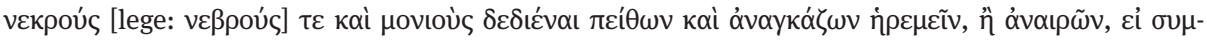

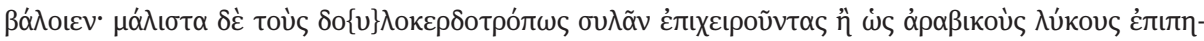

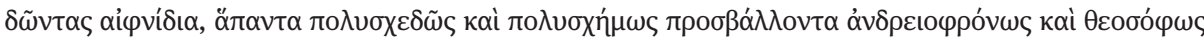

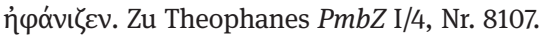

33 Vita des Nikon ho Metanoeite (BHG 1366), cap. 3 (ed. D. Sullivan, The Life of Saint Nikon. Brookline, Mass. 1987, 36, 8-12). Sein Vater war reicher Landbesitzer in Paphlagonien und Paroiken arbeiteten für ihn. Zu den Paroiken: G. Ostrogorsky, Pour l'histoire de la féodalité byzantine (Corpus Bruxellense Historiae Byzantinae Subsidia 1). Bruxelles 1953; IDEM, Quelques problèmes d'histoire de la paysanerie byzantine (Corpus Bruxellense Historiae Byzantinae Subsidia 2). Bruxelles 1956, 11-24, 40 - 74; J. KaRAYAnNopulos, Ein Problem der spätbyzantinischen Agrargeschichte. JÖB 30 (1981) 207237; A. HARVEY, Economic Expansion in the Byzantine Empire 900 -1200. Cambridge 1989 (Nachdruck 2002), 128-162; M. KAPLAN, Les hommes et la terre à Byzance du VIe au XIe siècle. Propriété et exploitation du sol (Byzantina Sorbonensia 10). Paris 1992, 264-273; J. LEFoRT, The Rural Economie, Seventh-Twelfth Centuries, in: The Economic History of Byzantium, 231-310. Zu Nikon Metanoeite PmbZ II/5, Nr. 26155.

34 Vita des Theodoros von Sykeon (BHG 1748), cap. 107 (ed. A.-J. Festugière, Vie de Théodore de

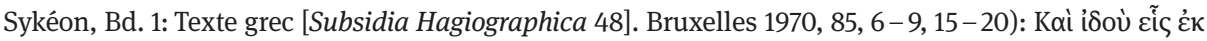

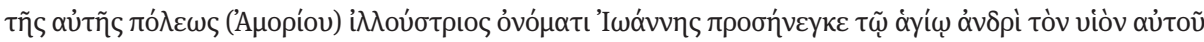

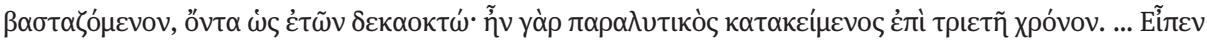

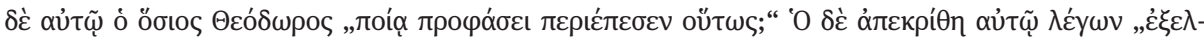

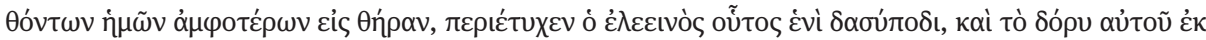

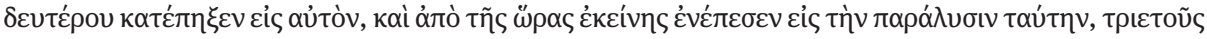

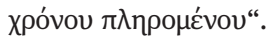


von dem er geheilt wurde. ${ }^{35}$ Die Texte zeigen deutlich eine soziale Differenzierung bei unterschiedlichen sportlichen Aktivitäten. Jagd $^{36}$ und Reiten gehörten zum Training Jugendlicher der sozialen Oberschicht städtischer bzw. ländlicher Herkunft, während offenbar Laufen, Springen, Ringen und Faustkampf für Jugendliche aller sozialen Schichten zugänglich waren, weil dies keine teure Ausrüstung wie Pferde ${ }^{37}$ und Waffen $^{38}$ erforderte. Mehrere Studien befassten sich bereits mit den Idealen und Wertvorstellungen der byzantinischen Aristokratie, ${ }^{39}$ wie etwa genos, oikos, eugeneia, philotimia, philodoxia, kenodoxia, philautia, epideiktikotita, hyperopsia, oiesis, physiosis und typhos. Unbeachtet blieben allerdings Ideale, die körperliche Kraft, militärische Ausbildung ${ }^{40}$ und Kampffähigkeit bzw. -bereitschaft betrafen und in der byzantinischen Literatur vielfach thematisiert werden. Diese Ideale übernahm offenbar der Nachwuchs der byzantinischen Aristokratie von der Ausbildung der Söhne der kaiserlichen Familie, da diese als Vorbild galt. Denn das körperliche und militärische Training war ein spezieller Bestandteil der Ausildung künftiger Kaiser. Es wurde wesentlicher Teil des kulturellen und literarischen Ideals kaiserlicher Kindheit und Adoleszenz ab der zweiten Hälfe des elften Jahrhunderts. Diese Entwicklung ist in

35 Vita des Theodoros von Sykeon (BHG 1748), cap. 107 (85-86, 20 - 42 FeSTUGIÈRE): Der Jugendliche (neaniskos) wurde nach dem Rat des Theodoros in die Theotokos - Kirche des Illoustrios Anastasios gebracht und dort von Theodoros mit Öl gesegnet und geheilt.

36 Zur ökonomischen und sozialen Differenzierung zwischen kaiserlicher bzw. aristokratischer Jagd

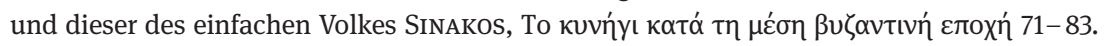

$37 \mathrm{Zu}$ Pferden, ihrer Bedeutung und Rolle in der Gesellschaft, im Krieg und in der Wirtschaft von der Antike bis Byzanz: Le cheval dans les sociétés antiques et médiévales: Actes des Journées d'étude internationales organisées par l'UMR 7044 (étude des civilisations de l'antiquité), Strasbourg, 6-7 novembre 2009, ed. St. Lazaris. Turnhout 2012; ST. LAZARIs, Rôle et place du cheval dans l'Antiquité

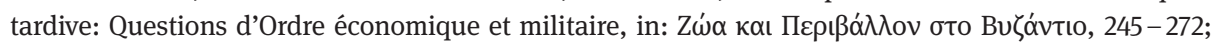
IDEM, Learning and Memorising Hippiatric Knowledge in Late Antiquity and in Byzantium (Sonderdruck von: Le cheval dans le culture médievale/textes réunis par B. Andenmatten - A. Paravicini Bagliani - E. Pibiri). Firenze 2007, 269-294; Zu Pferdekrankheiten: A. McCABE, A Byzantine Encyclopaedia of Horse Medicine. The Sources, Compilation, and Transmision of the Hippiatrika. Oxford New York 2007; J. HALDON, The Organisation and Support of an Expeditionary Force: Manpower and

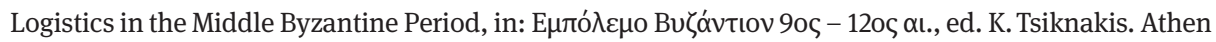
1997, 111-151, besonders 127.

38 T.G. KoLIAS, Byzantinische Waffen: ein Beitrag zur byzantinischen Waffenkunde von den Anfängen bis zur lateinischen Eroberung. Wien 1988.

39 K.-P. MATSChKE - F. Tinnefeld, Die Gesellschaft im späten Byzanz. Gruppen, Strukturen und Lebensformen. Köln - Wien 2001; P. MAgdalino, Byzantine Snobbery, in: The Byzantine Aristocracy IX - XIII Centuries, ed. M. Angold. Oxford 1984, 58-78; IDEM, The Byzantine Aristocratic Oikos, in: The Byzantine Aristocracy IX - XIII Centuries, ed. M. Angold. Oxford 1984, 92-111.

40 MATSChKE - TinnefELD, Gesellschaft 25: Wenn Sprösslinge der Oberschicht in der Palaiologen Zeit daran interessiert waren, konnten sie schon im jugendlichen Alter militärische und auch einflussreiche zivile Funktionen übernehmen, d.h. an die Spitzen nicht nur von Heeren und Flotten, sondern auch von Städten und Regionen treten. Vgl. mit der Ausbildung des aristokratischen Nachwuchses zum Ritter im Westen: G. Duby, Dans la France du Nord-Ouest au XIIe siècles: les ,jeunes“ dans la société aristocratique. Annales économies, sociétés civilisation 1964, 835-846. 
zahlreichen idealisierten Portaits der kaiserlichen Kindheit und Adoleszenz zu sehen. Die kaiserlichen Lobreden aus dem zwölften Jahrhundert heben neben dem militärischen Training als Teil der körperlichen Ausbildung eines Kaisers auch das Betreiben von Sport und Spiele hervor. ${ }^{41}$ Ein repräsentatives Beispiel dafür ist im 12. Jahrhundert das Gedicht des Theodoros Prodromos auf die Geburt des Alexios, Sohnes des Sebastokrators Andronikos. Dort erläutert der Autor detailliert, was Alexios in den beiden Phasen der Adoleszenz lernen und beherrschen müsse: als meirakion Ballspiel, Jagd, Reiten, Bogenschießen und sich militärischen Übungen für den Kampfeseinsatz unterziehen, als neaniskos tapfer gegen die Barbaren kämpfen und Siege davontragen. ${ }^{42}$ Anna Komnene im 12. Jahrhundert berichtet von der militärischen Ausbildung ihres Vaters und seiner Bereitschaft, als Jüngling in den Krieg zu ziehen, ${ }^{43}$ aber auch von der Liebe ihres Onkels Isaakios ${ }^{44}$ für die Jagd und seiner Freude vor allem an militärischen Aktivitäten. ${ }^{45}$ Johannes II. Komnenos (1118-1143) brachte seinem Sohn

41 D. G. Angelov, Emperors and Patriarchs as Ideal Children and Adolescents. Literary Conventions and Cultural Expectations, in: Becoming Byzantine: Children and Childhood in Byzantium, ed. A. Papaconstantinou-A.-M. Talbot (Dumbarton Oaks Byzantine Symposia and Colloquia). Washington D.C. 2009, 85-125, hier 105-111.

42 W. HörandnER, Theodoros Prodromos. Historische Gedichte (WBS 11). Wien 1974, Gedicht XLIV,

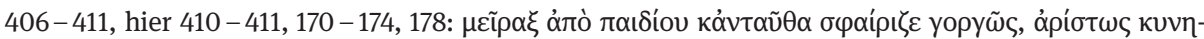

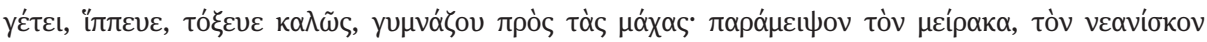

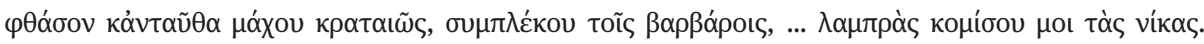
43 Anna Komnene, Alexias I 1 (ed. D. Reinsch - A. KambyLis, Annae Comnenae Alexias [CFHB 40/1].

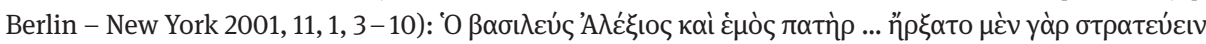

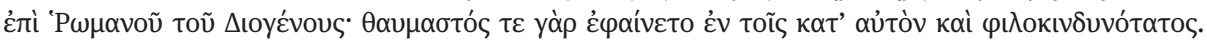

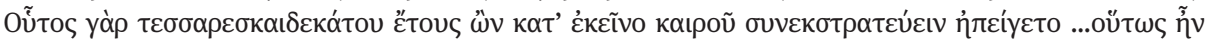

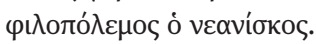

44 Es kommt auch in den Quellen vor, dass junge Söhne des Kaisers, wie Alexandros, der Sohn von Basileios, und Michael III., der Sohn des Theophilos I. (Ioannis Scylitzae Synopsis Historiarum, hrsg. I. THURN [CFHB]. Berlin-New York 1973, Alexandros: 2, 25-34; Michael: 7, 25-34; Georgius Monachus, Chronographia, chronicon breve (libri 1-6), vol. 110, p. 1124-1125), und Romanos II., der Sohn des Konstantinos VII. (ed. W.J. AERTs, Michaelis Pselli Historia Syntomos [CFHB Series Berolinensis 30]. Berlin - New York 1990, 2-108, hier 103, 19-26) von den Autoren kritisiert werden, weil sie Reiten und Jagd nicht als militärisches Training betrachteten, sondern nur als Spaß, der mit Pferderennen, Trinken und anderen Unterhaltungen eng verbunden war. Die Sucht nach solchen Freizeitaktivitäten verhinderte, dass diese Thronnachfolger ihre Verantwortung als Kaiser ernst nahmen und das Reich erfolgreich regierten. Zu Alexandros PmbZ II/1, Nr. 20228; Zu Michael III. PmbZ I/3, Nr. 4991.

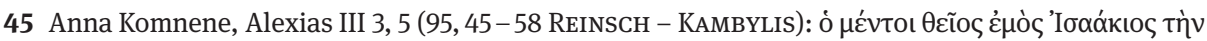

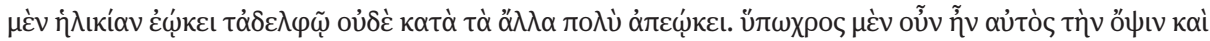

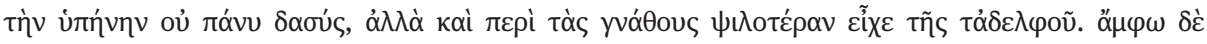

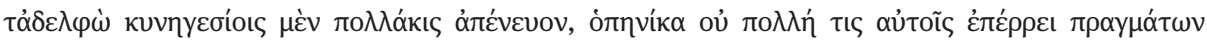

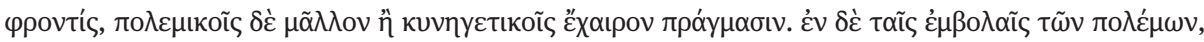

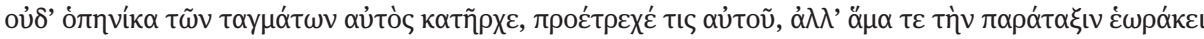

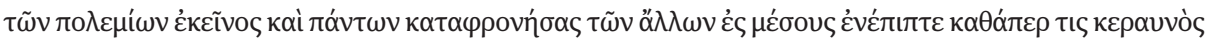

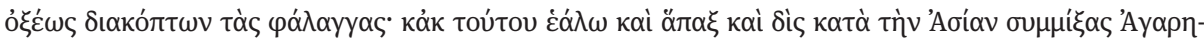

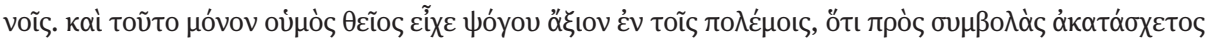
กิ้v. 
Manuel I (1143-1180) den Umgang mit Waffen und militärische Strategie bei. ${ }^{46}$ Die rhetorischen Werke am Hof der Komnenen heben besonders die Rolle des Vaters für die militärische Ausbildung seines Sohnes als künftigen Thronnachfolgers hervor. ${ }^{47}$ Auch im Epos des Digenis Akritas wird überliefert, dass der Protagonist bereits im Kindesalter erst von seinem Vater und dann drei Jahre lang von einem Lehrer Ausbildung in Reiten und Jagd bekam und als Zwölfjähriger mit wilden Tieren kämpfen wollte. Letzteres wurde ihm aber von seinem Vater verweigert, weil er noch nicht die hebe (Pubertät) erreicht habe und daher zu jung sei. ${ }^{48}$ Konstantinos Manasses schrieb im 12. Jahrhundert über die kaiserliche Jagd, wobei er deren positiven Einfluss besonders hervorhebt. Die Jagd und das Reiten schützen Menschen vor Krankheiten wegen der körperlichen Tätigkeit, die ihnen diese Aktivitäten abverlangen, aber auch wegen des seelischen Wohlbefindens, das sie ihnen schenken. Beides bereitet die jungen Leute auf das richtige Verhalten im Kampf vor, indem es Reitkunst, Anrücken und den Umgang mit Pferden lehrt. Das dürfte, könnte man sagen, eine Übung maßvoller Art zum Denken an Größeres sein. Das ist laut dem Text „ein Kampf, der nicht Männer vernichtet, das ist Ares ohne Eisen, der seine Rechte nicht blutbefleckt hat und die Lanze nicht von Blut triefend“. ${ }^{49}$

Es ist aus der byzantinischen Literatur bekannt, dass die byzantinischen Kaiser, vor allem die aus der Dynastie der Komnenen, die als Vertreter der militärischen Aristokratie galten, sich durch ihre außerordentliche Jagderfahrung wie auch militärische Fähigkeiten auszeichneten. ${ }^{50}$ Künstlerische Darstellungen von Jagdszenen

46 Michael Italikos (ed. P. GAUTIER, Michel Italikos: Lettres et Discours. Paris 1972, 282-284); Eustathios von Thessalonike, in: Fontes rerum byzantinarum: Rhetorum saeculi XII orations politicae, ed. V. Regel - N. Novosadskii. St. Petersburg 1892-1917 (repr. Leipzig 1982), 121 (= ed. P. WIRTH, Eustathii Thessalonicensis opera minora (CSHB). Berlin 2000, 285).

47 ANgelov, Emperors and Patriarchs as Ideal Chidren and Adolescents 109.

48 E. TRAPP, Digenis Akritas. Synoptische Ausgabe der ältesten Versionen (WBS 8). Wien 1971, G IV, 1017-1037.

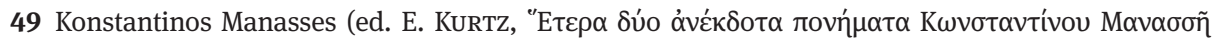

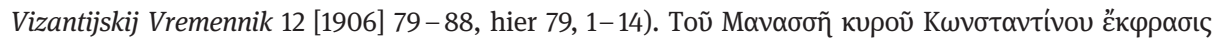

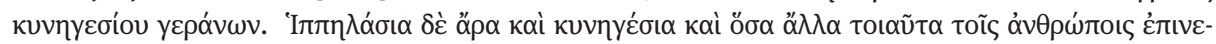

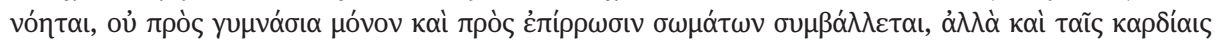

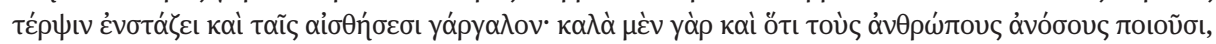

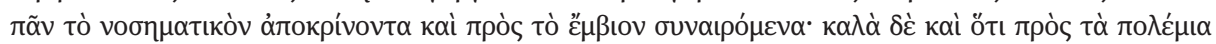

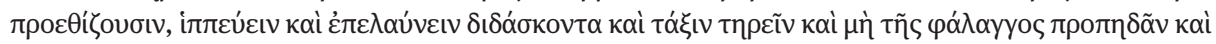

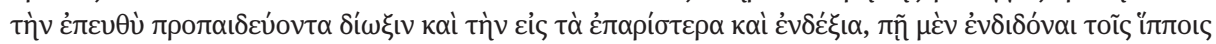

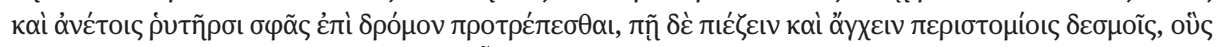

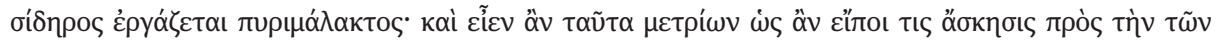

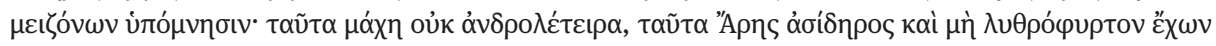

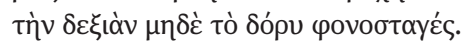

50 Die Jagd, die von Mitgliedern der Aristokratie organisiert war und an der auch der Kaiser teilnahm, verlangte zahlreiche Begleitpersonen und große Vorbereitungen. Diese Jagd konnte in einem offenen Feld oder an einem umzäunten Ort, in kaiserlichen Parkanlagen stattfinden (N. ŠEvčENKo, Wild Animals in the Byzantine Park, in: Byzantine Garden Culture, eds. A. Littlewood - H. Maguire - J. Wollschke-Bulmahn. Washington D.C. 2002, 69-86). Zu verschiedenen Arten der Jagd, die der sozialen 
waren mit der kaiserlichen Ideologie und dem Empfinden, dass Jagdfähigkeit und militärische Tugend zusammengehören, eng verbunden. Die Jagd und das Polospiel gehörten nicht nur zu sportlichen ${ }^{51}$ Aktivitäten des Kaisers und der Aristokratie, ${ }^{52}$ sondern auch zum Unterhaltungsprogramm und waren eine beliebte Veranstaltung, an der manchmal auch Frauen als Zuschauerinnen teilnahmen. ${ }^{53}$

Texte aus dem aristokratisch-höfischen Milieu der Komnenenzeit und hagiographische Texte früherer Jahrhunderte lassen deutlich einen gemeinsamen Erfahrungshorizont erkennen, in dem die körperliche Übung und Erlangung körperlicher Fähigkeiten als ein wichtiges Instrument und als Rahmen für Sozialisierungsvorgänge von Jugendlichen verstanden werden. Die Art der Betätigung war aufgrund der ökonomischen Voraussetzungen und der damit verbundenen Wertvorstellungen schichtspezifisch geprägt und hatte demgemäß eine hohe Signalwirkung sowohl nach außen gegenüber anderen Gruppierungen als auch nach innen im Sinne der Verfestigung der kollektiven sozialen Identität und inneren Kohärenz. Bestimmte Formen der Jagd und Übungen aus dem Bereich des Kriegshandwerks unterstrichen vor allem militärische Idealvorstellungen, die als Normen und Identifizierungsmerkmale junger Aristokraten akzeptiert waren. Demgegenüber standen weniger aufwendige körperliche Betätigungen für jugendliche Gruppen aus niedereren Gesellschaftsschichten, die ähnliche Funktionen der Selbstvergewisserung erfüllten. Darüber hinaus lassen die Texte eine Hierarchisierung der Tätigkeiten nach Anforderungen und Schwierigkeiten erkennen, die eine zunehmende Inanspruchnahme entsprechend dem voranschreitenden Reifungsprozess der Jugendlichen widerspiegelt. Dies deutet auf eine gewisse Ritualisierung des Eingliederungsprozesses von Jugendlichen im Sinne einer graduellen Einführung in zunehmend anspruchsvollere Betätigungen und die damit verbundenen Wertsysteme hin.

Ein zweiter Typus von Peergruppen bestand aus Jugendlichen aus städtischem Milieu und wohlhabenden Familien, die ihre Freizeit mit Amüsements verbrachten, ihren Geist den zügellosen Bestrebungen und jugendlichen Begierden hingaben. In den Quellen liest man über vielseitige Aktivitäten: Kneipen ${ }^{54}$ - und Bordellbesuch, ${ }^{55}$

Stellung der Menschen entsprach, siehe E. PALTAGEAn, De la Chasse et du Souverain. DOP 46 (1992) 257-263 und besonders 257, 259-260.

$51 \mathrm{Zu}$ Sport in Byzanz siehe A. KaRPozIlos - A. CutLER, „Sports“, in: ODB 3, 1939.

52 ANGelov, Emperors and Patriarchs as Ideal Children and Adolescents 110-111.

53 Nach einer Jagd folgte ein Fest bzw. ein Symposium, um den Jagderfolg zu feiern und Jagdbeute zu

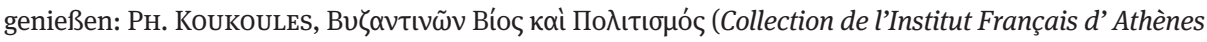

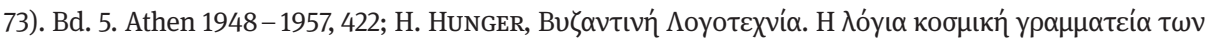

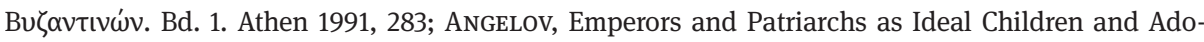
lescents 111 .

$54 \mathrm{Zu}$ Kneipe, ihrer Organisation und Kundschaft: E. KISLINGER, Gastgewerbe und Beherbergung in frühbyzantinischer Zeit. Eine realienkundliche Studie aufgrund hagiographischer und historiographischer Quellen. (Diss.) Wien 1982. IDEM, Taverne, alberghi e filantropia ecclesiastica a Bizanzio. Atti della Accad. delle Scienze di Torino, Cl. sc. mor. stor. e filol. 120 (1986) 84-96. Zu Kneipe und Kriminalität 
Weintrinken und Tanzen, ${ }^{56}$ Gesellschaft mit Tänzerinnen, Scherzen, Würfelspiele, ${ }^{57}$ Feste feiern, Teilnahme an Bühnenspektakeln ${ }^{58}$ und Theater. ${ }^{59}$ Das reizte auch verwaiste reiche Jugendliche, die sich in ihrem instabilen Alter durch ererbtes Geld die Möglichkeit zur Befriedigung ihrer Wünsche in den oben genannten Vergnügungen

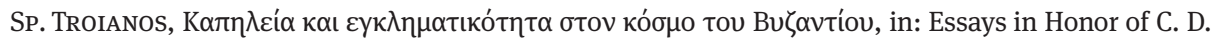
Spinellis, ed. M. Galanou. Athen - Komotene 2010, 1285-1300.

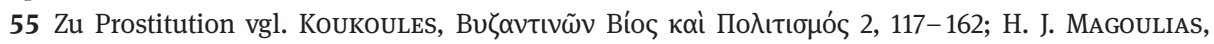
Bathhouse, Inn, Tavern, Prostitution and the Stage as seen in the Lives of the Saints of the Sixth and Seventh Centuries. EESB 38 (1971) 233-252. ST. LEonTsINI, Die Prostitution im frühen Byzanz (Diss. der Universität Wien 194). Wien 1989.

56 Die Byzantiner bewerteten kordax, einen meist von Gesang und kithara begleiteten Männertanz, und methe, die Trunkenheit, vorwiegend negativ, siehe dazu J. KodER, Kordax und Methe: Lasterhaftes Treiben in Byzantinischer Zeit. ZRVI 50/2 (2013) 947-958. Zum Tanz auf öffentlichen Orten wie Straßen oder Plätzen während eines Symposiums und eines religiösen Festes siehe R. WEBB, Demons and Dancers. Performance in Late Antiquity Cambridge, Mass. - London 2008.

57 Vita des Platon von Sakkudion (BHG 1553), cap. 6 ('Eлıт́́ $\varphi$ ıос. PG 99, 803-850, hier 808B-C-D). Sowohl das kirchliche (Apostolische Kanones; Trullanum Kanon 71; Balsamons Kommentar) als das weltliche Recht (Justinian Novelle 123) waren gegen das Würfelspiel. Wahrscheinlich ging es weniger um das Spiel als solches, als um das Glücksspiel mit all seinen Risiken für den einzelnen Spieler und für seine Familien. Das Würfelspiel wurde für Laien und Kleriker gleichermaßen verboten und sogar unverhältnismäßig hoch bestraft. Kleriker wurden mit der Absetzung und Laien mit der Exkommunikation (Trullanum Kanon 50) bedroht. In der Novelle Justinians 123 ist nur vom Klerus die Rede oder nur von hohen Würdenträgern. Vgl. H. - G. BECK, Orthodoxie und Alltag, in: Byzantium. Tribute to Andreas N. Stratos, II: Theology and Philology. Athen 1986, 329-346, hier 331-333. Zu Platon von Sakkudion PmbZ I/4, Nr. 6285.

58 Vita des Niketas von Medikion, cap. 5 (Acta sanctorum Apr. I, Appendix, XVIII-XXVII, 19).

59 Unter Theatron sind alle möglichen Spektakel und Veranstaltungen auf öffentlichen Schauplätzen zu verstehen; vgl. dazu W. Puchner, Zum „Theater“ in Byzanz. Eine Zwischenbilanz, in: Fest und Alltag in Byzanz, hrsg. von G. Prinzing - D. Simon). München 1990, 11-16, hier 12; Über den schlechten Ruf des „Theaters“ vgl. auch Johannes Chrysostomos, Homilie 42, 4 (in acta Apost.) PG 60, 301: 'Ev $\delta \varepsilon$

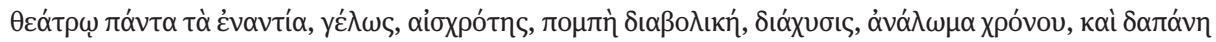

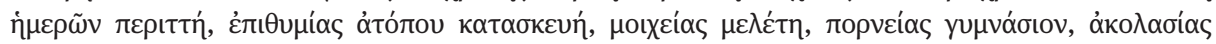

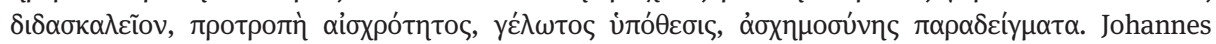

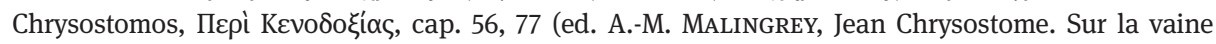
gloire l'education des enfants. Introduction, Texte critique, traduction et notes [SC 18]. Paris 1972, hier 733-734, 923-925); Johannes Chrysostomos nahm deutlich Stellung gegen das „Theater“, weil dort unmoralische Dinge passierten, und empfahl den Eltern, den Kindern nicht zu erlauben, dorthin zu gehen, damit sie durch die Dinge, die dort zu sehen oder zu hören waren, keinen Schaden erleiden. Er kam offenbar zu dieser Ansicht, weil die Welt des Theaters mit Kupplern, Mimen, Prostituierten und Päderasten in Verbindung gebracht wurde. Aus den frühbyzantinischen Quellen sind uns einige Miminnen bekannt, die zugleich als Prostituierte arbeiteten, darunter die spätere Kaiserin Theodora. Vgl. Leontsini, Prostitution 122-127; C. MANGo, Daily Life in Byzantium. JÖB 31/1 (1981) 337-353, hier 342343. Zum Mimos vgl. F. TinnEFeLD, Zum profanen Mimos in Byzanz nach dem Verdikt des Trullanums (691). Byzantina 6 (1974) 323-343. Vgl. Theatron. Rhetorische Kultur in Spätantike und Mittelalter (Millennium Studien 13), hrsg. M. Grünbart. Berlin - Boston 2007, VII. 
verschafften, wie die Vita des Platon von Sakkudion erzählt. ${ }^{60}$ Da ihnen offenbar die elterliche Betreuung und das Gefühl der Familienzugehörigkeit fehlten, erlebten sie die altersbedingten emotionalen Schwankungen besonders intensiv und versuchten daher Geborgenheit durch neue Kontakte $\mathrm{zu}$ Gleichaltrigen $\mathrm{zu}$ finden und in der Unterhaltung Trost für den Verlust ihrer Eltern zu suchen. In diesem Fall war der Einfluss der Gleichaltrigen von großer Bedeutung für die Lebensgestaltung der Jugendlichen und konnte zerstörerische Folgen haben, weil sie leicht zu beeinflussen und verführbar waren.

In der Vita des Symeon Salos wird über eine große Menge der männlichen Einwohner der Stadt Emesa berichtet, die ein zügelloses Leben führten. Sie besuchten Tavernen, sie tranken Wein, sie tanzten auf öffentlichen Plätzen, sie verführten Dienstmädchen und gingen mit ihren Gefährten dorthin, wo sie sich mit Dirnen vergnügen konnten. ${ }^{61}$ Obwohl der Text keinerlei Auskünfte über das Alter oder die Altersstufe der Männer gibt, lässt sich aus dem beschriebenen Verhalten durchaus erschließen, dass es sich ähnlich den aus anderen Viten bekannten Beschreibungen um typisches Benehmen von Jugendlichen handelt, die sich mit ihren gleichaltrigen Freunden amüsierten.

Aus der Vita des Andreas Salos erhalten wir ausführlichere Informationen über die Existenz, das Verhalten und das Alltagsleben von jugendlichen Peergruppen im Konstantinopel des 10. Jahrhunderts, auch wenn diese eine fiktive Erzählung ist und

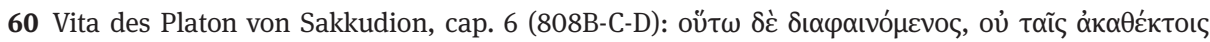

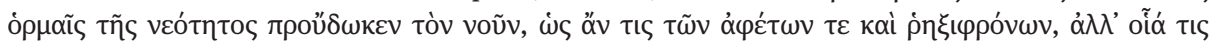

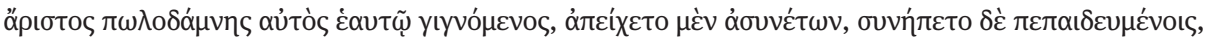

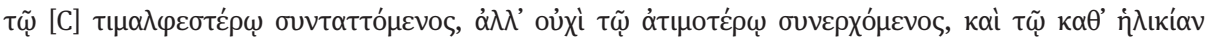

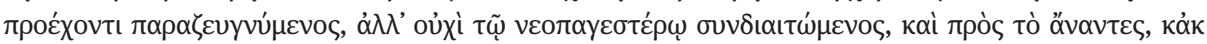

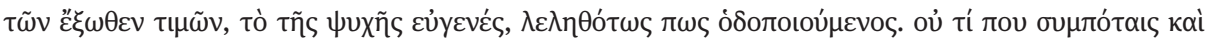

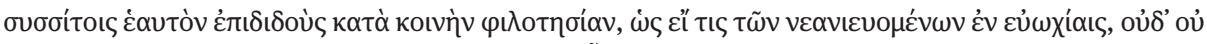

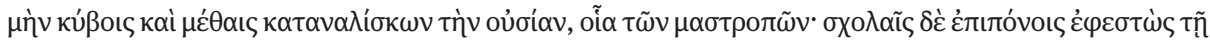

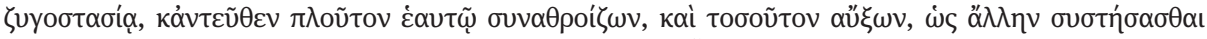

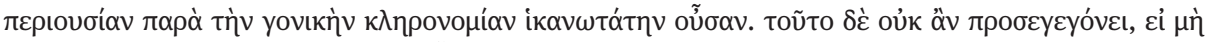

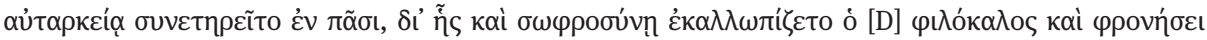

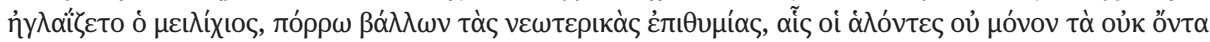

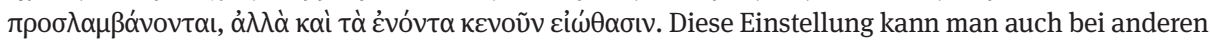
Viten finden. Die verwaisten Kinder bzw. Jugendlichen waren in Gefahr, durch ererbtes Geld und die daraus resultierenden Möglichkeiten zu einem ausschweifenden Leben auf die schiefe Bahn zu geraten, wie uns auch die Vita des Heiligen Markellos Akoimetes aus dem 5. Jahrhundert mitteilt. Markellos Akoimetes wählte allerdings als künftiger Heiliger, obwohl er ein Waisenkind war, einen ganz anderen Weg (ed. G. DAGRon, La vie ancienne de saint Marcel l'Acéméte. AB 86 [1968] 271-321, hier

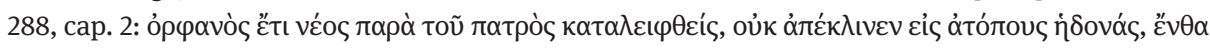

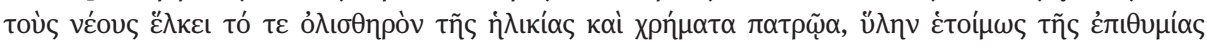

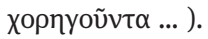

61 Vita des Symeon Salos (BHG 1677) (ed. L. RydÉN, Das Leben des heiligen Narren Symeon von Leontios von Neapolis [Studia Graeca Upsaliensia 4]. Uppsala 1963, 147, 12-14;151, 12; 164, 9; 155, 1). Vgl. dazu die Analyse L. RydÉN, Bemerkungen zum Leben des Heiligen Narren Symeon von Leontios von Neapolis. (Studia Graeca Upsaliensia 6). Uppsala 1970, 94-96. 
daher für die Interpretation des Textes die Intention des Autors berücksichtigt werden muss, die dessen persönliche Einstellung, gesellschaftliche Normen, Werte, soziale Vorbilder und Stereotype widerspiegelt. So führt uns der Autor in den Tagesablauf von Mitgliedern einer Peergruppe ein, die als jugendliche Unruhestifter bezeichnet werden, auf Unterhaltung bedacht waren und in Heißblütigkeit und jugendlichem Übermut nach Weingenuss sich anderen Menschen gegenüber respektlos und gewalttätig verhielten. Sie verbrachten den Tag in einer Kneipe, tranken, belästigten Andreas Salos zum Spaß und schlugen ${ }^{62}$ ihn, dann gingen sie zu Prostituierten und blieben dort bis spät in der Nacht. ${ }^{63}$ Auf dem Weg nach Hause wurden sie festgenommen, geschlagen und der Kleider beraubt, so dass ihnen bewusst wurde, dass sie wegen ihrer erniedrigenden Gewalttätigkeit Andreas gegenüber von Gott bestraft worden waren. In Diskussionen kamen sie zu dem Schluss, dass es nicht richtig war, mit dem Verrückten zu spielen, weil dieser unter Gottes Schutz stehe. ${ }^{64}$

Da körperliche Attraktivität mit der gesteigerten Sexualität ${ }^{65}$ während des Jugendalters eng verbunden war, galten Jugendliche als besonders verführerisch bzw. besonders anfällig für sexuelle Versuchungen. In der Vita des Gregorios Dekapolites

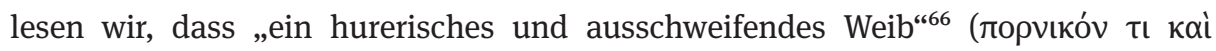

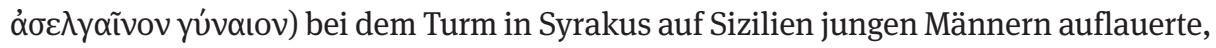
deren leidenschaftliche Triebe entzündete und die von Leidenschaft Entflammten

62 Vita des Andreas Salos (BHG 115z), cap. 5 (ed. L. RYDÉN, The Life of St. Andreas the Fool I: Text, translation and notes, appendices [Studia Byzantina Upsaliensia 4; Acta Universitatis Upsaliensis].

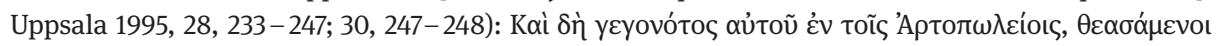

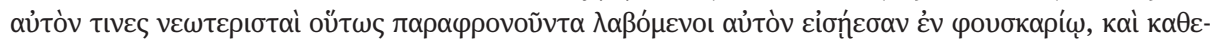

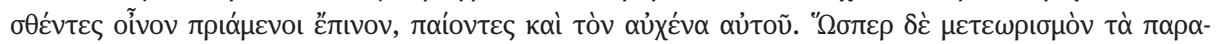

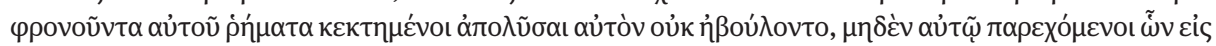

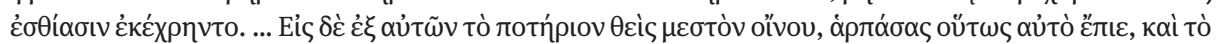

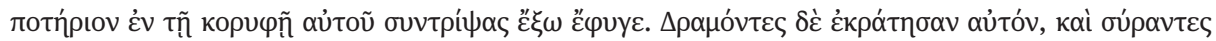

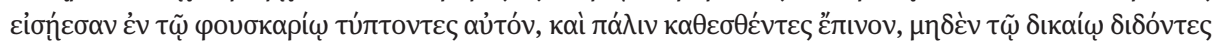

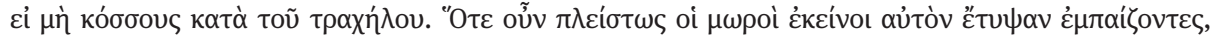

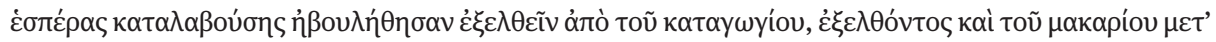

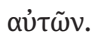

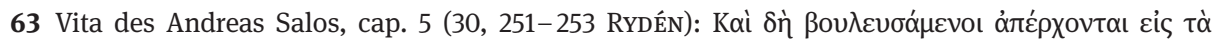

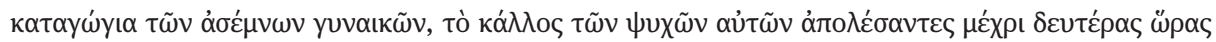

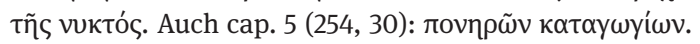

64 Vita des Andreas Salos, cap. 5 (30, 254-271 RYDÉN).

65 FlAMMER - AlSAKER, Entwicklungspsychologie der Adoleszenz 211; G. R. AdAMS - T. P. GulLottA - C. Markstrom-Adams, Adolescent Life Experiences. San Francisco 19943. Sexuelles Verhalten war nicht allein ein angeborener „Trieb“, sondern wurde von gesellschaftlichen Bedingungen entscheidend mitbestimmt In der Psychologie unterscheidet man zwei Hauptperspektiven der jugendlichen Sexualität, eine biologische und eine soziale. Die erste besagt, dass Jugendliche wegen der hormonalen Veränderungen, die in der Pubertät stattfinden, sexuell aktiv werden. Die zweite besagt, dass das Sexualverhalten gelernt und sozialisiert wird, wobei die soziale Kontrolle eine bedeutende Rolle spielt. 66 Zur verschiedenen Bezeichnung der Prostituierten in der frühbyzantinischen Literatur: LEONTSINI, Prostitution, 22-41. 
anlockte, besonders jene, die vom Meer kamen, d.h. Reisende und Seeleute. ${ }^{67}$ In der Vita des Andreas Salos versuchte eine Schauspielerin $(\mu \mu \alpha \dot{\alpha})^{68}$ zwei Jugendliche mit lasziven Bewegungen und dummen Tricks sexuell zu verführen. ${ }^{69}$ Einer der Jugendlichen, Epiphanios, bezeichnete die Frau als schamlosen Fuchs, der die Seele des Jugendlichen (neaniskos) wie einen Vogel erjagen wolle. In den Quellen werden sowohl Prostituierte als auch normale junge Frauen, die junge Männer begehrten und zu gewinnen suchten, als Jägerinnen, Jugendliche als ihre Opfer gleich schwachen Tieren wie etwa, Hirsche und Vögel, dargestellt. Um dies zum Ausdruck zu bringen, benutzen die Autoren Begriffe und Wendungen aus dem semantischen Feld der Jagd, wie Auflauern, Fallen stellen, oder Waffen einsetzen.

Dass Sexualität wegen der hormonalen Veränderungen in der Adoleszenz ihren Höhepunkt erreicht, wird auch in hagiographischen Texten vielfach thematisiert. Dies geschieht vor allem im Rahmen des hagiographischen Topos, dass Jugendliche als künftige Heilige außergewöhnliche Kräfte besaßen, um ihre fleischlichen Gelüste in den Griff zu bekommen. ${ }^{70}$ Manchmal kämpften allerdings auch Heilige als Jugendliche

67 Vita des Gregorios Dekapolites (BHG 711), cap. 28 (ed. G. MAKRIs, Ignatios Diakonos und die Vita des Hl. Gregorios Dekapolites. Mit einer Übersetzung der Vita von M. CHRonz [Byzantinisches Archiv 17].

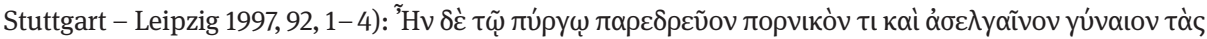

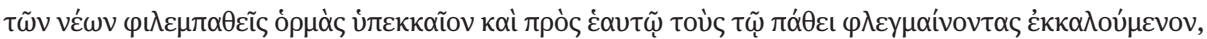

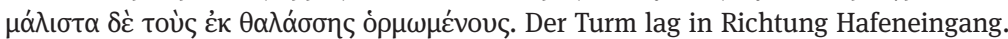

68 Leontsini, Prostitution 28: Die Gleichsetzung von Schauspielerinnen und Prostituierten wird im Falle von $\mu$ нó́ und $\theta v \mu \varepsilon \lambda ı n ́$ deutlich, Begriffe, die in den Texten synonym mit Prostituierten verwendet werden.

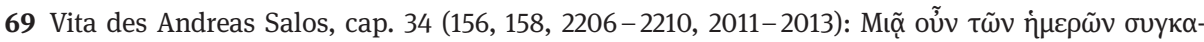

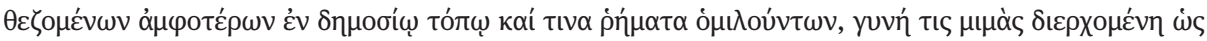

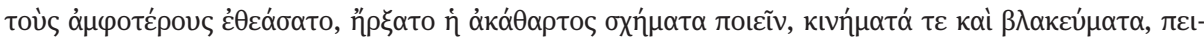

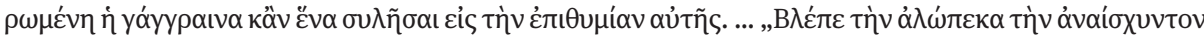

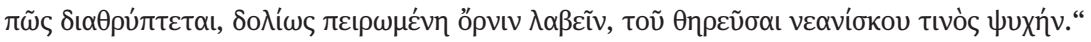

70 Vita des Euthymios (BHG 647) (A. E. Schwartz, Kyrillos von Skythopolis [Texte und Untersuchungen 49]. Leipzig 1939, 3-85, hier 12, 24-25): Als den jugendlichen Euthymios leidenschaftliche Gedanken überkamen, siegte seine Besonnenheit; Vita des Lukas Stylites (BHG 2239), cap. 5(ed. H. DeleHAYE. Les saints stylites [Subsidia Hagiographica 14]. Bruxelles 1923, 195-237, hier 200, 24-28): Lukas Stylites befand sich in der Blüte seiner Jugend, der akme neotetos, als er zum Mönch geschoren wurde, und gestaltete sein Leben dementsprechend, indem er sein Fleisch „mit Eisen nagelte“, und so die schwer zu zügelnden Aufwallungen der Leidenschaften, typisch für diese Altersstufe, bezwang. Zu Lukas Stylites PmbZ II/4, Nr. 24758. Vita des Elias des Jüngeren (BHG 580), cap. 10, 12-13 (ed. G. RossI TAIBBI, Vita di sant'Elia il Giovane. Testo inedito con traduzione italiana [Instituto siciliano di studi byzantini e neoellenici. Testi 7. Vite dei santi siciliani III.]. Palermo 1962, 16-18: Elias der Jüngere wurde als Gefangener von den Arabern nach Afrika gebracht und dort von einem reichen, arabischen Christen gekauft. Dieser liebte Elias' Charakter und so machte er ihn zum Aufseher über seine Güter. Die Frau des Hausherrn versuchte mit allen Mitteln, wie Schminken, Haareschütteln und Hochziehen des Gewandes, den jungen Elias zu verführen. Letzterer kämpfte dagegen an und erlag ihren Verführungen nicht. Deshalb verleumdete sie ihn aus Rache bei ihrem Mann, Elias habe sie vergewaltigen wollen. Vita des Elias Spelaiotes (BHG 581), cap.4 (AASS Sept. III, 848-888, hier 849C): Ein Mönch begegnete dem jugendlichen Elias Spelaiotes und ermutigte ihn, den Weg Gottes einzuschlagen. Als Elias nach Hause kam, war er durch diese Worte erschüttert und überlegte voll Sorge, wie er im jugendlichen Alter 
gegen sexuelle Versuchungen bzw. Phantasien im realen Leben oder in der Vorstellung an. Diese Texte thematisierten die besondere Intensität jugendlicher Passion, die der Heilige nur durch drastische Selbstzüchtigung bewältigen konnte. Es gibt in den Quellen aber auch Fälle von Heiligen, wie Symeon ${ }^{71}$ und Andreas Salos, ${ }^{72}$ die ihre eigenen körperlichen Bedürfnisse bereits abgetötet hatten und daher Prostituierte besuchten, um diese entsprechend der Idee der Nachfolge Christi zu bekehren und von Sündhaftigkeit und Ausschweifung zu retten. ${ }^{73}$

All diese Fälle machen deutlich, dass die „übermenschliche“ Widerstandsfähigkeit junger Heiliger als für normale Jugendliche beinahe unmöglich erachtet wurde. Die Intention der Autoren war, durch die Darstellung menschlicher Schwächen von Heiligen, diese ihren Zuhörern bzw. Lesern zugänglicher zu machen, damit sie diese als nachahmenswerte Vorbilder ihrer eigenen Lebensführung ansehen konnten. Der Heilige erscheint als beispielgebender Antitypus zum Gemeinplatz des kraftvollen, aber unbeherrschten, Leidenschaften und Vergnügungen ausgesetzten Jugendlichen. Hagiographische Texte thematisieren die Widersprüchlichkeit zwischen christlichen Moralvorstellungen und sozialer Wirklichkeit. Während der Heilige als Held und ideales Verhaltensvorbild ${ }^{74}$ fungiert, repräsentiert der normale Mensch menschliche Erfahrungen, soziale Lebensordnungen und gesellschaftliche Normen. Episoden der Kindheit und Adoleszenz stehen in diesem Spannungsfeld und verbinden literarische Darstellungsabsichten mit Elementen der soziokulturellen Realität, indem sie den Übergang von der normalen menschlichen zu heiligmäßigen Ebenen thematisieren. ${ }^{75}$

Texte berichten neben der oben erwähnten christlichen Einstellung der Heiligen zur Sexualität auch von einer anderen Realität, in der Jugendliche auf der Suche nach sexueller Identität sind und an sexuellen Kontakten reges Interesse haben. Byzantinische Autoren betonen besonders die gesteigerte sexuelle Leidenschaft als Verhalten prägendes Charakteristikum der Adoleszenten. Junge Männer waren wegen der gesteigerten sexuellen Gier, der unkontrollierten Triebe und der leichten Verführbarkeit

\footnotetext{
und mit kraftstrotzendem Körper die sexuelle Begierde löschen könne. Vita des Lazaros von Galesion (BHG 979) cap. 7 (AASS Nov. III., 508-588, 511 A-B): Als der Neunzehnjährige Lazaros von Galesion nach Chonai gekommen war, betete er in der Nacht in der Archangel-Kirche und schlief dort ein. Im Traum sah er eine vom bösen Teufel geschickte Frau, die, als Nonne verkleidet, auf ihn zuging und ihn zu schändlichem Geschlechtsverkehr zu verlocken suchte. Lazaros aber stand schnell auf, flüchtete vor ihr wie vor dem Feuer, sagte nichts zu ihr und verschwand. Er bat Gott, ihn vor den Tricks des Teufels zu schützen und ihm den Weg zu den heiligen Orten zu erleichtern. Vita des Andreas Salos, cap. 12 (66, 805 RYDÉN): Epiphanios, der Schüler des Andreas Salos widerstand den fleischlichen Genüssen.

71 Vita des Symeon Salos (154, 27-28-155, 1-18 RYDÉN).

72 Vita des Andreas Salos, cap. 6 (33, 298-305, 34, 305-335, 36, 337-340 RYDÉN).

73 Leontsini, Prostitution 102: Zahlreiche Beispiele sind in der Hagiographie nicht nur von Heiligen, sondern auch von Mönchen und Asketen zu finden, die Prostituierte aus demselben Grund besuchten. 74 Kiousopoulou, Xpóvos 17.
}

75 ARIANTzI, Adoleszenz in Byzanz 9-10. 
die bevorzugte Zielgruppe ${ }^{76}$ der Prostituierten. ${ }^{77}$ Die Orte ihrer Tätigkeit befanden sich in Bordellen ${ }^{78}$ und auf Straßen von Groß- und Hafenstädten sowie in Pilgerzentren, wo die Nachfrage groß war. Vor allem in der Hauptstadt Konstantinopel als Zentrum von Verwaltung, Handel, Gewerbe und Verkehr mit hoher Bevölkerungsfluktuation hatten Prostituierte besondere Vorteile. ${ }^{79}$ Neben dem Bordell galt auch das Theater ${ }^{80}$ als Ort mit „schlechtem Ruf“81. Die Welt des Theaters wurde mit Kupplern, Mimen, ${ }^{82}$ Prostituierten ${ }^{83}$ und Päderasten in Verbindung gebracht. ${ }^{84}$ In den hagiographischen Quellen findet man zahlreiche Fälle, in denen das Theater ein bei der Jugend beliebter Ort war, den der Heilige allerdings beharrlich mied..$^{85}$

In dieser zweiten Kategorie paaren sich gängige Stereotype von jugendlicher Zügellosigkeit mit der Darstellungsabsicht hagiographischer Texte, idealtypische Verhaltensnormen heiligmäßiger Männer durch die Kontrastierung bzw. durch die Betonung des Konflikts mit dem jeweiligen Gegentypus herauszustellen. Andreas Salos stellt insofern eine Extremform dieses Denkschemas dar, als sich seine Heiligkeit nicht nur im Gegensatz zu den „normalen“ Jugendlichen, sondern in der Verhöhnung durch sie konstituiert. Da diese Gruppen nur im Kontrast zum Heiligen in Erscheinung treten, stellt sich hier ganz besonders die Frage, ob sie als rein literarische Fiktion oder als literarisches Abbild von sonst kaum fassbaren Gruppierungen ungebändigter Jugendlicher zu verstehen sind. Immerhin lässt sich so viel sagen, dass der Aspekt der Sozialisierung über die gemeinsame Tätigkeit im Gruppenverband auch hier deutlich in Erscheinung tritt. Eine genauere Erfassung der sozialen Komponenten und Merkmale dieser Gruppierungen ist aus dem dritten Typus von Peers möglich.

76 LEONTSINI, Prostitution 99.

77 Da der voreheliche Geschlechtsverkehr mit anständigen jungen Mädchen nicht als angebracht galt.

78 Bezeichnungen für Bordelle in der frühbyzantinischen Literatur Leontsini, Prostitution 54-62. 79 LeONTSini, Prostitution 63-64.

$80 \mathrm{Vgl}$. Jean Chrysostome. Sur la vaine gloire l'education des enfants. Introduction, Texte critique, traduction et notes, hrsg. A.-M. MALIngRE (SC 18). Paris 1972, §56, $733-734$ und § 77, 923-925: Der Kirchenvater nahm deutlich Stellung gegen das „Theater“, weil dort unmoralische Dinge passierten, und empfahl den Eltern, den Kindern nicht zu erlauben, dorthin zu gehen, damit sie durch die Dinge, die dort zu sehen oder zu hören waren, keinen Schaden erleiden.

81 Zur Bedeutung des Theaters in Byzanz siehe Anm. 59.

82 Prokopios, Anekdota. Geheimgeschichte des Kaiserhofs von Byzanz. Übersetzt und herausgegeben von Otto Veh. Mit Erläuterungen, einer Einführung und Literaturhinweisen von M. MAIER - H. LEPPIN. Düsseldorf - Zürich 2005, Kapitel 9, 84-90 über Theodoras Herkunft und Vorleben. Vgl. LEONTSINI, Prostitution 126: Aus den frühbyzantinischen Quellen sind uns einige Miminnen bekannt, die zugleich als Prostituierte arbeiteten, darunter die spätere Kaiserin Theodora.

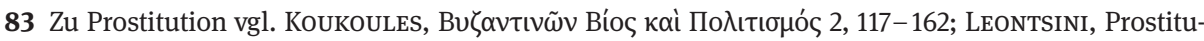
tion.

84 F. Tinnefeld, Mimos 327; A. Vogt, Le Théatre à Byzance dans l'Empire du IVe au XIIIe siècle. Rev. Quest. Hist. 59 (1931) 286.

85 Vita des Nikolaos von Myra, Vita per Michaelem (BHG 1348), cap. 8 (ed. G. ANRICH, Hagios Nikolaos. Der Heilige Nikolaos in der griechischen Kirche, Bd 1. Texte und Untersuchungen. Berlin 1913, 113-139, hier 117. Weitere Beispiele, ARIANTZI, Kindheit 161-162. 
Diesen bilden Jugendbanden vor allem städtischer Herkunft, die durch belästigendes bzw. gewalttätiges und kriminelles Verhalten gekennzeichnet sind. ${ }^{86}$ Aus den Quellen ergibt sich dabei eine deutliche Differenzierung. Einerseits gibt es Jugendbanden, die autonom und spontan aktiv und offenbar aus geistiger Unreife zum Spaß gewalttätig sind. Andrerseits erzählen die Texte über die Existenz von Jugendbanden, die quasi professionell im Auftrag von mächtigen Menschen handeln.

So erfahren wir aus der Salos-Vita über das gewalttätige Vorgehen zweier Jugendbanden, die im Konstantinopel des 10. Jahrhunderts unter dem Namen „Jugendliche der Stadt“ und „Jugendliche des Erzbischofs“ bekannt waren. Der Text erweckt den Eindruck, dass solche Jugendbanden durch ihre Aktivitäten den Bewohnern der Stadt bekannt waren. „Jugendliche der Stadt“ haben Andreas geschlagen und heftig geohrfeigt. Sie wickelten ein Seil um seinen Nacken, zogen ihn vor den Augen aller daran und beschmierten sein Gesicht mit schwarzem Ruß. ${ }^{87}$ Die „Jugendlichen des Erzbischofs“ benahmen sich wie skrupellose Räuber, überfielen Andreas nachts, zogen ihm seine Kleider aus, ließen ihn nackt zurück und liefen weg. ${ }^{88}$ Die genauen Hintergründe dieser Episode sind schwer zu erhellen. Die genannten Jugendlichen stammten aus armen Verhältnissen. Man könnte sich vorstellen, dass sie beim Erzbischof um Schutz und Unterstützung als Gegenleistung für ihre Dienste nachkamen.

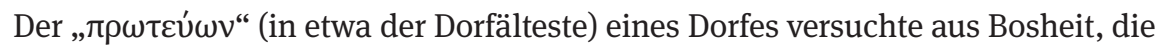
der Autor auf den Einfluss des Teufels zurückführt, den Ruf zu verbreiten, dass die Besuche einer verwitweten Frau namens Eirene, die Nonne geworden war, bei dem geistlichen Vater Lazaros von Galesion mit einer sexuellen Beziehung verbunden seien. ${ }^{89}$ Da er einsehen musste, dass die Dorfbewohner das nicht für glaubwürdig erachteten, beauftragte er einige Jugendliche (neous), die Frau und ihre beiden Begleiterinnen zu entführen und zu vergewaltigen. Das gelang ihnen nicht, da sie laut der Erzählung über Steine stürzten und in Ohnmacht fielen, und so kamen die Frauen

86 Über das gewalttätige Verhalten als verbreitetes Phänomen in den spätantiken Städten: T. E., GREGoRY, Urban Violence in Late Antiquity, in: Aspects of Graeco-Roman Urbanism. Essays on the classical city (BAR 188), hrsg. R.T. Marchese. Oxford 1983, 138-161. TH. SizGoRICH, Violence and Belief in Late Antiquity: Militant Devotion in Christianity and Islam, University of Pennsylvania Press 2009. Vgl. J.-U. KRAUSE, Kriminalgeschichte der Antike. München 2004, Kap. 8.

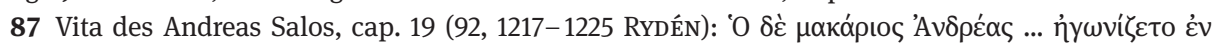

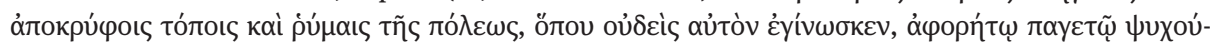

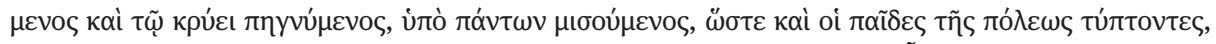

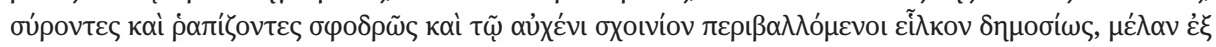

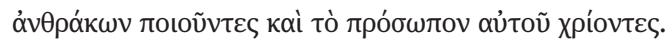

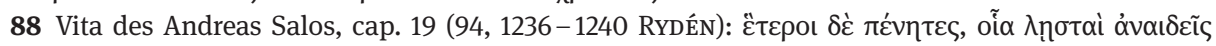

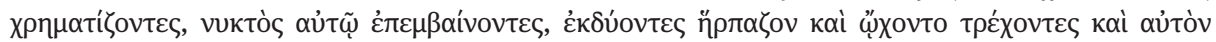

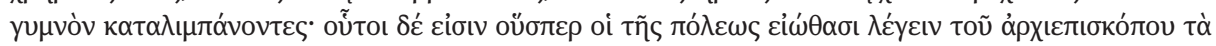
$\pi \alpha \iota \delta i \alpha$.

89 Vita des Lazaros von Galesion (BHG 979) cap. 52 (AASS Nov. III., 508-588, hier 527B-C). Eirene wollte den Kontakt mit ihm beibehalten, besuchte ihn oft und half ihm. 
unbehelligt an ihr Ziel. ${ }^{90}$ Die Bewohner der Stadt Kyzikos erhoben Anklage gegen den dortigen schlechten Bischof Eusebios und baten den Kaiser, ihn zu bestrafen. Als sie aber bemerkten, dass Eusebios der Strafe entkam und sehr mächtig wurde, verschworen sich Jugendliche und töteten ihn auf der Agora der Stadt. ${ }^{91}$

Das gewalttätige Verhalten von Jugendlichen wird auch von anderen Quellen bestätigt. Bei Prokopios ${ }^{92}$ und im Chronikon Paschale ${ }^{93}$ wird überliefert, dass Jugendliche als Mitglieder von Zirkusparteien in Konstantinopel am Nika-Aufstand teilnahmen, mit Gewalt auf den Straßen der Stadt gegen Justinians Politik protestierten und sogar in den Palast mit zerstörerischer Absicht eindrangen ${ }^{94}$. Im 8. Jahrhundert erscheinen Jugendliche in den anti-ikonoklastischen Texten als willige Werkzeuge für die Umsetzung der bilderfeindlichen Politik der isaurischen Dynastie. So lesen wir in der Vita des Theodoros Studites von einer Gruppe von Jugendlichen,

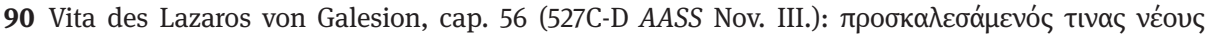

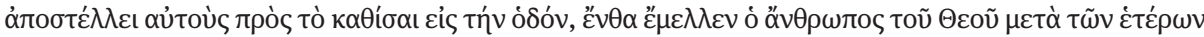

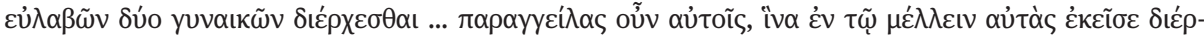

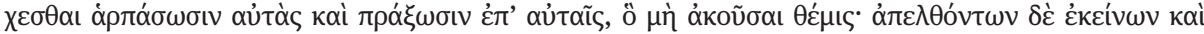

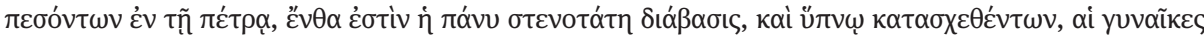

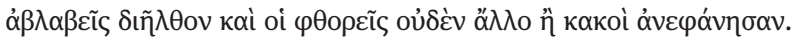

91 Prokopios, De Bellis (eds. G. WIRTH - J. HAURY, Procopii Caesariensis opera omnia. Vol.1-2. Leipzig

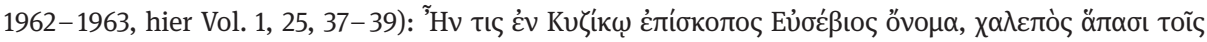

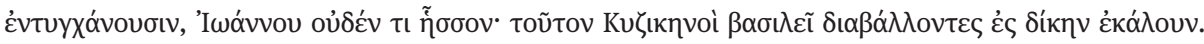

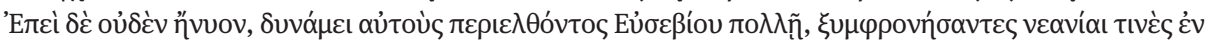

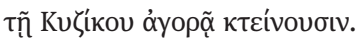

92 Procopios, Anekdota 7 (eds. G. WiRTH - J. HAURY, Procopii Caesariensis opera omnia. Vol. 3. Leipzig

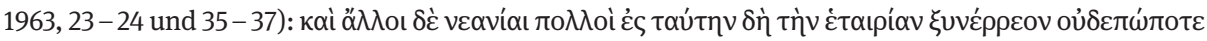

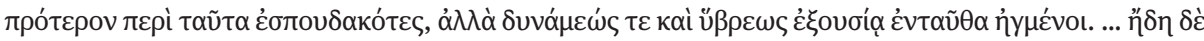

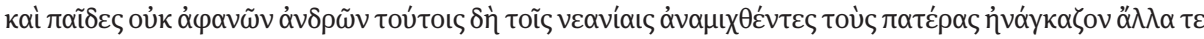

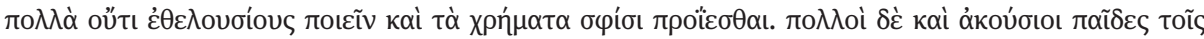

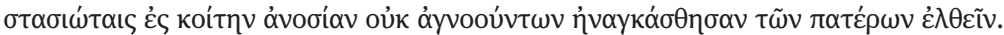

93 Chronikon Paschale (ed. L. Dindorf, Chronicon Paschale. Vol. 1 [CSHB] Bonn 1832, 625, 12-19):

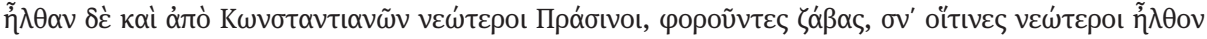

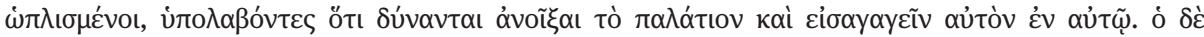

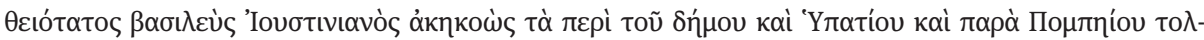

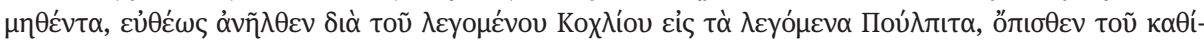

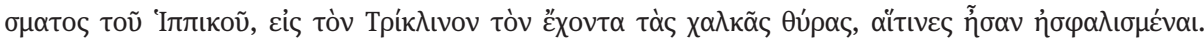
94 Zum Nika - Aufstand und speziell über die Teilnahme von Jugendlichen siehe A. CAMERon, Circus Factions. Blues and Greens at Rome and Byzantium, 1976, 75, 77, 99, 342. Es handelt sich in erster Linie um Jugendliche aus aristokratischen Familien, die als Mitglieder der Zirkusparteien aufgenommen wurden, siehe dazu M. JaczYNowsKa, Les Organisations de Iuvenes et l'aristocratie municipal, in: Recherches sur les structures sociales dans l'antiquité classique. Paris 1970, 265-274. Vgl. dazu die Organisationen junger Männer (Futuwwa: sportliche und soziale Organisationen) im Islam (im mittelalterlichen Syrien und Iran). Siehe dazu: SP. VRYonIs, Byzantine Circus Factions and Islamic Futuwwa Organizations (Nعqvíaı, Fityān, AḤDATH). BZ 58/1 (1965) 46-59. Vgl. M. BonNER, Aristocratic Violence and Holy War: Studies in Jihad and the Arab-Byzantine Frontier. New Haven 1996. B. JoKIsch, Islamic Imperial Law. Berlin 2008, 338-347, 453f, 457 f. M. ZAKERI, Sasanid Soldiers in Early Muslim Society: The Origins of Ayyarun and Futuwwa. Wiesbaden 1995. 
die als träge, zügellos, zur Unmoral verleitend und an der äußeren Erscheinung interessiert dargestellt wird und im Dienst Kaiser Leons III. (717-741) stand..$^{95}$ In der Vita des Stephanos des Jüngeren waren Jugendliche (neoteroi) zusammen mit anderen Altersgruppen an der Steinigung des Stephanos im Theater des Hippodroms beteiligt. ${ }^{96}$

In diesen Beispielen haben wir es mit Adoleszenten im Rahmen konkreter politischer Handlungen und sozialer Netzwerke zu tun. Jugendliche agieren in Verbänden, die im Kontext städtischer Einrichtungen und Gesellschaftsstrukturen bestanden und in mehr oder weniger deutlich erkennbarer Verbindung zu den Trägern und Repräsentanten politischer Macht im kirchlichen und weltlichen Bereich standen. Je nach den beschriebenen Umständen und der Aussageabsicht der Texte, die in allen genannten Beispielen stark normativ und vor allem im Falle der anti-ikonoklastischen Beispiele auch propagandistisch ist, erscheinen die Jugendgruppen als Unruhestifter und Schlägertrupps, als eine Art inoffizielle Ordnungsmacht, als Handlanger politisch motivierter Zielsetzungen oder als Schützlinge und Instrumentarien einer übergeordneten städtischen Autorität. Die Texte sagen kaum etwas über die innere Organisation dieser Gruppen. Im Vordergrund steht deren Wirkung nach außen. In allen Fällen wird jedenfalls deutlich, dass es sich um größere Verbände handelte, die im urbanen Sozialgefüge durch bestimmte aktions- und gewaltorientierte Verhaltensweisen bemerkbar waren und somit den Jugendlichen einen Rahmen der öffentlichpolitischen Betätigung und eine Art soziales Netzwerk boten.

Während die Autoren hagiographischer Texte normale Adoleszenten als gesellig, besonders sportlich, wild lebend, frech und manchmal respektlos und kriminell präsentieren, wird das Verhalten der Heiligen als Jugendliche als Gegentypus zum Normalfall dargestellt. Ziel der Autoren war es, die Zuhörer bzw. Leser zu unterhalten, $\mathrm{zu}$ belehren und moralische, religiöse und kirchenpolitische Werte $\mathrm{zu}$ propagieren. Um bei der Zuhörer- bzw. Leserschaft eine möglichst große Wirkung zu erzielen, betont der Verfasser bestimmte schicht-, geschlechts-, aber auch altersspezifische Haltungen, religiöse Einstellungen und die mit den Vorstellungen von Heiligkeit verbundenen Erwartungen. ${ }^{97}$ Die Autoren unterscheiden zwischen „guten“ und

95 Vita des Theodoros Studites (BHG 1755d) (ed. B. LATYŠEv, Vita S. Theodori Studitae in codice

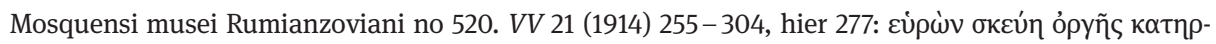

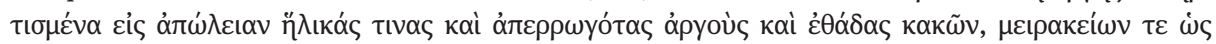

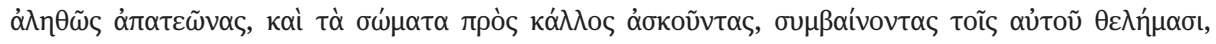

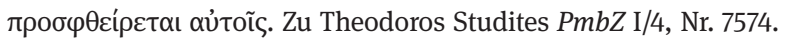

96 Vita des Stephanos des Jüngeren (BHG 1666) cap. 40 (ed. M.-Fr. AuZÉPY, La vie d'Étienne le Jeune par Étienne le diacre. Introduction, edition et traduction [Birmingham Byzantine and Ottoman Monographs 3]. Aldershot 1997, 139, 25, 140, 1-2). Zu Stephanos dem Jüngeren PmbZ I/4, Nr. 7012.

97 The Byzantine Saint. University of Birmingham Fourtheenth Spring Symposium of Byzantine Studies, hrsg. S. Hackel. Birmingham 1981; H. MAGUiRE, The Icons of their Bodies: Saints and their Images in Byzantium. Princeton 1996; Efthymiadis, Hagiographer, 59-80; B. ChevallieR-CaSEAU, Childhood in Byzantine Saints' Lives, in: Becoming Byzantine: Children and Childhood in Byzantium, 
„schlechten“ Adoleszenten. ${ }^{98}$ Die schlechten sind die normalen Jugendlichen, die noch unreif und auf der Suche nach sexueller, sozialer bzw. politischer Identität waren. Die guten sind die künftigen Heiligen, die sich von Vergnügungen fernhielten und auf Besonnenheit bedacht waren. Trotz ihrer Jugend waren sie geistig in der Lage, Richtlinien für ihr Leben zu erkennen, d.h. das Nützliche vom Nutzlosen und das Vollkommene vom Wertlosen zu unterscheiden. Sie genossen die Ruhe und die Lektüre von Büchern. Kontakte pflegten sie nur mit denjenigen Altersgenossen, welche dieselbe Einstellung teilten. Sie besuchten Kirchen und heilige Stätten statt Bordelle und Kneipen. In der Hagiographie sind unzählige Fälle zu finden, so etwa Kyrillos von Philea, ${ }^{99}$ Niketas von Medikion, ${ }^{100}$ Nikon ho Metanoeite, ${ }^{101}$ Euarestos, ${ }^{102}$ Platon von Sakkudion, ${ }^{103}$ oder Johannes, Bischof von Polyboton. ${ }^{104}$

ed. A. Papaconstantinou - A.-M. Talbot (Dumbarton Oaks Byzantine Symposia and Colloquia). Washington D.C. 2009, 127-166, hier 128-129.

98 Vgl. dazu N. Kalogeras, What do they think about Children? Perceptions of Childhood in Early Byzantine Literature. BMGS 25 (2001) 2-19.

99 Kyrillos von Philea (BHG 468) cap. 2.2 (ed. E. SARgologos, La vie de Saint Cyrille de Philéote,

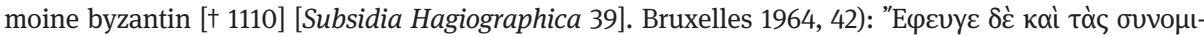

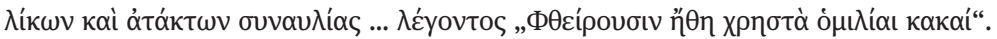

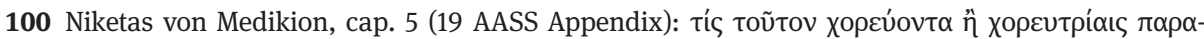

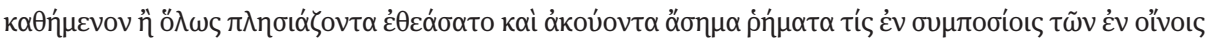

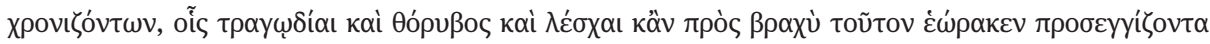

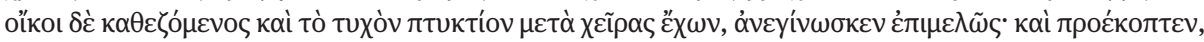

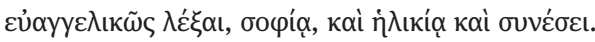

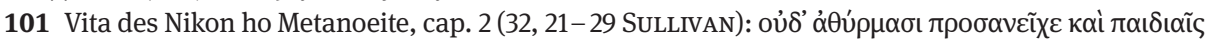

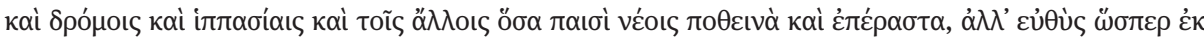

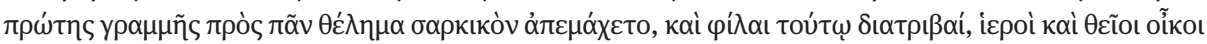

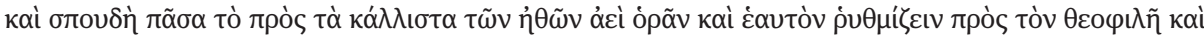

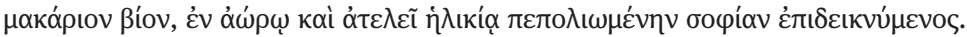

102 Vita des Euarestos (BHG 2153), cap. 4 (ed. CH. vAN DE VoRST. La vie de S. Évariste, higoumène à

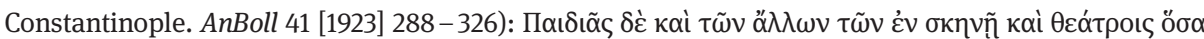

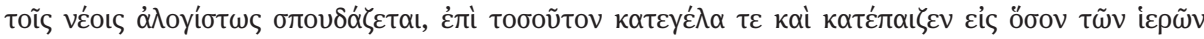

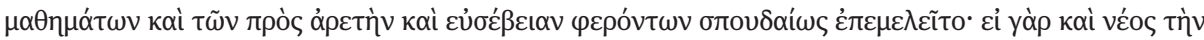

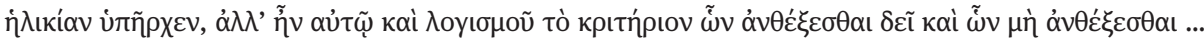

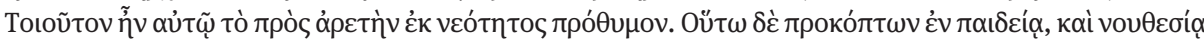

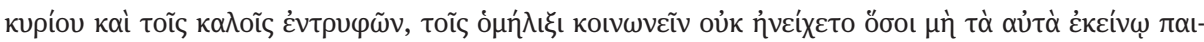

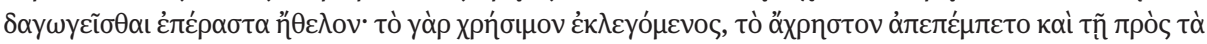

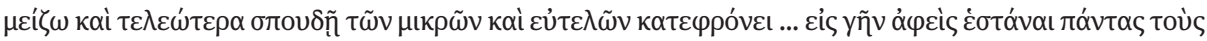

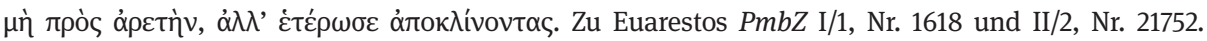

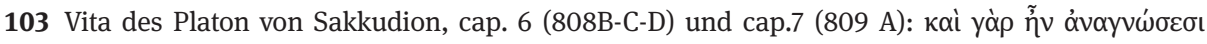

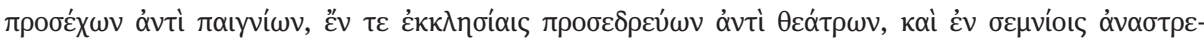

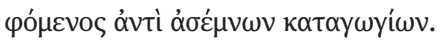

104 Vita des Johannes, Bischof von Polyboton, cap. 3 (H. Delehaye, Synaxarium ecclesiae Constantinopolitanae. Propylaeum ad Acta sanctorum Novembris. Bruxelles 1902, 277-280, hier, 277,

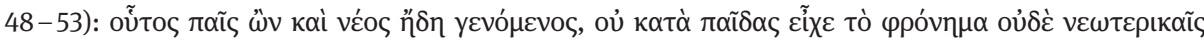

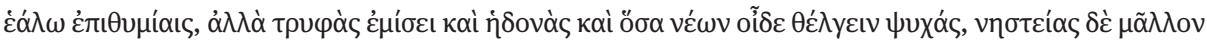

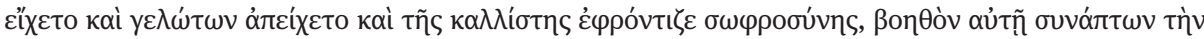


Fassen wir also zusammen: Was erfahren wir von jugendlichen Peergruppen in Byzanz? In den Quellen findet sich kein Terminus für die Gruppe der Peers. Um sie zu bezeichnen, verwenden die Autoren die für die Adoleszenten üblichen Begriffe ${ }^{105}$ im Plural. Für Peergruppen charakteristisch war, dass sie aus mehreren Mitgliedern aus städtischem Milieu bestanden, die laut den Quellen gemeinsam Sport betrieben, sich bei Unterhaltungen vergnügten, kriminelle Aktivitäten setzten und dabei eine Gruppenidentität aufbauten. Adoleszenten waren beweglicher als Kinder und bekamen ohne Einbeziehung der Eltern Kontakte mit Gleichaltrigen, die nicht nur in ihrer unmittelbaren Nachbarschaft wohnten. Sie trafen sich von den Erziehungsberechtigten unkontrolliert an verschiedenen öffentlichen Orten.

Die Zugehörigkeit zu diesen Gruppierungen war für die Identität des einzelnen Jugendlichen wichtig. Mit der Integration in diese war die Übernahme von darin üblichen Verhaltensformen verbunden. Peers dienten zu harmonischer Sozialisierung in der Gesellschaft und schrittweise emotionalen Ablösung von den Eltern und zunehmender Erlangung der Unabhängigkeit. Denn das Individuum versuchte außerhalb der Familie einen Platz in einer Clique zu finden und von den anderen Gleichaltrigen wegen seines Charakters bzw. seiner Interessen und nicht seiner Familienzugehörigkeit anerkannt bzw. respektiert zu werden. ${ }^{106}$ Der besondere Reiz der Gleichaltrigengruppen lag in ihrer symmetrischen Konstitution, die ganz anders angelegt war als die hierarchischen, durch Generationsunterschiede gekennzeichneten Beziehungen innerhalb der Familie. ${ }^{107}$

Die Mehrheit der Aussagen sowohl hagiographischer als auch historiographischer Quellen betrifft jugendliche Peers aus städtischen Zentren, vor allem aus der Hauptstadt Konstantinopel und aus wohlhabenden Familien, auch wenn nicht immer das soziale Milieu der Peers zu erkennen ist. Dies hat zweifellos mit den verschiedenen sozioökonomischen Strukturen zwischen Stadt und Land bzw. Dorf zu tun. In einer Stadt und besonders in Konstantinopel als Zentrum jedes sozialen und politischen Ereignisses konnten Jugendliche aktiv werden. Zusätzlich bot ihre Infrastruktur ihnen mehr Unterhaltungsmöglichkeiten an. Auch die familiäre Situation und finanzielle Lage war ausschlaggebend. Denn Adoleszenten aus bäuerlichen bzw. ärmeren Familien mussten schon vom Kindesalter an in der Landwirtschaft, als Hirten oder im Betrieb des Vaters arbeiten oder eine berufliche Ausbildung durchmachen. So waren sie als Arbeitskraft unersetzlich und leisteten einen wichtigen Beitrag zum Familieneinkommen. Sie hatten daher weder Zeit noch Geld, an teuren Unterhaltungen und Vergnügungen teilzunehmen. Die Quellen zeigten deutlich, dass Jugendliche aus ländlichen Verhältnissen früher als Jugendliche aus städtischen Milieus in die Er-

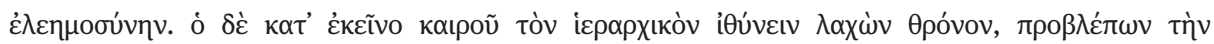

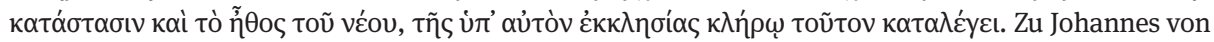
Polyboton PmbZ I/2, Nr. 2753.

105 Zur Terminologie der Adoleszenz und Adoleszenten: ARIANTZI, Adoleszenz in Byzanz 30-31.

106 Flammer - AlsaKer, Entwicklungspsychologie der Adoleszenz 195-196.

107 HurRelmanN, Jugendforschung, Kap. 3.3.
} 
wachsenenwelt kamen und Verantwortungen übernahmen. Sie erfüllten schon früh zunehmend Aufgaben der Erwachsenen und leisteten teilweise durch ihre Arbeit einen wichtigen Beitrag zum ökonomischen System des byzantinischen Reiches.

Évelyne Patlagean zeigte, dass Jugendliche in der frühbyzantinischen Zeit wegen der soziopolitischen Umstände und militärischen Aktionen als Verteidiger ihrer Heimatstädte gegen Perser und Araber aktiv wurden. ${ }^{108}$ Auch aus der Untersuchung der Literatur der mittelbyzantinischen Zeit ergibt sich, dass jeweilige sozio- und kirchenpolitische Ereignisse wie der Nika-Aufstand und Unruhen im Ikonoklasmus Jugendliche in Bewegung setzten. Jugendliche Peers in Byzanz bildeten eine separate soziale Gruppe im Rahmen der Gesellschaft mit entsprechenden physiologischen und intellektuellen Charakteristika und übernahmen eine soziopolitische Rolle. Darüber hinaus treten in den oben präsentierten Quellen Formen der Gruppenidentität und ein auf schichtspezifische Wertsysteme ausgerichtetes, Gemeinschaft begründendes Zusammengehörigkeitsgefühl zutage. Durch gemeinsame sportliche Aktivitäten, Vergnügungen und innerhalb der Gruppe akzeptierte Normvorstellungen konnten sich Peergruppen verfestigen und erreichten einen in den Quellen deutlich reflektierten Grad an Eigenständigkeit gegenüber dem sozialen Gesamtgefüge. Der Heilige blieb ihnen gegenüber meist isoliert und erscheint oft als deren moralischer Gegenpol. Es handelte sich also um Gruppen, die sich in ihrem Verhalten deutlich von kirchlichen und monastischen Idealvorstellungen abgrenzten.

108 E. Patlagean, Les ,jeunes“ dans les villes byzantines: émeutiers et miliciens, in: Le charivari,

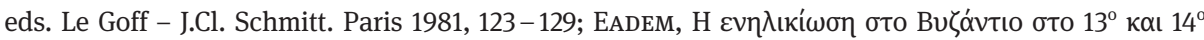

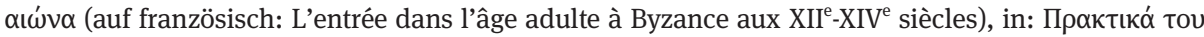

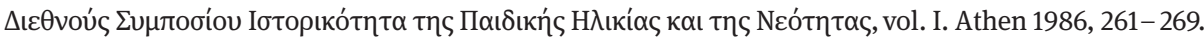




\section{Images of Byzantine Adolescents}

Byzantine teen-agers are virtually invisible. Their bones are noted - though rarely discussed - in archaeological excavations of burial grounds, but they are hard to track in the written sources, ${ }^{1}$ and they have been ignored in discussions of Byzantine imagery: unlike women and children, no one has ever even looked for the portrayal of Byzantine adolescents in the visual evidence. ${ }^{2}$ Portraits of Byzantine teen-agers are, however, important to find and to evaluate, not just because no one has ever done this, but because they illuminate an area of Byzantine social history that has been neglected and is in itself significant. How Byzantine authors represented the transition and transformation of the Byzantine male or female across his or her lifetime is beginning to be studied, but how that transition and transformation was represented visually is even more important to understand, because images provide a data set produced by a different community than the mostly urban and mostly elite males who provide the bulk of textual evidence preserved from the Byzantine empire, and for a much broader constituency. We mostly hear the voices of a small and select group of Byzantines; images, in contrast, allow us to see with everyone in Byzantium who had not lost their sight. While we have not learned (and can never learn) to see in the same way as a Byzantine did, looking at portrayals of adolescents nonetheless adds valuable new layers of information, from different social and cultural perspectives, to the evidence about the Byzantine life course - how people, from ordinary peasants to the imperial family, experienced growing up - provided by words.

The Byzantine life course itself has only recently received scholarly attention. In a PhD thesis awarded in 2012, Eve Davies looked at how the Byzantines described and valued infancy, youth, maturity and old age; and she compared and contrasted

I am deeply grateful to Despoina Ariantzi for inviting me to speak at the conference that generated
this volume, and to the participants for stimulating discussion. I am also, as always, grateful to Chris
Wickham, who read and commented on an earlier draft of this chapter. Please note that I have used
Hellenised spellings for Greek names except when - as is the case for, e.g., Constantine, Constans
and Justinian - their anglicised forms are in virtually universal use in the English-speaking world.

1 But see the recent publications D. ARIANTZI, Terminologische und sozialhistorische Untersuchungen zur Adoleszenz in Byzanz (6.-11. Jahrhundert). Teil I. Theorien, Konzepte, narrative Quellen. JÖB 63 (2013) 1-31; and E. DAviEs, Age, gender and status: a three-dimensional life course perspective of the Byzantine family, in: Approaches to the Byzantine family, eds. L. Brubaker and S. Tougher. Farnham - Burlington 2013, 153-76, for a start. The archaeology is evaluated in DAVIES' PhD thesis, referenced in note 3 below.

2 For images of women, see M. MEYER, An obscure portrait. Imaging women's reality in Byzantine art. London 2009; for images of children, see C. HenNEssy, Images of children in Byzantium. Farnham-Aldershot 2008.

Ә OpenAccess. (c) 2018 Leslie Brubaker, published by De Gruyter. (c) BY-NC-ND This work is licensed under the Creative Commons Attribution-NonCommercial-NoDerivatives 4.0 License. 
the ways that male and female experiences of childhood, adolescence, adulthood and old age were discussed. ${ }^{3}$ Her focus was on the archaeological and textual evidence for family roles across the life courses of both men and women from late antiquity until the thirteenth century.

Davies' key findings about Byzantine adolescents may be summarised as follows. ${ }^{4}$ Byzantine authors saw adolescence somewhat differently than we do, though then as now the potential emotional and physical eruptions of male adolescents were frequently noted, decried, and (often) later forgiven as youthful indiscretions. The idea of the 'teenager', however, is not found; the Byzantines seem to have viewed 'youth' as beginning roughly around the age of 12 or 13 (for girls) or 14 or 15 (for boys) and ending, at least for males, around the age of 25. As this indicates, the ways that Byzantine authors discussed 'youth' were gendered. Normally, they described female youth in terms of beauty, with marriage the signal marker of transition from youth to maturity. Male youth, in contrast, was described in terms of activity, physical strength, and socialisation. When the age of adolescents is specifically recorded (which is not common), there are no years that are especially prominent for males; for females, however, virtually the only age mentioned is 12 , which was apparently the symbolic turning point for female sexual awakening: Mary of Egypt, for example, was 12 when she rejected her family to run riot in Alexandria. ${ }^{5}$ In short, a series of distinctive textual conventions cluster around descriptions of both male and female adolescence.

Davies was not able to look at how the visual evidence from Byzantium intersects with the written and archaeological record, and, as it happens, the distinctive nature of adolescence noted in Byzantine written sources is only partially seen in Byzantine visual sources. How and why this is the case, and what this tells us about the significance of the Byzantine experience of, and social response to, adolescence, are the subjects of this chapter.

The first thing to be said is that the Byzantine painters could and did clearly distinguish between various stages of the life course trajectory, sometimes quite intentionally. The scene of Gregory's burial in the copy of the Homilies of Gregory of Nazianzos in Paris ( $c a$ 880), for example, surrounds the dead Gregory with figures representing the three ages of, in this case, man: a beardless youth lowers the saint's feet into the sarcophagus; a bearded middle-age man lowers his head; and an elderly priest, with grey hair and beard, presides (fig. 1). ${ }^{6}$ Though they are normally less schematically

3 E. DAviEs, 'From womb to the tomb: the Byzantine life course AD 518-1204', PhD thesis. University of Birmingham 2012. Some of her conclusions have appeared in the article cited in note 1.

4 DAvies, 'Womb to the tomb' 91-131.

5 M. Kouli (transl.), Life of St. Mary of Egypt, in: Holy women of Byzantium: ten saints' lives in English translation, ed. A.-M. Talbot. Washington DC 1996, 70 -93, here 80.

6 Paris, BNF, gr. 452r. For discussion of this image, see L. BRUBAKER, Vision and meaning in ninthcentury Byzantium: Image as exegesis in the Homilies of Gregory of Nazianzus in Paris. Cambridge 
displayed, almost all other facets of the Byzantine life course also find visual expression. Infants appear in representations of the Nativity, as well as in other examples such as the votive images at Hagios Demetrios; ${ }^{7}$ small children are shown in many representations of the Crossing of the Red Sea, the toddler Benjamin is depicted in some pictorial sequences of Joseph's life, and a young boy (perhaps intended to represent the brother of Jesus) also occurs in the Kokkinobaphos manuscripts. ${ }^{8}$ Young men - for example Joseph before his sale to Potiphar - and young women such as Jairos's daughter appear regularly, ${ }^{9}$ though less frequently than mature adults of both sexes, who are visualised with specific attributes: the males bearded to signify maturity and the females veiled to signal matronly modesty ${ }^{10}$. Old men are distinguished by their grey hair and beards; only old women do not appear in the Byzantine visual record. ${ }^{11}$

But although painters could clearly distinguish between the different stages of the life course, they did not always do so: in a ninth-century miniature illustrating the life of St Basil (fig. 2), for example, the child St Basil hiding in a cave with his parents is shown as an adult, though elsewhere in this manuscript the painter portrays children, and elsewhere on the same page the miniaturist has been happy to portray Basil as a youth, an adult, and as an old man. ${ }^{12}$ There are two key points to take from this example. First, what we might see as an issue of verisimilitude this is the image selected to represent Basil's childhood and family, so he should

1999, 136 - 37, fig. 46; on the 'three age system' more generally, see E. SEARs, The Ages of Man. Medieval Interpretations of the Life Cycle. Princeton NJ 1986.

7 Images of the Nativity, which invariably show Christ as a baby, often once dressed in swaddling clothes and once naked, in the bath, are ubiquitous; for Hagios Demetrios, see, e.g., C. HENNEssy, Iconic images of children in the church of St Demetrios, Thessaloniki, in: Icon \& word. The power of images in Byzantium, eds. A. Eastmond - L. James. Aldershot 2003, 156-72, though I disagree with some of her conclusions (L. BRUBAKER, Elites and patronage in early Byzantium: the evidence from Hagios Demetrios at Thessalonike, in: Elites old and new in the Byzantine and early Islamic Near East [The Byzantine and early Islamic Near East 6], eds. J. Haldon - L. Conrad. Princeton NJ 2004, 63-90).

8 For discussion, see Meyer, An obscure portrait, 102-5. For the Kokkinobaphos child see C. HeNNESSY, The stepmum and the servant: the stepson and the sacred vessel, in: Wonderful things: Byzantium through its art, eds. A. Eastmond - L. James. Farnham 2013, 79-98.

9 Both found, for example, in the Paris Gregory (Paris.gr.510, ff.69v, 143r): BRUBAKER, Vision and meaning, figs 12, 19.

10 For beards, see I. KALAvrezou-MaXeIner, The Portraits of Basil I in Paris gr. 510. JÖB 27 (1978) 19-24; for veiling, see MEYER, An obscure portrait, 120: 'According to Byzantine protocol, older or married women are depicted with head coverings and maidens are not'.

11 See, e. g., representations of the Old Testament prophets Isaiah and Ezekiel: for ninth-century examples, BRUBAKER, Vision and meaning, figs 11, 44. With the occasional exception of Mary of Egypt, old women were not represented until the late Byzantine period, but older women were sometimes distinguished from younger ones, as in examples of the Nativity where the older midwife is helped by a younger assistant: see MEYER, An obscure portrait, 119-20, 218. I thank Tony Eastmond for discussion on this point.

12 Paris, BNF, gr.510, f.104r: BRUBAKER, Vision and meaning 137-41, fig. 17 (as in n. 6). 
be represented as a child - was not necessarily relevant to the Byzantines. The painter or patron chose whether or not to depict someone as a child, an adult, or aged; so (and this is the second key point) when this decision resulted in a portrait that seems to us to contradict the actual age that we know the person portrayed was, we should not assume, as scholars sometimes do, that the painter was inept, or that the Byzantines saw children as miniature adults. We need instead to ask why the painter made this choice. In this case, the child-adult Basil is probably a visual reference to his mature behaviour even as a child (the puer senex trope), which is carefully elucidated in the accompanying text, Gregory of Nazianzos' funeral oration to Basil. ${ }^{13}$ The important issue for us here, however, is that depictions of individuals at any given stage of their life cycle represents a choice based on criteria other than 'naturalism': that there are images of old men but not of old women does not mean that no Byzantine women grew old, ${ }^{14}$ but that old men were important to depict while old women were not. That social conventions, conscious or subconscious, underpinned this decision needs to be recognised, and we will return to this issue at a later stage in this chapter.

A second qualification that we need to consider is the impact of status on representations of Byzantine men and women. The presence or absence of a beard is a key distinguishing mark between adolescent and adult males in Byzantine imagery, but it is also a sign of status. ${ }^{15}$ In imperial portraits after the mid-seventh century, for example, the junior emperor is portrayed as beardless no matter what his age, in contrast to the bearded senior emperor. ${ }^{16}$ Similarly, the presence or absence of head covering can distinguish adolescent and adult females in Byzantine representations, but head covering too is a sign of status. Prostitutes and, often, female servants, whatever their actual age, are often depicted without veils. ${ }^{17}$ Because most generic depic-

13 PG 36, 509, 512 (Eng.trans. in NPNF 7, 399). On the puer senex trope in Byzantium, see DAviEs, From womb to the tomb, 81-82.

14 See note 11 above.

15 Lack of facial hair may also, of course, signal that the male portrayed is a eunuch, but context usually allows us to age the man appropriately: see, e.g., the eunuch patriarch Ignatios portrayed in a ninth-century mosaic at Hagia Sophia, and the eunuch Leo shown gifting his bible to the Virgin Mary in the Leo Bible: for the mosaic, see C. MANGo - E. J. W. HAwkins, The Mosaics of St. Sophia at Istanbul. The Church Fathers in the North Tympanum. DOP 26 (1972) 3-41, at 9-11, fig. 12; for the manuscript (Vat.reg.gr.1, f. 2v) see S. Dufrenne - P. CAnArt, Die Bibel des Patricius Leo. Codex Reginensis Graecus IB. (Codices e Vaticanis Selecti 75). Vatican City 1988.

16 See Kalavrezou, Portraits of Basil I; and note that on the coins minted during the period when the empress Eirene was in control of the empire her son Constantine VI, though an adult, was portrayed without a beard (see note 48 below), as had been Heraklonas Constantine when Martina was evidently the power behind the throne (see note 37 below). Similarly, very young co-emperors were often depicted as youths rather than the small children they actually were, as with Constantine, toddler son of Michael VII Doukas, on the Royal Crown of Hungary: E. KovÁcs - Z. LovAG, The Hungarian crown and other regalia. Budapest 1980, 34.

17 See MEyER, An obscure portrait, 134-53 (female servants), 182-200 (prostitutes), and 275-78 (hair). 
tions of figures who appear to be Byzantine youths - men without beards; women with uncovered heads - also represent workers in the field or servants, male or female ${ }^{18}$ it is hard to determine whether the beardless males shown (say) pruning vines, holding washing implements or running errands, and the female servants portrayed without veils, are actually meant to represent young men and women or are beardless and veil-less simply to indicate their lesser status. I will therefore focus on portraits of identifiable adolescents for the remainder of this chapter, which, sadly, effectively obliges us to look at saints (like St Basil), aristocrats, and the imperial family. This means that the run of portraits we are about to survey overlaps to a considerable extent with the evidence of the written sources in that the images are urban productions associated, in many cases, with the court or with a courtly milieu. I will, however, open out the data set at the end of the chapter, and draw some conclusions applicable to a larger and less restricted viewing audience.

The following several paragraphs present a gallery of portraits, arranged chronologically by the age of the youth portrayed. Even this brief survey illuminates the issues that portraits of Byzantine youth require us to confront. ${ }^{19}$

The frontispiece of the Paris Gregory (Paris, BNF gr.510, f.Br), dated to $\mathrm{ca} 880$, portrays the (eventual) emperor Alexander at around age 10 (fig. 3), ${ }^{20}$ in a portrait quite distinct from his mosaic portrait in the north gallery at Hagia Sophia where, at age 23 or 24 , he wears the beard of maturity as well as imperial vestments. ${ }^{21} \mathrm{~A}$ slightly older male youth appears in a miniature in the copy of Dionysios the Areopagite in the Louvre, which depicts Manuel II Palaiologos and his wife Helena with their three children, the eldest of whom, John, was born in 1392, and was therefore 11, 12 or 13 when the family portrait miniature was painted between 1403 and 1405 (fig.

18 I know of no study dedicated to depictions of Byzantine male servants, but a representative sampling of images may be found in: Everyday life in Byzantium, ed. D. PAPANIKola-BAKIRTzI. Athens 2002. Servants also often attend rulers in later Byzantine images (e.g. a servant may hold a basin jug in images of Pilate washing his hands after the condemnation of Christ), and these men are virtually always shown without beards.

19 I have made no attempt to cluster images in one century, or even one period: there is not enough preserved material to do this effectively, and I am not sure that portrayals of adolescents changed significantly between the $4^{\text {th }}$ and the $14^{\text {th }}$ century. We will return to this issue at the close of the chapter. Note too that many of my examples come from manuscripts. This is due simply to the fact that in Byzantium manuscripts had a greater range of images than other media. Because of arguments about its date, I have omitted the Vatican epithalamion (Vat.gr.1851) from this overview, though we will return to its miniatures at the end of this chapter: on the manuscript, see most recently C. HENNESSY, A child bride and her representation in the Vatican Epithalamion, cod. gr. 1851. BMGS 30 (2006) 115150 .

20 BRUBAKER, Vision and meaning, fig. 2.

21 P. Underwood - E. J. W. HAWkins, The mosaics of Hagia Sophia at Istanbul. The portrait of the emperor Alexander. A report on work done by the Byzantine Institute in 1959 and 1960. DOP 15 (1961) $187-217$. 
4). ${ }^{22}$ For another 12-year-old, we need only look at images of Christ in the temple, as for example in the Paris Gregory again (f.165r), which presents an extended version of this episode that portrays Christ three times (fig. 5): once hurrying to Jerusalem, once preaching in the synagogue, and finally reunited with his anxious parents. ${ }^{23}$ For a $12-$ year-old female, we may look to images of the Virgin Mary when she is betrothed to Joseph in the Kokkinobaphos manuscripts (fig. 6). ${ }^{24}$ A 13- (or possibly 16-) year-old Alexios is portrayed in a mosaic in the south-west gallery of Hagia Sophia to the side of his parents John II Komnenos and Eirene (fig. 5 in Hennessy article); Alexios was born in 1106, and was named co-emperor in 1119 , aged $13 ;^{25}$ my belief is that the mosaic commemorates his elevation, but the mosaic is usually dated to 1122, in which case he is 16 in this image. ${ }^{26}$ The Paris Gregory - on the same page as we have just seen Alexander at 10 - also portrays his elder brother Leo VI, born in 866, at around age 14 (fig. 3). All of these youths in their early teens are distinguished from both younger children and adults by their height (with children shorter and adults taller than the youths portrayed) and their slight builds, which usually contrast with the slightly chubbier children and the somewhat stockier adults.

Aside from the Virgin, the examples we have seen thus far have all been male, but the Lincoln College typikon (Oxford, Bodleian Library, Lincoln College, f. 11r), in a miniature dated around the year 1300, introduces another female, with Theodoule, the foundress of the monastery for which this is the typikon, leading her daughter Euphrosyne (born 1285/6), who took over the monastery at her mother's death. ${ }^{27}$ Euphrosyne is aged about 16 in this portrait (fig. 7). For the end of the teen-age decade, the Paris Gregory, source of many of our examples, depicts St Basil as a youth aged 18 to 20 with his good friend Gregory studying in Athens (fig. 2). The faces are abraded, but the figure on the left has clearly grown a beard to indicate his increased maturity; it contrasts with the grey beard of the wise old philosopher who teaches the pair. The significance of the beard as a sign of mature authority may be underlined by the Trier ivory, which has recently been interpreted as a depiction of the translation of relics to, and dedication of, Hagia Euphemia in 796 by Constantine VI and his

22 Byzance. L’art byzantin dans les collections publiques françaises. Paris 1992, 463-64.

23 BRUBAKER, Vision and meaning, fig. 21.

24 BAV gr.1162, f.100r and Paris, BNF gr.1208, f.135r. The best discussion of this sequence of images appears in K. LINARDOU, Reading two Byzantine illustrated books: the Kokkinobaphos manuscripts (Vaticanus graecus 1162 and Parisinus graecus 1208) and their illustration, unpub. PhD dissertation. University of Birmingham 2004, 92-118, for this image figs 81-82; see also her The couch of Solomon, a monk, a Byzantine lady and the Song of Songs, in: The church and Mary (Studies in church history 39), ed. R. Swanson. Suffolk 2004, 73-85, which deals with one miniature in this sequence. Further discussion of these manuscripts, with additional bibliography, appears below.

25 His details are tracked in DOC 4, 244.

26 So Hennessy, Images of children 160.

27 I. HutTER, Die Geschichte des Lincoln College Typikons. JÖB 45 (1995) 79-114; L. BRUBAKER, Pictures are good to think with: looking at Byzantium, in: L'écriture de la mémoire. La littérarité de l'historiographie, eds. P. Odorico - P. Agapitos - M. Hinterberger. Paris 2006, 221-40. 
mother Eirene: ${ }^{28}$ if this is correct, it portrays Constantine VI (born 771) beardless at 25. This would be unusual, but given the relationship between Constantine and his mother, and her apparent attempts to share the throne - which she would succeed in taking a year later after Constantine was blinded - it is perhaps not surprising. Certainly we see the same suppression of Constantine's authority on the coin issues of the same year, which suggests that Eirene and her supporters had control of the mints. ${ }^{29}$

Coins are, in fact, interesting for our purposes, for three reasons. First, coins and particularly low denomination coins - often circulated widely. They were thus viewed, or had the potential to be viewed, by a wide swath of the Byzantine populace, and had legal standing, so any representations on them had specific requirements: images of the emperor(s) validated the worth of the coinage. Though the Byzantines never tell us this, the function of coinage requires their ornamentation to be sufficiently conservative that when (and if) people actually looked closely at their money they would not distrust the coins. We are probably safe to assume, therefore, that the images we find on coins would be broadly acceptable to the Byzantine populace, and represent conventional images. In our case, that means that when we see adolescents represented on coins, they are likely to show us portraits of youths that the Byzantines accepted as suitable and appropriate.

Coins are also interesting to us here because they could be used to convey particular messages from the emperor or empress to his or her people. Whether or not those people paid any attention is a moot point; all the same, we may regard images of adolescents on Byzantine coins as representing what was thought to be a good public face of imperial youth.

The third reason coins provide interesting evidence for understanding Byzantine teenagers is perhaps the most important one: during the seventh century, and (although less vividly) into the eighth, some coin sequences visualise the aging process across adolescence. The Herakleian house provides particularly good examples, well exemplified in the coins of Herakleios' grandson Constans II, who was born in November 630, named co-emperor in 641, and ruled as sole or senior emperor from 641/2 until 668. We can track his maturation in a series of coins portraying him at age 11 to 15 , when he is shown without a beard (fig. 8 ) $;^{30}$ at age 17 , when he wears

28 P. NiEwöHNER, Historisch-topographische Überlegungen zum Trierer Prozessionselfenbein, dem Christusbuld an der Chalke, Kaiserin Irenes Triumph im Bilderstreit und der Euphemiakirche am Hippodrom. Millennium 11 (2014) 261-87.

29 See note 16 above.

30 DOC 3, 420-421, pl. XXIV, 1a-4b (minted 641-646). I illustrate this progression with coins from the Whitting Collection held at the Barber Institute of Fine Art, Birmingham, and am extremely grateful to Dr Rebecca Darley and Dr Jonathan Jarrett for their assistance in location the coins and obtaining photographs of them; because the Barber coins are not yet catalogued, I cite the relevant sections of the Dumbarton Oaks catalogue. 
a short beard, often, as here, simply indicated by a row of dots (fig. 9); ${ }^{31}$ at age 18 to 19 , with a short but more fully developed beard (fig. 10); ${ }^{32}$ at age 20 to 21 , with - on many but not all coins - a slightly longer beard (fig. 11);3 and from age 21 onward, with a full beard and moustache (and often with, from 654, his son Constantine IV, proclaimed co-emperor in that year, aged 4: fig. 12). ${ }^{34}$ The most notable development is, clearly, Constans' beard, which becomes progressively more prominent until, at the age of 21 - four years before the 'legal' year of male adulthood in Roman law - it becomes the dominant feature of his portrait, thus earning him the nickname pogonatos (the bearded one). ${ }^{35}$

Other examples can be adduced from the numismatic evidence, though they rarely visualise the stages of the life course as graphically as the coins of Constans II. ${ }^{36}$ But the coins of Constans II's grandfather, Herakleios (610 - 641), anticipate the sequence, with Herakleios Constantine (son of Herakleios and father of Constans II, born in 612 and crowned in 613) shown small on the early coins, then gradually becoming larger and, finally, bearded; his younger brother, Heraklonas (born of Herakleios' second wife, Martina, in 626 and named augustus at age 12), remained small and beardless, both because he was the junior co-emperor and because he was only 15 when Herakleios died and he and his mother were, shortly thereafter, exiled. ${ }^{37}$ And coins minted toward the end of Constans II's reign show Constantine IV growing older, accompanied, between 659-668, by his younger brothers Herakleios and Tiberios, with the ages of the three sons clearly differentiated by their size. ${ }^{38}$ During

31 DOC 3, 422, pl. XXIV, 5c (minted 647).

32 DOC 3, 423, pl. XXIV, 13c (minted 648/9).

33 DOC 3, 424, pl. XXIV, 16a, 17b (minted 650/1).

34 DOC 3, 425-27, pl. XXIV, 19a-30a (minted 651-659).

35 In fact, his grandfather, Herakleios, was often depicted on coins with an equally impressive beard and moustache: see, e.g., DOC 2, pl. VIII, 26e and 27 (both minted 629-631 in Constantinople, when Herakleios was about 55).

36 In addition to the coins cited in the following paragraph, Maurice's son Theodosios - born in 583 or 585, named augustus in 590 and killed in 602 (PLRE 3, 1293-94) - appears on coins in 591/2, when he was not yet an adolescent (ibid., 1294), and on a series of coins that are, sadly, of indeterminate date (DOC $1,373-76)$, but the coins are too badly abraded to allow us to speculate about his appearance.

37 On this extremely complicated sequence of coins, see DOC 2, 216-383, 389-401. During the few months that Heraklonas remained on the throne after his father's death, he remained beardless on the coins, perhaps because of his age, or perhaps because of his mother's (apparently unofficial) regency. In this respect, these coins anticipate some of those minted during the reign of Constantine VI and Eirene (on which see notes 16 above and 48 below).

38 See, e.g., DOC 2, 434-35, pl. XXV, 40c, 41d, 42e, 43b (minted 663-668). The three brothers remained on the coinage after the death of Constans II in 668, until Constantine IV deposed his brothers in 681 to clear the way for his own son, Justinian II; because it is uncertain when Herakleios and Tiberios were born, however, their ages on the coinage are equally unclear and I have therefore not considered them here: see PmbZ I/2, Nr. 2556 and I/5, Nr. 8484; for the coins, see e.g. DOC 2, 525-29, pls XXXII-XXXIII, 1c-10 g, 23.9-27.1. 
his first reign, the coinage of Constantine IV's son, Justinian II (685-695), followed the pattern established by his grandfather (Constans II): at 16, he appears beardless; at 17 or 18, his beard is formed of small dots; at 18 or 19 his beard, though still short, is more fully developed; and by the last years of his first reign - famously also signalled by the introduction of Christ on the gold coinage - he wears a full beard. ${ }^{39}$

After the demise of the Herakleian dynasty, the importance of the beard stops being primarily a signifier of maturity and becomes instead an indication of rank: under the Isaurians, beginning with Leo III (717-741), junior emperors on coins are shown without beards, no matter what their age, and the senior emperor is bearded, as are the Isaurian ancestors who appear on the reverse of coins from the reign of Leo III's son Constantine V (741-775) onward. ${ }^{40}$ On Leo III's gold coins, Constantine V appears, aged 2, as soon as he was named augustus in 720, and Philip Grierson tracks his increasing maturity on coins minted from 720 ('head very small, his hair not covering his ears': fig. 13); ${ }^{41}$ to the years $720-725$, when Constantine V was between 2 and 7 ('head slightly larger and older, with longer hair': fig. 14); ${ }^{42}$ to $725-$ 732, when he was 7 to 14 ('head still larger'), ${ }^{43}$ through the rest of Leo III's reign, 732-741, by which time Constantine V was between 14 and 22 ('bust more mature': fig. 15). ${ }^{44}$ The key developments here are, as Grierson noted, the size of the head, which becomes more or less the same size as Leo III's only in the coins minted when Constantine was between 14 and 22; hair length; and, though Grierson does not mention this, facial shape: Constantine $\mathrm{V}$ acquires the almost pointed chin of his father only in the latest coins of this sequence. At this point, perhaps to indicate dynastic unity, the portraits of Leo III and Constantine V are virtually indistinguishable except for the small beard worn by Leo.

Coin portraits of Constantine V's son, Leo IV (born 749 and named co-ruler in 751), follow a similar pattern: his round face with short hair gradually elongates and the hair lengthens until he closely resembles his father, and, on the reverse, his grandfather. ${ }^{45}$ This changed, however, when Leo IV became senior emperor in 775, and named his own son, Constantine VI, augustus in 776. Constantine VI was then 5, and - following the precedent of the coins of Leo III and Constantine V one would expect to see the child's face and hair lengthen as he grew. This does not happen. Constantine VI appears on his father's gold coinage immediately after his elevation, but is distinguished from him only by his lack of facial hair (fig. 16):

39 DOC 2, 568-609, pls XXXVII-XXXIX. During his second reign (705-711) Justinian II sometimes appears with his son Tiberios, but he had not yet reached his teens when he was killed following the downfall of his father: see DOC 2, 644-63.

40 Coins and discussion in DOC 3, 225-324, pls I-IX.

41 DOC 3, 242, pl. I, 3a, 3e.1, 3e.3.

42 DOC 3, 243, pl. I, 4a, 4c-f.

43 DOC 3, 244, pl. I, 5.2-5.10.

44 DOC 3, 245, pl. I, 6-7.

45 DOC 3, 299-301, pl. VIII. 
we never see Constantine VI as a child on the coins. ${ }^{46}$ It is possible that this was due to Leo IV's ill health, or to his apparent wish to ensure the succession of his son rather than of his brothers, ${ }^{47}$ either of which may have urged that dynastic succession was stressed above any indication of his heir-designate's youth. In fact, Constantine VI's portrait changed remarkably little across the 20 years he appeared on coins: after his father's death in 780, when Constantine VI was 9, he appeared, beardless, with his mother, the regent empress Eirene, and he remained beardless on the gold coinage until his deposition in 797 (fig. 17), when he was $26 .^{48}$

Political circumstances may have prompted Constantine VI's extended numismatic youth, but the absence of a portrait sequence visualising his adolescent growth and increasing maturity continued under Nikephoros I, whose son Staurakios joined him on coins after he was named augustus in 803 , aged 10 or 12, and remained there until Nikephoros' death in battle in 811, but whose features never varied from the beardless but mature facial type that had previously typified rulers in their late teens. ${ }^{49}$ The same is true of Theophylact, son of the emperor Michael I, who ruled with his father from 811 to 813 , aged 8 to $10 ; ;^{50}$ and it is also true of Smbat/Constantine, born sometime between 800 and 810 , who ruled with his father Leo V from 813 $820 . .^{51}$ What seems to be happening here is that the age-specificity we found under the Herakleians is fading: the beard becomes the dominant signifier of status, and it was apparently more important to signal that there was an heir-designate who looked the part than to track that heir's growth spurts. The insecurity of the period seems to have suggested that dynastic stability was more important to display than the growing maturity of the co-ruler.

Coins minted under Michael II the Amorian which show Theophilos, born in 812/ 3 and elevated to the throne by his father in 821, appear to continue this pattern, but are problematic. They have not been dated more precisely than '821-829' so that while some examples show the junior emperor with a slightly more rounded face than others, it is impossible to establish whether or not these were minted earlier than coins that show him with a slightly more elongated face - whether, in short, the variation (which is in any case slight) represents an attempt to indicate aging or whether it is simply due to the use of different dies. ${ }^{52}$ Theophilos' first son, Constantine (who was born in the 820s but who died young), appears on the coinage as a

46 See $D O C$ 3, 328-30, pl. XII.

47 See $P m b Z$ I/2, Nr. 4243.

$48 D O C$ 3, 340 - 46, pls XIII-XIV. For discussion, see ibid., 336-39; and, with additional bibliography, L. BRUBAKER - J. HALDON, Byzantium in the iconoclast era c. 680 - 850: a history. Cambridge 2011, 352-54.

49 On Staurikios, see PmbZ I/4, Nr. 6866; for the coins, see DOC 3, 355-61, pls XV-XVII.

50 On Theophylact, see PmbZ I/4, Nr. 8336; for the coins, see DOC 3, 363-70, pl. XVII.

51 On Smbat/Constantine, see PmbZ I/2, Nr. 3925; for the coins, see DOC 3, 371-86, pls XVIII-XIX. 52 DOC 3, 394-405, pls XX-XXI. DOC 2b and 3a show Theophilos with a slightly rounder face, which elongates a bit on nomisma 4, and settles into the standard pointed format on nomisma $5 \mathrm{a}$. 
child, but (like Staurakios, Theophylact and Smbat/Constantine before him) is never shown aging; ${ }^{53}$ his daughters appear on the rare class IV nomismata of the late 830s with their relative ages indicated by scale but not physiognomic details (which would, in any case, be difficult given the size of the coins) $;^{54}$ and Michael III appears virtually immediately after his birth, portrayed - despite his infancy - with the beardless but mature facial type that characterised images of the young Constantine VI, Staurakios and Smbat/Constantine earlier in the century ${ }^{55}$. Once again, the appearance of a male heir, presented in the appropriate and approved fashion sanctified by the last five generations of rulers, was more important to signal than Michael III's increasing maturity.

After his father's death in 842, however, Michael III appears as a child (with his older sister Thekla) on the reverse of the coin; as regent, his mother, the empress Theodora, is shown on the obverse. ${ }^{56}$ Soon thereafter, Michael III - now once more with the beardless but mature facial type he displayed during his father's lifetime - is joined by his mother, and an image of Christ occupies the obverse. ${ }^{57}$ In 856 , when Michael III was 16, his mother disappears, and the emperor is shown as a bearded adult ${ }^{58}$. This tells us two things, neither of which is surprising. First, as is clear from the coins of co-rulers from Constantine VI onwards, coin imagery shows what is believed to be appropriate; it is not visual reportage. Theodora's mint-master followed the precedent that had been established most recently when Eirene became regent in association with the young Constantine VI, and visualised this relationship on the coinage by presenting the pair as an easily recognisable mother and young son. Second, the beardless but mature facial type has become the normative way to represent imperial youth, and this continues until the end of the Empire. Henceforth, adolescents on coins follow the pattern established by Constantine VI, Staurakios, Smbat/Constantine and, except on the coins minted immediately after his father's death, Michael III: they may or may not be smaller in scale than the senior emperor, and they are beardless, but they do not display any other signs of aging. ${ }^{59}$ Never again will their progressive maturation be portrayed as vividly as under the Herakleians and, though to a lesser extent, the Isaurians.

53 For Constantine, see $P m b Z$ I/2, Nr. 3931; for the coins, DOC 3, 425-28, pl. XXII, 2a-3e.

54 DOC 3, 428, pl. XXII, 4; discussion in ibid., 407, 415-16.

55 DOC 3, 428-29, pl. XXII, 5.

56 DOC 3, 461-62, pl. XXVII, 1a-d.

57 DOC 3, 463, pl. XXVII, 2.

58 DOC 3, 463-64, pl. XXVII, 3.

59 Adolescents and sons on coins after 867: Basil's sons Constantine, Leo VI and Alexander (DOC 3, 487-505); Leo VI's son Constantine VII (DOC 3, 513-14); Constantine VII during his mother's regency (DOC 3, 541-42); Romanos I's sons Christoher, Stephen and Constantine Lekapenos, sometimes with Constantine VII, who from 921 is sometimes presented as a bearded adult and sometimes not, apparently depending on the political tide (DOC 3, 544-57); Constantine VII's son Romanos II (DOC 3, 551-69); Nikephoros II's stepson Basil II (DOC 3, 582-83); Eudokia's sons Michael VII and Constantios (DOC 3, 783-84); Romanos IV's stepsons (DOC 3, 789-91); Alexios I's son John II (DOC 4, 
The run of portraits we have just examined allows us to draw a number of conclusions, one of which is that the visualisation of adolescence is heavily gendered. For males, from the mid-seventh century on, the key indicator is the presence or absence of a beard, which is, as we have seen, a signal of both maturity and of a shift in status. For females, the key indicator is less blatant. Although we do not have a full run of female portraits, the distinction between the just-turned-12-year-old Mary and the 16-year-old Euphrosyne is, however, striking. We may add to it the image of a princess in the Vatican epithalamion (Vat.gr.1851), for, whomever the girl represents, she is presented as a pre-pubescent female (fig. 18). ${ }^{60}$ The epithalamion child is slight, raises her hands in supplication, and is bareheaded, allowing us to admire the pearls in her blond hair. The 16-year-old Euphrosyne is far more solid, stands nearly frontal, and is portrayed with covered head. The child-princess, even when she is shown dressed in Byzantine imperial regalia and enthroned amidst her court, displays the top of her head, and her hair remains visible, unlike the presumably older girls and women who attend to her. Euphrosyne is solemn and displays due decorum; the princess appears to smile. In part, the differences between the child and Euphrosyne have to do with status: the younger girl is a princess, the elder is a nun. But if we look at other young but married females in the Lincoln College typikon some of the distinctions continue. A sequence of family portraits was added to the typikon around 1335, displaying eight married couples in the family, some of whom are mentioned in the typikon. ${ }^{61}$ The older married couples - fig. 19 portrays Euphrosyne's elder brother Theodore Komnenos Doukas Palaiologos Synadenos and his wife Eudokia Doukaina Komnene Synadene Palaiologina - are blessed by the Virgin of Sure Hope, to whom the monastery was dedicated. The younger married couples - fig. 20 depicts the protosebastos Constantine Komnenos Raul Palaiologos with his wife, and Euphrosyne-the-nun's niece, Euphrosyne Doukaina Palaiologina - are blessed by the youthful Christ. We do not have accurate dates for the second Euphrosyne, but she is probably slightly younger than Euphrosyne-thenun, so in terms of chronological age she is well within our category of Byzantine female adolescents, though she is clearly married. Like the epithalamion princess, the typikon young women are dressed in courtly finery, but they all differ distinctly from the princess in their head covering and solemn demeanor. The princess, though arriving in Constantinople for her wedding, is not yet married age; the typikon women are, either to elite men or to Christ. The defining difference between how adolescent females were depicted had little to do with actual age-in-years, but was in-

224-26); Michael VIII's son Andronikos II (DOC 5, nos 36, 44, 197-219); Andronikos II's sons Michael IX and Andronikos III (DOC 5, nos 235-503, 560, 595-623, 633-716, 853-56); John V's sons Andronikos IV and Manuel II (DOC 5, 200 and no. 1196).

60 The problematic dating of this manuscript and its illustrations are discussed in HENNEssy, A child bride.

61 See the references in note 27 above. 
stead dependent on whether or not they were married (to a terrestrial man or, as in Eudokia's case, to Christ).

Two additional examples drive this point home. Eudokia Doukaina Komnene Synadene Palaiologina (wife of Euphrosyne-the-nun's elder brother Theodore), whom as we have already noted was pictured in the Lincoln College typikon around 1335 (fig. 19), was, apparently before her marriage, also portrayed in an unpublished prayer scroll made for her personal and private use, where she is identified by inscription (fig. 21). The young Eudokia is strikingly similar to the Vatican princess, down to the pearls interwoven into her long and loosely flowing hair. She is, equally, strikingly different from her later portrait. Although we cannot see the top of her head, her hair is unbound and her facial features appear much more relaxed than they do in the later image. We do not know how old Eudokia was when the prayer scroll was made (which is why I did not include her in our rogues' gallery earlier), but her size relative to John Chrysostom, before whom she anachronistically stands in prayer, and her bulk indicates that she is not a small child. She is evidently not, however, yet married, and so appears as a female youth. Marriage, not age-in-years, and - as the next example will demonstrate - not even puberty itself, determines whether a young woman of status is presented as an adolescent or a matron.

This convention of female adolescent portraiture is brought out sharply in the two twelfth-century manuscripts illustrating the life of the Virgin Mary attributed to the monk James of the Kokkinobaphos monastery. ${ }^{62}$ Mary appears in about three-quarters of the miniatures, which depict her life from her birth until her virginal purity, despite being pregnant, is vindicated by trial by water. She is shown as an infant in the bath at her Nativity, ${ }^{63}$ but as soon as she is accorded any agency she is presented as a child, and her appearance changes little as she grows up in the temple. According to the Protevangelion of James, after her precocious seven first steps at six months, her mother Anna vowed that Mary's feet would never touch the ground until she reached the age of 3, when she would be dedicated to the temple. ${ }^{64}$ The first seven steps are not illustrated in the Kokkinobaphos manuscripts, but she appears in several miniatures that picture episodes that took place before she was 3, and the miniaturist adheres to the prescription of the Protevangelion: Mary is always

62 See note 24 above, and H. Omont, Miniatures des homélies sur la Vierge du moine Jacques (MS grec 1208 de Paris). Bulletin de la Société française de reproductions de manuscrits à peintures 11 (1927), 1-24; I. HutTER - P. CANART, Das Marien homiliar des Mönches Jakobos von Kokkinobaphos, codex Vaticanus graecus 1162 (Codices Vaticanis selecti 79). Vatican City 1991; J. C. ANDERSON, The Illustrated Sermons of James the Monk: Their Dates, Order, and Place in the History of Byzantine Art. Viator 22 (1991) 69-120; and for substantial revisions to the dating of the two manuscripts, K. LINARDoU, The Kokkinobaphos manuscripts revisited: the internal evidence of the books. Scriptorium 61 (2007) 384-407.

63 Vat.gr.1162, f.29r; Paris.gr.1208, f. 38v: reproductions in the facsimiles cited above, and in LINARDou, Reading two Byzantine illustrated books 42-43, pls 18-19.

64 E. Hennecke - W. Schneemelcher, New Testament Apocrypha 1. Philadelphia PA $1965,377$. 
shown either in bed or in the arms of her mother. ${ }^{65}$ Between the ages of 3 and 12 she is shown several times, always as a slight young woman considerably shorter in height than the adult figures in the images (fig. 22), but with no attempt to portray the various stages of her physical development as she grew older. ${ }^{66}$ When Mary turned 12, the high priest Zacharias began to worry about what to do with her, as she could not stay in the temple once she reached puberty. He prayed; God told him to summon widowers; and from among the widowers Joseph was selected to be Mary's husband and guardian. ${ }^{67}$ Seven miniatures accompany this narrative, and Mary appears in all but the two scenes that depict Zacharias in prayer and the summoning of the widowers. ${ }^{68}$ In all of them, including the miniature that pictures her presentation to Joseph (fig. 6), she is presented in the child-like format familiar from earlier episodes in the manuscript. Minutes later, Mary bids farewell to the priests, and leaves the temple with Joseph to travel to his house: she is here, and henceforth, portrayed as an adult woman (fig. 23). ${ }^{69}$ This is a graphic demonstration of the role of marriage in determining how young women were portrayed in Byzantium: what defines Mary as a young woman rather than an adolescent girl is neither puberty nor her betrothal to Joseph, but her actual partnership with him. As visualised in Middle and Late Byzantium, growing up, for women, was not a gradual process, but an instant transformation effected by marriage.

Whether or not this was true before the social shifts highlighted by Iconoclasm is impossible to say, for we simply do not have sufficient evidence of youthful female

65 Vat.gr.1162, ff.38v, 41r, 43r, 44v, 46v; Paris.gr.1208, ff.52r, 56r, 59r, 61r, 63r: reproductions in the facsimiles cited above, and in LINARDOU, Reading two Byzantine illustrated booksn 53-62, pls 28-37. The scenes present her parents giving thanks for her birth, with Mary in a cradle; Anna's invocation of David, with Mary in her mother's lap; Mary in her bed; the banquet of the priests, with Mary held by Anna and in her cradle (only the second scene appears in the Paris manuscript); and the end of the banquet, with Mary in Joachim's arms reaching out for her mother (scene omitted in Paris), and Mary tucked into bed with a second image of her held by her mother.

66 Vat.gr.1162, ff.57v, 59v, 62v, 65r, 67v, 68v, 76v; Paris.gr.1208, ff. 77v, 80r, 87v, 91r, 92v, 103v: reproductions in the facsimiles cited above, and in LINARDou, Reading two Byzantine illustrated books, pls $46-50,53-58,61-62$. The only exception is the image showing Mary's parents, Joachim and Anna, visiting her in the temple when they had not seen her for some time, when she is taller and stockier, and almost resembles her portrait as an adult and it may be - as Linardou suggested that the shift in scale is intended to indicate Mary's growth since her parents last saw her: Vat.gr.1162, f.74v; Paris.gr.1208, f.100v; LINARDou, Reading two Byzantine illustrated books, 89-91, pls 59-60. This image is, however, preceded and followed by miniatures with the short, slight Virgin-as-child, so if the painter intended to picture a growth-spurt, it did not last.

67 Hennecke - SchneEmelcher, New Testament Apocrypha 1, 378-79.

68 Vat.gr.1162, ff. 85r, 87v, 90r, 97v, 100r; Paris.gr.1208, ff. 113v, 117r, 120r, 131v, 135r: reproductions in the facsimiles cited above, and in LINARDOU, Reading two Byzantine illustrated books, pls 67-72, 79-82. An eight, extra-narrative scene in the middle of this sequence shows an adult Mary triumphing over Satan (ibid., pls 73-74).

69 Vat.gr.1162, f.105r; Paris.gr.1208, f.142r: reproductions in the facsimiles cited above, and in LINARDoU, Reading two Byzantine illustrated books 115-16, pls 83-84. 
portraiture. But for portraits of adolescent males we have the coins, and these, as we have seen, demonstrate a sharp break after the Herakleians and Isaurians, which at least suggests that different attitudes toward visualising changes in the Byzantine life course prevailed before and after the eighth century.

Indeed, after Iconoclasm, young (pre-married) females look remarkably the same. The epithalamion princess (fig. 18) and the young Eudokia (fig. 21) are almost interchangeable; and Mary looks the same at 3 (fig. 22) as she does at 12 (fig. 6). As the coin evidence demonstrated, during and after Iconoclasm, the same is true of adolescent (pre-beard) males. This is not because Byzantine painters (or coin die strikers, for that matter) were incapable of depicting different facial types: adult males are often sharply distinguished one from another, and even when they were clearly reproducing types rather than painting portraits, painters were usually careful to make adult men in the same scene look different. ${ }^{70}$ In contrast, after Iconoclasm, even portraits of actual adolescents look exactly like all other male youth: the 10-year-old Alexander and 14-year-old Leo VI from the Paris Gregory (fig. 3) are distinguished from one another only by their height and attributes, and look remarkably similar to the 12-year-old Christ (fig. 5) as well.

Though Byzantine texts present adolescents with a series of distinctively defined characteristics, these characteristics are themselves topoi (e.g. impetuous youth, saintly youth). In stories - hagiography and the twelfth-century (and later) Byzantine novels - adolescents play well-defined roles, ${ }^{71}$ but they are otherwise rarely described as differentiated actors: once Byzantine males become active participants in the political or social narrative, they are treated as adults. Clearly, the Byzantines were well aware of the way that 'real' males age across adolescence; we could assume it, but the coins of the seventh century confirm it for emperors. Nevertheless, in terms of representations, in all other contexts, the overwhelmingly dominant way youths are portrayed is the generic. The visual evidence corroborates the suspicion that adolescent males were simply not important enough as characters for Byzantine painters to want to distinguish them. This gives some context to textual representations of unruly or saintly youth; there were of course clichés, but also clichés which, thanks to the visual evidence, we can see were the work of writers who did not care how accurate they were, for in a sense all adolescents were much the same. ${ }^{72}$ (In this sense, on suspects that adolescents, like women, were good to think with). This was,

70 See, e.g., the two older men in figure 1, or the miniature of martyrdom of the twelve apostles in the same manuscript: Paris.gr.510, f.340r (BRUBAKER, Vision and meaning, fig. 34).

71 DAviEs, Womb to the tomb 91-131. On adolescents in the novels, see the contribution of Catia Galatariotou to this volume. For a structural analysis of male saints' lives, see E. PATLAGEAN, Ancienne hagiographie byzantine et histoire sociale. Annales. Économies, sociétés, civilisations 1 (1968) 106-26; repr. in: EADEM, Structure sociale, famille, chrétienté à Byzancem IVe-XIe siècles. London 1981, essay 5; Eng. trans. EADEM, Ancient Byzantine hagiography and social history, in: Saints and their cults: studies in religious sociology, folklore and history, ed. S. Wilson. Cambridge 1983, 101-21. 72 In this sense, on suspects that adolescents, like women, were good to think with. 
definitely, not a culture which at any point across its millennium of history paid much attention to the experience of living through adolescence.

My final point is related to this. Women, like youthful males, all look the same. There are young, unmarried women, and there are older, married women (brides of men, brides of Christ, and occasionally widows), but none of them have any distinguishing physiognomic characteristics except for the colour of their hair. After Iconoclasm, only men were significant enough players to be individualised, and this fits with my earlier observation that only men grow old in Byzantine imagery. So: Females appear as children (rarely), unmarried adolescents (rarely), servants (age uncertain) and generic adults, but undifferentiated inside each group. Males appear as generic children, generic adolescents, generic servants (age uncertain), but then differentiated adults, including eunuchs, and often (even if not always) differentiated old men. The conventions of gendered social status and interpersonal relations in Byzantium could hardly be clearer. 


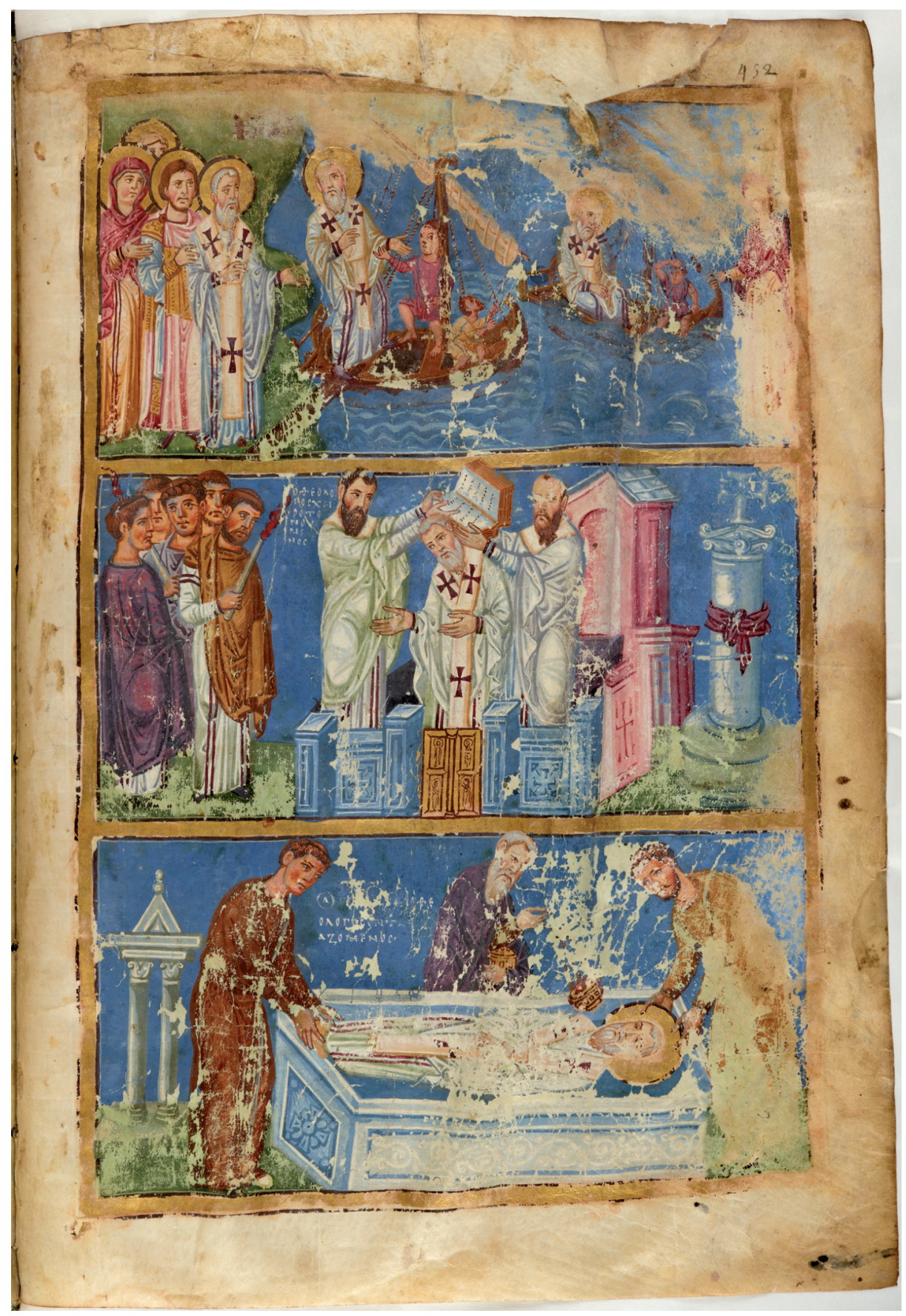

Fig. 1. Burial of Gregory of Nazianzos (Paris.gr.510, f.452r); (C) Bibliothèque Nationale de France 


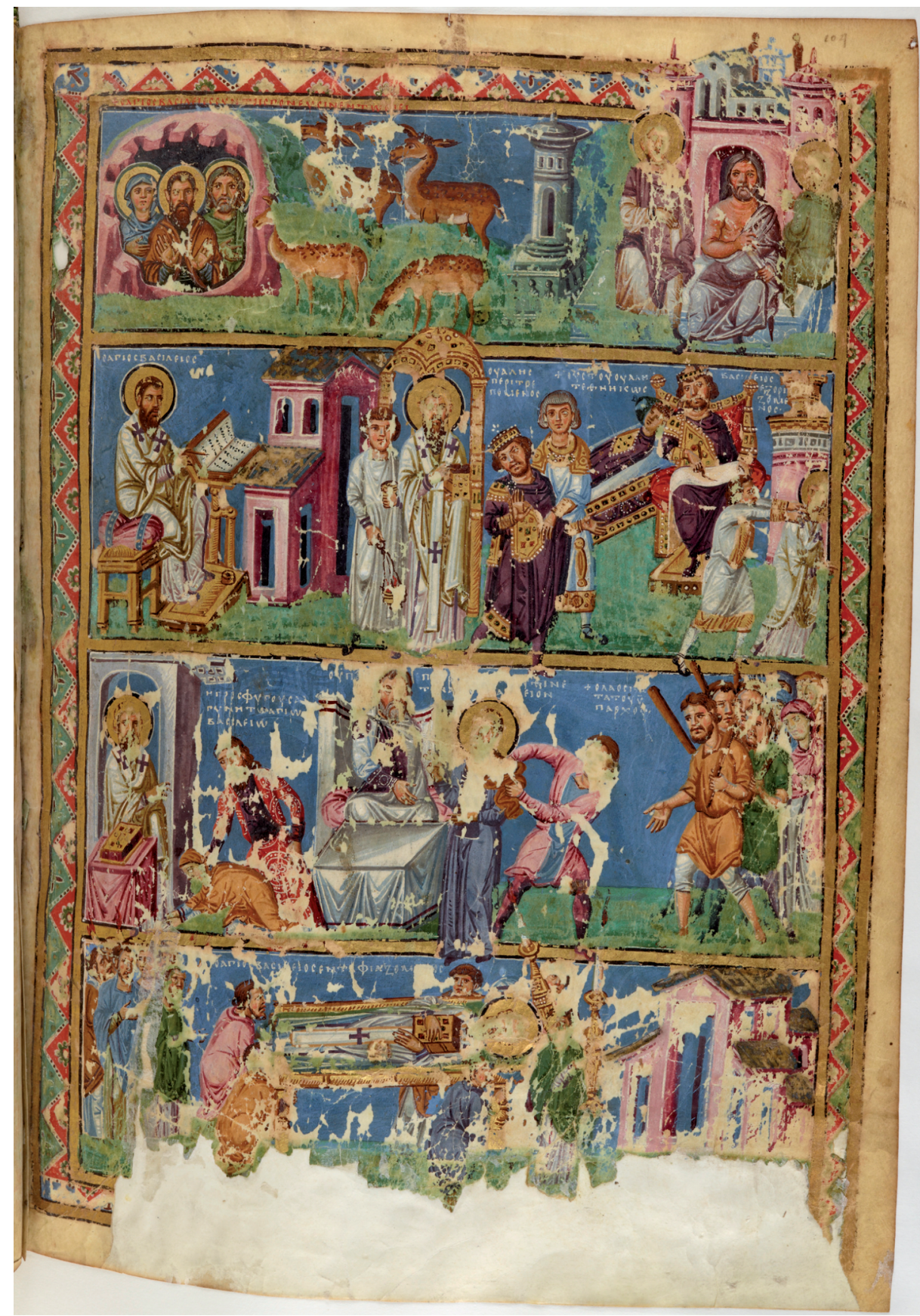

Fig. 2 Life of St Basil (Paris.gr.510, f.104r); @ Bibliothèque Nationale de France 


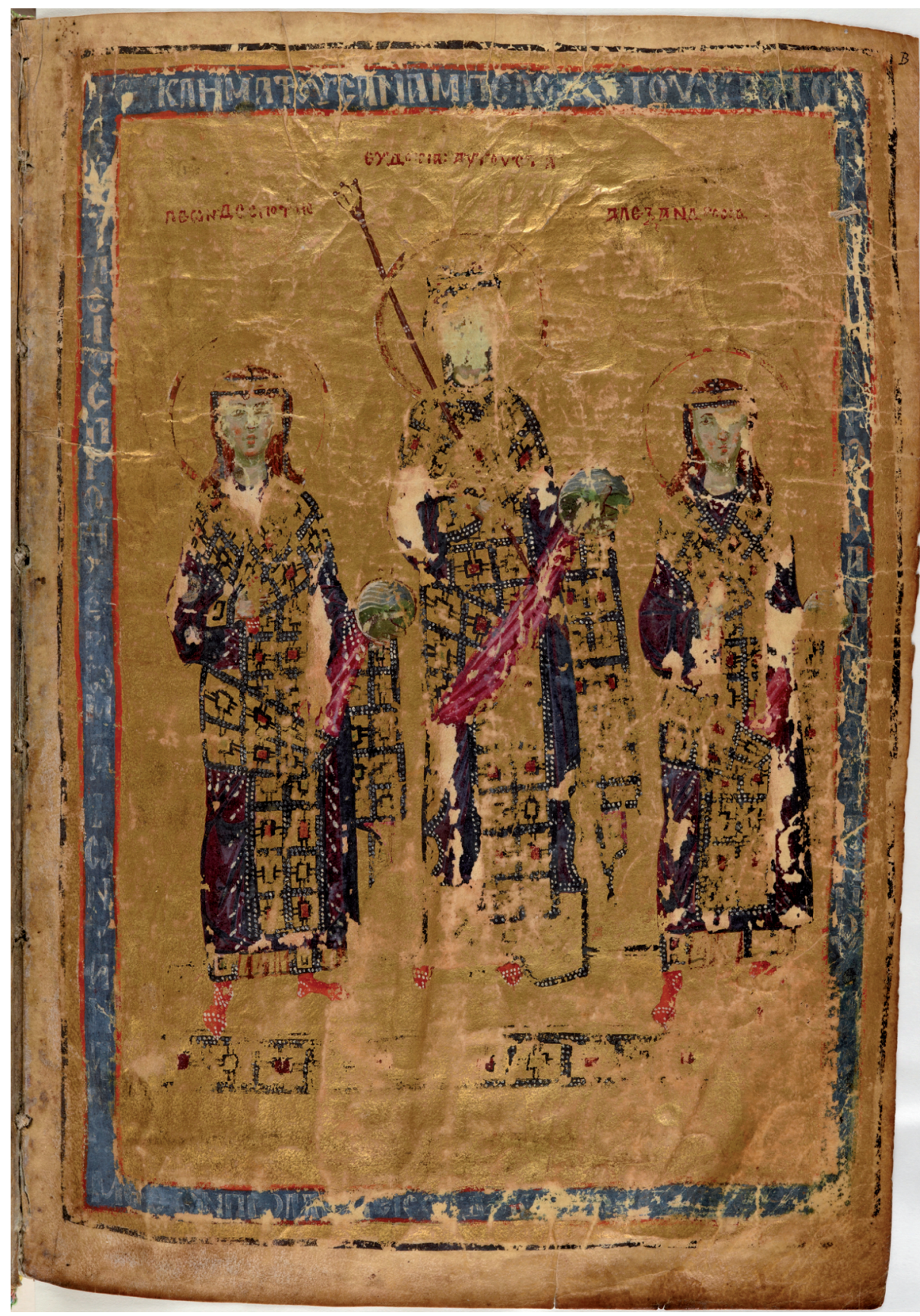

Fig. 3 Leo VI, Eudokia and Alexander (Paris.gr.510, f.Br); ( Bibliothèque Nationale de France 


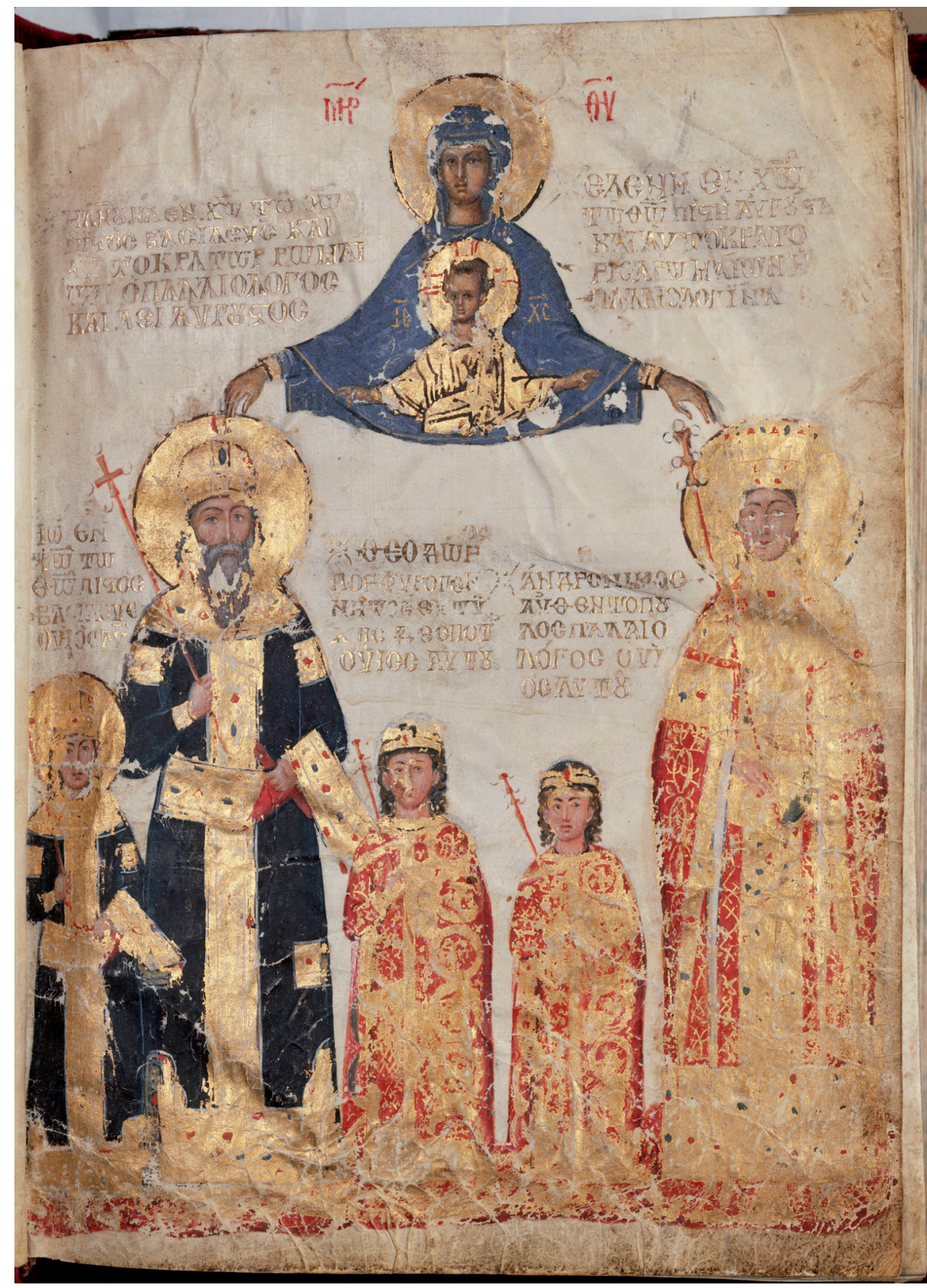

Fig. 4 Manuel II Palaiologos, his wife Helena and their three children (Paris MR 416, f.2r) photo () RMN-Grand Palais (musée du Louvre) / Daniel Arnaudet 


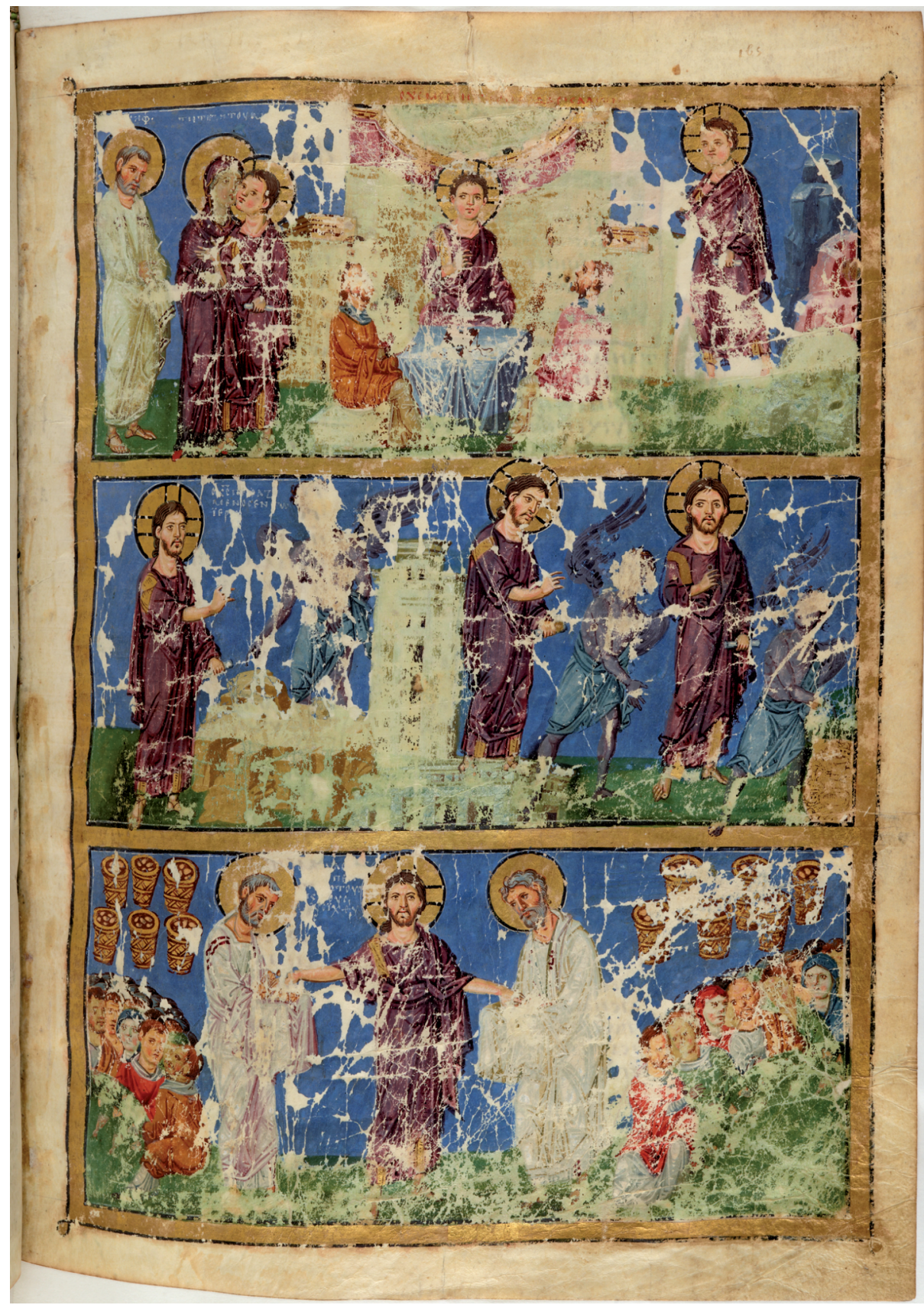

Fig. 5 Christ preaching in the temple (Paris.gr.510, f.165r); (c) Bibliothèque Nationale de France 


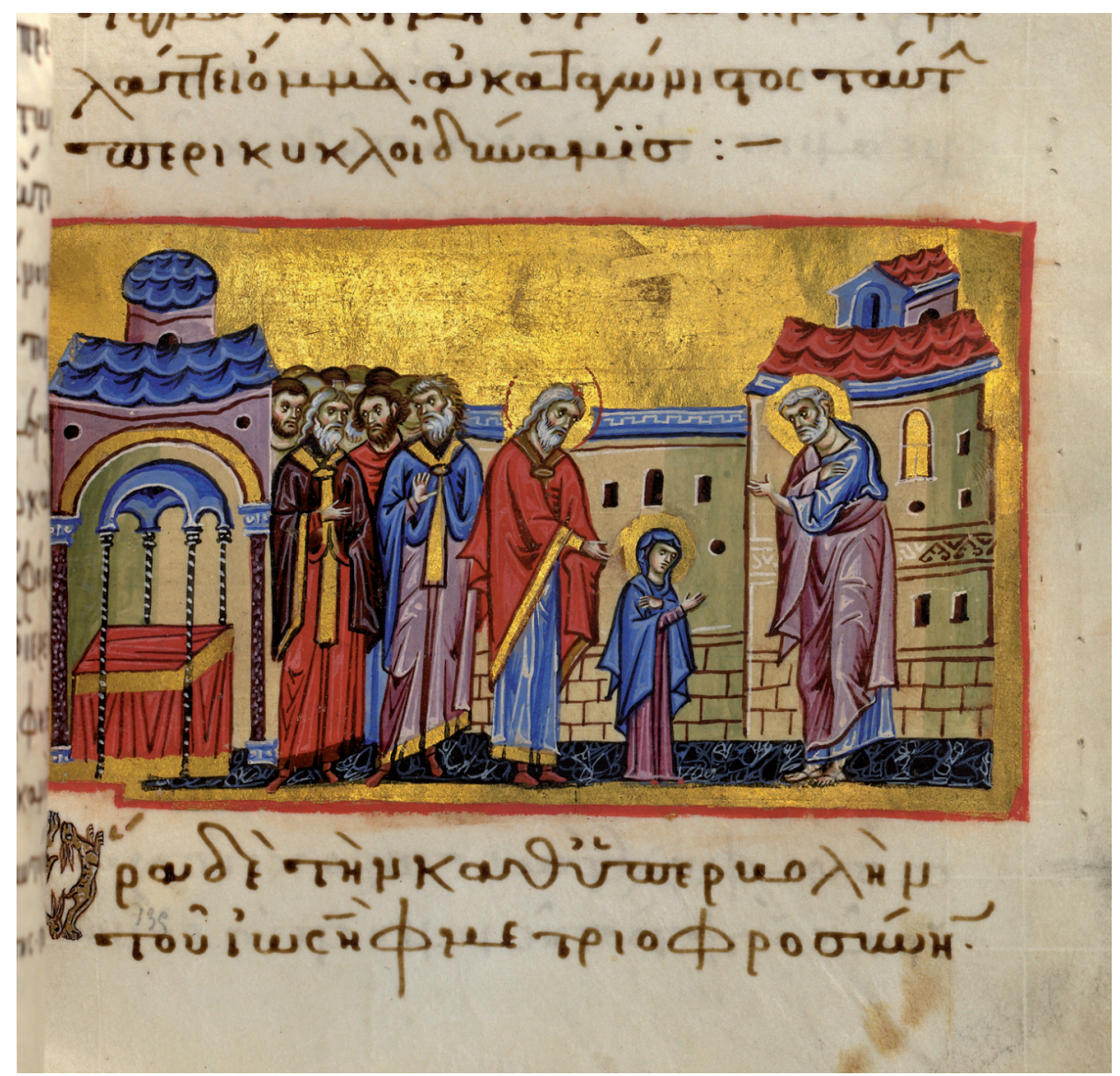

Fig. 6 Mary’s betrothal to Joseph (Paris.gr.1208, f.135r); (C) Bibliothèque Nationale de France 


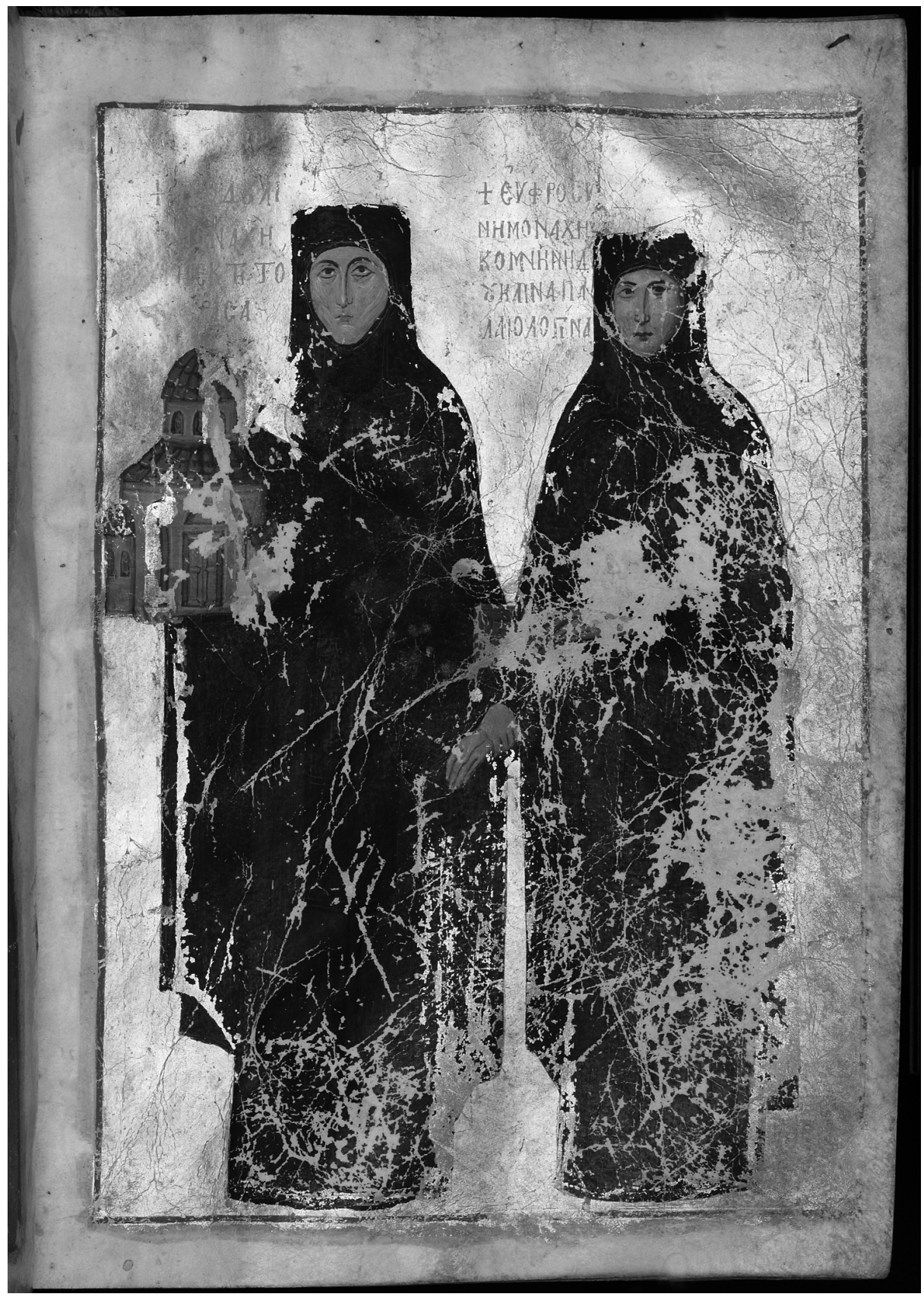

Fig. 7 Theodoule and Euphrosyne offer the monastery and typikon to the Virgin (Oxford, Bodleian Library, Lincoln College gr.35, f.11r); () Lincoln College, Oxford 

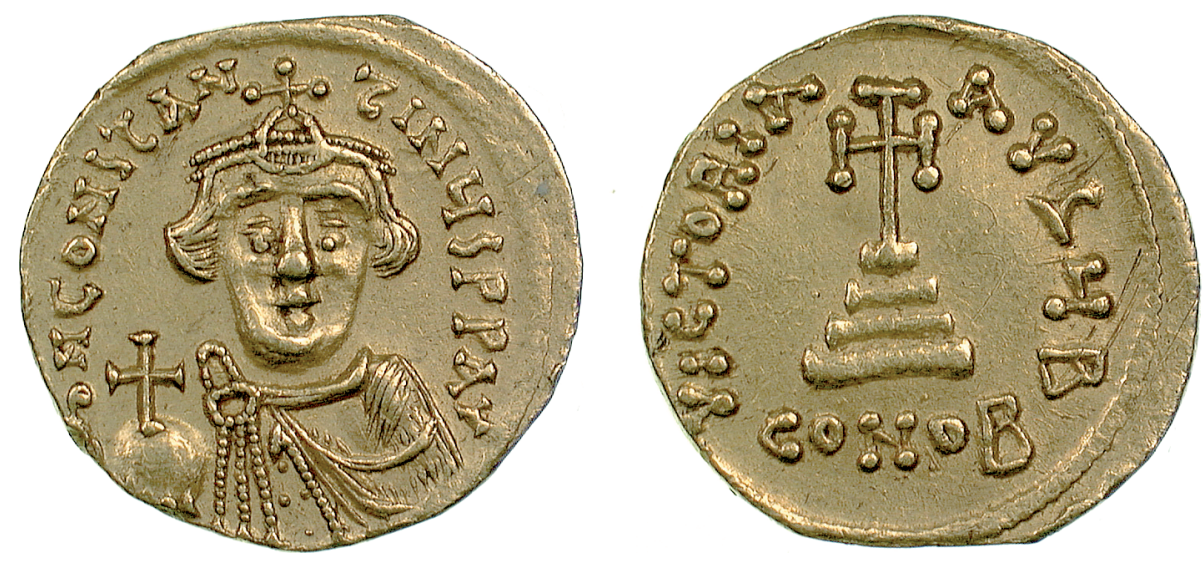

Fig. 8 Constans II, age 11-15 (Birmingham, Barber Institute of Fine Arts, inv. no. B3746); (CThe Barber Institute of Fine Arts, University of Birmingham
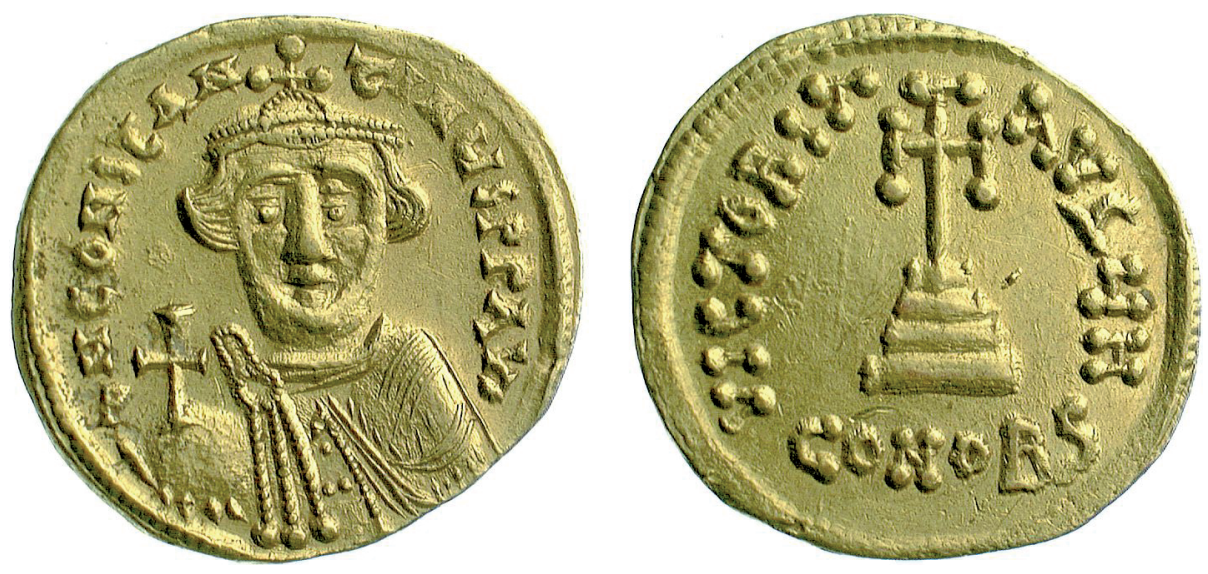

Fig. 9 Constans II, age 17 (Birmingham, Barber Institute of Fine Arts, inv. no. B3773); ( The Barber Institute of Fine Arts, University of Birmingham 

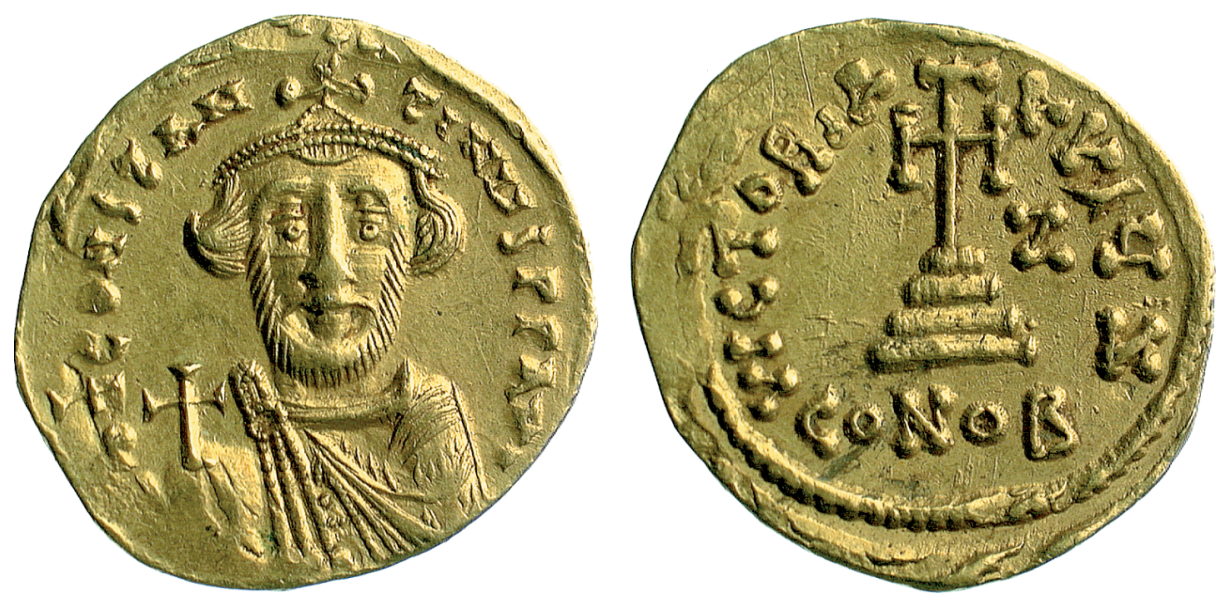

Fig. 10 Constans II, age 18-19 (Birmingham, Barber Institute of Fine Arts, inv. no. B3791); (C The Barber Institute of Fine Arts, University of Birmingham
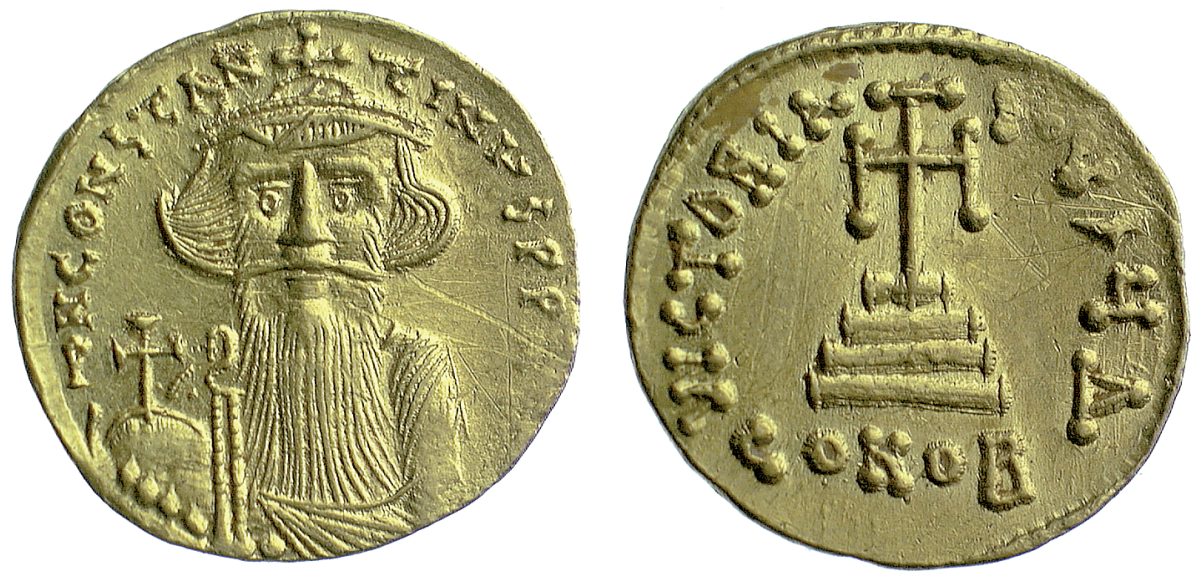

Fig. 11 Constans II, age 20-21 (Birmingham, Barber Institute of Fine Arts, inv. no. B3812); ( The Barber Institute of Fine Arts, University of Birmingham 

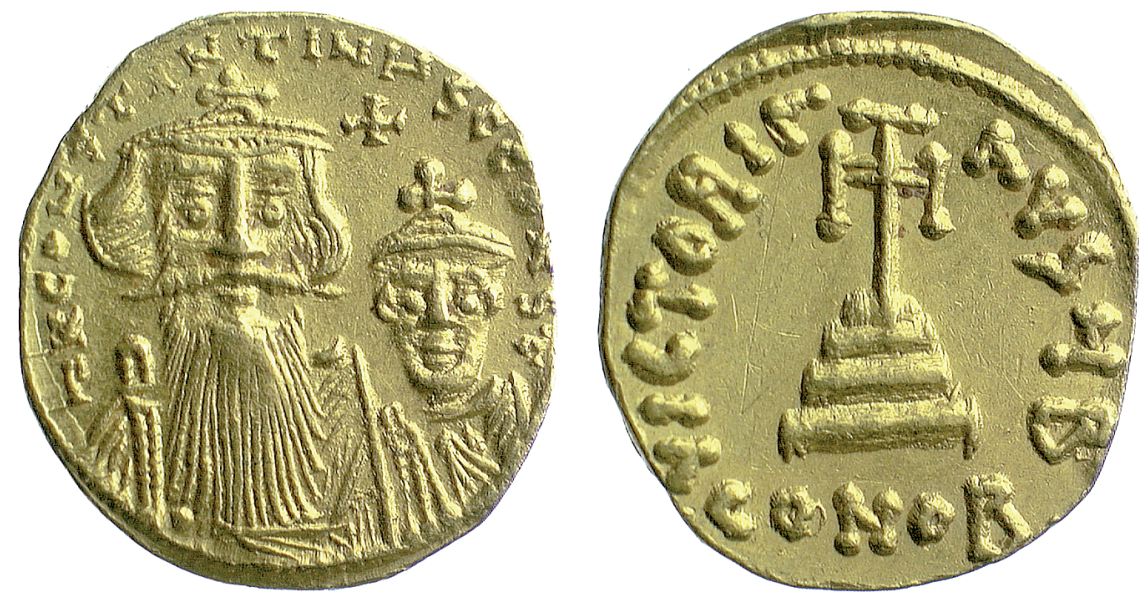

Fig. 12 Constans II with son Constantine IV (Birmingham, Barber Institute of Fine Arts, inv. no. B3832); (C) The Barber Institute of Fine Arts, University of Birmingham
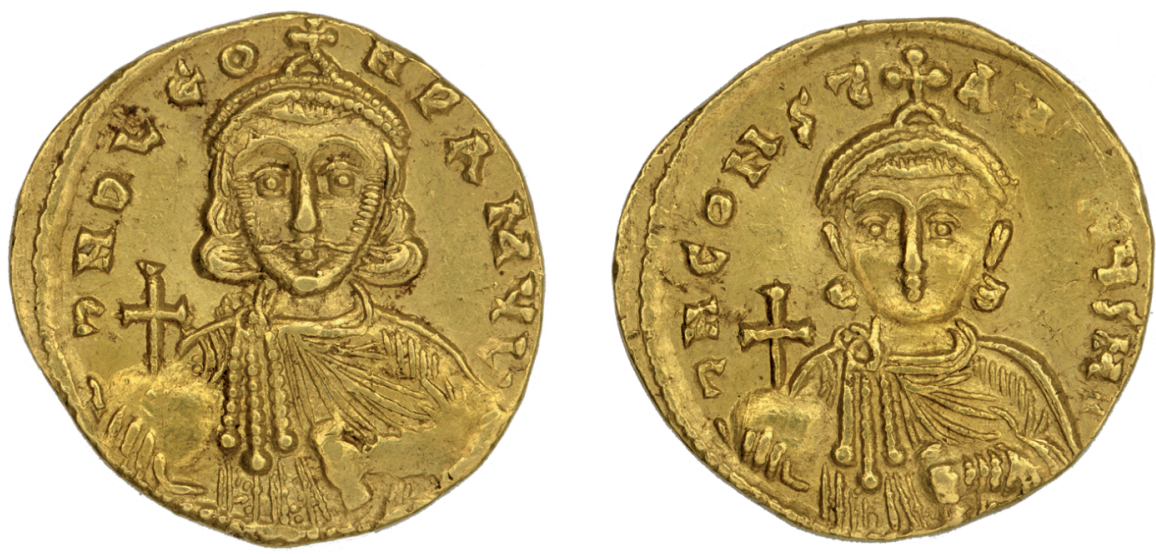

Fig. 13 Leo III and Constantine V, age 2 (Washington, DC, Dumbarton Oaks, inv. no. BZC.1948.17. 2445); ( D Dumbarton Oaks, Byzantine Collection, Washington, DC. 

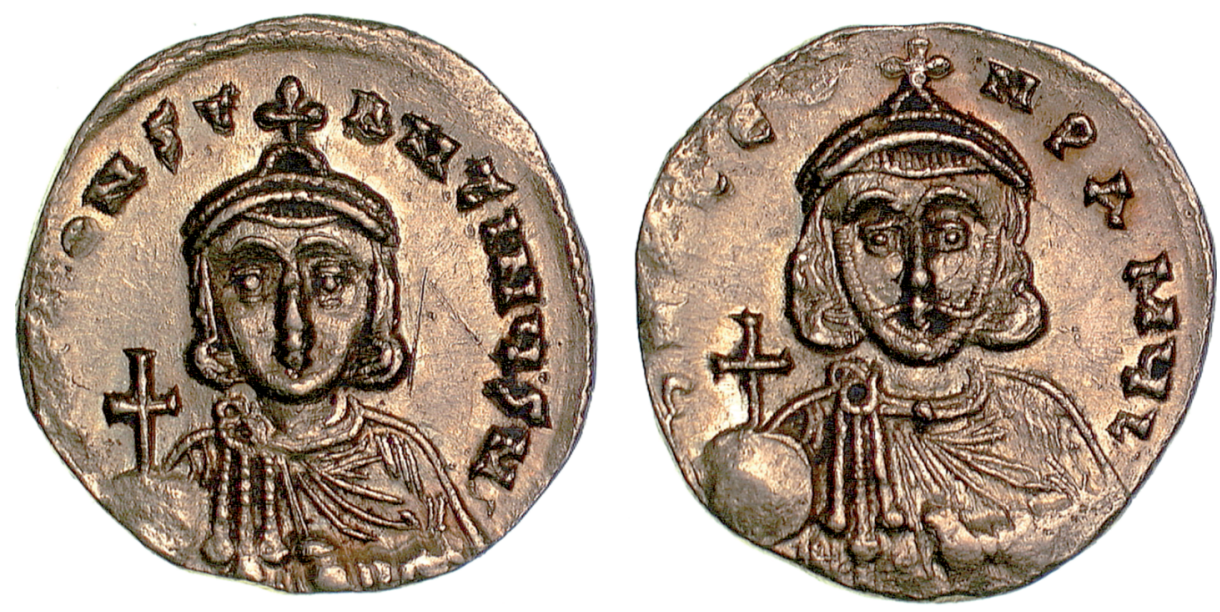

Fig. 14 Leo III and Constantine V, age 2-7 (Birmingham, Barber Institute of Fine Arts, inv. no. B4511); (C) The Barber Institute of Fine Arts, University of Birmingham
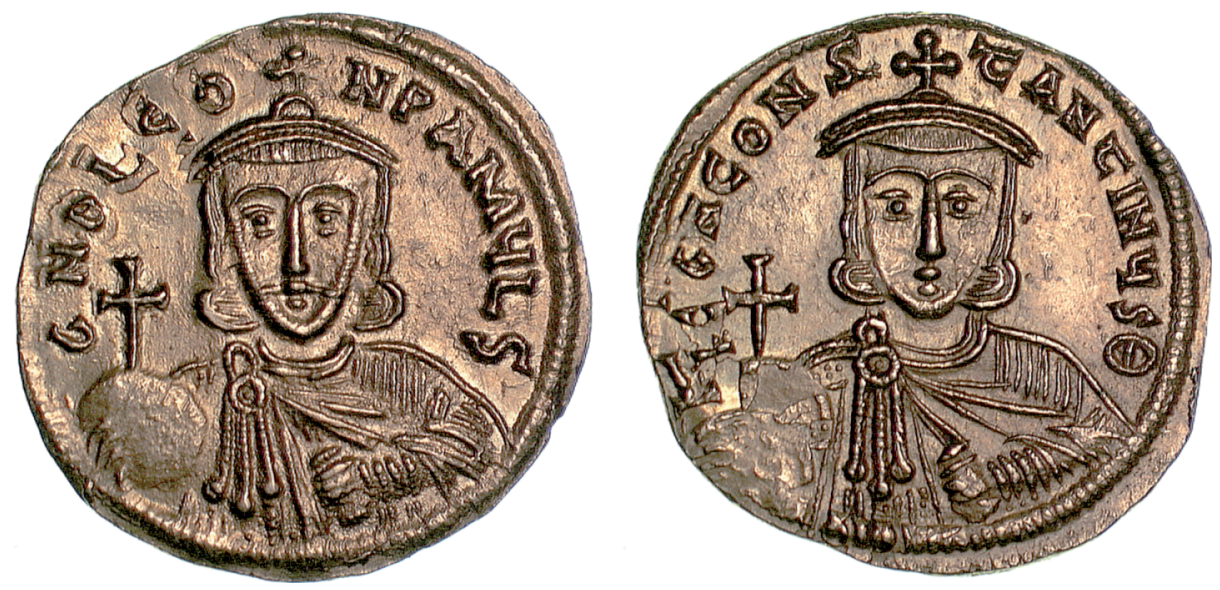

Fig. 15 Leo III and Constantine V, age 14-22 (Birmingham, Barber Institute of Fine Arts, inv. no. B4510); ( The Barber Institute of Fine Arts, University of Birmingham 

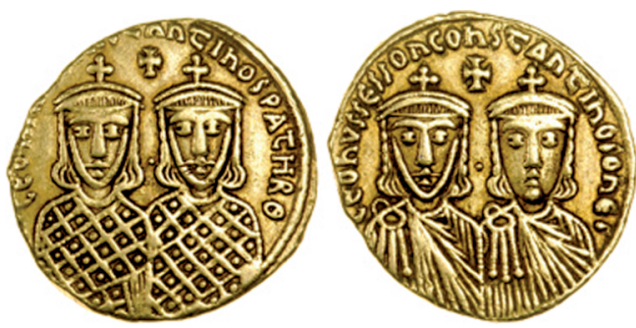

Fig. 16 Leo IV and Constantine VI, age 5 (Birmingham, Barber Institute of Fine Arts, inv. no. B4583); (C) The Barber Institute of Fine Arts, University of Birmingham
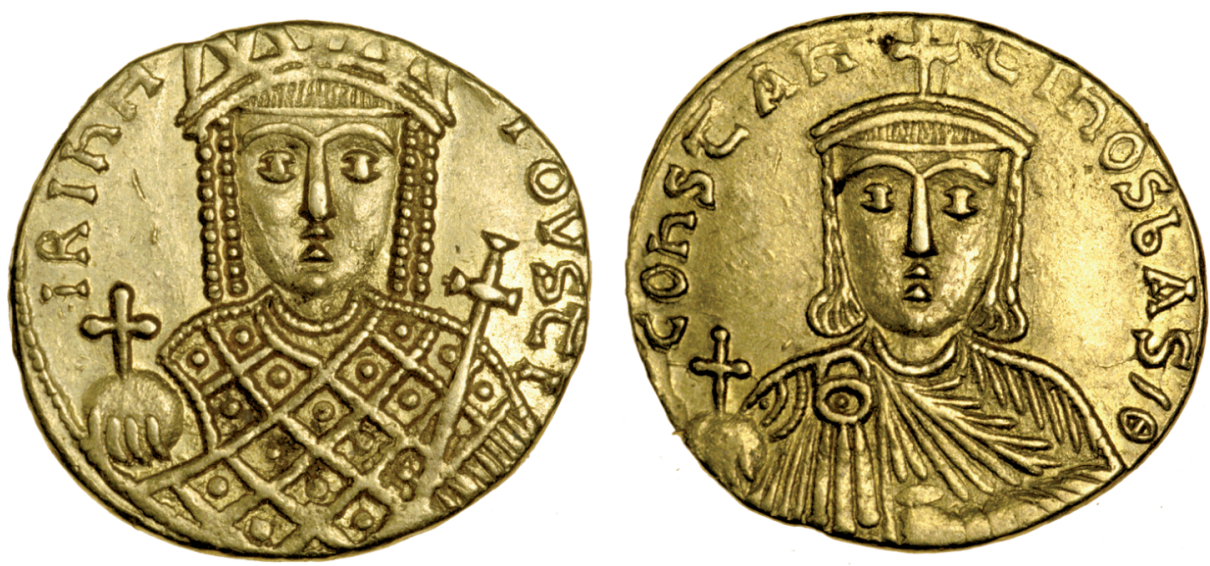

Fig. 17 Constantine VI, age 26 (Birmingham, Barber Institute of Fine Arts, inv. no. B4597); ( The Barber Institute of Fine Arts, University of Birmingham 


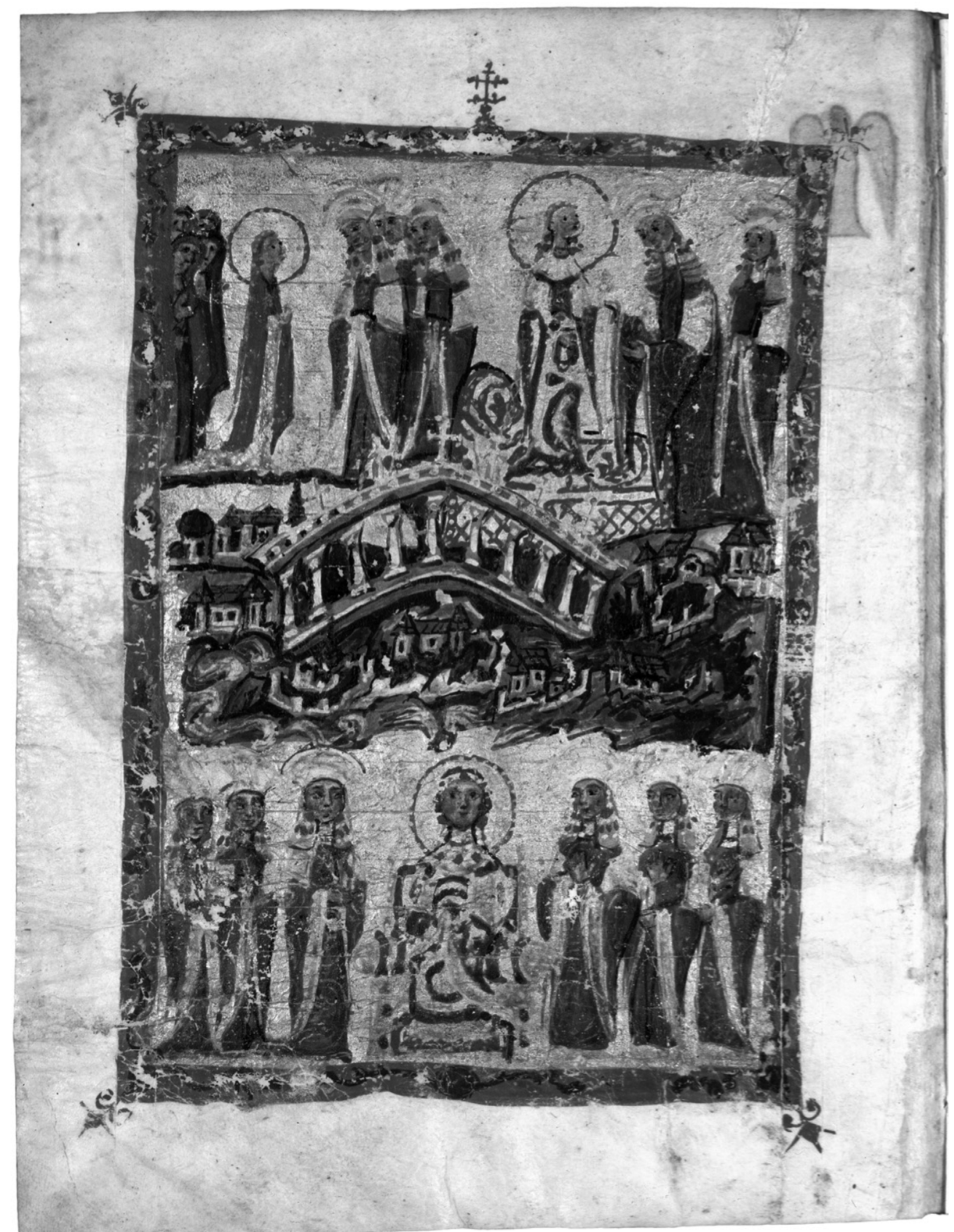

Fig. 18 A foreign princess arrives in Constantinople (Vat.gr.1851, f.3v); @ Biblioteca Apostolica Vaticana 


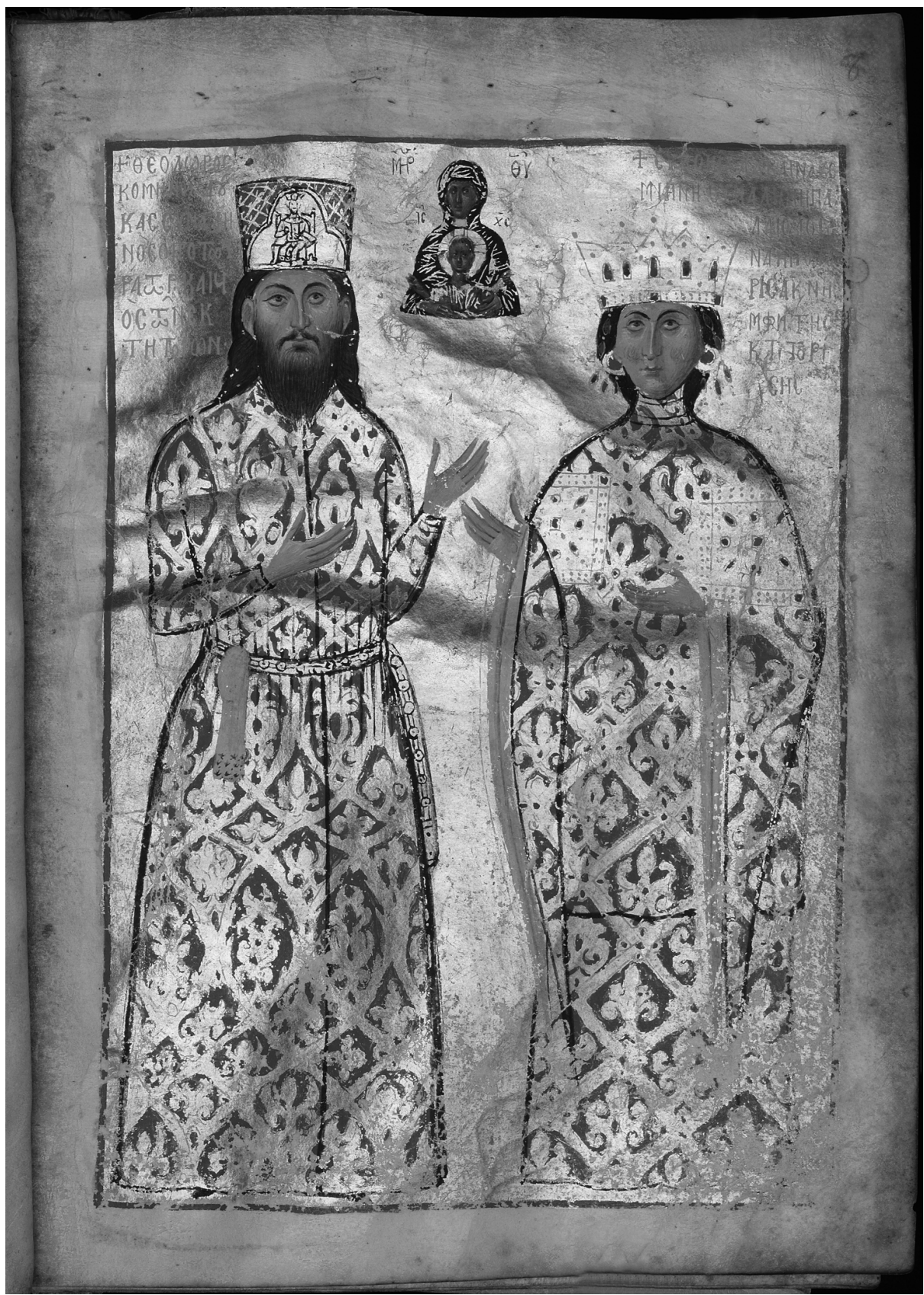

Fig. 19 Theodore Komnenos Doukas Palaiologos Synadenos and Eudokia Doukaina Komnene Synadene Palaiologina (Oxford, Bodleian Library, Lincoln College gr. 35, f.8r); ( Lincoln College, Oxford 


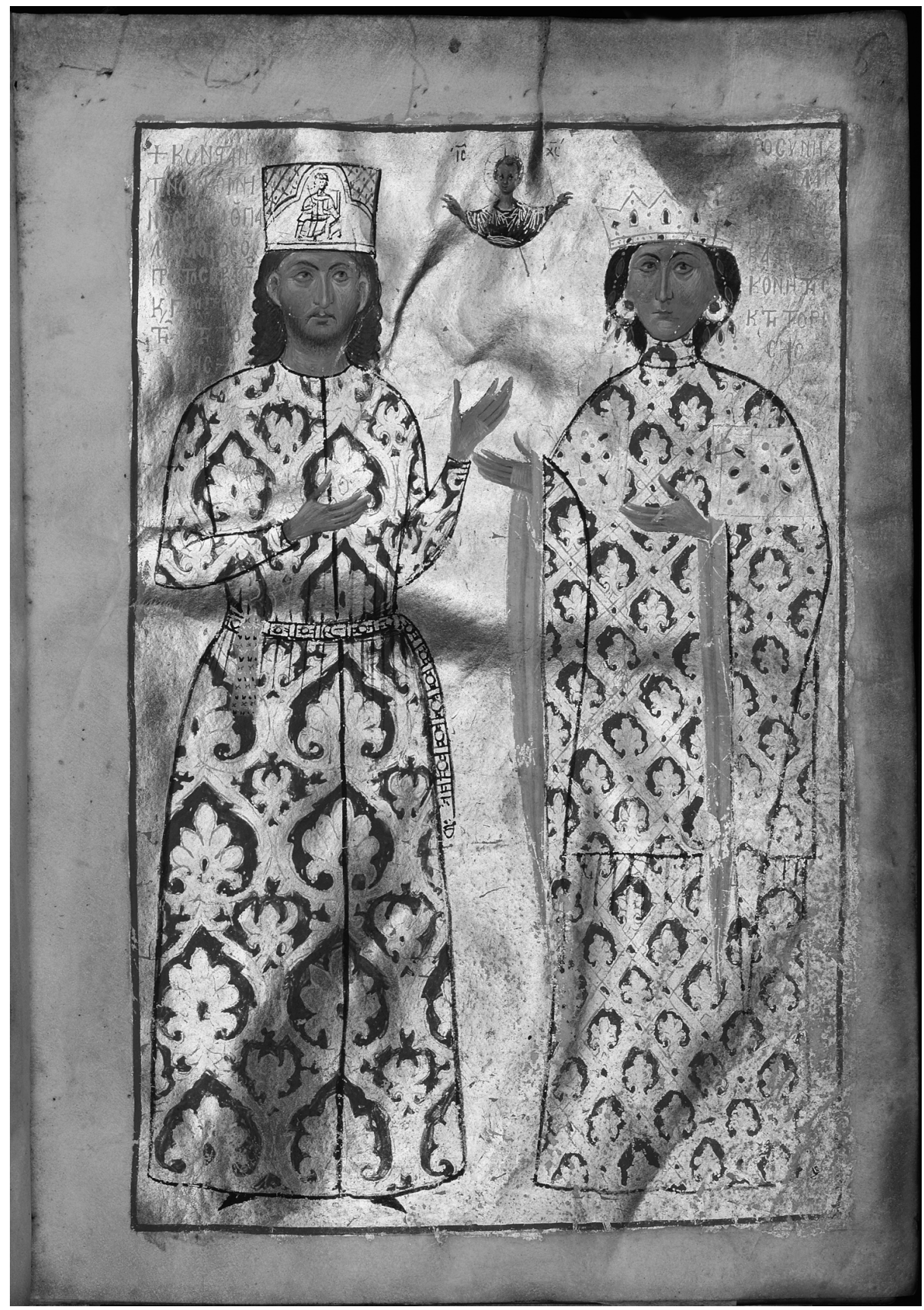

Fig. 20 Constantine Komnenos Raul Palaiologos and Euphrosyne Dukaina Palaiologina (Oxford, Bodleian Library, Lincoln College gr. 35, f.6r); (c) Lincoln College, Oxford 


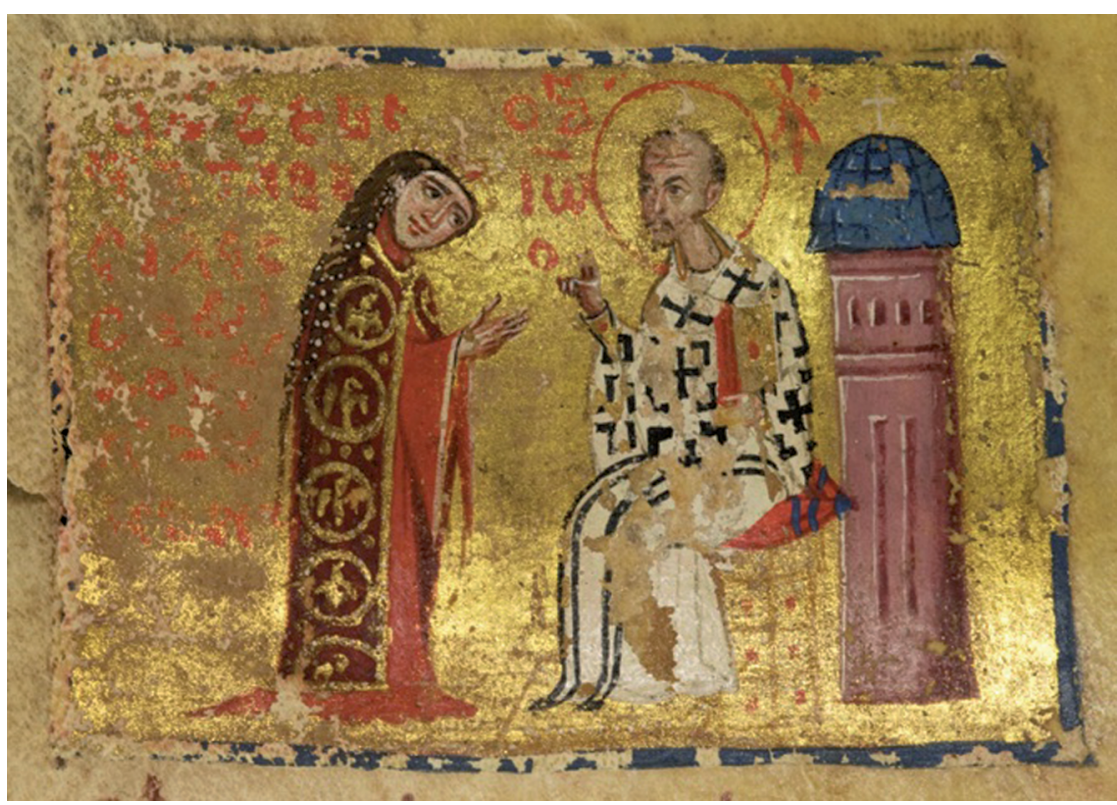

Fig. 21 Eudokia Doukaina Komnene Synadene Palaiologina with John Chrysostom (Private Collection) By permission of the owner 


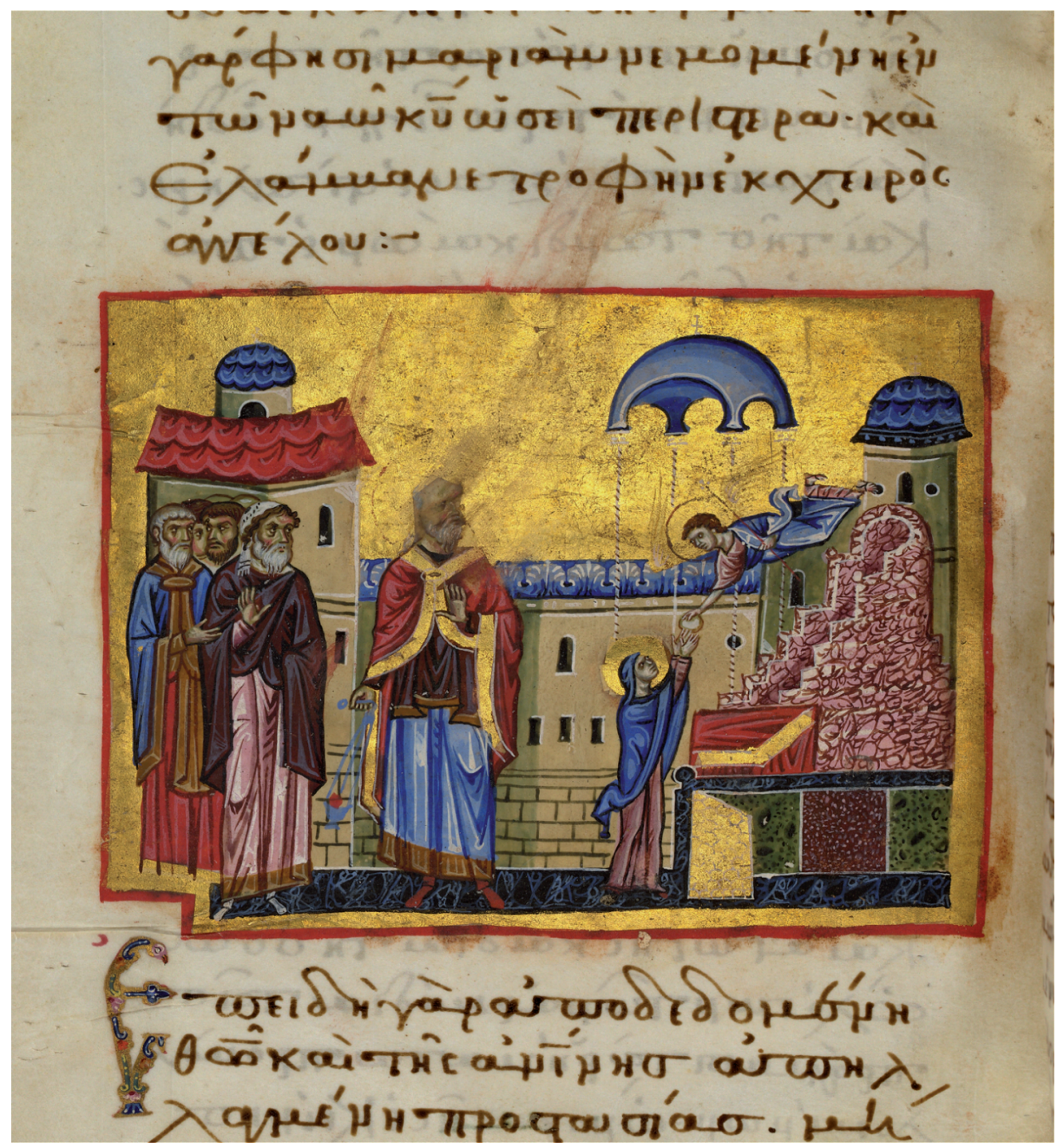

Fig. 22 Mary being fed by an angel in the temple (Paris.gr.1208, f.103v); @ Bibliothèque Nationale de France 


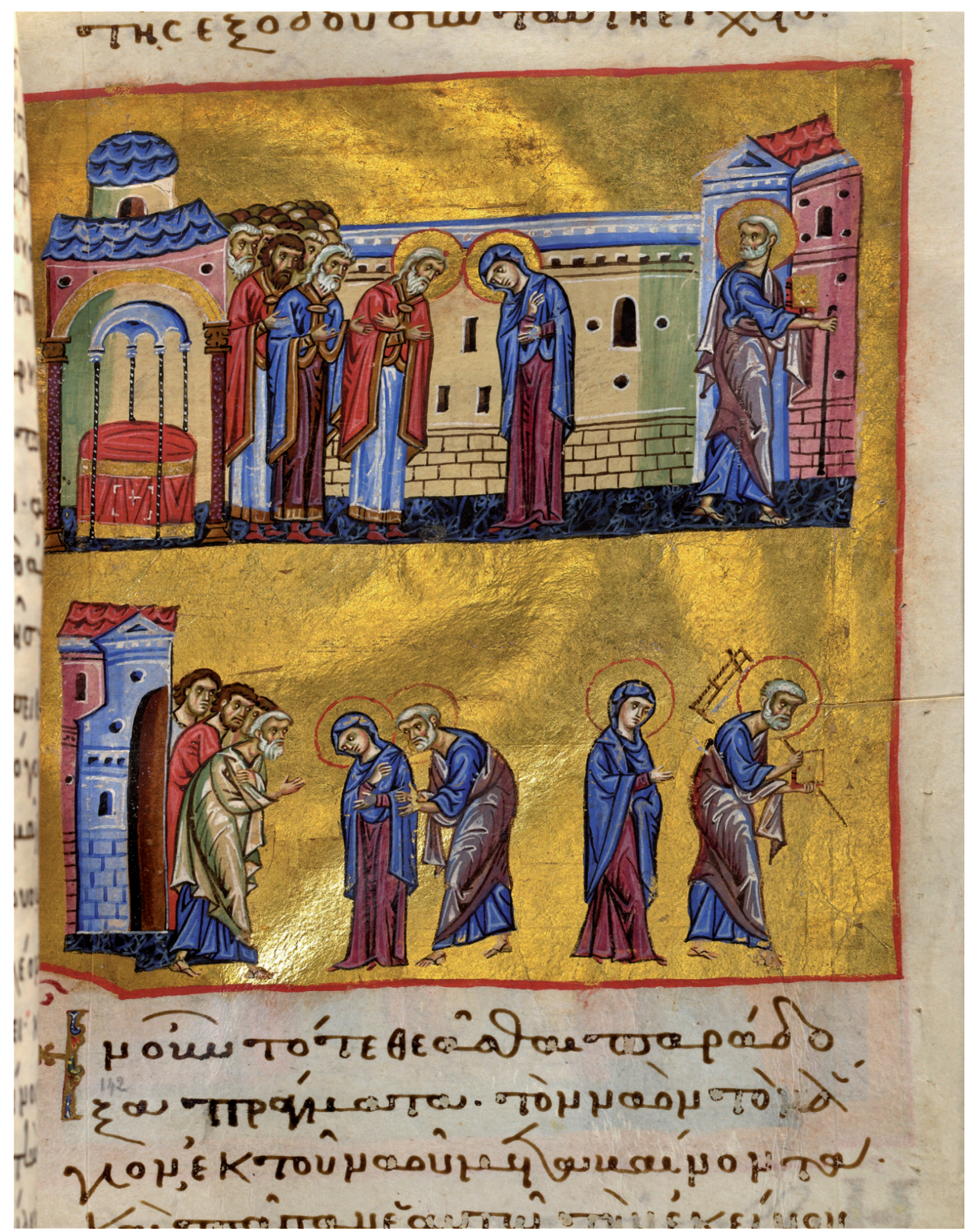

Fig. 23 Mary leaving the temple with Joseph (Paris.gr.1208, f.142r); ( B Bibliothèque Nationale de France 


\section{Representations and Roles of Adolescence with a Focus on Apocryphal Imagery}

The aim of this paper is to explore how adolescents are shown in imagery derived from apocryphal writings. ${ }^{1}$ To set this in context, first, it discusses approaches to interpreting adolescence; second, it looks at some of the complexities of defining adolescence, at a brief survey of legal issues and at areas of responsibility; third, it turns to pictorial representation and reviews how adolescence is depicted in contexts other than apocryphal ones, both biblical and historical. Finally, it focuses on a selection of imagery related to the Virgin, Christ, and their family from apocryphal stories not included in the canonical texts.

Anthropologists have often analysed adolescence as a period of preparation for adulthood, seeing it in adult terms, yet a more recent trend is to focus on the agency of youth and to assess the cultural practice of youth apart from its adult framework. ${ }^{2}$ In a related way, in terms of viewing images, one can focus on the agency of the young and attempt to understand an image from the perspective of the children and adolescents depicted. Yet how should the terms child and adolescent be defined or used and how should the identification of a child or an adolescent be undertaken? The terms are applied in a broad sense to refer to those people who have not reached maturity, yet when does this occur and what conditions identify it? How are such stages in life depicted? Is it possible to visually distinguish a child from an adolescent or an adult, or to differentiate an adolescent from an adult? It is only generally with datable portraiture that one can calculate the age of the child or adolescent depicted and, in most cases, one is left to rely on inferred information to establish age. When some identification of adolescence is achieved then the image can be interpreted through an understanding of the historical setting, with the aim of focusing on the standpoint of youth.

Contemporary definitions of childhood and adulthood differ, geographically and in a range of contexts, and similarly classifications of stages in life in Byzantium appear to have fluctuated and to have had various readings. Present day distinctions

I would like to thank Despoina Ariantzi for inviting me to present this paper and kindly giving me access to her research on adolescence.

1 I am accepting the common use of Apocryphal as those Early Christian writings not accepted into the canon of New Testament texts as compiled mid fourth century. For a definition, see J. K. ELLIOTT, The Apocryphal New Testament: a collection of apocryphal Christian literature in an English translation. Oxford 1993, xi-xiii.

2 See, for instance, M. Bucholtz, Youth and Cultural Practice. Annual Review of Anthropology 31 (2002) 525-552, here 525.

Ә OpenAccess. (c) 2018 Cecily Hennessy, published by De Gruyter. (c) BY-NC-ND This work is licensed under the Creative Commons Attribution-NonCommercial-NoDerivatives 4.0 License. 
between maturity and immaturity are altered in certain legal and moral contexts, as well as societal ones. Society is comfortable with this multifaceted view, though it is often discussed, and childhood or adolescence is not perceived as a defined entity. In Byzantium, there are a range of terms used for those between childhood and adulthood. ${ }^{3}$ There is a tendency to think that we should be able to make hard and fast distinctions in historical cultures and yet in them we see the same variable use of terminology, regulations and expectations. In present culture, adolescence is often associated with a period of adjustment, alienation or rebellion, but there is no justification in necessarily transferring such associations to a historical setting. Adolescence should perhaps be seen as a period of physical, cognitive and societal change with a recognition that humans continue changing in all these ways, though perhaps less rapidly, throughout their lives. ${ }^{4}$

Byzantine religious and secular law epitomised the manifold interpretations of maturity in children. Boys and girls were deemed to gain religious maturity at the age of ten, although this was not applied consistently, and it was only at the age of twelve that they had to confess before taking communion, implying perhaps that before then they were not responsible for their actions, although there were several variances on this rule. ${ }^{5}$ Byzantine law followed Roman in determining that puberty began at the age of fourteen for boys and twelve for girls, and at this time they could marry. ${ }^{6}$ Not strictly adhered to, however, children could be betrothed and married younger than this and at the age of seven they were deemed responsible to approve a prospective marriage. ${ }^{7}$ They remained minors until the age of twenty-five, subject to the authority of a legal guardian, unless released by special appeal. ${ }^{8}$

3 For recent analysis on the terminology and understanding of adolescence in Byzantium, see D. ARIANTZI, Terminologische und sozialhistorische Untersuchungen zur Adoleszenz in Byzanz (6.11. Jahrhundert). Tel I. Theorien, Konzepte narrative Quellen. JÖB 31 (2013) 1-31, with a summary of terms $30-31$.

4 See Ariantzi, Adoleszenz 14-28.

5 On this, see E. Patlagean, L'enfant et son avenir dans la famille byzantine (IVe-XIIe siècles). Annales de démographie historique (1973) 85-93, here 88 [= Structure sociale, famille, chrétienté à Byzance IVe-XIe siècle (Variorum Reprints) London 1981, Nr. X]; on communion, see Nikolas Grammatikos (ed. V. GRUMEL, Les regestes des actes du patriarcat de Constantinople [Le patriarcat byzantin; série 1, Vol. 1, part 3, Les régestes de 1043 à 1206]. Paris 1947, no. 995, paragraph 10).

6 Codex Justinianus V. IV. 24 (dated 530) (eds. P. KRUEGER - T. MommsEn, Corpus iuris civilis: Institutiones, Digesta, Codex Justinianus, Novellae. 3 vols. Berlin 1928); Prochiron IV. 3 (ed. E. H. FrESHFIELD, A Manual of Eastern Roman Law: The Procheiros Nomos, Published by the Emperor Basil I at Constantinople, between 867 and 879 A.D. Cambridge 1928); some texts, such as Ecloga II. 1 state the ages as fifteen and thirteen (ed. E. H. Freshfield, A Manual of Roman Law: The Ecloga Published by the Emperors Leo III and Constantine V of Isauria at Constantinople AD 726. Cambridge 1926).

7 Ecloga I. 1 and repeated in the Prochiron I. 7.8; in: E. PatLagEAn, L'entrée dans l'âge adulte à Byzance au XIIIe-XIVe siècles, in: L'Historicité de l'enfance et de la jeunesse. Paris 1986, 263-270, here 265; Patlagean, L'enfant 87-89.

8 See Patlagean, L'entrée 264-5; G. Prinzing, Observations on the Legal Status of Children and the Stages of Childhood in Byzantium, in: Becoming Byzantine. Children and Childhood in Byzantium, 
Adolescence was a period in which much responsibility could be granted. Byzantine children often participated in work, either at home (particularly the girls), or in a family workshop or beyond the family arena. ${ }^{9}$ Frequently, young men and women took on obligations at an early age. However, boys from affluent families appear to have had, in certain contexts, an extended period of what we would call adolescence, during which they were educated, before they assumed full adult responsibility. This is less apparent in girls who often married young and apparently participated in adult life in terms of bearing children. But perhaps a period of cultural adolescence in Byzantium could be experienced by young mothers? Issues such as this are very hard to determine. However, imperial practice gives an indication of cultural attitudes, at least in court circles, to responsibility. Several emperors came to the throne when adolescent. Sixteen seems the most acceptable age for a boy to succeed, although sometimes with the assistance of an overbearing mother or adviser. ${ }^{10}$ To give examples of how imperial adolescents could have many commitments, John $\mathrm{V}$ was aged nine when he succeeded to the throne with regents and fifteen when he married. ${ }^{11}$ His first son, Andronikos IV, was born in 1348 when he was aged sixteen. ${ }^{12}$ Andronikos was then made co-emperor in 1355 aged seven and the next year he married Maria, the nine-year-old daughter of the tsar of Bulgaria, when John V himself was still only twenty-four. ${ }^{13}$

Having summarised some of the complications in defining adolescence, for the sake of clarification, one could argue that a biological approach is the least subjective and, although ages of puberty vary historically and geographically, that the term child should be used for those who are generally below the onset of puberty, that is twelve for girls and fourteen for boys (the Ancients and the Byzantines recognised that girls matured earlier than boys), and the term youth or adolescent for those beyond that age. However, the age of twelve in cultural terms for both boys and girls indicates a transition to increased responsibility and is perhaps a more judicious choice. Adolescence may be seen as continuing until the age of majority, twenty-

\footnotetext{
ed. A. Papaconstantinou-A.-M. Talbot (Dumbarton Oaks Byzantine Symposia and Colloquia). Washington D.C. 2009, 15-34; D. ARIANTZI, Kindheit in Byzanz. Emotionale, geistige und materielle Entwicklung im familiären Umfeld vom 6. bis zum 11. Jahrhundert (Millennium-Studien 36). Berlin-Boston 2012, 41-47.

9 About working children in the framework of the family see D. ARIANTZI, Kinderarbeit in Byzanz im Rahmen der Familie auf Grund der hagiographischen Quellen vom 6. bis zum 11. Jahrhundert, in: Laetae Segetes Iterum. Brno 2008, 405-413; EADEM, Kindheit 135-141; Y. Rotman, Working kids. Forthcoming.

10 For further examples and references, see C. Hennessy, Images of Children in Byzantium. Farnham - Burlington 2008, 25-6.

11 A. T. Papadopoulos, Versuch einer Genealogie der Palaiologen, 1259-1453. Speyer 1938, no. 73.

12 Papadopoulos, Versuch no. 81

13 On the date of Andronikos's coronation, see A. LUTTRELL, John V's Daughters: A Palaiologan Puzzle. DOP 40 (1986) 103-112, here 104.
} 
five, but it is necessarily a flexible time period, with variations for boys and girls and children from different economic and cultural backgrounds. ${ }^{14}$

Turning to the pictorial depiction of adolescence, I would like to review three aspects; first, beauty and its relation to youth, second, Christ' youth, and third, imperial offspring in their youth. I have looked at parts of this material before in the context of the depiction of children, perhaps justifiably as childhood and adolescence as discussed are challenging to delineate. ${ }^{15}$ However, a further appraisal is helpful in refining and defining aspects of adolescence and its connotations and, in relation to girls, my views have changed. ${ }^{16}$

However, before looking at the depiction of adolescence in more detail, it is useful to establish some principal tenets about the depiction of youth and age in Byzantium and to recognise that there are certain formulas. For instance, regarding the portrayal of individual prophets, apostles and saints, as is well-known, Byzantine artists tended to adhere to an iconographic tradition. These figures are usually shown consistently as old, young, or middle aged with uniform features. In Dionysius of Fourna's eighteenth-century description of the appearance of the twelve apostles, the four evangelists and the seventy apostles, the main iconographic feature supplied is the absence or length of the beard, followed by the colour of the hair. ${ }^{17}$ Dionysius does not refer to age, but the presence of white hair and a long beard must indicate old age and the absence of a beard implies youth, as for the most part the men are not eunuchs.

There are numerous examples in various media, metalwork, ivories, panel paintings, mosaics, wall paintings and manuscripts in which the iconography is often very consistent, suggesting the practitioners shared iconographic and stylistic devices. This essay uses examples from several media but to give some continuity often returns to illuminations from an eleventh-century lectionary, Dionysiou, cod. gr. 587 m., which was probably made in Constantinople. ${ }^{18}$ In this manuscript, the apostles are shown at various ages and can be identified by comparisons with other examples. For instance, in the scene of the Washing of the Disciples' Feet, Peter and Andrew are depicted advanced in years (Peter is next to Christ and Andrew

14 For Patlagean, boys from the ages of 14 to 25 were 'jeunes gens' (neoi), see PATLAGEAN, L'entrée 264.

15 Hennessy, Children 3, 6, 7, 11, 14, 35, 36-7, 38, 39, 42, 53, 62-3, 73, 91, 107, 180, 199, 210, 214 and also under specific topics.

16 See Hennessy, Children 68.

17 In translation, P. Hetherington, The Painter's manual of Dionysius of Fourna: an English translation, with commentary, of cod. gr. 708 in the Saltykov-Shchedrin State Public Library, Leningrad. London 1974, 52-59. While this text is late in date, it indicates the traditions.

18 Kurt Weitzmann has argued that it was most likely made ca. 1059 in Constantinople and commissioned by Isaac Komnenos for the St John Stoudios monastery since it has several images relating to St. John the Baptist, whose head was held at the monastery at that time, although there is no proof of this; see K. WeITZMAnN, The Narrative and Liturgical Gospel Illustrations, in: Studies in Classical and Byzantine Manuscript Decoration, ed. H. Kessler. Chicago 1971, 247-270, here 269. 


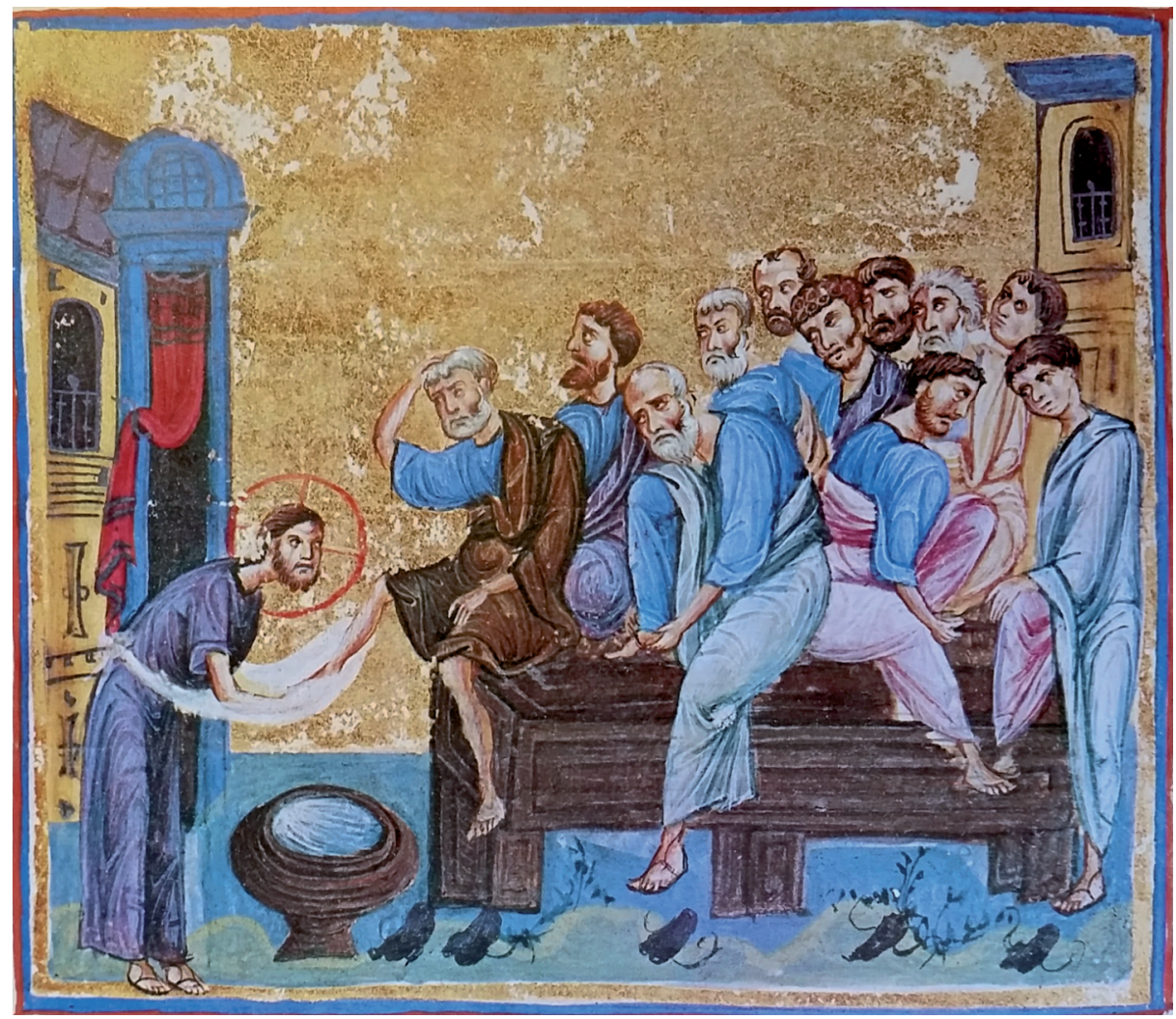

Fig. 1 Christ washing the disciples' feet, Athos, Dionysiou, cod. gr. 587 m, fol. $52 r$ (Source: Treasures of Mount Athos: illuminated manuscripts. 1. The Protaton and the monasteries of Dionysiou, Koutloumousiou, Xeropotamou and Gregoriou [Treasures of Mount Athos: Illuminated manuscripts 1] Athens 1974, fol. 52r, fig. 223, p. 439)

is nearby taking his sandal off), and John and Philip are typically young (John is at the far right and Philip just above him), beardless and boyish, even one might say adolescent (fig. 1). ${ }^{19}$ Judas is also shown as young, as for example in the same manuscript in the Betrayal scene. ${ }^{20}$

Often a feature of youth is beauty. It is striking how many representations of male saints, and a few Old Testament figures also, are shown as consistently young and good looking (clearly a subjective assessment, but appearing fine featured and well-formed). It seems that there was a cultural affinity towards aesthetically pleasing males who had not yet assumed facial hair and a look of maturity, what

19 Treasures of Mount Athos: illuminated manuscripts. 1. The Protaton and the monasteries of Dionysiou, Koutloumousiou, Xeropotamou and Gregoriou. (Treasures of Mount Athos: Illuminated manuscripts 1) Athens 1974, fol. 52r, fig. 223, p. 439.

20 Treasures of Mount Athos, vol. 1, fol. 104v, figs. 233-4, p. 440. 


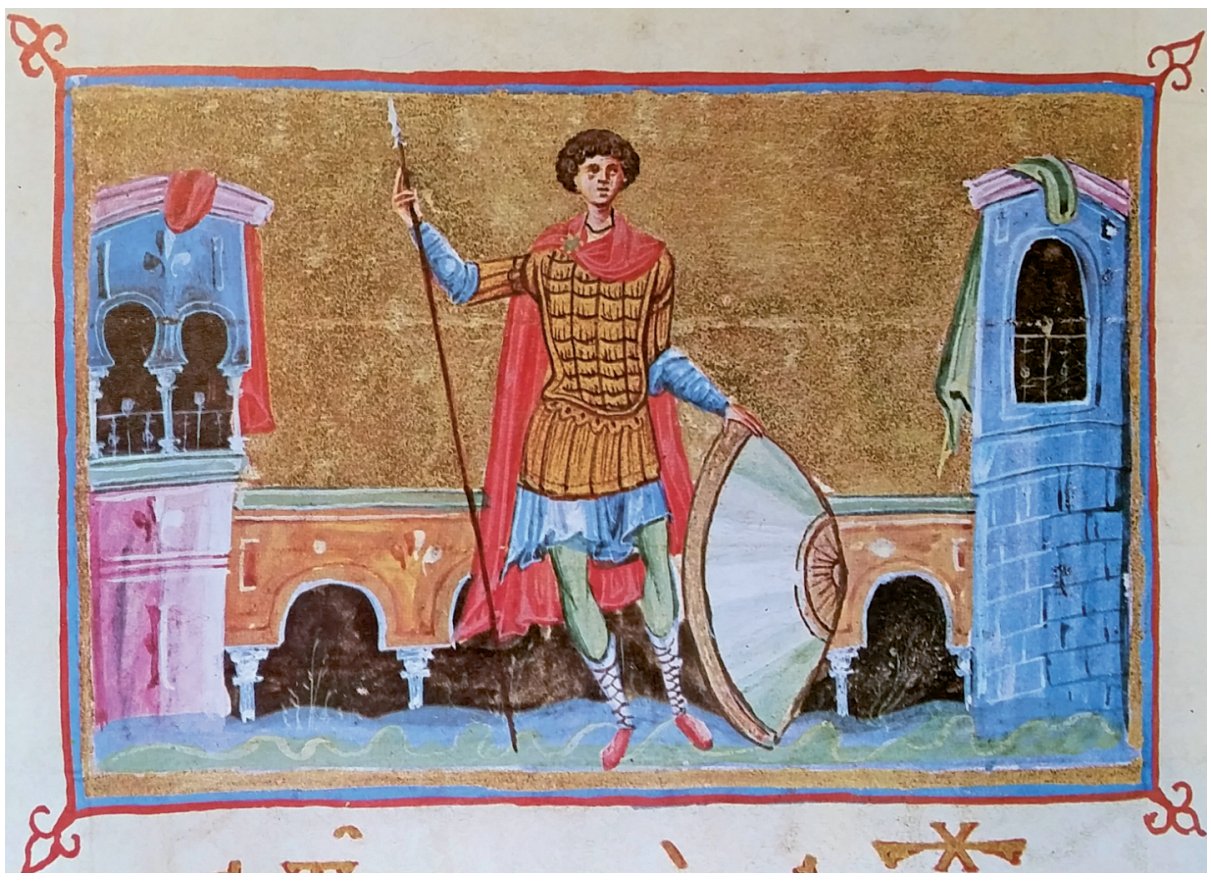

Fig. 2 Saint George, Athos, Dionysiou, cod. gr. 587 m, fol. 151v (Source: Treasures of Mount Athos, vol. 1, fol. 151v, fig. 265 , p. 444)

one might potentially call adolescence. The beardless face, smooth skin and short hair indicate youth. In literature, beauty is associated with closeness to God, and often it is also linked with youth. The martyrs Demetrios, George, Prokopios, Panteleimon, Sergios and Bakchos are all shown as young, perhaps to convey the idea that their lives were cut short for their love of Christ. ${ }^{21}$ Males served in the army from a young age, often from sixteen, so a youthful warrior saint is not necessarily incongruous. One could argue that these figures are aged between sixteen and twenty five, the age of maturity. To return to the lectionary, Dionysiou cod. gr. 587 m., in individual standing portraits of the saints, George is shown as young and elegant and Demetrios as very youthful (fig. 2). ${ }^{22}$ In the mid-twelfth-century church dedicated to Saint Panteleimon at Nerezi, several young male saints are exquisitely and attractively portrayed. The church was built in 1164 by a grandson of Alexios I Komnenos, named Alexios Komnenos Angelos. Panteleimon himself is positioned to the right of the apse and appears very young, delicate and sensitive, with pale skin, scarcely more

21 For George, see $B H G$ I, 669y-691y; for Prokopios, $B H G$ II, 1576-1584; for Panteleimon, $B H G$ II, 1412z-1418c; for Sergios and Bakchos, BHG II, 1624-25; on male beauty in Byzantium, see M. HATZAKI, Beauty and the Male Body in Byzantium: Perceptions and Representations in Art and Text. New York 2009.

22 Treasures of Mount Athos, vol. 1, fol. 151v, fig. 265, p. 444; fol. 123r, fig. 241, p. 441. 
than a boy, not yet having reached manhood. ${ }^{23}$ It is apparent that beauty is associated with spiritual purity and with youth through texts. Panteleimon's Vita draws attention to his youth and his great beauty. ${ }^{24}$ Also, Bishop Theodore of Edessa is described as elegant, with a comely body, cheeks 'just blooming with down', which show the beauty of his soul. ${ }^{25}$ It is this stage in life, verging on manhood but still in boyhood, that seems to be attractive.

This beauty has threatening aspects. There are many texts that warn against the presence of boys and young men in monasteries for fear of tempting the monks, indicating that child and adolescent boys were viewed as sexually attractive. This cautioning stems from early typika, such as Christodoulos' foundation typikon for St. John on Patmos. ${ }^{26}$ Registering that boys and beardless young men as well as girls and young women were seen as sexually tempting, the question must arise whether the images of the young male saints depict a sexual element of attractiveness, perhaps to both men and women. As far as I know, there are no texts that describe such images as having sexual appeal, but then such references may have been taboo. ${ }^{27}$

Other cases suggest an attraction towards youth, since certain religious figures are depicted as much younger than indicated in the main texts about them. For instance, Moses is often portrayed as a very young man, with a hairless face, youthful features and a general air of grace and loveliness. This occurs from the very earliest images in the catacombs and on sarcophagi. However, in other early instances he is shown as either young and bearded or as mature. ${ }^{28}$ Two thirteenth-century icons from Saint Catherine's monastery in Sinai, showing Moses before the burning bush and receiving the tablets of the law, depict him as very youthful and, in the latter, in Doula Mouriki's words, 'as almost an ephebe'. ${ }^{29}$ In the first, untying his sandal before the burning bush, he has a smooth, serious face and short slightly untidy hair but an apparently strong body. In the second, reaching up to God to receive the tab-

23 See I. Sinkević, The Church of St. Panteleimon at Nerezi: architecture, programme, patronage. Wiesbaden 2000, fig. 69.

24 PG 115:448.

25 See BHG II, 1744, 14.11-15, 18.20 - 23; in A. KAZHDAN - H. MAGUIRE, Byzantine Hagiographical Texts as Sources on Art. DOP 44 (1991) 1-2.

26 F. R. MikLosich - I. MÜLler, Acta et diplomata graeca medii aevi sacra et profana collecta, vol. 6. Patmos. Vienna 1860 -90, rep. 1968, 65; J. P. ThomAS - A. C. HERo, Byzantine monastic foundation documents: A complete translation of the surviving founders' typika and testaments (Dumbarton Oaks Studies 35). Washington, DC 2000, A10, 583.

27 On sexuality in hagiography, see ARIANTzI, Adoleszenz 20.

28 The earliest example may be in the synagogue at Dura Europas, see C. H. KrAELING, The Synagogue (The Excavations at Dura Europas. Final Report VIII). New Haven - London 1956, plate 76. For numerous examples of various types, see Lexikon der christlichen Ikonographie, vol. 3. Rome - Freiburg - Basel - Vienna 1971, 283.

29 D. MourikI, A Pair of Early 13th-century Moses Icons at Sinai with the Scenes of the Burning Bush and the Receiving of the Law. Deltion 16 (1992) 171-184, here 178. 
lets, his face is equally serious, but slightly more rounded, his body is entirely enveloped in his garment and he appears boyish. Other Old Testament figures traditionally represented as young and attractive are Daniel and Solomon.

A further figure who without textual basis is consistently shown as a youth is Prochoros, traditionally one of the deacons assigned to take care of the poor (Acts 6:5), and depicted as the scribe for John the Evangelist on Patmos. This tradition is associated with the Acts of John of Prochoros, an apocryphal text, and so perhaps should come later in the discussion but is placed here in the context of youthful male saints. ${ }^{30}$ Kurt Weitzmann has suggested that the image was created for the Vita of John by Symeon Metaphrastes at the end of the tenth century. ${ }^{31}$ From the menologion it was incorporated into lectionaries and Gospel Books. ${ }^{32}$ In this and all pairings of John and Prochoros, John is shown as very old and Prochoros as a tender adolescent. An example from a menologion is in an eleventh- or twelfth -century manuscript, BL Add. Ms. 11870, where Prochoros sits at John's feet inscribing the words received by John as the old man looks up to the hand of God. ${ }^{33}$ An example from a lectionary, in which John and Prochoros appear in the first evangelist portrait, is in Dionysiou cod. gr. $587 \mathrm{~m}$. (fig. 3). ${ }^{34}$ Prochoros is seated on a red cushion on the left as he writes on a scroll. He wears a blue chiton and white himation with a purple sash. His hair is short, though longer in the back, and his youth is emphasised by a narrow neck and perhaps a touch of downy hair on his chin. In contrast, John, standing on the right and looking back and up to God's hand is white haired, balding and bearded. An example from a Gospel Book is in the thirteenth-century, Dionysiou, cod. 4, where a very small Prochoros is seated humbly beneath John's pointing hand, recording his words that are being inspired from on high. Prochoros is slight, with short brown hair and wears a long blue chiton and green himation. ${ }^{35}$ On the facing page, they again appear together, similarly aged and youthful, in an initial on the opening page of John's Gospel. ${ }^{36}$ While clearly not exclusively found in monastic manuscripts, the pairing seems to be particularly popular in them. It perhaps serves to idealise the elderly and youthful male relationship that was forbidden in monasteries, but also shows the significant role of scribe held by an adolescent. In accordance with other young saints, Prochoros is attractively depicted with fine features and a graceful air.

30 See ElLiotT, Apocrypha 303, 347. The text is dated to the fifth or sixth century and recounts John's miracles on Patmos.

31 K. Weitzmann, The Constantinopolitan Lectionary, Morgan 639, in: Studies in art and literature for Belle da Costa Greene, ed. E. D. Miner. Princeton 1954, 358-73 and plates after 374, p. 373. 32 Weitzmann, Morgan 639, p. 363.

33 Fol. 197v, WeitzmanN, Morgan 639, fig. 292; also: http://www.bl.uk/manuscripts/Viewer.aspx?ref= add_ms_11870_f197v.

34 Treasures of Mount Athos, vol. 1, fol. 1v, fig. 189, p. 435.

35 Treasures of Mount Athos, vol. 1, fol. 278v, fig. 25, p. 395.

36 Treasures of Mount Athos, vol. 1, fol. 279r, fig. 27, p. 396. 


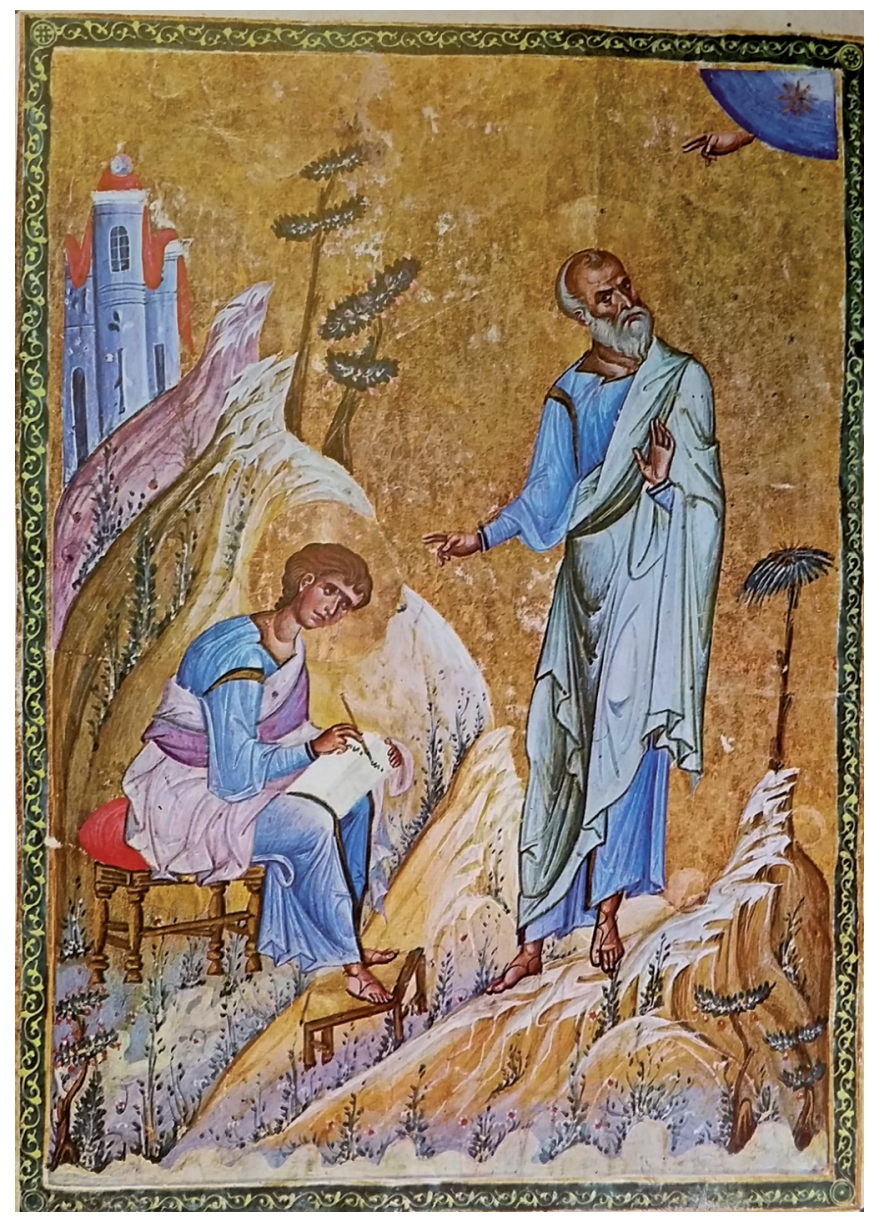

Fig. 3 Saints John and Prochoros, Athos, Dionysiou, cod. gr. 587 m, fol. 1v (Source: Treasures of Mount Athos vol. 1, fol. 1v, fig. 189, p. 435)

As with the male saints, texts often emphasise the beauty of female saints and this beauty is also associated with being young. In images, determining the age of the sanctified girls and women is often problematic. They tend to be portrayed with no signs of aging, perhaps just a certain maturity conveyed through the covering of their hair, but except in unusual cases, with an air of timelessness. Typical examples are the various female saints depicted on the west wall of the narthex at Hosios Loukas, probably datable to the eleventh century, which include Saints Anastasia, Thecla, Febronia, Eugenia, Agatha, Irene, Catherine, Barbara, Euphemia, Marina and Juliania, all relatively young and attractive with smooth skins and large eyes. Could these male and female examples suggest that there are certain conventions in depicting idealised beauty which perhaps can be defined as adolescence? 
Turning to portrayals of Christ, the only canonical event that occurs in his adolescent years is when, at the age of twelve, he goes up to Jerusalem with his parents and teaches the doctors in the temple (Luke 2:41-50). The age of thirteen, in Judaism now linked with the Bar Mitzvah, was from the first or second century associated with the age of majority in respect to following the Torah. In this sense he is gaining moral responsibility. As mentioned, in Byzantine society twelve was, in some contexts, the age that children assumed moral accountability. Christ teaching in the temple is not a particularly widespread image. Perhaps the earliest extant example is in Paris, cod. gr. 510, a manuscript in which many of the Old Testament figures are shown as adolescent. ${ }^{37} \mathrm{~A}$ further early example is in Tokalı New Church in Göreme, Cappadocia, dated to about 960, in which the painting is damaged but Christ looks mature, having a substantial body and robust knees with no reference to adolescence. ${ }^{38}$ In this scene in Dionysiou, cod. gr. 587 m., a young Christ with short hair and round face, dressed in dark and mid-blue sits centrally on a curved marble bench like a synthronon and teaches four older men, two on each side. He is depicted with the puer senex features, with his hairline slightly receding to suggest a maturity beyond his years. ${ }^{39}$ A later example is in St Nicolaos Orphanos in Thessaloniki, dated to the fourteenth century, where the scene is shown in relation to the Akathistos Hymn. ${ }^{40}$ Christ is seated looking youthful, with a full head of short hair and delicate features.

Particularly from the eleventh century, this visual appreciation of youth extends to images of the adolescent Christ in the form known as Christ Emmanuel. For instance, in Dionysiou cod. gr. 587 m., a boy Christ is employed in an initial letter omicron. ${ }^{41}$ Dressed in a blue chiton and purple himation, he again has puer senex features but still seems very young, perhaps about aged ten (fig. 4). In another initial, the letter $\mathrm{T}$ at the beginning of the Gospel of Luke, he appears once more in the

37 Fol. $165^{\mathrm{r}}$, L. BRUBAKER, Vision and meaning in ninth-century Byzantium: image as exegesis in the homilies of Gregory of Nazianzus. Cambridge 1999, fig. 21, 83-6; for further discussion, see E. ANTO-

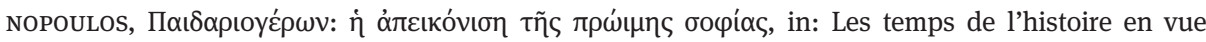
d'une histoire de l'enfance et de la jeunesse. Athens 1998, 215-231, on the scene, 219 and on Paris cod. gr. 510, 225.

38 G. DE JERPHANION, Une nouvelle province de l'art byzantin: les églises rupestres de Cappadoce (Bibliothèque archéologique et historique 5-6). 5 vols. in 7 parts. Paris 1925-1942, vol, 2.1, 332-3; A. Wharton Epstein, Tokalı Kilise: Tenth-century Metropolitan Art in Byzantine Cappadocia. Washington, DC 1986, figs. 66-7. Jerphanion points out that Christ looks adult and suggests this is because two scenes are confused, Luke 2:42 and John 7:10, the first when he goes to Jerusalem with his parents, the second when he goes as an adult; see JERPHANION, Églises rupestres 333, fn. 2.

39 Treasures of Mount Athos, vol. 1, fol. 135r, fig. 252, p. 443; on the puer senex, see ANTONOPOULOS,

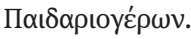

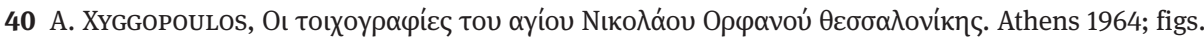
101, 103. On the Akathistos hymn, see J. LAFonTAINE-Dosogne, Iconography of the cycle of the infancy of Christ, in: The Kariye Djami, ed. P. Underwood. New York, 1966, 4 vols., vol. 4, 201, fn. 30. 41 Treasures of Mount Athos, vol. 1, fol. 14v, fig. 200, p. 436. 


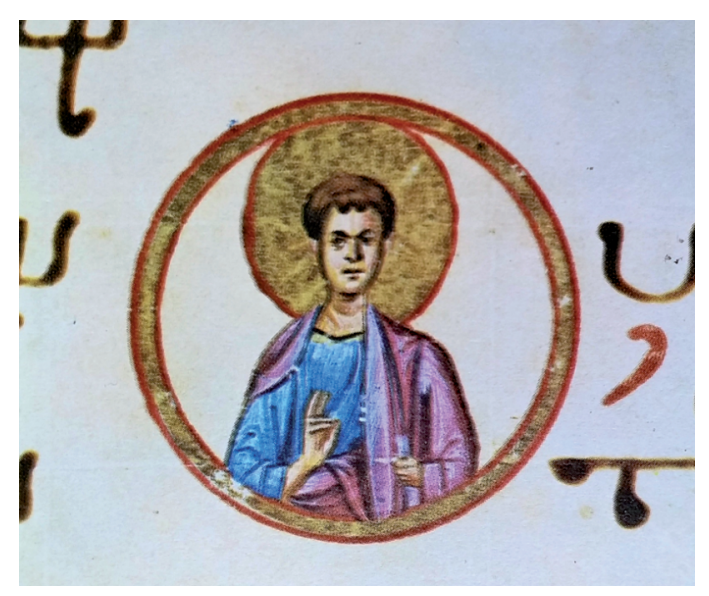

Fig. 4 Christ Emmanuel, Athos, Dionysiou, cod. gr. 587 m, fol. $14 \mathrm{v}$ (Source: Treasures of Mount Athos vol. 1 , fol. $14 \mathrm{v}$, fig. 200 , p. 436 )

same clothes. ${ }^{42}$ Other examples from about the same date are in the domes at Elmal and Karanlık churches in Cappadocia, Göreme, dated probably to the mid-eleventh century. $^{43}$

Turning to representations of imperial youth, there are several portrait examples that show the sons of emperors in adolescence, such as the mosaic figure of Alexios, the eldest son of John II, on the southeast wall of the upper gallery at Hagia Sophia (fig. 5). ${ }^{44}$ This mosaic, displaying budding manhood, was probably made in about 1122 when Alexios became co-emperor and was about sixteen and showing his first downy moustache. There are fewer examples of female imperial portraits. One that shows the extended family of Michael VIII's half brother is an illuminated copy of a foundation typikon of the Convent of our Lady of Certain Hope, Tis Bebaias Elpidos. The manuscript, Oxford, Lincoln College, gr. 35, now in the Bodleian Library,

42 Treasures of Mount Athos, vol. 1, fol. 116r, fig. 237, p. 441.

43 For Karanlık, also known as chapel 23, see JERPHANion, Églises rupestres vol. I.2, 393-430, pls. II, 96-110; M. RESTLE, Die byzantinische Wandmalerei in Kleinasien. 3 vols. Recklinghausen 1967, vol. I, 128-129, II, figs. 218-244; L. RodLey, Cave monasteries of Byzantine Cappadocia. Cambridge 1985, 2010², 48-56; C. JoLIVET-LÉVy, Les églises byzantines de Cappadoce: le programme iconographique de l'abside et de ses abords. Paris 1991, 132-135. It is thought to have been decorated by the same workshop as the other so-called column churches, Elmalı and Çarıklı; on Elmalı, see JERPHANION, Églises rupestres vol. I.2, 431-454, pls. II, 113-124; on Çarıklı, see JERPHANION, Églises rupestres vol. I.2, 455-473, pls. II, 125-132; on dating and style, see A. EPSTEIN, The Fresco Decoration of the Column Churches, Göreme Valley, Cappadocia: A Consideration of Their Chronology and Their Models. CahArch 29 (1980-81) 27-45; A. WHARTON, Art of empire: painting and architecture of the Byzantine periphery: a comparative study of four provinces. University Park, PA - London 1988, 45- 51 .

44 For further examples of imperial youth and references, see HENNEssy, Children 143-178. 


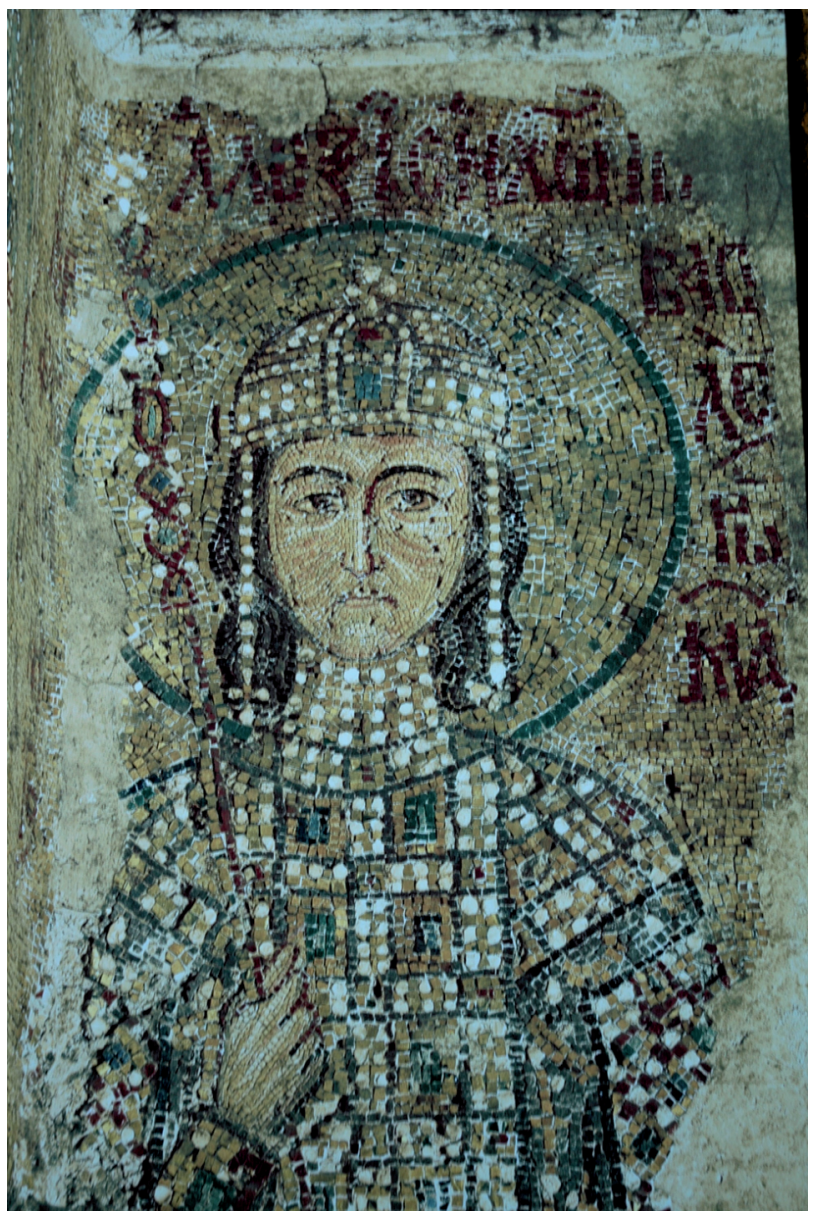

Fig. 5 Alexios, upper gallery, Haghia Sophia, Istanbul (Source: Cecily Hennessy)

shows Euphrosyne, who is the great niece of Michael VIII and the daughter of the foundress as an adolescent. ${ }^{45}$ It has a series of portraits on twelve folios. In one, Euphrosyne is depicted with her mother, Theodora, and is shown shorter than Theodora, with a smaller, more youthful face, clearly not fully grown, yet not a child

45 For the text, see H. Delehaye, Deux typica byzantins de l'époque des Paléologues. Brussels 1921; for comment and translation, see THOMAS - HERo, Founders' Typika, 1512-1578; for analysis and plates, see I. SpatharaKis, The portrait in Byzantine illuminated manuscripts. Leiden 1976, 190 207, pls. 143-154; I. HUTTER, Die Geschichte des Lincoln College Typikons. JÖB 45 (1995) 79-114; see also Cutler - P. Magdalino 1978, 179-198; Connor 2000, 107-108; C. Hennessy, The Lincoln College Typikon: The Influence of Church and Family, in: Under the Influence: The Concept of Influence and the Study of Illuminated Manuscripts, eds. J. Lowden and A. Bovey. Brepols 2008, 97-109. 
(fig. 8 in Brubaker article). ${ }^{46}$ Her reliance on her mother, and the direction she receives from her, is emphasised by the way Theodora grasps her wrist, as if leading her forward to present her to the Virgin, imaged on the facing page. It seems likely that the portrait was made when Euphrosyne was about fifteen or sixteen (she was born in 1285 or 1286), at the time the convent was first functional ca. 1300. A final image shows the assembled thirty or so nuns of the community, grouped together in rows one above the other (folio 12r). In the front row are five young nuns: although they are dressed similarly to their elders, they are clearly smaller and wear a different headdress. ${ }^{47}$ A further example of a series of illuminations showing adolescence and changes in life, is in Vatican, gr. 1851, which contains the partial text of a poem, describing a foreign princess coming to wed the son of the Byzantine emperor, and seven illuminations. ${ }^{48}$ In a scene showing the girl's arrival, she appears at the top left in a simple gold dress with a red cloak around her shoulders and loose blonde hair hanging down her back, slender and shorter than the women who greet her (fig. 19 in Brubaker article). ${ }^{49}$ In the adjacent scene, she appears to be standing on a splendid red and gold platform or perhaps couch, being dressed by the women of the court in an elaborate gown of the same colours, and in the register below she wears a coronet in a formal frontal seated pose. In a further image, she is shown in a tent meeting the daughter of the emperor, who is slightly taller than her but who also has hair falling down her back in a black plait, implying that she also is young. ${ }^{50}$ The scene below shows them sitting together apparently talking. The bride's youth however, is again suggested by her slightly smaller face and figure. The groom also, in an earlier miniature, is shown as youthful, standing by the side of his father, dressed in a similar loros, and although only half a head shorter than the emperor,

46 Folio 11 .

47 The original typikon does not mention the inclusion of children, although Euphrosyne's textual addition states that lay children should not be educated at the convent, except girls intending to be tonsured; see Delehaye 1921, § 48; Thomas - Hero, Founders’ Typika 1564.

48 J. StRzYgowski, Das Epithalamion des Paläologen Andronikos II. BZ 10 (1901) 546-567; S. PAPA-

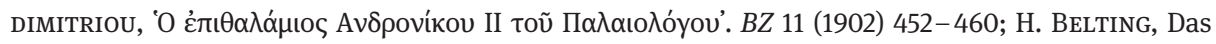
illuminierte Buch in der spätbyzantinischen Gesellschaft. Heidelberg 1970, 26-29; P. CANART, Codices Vaticani Graeci: codices 1745-1962. Vatican 1970, 324; IDEM, Sussidi bibliografici per I manoscritti greci della Biblioteca Vaticana (studi e testi). Vatican 1970, 650; IDEM, Codices Vaticani Graeci: codices 1745-1962, II, Introductio, addenda, indices. 1973, XLVI; SPATHARAKIS, Portrait 210 - 230, figs. 158173; M. JefFreys, The vernacular cioıtípı for Agnes of France, in: Byzantine Papers: Australian Byzantine Studies Conference. Canberra 1981, 101-15; A. IAcoBINI, L'epitalamio di Andronico II. Una cronaca de nozze dalla Constantinopoli Paleologa, in: Arte profana e arte sacra a Bisanzio, eds. A. Iacobini - E. Zanini. Rome 1995, 361-409; C. Hennessy, A Child Bride and Her Representation in Vatican, cod. gr. 1851, in: Byzantine Studies Conference: Abstracts of Papers. Notre Dame 2001; C. HiLsDALE, Constructing a Byzantine “Augusta:” A Greek Book for a French Bride. Art Bulletin 87 (2005) 458-483; C. Hennessy, A Child Bride and her Representation in the Vatican Epithalamion, cod. gr. 1851. BMGS 30 (2006) 115-150.

49 Fol. 3v.

50 Fol. 6r. 
slight and fine featured with a long plait laying on each shoulder. ${ }^{51}$ This unusual manuscript, which has no real parallels, gives a nuanced picture of a boy and two girls involved in diplomatic alliances. However, these figures may well, according to our earlier definitions, be children and not adolescents. It has been argued that the boy might be one of the three figures: either Alexios II Komnenos who married in 1179, when he was aged ten and his bride nine; or Andronikos II Palaiologos who first married in 1272, when he was aged 14 and his bride 12; or Andronikos IV who married in 1356, when he was aged eight and his bride nine. Regardless of specific age, the illustrations show a distinct recognition of the individuals' liminal place in life. ${ }^{52}$

Having reviewed some reasonably well-known examples, it is apparent then that in representations of saints, both male and female, of certain Old Testament figures, of Christ and of members of the imperial family, youthfulness is distinctly portrayed, with qualities between childhood and maturity. The example of Prochoros showed how an apocryphal role was highly developed in imagery with an emphasis on adolescence. As seen in Prochoros, compared to illustrations of canonical texts, those of apocryphal ones perhaps have less rigid iconographical traditions and may convey lifelike portrayals that reflect cultural realities or suggest biological, spiritual and societal change in the transitions from childhood to adulthood.

This material is explored here through depictions of Christ and his family. Quite who is part of his family is a little complex. According to the Gospels of Mark and Matthew Christ was said to have had four brothers and some sisters: 'Is not this the carpenter, the son of Mary, the brother of Jacob, and Joses, and of Juda, and Simon? And are not his sisters here with us?' (Mark 6:3). ${ }^{53}$ 'Jacob the Younger' and Joses are also mentioned as the children of a Mary, who is commonly held to be the Virgin, present at the crucifixion (Mark 15:40). In both contexts Jacob is mentioned first, perhaps implying either that he is the eldest or his importance as a disciple, to be inferred from canonical texts (Galatians 1:19) as well as the Gospel of Thomas (logion 12). ${ }^{54}$ He was the first Bishop of Jerusalem. ${ }^{55}$ The men named as Jacob the

51 Fol. 7r.

52 For a brief summary of the arguments, see, C. Hennessy. The Vatican Epithalamion, in: A Companion to Byzantine Illustrated Manuscripts, ed. V. Tsamakda. Leiden 2017, 177-182.

53 Also Matthew 13: 55-6.

54 The Gospel of Thomas is a second-century text found at Nag Hammadi, Egypt. The logion reads: The disciples said to Jesus: We know that You will depart from us. Who is to be out leader? Jesus said to them, Wherever you are, you are to go to James the Righteous for whose sake heaven and earth came into being; The Nag Hammadi Library IN English. Leiden 1977, 119; for the Coptic, see U.K. PLISCH, Das Thomasevangelium: Origininaltext mit Kommentar. Stuttgart 2007, 63.

55 Several recent books focus on James and his role in the early Church: P.-A. BERnHEIM, Jacques, frère de Jésus. Paris 1996; in English, James, Brother of Jesus (trans. J. Bowden). London 1997; most controversially, R. EISENMAN, James, the brother of Jesus: the key to unlocking the secrets of early Christianity and the Dead Sea Scrolls. London 1998; J. PAINTER, Just James: the brother of Jesus in history and tradition. Minneapolis 1999. 
Just and Jacob the son of Alphaeus are also conflated with Jacob the Less/Younger (Mathew 10:3, Mark 3:18, Luke 6:15). Who exactly were these siblings? In Byzantium, the common interpretation was that they were the sons of Joseph, born to him by a prior marriage, thus conserving Mary's virginal state. There are three other main interpretations. Helvidius, in the fourth century, suggested the siblings were born to Joseph and Mary, which was refuted by Jerome who developed the theory of a line coming through Mary of Cleophas, the proposed daughter of Anna's second marriage to Cleophas; thus they were cousins, not siblings, to Christ. A further branch to the family was added in the ninth century, which included James (Jacob) the Great and John the Evangelist as sons of Mary Salome and Zebedee, Mary Salome being the daughter of Salome (a man) who was Anna's third husband..$^{56}$ In the west, these three interpretations are illustrated in several contexts.

In Byzantine illustrations of Christ's Infancy, the six children of Joseph by his deceased wife, four sons and two daughters, are not depicted. Rather one, sometimes two and occasionally four brothers are included in various scenes. However, one, occasionally labelled Iakobos (Jacob/James), takes a more prominent role. Jacob's role is partially derived from the Infancy Gospel of James (Jacob) a text probably originating in the second century, which only mentions two sons and no daughters, and in the narrative does not highlight Jacob. ${ }^{57}$ The text was interpreted as being written by Jacob the brother of Jesus due to an addition at the end of the text, which states that it was written by him in Jerusalem (Infancy Gospel 25:1-3). ${ }^{58}$ A further text, the Story

56 Jerome, De perpetua virginitate B. Mariae, adversus Helvidium. PL XXIII, 183-206; for further references and detail, see M. NAydenova SlADE - D. PARK, The earliest Holy Kinship image, the Salomite controversy, and a little-known centre of learning in northern England in the twelfth century. Journal of the Warburg and Courtauld Institutes 71 (2008) 95-119, here 96.

57 C. von TISCHENDORF, Evangelia apocrypha, adhibitis plurimis codicibus graecis et latinis maximam partem nunc primum consultis atque ineditorum copia insignibus. Leipzig 1853, 1-49; E. Amman, Le Protévangile de Jacques et ses remaniements latins. Paris 1910; M. R. JAmEs, The apocryphal New Testament: being the apocryphal gospels, acts, epistles, and apocalypses with other narratives and fragments newly translated by Montague Rhodes James. Oxford 1924, 38-49; E. HENNECKE, New Testament Apocrypha, ed. W. Schneemelcher (English trans. R. McL. Wilson). London 1959, vol. 1, 370 - 388. For the most recent text and commentary, see R. F. Hock, The Infancy Gospels of James and Thomas: with introduction, notes, and original text featuring the New Scholars Version translation. Santa Rosa, CA 1997, used here for references. The earliest known manuscript is dated to the fourth century, Papyrus Bodmer V; on the manuscripts, see Hock Infancy Gospels 28-29; the original version probably dates to the late-second century, see Hock Infancy Gospels 11-12. For a discussion of the textual tradition, see LAFONTAINE-Dosogne, Iconographie de l'enfance de la Vierge dans l'empire byzantin et en Occident. Brussels, 1964, vol. 1, 13-23 with summary at 23; on Joseph's sons, see Amman Protévangile 37-39, 52, 82, 131, 142, 208, 216-17; and Hock, Infancy Gospels 49, 63, 67, 77; also on dating and acceptance by the early church, see M. B. Cunningham, The Use of the Protoevangelion of James in Eighth-Century Homilies on the Mother of God, in The Cult of the Mother of God in Byzantium: Texts and Images, eds. L. Brubaker - M. J. Cunningham. Farnham - Burlington 2011, $163-7$.

58 Chapter 25: 1-3, 'Now I, James, am the one who wrote this account at the time when an uproar arose in Jerusalem, at the death of Herod. I took myself off into the desert until the uproar in Jeru- 
of Joseph the Carpenter, originated in Egypt, perhaps in the fifth century. Written in the voice of Christ, it refers to the four sons and two daughters (Carpenter 2). ${ }^{59}$ Here Jacob is the youngest, for 'Now Justus and Simeon, the elder sons of Joseph, were married, and had families of their own. Both the daughters were likewise married, and lived in their own houses. So there remained in Joseph's house, Judas and Jacob the Less, and my virgin mother' (Carpenter 11). No age of the children is given but Joseph's wife has only just died when the Virgin turns twelve, as 'Now when righteous Joseph became a widower, my mother Mary, blessed, holy, and pure, was already twelve years old (Carpenter 3). In the Pseudo-Matthew, which was popular in the west and probably developed at the beginning of the seventh, eighth or even ninth century, there are also four sons, and two daughters (PseudoMatthew 42:1). ${ }^{60}$ The text was composed from the Infancy Gospel of James and the Infancy Gospel of Thomas in addition to some other influences. ${ }^{61}$ However, the PseudoMatthew was not a major source of iconography in Byzantium.

Perhaps the earliest representation of the brothers is on the sixth-century ivory front cover of the Etchmiadzin Gospels, in the scene of the Journey to Bethlehem. ${ }^{62}$ In this unusual image, Joseph is walking beside the donkey, clasped by the Virgin, while one son walks behind and another is on the far side of the donkey at the front. The brothers seem to be beardless, which might suggest they are youthful. The figure on the far side of the donkey, while described as a son by Lafontaine-Dosogne may be an angel. ${ }^{63}$ This tradition reappears in the Gospel of Pseudo-Matthew, which describes how when Joseph and Mary were going to Bethlehem (no sons mentioned), a beautiful boy in white raiment appeared, and interpreted a vision had by

salem died down. There I praised the Lord God, who gave me the wisdom to write this account', Hock Infancy Gospels 76-77; for a summary of views on who actually wrote the text, see Hock, Infancy Gospels 8-11.

59 Derived from the Infancy Gospel, TischendoRf, Evangelia 115-133; JAMEs, Apocryphal, 84-86; J. K. ELLiotT, The Apocryphal New Testament: a collection of apocryphal Christian literature in an English translation. Oxford 1993, 111-117; LAfontAINE-Dosogne, Iconographie, vol. 1, 22; for the Arab text, see A. Battista - B. BagatTi, Edizione critica del testo arabo della Historia Iosephi Fabri Lignarii e ricerche sulla sua origine. Jerusalem 1978.

60 J. GIJSEL - R. BEYERS, Libri de Nativitate Mariae, Pseudo-Matthaei Evangelium Textus et Commentarius (Corpus Christianorum Series Apocryphorum 9 and 10), vol. 1. Turnhout 1997, 13; Elliott gives the eighth to ninth century date, which is more common, see ElLIotT, Apocryphal, 86. James is mentioned as the eldest, Et die quadam vocavit Ioseph ad se filium suum primogenitum Iacobum....; 'And on a certain day Joseph called to him his first-born son James...'. (Pseudo-Matthew 41-1), TISCHENDORF, Evangelia 103. In the ninth century, the Pseudo-Matthew text was used as a model for the Libellus de nativitate sanctae Mariae, GIJSEL - BEYERS, Libri de Nativitate 21.

61 T. BuRKE, De Infantia Iesu Evangelium Thomae Graece. (Corpus Christianorum Series Apocryphorum 17). Turnhout 2010.

62 Yerevan, Matenadaran 2374, in: W. F. VolBACH, Elfenbeinarbeiten der Spätantike und des frühen Mittelalters. Mainz 1976, pl. 75, no. 142; L. A. DouRnovA, Armenian miniatures. London 1961, preface photograph.

63 J. Lafontaine-Dosogne, Iconography of the cycle 205, n. 57. 


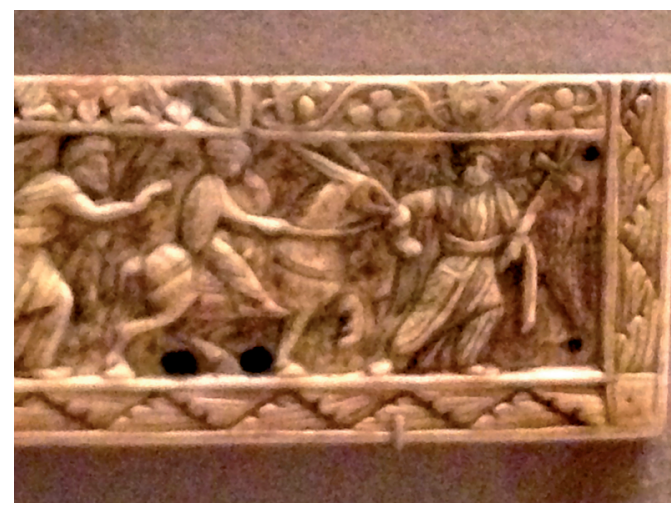

Fig. 6 An angel leading the donkey in the Journey to Bethlehem, Ivory panel, sixth century, Louvre OA 11149 (Source: Cecily Hennessy)

Mary and then 'the angel ordered the beast to stand, for the time when she should bring forth was at hand'. ${ }^{64}$ The angel is pictured in a similar location and pose on the ivory on the Chair of Maximianus, a scene where there are no sons, and on an ivory panel in the Louvre, the angel strides forward holding a cross and leading the donkey (fig. 6) ${ }^{65}$ It is possible that the angel in this type of image is transposed into Jacob in later examples. A further early example where Jacob is shown is in the paintings at Castelseprio, which are probably dated to the ninth century and include apocryphal scenes. ${ }^{66}$ In the Journey to Bethlehem, a boy is in front of the donkey, now only partially visible, and so again his age is indeterminate, although he appears to be wearing a short tunic, something usually worn by servants, children, or youths. In a further ninth-century example, an enamel reliquary box, the Paschal Cross in the Vatican, dated 817-24, one son is shown (fig. 7). ${ }^{67} \mathrm{He}$ is unnamed, as are

64 Pseudo-Matthew 13:2. The Pseudo-Mathew text is later than the ivory, but compiles early sources. 65 The Maximinianus chair, illustrated in: C. R. MoReY, Castelseprio and the Byzantine "Renaissance”. The Art Bulletin 34 (1952) 173-201, fig. 29; the Louvre example, OA 11149, J. DuRAND, Byzance: L'art byzantine dans les collections publiques françaises. Paris 1992, cat. 24.

66 BognetTI ET AL 1948; the dating is controversial, ranging from the sixth to tenth centuries; for ninth century, see P. D. LEveto, The Marian Theme of the Frescoes in S. Maria at Castelseprio. The Art Bulletin 72 (1990) 393-413, here 393; for others, see K. Weitzmann, The fresco cycle of S. Maria di Castelseprio. Princeton 1951; Morey, Castelseprio, 189-201; M. SchaPIRo 1952, Book Review: Kurt Weitzmann, The Fresco Cycle of S. Maria di Castelseprio. The Art Bulletin 44 (1952) 147-163; M. SCHAPIRO, Notes on Castelseprio. The Art Bulletin 39 (1957) 292-299.

67 C. R. Morey, The Inscription on the Enameled Cross of Paschal I. The Art Bulletin 19 (1937) 595-6; A. LEgner, Ornamenta ecclesiae: Kunst und Künstler der Romanik, Katalog zur Ausstellung des Schnütgen-Museums in der Josef-Haubrich-Kunsthalle. Cologne 1985, 82-84 with bibliography; R. P. BERGMAN, Splendor of the popes: treasures from the Sistine Chapel and the Vatican museums and library. Baltimore, MD 1989, 4, 6; C. StiegemanN - M. Wemhoff, Kunst und Kultur der Karolin- 


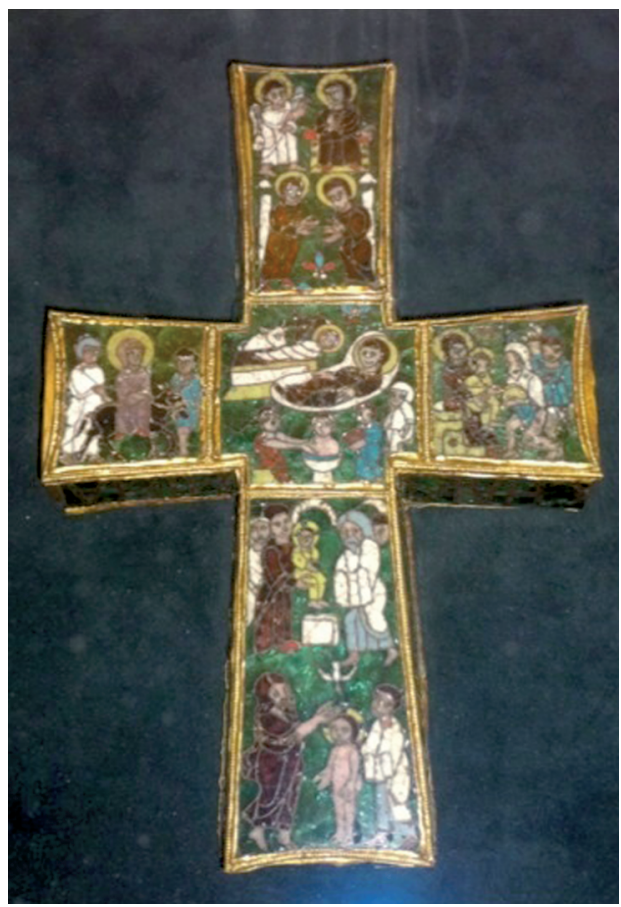

Fig. 7 Paschal Cross, Vatican (Source: Cecily Hennessy)

all the figures on the box, and he appears to be a youth, shown as smaller than Joseph, since size is often used to distinguish stature, and with a beardless face, cropped hair and short tunic. The boy is placed in a position of servility or responsibility guiding the donkey. Moving to the early tenth century, in Cappadocia, in the Old Church at Tokalı kilise, Jacob is depicted and named, simply as Iakobos, leading the donkey on the road to Bethlehem and also in the Flight to Egypt. ${ }^{68}$ The Flight to Egypt is not included in the Infancy Gospels, but none the less, Jacob has kept up his previous role. There are numerous other examples. In each, his adolescent stage is apparent, shown too tall to be a boy, but without facial hair and wearing the tunic of youth. No examples in Cappadocia, however, show more than one

gerzeit: Karl der Grosse und Papst Leo III. in Paderborn: Katalog der Ausstellung, Paderborn 1999. Mainz 1999, $650-51$.

68 Perhaps the earliest representation of Jacob in Cappadocia is in Ayvalı kilise in Güllü Dere, Çavuşin, which is dated to 913-920; see RESTLE, Wandmalerei no. 29, vol. 3, figs. 340 - 341; Göreme Chapel 7, JERPHANion, Églises rupestres vol. 1.1, 271, 273-4; RESTLE, Wandmalerei vol. 1, 110-116, vol. 2, figs. 66, 86; on the iconography of the journey to Bethlehem, see JERPHANION, Églises rupestres vol. 1.1, 76 and on the flight to Egypt, vol. 1.1, 79; on the depiction of Jacob (James the Less) in the medieval and later periods, primarily in the west, see R. P. BEDFoRD, St. James the Less: a study in Christian iconography. London 1911. 
son. A son of Joseph also appears in manuscript illuminations, such as on the opening page of the Gospel of Mathew in Dionysiou cod. gr. $587 \mathrm{~m}$, leading the donkey in the Flight to Egypt. ${ }^{69} \mathrm{He}$ is on the right wearing a short chiton and high boots and walks in front leading the donkey and holding a staff on which hangs a knapsack over his left shoulder. Jacob is as tall as Joseph, who follows behind, but clearly youthful with short brown hair and beardless face. A further example is in another eleventh-century text, a menologion, on Athos, Esphigmenou, cod. gr. 14, in which there are several unusual scenes drawn from apocryphal texts. A beardless but fully grown son leads the horse/mule on the Flight to Egypt and Joseph follows behind with the Christ Child on his shoulders ${ }^{70}$. In Paris cod. gr. 74, also eleventh century, a son in a full length garment leads the donkey on the Journey to Bethlehem, illustrating the Gospel of Luke, and also in a short tunic follows the donkey which is led by Joseph in the Flight to Egypt, illustrating the Gospel of Matthew. ${ }^{71}$ Although in several of these, Jacob is not named, his identity can perhaps be inferred from those others where he is named.

The reason for Jacob's consistent inclusion in the iconography may be that he serves as a witness to the events, a feature that appears in other contexts where subsidiary figures act as witnesses, such as Anna's servant in attendance at the Annunciation to Anna and a servant present at the Visitation. ${ }^{72}$ These figures do often seem young, as for instance, the young maid watching the Visitation in the bema at the sixth-century Basilica Eufrasiana, Poreč and a boy witnessing the Annunciation to Anna in the fourteenth-century mosaics at the former church of Our Saviour in the Chora, the Kariye Camii, Istanbul (fig. 8). ${ }^{73}$ Alternatively, Jacob's presence may put a mantle of propriety over Joseph escorting the Virgin. Jacob does not appear in scenes of the Nativity or the Adoration, where other figures are also present, suggesting that his role is that of donkey hand or protector/witness when Joseph is alone with the Virgin. His presence also emphasises the age of the widowed Joseph (in

69 Treasures of Mount Athos, vol. 1, fol. 133v, fig. 251, pp. 442-3.

70 Treasures of Mount Athos, illuminated manuscripts. 2. The Monasteries of Iveron, St. Panteleimon, Esphigmenou, and Chilandari. Athens 1975, fol. 384v, fig. 343, p. 370. Other scenes are connected with the Virgin's youth. She is shown walking out from beneath a ciborion to be given to Joseph, Treasures of Mount Athos, vol. 2, fol. 389r, fig. 350, p. 371; walking with Joseph (no sons) on the road to Bethlehem, Treasures of Mount Athos, vol. 2, fol. 391r, fig. 353, p. 372 (the latter has beneath it Mary's vision, associated with a homily by John of Damascus, not directly an apocryphal text). It has been suggested that this manuscript was from the Studios Monastery, Constantinople, see Treasures of Mount Athos, vol. 2, 361.

71 H. Omont, Évangiles avec Peintures Byzantines du XIe Siècle: Reproduction des 361 miniatures du Manuscrit grec 74 de la Bibliothèque nationale. Paris 1908, vol. 2, fol. 108, pl. 96, vol. 1, fol. 4v, pl. 7. 72 On this, see R. Deshman, Servants of the mother of God in Byzantine and medieval art. Word and Image 5 (1989) 33-70, here 50-52.

73 For Poreč, Deshman, Servants fig. 24; A. Terry - H. Maguire, Dynamic Splendor: The Wall Mosaics in the Cathedral of Eufrasius at Poreč. University Park, PA 2007, vol. 1, 102-104, 174, vol. 2, fig. 126; for Kariye Camii, Underwood, Kariye pls. 92-93, vol. 1, 64. 


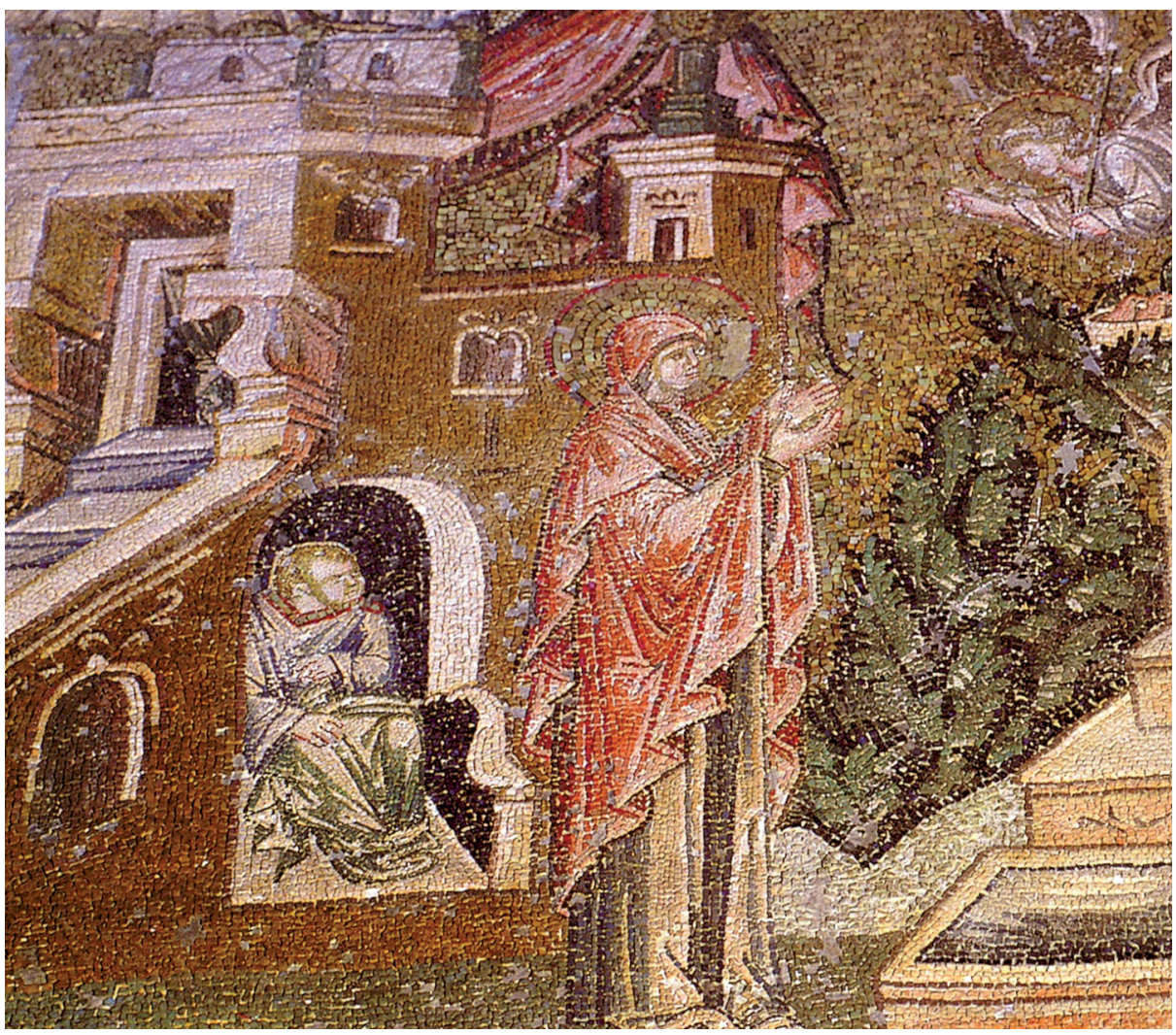

Fig. 8 Annunciation to Anna, former Church of Our Saviour in the Chora, the Kariye Camii, Istanbul (Source: Cecily Hennessy)

the Story of Joseph the Carpenter, he is described as 'the pious old man' and he is supposedly 90 years old at the time of Christ's birth (Carpenter 4, 14).

In the Kariye Camii the sons of Joseph are depicted at several stages of life but also in different configurations, perhaps suggesting that various models were used for the programme. The first time one appears is in the scene where Joseph takes the Virgin to his home (fig. 9).$^{74}$ Joseph and the son turn to look back at the Virgin, and the son is slighter and less tall than his father, short haired and beardless, clearly a youth. Then, in the Journey to Bethlehem a mature son leads the donkey, mature in that he has a short beard and wears a full-length chiton. ${ }^{75}$ Next, when Joseph and Mary are counted in the census, all four brothers crowd behind them, one mature and bearded, two youthful and one rather indistinct. ${ }^{76}$ In the return from Egypt, Jo-

74 UNDERWOOD, Kariye scene 97, pls. 143-145, vol. 1, 81-2.

75 UNDERWOOD, Kariye scene 100, pls. 155, 158, vol. 1, 86-8.

76 UNDERWOod, Kariye scene 101, pls. 163, 165, vol. 1, 88-9. 


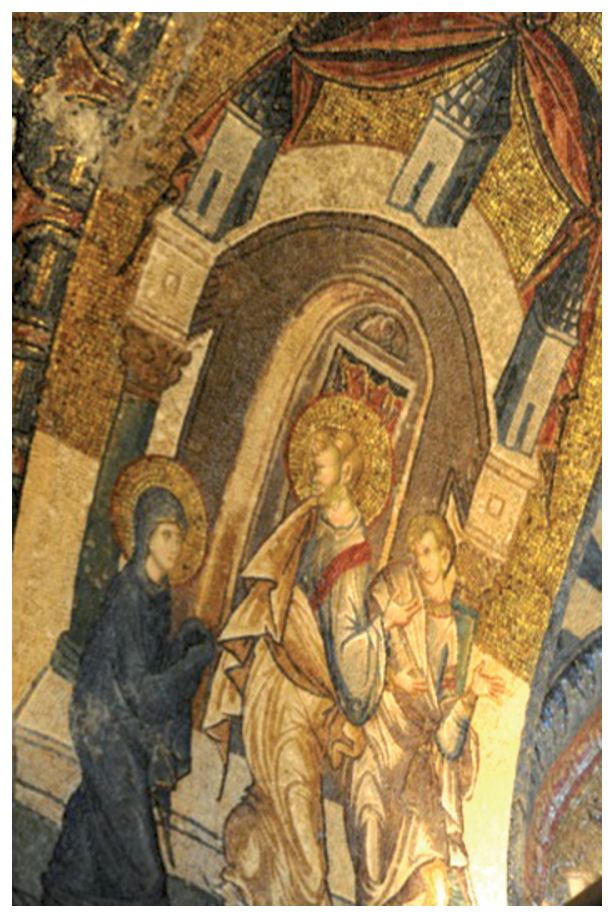

Fig. 9 Joseph takes the Virgin to his home, former Church of Our Saviour in the Chora, the Kariye Camii, Istanbul (Source: Cecily Hennessy)

seph carries the child on his shoulders and a son, clearly youthful and dressed in a short tunic, follows the donkey. ${ }^{77}$ When Christ is taken by his parents to Jerusalem at the age of twelve, he is accompanied by two of the brothers, one bearded and one beardless who wears a short tunic. ${ }^{78}$ Christ is dressed in a long gold garment, which has no reference to youth but rather to his importance or sanctity. However, he appears fresh faced and youthful.

A further representation of the four brothers is in the paintings in two illustrated Homilies of James Kokkinobaphos, Vatican, gr. 1162 and Paris, gr. $1208 .^{79}$ The manu-

77 UNDERWOOD, Kariye scene 111, pls. 200, 202, vol. 1, 104-6.

78 UnderWOOD, Kariye scene 112, pls. 206, 208-9, vol. 1, 106-7.

79 PG 127: 543-700; H. OMONT, Miniatures des homélies sur la Vierge du moine Jacques. Paris 1927; C. Stornajolo, Miniature delle omilie di Giacomo monaco (Cod. vatic. gr. 1162) e dell'Evangeliario greco urbinate (Cod. vatic. urbin. gr. 2). Rome 1910; ANDERSON, The Illustrated Sermons of James the Monk: Their Dates, Order, and Place in the History of Byzantine Art. Viator 22 (1991) 69-120; HuTTER - CANART, Das Marienhomiliar des Mönchs Jakobos von Kokkinobaphos: Codex vaticanus graecus 1162. 2 vols. Zurich 1991; MAGUIRE, The icons of their bodies: saints and their images in Byzantium. Princeton, NJ 1996, 159-166, figs. 141-145; R. NELson, Theoktistos and Associates in Twelfth-Century Constantinople: An Illustrated New Testament of A.D. 1133. The J. Paul Getty Museum Journal 53 (1987) 53-78, p. 76; K. LINARDOU, Reading two Byzantine illustrated books: the Kokkinobaphos manuscripts (Vaticanus graecus 1162 and Parisinus graecus 1208) and their illustration. University of Birmingham PhD thesis, 2004; on the text, see E. JEFFrEYs, The Sevastokratorissa Eirene as 
scripts illustrate six homilies on major events in the Virgin's life. Their date is normally given as between 1130 and the 1150s. ${ }^{80}$ They depict rich details of the Virgin's life (from her conception to the declaration of her innocence after her pregnancy is discovered), with evidence of then contemporary childhood and adolescence. It is often said that the illustrations derive from an illustrated Infancy Gospel, but no examples of such a manuscript survive and in fact the presence of four sons in several scenes would suggest that this is not the case. In the illustrations (not in the Homilies), it is the youngest son who takes up a key role. It is possible the images are related to the Story of Joseph the Carpenter and derive from an illustrated version of it. Yet, in the Carpenter text, the daughters are said to have left home, so their absence is justified, but two sons have left as well, and in the Kokkinobaphos manuscripts, four brothers are present in Joseph's house. ${ }^{81}$ However, the Carpenter text does give Jacob a special role, that of a bereft child who is adopted by his new stepmother, Mary. The text says she 'found James the Less in his father's house, broken-hearted and sad on account of the loss of his mother, and she brought him up' (Carpenter 4). The youngest child in the Homilies' illustrations is interpreted as being Jacob the Less, though there is really no evidence for this. He is neither named in the text nor in the illustrations. In the scene where the Virgin first enters Joseph's house, the two older sons look mature with beards, the third son looks what one might justifiably call an adolescent, beardless, a little shorter than his brothers and a little slighter. The fourth son appears as a boy, significantly smaller with a small round head, a slightly receding hairline and an appearance similar to the puer senex features used for Christ (fig. 10). ${ }^{82}$ Throughout the illustrations in these manuscripts he remains a boy, distinguished from his brothers. He sometimes wears a similar long tunic, at other times a short one. This boyishness is perhaps because in many situations he is alone with the Virgin as a protector, and so his lack of sexual maturity needs emphasis, but the puer senex appearance gives him the appearance of wisdom. One could argue that he is about twelve and so on that cusp between childhood and adolescence.

Often it is said that the Virgin before her maturity is depicted as a small adult, but this is clearly far from accurate. ${ }^{83}$ She is frequently shown at distinct stages of childhood and adolescence, as in the Kokkinobaphos manuscripts. For instance, she appears as a little girl in bed and again being tucked into her cot, when present-

Literary Patroness: The Monk Iakovos. JÖB 31/2 (1982) 63-71; on the group of manuscripts by the 'Kokkinobaphos Master', see E. JEFFreYs, Aristocratic Book Patronage in Twelfth-Century Byzantium. Forthcoming.

80 For a brief summary of the dating issues, see C. Hennessy, The Stepmum and the Servant: The Stepson and the Sacred Vessel, in Wonderful things: Byzantium through its art, eds. A. Eastmond - L. James. Farnham - Burlington 2013, 79-98, here 89-90.

81 Lafontaine-Dosogne points out how unique this is and an invention of the miniaturist, LAFONTAINE-Dosogne, Iconographie vol. 1, 180.

82 Paris gr. 1208, fol. 142v; Vatican gr. 1162, fol. 105v.

83 For instance, $O D B$ 1, 421 under 'Childhood'. 


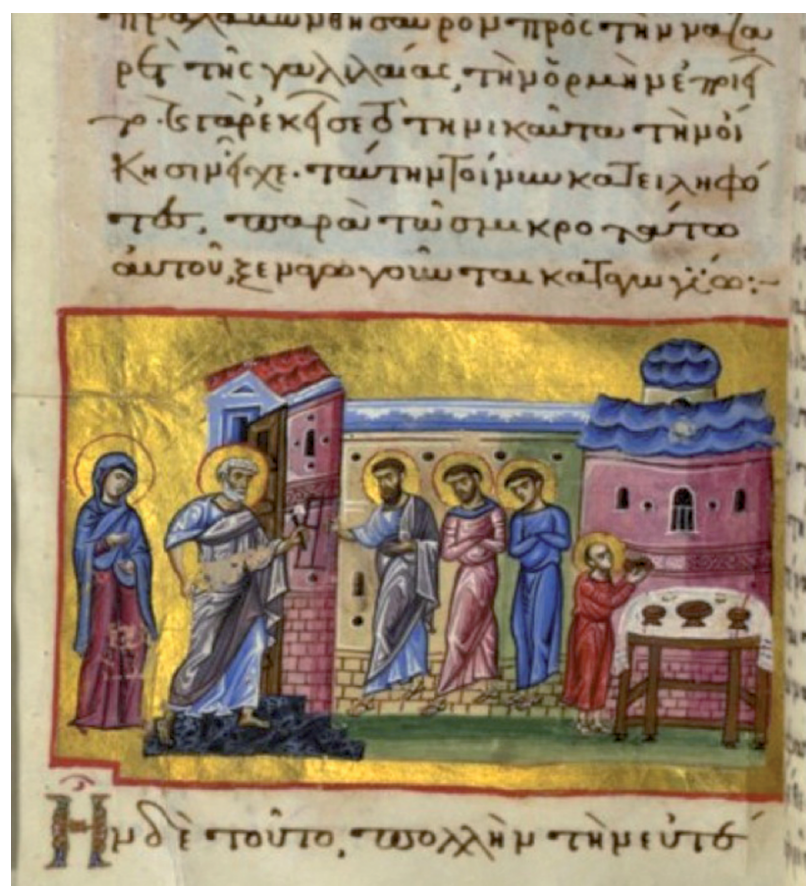

Fig. 10 The Virgin enters Joseph's house, Paris gr. 1208, fol. 142v (Source: Bibliothèque nationale)

ed to Zachariah in the temple and when installed on the altar. ${ }^{84}$ Interestingly when she says goodbye to her parents, she seems fully grown (fig. 11). ${ }^{85}$ But then when fed by the angel on the altar she is again a little girl (fig. 12). ${ }^{86}$ Zachariah decides that when she has reached the age of twelve, it is no longer proper for her to be in the temple, so Joseph is chosen as her betrothed. When she is given to Joseph, she is a child, but when shortly thereafter she leaves with Joseph through Jerusalem, she has become much more grown up, and when she enters his home and meets the sons, she is mature. ${ }^{87}$ The text of the homilies refers to her with various terms, aware of the delicate situation evoked by her reaching the age of twelve. For instance, in a speech made by Joseph after he has been chosen to betroth the Virgin,

84 For the cot scenes, Paris gr. 1208, fol. 52r, Vatican gr. 1162, fol. 38v; Paris, gr 1208, fol. 59r, Vatican gr. 1162 fol. 43r; Vatican gr. 1162, fol. 44r (this is not in the Paris manuscript); Paris gr. 1208, fol. 63v; Vatican gr. 1162, fol. 46v; for the altar scene, Paris gr. 1208, fol. 92v; Vatican gr. 1162, fol. 68v.

85 Paris gr. 1208, fol. Bis 100v; Vatican gr. 1162, fol. 74v.

86 Paris gr. 1208, fol. 103v; Vatican gr. 1162, fol. 76v.

87 For reception of Mary by Joseph, Paris gr. 1208 fol. 135r, Vatican gr. 100r; for Joseph and Mary leaving Jerusalem, Paris gr. 1208, 142r, Vatican 105r; for entering the house, Paris gr. 1208, fol. 142v, Vatican $105 \mathrm{v}$. The age of the Virgin varies in certain texts, see LAFONTAINE-DosognE, Iconographie vol. 1, 167. 


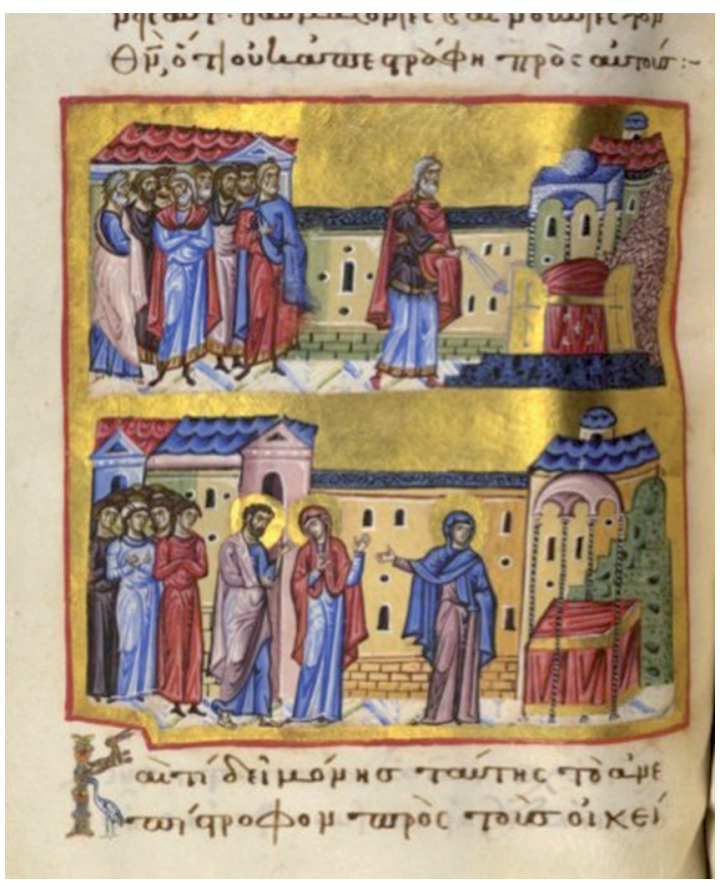

Fig. 11 The Virgin says goodbye to her parents, Paris gr. 1208, fol. Bis 100v; Paris gr. 1208, fol. 103v (Source: Bibliothèque nationale)

he refers to her as 'a young girl with a divine appearance'. ${ }^{88}$ When Joseph returns from his work and sees her pregnant, he is perplexed, but he still calls her 'girl' until he realises that indeed she is pregnant, when he addresses her as 'woman'. ${ }^{9}$ In this image, she looks full grown in height (though not ostensibly pregnant). ${ }^{90}$ However, later she is referred to again as 'girl'. As mentioned, many details in the images are not derived from the text and the phrasing may not be directly relevant to the pictorial representation.

The subtle recognition of adolescence may be attributable to patronage. Much recent reflection on the Kokkinobaphos manuscripts has lent towards the Sebastokratorissa Eirene as the patroness of the manuscript. She was the widow of Andronikos (1108/9-1142), the second son of John II. ${ }^{11}$ However, the iconography may be related

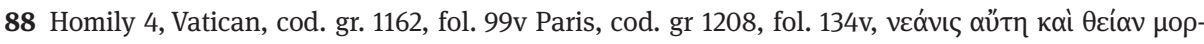

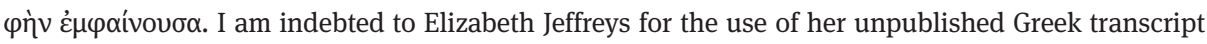
and translation of the texts.

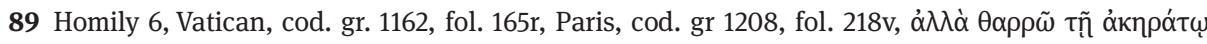

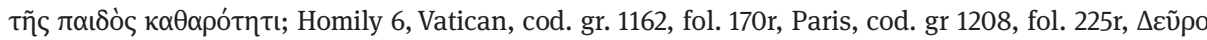

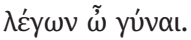

90 Vatican, cod. gr. 1162, fol. 170r, Paris, cod. gr 1208, fol. 225r.

91 See Hennessy, Stepmum for more on this and full bibliography. 


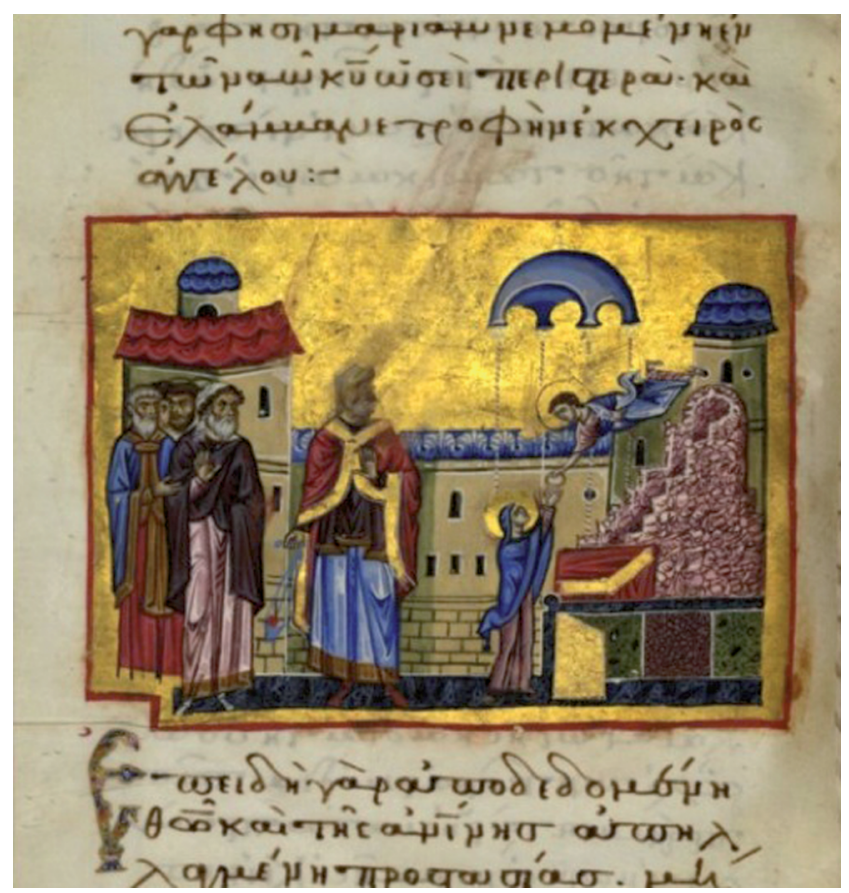

Fig. 12 The Virgin is installed on the altar, Paris gr. 1208, fol. 103v; Paris gr. 1208, fol. 103v (Source: Bibliothèque nationale)

to Emperor Manuel I, the youngest of four sons, who ascended the throne in 1143 aged 25. If this were so, his youth in relation to his brothers might be emphasised. Manuel was twelve years younger than his eldest brother, Alexios (born 1106), and five years younger than the third son, Isaac (born 1113). The inclusion of an image of Christ Emmanuel, in the burning bush might reinforce this theory since Manuel had a particular interest in Christ Emmanuel and put the image on his coins. ${ }^{92}$

Returning to the Kariye Camii, there is also a well-developed sense of the Virgin's coming of age. She appears in various scenes as a baby and small child, easily recognisable as she wears the familiar blue maphorion. She looks like a small girl when she takes her first seven steps at the age of six months, after which time she is not allowed to touch the ground until she is taken to the temple. ${ }^{93}$ She appears as a baby when carried by Joachim to be blessed by the priests on her first birthday and when

92 Paris gr. 1208, fol. 73v; Vatican fol. 54v; for the coins, see M. F. Hendy, Coinage and money in the Byzantine Empire, 1081-1261. Dumbarton Oaks, DC 1969, 111-112, 114, pls. 12:1-12; 13:1-2, 10 -12; in part in A. W. CARR, Gospel Frontispieces from the Comnenian Period. Gesta 21 (1982) 3-20, here 9. Hendy does not reference the coins on pl. 12 as Emmanuel, but they appear to be so.

93 Underwood, Kariye scene 88, pls. 104-7, vol. 1, 68-9; Infancy Gospel (6:1). 


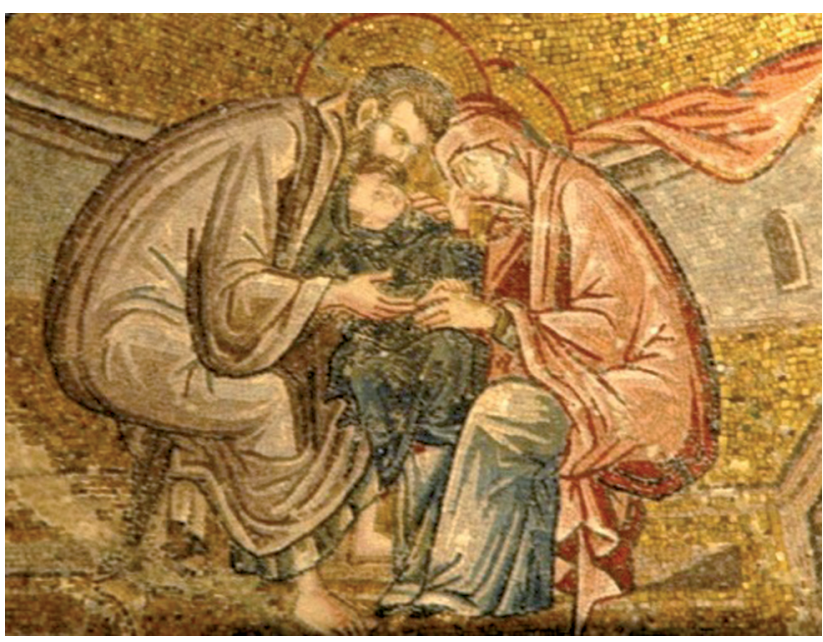

Fig. 13 'The fondling of the Theotokos' former Church of Our Saviour in the Chora, the Kariye Camii, Istanbul (Source: Cecily Hennessy)

cuddled by her parents in a scene called 'the fondling of the Theotokos' (fig. 13)..$^{94}$ She then appears as a little girl when at the age of three she is presented in the temple and fed by an angel and still as a little girl when Joseph is chosen to betroth her by the sprouting of his rod when she is aged twelve (Infancy Gospel 8:3; Carpenter 3). ${ }^{95}$ But suddenly then, in departing with Joseph to his house in the scene mentioned above, she has entered what one might term adolescence, since she is no longer a little girl, is taller while not full height and slight in body (fig. 9 ). ${ }^{96}$ So she is not fully mature when leaving for Joseph's house, but when he departs, again with a son, to engage in some carpentry work, she has become mature, although according to the Infancy Gospel text this occurred only shortly after. ${ }^{97}$ According to the Story of Joseph the Carpenter, she conceives Christ at the point when 'after the holy virgin had spent two years in his house her age was exactly fourteen years' (Carpenter 4). And

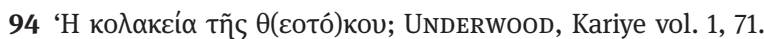

95 Underwood, Kariye scenes 89, 90, 91, 92, 96, pls. 108-9, 114-5, 119-20, 122, 126-127, 138, 140, 142; vol. 1, 69-74, 79-80. The caressing of the Virgin is perhaps incorrectly placed here and should come after her first steps, when she is gathered up by her mother who declares she shall no longer walk on the ground, so that scene 90 follows scene 88. The Presentation in the Temple is the first of these scenes to feature not only in the Infancy Gospel (7:2), but also in the Pseudo-Matthew (4) and the Gospel of the Nativity of Mary (6). The Feeding by the Angel and the Virgin Entrusted to Joseph are also in all three, Infancy Gospel (8:1, 9:1-2), Pseudo-Matthew (6, 8), Gospel of the Nativity of Mary $(7,8)$.

96 UnDERWOOD, Kariye scene 97, pl. 97, pls. 143-4, vol. 1, 81-2. This scene is only in the Infancy Gospel (9:2) and Joseph the Carpenter (4).

97 UnDERWOOD, Kariye scene 99, pls. 148-9, vol. 1, 83-5; Infancy Gospel (9:11). 


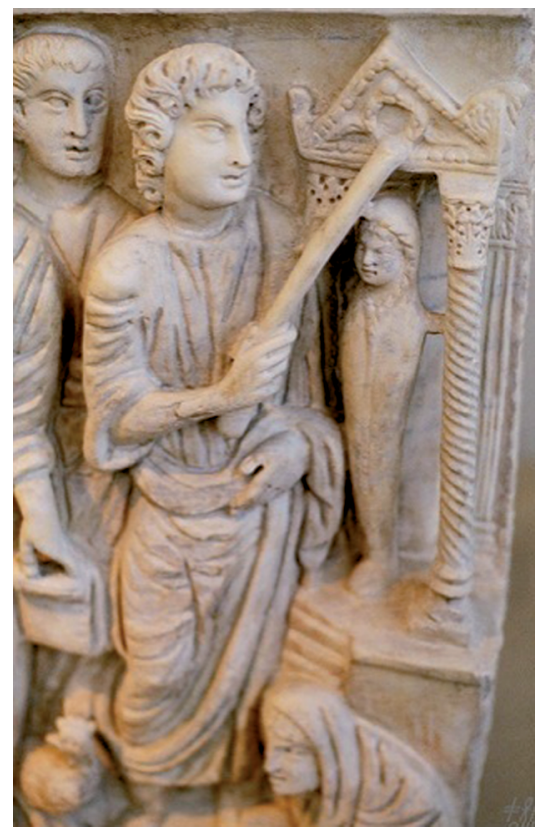

Fig. 14 Sarcophagus of Marcus Claudianus, in the Museo nazionale delle Terme in Rome, dated to 330-40, Museo nazionale delle Terme, Palazzo Massimo, Rome (Source: Cecily Hennessy)

indeed she is mature in the census scene viewed before. ${ }^{98}$ These representations all seem to show a sensitive awareness of coming of age and the transitional phases in life. While the Virgin is dressed from the time of her babyhood in the same blue dress and veil, the nuanced distinctions to her size and facial features reveal a distinct recognition of the stages of maturation.

Finally, turning back to the Early Christian period in which the non-canonical texts developed that gave rise to these diverse images, it is intriguing to look at the portrayal of Christ and to consider to what extent his depiction was dependent on apocryphal texts. It was discussed how he appears youthful, as an adolescent or a 'wise beyond his years' boy, at the age of twelve teaching in Jerusalem, but in a broad range of Early Christian and Early Byzantine representations in other scenes, within the canonical events that occur during his maturity (that is after the age of 30), he appears beardless and with a youthful body, even in some contexts adolescent. Many examples exist prior to the sixth century in various media. On numerous sarcophagi, in many of which he is performing miracles, he has short curly hair, fine features and no sign of a beard, whereas all the mature characters such as Peter and Paul are bearded. An example is the sarcophagus of Marcus Claudianus, in the Museo nazionale delle Terme in Rome (fig. 14). ${ }^{99}$ Another striking example is the mo-

98 Underwood, Kariye scene 101, pls. 159, 161, 163, vol. 1, 88-9, Luke (2:4-5), Infancy Gospel (17:1), Pseudo-Matthew (13).

99 Dated to 330 -40, Museo nazionale delle Terme, Palazzo Massimo, Rome. 
saic of the Baptism in the dome at the Arian baptistery in Ravenna, dated 500 - 530, where Christ appears as a fleshy youth with round shoulders and curving hips, wavy hair and beardless. ${ }^{100}$ This type of youthful portrayal has been discussed frequently, perhaps most persuasively by Thomas Mathews who argues that the likenesses are related to pagan gods such as Dionysius and Apollo, among other influences. ${ }^{101}$ The development of the iconography of Christ is beyond the scope of this essay, but it is possible that this type of youthful depiction was associated with a selection of texts that either mention Christ appearing 'in the form of' a boy or a child or he is described as a youth, as for example, 'The saviour appeared in the form of a boy of twelve years', (Acts of Peter and Andrew) and 'I saw a beautiful youth covering her with this cloak' (Acts of John 76). ${ }^{102}$ His youthful depiction is possibly explained by texts or, alternatively, the texts respond to images already prevalent. On the other hand, texts and images may reflect a cultural esteem for idealised youth and the visual appreciation of youthfulness in boys and girls in their transition towards adulthood.

Having looked at some of the problems of defining what adolescence was in Byzantium and having acknowledged that just in our culture it is not clear-cut, so it seems it was not then. Representations of youth, of girls and boys in the period between childhood and adulthood show subtle gradations between boy and man, girl and woman and appear to have been both customary and appreciated. In imagery of Christ's family, his brothers and his mother, various portrayals also emphasise childhood and adolescence. In apocryphal iconography, the origin of the imagery is still not clear. This is a subject that could well be explored further as it seems that either various textual traditions are contributing to it or that the iconography seems to arise somewhat independently from texts and perhaps from more personalised issues associated with patronage or societal influences.

100 D. M. Deliyannis, Ravenna in Late Antiquity. New York 2010, figs. 61-2.

101 T. Mathews, The Clash of Gods: A Reinterpretation of Early Christian Art. Princeton 1993.

102 Elliott, Apocrypha 300, 332; on the Acts of Peter and Andrew, see Elliott, Apocrypha 241, 242-3; on the Acts of John, see ELLiotT, Aprocrypha 303-310; for these and further examples, see D. Cartlidge - J. K. Elliott, Art and the Christian Apocrypha. London 2001, 58-9; for a summary of the various views, see Cartlidge, Art 53-61; also Mathews, Clash 139. Theories that the youthful appearance may have arisen in a Gnostic environment do not seem supported, and the Acts of John is no longer seen as a Gnostic text, see Elliott, Apocrypha 306-307; CARTLIDGE - ElliotT, Art 60. 


\section{The Byzantine Adolescent: Real or Imaginary?}

This paper seeks to answer the question of whether there was a Byzantine conceptualization of adolescence as a separate and distinct phase of the life cycle. It first takes a long (yet also necessarily concise) look at data collected by two main fields of inquiry into adolescence - Social Anthropology and Psychoanalysis - before using it to contextualize and hopefully illuminate the evidence yielded by a particular set of Byzantine sources.

Adolescence is a particularly transient and elusive phase: we know when one is born, marries, has children, dies; but when does adolescence begin and end?

You might think that the first appearance of the physical signs of puberty provides a clear enough starting date; yet difficulties arise the minute you begin to consider the communal, societal level. For example, menarche varies greatly in different societies and amongst females within the same society. Researchers suggest that this is so because menarche depends on a combination of circumstances such as climate, diet, social class, heredity. However, they are unable to be more specific regarding this recipe's ingredients; and the available evidence does not permit the easy drawing of inferences. For instance, researchers have concluded that since the 1850 s the mean starting age of adolescence in the West has inched back by one year every twenty-five, so that it now occurs six years earlier than it did in the 1850s (at eleven or twelve for girls, twelve or thirteen for boys). They may be right, but their further suggestion that this is perhaps due to greater affluence and better nutrition does not stand up to comparative examination. ${ }^{1}$ For instance, earlier studies of a number of societies from Japan to Africa found that the mean age for menarche ranged from ten to sixteen years, including between societies that were physically and culturally close to each other. ${ }^{2}$ And what are we to make of the fact that for centuries the legal age for marriage in Byzantium was set at seven years of age? Granted that the explicit aim of marriage was procreation, does this imply that the mean age for menarche occurred at about the same age? And when in the ninth century the age of hebe and marriage was raised to twelve for girls and fourteen for boys, ${ }^{3}$ are we to infer from this that the Byzantines were now poorer, less well fed, therefore girls got their periods later? This is clearly nonsense.

The more complex fact is that, as social anthropologist Arnold van Gennep concluded, "We must distinguish between physical puberty and social puberty, just as we do between physical kinship (consanguinity) and social kinship, between physical ma-

1 'The invention of adolescence'. Psychology Today (1 January 1995).

2 ARnold van Gennep, The Rites of Passage. Routledge 1960 (repr. Routledge - Abington - N. York 2004), 65-70.

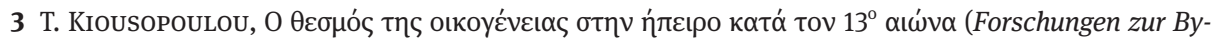
zantinischen Rechtsgeschichte). Athen 1990, 27-28, 74. 
turity and social maturity". ${ }^{4}$ Or, as a psychoanalyst would say, between biological and psychological maturity.

Van Gennep has shown that the physical manifestations and the cultural definition of puberty rarely coincide. In many pre-industrial societies, ceremonies and rituals associated with puberty extend over a long - sometimes very long - period. Age is not rigidly taken into account whereas other attributes, of a socialized nature, are.

Anthropological literature abounds with examples supporting van Gennep's understanding. For instance, Masai boys are circumcised not when they reach a specific age but when they are sufficiently strong: any time between twelve and sixteen, sometimes earlier if the parents are rich, later if they are poor. Amongst some American Indian tribes, entry into the grown-ups' group depends on prowess in war or raiding. In one such tribe (the Fox) initiation into manhood lasts nine years, in the last night of which the boy sleeps on the floor of the dance house and awakes a man. In some societies marriage ceremonies end only with the birth of the first child of the couple. Amongst the Cameroons the girl enters the group of women not when she reaches a certain age or loses her virginity or marries but when she gives birth ${ }^{5}$. One wonders whether there is an echo of this in Digenes Akrites where his wife is consistently described as a kore, despite marriage and the evident loss of her virginity. Does this signify that she never really became a woman because she never became a mother?

Van Gennep showed that every society indicates transitions between states through rituals. He defined rituals as performances replete with symbolism referring to processes of change and transformation, concerning both the individual at their centre and society at large. He specified a particular class of rituals as "rites which accompany every change of place, state, position and age" at birth, puberty, marriage and death. He called these rituals rites de passage and showed that they are comprised of three stages. ${ }^{6}$

The first is the brief phase of separation, in which symbolic behavior signifies the detachment of the individual from a hitherto fixed point in the social structure.

This ushers in the phase of marginality or liminality (from the Latin limen, 'thershold'). The liminal persona is regarded as embodying and existing in a fluid, transitional state between-and-betwixt other, stable states. In rituals marking adolescence, he or she is considered to be neither child nor adult; and in many societies also as neither male nor female (yet). Much that is normative is inverted. Usual everyday activities and notions of space and time are suspended and replaced by other, corresponding cultural values. Individuals can exhibit behaviour that would normally be regarded as bizarre and pathological, yet the rest of the tribe does not regard it

4 VAN GenneP, Rites 65-70 (note. 2).

5 VAN GENNEP, Rites 65-70, 85, 87, 177, 183.

6 VAn GenneP; W. Lloyd Warner 1959, in: V. Turner, The Forest of Symbols. Aspects of Ndembu Ritual. Ithaka, London, 1967, 94-95. V. Turner, The Ritual Process. Structure and Anti-Structure. New Brunswick -London, 1969 (repr. 2009). 
as such. If anything, it is the absence of such behaviour that is looked upon as abnormal. Thus, ambiguity reigns: it is not only accepted but expected. Such a state of affairs can clearly only be tolerated within set, specifically transient boundaries, if only because in all cultures - albeit in different degrees - what is socially ambiguous is also regarded as deeply unsettling and potentially dangerous. ${ }^{7}$

Sooner or later, therefore, liminality is followed by the final phase of reintegration, during which the hitherto liminal person is reincorporated in society, in a new individual and social position that is once again relatively stable and unambiguous. ${ }^{8}$

Not all societies mark adolescence with elaborate rituals. Some may instead employ short initiation rites. These are always of a sexual nature, deemed to make one a man or a woman, or at least fit to be one. ${ }^{9}$ Viktor Turner notes that such rituals emphasize hunting and sex for boys; sex and motherhood for girls; respect for the elders and other social superiors for both. ${ }^{10}$

Turning to Psychoanalysis, it comes as no surprise to find that the first contribution to the study of adolescence was made by Sigmund Freud. ${ }^{11}$

Freud described psychosexual development as a biphasic process, with a long interruption between the two. The first phase extends from birth to about the third year of life. During this period all the essential elements of one's internal world and his or her ways of relating to the external world are established: the three agencies of the mind (id, ego, super-ego), attachments, identifications, oedipal configurations and conflicts, basic socialization skills. This phase comes to an end around the third year of life, when a wave of repression, particularly of the oedipal desires and conflicts, ushers in the long period of latency. During latency psychosexual growth essentially stops, to resume in earnest with the onset of puberty. Adolescence marks the second and final phase of psychosexual development. It is the critical, decisive period during which one's identity assumes its final form. It is also a time of upheavals often summarily referred to as 'the adolescent crisis'. (It is worth noting that anthropologist Viktor Turner uses the term 'life crisis' to denote times of important change, including puberty; and that the word krisis also - and literally - means 'decision').

Freud's theory of adolescence is intimately linked to his theory of narcissism. ${ }^{12}$ He postulated that every human being is born with a reservoir of narcissism (love

7 M. Douglas, Purity and Danger. An Analysis of Concepts of Pollution and Taboo. London 1969.

8 TURnER, The Forest of Symbols 95.

9 VAn Gennep, Rites 65-70 (note 2).

10 TURner, The Forest of Symbols 7-8 (referring to the Ndembu).

11 Essentially in Sigmund Freud, Three Essays on the Theory of Sexuality (1905), English transl., The Standard Edition of the Complete Psychological Works of Sigmund Freud. London 1974, VII, $123-246$. See generally M. Perret-Catipovic - F. Ladame (ed.), Adolescence et psychanalyse: une histoire. Lausanne 1997. Transl. PH. SoRKIN, Adolescence and Psychoanalysis. The Story and the History. London 1998).

12 Essentially in Sigmund Freud, On Narcissism: An Introduction (1914), in op. cit., XIV, 67-104. 
for one's self), itself an expression of the Life and self-preservative instincts, enlivened by a particular type of energy he called 'narcissistic libido'. Being entirely self-centred, for a short while the infant continues to invest (cathect) its narcissistic libido in the only object that matters - its own self. To the extent that it recognizes other objects, it is interested in them only in so far as they serve its survival needs. Soon however the infant begins to invest quantities of narcissistic libido in something other-than-me. The first such investments will be on the part-object of the breast that feeds it; then on the whole-object of the mother. These will be the first of a lifetime's emotional attachments, involving a range of feelings of fluctuating intensities. Throughout life, from time to time the ego will be withdrawing cathexes from an object to reinvest them in the ego or in another object. Such movements are particularly frequent and intense during puberty, when the hitherto dispersed sexual instincts coalesce to acquire a new, overarching sexual reproductive aim, and the ego seeks for the first time an explicitly sexual object in the external world. This new object may be of the 'attachment type', based on early infantile prototypes; or of the more ancient, 'narcissistic type', which seeks the subject's own self in another person. The representations of the first objects and everything associated with them are "a prototype of every kind of love": "The finding of an object is thus in fact a re-finding of it”.

The choice of an object is at first accomplished in the imagination: to begin with, the adolescent's sexual life is almost entirely filled with phantasies. This soon comes to an end. A partial ego regression leads to the lifting of some of latency's repressions and to a rekindling of early psychic modes of being. Infantile oedipal impulses and modes of relating to the parents re-emerge, in usually unconscious fantasies. However, in infancy incestuous unconscious phantasies were destined to remain just that; whereas in adolescence the body is capable of realizing them - with terrible end results, as the tragedy of Oedipus exemplifies. The taboo of incest, a bulwark built during latency, leads to the repudiation of incestuous thoughts and compels the individual to seek a sexual object in the real, external world, beyond phantasy and beyond parents and parental substitutes. The detachment of the individual from parental authority represents the greatest and most difficult achievement of adolescence.

Adolescence is thus a time of enormous psychic reorganization. Everything that is important is laid down in infancy, and stored and enriched in latency; but it is in adolescence, with the dissolving of the parental ties through the so-called 'dissolution of the Oedipal complex', that the personality acquires its final, definite shape, ${ }^{13}$ at the end of this "second (and final) individuation process". ${ }^{14}$

13 E. Kestemberg, 'Notule sur la crise de l'adolescence. De la description a la conquête'. Revue Française de Psychoanalyse 44 (1980) 623-30.

14 P. Blos, 'The Second Individuation Process of Adolescence'. Psychoanalytic Study of the Child 22 (1967) 162-86; repr. in: PeRret-CATIPOVIC - LAdAME, op. cit., 77-102 (note 11). 
Amongst the main psychological characteristics of adolescence, the most obvious is the intense, biologically-bound upsurge of sexual excitation. It is not easy for the adolescent to know what to do with it. Sexual fore-play is also a fore-pleasure, involving all main organs (the eyes, at the sight of the desired object, the tactile sensation when that object is touched, and so on). But it is a pleasure that also leads to an increase in sexual tension. When - as so often in adolescence - it finds no satisfaction through consummation, it turns into the intense un-pleasure of sexual frustration. To use Freud's parallel, foreplay without consummation is like a joke with no punch line.

But sexual frustration is hardly the greatest problem adolescents face. Other and much more pressing difficulties have been the focus of post-Freudian research.

Anna Freud saw that by the end of latency a character structure has emerged. This is based on whatever equilibrium has formed between ego, id and super-ego within the entailed nexus of identifications, conflicts, defenses, and generally all significant factual and psychic experiences. In adolescence all this will shift, change and transform as the adolescent detaches libido from the parents, partially abandoning them and other hitherto stable objects. For a time the ego will be temporarily weakened, due to the combination of the emotional disengagement from internalized infantile objects, partial regression, and increased pressure of drives. Hitherto, the parental ego had been making itself available to the child, lending structure and organization to the child's ego so that it could function. As such, it had constituted an essential component of the child's ego. Adolescence disrupts this dyadic alliance, propelling the adolescent to partly abandon ego states that had been citadels of safety and security or had provided ways of coping with stress. ${ }^{15}$

The process of adolescence therefore inevitably involves loss: the loss of the now diminished love-objects of the past; but also, the loss of a part of the self. ${ }^{16}$ The sense of loss, and of being lost, can be further exacerbated by disappointment when the expected experience does not live up to one's fantasies of what adolescence would or should be like. Coming to terms with reality therefore involves the further loss of the phantasy. These losses, like any other, have to be mourned. A type of mourning frequently presents as the adolescent tendency towards melancholia, as Shakespeare embodied in Hamlet. This tendency carries the danger of turning into a depression; which, compounded with impetuosity and heightened emotionality, can lead to tragic end results: adolescence carries a greatly increased risk of suicide.

The weakening of the ego also causes huge anxieties. In order to protect the self, the ego strengthens existing psychic defences and may also develop new ones. If, for whatever reasons, coming of age is too frightening to the adolescent, such defensive

15 Essentially in Anna Freud, ‘Adolescence'. Psychoanalytic Study of the Child 13 (1958)255-78. See also BLos, Second Individuation.

16 Freud, art. cit.; Blos, art. cit. 
maneuvers can become extreme. ${ }^{17}$ One might employ disavowal to retreat from adolescence altogether, denying it and trying to obliterate it and turn the clock and the body back into latency. An example of this is provided by the 'ascetic' adolescent who fights off all impulses, including physiological ones for food, sleep, comfort. Anorexia, for example, is a particularly adolescent illness. ${ }^{18}$ Another adolescent may react in ways that seem to be the very opposite of a retreat but are in fact propelled by equally high anxieties. Instead of resisting the change, this adolescent rushes to embrace it, in an often violent psychic move. Unable to negotiate a gradual separation from internal infantile objects, he or she may resort to flight, suddenly cutting off ties, becoming detached, rejecting and debasing towards the parents. This leads to a conscious sense of alienation - another frequent characteristic of the adolescent experience; but also to guilt towards the debased parents. This guilt often remains unconscious, and may emerge into consciousness only after adolescence is over. ${ }^{19}$

In short, when things go wrong adolescence is not only a critical but also a traumatic time, due to the accumulation of conflicting pressures: the vacuum created by the psychically ousted objects; the pressure to find new ones to fill their place; the urge to move on while fighting regressive tendencies against the relinquishing of infantile ties. All this and more propels some characteristic adolescent stances, such as the experiencing of sudden 'crushes' on people, or entering into emotionally quasimerged states with others in an attempt to satiate a typical "affect and object hunger”. ${ }^{20}$ Hence the creation of near-instant erotic relationships seeking not a personal bond but sharpness of affect and emotional agitation; or the creation of instant and intense friendships and the embracing of very tight groups or gangs of contemporaries. These are in effect substitute family formations. They offer stimulation, sharing, comradeship, understanding, loyalty, devotion, empathy, and thus replace the parents in supporting the adolescent ego while it struggles to orientate itself towards a mature, personal identity. ${ }^{21}$ Crushes and mergers can also occur in relation to abstractions (Truth, Nature and Beauty), ideas and ideals, the idolization of famous people or a new object.

Conversely, when no new external object is invested, the ego libido, withdrawn from the infantile objects and now unspent in the adolescent, makes him or her withdrawn, moody, secretive, alone, selfish, uncaring. He or she will also have little tolerance of frustration, due to the partial regression of their weakened ego and increased drive energy. Furthermore, intolerance of frustration, and a revived nearinfantile sense of omnipotence, can lead adolescents to take risks in denial of danger and death; and impels them to seek instant gratification, as if they were infants

17 FREUD, art. cit.

18 Kestemberg, Notule.

19 BLos, Second Individuation.

20 IDEM, art. cit.

21 IDEM, art. cit. 
anew: 'I want it, and I want it now', seems to be the order of the day, as Aristotle already knew: "Young men have strong passions and tend to gratify them indiscriminately. Of the bodily desires the sexual is the one by which they are most swayed and in which they show absence of self control".22

The combination of intolerance of frustration, need to enrich a depleted ego, find a new object, but also keep in touch with reality, often leads adolescents to immerse themselves in the external world with a pressing need to be active, keep moving, do things. Hence an often seen return to 'action language' and 'body language', to somatization of affects, conflicts and drives. ${ }^{23}$

Through all this sturm und drang, the essential question which the adolescent seeks to answer is, 'Who am I?'. Not so much 'To be or not to be', but rather 'Who to be and who not to be'. Normally, by the end of adolescence he or she has arrived at an answer. A new psychic equilibrium has been established, affording a higher level of psychic organization and functioning, autonomy of thought, a personal life style, a stable sexual identity. The ego recovers its strength, enriched by the journey. The psychic defences employed during adolescence can now relax, making reconciliation with parental and other previously abandoned objects possible.

The psychoanalytic understanding of the adolescent process is parallel to the social anthropological description of the rites de passage and their phases of separation, liminality and re-integration. The focus is different, of course. Psychoanalysis sees adolescence as a process of individuation; Anthropology sees it as a social process, expressed and enacted in the communal sphere.

One of the striking similarities between the two is the acceptance of bizarre behaviour as normal during the period of liminality (for the anthropologist) or adolescence (for the psychoanalyst). Psychoanalysts insist that the upheavals of adolescence are expressions of the momentous adjustments taking place in the internal world. "There is no adolescence without a crisis of adolescence", they insist. ${ }^{24}$ In parallel to the anthropologists, they maintain that this crisis is not pathological but necessary, inevitable and ultimately beneficial; and that it is the maintenance of a steady equilibrium throughout it is that is problematic. As Blos put it, the adolescent may be doing all the wrong things but he is doing them for the right reasons. For the parents, this can also be a very trying time, if they see their child transform into a rejecting, obsessional, ascetic, schizoid, secretive, paranoid, promiscuous, hysterical, even suicidal adolescent, who transfers his or her allegiance outside the family while treating the parents with an apparent rejecting indifference that borders on callousness. But, to quote Anna Freud: "It is normal for an adolescent to be inconsistent and unpredictable; to fight his impulses and to accept them; toward them off and then suc-

22 M. Perret-Catipovic - F. Ladame, 'Adolescence and Psychoanalysis: the story of the history', in: M Perret-CATipociv - LAdAme, op. cit., 1-16, esp. 4 (as note 11).

23 Blos, art. cit.

24 F. Ladame - M. Perret-Catipovic, 'Normality and Pathology in Adolescence', in: Perret-CatiPOVIC - LADAME, op. cit., 161-72, esp. 162. 
cumb to them; to imitate others while searching for his own identity; to love and hate his parents; to revolt against them and be dependent on them; to be more idealistic artistic, generous, unselfish than he will ever be again, while also self-centred, egotistical, calculating”. All this until, hopefully, a solid adult structure of the personality emerges and takes permanent hold. Instead of rushing the adolescent into psychotherapy, Anna Freud gently suggests that it is more likely the parents who may be in need of professional help during this period.

The view that strife is a normal and inevitable part of adolescence has been contested. In her influential book on Samoa anthropologist Margaret Mead postulated that the 'adolescent crisis' is not universal in all societies and therefore not accounted for by innate psychobiological processes. It is specific to modern western societies, due to the pressures they impose on their adolescents. She found no trace of it amongst Samoa's adolescents. ${ }^{25}$

Is Mead right? If so, what was going on in the mind of the Byzantine adolescent? Or perhaps the question should be, Was there a Byzantine adolescent? Was there a social recognition that the passage from childhood to adulthood was a different and special life phase, with its own characteristics?

At first blush, the evidence seems to suggest a negative answer. As death came early, it seems that ages were telescoped in a Byzantine population of essentially (by our standards) young people. ${ }^{26}$ With death expected at between fifty and sixty years of age, it is no surprise that in our sources fifty-two year old Filopappous is described as a geron. ${ }^{27}$ Fifty-four year old Cyril of Skythopolis as a "revered old man". ${ }^{28}$ Children were asked to be older than their years - marry, have babies, work or go to war as early as possible. Even with the ninth-century increase of the legal age of marriage girls could still be married off at twelve, boys at fourteen, ${ }^{29}$ while parents exerted strict control over their offspring: they confined girls, severely punished disrespectful behaviour, chose their child's spouse (albeit with the child's consent), drew up marriage contracts, often enough arranged a marriage while the girl, especially, was below the legal limit. Justinianic legislation set adulthood at twenty-five years (at which point e.g. an unmarried female could bypass her parents and marry of her own accord) yet even after that age offspring remained hypexousia to parents and grandparents. ${ }^{30}$

25 M. MEAD, Coming of Age in Samoa: A Psychological Study of Primitive Youth for Western Civilisation. 1928.

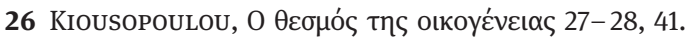

27 Ed., transl., E. JEFfreys, Digenis Akritis. The Grottaferrata and Escorial versions. Cambridge 1998. I shall be following Jeffrey's translation for consistency's sakes.

28 E. DAvies, 'Age, Gender and Status: A Three-Dimensional Life Course Perspective of the Byzantine Family', in: Approaches to the Byzantine Family (Birmingham Byzantine and Ottoman Studies 14), ed. L. Brubaker - S. Tougher. Farnham - Burlington 2013, 153-71, esp. 167-8.

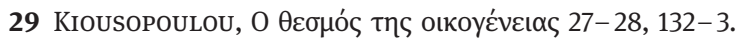

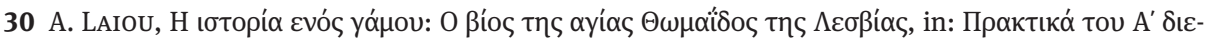

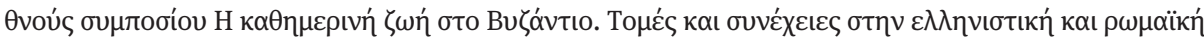


Granted all this, one may wonder whether a Byzantine child had time to be an adolescent, and whether any adolescent expression would have been thinkable, let alone permissible.

In turning to this question, we do well to remember that all these terms - 'childhood', 'adolescence', 'marriage' 'adulthood' - carried a different significance and set of cultural values in Byzantium than they do with us; that within Byzantium itself they carried a different significance at different times, and at different places according to local custom; that legislation set parameters rather than reflected social realities; that the factual evidence from Byzantine sources is not only relatively scarce but also necessarily inconsistent. For instance: Davies' study of Saints' Lives from the sixth to the twelfth century suggests that the upper markers of youth were at twenty-four, twenty-five years for women, the late twenties for men; in twelfth-century Cyprus Neophytos the Recluse was eighteen when his parents drew up his marriage contracts; Laiou concludes that the usual marriage age for a tenth-century girl was between thirteen and fifteen; Kiousopoulou finds that newlyweds in thirteenthcentury Epirus were between fifteen and twenty. ${ }^{31}$

It is very difficult to gage much about the Byzantine adolescent experience (or absence thereof) from the meager factual information our sources yield. I suggest, however, that in surprising and sharp contrast, Byzantine sources register the internal, psychic world of adolescence, and do so with gusto, exhibiting every single one of its unmistakable characteristics. The locus of this psycho-cultural registration is the fictional literature of twelfth-century Constantinople and the reduction of epic poetry that preceded it. The surprising thing is that the Byzantine understanding and depiction of adolescence in these works is astonishingly close to the psychoanalytic description of adolescence. Less surprisingly perhaps, it also carries echoes of anthropological descriptions of adolescence in pre-industrial societies.

Let us turn then to the novels of the twelfth century - a century which opens, more or less, with the first reduction of the ballads of Digenes Akrites into a single romance. ${ }^{32}$

All these works (including Digenes which is distinguished from the slightly later novels by a less sophisticated expression) contain a psychological layer of signifi-

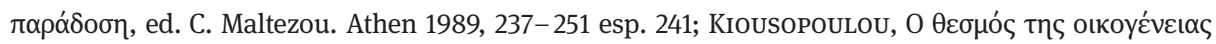
28, 131-9. D. ARIANTZI, Kindheit in Byzanz. Emotionale, geistige und materielle Entwicklung im familiären Umfeld vom 6. bis zum 11. Jahrhundert (Millennium Studien 36). Berlin - Boston 2012, $41-47$. 31 Davies, Age, Gender and Status esp. 167 (note 28); C. Galatariotou, The Making of a Saint. The

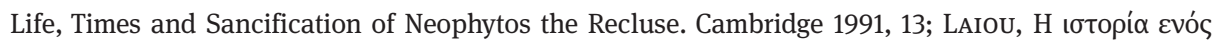

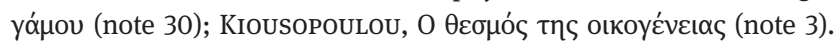

32 P. Magdalino, The Empire of Manuel I Komnenos 1143-1180. Cambridge University Press 2002, 421. For consistency's sakes I will be following the translation in: E. JEFFREYs, Four Byzantine Novels. Theodore Prodromos, Rhodanthe and Dosikles. Eumathius Makrembolites, Hysmine and Hysminias. Constantine Manasses, Aristandros and Kallithea. Niketas Eugenianos, Drosilla and Charikles. Liverpool 2012. 
cance which is culturally extremely important, and of which their authors were naturally not fully - if at all - consciously aware. At this psycho-social level, Digenes and the novels are nothing if not depictions of the psychic sturm und drang of adolescence, to which they give external symbolic form through the mediation and safety of a 'fictional' tale.

The protagonists are all adolescents, and very young ones at that. This is emphatically depicted in general references to the bodily characteristics of first-blooming youth, which is at times also specifically described: Dosikles was "comely in appearance, / with his beard just blooming on his chin / and his face gracefully surrounded by the first down on his jaw"; 33 Charikles "down" "[had not] begun to shadow [his] chin", "he was a 'ruddy-complexioned youth, / his cheeks smooth"; 34 Dryas' "chin [was] wreathed with its first down"; ${ }^{35}$ Digenes is twelve when he abducts and marries the girl, who seems to be even younger.

How much younger the desired female could be becomes disturbingly explicit in Kleandros' love story in Drosilla and Charikles. The object of his desire, the maiden Kalligone to whom he sends very explicit erotic letters, is of "tender youthfulness", "a child, a tender child"; yet "The sight of her drove even old men, impotent from excessive age, to passion". Kleandros becomes "possessed" both by "savage desire" and "a deep affection for her childish innocence / and compassion for everything it gives rise to". She was a "childish, fragile girl", so young that when she did not respond to one of his love letters he thought, "Either she did not receive that letter / or she was too busy with her playmates". ${ }^{36}$

Byzantine societies do not seem to have acknowledged the transition from "tender child" to adolescent through explicit puberty rituals or initiation rites of the type anthropologists describe. However, the novels contain references to markers or entrypoints from boyhood to manhood. In Digenes this comes very close to an initiation rite proper, consisting of the spectacular display of his riding and especially hunting skills before the elders of his tribe - his father and uncles - when he kills in quick succession a bear, a deer, a lion. Prior to this his father had laughed at his son's suggestion to go hunting with the men, Digenes being but a prepubescent child: "and you are a boy" (pais) 'of twelve, not yet in your prime" (hebes ektos hyparheis). Digenes enters the hunt an agouritzes and anaxios, and exits it a man: andreiomenos. This is a first initiation rite marking the passage from childhood into adolescence. It is completed and emphasised with the change of clothes immediately after it, his discarding of simple clothes and his investiture, as it were, with resplendent clothing. ${ }^{37}$

In a more urbane mind set, Charikles also describes arriving at the cusp of adolescence just before "down begun to shadow [his] chin", with the emphasis - so fa-

33 JEFFreys, Byzantine Novels 117.214 - .217, and see also the references to Dionysus.

34 JefFreys, Byzantine Novels 375.60, 388.79 - .80 (note 32).

35 JEFFREYS, op. cit., 40.130.

36 JefFreys, op. cit., 362-73, esp. 363.70, 364.76, 364.81 - .84, 364.97, 365.112, 367.193 - .195.

37 JeFFREYs, Digenis Akritis 72-80 (note 27). 
miliar to the anthropologist and the psychoanalyst - on socialization, friendship, induction in a peer group, and the joining in the pursuits that make a man of a boy horse-riding and hunting: "I had already reached adolescence, / brought up according to the norms of well-born youths; / I was happy in the company of the young men with whom I associated; / I rode, I joined in sports, as is customary for young men, / I hunted hare, I became a skilled equestrian / - for I had highly skilled companions". 38

38 JefFreys, Byzantine Novels 375.53-60 (note 32). 

Ulrike Sirsch

\title{
Erwachsenwerden oder Erwachsensein?
}

\author{
Ausgewählte Aspekte zu Jugend, „Emerging Adulthood“ und \\ jungem Erwachsenenalteraus Sicht der Entwicklungspsychologie
}

\section{Einleitung}

In diesem Beitrag wird auf die Anfänge einer wissenschaftlichen Psychologie bzw. Entwicklungspsychologie im deutschen Sprachraum eingegangen. In weiterer Folge werden sowohl ausgewählte „klassische“ Theorien als auch „neuere“ Ansätze der Entwicklungspsychologie bezogen auf die Altersphase „Jugend“ und ,junges Erwachsenenalter“ dargestellt.

Ausgewählte Themen dieses Beitrages sind Grundelemente der psychoanalytischen Entwicklungstheorie von Sigmund Freud, der psychosozialen Entwicklungstheorie von Erik H. Erikson, der Theorie der Entwicklungssaufgaben von Robert J. Havighurst und schließlich das Konzept „Emerging Adulthood“ von Jeffrey Jensen Arnett.

\section{Historische Entwicklung einer wissenschaftlichen Psychologie}

Der Ursprung westlicher Psychologie basiert auf Sokrates, Platon und Aristoteles und deren Dialogen über Geist, Wille und der Beziehung zwischen Individuum und Gemeinschaft/Staat. Erst gegen Ende des 19. Jahrhunderts entwickelte sich die Psychologie zu einer eigenen Fachdisziplin - Fragen aus der Philosophie wurden mittels Methoden aus z.B. Physiologie und Physik empirisch im experimentellen Kontext untersucht. Wilhelm Wundt gründete 1879 das erste Labor für experimentelle Psychologie in Leipzig. ${ }^{1}$

Im weiteren Verlauf wurde 1904 die „Gesellschaft für experimentelle Psychologie“ in Gießen etabliert. 1929 wurde sie in Wien in „Deutsche Gesellschaft für Psychologie“ umbenannt, um eine Einengung der Psychologie auf „experimentell“ und „empirisch“ zu vermeiden - wie die Website der Deutschen Gesellschaft für Psychologie (DGPs) zu ihrer Geschichte anführt. ${ }^{2}$ Die Deutsche Gesellschaft für Psychologie, mit ihrem Sitz in

1 R. J. GeRRIG, Psychologie. Hallbergmoos ${ }^{20} 2015,9$.

2 Deutsche Gesellschaft für Psychologie (DGPs), Geschichte der DGPs. 2014c. Abgerufen unter http:// www.dgps.de/index.php?id=111 [14.10.2014]

Ә OpenAccess. (c) 2018 Ulrike Sirsch, published by De Gruyter. (cc))BY-NC-ND This work is licensed under the Creative Commons Attribution-NonCommercial-NoDerivatives 4.0 License. 
Göttingen, definiert sich als „Vereinigung der in Forschung und Lehre tätigen Psychologen und Psychologinnen“ und zielt darauf ab, die wissenschaftliche Psychologie $\mathrm{zu}$ fördern und zu verbreiten. ${ }^{3}$

\section{Traditionelle und moderne Entwicklungspsychologie}

Bestrebungen, eine „Fachgruppe Entwicklungspsychologie“ innerhalb der Deutschen Gesellschaft für Psychologie zu etablieren, erfolgten in den 1970er Jahren, gegründet wurde die „Fachgruppe Entwicklungspsychologie der Deutschen Gesellschaft für Psychologie“ 1982. ${ }^{4}$ Zur Entstehung trug ein Koordinationskreis von Personen bei, dem u.a. auch Brigitte Rollett angehörte, die als Professorin von 1979 bis 2003 am (damaligen) Institut für Psychologie in Wien die Abteilung Kinder- und Jugendpsychologie leitete und auch Gründungspräsidentin der „Österreichischen Gesellschaft für Psychologie (ÖGP)“ im Jahre 1993 war. $^{5}$

Als Gegenstand der Entwicklungspsychologie gibt die Fachgruppe auf ihrer Website $^{6}$ folgende Definition an: „Die Entwicklungspsychologie befasst sich mit der Beschreibung, Erklärung und Optimierung von relativ überdauernden intraindividuellen Veränderungen im menschlichen Erleben und Verhalten über die gesamte Lebensspanne hinweg, d.h. ab vorgeburtlicher Entwicklung bis ins hohe Alter und zum Tod“.

Diese Fokussierung auf die gesamte Lebensspanne war innerhalb der Entwicklungspsychologie nicht immer gegeben. Die Entwicklungspsychologie blickt zwar auf eine etwa hundertjährige Tradition zurück, konzentrierte sich aber zunächst darauf allgemeine Veränderungen $\mathrm{zu}$ untersuchen. Phasen und Stufenmodelle wurden erstellt. ${ }^{7}$ Bekannte Beispiele diesbezüglich sind Freuds (1856-1939) Phasentheorie und Eriksons (1902-1994) Stufenmodell der Entwicklung. ${ }^{8}$

3 Deutsche Gesellschaft für Psychologie (DGPs, 2014d), Was ist die DGPs? Abgerufen unter http:// www.dgps.de/index.php?id=83 [14.10.2014].

4 Deutsche Gesellschaft für Psychologie (DGPs, 2014a) Fachgruppe Entwicklungspsychologie. Abgerufen unter http://www.dgps.de/index.php?id=159 [14.10.2014].

5 G. H. Fischer, Ein halbes Jahrhundert Geschichte des Instituts für Psychologie. Abgerufen unter http://psychologie.univie.ac.at/fileadmin/user_upload/fak_psychologie/files/Ein_halbes_Jahrhun dert_Geschichte_des_Instituts_f\%C3\%BCr_Psychologie.pdf [14.10.2014]; Österreichische Gesellschaft für Psychologie (ÖGP, 2014). Vereinsgeschichte. Abgerufen unter http://www.oegp.net/vereinsgeschich te.htm [14.10.2014].

6 Deutsche Gesellschaft für Psychologie (DGPs, 2014b). Fachgruppe Entwicklungspsychologie. Gegenstand der Fachgruppe. Abgerufen unter http://www.dgps.de/index.php?id=2000615 [14.10.2014]. 7 L. MontadA, Fragen, Konzepte, Perspektiven, in: Entwicklungspsychologie, hrsg. von R. Oerter - L. Montada. Weinheim ${ }^{6} 2008,3-48$, hier 3.

8 E. H. ERIKSON, Identity: Youth and crisis. New York 1968; S. FreUd, Über Psychoanalyse: Fünf Vorlesungen gehalten zur 20jährigen Gründungsfeier der Clark University in Worcester Mass., Sep- 
Beispielhaft zeigt sich in Eriksons Stufenmodell „die Entwicklung einer Entwicklungspsychologie“ als Erweiterung einer traditionellen Entwicklungspsychologie, die auf allgemeine Veränderungen abzielte und auf eine Kinder- und Jugendpsychologie beschränkt war, ${ }^{9}$ hin zu einer Entwicklungspsychologie, die Entwicklung über die Lebensspanne betrachtet. Eine moderne Entwicklungspsychologie ist zudem fokussiert auf die Untersuchung differentiell unterschiedlicher und kontextbeeinflusster Entwicklungen. Das Wissen über mögliche Einflussfaktoren bzw. moderierende Bedingungen auf die Entwicklung liefert dabei die Basisinformationen, die für die Förderung von Entwicklung und die Prävention von Fehlentwicklungen notwendig sind. ${ }^{10}$

\section{Jugend als eigenständige Entwicklungsperiode}

Entsprechend den Wurzeln der Psychologie betrachten Oerter und Dreher (2008) auch den Ursprung der Vorstellung einer eigenständigen Jugendphase als weit in der Zeit zurück. Gesellschaftlich und sozialpolitisch eingebettet wurde eine Phase der Jugend aber erst im 19./20. Jahrhundert, verbunden mit einer ansteigenden Industrialisierung - da auf jugendliche Erwerbstätige zunehmend verzichtet werden konnte - und der Ausweitung der allgemeinen Schulpflicht. ${ }^{11}$

Als Begründer der psychologischen Jugendforschung gilt G. Stanley Hall, der 1904 ein zweibändiges Werk zu „Adolescence“ herausbrachte. ${ }^{12}$ Als Professor für Psychologie und Pädagogik war Hall Präsident der Clark University (Worcester, Massachusetts, USA), als Freud 1909 seine fünf Vorlesungen über Psychoanalyse zur 20jährigen Gründungsfeier der Clark University hielt. ${ }^{13}$

Neben der Psychologie beschäftigen sich weitere Fachdisziplinen in den Sozialwissenschaften mit der Lebensphase Jugend, wie z.B. die Soziologie oder die Päda-

tember 1909 (Projekt Gutenberg Ebook 2007). Wien 1910/2007. Abgerufen unter http://www.gutenberg. org/ebooks/20613 [29.10.2014]. Für einen Überblick siehe W. DATLER - M. WiningER, Psychoanalytische Zugänge zur frühen Kindheit, in: Theorien in der Entwicklungspsychologie, hrsg. von L. Ahnert. Wien 2014, 354-379; A. Flammer, Entwicklungstheorien. Psychologische Theorien der menschlichen Entwicklung. Bern 2009, Kap. 5 und 6.

9 T. KREtTEnauer, Der Entwicklungsbegriff in der Psychologie, in: Theorien in der Entwicklungspsychologie, hrsg. von L. Ahnert. Wien 2014, 2-25, hier 21.

10 Montada, Fragen, Konzepte, Perspektiven 5 (wie Anm. 7).

11 R. OERTER - E. DREHER, Jugendalter, in: Entwicklungspsychologie, hrsg. von R. Oerter - L. Montada. Weinheim ${ }^{6} 2008,271-332$, hier 271; M. PINQUART - A. GROB, Soziale Übergänge von der Kindheit bis ins frühe Erwachsenenalter, in: Entwicklungspsychologie des Jugendalters (Enzyklopädie der Psychologie, Serie Entwicklungspsychologie), hrsg. von R. K. Silbereisen - N. Birbaumer. Göttingen 2008, V 109-132, hier 112.

12 J. J. ARNETT, Emerging adulthood. A theory of development from the late teens through the twenties. American Psychologist 55 (2000) 469-480, hier 476.

13 Freud, Psychoanalyse (wie Anm. 8). 
gogik. Deutlich wird dies z. B. im 6. Bericht zur Lage der Jugend in Österreich, ${ }^{14}$ in dem der Jugendbegriff aus soziologischer ${ }^{15}$, entwicklungspsychologischer ${ }^{16}$ und pädagogischer Perspektive ${ }^{17}$ betrachtet wird. Ergänzend wird Jugend in der Jugendarbeit ${ }^{18}$ und aus juristischer Sicht ${ }^{19}$ beschrieben.

Als disziplinübergreifenden Konsens in den Sozialwissenschaften beschreibt Dreher ${ }^{20}$ das „Verständnis der ,Jugendphase“ als soziohistorisch und gesellschaftspolitisch verankertes Phänomen, das dem epochalen Wandel, der sozioökonomischen und multikulturellen Veränderung von Lebensbedingungen unterliegt, letztlich aber auch das Selbstverständnis der jeweiligen Erwachsenengeneration widerspiegelt.“

\subsection{Beginn und Ende der Jugend und des jungen Erwachsenenalters}

In Lehrbüchern der Entwicklungspsychologie, wie Oerter und Montada oder nachfolgend Schneider und Lindenberger, und deren Beiträgen zur Jugend ${ }^{21}$ und zum jungen Erwachsenenalter ${ }^{22}$ findet man übereinstimmend körperliche Veränderungen, bedingt durch die Pubertät als Beginn der Jugend. ${ }^{23}$

$\mathrm{Zu}$ den körperlichen Veränderungen in der Pubertät ist allerdings anzumerken, dass diese seit Ende des 19. Jahrhunderts immer früher einsetzen mit stagnierendem Trend. 1840 lag das Menarche-Alter bei durchschnittlich 17 Jahren während aktuell

14 Bundesministerium für Wirtschaft, Familie und Jugend. 6. Bericht zur Lage der Jugend in Österreich. Wien 2011.

15 R. RICHTER, Soziologischer Jugendbegriff - Was ist Jugend? in: Bundesministerium für Wirtschaft, Familie und Jugend. 6. Bericht zur Lage der Jugend in Österreich. Wien 2011, 27-32.

16 E. DREHER, Jugend aus entwicklungspsychologischer Perspektive. In Bundesministerium für Wirtschaft, Familie und Jugend. 6. Bericht zur Lage der Jugend in Österreich. Wien 2011, 33-37.

17 S. Sting, Jugend aus pädagogischer Sicht. In Bundesministerium für Wirtschaft, Familie und Jugend. 6. Bericht zur Lage der Jugend in Österreich. Wien 2011, 39-42.

18 L. ABUZAHRA, Jugendbegriff in der Jugendarbeit: Der junge Mensch als Mensch, in: Bundesministerium für Wirtschaft, Familie und Jugend. 6. Bericht zur Lage der Jugend in Österreich. Wien 2011, 43- 44.

19 A. BirKLbaUER, Überlegungen zum juristischen Jugendbegriff. In Bundesministerium für Wirtschaft, Familie und Jugend. 6. Bericht zur Lage der Jugend in Österreich. Wien 2011, 45-47.

20 DREHER, Jugend aus entwicklungspsychologischer Perspektive 33 (wie Anm. 16).

21 OERTER - DREHER, Jugendalter 271-332 (wie Anm.11); R. K. SILBEREISEN - K. WeICHHOLD, Jugend (12-19 Jahre), in: Entwicklungspsychologie, hrsg. von W. Schneider - U. Lindenberger. Weinheim 2012, $259-282$.

22 A. M. FreUnd - J. Nikitin, Junges und mittleres Erwachsenenalter, in: Entwicklungspsychologie, hrsg. von W. Schneider - U. Lindenberge. Weinheim ${ }^{\top} 2012$, 259-282; G. KRAMPEN - B. REICHLE, Entwicklungsaufgaben im frühen Erwachsenenalter, in: Entwicklungspsychologie, hrsg. von R. Oerter - L. Montada. Weinheim ${ }^{6} 2008$, 333-365.

23 Oerter - Dreher, Jugendalter 272 (wie Anm.11); Silbereisen - WeichHold, Jugend 237 (wie Anm. 21); vgl. Dreher, Jugend aus entwicklungspsychologischer Perspektive 34 (wie Anm. 16). 
12 Jahre angeführt werden. ${ }^{24}$ Begründet wird dies mit besseren allgemeinen Umweltbedingungen, kalorienreicherer Ernährung und verbesserten medizinischen Bedingungen. ${ }^{25}$

Die Angaben bezogen auf ein konkretes Alter betreffend das Ende der Jugend bzw. den Beginn des Erwachsenenalters sind weit weniger klar. Oerter und Dreher ${ }^{26}$ verweisen als Abgrenzungskriterien von der Lebensphase Jugend zu der des frühen Erwachsenenalters einerseits auf Rollenübergänge (z.B. Berufseinstieg) und andererseits auf Aspekte sozialer Reife (z. B. Eigenverantwortlichkeit). Krampen und Reichle ${ }^{27}$ führen 1. formale und rechtliche Kriterien, z. B. Volljährigkeit, 2. objektive Kriterien, z.B. Elternschaft, 3. psychologische Kriterien wie Ablösung, Autonomie und psychologische Reife, und schließlich 4. subjektive Kriterien, nämlich die Selbstklassifikation zu einer bestimmten Altersgruppe, als Kriterien für den Übergang zum frühen Erwachsenenalter an. Silbereisen und Weichhold ${ }^{28}$ verweisen auf einen graduellen Eintritt ins Erwachsenenalter vom Auszug aus dem Elternhaus hin zu Partnerschaft und Familiengründung. Die beiden letztgenannten werden auch von Freund und Nikitin neben Volljährigkeit, Autonomie (verstanden als finanzielle und emotionale Unabhängigkeit, mit schon erfolgtem Auszug aus dem Elternhaus sowie Verantwortung für eigene Entscheidungen) und Berufseinstieg als Kriterien zur Definition des jungen Erwachsenenalters genannt. Sie betonen, dass es für den Beginn des jungen Erwachsenenalters (und damit für das Ende der Adoleszenz) keine klaren Kriterien gibt, sondern dass hier „historische Veränderungen und kulturelle Variationen“ ${ }^{\text {29 }}$ eine Rolle spielen.

Somit lässt sich zusammenfassen, dass der Beginn des Erwachsenenalters angelehnt an die soziologische Literatur anhand von sozialen Übergängen definiert wird. ${ }^{30}$ Es werden Übergänge in den produktiven Bereich der Kultur und Gesellschaft, wie Abschluss der Schule, Auszug aus dem Elternhaus sowie Beginn der Berufstätigkeit dargestellt, und Übergänge in den reproduktiven Bereich der Kultur und Ge-

24 Silbereisen - WeichHold, Jugend 239 (wie Anm. 21); siehe dazu auch E. DREHER - U. SiRSCH - S. STRoвL - S. MucK, Das Jugendalter - Lebensabschnitt und Entwicklungsphase, in: Bundesministerium für Wirtschaft, Familie und Jugend. 6. Bericht zur Lage der Jugend in Österreich. Wien 2011, 49-81, hier 60.

25 Silbereisen - WeichHold, Jugend 239 (wie Anm. 21); für eine zusammenfassende Beschreibung biologischer, kognitiver und sozialer Veränderungen im Jugendalter siehe DREHER - SIRSCH - STROBL

- MucK, Das Jugendalter - Lebensabschnitt und Entwicklungsphase (wie Anm. 24).

26 OERTER - DREHER, Jugendalter 273 (wie Anm. 11).

27 Krampen - Reichle, Entwicklungsaufgaben im frühen Erwachsenenalter 333f. (wie Anm. 22).

28 Silbereisen - WeichHold, Jugend 243 (wie Anm. 21).

29 FREUND - Nikitin, Junges und mittleres Erwachsenenalter 260 f. (wie Anm. 22).

30 PINQUART - GROB, Soziale Übergänge 110 (wie Anm.11). 
sellschaft, wie Partnerschaft und Elternschaft, beschrieben. ${ }^{31}$ Ablösung und Autonomie waren und sind dabei wesentliche Themen psychologischer Forschung.

Aus der Sicht der Betroffenen ist Erwachsenwerden mit positiven und negativen Aspekten verbunden, eine gewisse Ambivalenz hinsichtlich dieses Entwicklungsschritts ist zu erkennen. Untersuchungen zu Vorstellungen von Jugendlichen das Erwachsenwerden betreffend, erfasst aus der Sicht von 12- bis 16-Jährigen, zeigen, dass zwar Unabhängigkeit die Liste der positiven Aspekte anführt, aber Verantwortung auch als wichtiger negativer Aspekt genannt wird. ${ }^{32}$ Verantwortung und Unabhängigkeit konnte auch Kalausek ${ }^{33}$ als die am häufigsten genannten Antworten auf die Frage „Was bedeutet es für Sie erwachsen zu sein?“ bei jungen Erwachsenen zwischen 18-29 Jahren und Erwachsenen zwischen 30 - 45 Jahren feststellen. Beide Gruppen gaben jeweils einen höheren Anteil an positiven Aspekten verglichen mit negativen Aspekten an, jedoch verbindet die jüngere Gruppe das Erwachsensein tendenziell mit mehr negativen Aspekten als die ältere Gruppe. An erster Stelle der positiven Aspekte steht in beiden Altersgruppen „Freiheiten/Entscheidungen treffen“ während „Verantwortung“ die Liste der negativen Aspekte in beiden Gruppen anführt. In der älteren Gruppe wurde ähnlich häufig auch noch fehlende Spontaneität genannt.

\subsection{Ausweitung der ,Jugend“ bzw. die Einführung einer neuen Lebensphase „Emerging Adulthood“}

Dreher $^{34}$ postuliert zu der Diskussion einer Ausdehnung der Jugendphase, mit einem früheren Beginn und einem späteren Abschluss, „dass die Jugendphase innerhalb komplexer und sich rasch verändernder Lebensbezüge als ,Lernphase für den Aufund Umbau von Kompetenzen, Rollen und Funktionen der Lebensbewältigung eine zeitliche Ausdehnung rechtfertigt“"

Ein „Spezifikum“ bezüglich Ausweitung der Jugend ist die Präsentation einer neuen Entwicklungsphase „Emerging Adulthood“ (dt. „aufkommendes Erwachsenenalter"), ${ }^{35}$ die Jeffrey Jensen Arnett gegen Ende des alten und Anfang des neuen Jahrtausends der Fachwelt präsentierte. ${ }^{36}$ Arnetts Beitrag, publiziert im Jahr 2000 im

31 J. E. NuRmi, Socialization and self-development. Channeling, selection, adjustment and reflection, in: Handbook of adolescent psychology, eds. R. M. Lerner - L. Steinberg. New York 2004, 85-124; PINQUART - GROB, Soziale Übergänge (wie Anm. 11).

32 E. Dreher - M. Dreher, Familientabus und Ablösung, in: Klinische Entwicklungspsychologie der Familie, hrsg. von. B. Rollett - W. Werneck. Göttingen 2002, 185- 205.

33 S. KALAUSEK, Subjektive Vorstellungen der Einflussfaktoren auf das Erwachsenwerden bei emerging adults und Erwachsenen. Wien: Unveröffentlichte Diplomarbeit, Fakultät für Psychologie der Universität Wien 2014.

34 DREHER, Jugend aus entwicklungspsychologischer Perspektive 33 (wie Anm. 16).

35 siehe FREUND - Nikitin, Junges und mittleres Erwachsenenalter 263 (wie Anm. 22).

36 J. J. ARNETT, Are college students adults? Their conception of the transition to adulthood. Journal of Adult Development 1 (1994) 213 - 224; J. J. ARNETT - S. TABER, Adolescence terminable and interminable: 
Journal „American Psychologist“ mit dem Titel „Emerging Adulthood. A theory of development from the late teens through the twenties“ erweckte Aufsehen in der Fachwelt. In diesem Beitrag beschreibt Arnett die Notwendigkeit einer ,distinct period of the life course ${ }^{\text {*37 }}$ abgegrenzt von Jugend und frühem Erwachsenenalter und fokussiert auf das Alter 18-25 Jahre.

Arnett beruft sich in seiner Konzeption auf Erikson, ${ }^{38}$ der bereits - allerdings als Ausnahme und nicht wie Arnett mehr oder weniger als Norm für eine ganze Alterskohorte $^{39}$ - eine verlängerte Adoleszenz einräumt, wenn die Gesellschaft die Möglichkeit eines psychosozialen Moratoriums bietet. ${ }^{40}$ Erikson $^{41}$ führt als Beispiele in diesem Zusammenhang die „Wanderschaft“ [gemeint sind die Wanderjahre von Handwerksgesellen nach Abschluss der Lehrzeit] und „academic life“ an, erwähnt aber weder eine eigene Stufe noch eine neue Bezeichnung für diesen Zeitabschnitt der Entwicklung in seiner psychosozialen Entwicklungstheorie, die in Stufen gegliedert ist. $^{42}$

\section{Ausgewählte Phasen- und Stufenmodelle der Entwicklungspsychologie im Überblick - Freud, Erikson und Havighurst}

Ein Charakteristikum von Phasen- bzw. Stufenmodellen ist deren Zusammenhang mit dem Alter. Im folgenden Abschnitt werden ausgewählte Phasen- bzw. Stufenmodelle im Überblick beschrieben. Diejenigen Anteile, welche zentral für das Jugend- und das frühe Erwachsenenalter sind, werden ausführlicher dargestellt.

\subsection{Freuds psychoanalytische Entwicklungstheorie}

Freud nahm in seiner psychoanalytischen Entwicklungstheorie an, dass Entwicklung durch ein Hinzukommen neuer erogener Zonen in jeder (Alters)Phase (von oral, anal,

When does adolescence end? Journal of Youth and Adolescence 23 (1994) 517- 537; J. J. ARNETT, Learning to stand alone: The contemporary American transition to adulthood in cultural and historical context. Human Development 41 (1998) 295-315; ARNETT, Emerging adulthood 469-480 (Anm. 12).

37 ARNETT, Emerging adulthood 469 (wie Anm. 12).

38 ERIKSON, Identity (wie Anm. 8) zitiert nach ARNETT, Emerging adulthood 470 (wie Anm. 36).

39 Vgl. J. E. CôtÉ, Emerging adulthood as an institutionalized moratorium: Risks and benefits to identity formation, in: Emerging adults in America: Coming of age in the 21st century, eds. J. J. Arnett J. L. Tanner. Washington, DC 2006, 85-116, hier 86.

40 ERIKSON, Identity 156 „psychosocial moratorium“ (wie Anm. 8)

41 ERIKSON, Identity 157 (wie Anm. 8).

42 Für einen Überblick siehe L. E. BERK, Entwicklungspsychologie. München ${ }^{5} 2011$; A. FLAMMER, Entwicklungstheorien. Psychologische Theorien der menschlichen Entwicklung. Bern 2009, Kap. 6. 
phallisch, über die Latenz, bis zu genital) zu beschreiben ist..$^{43}$ Libido (Lustansprüche) ist (sind) an erogene Zonen gebunden, d.h. an Mundschleimhäute, die Afterregion und an Genitalien. Erogene Zonen werden im Laufe der Entwicklung zu unterschiedlichen Zeiten aktuell, d.h. es kommt zu einer Verlagerung vom Oralen zum Analen und dann zu den Genitalien. Negativ kritisiert wurde Freud aufgrund der Überbetonung des Einflusses der Sexualität. Positiv anzumerken ist, dass Freuds Phasentheorie die erste Theorie war, die früher Entwicklung eine wichtige Rolle einräumte (d.h. kindliche Erfahrungen prägen das spätere Leben bzw. negative Erfahrungen führen zur Entwicklung psychischer Störungen).

Ausgehend von einem Entwicklungsbegriff basierend auf Reifung, d.h. einem Wachstum des Organismus nach inneren Gesetzmäßigkeiten ${ }^{44}$ bzw. eine durch Erbanlagen gesteuerte Entwicklung ${ }^{45}$, endet Freuds Phasenmodell mit der Jugend - eine Weiterentwicklung des Individuums im Erwachsenenalter war zu dieser Zeit noch nicht etabliert.

Oft wird Pubertät und Adoleszenz ${ }^{46}$ gleichbedeutend benutzt. Der Begriff Adoleszenz betont, dass Jugend als von Gesellschaft und Kultur beeinflusst betrachtet wird - „Jugend als soziale Konstruktion“, ${ }^{47}$ während die Verwendung des Begriffes Pubertät die Geschlechtsreife meint ${ }^{48}$ und damit körperliche Veränderungen anspricht.

\subsection{Jugend und junges Erwachsenenalter - psychoanalytische Theorien und Individuation}

Körperliche Reifungsprozesse, eine Zunahme an kognitiven Fähigkeiten und eine Veränderung von gesellschaftlichen Rollen und Erwartungen führen dazu, dass Jugendliche zunehmend nach mehr Unabhängigkeit streben. Historisch betrachtet befassten sich Vertreter/innen psychoanalytischen Theoriengutes als erste mit dem Prozess der Ablösung der Jugendlichen von den Eltern. Unter einer psychoanalytischen Perspektive stellen Konflikte und verstärkte Spannungen in der Familie einen

43 Freud, Psychoanalyse (wie Anm. 8). Für einen Überblick siehe W. DATler - M. Wininger, Psychoanalytische Zugänge zur frühen Kindheit, in: Theorien in der Entwicklungspsychologie, hrsg. von L. Ahnert. Wien 2014, 354-379; FlAMmER, Entwicklungstheorien Kap. 5 (wie Anm. 42).

44 T. Krettenauer, Der Entwicklungsbegriff in der Psychologie, in: Theorien in der Entwicklungspsychologie, hrsg. von L. Ahnert. Wien 2014, 2-25, hier 3.

45 M. KAvšEK, Reifung, in: Dorsch. Lexikon der Psychologie, hrsg. von M. Wirtz - J. Strohmer. Bern ${ }^{17} 2014,1409$.

46 Als Synonym für das Jugendalter: B. SchwARz, Adoleszenz, in: Dorsch. Lexikon der Psychologie, hrsg. von M. Wirtz - J. Strohmer. Bern ${ }^{17} 2014$, 99.

47 Silbereisen - WeichHold, Jugend 236 (wie Anm. 21).

48 K. Weichold, Pubertät, in: Dorsch. Lexikon der Psychologie, hrsg. von M. Wirtz - J. Strohmer.

${ }^{17}$ Bern 2014, $1358-1359$. 
zentralen Stellenwert für die Entwicklung einer reifen Persönlichkeit dar. ${ }^{49}$ Margaret Mahler schlug ein Mehr-Phasen-Modell der Separation-Individuation vor und verstand unter „Individuation“ einen Prozess in der frühen Kindheit, wenn sich das Kind erstmals als unabhängig existierend von seiner Mutter begreift und von sich und der Mutter stabile mentale Repräsentationen entwickelt. ${ }^{50}$ Peter Blos (1973) postulierte schließlich eine „zweite Individuation“ im Jugendalter. ${ }^{51}$

In neueren Ansätzen zur Individuation, die nicht mehr von „der klassischen Konfliktthese der Psychoanalyse“ ${ }^{\text {“52 }}$ geprägt sind, stehen Veränderungen in den Interaktionen im Jugendalter zwischen Jugendlichen und Eltern im Fokus unter der Annahme einer zunehmenden Autonomieentwicklung bei gleichzeitig weiter bestehender Verbundenheit mit den Eltern..$^{53}$

Da in den letzten Jahrzehnten die Phase des Übergangs in Richtung Erwachsenenalter durch ein Hinausschieben von Erwachsenenrollen in westlichen Ländern zunehmend länger zu werden scheint bis hin zur Einführung einer eigenen Lebensperiode bezeichnet als „Emerging Adulthood“54, ist auch eine Ausweitung der Forschung zur Individuation im Jugendalter auf Individuation im jungen Erwachsenenalter bzw. „Emerging Adulthood“ zu verzeichnen. ${ }^{55}$

\subsection{Eriksons psychosoziales Stufenmodell der Entwicklung}

In der Erweiterung von Freuds Phasenmodell übernimmt Erikson Freuds Grundannahmen und postuliert sein psychosoziales Stufenmodell ${ }^{56}$ mit insgesamt acht Stufen. Das Modell berücksichtigt Entwicklung bis ins Alter. ${ }^{57}$

49 J. G. MASche - S. WALPER, Facetten und Funktionen der Individuation, in: Eltern-Kind-Beziehungen im Jugend- und Erwachsenenalter. Entwicklungsverläufe, Einflussfaktoren und Konsequenzen der Individuation (Zeitschrift für Familienforschung, Sonderheft 3), hrsg. von J. G. Masche - S. Walper. Opladen 2003, 7-17; L. Steinberg, Adolescence. New York ${ }^{8} 2008$, Kap. 9.

50 M. MAhler - F. Pine - A. Bergman, Die psychische Geburt des Menschen: Symbiose und Individuation. Frankfurt am Main ${ }^{19} 2008$; siehe auch DATLER - WinINGER, Psychoanalytische Zugänge zur frühen Kindheit (wie Anm. 43); Flammer, Entwicklungstheorien Kap. 5 (wie Anm. 42).

51 P. BLos, The second individuation process of adolescence. The Psychoanalytic Study of the Child 22 (1967) 162-186; IDEM, Adoleszenz. Eine psychoanalytische Interpretation. Stuttgart 1973.

52 MASCHE - WALPER, Facetten und Funktionen der Individuation 8 (wie Anm. 49).

53 H. D. Grotevant - C. R. Cooper, Patterns of interaction in family relationships and the development of identity exploration in adolescence. Child Development 56 (1985) 415-428; J. YounISS J. SMOLlaR, Adolescent relationships with mothers, fathers, and friends. Chicago 1985.

54 ARnetT, Emerging adulthood (wie Anm. 12).

55 Für einen Überblick bezüglich Identität und Individuation in der Adoleszenz und in der Phase „Emerging Adulthood“ siehe S. Koepke - J. J. A. Denissen, Dynamics of identity development and separation -individuation in parent - child relationships during adolescence and emerging adulthood - A conceptual integration. Developmental Review 32 (2012) 67-88.

56 ERIKSON, Identity (wie Anm. 8). 
Der Lebenszyklus wird von Erikson (1968) als Abfolge psychosozialer Krisen verstanden, die es zu lösen gilt und die eine gute oder weniger gute Lösung erfahren können. Die ersten fünf Stufen Eriksons lassen sich altersmäßig den Phasen Freuds zuordnen ${ }^{58}$ : „Urvertrauen versus Misstrauen“ (Freud: oral) und „Autonomie versus Scham und Zweifel“ (Freud: anal) in der Säuglings- und Kleinkindphase, „Initiative versus Schuldgefühl“ in der frühen Kindheit (Freud: phallisch) und „Fleiß versus Minderwertigkeitsgefühl“ in der mittleren Kindheit (Freud: Latenz). „Identität versus Identitätsdiffusion“ (Freud: genital) sieht Erikson als Hauptthema der Adoleszenz, „Intimität versus Isolierung“ als das Thema für das junge Erwachsenenalter. Im mittleren Erwachsenenalter schließlich gilt es die Krise der „Generativität versus Selbstabsorption“, im höheren Erwachsenenalter „Integrität versus Verzweiflung“ zu bewältigen.

Eriksons Entwicklungstheorie erhebt den Anspruch, eine universale Stufentheorie darzustellen, gültig für alle Kulturen und historische Zeiten. Erikson betrachtet Entwicklung im Kontext der Umwelt, der Umkreis der Bezugspersonen erweitert sich zunehmend von der primären Bezugsperson, über Eltern, Familie, Schule, Gleichaltrige, Freunde, sexuelle Partner/innen, Arbeitskollegen/innen hin zu gemeinsamer Arbeit, dem Zusammenleben in einer Partnerschaft und der Menschheit generell. ${ }^{59}$ Während die Reihenfolge der Krisen ${ }^{60}$ festgelegt ist - alles folgt einem Grundplan ${ }^{61}$ wurde zur Reihenfolge der gelungenen Lösungen von Erikson kein entsprechendes Postulat aufgestellt. ${ }^{62}$

\subsection{Jugend und junges Erwachsenenalter in Eriksons psychosozialem Stufenmodell der Entwicklung}

Identitätsentwicklung, die letztlich lebenslang ein Thema ist, wird von Erikson als die zentrale Aufgabe der Adoleszenz betrachtet. ${ }^{63}$ Jugendliche versuchen die Fragen zu beantworten, „Wer bin ich?“ und „Wo ist mein Platz in der Gesellschaft?“ Aufgrund körperlicher Veränderungen durch die Pubertät und neuer Erwartungen der Umwelt werden neue Rollen ausprobiert bzw. (intensiv) exploriert (z. B. Verliebtheit, Anschluss an Jugendgruppen). Die vorausgehenden Stufen Eriksons liefern zudem Elemente, die

57 Für einen Überblick siehe BERK, Entwicklungspsychologie (wie Anm. 42); FlAMmER, Entwicklungstheorien Kap. 6 (wie Anm. 42).

58 Siehe dazu z.B. BERK, Entwicklungspsychologie 19; Flammer, Entwicklungstheorien 97 (wie Anm. 42).

59 Flammer, Entwicklungstheorien 97 (wie Anm. 42).

60 Invariante Stufentheorie: Flammer, Entwicklungstheorien 105 (wie Anm. 42).

61 Epigenetisches Prinzip: ErIKson, Identity 92 (wie Anm. 8).

62 Flammer, Entwicklungstheorien 106 (wie Anm. 42).

63 ERIKSON, Identity (wie Anm. 8); siehe auch BERK, Entwicklungspsychologie (wie Anm. 42); Flammer, Entwicklungstheorien Kap. 6 (wie Anm. 42). 
jetzt in ein konsistentes Selbst integriert werden müssen, um eine gelungene Erwachsenenidentität herauszubilden. ${ }^{64}$ Eriksons intensive Beschäftigung mit dieser Stufe regte seinen Schüler James Marcia ${ }^{65}$ an, ein Ablaufmodell der Identitätsentwicklung vorzustellen. Marcia beschreibt vier Identitätszustände: 1. „Foreclosure“ als (eventuell vorschnell) von anderen (z. B. Eltern) übernommene Identität, wobei ohne oder mit wenig Exploration Verpflichtungen (sogenannte „commitments“) eingegangen werden, wie z. B. im Bereich von Rollen, die der Partnerschaft und Elternschaft oder im Bereich von Wertvorstellungen, z. B. solche betreffend Religion, oder auf Lebensstile bezogene commitments. Weitere Identitätszustände sind 2. „Identity Diffusion“ als die Phase der Identitätsverwirrung mit mangelnder Exploration und Verpflichtung, dann 3. das „Moratorium“ als eine Phase der intensiven Exploration aber (noch) keiner Verpflichtungen, und abschließend 4. „Identity Achievement“ als erarbeitete Identität, wobei nach ausführlich erfolgter Exploration Verpflichtungen eingegangen werden. ${ }^{66}$ Marcias Modell ist heute eher als Typenmodell zu verstehen, da es keine empirische Unterstützung für den Ablauf fand. ${ }^{67}$

Die Forschung der letzten drei Jahrzehnte zu Marcias Identitätsaspekten sieht Seiffge-Krenke ${ }^{68}$ in der großangelegten Metaanalyse von Kroger, Martinussen und Marcia (2010) zusammengefasst. Kroger et al. benutzten eine Basis von 500 Studien und wählten 124 davon nach vordefinierten Qualitätskriterien zur weiteren Analyse aus. Sie konnten feststellen, dass ein Teil der Jugendlichen sowohl Progression in ihrer weiteren Identitätsentwicklung (hin zu einer „reiferen“ Identität), ein geringer Teil Regression (weg von einer „reiferen“ Identität) und mehr als die Hälfte Stabilität und damit keine Veränderung zeigen. Kroger et al. fanden weiters in Studien, die Identitätsentwicklung im Längsschnitt bzw. im zeitlichen Verlauf untersuchten, heraus, dass etwa $50 \%$ der untersuchten Personen erst zwischen 30 und 36 Jahren eine „reife“ (erarbeitete) Identität erreicht hatten.

In weiterer Folge wurden im letzten Jahrzehnt zwei Modelle vorgestellt, die auf Marcias Konzeption von Exploration und Verpflichtung aufbauen bzw. sie erweitern. Einerseits ein Zwei-Zyklen-Modell mit Exploration in die Breite, Verpflichtung, Exploration in die Tiefe, Identifikation mit Verpflichtung und grübelnde Exploration ${ }^{69}$

64 Flammer, Entwicklungstheorien Kap. 6 (wie Anm. 42).

65 J. E MARCIA, Development and validation of ego-identity status. Journal of Personality and Social Psychology 3 (1966) 551-558.

66 Für Beispiele von Antworten zu Marcias Identitätszuständen, die bestimmten Bereichen wie der Berufswahl oder Wertvorstellungen zugeordnet sind, siehe MARCIA, Development and validation of ego-identity status 553 (wie Anm. 65).

67 Flammer, Entwicklungstheorien 101 (wie Anm. 42).

68 I. SeIfFGe-Krenke, Psychoanalytische Zugänge zur frühen Kindheit, in: Theorien in der Entwicklungspsychologie, hrsg. von L. Ahnert. Wien 2014, 380 -403, hier 399.

69 K. LuYCKX - L. Goossens - B. SoenEnS, A developmental contextual perspective on identity construction in emerging adulthood: Change dynamics in commitment formation and commitment evaluation. Developmental Psychology 42 (2006) 366-380; K. LuYCKX - S. J. SCHWARTZ - M. D. BERZONSKY - B. Soenens - M. VANSTEENKISTE -I. SMits - L. Goossens, Capturing ruminative exploration: 
und andererseits ein 3-Faktor-Modell mit Verpflichtung, Exploration in die Tiefe und Überdenken der Verpflichtung. ${ }^{70}$

Erikson postulierte im Anschluss an „Identität versus Identitätsdiffusion“ in der Adoleszenz die Krise „Intimität versus Isolierung“ für das junge Erwachsenenalter ${ }^{71}$. Eine gelungene Lösung der Identitätsaufgabe sieht Erikson ${ }^{72}$ als Voraussetzung für das Eingehen einer tragfesten Partnerschaft und Intimität an.

Befunde zur Entwicklung von Identität und Intimität in der Adoleszenz und im jungen Erwachsenenalter im Längsschnitt über 10 Jahre (im Alter von 15 bis 25 Jahre), wobei beide Einflussrichtungen (Identität beeinflusst Intimität und Intimität beeinflusst Identität) getestet wurden, zeigen, dass Identität zur Intimitätsentwicklung bedeutsam beiträgt und nicht umgekehrt. ${ }^{73}$ Damit unterstützt dieses Ergebnis Eriksons (1968) Überlegungen zur Abfolge.

\subsection{Havighursts Entwicklungsaufgaben}

Die Theorie der Entwicklungsaufgaben stammt von Robert J. Havighurst (1900 - 1991) aus dem Jahre 1948. ${ }^{74}$ Entwicklung wird in diesem hierarchisch aufgebauten Stufenmodell als Lernprozess über die gesamte Lebensspanne betrachtet. Havighurst definiert dazu altersspezifische Entwicklungsaufgaben und nennt jeweils sechs bis zehn Entwicklungsaufgaben pro Altersabschnitt, beginnend mit der frühen Kindheit bis hin zum höheren Alter. Einige seiner Entwicklungsaufgaben sind zeitlich begrenzt (z.B. Gehen lernen), andere wiederum kommen in mehreren Entwicklungsaufgaben über verschiedene Lebensabschnitte vor (z. B. Gleichaltrige und deren Rolle für die Entwicklung).

Entwicklungsaufgaben nach Havighurst sind kulturabhängig. Die Bewältigung der Entwicklungsaufgaben einer Altersphase ist wichtig für die Bewältigung von Entwicklungsaufgaben darauffolgender Altersphasen - die erfolgreiche Bewältigung

Extending the four-dimensional model of identity formation in late adolescence. Journal of Research in Personality 42 (2008) 58-82.

70 E. Crocetti - M. Rubini - W. Meeus, Capturing the dynamics of identity formation in various ethnic groups: Development and validation of a three-dimensional model. Journal of Adolescence 31 (2008) 207-222.

71 ERIKSON, Identity (wie Anm. 8); siehe auch BERK, Entwicklungspsychologie (wie Anm. 42); Flammer, Entwicklungstheorien Kap. 6 (wie Anm. 42).

72 ERIKSON, Identity (wie Anm. 8).

73 W. Beyers - I. SEIFFGE-KRENKE, Does identity precede intimacy? Testing Erikson's theory on romantic development in emerging adults of the 21st century. Journal of Adolescent Research 25 (2010) 387-415.

74 R. J. HAVIGHURST, Developmental tasks and education. New York ${ }^{3} 1981$; siehe auch E. DREHER - M. DREHER, Entwicklungsaufgaben als Zugang zur Biographie, in: Biographische Diagnostik, hrsg. von G. Jüttemann. Lengerich 2011, 119-126; FlammER, Entwicklungstheorien Kap. 16.3 und 16.4 (wie Anm. 42); FREUnd - Nikitin, Junges und mittleres Erwachsenenalter 263 (wie Anm. 22). 
einer Entwicklungsaufgabe verhilft $\mathrm{zu}$ Erfolg in einer anderen wiederkehrenden Aufgabe (Interdependenz), sie erleichtert aber auch den Erfolg bei zusammenhängenden Entwicklungsaufgaben. ${ }^{75}$ Die Bewältigung von Entwicklungsaufgaben führt zu gesellschaftlicher Akzeptanz bzw. Missbilligung, beispielsweise sieht Havighurst (1981) als eine Aufgabe für das frühe Erwachsenenalter „ein Heim herstellen, Haushalt organisieren“, geschieht dies sehr spät oder gar nicht - z. B. ein 35- bis 40-Jähriger lebt noch zu Hause bei den Eltern (bzw. bei der Herkunftsfamilie) - erzeugt dies auch noch in der heutigen Zeit Verwunderung.

Als Quellen der Entwicklungsaufgaben Havighursts sind 1. biologische Anforderungen (z. B. Pubertät), 2. gesellschaftliche Erwartungen (z. B. Lesen lernen im Rahmen des Erlernens von Kulturtechniken wie Lesen, Schreiben und Rechnen) und 3. individuelle Zielsetzungen (z.B. eine bestimmte Musikgruppe kennenzulernen) zu nennen. ${ }^{76}$

E. Dreher und M. Dreher ${ }^{77}$ erstellten eine phasenübergreifende Zuordnung aller Entwicklungsaufgaben von Havighurst. Sie unterscheiden vier Qualitäten: 1. Erwerb von Fähigkeiten und Kompetenzen in spezifischen Funktionsbereichen einschließlich der Kulturtechniken, 2. Auseinandersetzung mit der eigenen Person (Körperkonzept, Selbstkonzept und Identität, Komponenten der Selbstregulation), 3. Gestaltung von Beziehungen und Rollen (Familie, Peergruppe, Freundschaften und Partnerschaft, Nachbarschaft, soziales Engagement) und 4. Konkretisierung von Lebensentwürfen (private, berufliche, gesellschaftliche Funktionen, Wertorientierungen, Verantwortungsübernahme, Zeit- und Planungsperspektiven sowie Komponenten des Lebensstils).

\subsection{Jugend und junges Erwachsenenalter in Havighursts Entwicklungsaufgaben}

Havighurst $^{78}$ definierte als Entwicklungsaufgaben für die Adoleszenz (12-18 Jahre): 1) Aufbau neuer und reiferer Beziehungen zu Altersgenossen beiderlei Geschlechts, 2) Übernahme der männlichen und weiblichen Geschlechtsrolle, 3) Akzeptanz des eigenen Körpers, 4) Emotionale Unabhängigkeit von den Eltern und von anderen Er-

75 z.B. Ablösung von den Eltern und Partnerschaft, siehe E. DrEHER - M. DrEHER, Entwicklungsaufgaben im Jugendalter, in: Entwicklungsaufgaben und Bewältigungsprobleme in der Adoleszenz, hrsg. von D. Liepmann - A. Sticksrud. Göttingen 1985, 56-70.

76 R. J. HAVighurst, Developmental tasks and education. New York ${ }^{3} 1981$; siehe auch E. DREHER - M. DreHER, Entwicklungsaufgaben (wie Anm. 74); FlAMMER, Entwicklungstheorien Kap. 16.3 und 16.4 (wie Anm. 42); FREUND - Nikitin, Junges und mittleres Erwachsenenalter 263 (wie Anm. 22).

77 DREHER - DreHER, Entwicklungsaufgaben 121 (wie Anm. 74).

78 Deutsche Bezeichnungen siehe DREHER - DREHER, Entwicklungsaufgaben im Jugendalter (wie Anm. 75). 
wachsenen, 5) Vorbereitung auf Ehe und Familie und 6) auf Beruf 7) die Entwicklung eines eigenen Wertesystems sowie 8) sozial verantwortliches Handeln.

Im frühen Erwachsenenalter (18 - 30 Jahre) sind nach Havighurst (1981) vor allem Rollenübergänge, wie Heirat, Familiengründung und Berufseinstieg bedeutsame Entwicklungsaufgaben. Konkret definiert Havighurst ${ }^{79}$ folgende Entwicklungsaufgaben:

1) Auswahl eines Partners, 2) Mit dem Partner leben lernen, 3) Gründung einer Familie, 4) Versorgung und Betreuung der Kinder, 5) ein Heim herstellen; Haushalt organisieren, 6) Berufseinstieg, 7) Verantwortung als Staatsbürger ausüben sowie 8) eine angemessene soziale Gruppe finden.

Havighurst (1981) betont in Zusammenhang mit der Beschreibung der Entwicklungsaufgabe Unabhängigkeit im Jugendalter eine mögliche zeitliche Verschiebung aufgrund von „kulturellen“ Gegebenheiten: „Boys and girls often cannot get married until they are economically independent. This postpones marriage, and lengthens the period of residence within the family. It keeps young people in a position of dependence for several years beyond the time when they want to be independent" ${ }^{80}$

Eva Dreher und Michael Dreher, die sich intensiv mit Entwicklungsaufgaben des Jugendalters beschäftigten, ${ }^{81}$ untersuchten unter der Annahme, dass die Bedeutsamkeit von Entwicklungsaufgaben durch kulturelle, sozioökonomische und ökologische Bedingungen beeinflusst wird, deren Bedeutungsveränderung über einen Zeitraum von 10 Jahren. Bei der ersten Untersuchung in den 1980igern zeigten sich einerseits neue Bereiche, wie intime Partnerbeziehung, Zukunftsplanung und Selbstkenntnis, und andererseits eine Integration zweier Entwicklungsaufgaben, nämlich „Wertesystem“ und „sozialverantwortliches Verhalten“, in eine einzige Aufgabe. Sowohl Beruf als auch Peers sind 1985 und 1997 bei beiden Geschlechtern unter den drei bedeutendsten Bereichen angeführt. Die Wichtigkeit von Entwicklungsaufgaben ist 1997 generell geringer, es zeigen sich auch weniger bedeutsame Geschlechtsunterschiede. Der Bereich „Selbst“ zeigt 1997 eine geringere Wichtigkeit bei Jugendlichen beiderlei Geschlechts, der Bereich „Beziehung“ eine höhere bei den weiblichen Jugendlichen. ${ }^{82}$

Ergebnisse von Seiffge-Krenke und Gelhaar ${ }^{83}$ bieten Hinweise darauf, dass einige der von Havighurst bereits vor mehr als einem halben Jahrhundert postulierten Ent-

79 Deutsche Bezeichnungen siehe DREHER - DREHER, Entwicklungsaufgaben im Jugendalter 56-70 (wie Anm. 75).

80 HAVIGHURST, Developmental tasks and education 56.

81 DREHER - DREHER, Entwicklungsaufgaben im Jugendalter 56-70 (wie Anm. 75); E. DREHER - M. DREHER, Wahrnehmung und Bewältigung von Entwicklungsaufgaben im Jugendalter: Fragen, Ergebnisse und Hypothesen zum Konzept einer Entwicklungs- und Pädagogischen Psychologie des Jugendalters, in: Lebensbewältigung im Jugendalter, hrsg. von R. Oerter. Weinheim 1985, 30 - 61; OERTER - DREHER, Jugendalter (wie Anm. 11).

82 OERTER - DREHER, Jugendalter 282f (wie Anm. 11).

83 I. SEIFFGE-KRENKE - T. GELHAAR, Entwicklungsregulation im jungen Erwachsenenalter: Zwischen Partnerschaft, Berufseinstieg und der Gründung eines eigenen Haushalts. Zeitschrift für Entwick- 
wicklungsaufgaben auch heutzutage noch Gültigkeit besitzen, aber auch, dass Entwicklungsaufgaben des Jugendalters und auch solche des frühen Erwachsenenalters sich in ihrem zeitlichen Rahmen verschoben haben. Aufgaben der Adoleszenz (12-18) werden schon mit etwa 14 Jahren bewältigt, die meisten Aufgaben des frühen Erwachsenenalterns (18-30) mit Beginn der 20iger noch nicht, d.h. Aufgaben der Adoleszenz verschieben sich nach vorne, Aufgaben des frühen Erwachsenenalters nach hinten.

\section{6 „Emerging Adulthood“ - eine neue Entwicklungsphase}

\subsection{Definition und Begründung von „Emerging Adulthood“}

Arnett (2000) sieht die Lebensphase junger Menschen zwischen 18 Jahren und den Mitt- bis Endzwanzigern fokussiert auf 18-25 Jahre als „Emerging Adulthood“ und definiert sie als „a normative period of development in industrialized societies“. ${ }^{84} \mathrm{Er}$ führt die Notwendigkeit einer neuen Bezeichnung an, da er Begriffe wie „late adolescence“, „youth“, ${ }^{55}$ „young adulthood“, „early adulthood“ , „transition to adulthood“ und auch „novice phase“"86 für nicht passend hält. ${ }^{87}$

Mit dem Begriff „Emerging Adulthood“, der im deutschen Sprachraum keine gebräuchliche Übersetzung fand, gelang es Arnett, Forschung im Altersbereich 18 bis Mitte bzw. Ende der 20iger Jahre unter einem Oberbegriff zu sammeln. 2003 wurde die erste Konferenz zu „Emerging Adulthood“ in Harvard abgehalten. ${ }^{88}$ Seitdem finden Konferenzen alle zwei Jahre in den USA, organisiert von der „Society for the Study of

lungspsychologie und Pädagogische Psychologie 38 (2006) 18-31; IDEM, Does successful attainment of developmental tasks lead to happiness and success in later developmental tasks? A test of Havighurst's (1948) theses. Journal of Adolescence 31 (2008) 33-52.

84 J.J. ARNETt, Emerging adulthood, a $21^{\text {st }}$ century theory: A rejoinder to Hendry and Kloep. Child Development Perspectives 1 (2007) 80 -82, hier 81.

85 In Anlehnung an K. KEniston, Youth and dissent the rise of a new opposition. New York 1971; zitiert nach J. J. ARNETT, Emerging adulthood: The winding road from late teens through the twenties. Oxford 2004, 20.

86 In Anlehnung an D. J. LEVInSON, The seasons of a man's life. New York 1978; zitiert nach ARNETT, Emerging adulthood 470 (Anm. 12).

87 ARnetT, Emerging adulthood (wie Anm. 84); IDEM, Debate. Emerging Adulthood in Europe: A response to Bynner. Journal of Youth Studies 9 (2006) 111-123; IDEM, Emerging adulthood: What is it, and what is it good for? Child Development Perspectives 1 (2007) 68-73; IDEM, New horizons in research on emerging and young adulthood, in: Early adulthood in a family context, eds. A. Booth - S. L. Brown - N. Landale - W. D. Manning - S. McHale. New York, 2012, 231-244.

88 J. J. ARnett - J. L. TANnER, Preface, in: Emerging adults in America. Coming of age in the 21st century, eds. J. J. Arnett - J. L. Tanner. Washington, D.C 2006, xvii-xxii. 
Emerging Adulthood“ (SSEA, 2014) ${ }^{89}$ statt. Seit 2013 existiert auch eine eigene Zeitschrift „Emerging Adulthood“. ${ }^{90}$ Zusätzlich sei erwähnt, dass Forschung zu Adoleszenz und „Emerging Adutlhood“ im europäischen Raum auf alle zwei Jahre stattfindenden Konferenzen der „European Association for Research on Adolescence (EARA)“91 referiert und diskutiert wird (EARA, 2014), die zugehörige Zeitschrift ist das „Journal of Adolescence“.

Arnetts intensive Beschäftigung mit der Lebensphase Jugend ließ ihn $2007^{92}$ ein zweibändiges internationales Enzyklopädie-Werk (Teil 1 mit den Länder A-J und Teil 2 mit den Ländern K-Z) herausgeben, ausgewählte Länder wurden einige Jahre später überarbeitet. ${ }^{93}$ Die Beiträge liefern einen Überblick über die Lebensbedingungen und die Lebenswelten von Jugendlichen in mehr als 100 Ländern. ${ }^{94}$ Ergänzend seien für Österreich zur Lage der Jugend die Jugendberichte des jeweils zuständigen Bundesministeriums (z. B. Bundesministerium für Wirtschaft, Familie und Jugend, 2011) und für Deutschland die SHELL Jugendstudien angegeben (z. B. Shell Deutschland Holding, 2010).

Die Notwendigkeit einer neuen Entwicklungsphase, abgrenzbar von Jugend- und Erwachsenenalter, sieht Arnett (2000) durch demographische Veränderungen in westlichen Industriegesellschaften begründet. Er betrachtet gesellschaftliche Veränderungen in westlichen Industriegesellschaften in den letzten Jahrzehnten als Ursache dafür, dass junge Menschen soziale Übergange - in der soziologischen Literatur als Beginn des Erwachsenenalters definiert ${ }^{95}$ - zunehmend hinausschieben, wie $\mathrm{Ab}$ schluss der Schule, Auszug aus dem Elternhaus, Beginn der Berufstätigkeit sowie Heirat und Geburt des ersten Kindes. Dies ist auch die Folge von einem zunehmend größeren Anteil an jungen Menschen, die mehr Bildung anstreben ${ }^{96}$ gefolgt von einem

89 SSEA (2014): Society for the Study of Emerging Adulthood.

90 Siehe auch M. H. M. van Dulmen, Emerging adulthood - the journal. Emerging Adulthood, 1/1 (2013) 3-4.

91 EARA (2014): European Association for Research on Adolescence. Biennial Meetings. Abgerufen unter http://www.earaonline.org/biennial-meetings/ [16.10.2014].

92 J. J. ARnETT (ed.), International encyclopedia of adolescence (Vol.1, A-J). New York 2007; IDEM (ed.), International encyclopedia of adolescence (Vol. 2, K-Z). New York 2007.

93 J. J. ARnEtT (ed.), Adolescent psychology around the world. New York 2012.

$94 \mathrm{Zu}$ Österreich siehe E. MAYR - M. ADAMEK, Austria. in: International encyclopedia of adolescence (Vol.1, A-J), ed. J. J. Arnett. New York 2007, 45-52; Mayr \& Adamek, 2007; zu Deutschland siehe E. Dreher - H. ZuMKLEY, Germany, in: International encyclopedia of adolescence (Vol. 1, A-J), ed. J. J. Arnett. New York 2007, 321-343; sowie E. Dreher - U. SIRSCH - S. STRobL, Germany, in: Adolescent psychology around the world, ed. J. J. Arnett. New York 2012, 273-289.

95 Nurmi, Socialization and self-development 85-124 (wie Anm. 31); PINQUART - Grob, Soziale Übergänge 109-132 (wie Anm. 11).

96 ARnETT, Debate. Emerging Adulthood in Europe 111-123 (wie Anm.87). 
späteren Berufseinstieg. Diese Entwicklung steht natürlich auch in Zusammenhang mit sich verändernden Geschlechtsrollen. ${ }^{97}$

Das Hinausschieben sozialer Übergänge verdeutlicht Arnett durch demographische Kennzahlen, z. B. zum Heiratsalter in unterschiedlichen europäischen Ländern und den USA. Diese Kennzahlen zeigen in einigen europäischen Ländern eine Verschiebung des Heiratsalters für Männer und Frauen von 1980 auf 2000, z. B. bezogen auf Deutschland und Österreich macht die Verschiebung etwa 5 Jahren aus. ${ }^{98}$ Betrachtet man neuere Zahlen zu Österreich, ist deutlich zu sehen, dass sich dieser Trend auch fortsetzt: 2013 betrug das mittlere Erstheiratsalter (Median) in Österreich für Männer 32, 2, für Frauen 29, 8 Jahre (Statistik Austria, 2014).

Von einigen Wissenschaftlern und Wissenschaftlerinnen wird eine Fokussierung Arnetts auf 18-25 Jahre für „Emerging Adulthood“ als zu knappes Zeitfenster angeführt. Côté ${ }^{99}$ schlägt beispielsweise eine Erweiterung auf 18-30 vor (speziell für Personen, die eine höhere Ausbildung anstreben), auch Buhl und Lanz ${ }^{100}$ treten für eine Erweiterung der Zeitspanne bezogen auf Europa ein. Douglass ${ }^{101}$ meint „If marriage and parenthood mark the end of emerging adulthood, populations in many European countries have pushed the age of that ending from 25 to 30“. Arnett selbst räumt auch durchaus ein, dass sich in den USA, verglichen mit anderen Industrieländern, ein durchschnittlich eher geringeres Heiratsalter zeigt bzw. auch das Alter bei der Familiengründung geringer ist, während in anderen Ländern und in Europa diese Altersangaben höher liegen, und schlägt deshalb folgende Altersbereiche für „Emerging Adulthood“ und „young adulthood“ in den USA und anderen Ländern vor: ${ }^{102}$ „18-25 and 25- 45 may be more fitting in the USA, and 18-29 and 30 - 45 more fitting in the rest of the industrialized world".

Arnett (2000) begründet die Notwendigkeit einer eigenen Lebensphase auch mit der Tatsache, dass sich ein großer Anteil an „Emerging Adults“ in seinen Studien selbst zwischen jugendlich und erwachsen einschätzen. ${ }^{103}$ Durch seine Untersuchungen angeregt stellten weitere Studien ${ }^{104}$ zum „perceived adult status“ bzw. sub-

97 R. LARSON - S. Wilson, Adolescence across place and time. Globalization and the changing pathways to adulthood, in: Handbook of adolescent psychology, eds. R. Lerner - L. Steinberg. New York 22004 299-330, hier $320 \mathrm{f}$.

98 ARnETT, Debate. Emerging Adulthood in Europe 112 (wie Anm.87).

99 CôtÉ, Emerging adulthood as an institutionalized moratorium 85-116 (wie Anm. 39).

100 H. M. BuHL - M. LANZ, Emerging Adulthood in Europe: Common traits and variability across five European countries. Journal of Adolescent Research 22 (2007) 439-443.

101 C. B. Douglass, From duty to desire: Emerging adulthood in Europe and its consequences. Child Development Perspectives 1 (2007) 101-108, hier 106.

102 ARnETt, Young adulthood 237 (wie Anm. 87).

103 ARnEtT, Emerging adulthood 469-480 (wie Anm. 12); idem, Conceptions of the transition to adulthood: Perspectives from adolescence through midlife. Journal of Adult Development 8 (2001) 133143.

104 S. BAdGER, - L. J. NELSON - C. M BARRY, Perceptions of the transition to adulthood among Chinese and American emerging adults. International Journal of Behavioral Development 30 (2006) 84-93; C. S. 
jektiven Erwachsenenstatus fest, dass sich auch „Emerging Adults“ zwischen 18 und 29 Jahren anderer Länder zu einem großen Anteil (35-69\%; 55\% in Österreich siehe Sirsch et al., 2009) zwischen ,jugendlich und erwachsen“ einschätzen. Dementsprechend ist der Übergang von der Selbstdefinition „jugendlich“ zur Selbstdefinition „erwachsen“105 bei einem großen Anteil Betroffener noch nicht vollzogen, während der Anteil derer, die sich als definitiv nicht erwachsen sehen, sehr gering ist (3-12\%; $7 \%$ in Österreich siehe Sirsch et al., 2009) und sich zwischen einem Viertel und mehr als der Hälfte (25-59\%) als erwachsen betrachten (38\% in Österreich siehe Sirsch et al., 2009).

\subsection{Merkmale von „Emerging Adulthood“}

Zentrale Merkmale der Entwicklungsphase „Emerging Adulthood“ sind: ${ }^{106}$

1) Identitätsexploration im Bereich Arbeit, Liebe und Weltanschauung („Emerging Adulthood“ als „age of identity explorations“ - eine Zeit, in der man herausfindet, wer man ist), 2) viele Möglichkeiten ausprobieren (Emerging Adulthood“ als „age of possibilities“ - eine Zeit vieler Möglichkeiten), 3) vorrangig mit einem starken Fokus auf sich selbst („Emerging Adulthood“ als „,self-focused age“), da ja noch kaum oder keine Verpflichtungen, wie fixe Partnerschaft, Elternschaft, eingegangen werden. Für viele ist es 4) eine Phase der Instabilität („Emerging Adulthood“ als „age of instability“ - „eine Zeit der Unbeständigkeit“), da oftmals Partnerschaften, aber auch die Wohnsituation (verbunden mit der Ausbildung) rasch wechseln. Darüber hinaus ist es 5) eine Phase des sich „Dazwischen Fühlens“ („Emerging Adulthood“ als „,age of feeling in-between“ - eine Zeit, in der man sich nicht sicher ist, ob man schon er-

L. CHEAH - L. J. NELSON, The role of acculturation in the emerging adulthood of aboriginal college students. International Journal of Behavioral Development 28 (2004) 495-507; A. FACIO - F. MICocCI, Emerging adulthood in Argentina. New Directions for Child and Adolescent Development 100 (2003) 21-31; A. FACIO - S. RESETT - F. MiCOCCI - C. MistroRigo, Emerging adulthood in Argentina: An age of diversity and possibilities. Child Development Perspectives 1 (2007) 115-118; L. J. NELSON - S. BADGER B. Wu, The influence of culture in emerging adulthood: Perspectives of Chinese college students. International Journal of Behavioral Development 28 (2004) 26-36; L. J. NELSON - C. M. BARRY, Distinguishing features of emerging adulthood. The role of self-classification as an adult. Journal of Adolescent Research 20 (2005) 242-262; U. SIRSCH - E. DREHER - E. MAYR - U. WILLINGER, What does it take to be an adult in Austria? Views of adulthood in Austrian adolescents, emerging adults and adults. Journal of Adolescent Research 24 (2009) 275-292.

105 G. KRAMPEN - B. Reichle, Entwicklungsaufgaben im frühen Erwachsenenalter, in: Entwicklungspsychologie, hrsg. von R. Oerter - L. Montada. Weinheim ${ }^{6} 2008$, 333-365.

106 ARNETT, Emerging adulthood: The winding road from late teens through the twenties. Oxford 2004; ARNETT, Emerging adulthood: Understanding the new way of coming of age, in: J. J. Arnett - J. L. Tanner (ed.), Emerging adults in America. Coming of age in the 21st century. Washington, D.C. 2006, $3-19$. 
wachsen ist). Der Fragebogen „Inventory of Dimensions of Emerging Adulthood (IDEA) erhebt diese fünf Bestimmungsstücke. ${ }^{107}$

Aufgaben, die Arnett als mögliche Entwicklungsaufgaben für diese Altersphase betrachtet und aus den bestimmenden Merkmalen ableitet, sind: 1) eine klarere Identität entwickeln, 2) eine geringere Breite an Möglichkeiten akzeptieren, 3) weniger selbst-fokussiert werden (teilweise durch das Eingehen von Verpflichtungen anderen gegenüber), 4) Lebensentscheidungen treffen, die Instabilität reduzieren und schließlich 5) sich mehr als Erwachsener fühlen. Arnett verweist zusätzlich aber auch auf die Kriterien, um als erwachsen zu gelten, die er in seinen Untersuchungen als besonders wichtig ermittelt hat. ${ }^{108}$

Werden junge Menschen nach den wesentlichen Kriterien, die erfüllt sein müssen, um als erwachsen zu gelten, gefragt, so werden „Verantwortung für die Konsequenzen der eigenen Handlungen übernehmen“ und „Entscheidungen auf der Basis persönlicher Einstellungen und Wertorientierungen treffen, unabhängig von elterlichen oder anderen Einflüssen“ als wesentlichste Kriterien genannt, während Heirat und Elternschaft wenig Bedeutung zugeschrieben wird ${ }^{109}$. Auch für Österreich sind ähnliche Ergebnisse feststellbar. ${ }^{110}$

\subsection{Kritik an „Emerging Adulthood“}

Kritiker/innen ${ }^{111}$ sehen Arnetts Stufenkonzept als nicht universell gültig, sondern stark auf gut gebildete junge Menschen in westlichen Industriegesellschaften begrenzt, sie kritisieren die Nichtberücksichtigung unterschiedlicher sozioökonomischer Bedingungen und bemängeln, dass unterschiedliche Verläufe im Erwachsenwerden in verschiedenen Dimensionen z. B. bezogen auf Beruf und Beziehungsbereich nicht angedacht sind.

107 A. ReIfMAN - J. J. ARnetT - M. J. Colwell, Emerging adulthood: Theory, assessment and application. Journal of Youth Development 2(1) (2007 Summer) Article 0701FA003. Abgerufen unter http:// data.memberclicks.com/site/nae4a/JYD_070201_final.pdf [20.09.2014]

108 Arnett, pers. Mitteilung, 25.11.2013

109 J.J. ARnETT, Conceptions of the transition to adulthood: Perspectives from adolescence through midlife. Journal of Adult Development 8 (2001) 133-143.

110 U. Sirsch - E. DREHER - E. MAYR - U. Willinger, What does it take to be an adult in Austria? Views of adulthood in Austrian adolescents, emerging adults and adults. Journal of Adolescent Research 24 (2009) 275-292.

111 J. Bynner, Rethinking the youth phase of the life-course: The case for emerging adulthood. Journal of Youth Studies 8 (2005) 367-384; L. B. HENDRY - M. KLOEP, Conceptualizing emerging adulthood: Inspecting the emperor's new clothes? Child Development Perspectives 1 (2007) 74-79; IIDEM, Redressing the emperor! A rejoinder to Arnett. Child Development Perspectives 1 (2007) 83-85; IIDEM, How universal is emerging adulthood? An empirical example. Journal of Youth Studies 13 (2010) $169-179$. 
Die Ergebnisse der Untersuchung von Gollubits (2010) zeigen, dass Personen mit verschiedenen Ausbildungen unterschiedliche Wertungen der Kriterien, die sie für wichtig erachten, um als erwachsen zu gelten, angeben. Berufstätige, die eine Lehre als Ausbildung absolviert haben, sehen im Vergleich zu Studierenden eine höhere Wichtigkeit von Verhaltensweisen, die der Norm entsprechen (z. B. Vermeidung von kleinen Verbrechen wie Ladendiebstahl und Vandalismus). Sie werten biologische Übergänge (z. B. das Erreichen der vollen Körpergröße, sowie die Fähigkeit, Kinder zu zeugen bzw. zu bekommen), rechtliche und altersbezogene Übergänge (z. B. Erreichen des 18. Lebensjahres, Besitz eines Führerscheines), Rollenübergänge (z. B. verheiratet sein) und Übergänge, die sich auf längerfristige Verpflichtungen beziehen (z. B. lebenslange Verpflichtungen für andere eingehen, ein Haus kaufen), höher. In der Bedeutsamkeit von individualistischen (z. B. Verantwortung für die Konsequenzen der eigenen Handlungen übernehmen, Treffen unabhängiger Entscheidungen) und familienbezogenen Kriterien (z. B. Fähigkeit für Kinder zu sorgen) unterscheiden sie sich jedoch nicht. Zudem fühlen sich Studierende tendenziell weniger erwachsen, sie explorieren mehr, probieren mehr Möglichkeiten aus und geben eine höhere Fokussierung auf sich selbst an als Berufstätige mit Lehrausbildung. ${ }^{112}$

Eine weiterführende Studie, bei der differenziert Kriterien des Erwachsenseins in drei Altersgruppen (Jugendliche, jüngere „Emerging Adults“, ältere „Emerging Adults“) mit unterschiedlichen Ausbildungshintergründen (Lehre, Schule, Studium - jeweils mit und ohne erfolgtem Berufseinstieg) untersucht wurden, zeigt, dass alle drei Altersgruppen individualistische Kriterien am wichtigsten finden, darüber hinaus jedoch scheint auch in dieser Untersuchung der Bildungshintergrund eine entscheidende Rolle bei der Beurteilung anderer Bereiche der Kriterien des Erwachsenseins zu spielen. ${ }^{113}$

Arnett und Taber räumten schon 1994 ein, dass „Emerging Adulthood“ von Sozialisation beeinflusst ist: „Emerging adulthood is intended to apply mainly to cultures characterized by broad socialization, where the achievement of adult status is individually defined“. ${ }^{114}$ Damit wurden bereits zu diesem Zeitpunkt Kontexteinflüsse angedacht. Auch Douglass (2007) beschreibt unterschiedliche Bedingungen für „Emerging Adults“ in Nord-, Süd-, Ost- und Westeuropa, wie beispielsweise ein sehr spätes Auszugsalter für Italien, das durch soziale Erwartungen und sozioökonomische Bedingungen beeinflusst ist.

Arnett beantwortet die Frage danach, ob „Emerging Adulthood“ als „Stufe“ - im Sinne eines Stufenkonzeptes - zu betrachten ist, mit ja, betont aber die Heterogenität

112 S. Gollubits, Wird das Erwachsenwerden in Abhängigkeit von der Ausbildung unterschiedlich erlebt? Ein Vergleich zwischen Studierenden und Berufstätigen. Wien: Unveröffentlichte Diplomarbeit, Fakultät für Psychologie der Universität Wien 2010.

113 S. Keiblinger - C. TAlaska - N. Filip - U. Sirsch, Erwachsenwerden - Die Bedeutung der Kriterien des Erwachsenseins für Personen mit unterschiedlichen Ausbildungsarten. Poster auf der 11. Tagung der Österreichischen Gesellschaft für Psychologie, April 24-26, Wien 2014.

114 ARnett - TABER, Adolescence terminable and interminable 534 (wie Anm.36). 
von „Emerging Adulthood“ als „one stage, many paths“ und auch, dass Stufenkonzepte generell nicht als „universal“ und „uniform“ betrachtet werden sollten ${ }^{115}$, da sie immer beeinflusst sind „by contexts of social class, ethnicity, culture, nationality, and other influences“.116

\section{Ausblick und Zusammenfassung}

Emerging Adulthood“ wird sich gemäß Arnett ${ }^{117}$ im Zuge der Globalisierung weiter ausweiten. Nimmt man an, dass der Anteil besser gebildeter Bevölkerungsgruppen auch in Ländern, in denen das momentan noch nicht so ist, zunehmen wird, so mag dies die Lebensbedingungen für 18- bis 25-Jährige in verschiedenen Ländern wohl noch ähnlicher machen als sie es ohnehin schon sind - denkt man an studierende junge Menschen - doch wird das auch die Unterschiede in der Einschätzung des Erwachsenseins zwischen solchen, die sehr früh arbeiten (müssen), verglichen mit solchen, die im selben Land die Möglichkeit zu studieren nutzen können (dürfen), beeinflussen?

Zusammenfassend lässt sich festhalten, dass Arnett die von Erikson (1968) und auch Havighurst (1981) angedachten Ausnahmen von zeitlichen Verschiebungen in der Entwicklung hin zum Erwachsensein zur Normentwicklung deklariert. Er prägte dafür die Bezeichnung „Emerging Adulthood“. Es scheint, als ob in jüngeren Beiträgen Arnetts eine stärkere differentielle Betrachtung von „Emerging Adulthood“ festzustellen ist - das Vorhandensein von „many emerging adulthoods between and within countries“118 deutet darauf hin - womit er moderneren Ansätzen in der Entwicklungspsychologie wieder etwas näher rückt.

115 ARNETT, Young adulthood 241-242 (wie Anm. 87).

$116 \mathrm{Zu}$ Details betreffend Einflüsse von „social class“ siehe J. J. ARNETT - J. L. TANNER, Themes and variations in emerging adulthood across social classes, in: Debating emerging adulthood. Stage or process? Eds. J. J. ARNETT - M. KLOEP -L. B. HENDRY - J. L. TANNER. New York 2011, 31-50; unter „andere“ Einflüsse sind solche bezogen auf das Geschlecht und religiöse Gruppierungen gemeint, siehe J. J. ARNETT, Emerging adulthood(s). The cultural psychology of a new life stage, in: Bridging cultural and developmental approaches to psychology. New syntheses in theory, research, and policy, ed. L. J. Arnett. Oxford 2011, 255-275, hier 265.

117 ARNETT, Emerging adulthood(s). The cultural psychology of a new life stage, hier 265 (Anm. 116). 118 ARnetT, Young adulthood 242 (wie Anm. 87). 



\section{Thomas Pratsch \\ Jugendliche und Heilung}

Mit der spätantiken „Transformation der Antike“ vom polytheistischen Götterkult zum monotheistischen Christentum und der damit verbundenen Übernahme und Integration vorchristlicher kultischer Praktiken in den jungen christlichen Kultus, hatte auch der antike Heilschlaf im Tempel, die Inkubation, eine christliche Form erhalten. Die oder der spätantike Kranke und Hilfesuchende ging nun nicht mehr in einen heidnischen Tempel, sondern in eine christliche Kirche, um dort einige Tage und Nächte im Gebet mit der Bitte um Heilung zu verbringen und hoffentlich geheilt zu werden. In den Nächten oder auch am Tage, im Traum oder auch in einem tranceartigen Wachzustand, erschien ihm nun nicht mehr Asklepios, Apollon oder eine andere pagane heilende Gottheit, sondern eine christliche Heilige oder ein christlicher Heiliger mit denselben heilenden Fähigkeiten und Gaben. An einigen Stätten, an denen heidnische Tempel in christliche Kirchen umgewandelt wurden, ${ }^{1}$ ist die nahezu bruchlose Fortführung der kultischen Praxis bzw. der Sitte und Gewohnheit auch archäologisch evident, bei neu erbauten christlichen Kirchen ist diese Tradition weit weniger augenfällig. An die Stelle der früheren paganen heilenden Gottheiten traten nunmehr christliche heilende Heilige. Einige dieser Heiligen - wie etwa Kosmas und Damianos $^{2}$ oder Febronia ${ }^{3}$ - besaßen dabei neben ihrer allgemeinen Heilwunderkraft eine gewisse Spezialisierung auf die Heilung bestimmter Krankheiten im Besonderen, generell wurde aber bei jedem Heiligen die Fähigkeit zur Fürsprache bei Gott und damit zur Herbeiführung der Heilung einer Krankheit angenommen. ${ }^{4}$

Da es in dem vorliegenden Beitrag um Wunderheilungen geht, wurden diejenigen Quellen, die der „weltlichen Konkurrenz“ zuzuordnen sind, also die medizinischen Texte, nicht herangezogen; bei ihnen ist der Übergang vom heidnischen zum christlichen Weltbild auch weniger ausgeprägt. Die Dokumentation der erfolgreichen Hei-

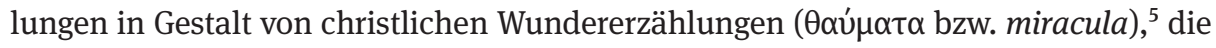
entweder als eigenständige Sammlungen oder integriert in die längeren Lebensgeschichten ( $\beta$ ío bzw. vitae) der Heiligen überliefert sind, diente verschiedenen Zwecken: zum einen stärkte die Lektüre dieser Geschichten, so ist jedenfalls anzunehmen,

1 E.g. zum Athena-Parthenos-Tempel in Athen (dem Parthenon) und seiner Umwandlung in eine Maria-Parthenos-Kirche vgl. A. C. ORLAndos avec la collaboration de L. I. VRANoussis, Les Graffiti du Parthénon: Inscriptions gravées sur les colonnes du Parthénon à l'époque paléochrétienne et byzantine (Académie d'Athènes. Centre de Recherches Médiévales et Néo-Helléniques). Athen 1973.

2 Vgl. ODB 2, 1151; W. ARTELt, Kosmas und Damian, in: LCI 7. Freiburg 1994 (= Sonderausgabe der Originalausgabe, Freiburg 1968-1976), 344-352 (dort jeweils weitere Literatur).

3 S. dazu noch unten.

4 Vgl. Th. Pratsch, Der hagiographische Topos: Griechische Heiligenviten in mittelbyzantinischer Zeit (Millennium-Studien 6). Berlin 2005, 228-247.

5 Vgl. Pratsch, Topos 225-297.

2 OpenAccess. (c) 2018 Thomas Pratsch, published by De Gruyter. (cc) BY-NC-ND This work is licensed under the Creative Commons Attribution-NonCommercial-NoDerivatives 4.0 License. 
beim Kranken das Vertrauen in die wunderwirkenden und heilenden Kräfte des jeweiligen Heiligen, zum anderen erwiesen erst erfolgreiche Wunder die Heiligkeit eines Verstorbenen und begründeten dessen Heiligenkult. Beide Genera, also Wundersammlungen und Lebensgeschichten von Heiligen mit integrierten Wundern, rechnen zum literarischen Genre der Hagiographie, dem Schrifttum über die Heiligen. ${ }^{6}$ Hagiographische Texte wurden im Byzantinischen Reich zwar in verschiedenen Sprachen abgefasst, so etwa in lateinischer, armenischer, syrischer und altkirchenslawischer Sprache, die meisten erhaltenen Texte dieser Art sind jedoch griechisch. Albert Ehrhard, einer der besten Kenner der hagiographischen Literatur der griechischen Kirche, zählte rund 2.750 erhaltene Handschriften mit Sammlungen hagiographischer Texte. ${ }^{7}$ Der Inhalt der Sammlungen überschneidet sich zwar weitgehend, das heißt, sie enthalten $\mathrm{zu}$ einem großen Prozentsatz dieselben Texte oder Varianten bereits bekannter Texte, ${ }^{8}$ dennoch ist die Zahl der erhaltenen hagiographischen Stücke immer noch erstaunlich groß. Entsprechend groß ist auch die Zahl der in diesen Texten berichteten Wunder, und unter diesen Wundern finden sich auch zahlreiche Darstellungen von Heilungen durch Inkubation in einer Kirche oder an einem anderen heiligen Ort. Und unter diesen wiederum finden sich auch Heilungen von Jugendlichen oder jungen Erwachsenen oder auch Heilungen, bei denen das Alter der Geheilten zwar nicht konkret genannt wird, aber aus den äußeren Umständen heraus anzunehmen ist, dass es sich wohl um Jugendliche oder junge Erwachsene gehandelt haben dürfte. Hier folgen zunächst die „gesicherten“ Fälle:

Gegen Ende des 7. Jahrhunderts wurden die Wunder des heiligen Artemios abgefasst, eines Märtyrers des 4. Jahrhunderts, der unter Kaiser Julian Apostata (361363) zu Tode gekommen sein soll. Sie enthalten zahlreiche Darstellungen von Inkubationen in der Kirche Johannes des Täufers und Vorläufers, die sich im Stadtteil

6 Die Literatur zu diesem Schrifttum ist kaum überschaubar, man vgl. vor allem die Zeitschrift der Bollandisten AnBoll, Brüssel 1882ff., sowie die Bibliotheca hagiographica Graeca (BHG) mit ihren Auctuaria, ferner in Auswahl vgl. Pratsch, Topos; A. Angenendt, Heilige und Reliquien: Die Geschichte ihres Kultes vom frühen Christentum bis zur Gegenwart. München ${ }^{2} 1997$; R. AIGRAIN, L'hagiographie. Ses sources - Ses méthodes - Son histoire (Subsidia Hagiographica 80). Brüssel 1953; Ndr. 2000; H. Delehaye, Les passions des martyrs et les genres littéraires (Subsidia Hagiographica 13B). Brüssel ${ }^{2} 1966$; IDEM, L'ancienne hagiographie byzantine: les sources, les premiers modèles, la formation des genres, conférences prononcées au Collèges de France en 1935 (Subsidia Hagiographica 73). Brüssel 1991; IDEM, Sanctus. Essai sur le culte des saints dans l'antiquité (Subsidia Hagiographica 17). Brüssel 1927; Ndr. 1954; IDEM, Cinq leçons sur la méthode hagiographique (Subsidia Hagiographica 21). Brüssel 1934; IDEM, Les saints Stylites (Subsidia Hagiographica 14). Brüssel 1923; Ndr. 1989; IDEM, Mélanges d'hagiographie grecque et latine (Subsidia Hagiographica 42). Brüssel 1966; F. HaLKIN, Hagiologie byzantine: Textes inédits publiés en grec et traduits en français (Subsidia Hagiographica 71). Brüssel 1986; IDEM, L'hagiographie byzantine au service de l'histoire, in: Proceedings of the $13^{\text {th }}$ International Congress of Byzantine Studies, Oxford, 5-10 September 1966, hrsg. von J. M. Hussey. London 1967, Main Papers, Nr. XI, 345-354.

7 Vgl. A. EHRHARD, Überlieferung und Bestand der hagiographischen und homiletischen Literatur der griechischen Kirche 1-3 (TU 50 - 52). Leipzig 1937-1952, I. III-XVII, bes. XVII.

8 Vgl. Ehrhard, Überlieferung und Bestand, I. 154-717; II. 1-305; РRATsCH, Topos 372-377. 
Oxeia in Konstantinopel befand. Auffällig an diesem heilenden Heiligen ist, dass er sich besonders auf die Heilung von Erkrankungen der Geschlechtsorgane sowie von Brüchen (Hernien) spezialisiert hat. ${ }^{9}$ Ein sehr interessantes Beispiel daraus ist das folgende, das zum einen zeigt, dass nicht nur die erkrankte Person selbst die Heilungsvision haben kann, sondern auch nahe Angehörige, zum anderen verdeutlicht es eine gewisse geschlechtsspezifische Arbeitsteilung zwischen dem heiligen Artemios und der heiligen Märtyrerin Febronia, was die Heilung von Geschlechtsorganen und Brüchen angeht:

Eine gewisse Frau hatte eine Tochter, die zur Ehe versprochen war, aber nach der Verlobung

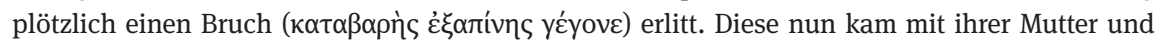
machte dem Märtyrer ihre Aufwartung und erwartete von ihm die Heilung. Nachdem sie dort aber fünfzehn Tage verbracht hatten, verließ sie die Zuversicht und sie kehrten unverrichteter Dinge in ihr Haus zurück. In jener Nacht erschien der Mutter der Heilige und sprach zu ihr: 'Geh' zur Schwester Febronia, und diese heilt sie.' Nachdem sie erwacht war und über das Wunderbare der Traumerscheinung nachgedacht hatte, nahm sie ihre Tochter und machte sich auf den Weg zur Kirche des heiligen Vorläufers und Täufers Ioannes. In dieser Kirche nämlich ist der Sarg des heiligen Märtyrers unter dem großen Altar, und rechts vom Altar ist ein Schrein der heiligen Märtyrerin Febronia. In derselben Nacht nun sah die Mutter der Tochter eine sehr gutaussehende Frau, die das Gewand einer Nonne trug, wie sie einen Verband aus Wachssalbe herstellte und an der Scham des Mädchens anbrachte und zu ihr sprach: 'Geh', und danke Gott und dem heiligen Vorläufer und dem heiligen Artemios.' Und während sie ihr dies sagte, erwachte die Mutter des Mädchens, und als sie sich von ihrem Lager erhoben, eine kleine Kerze angezündet und ihre Tochter betastet hatte, fand sie den Verband angebracht, wie sie es (im Traum) gesehen hatte. Ihre Tochter aber war gesund geworden, als sei sie neu geboren. ${ }^{10}$

Offenbar stellte der Bruch der jungen Frau ein echtes Ehehindernis dar, das durch das Wunder beseitigt wurde. Es könnte sich eventuell um einen (partiellen) Prolaps uteri et vaginae ${ }^{11}$ handeln, der zum einen die Gebärfähigkeit der jungen Frau in Frage gestellt hätte und zum anderen nach dem damaligen Stand des medizinischen Wissens leicht für eine Hernie gehalten werden konnte. Ein Bruch ganz anderer Art, wohl ein Nabelbruch, findet sich in dem folgenden Wunder:

9 Zur Heilung von Brüchen vgl. auch PRATSCH, Topos 242; zur Heilung eines Leistenbruchs bei einem Kind vgl. D. ARIANTZI, Kindheit in Byzanz. Emotionale, geistige und materielle Entwicklung im familiären Umfeld vom 6. bis zum 11. Jahrhundert (Millennium-Studien 36). Berlin-Boston 2012, 315f. Die Gründe für diese Spezialisierungen sind nicht immer auszumachen. Mitunter liegen sie aber in der Lebensgeschichte des oder der Heiligen selbst, etwa wenn der oder die Heilige eine solche oder ähnliche Erkrankung im Laufe seines oder ihres Lebens selbst erfahren und überwunden hatte oder bereits zu Lebzeiten eine solche Erkrankung selbst geheilt hatte oder in irgendeiner Weise im Laufe des Lebens mit dieser Erkrankung in Berührung gekommen war.

10 Miracula Artemii 24 (ed. V. S. CrisAfulli - J. W. NesBitt, The Miracles of St. Artemios: A Collection of Miracle Stories by an Anonymous Author of Seventh-Century Byzantium [The Medieval Mediterranean 13]. Leiden 1997, 140, 142); übers. von Th. PRATSCH.

11 Vgl. Pschyrembel, Klinisches Wörterbuch. Berlin - New York ${ }^{258} 1998,1295$. 
Ein ganz junger Mann namens Platon, der auf seine Jugend vertraute und, wie die jungen Leute es gern tun, mit dem Ausmaß seiner Stärke prahlte, schloss eine Wette ab, dass er den Stein der Waage des Holzverkäufers hochheben und auf seine Schulter nehmen werde. Nachdem der Wetteinsatz vereinbart worden war, hob er den Stein an. Und als er sich anstrengte, um ihn auf die

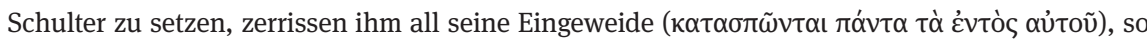
dass die Zuschauer von dem Anblick völlig überrascht waren. Einige gute Menschen gaben ihm nun Ratschläge und sagten: 'Vertraue dich keinem Arzt an, sondern gehe in die Kirche des Hagios Ioannes nach Oxeia ${ }^{12}$ zum heiligen Artemios, und dieser wird dich heilen. Denn er wirkt nämlich jeden Tag Wunder in solchen Fällen.' Nachdem er von einigen von ihnen aufgehoben worden war, wurde er auf einer Trage fortgebracht und fürchtete um sein Leben. Er verbrachte nun einige Tage unter unerträglichen Schmerzen und Leiden, ehe er den heiligen Artemios im Schlaf sah, der zu ihm sagte: 'Und warum gefällt es dir, Wetten abzuschließen? Siehe, sowohl gegen deine Seele als auch gegen deinen Körper hast du dich verschworen.' Und er ermahnte ihn, von nun an keine Wette mehr abzuschließen. Und während er dies sagte, trat er ihm auf seinen Bauch. Der Wetteiferer aber erwachte und war befreit von dem Schmerz und auch von der Krankheit. ${ }^{13}$

So weit also zwei eindeutige Beispiele von Heilungen Jugendlicher bzw. junger Erwachsener, wesentlich zahlreicher finden sich allerdings Heilungen von Patienten, deren Leben offenbar noch weitgehend vor ihnen liegt, deren genaues Alter allerdings in den Texten nicht genannt wird.

So verfasste etwa im 9. Jahrhundert Ignatios Diakonos ${ }^{14}$ die Vita des heiligen Gregorios Dekapolites, ${ }^{15}$ eines Mönchsvaters, der gegen Ende des 8. Jahrhunderts geboren wurde und spätestens im Jahre 842 verstarb. Typisch für die zahlreichen Wunder, die auch in dieser Vita berichtet werden, ist ihre auffallende Kürze der Darstellung. Unter diesen Wundern findet sich auch eine Inkubation, die zur Heilung (möglicherweise von Migräne oder psychosomatischen Stresssymptomen) eines Mannes führte, dessen Alter offen bleibt:

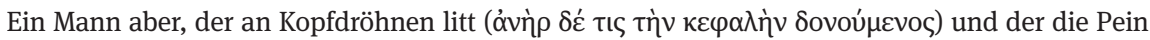
des Schmerzes nicht ertragen konnte, näherte sich bei Einbruch der Nacht dem Sarg des Heiligen und sah im Traum, wie der Heilige seinen Scheitel berührte und in zwei Teile zerlegte, verfaulte Fleischstücke herauszog und zu ihm sagte: 'Siehe, du bist gesund geworden, ziehe hin in Frieden'. ${ }^{16}$

12 Gemeint ist der Stadtteil Oxeia in Konstantinopel.

13 Miracula Artemii 7 (wie Anm. 10), 90, 92; übers. von Тн. РRATsch. Zur Selbstüberschätzung der Jugend vgl. jetzt auch D. ARIANTZI, Terminologische und sozialhistorische Untersuchungen zur Adoleszenz in Byzanz (6.-11. Jahrhundert). Teil I. Theorien, Konzepte, narrative Quellen. JÖB 63 (2013) 1-31, hier 22.

14 Vgl. PmbZ I/2, Nr. 2665.

15 Vgl. PmbZ I/2, Nr. 2486. Gregorios stammte aus der isaurischen Dekapolis in Kleinasien.

16 Vita Gregorii Decapolites 86 (ed. G. MAKRIs, Ignatios Diakonos und die Vita des Hl. Gregorios Dekapolites. Mit einer Übersetzung von M. Chronz [Byzantinisches Archiv 17]. Stuttgart - Leipzig 1997, 146, 148). 
Die Darstellung erinnert frappierend an die gelegentlichen, paranormalen Wahrnehmungen einiger moderner Patienten, die sich einer Operation unterziehen mussten und im Nachhinein berichteten, sich während der Dauer der Operation von außen, von einem Standpunkt über dem Operationstisch, also gleichsam über ihrem eigenen Körper schwebend beobachtet zu haben. ${ }^{17}$

In der ersten Hälfte des 10. Jahrhunderts wurde von einem anonymen Verfasser die Vita des Nikolaos Studites ${ }^{18}$ abgefasst. Nikolaos war Mönch und Abt des Studiosklosters in Konstantinopel gewesen und im Jahre 868 verstorben. Auch diese Vita enthält unter den Wunderberichten eine Inkubation, allerdings diesmal nicht in der Kirche, sondern in der ehemaligen Mönchszelle des verstorbenen Abts Nikolaos. In diesem Falle dürfte es sich wohl um einen jüngeren Mönch gehandelt haben, da er nach der erfolgreichen Heilung noch mindestens vierzig Jahre lebte:

Der Schüler unseres heiligen Vaters Nikolaos namens Antonios, ${ }^{19}$ der von einigen wegen seines Aussehens Mauros (zu Deutsch: „Schwarzer“) genannt wurde, wurde viele Jahre von der Krankheit des Blutflusses bedrückt, ${ }^{20}$ wobei der Verlauf der Erkrankung die Ärzte zur Verzweiflung gebracht hatte. Ihm wurde nun von dem vorgenannten Vater Hilarion ${ }^{21}$ erlaubt, sich in der Zelle, in der früher der Diener Christi Nikolaos gelebt hatte, niederzulassen und (dort) die Trennung vom Körper zu erwarten. Nachdem er mit großer Freude in dieser Zelle sein Lager aufgeschlagen hatte, erschien ihm im Schlaf der Heilige (sc. Nikolaos) und sprach auf diese Art zu ihm: 'Bescheidener Antonios, welche Krankheit behelligt dich?' 'Als ich das Leiden nannte', sagte er (sc. Antonios), 'verschwieg ich nicht, dass es unheilbar ist.' 'Hab' keine Furcht mehr', sagte der Große (sc. Nikolaos), 'du sollst nämlich von diesem Augenblick an gesund sein und nicht mehr durch diese Krankheit behelligt werden.' 'Und nachdem ich zu mir gekommen war und nicht mehr im Schlaf, (sc. sagte Antonios) sah ich mit eigenen Augen, wie er im hinteren Teil der Zelle verschwand, von wo er den Blutfluss umgehend stillte. Großer Wohlgeruch breitete sich in der Zelle aus und erfüllte mein Herz mit unaussprechlicher Freude.' Von da an sind nun vierzig Jahre vergangen, und er wurde durch die Gnade Gottes nicht wieder von dieser Krankheit heimgesucht. ${ }^{22}$

So weit der anonyme Verfasser des 10. Jahrhunderts, der in der zweiten Hälfte seines Berichts stilistisch etwas aus dem Rahmen fällt: Nikolaos spricht in diesem PseudoDialog zwar zu Antonios, Antonios aber spricht nicht zu Nikolaos, sondern zum Verfasser bzw. Leser oder Hörer der Vita! Über die Krankheit des Antonios kann man

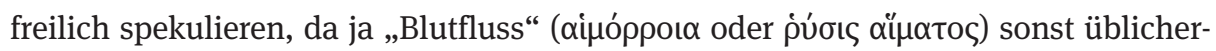
weise und bereits in den Evangelien ${ }^{23}$ als typische Frauenkrankheit gilt und im All-

17 Vgl. dazu jetzt T. StukEnBERg, Nahtod-Erlebnisse: Schweben über dem OP-Tisch, in: Spiegel-Online vom 09.10.2014: http://www.spiegel.de/wissenschaft/mensch/nahtoderlebnisse-studie-belegt-be wusstsein-bei-herzstillstand-a-995996.html (zuletzt geprüft am 14.11.2014)

18 Vgl. PmbZ I/3, Nr. 5576.

19 Vgl. PmbZ II/1, Nr. 20485.

20 Vgl. zum „Blutfluss“ auch PRATsCH, Topos $228 \mathrm{f}$.

21 Vgl. PmbZ II/2, Nr. 22601.

22 Vita S. Nicolai Studitae (BHG 1365) 924 (ed. J.-P. MignE, Anonymi Vita S. Nicolai Studitae, in: PG 105); übers. von TH. PRATSCH.

23 Mt 9. 20 -22; Mk 5. 25-29; Lk 8. 43-48. 
gemeinen mit Menstruationsstörungen gleichgesetzt wird. In diesem Falle könnte es sich aber etwa um Hämophilie, die sogenannte Bluterkrankheit, eine Gerinnungsstörung des Blutes, handeln ${ }^{24}$ oder auch um ein Hämorrhoidalleiden mit Blutungen im Analbereich. ${ }^{25}$ Interessant an diesem Beispiel ist auch, dass die Inkubation und die Heilung im Schlaf nicht in einer Kirche erfolgen, wie dies meist der Fall ist, sondern in der ehemaligen Mönchszelle des Nikolaos im Studioskloster in Konstantinopel, einem Ort, der durch die frühere Präsenz des Heiligen dortselbst quasi geheiligt ist. ${ }^{26}$

Manchmal ist nicht einmal wirklicher Schlaf vonnöten, um im Schlaf geheilt zu werden, wie das folgende Beispiel zeigen wird. In der Mitte des 11. Jahrhunderts wurde die Vita des Nikon „Metanoeite“ 27 verfasst, eines Mönchsvaters auf der Peloponnes, der in der zweiten Hälfte des 10. Jahrhunderts in Lakedaimon und Umgebung mehrere Klöster gründete. Auch in den Wunderberichten dieser Vita findet sich eine Inkubation und Heilung einer Frau, die anscheinend noch nicht sehr alt war: ${ }^{28}$

Eine Frau nämlich, eine Einwohnerin von Sparta, war durch den Neid des bösen Dämons an den Füßen gelähmt, so dass die Füße ihre Hinterbacken berührten, und sie nicht aufrecht gehen

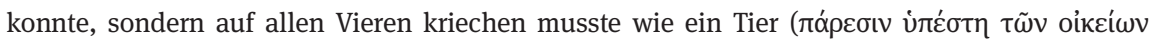

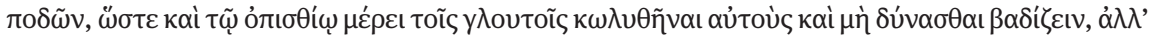

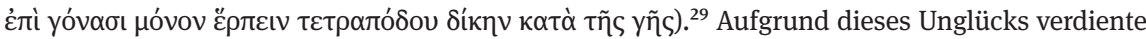
diese Frau großes Mitleid und Trost, beides erhielt sie von diesem unbezahlten Arzt und Heiler (sc. Nikon). Wie dies geschah, wird die Rede mitteilen. Die bedauernswerte Frau lag unterhalb der Gewölbe des heiligen Klosters und bat den Heiligen um Hilfe. Sie vergoss dabei Tränen und flehte um Erbarmen. Eines Nachts, als das Mondlicht die Erde beleuchtete, schien sie einen Mönch zu sehen, der die Stufen der göttlichen Kirche herabkam und sich ihr näherte. Als die Erscheinung des Mannes nahe genug herangekommen war, stieß er die Frau mit dem Stab an, den er bei sich trug, und sagte: 'Steh' auf, Weib, und stehe auf deinen Füßen.' Sie antwortete, dass sie das nicht tun könne, da sie seit langer Zeit gelähmt und unfähig sei zu stehen. Er aber sagte mit fester Stimme: 'Steh' auf, unglückliches Weib, denn der Herr Jesus Christus hat dich auf Bitten des Metanoeite geheilt'. Und sogleich hatte sie die Bande der Krankheit abgestreift und stand auf. Mit jeder Bewegung wurden ihre Füße kräftiger, und schon sprang sie wie eine Gazelle umher und stieg die Stufen (zur Kirche) hinauf. Der aber, der sie geheilt hatte, war verschwunden. Da rief sie mit lauter Stimme: 'Herr, erbarme dich' und 'Ehre sei dir, heiliger Mann Gottes'. ${ }^{30}$

24 Zur „Hämophilie“ vgl. PschYREMBEL, Klinisches Wörterbuch, s. v.; MSD-Manual der Diagnostik und Therapie, hrsg. von Sharp \& Dohme GmbH, München, ein Unternehmen der Merck \& Co., Inc., Rahway, N. J., U. S. A. Deutsche Bearbeitung: Prof. Dr. K.WIEMANN. München - Wien - Baltimore ${ }^{4} 1988$, s. v. „Hämophilien“.

25 So ein Hinweis von J. Koder.

26 Zu heiligen Orten vgl. auch PRATsCh, Topos 158-159; AngenendT, Heilige und Reliquien 125-128, auch 155-158 zur Übertragung der einwohnenden virtus.

27 Vgl. PmbZ II/5, Nr. 26155.

28 Dies geht aus dem Text zwar nicht eindeutig hervor, ihre Jugend wird jedoch dadurch wahrscheinlich, dass sie nach der Heilung „wie eine Gazelle“ umhersprang.

$29 \mathrm{Zu}$ weiteren Beispielen von Lähmungen vgl. Pratsch, Topos 232-234; zu Heilungen von Lähmungen bei Kindern vgl. ARIANTZI, Kindheit 299-303.

30 Vita Niconis 53 (ed. D. F. Sullivan, The Life of Saint Nikon: Text, Translation and Commentary. Brookline, MA 1987); übers. von TH. PRATSCH. 
Das Besondere an dieser Inkubationsdarstellung ist, dass hier nicht der Heilige der Kranken im Schlaf bzw. Traum erscheint, sondern ein Abgesandter des Heiligen erscheint im Mondlicht, er wird also in einer Art halbschlaf- oder tranceähnlichem Wachzustand wahrgenommen.

Wohl ebenfalls in das 11. Jahrhundert zu datieren ist die Vita des Elias Spelaiotes. ${ }^{31}$ Dieser Mönchsvater wurde zwischen 860 und 870 in Rhegion in Kalabrien geboren und gründete - wie sein Beiname schon verrät - ein bekanntes Höhlenkloster, also ein Kloster, das wenigstens zum Teil in eine große Höhle gebaut war. Elias starb am 11. September des Jahres 960. Die umfangreiche Wundersammlung seiner Vita enthält ebenfalls zahlreiche Inkubationsgeschichten; eine typische daraus sei hier vorgestellt:

Ein weiteres Wunder hört nun, wie sich der Heilige dem Glaukeias, ${ }^{32}$ dem Einwohner von Moros zeigte. Diesem waren einst durch das Wirken der Dämonen die Eingeweide heftig in Brand gesetzt

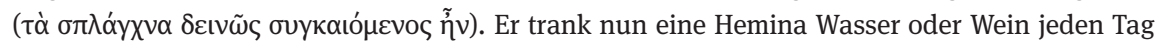
und jede Nacht und konnte den Durst nicht stillen. Sehr oft hatte er die medizinischen Gelehrten aufgesucht, ohne dass es ihm etwas genützt hätte. Da flüchtete er sich zu dem geistlichen Arzt, ich meine den Sarg des Heiligen. Und nachdem er zwei Tage und Nächte dort zugebracht hatte, sah er den Heiligen im Schlaf, der zu ihm sprach: 'Öffne deinen Mund'. Nachdem jener eilig den Mund geöffnet hatte, zog der Heilige aus dessen Bauch eine sich krümmende Schlange hervor. Und nachdem er diese vor den Augen des Mannes getötet hatte, sprach er zu ihm: 'Gehe nun, Bruder Glaukeias, gesund in dein Haus zurück und erzähle, was Gott, der Herr, mit dir gemacht hat, und dass er Erbarmen mit dir hatte'. Am Morgen erhob er sich und spürte die Wirkung des elenden Dämons nicht mehr. Er ging nachhause und pries Gott. ${ }^{33}$

Das römische Hohlmaß der Hemina fasst mit ca. 0,272 Litern keine allzu große Flüssigkeitsmenge angesichts der Tatsache, dass heutige Mediziner von einem täglichen Bedarf von 2 bis 3 Litern Flüssigkeit ausgehen, wobei Alkohol gar nicht und Kaffee nur zur Hälfte mitgerechnet wird. Allerdings ist das Hohlmaß dieses Namens wohl in byzantinischer Zeit nicht mehr mit dem antiken Hohlmaß gleichzusetzen. ${ }^{34}$ Der Autor will wohl zum Ausdruck bringen, dass Glaukeias sehr viel getrunken hatte, es aber dennoch nichts half.

Abgesehen davon, finden wir hier das typische Motiv der Verkörperung von Krankheiten durch Dämonen in Tiergestalt. Diese Tiere sind - wie im angeführten Beispiel - vorzugsweise Schlangen, aber auch Echsen, Frösche, Insekten, etwa Wes-

31 Vgl. $\mathrm{PmbZ}$ II/2, Nr. 21646.

32 Vgl. PmbZ II/2, Nr. 22311.

33 Vita Sancti Eliae 91 (ed. J. StiLting, in: AASS, Septembris 3 [nach Cod. Messin. S. Salvatoris 42]. Paris 1868, 884); übers. von Th. Pratsch.

34 Vgl. E. SchilBaCH, Byzantinische Metrologie (Handbuch der Altertumswissenschaften XII, 4; Byzantinisches Handbuch 4). München 1970, 114: „Allerdings ist die $\mu^{\prime} v \alpha$ als Maß durchaus nicht mit der antiken hemina zu 0,272 l gleichzusetzen, sondern sie scheint ein erst in byzantinischer Zeit ent-

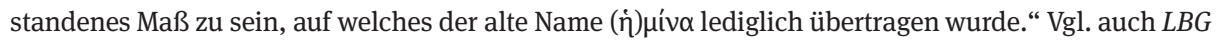
s. v. „„uiva“ zu den verschiedenen Vorstellungen von dem Hohlmaß. 
pen und Fliegen, kleine Nager wie Mäuse und Ratten oder Vögel wie Krähen und Raben kommen dafür in Betracht, also ganz allgemein Tiere, die mit dem Dunkel und der Unterwelt in Verbindung gebracht werden. ${ }^{35}$

Nicht so sehr aus den hier betrachteten Beispielen, aber aus anderen Inkubationsdarstellungen und generell aus der hagiographischen Literatur erfahren wir auch einiges über die praktischen Gepflogenheiten des christlichen Heilschlafs in der Kirche. Auch wenn diese Gepflogenheiten von Ort zu Ort und über die Jahrhunderte im Detail variiert haben dürften, lässt sich doch einiges allgemein feststellen: So hatten etwa die Kranken in der Regel ihre Schlafstatt für die Übernachtungen, also eine Matratze oder Matte, eventuell Decken und Kissen, selbst mitzubringen. Schwerkranke und bettlägerige Personen wurden häufig von ihren Verwandten gleich auf einer Trage in die Kirche gebracht und diese dort abgestellt. Besonders begehrt waren die Schlafplätze nahe am Grab des jeweils aufgesuchten Heiligen. Damit kein Streit um die besten Plätze entsteht, wurden die Schlafplätze von Diakonen, Priestern oder Mönchen der Kirche oder des Klosters den Inkubanten zugewiesen. In Klöstern wurden die Kranken üblicherweise von der Klosterküche mit Speisen versorgt. Es liegt auf der Hand, dass sie sich daher nach dem Speiseplan des Klosters richten mussten. Bei bestimmten Erkrankungen wurde mitunter aber auch eine spezielle Diät angeordnet. Nachts war die Kirche normalerweise verschlossen. Damit dies nicht zu Problemen bei plötzlichen Bedürfnissen der Kranken führte, wurde ein Wachdienst eingerichtet, der die Kirchentür auf ein Klopfzeichen hin von außen öffnete. Es dürften auch regelmäßige Durchgänge durch die Kirche bzw. den Krankensaal stattgefunden haben, auch wenn diese in der Darstellung der Wunderberichte vornehmlich von den Heiligen selbst vorgenommen wurden.

Die hier vorgeführten Beispiele weisen eine chronologische Ordnung auf. Die erste Inkubationsdarstellung stammte aus den Miracula des heiligen Artemios aus dem 7. Jahrhundert, ein weiteres aus der Vita des heiligen Gregorios Dekapolites aus dem 9. Jahrhundert, eines aus der Vita des heiligen Nikolaos Studites aus der ersten Hälfte des 10. Jahrhunderts und zwei Beispiele aus den Viten des heiligen Nikon „Metanoeite“ und des heiligen Elias Spelaiotes aus dem 11. Jahrhundert. Diese Eckdaten umreißen auch schon in etwa den Zeitraum der Blütezeit der Erwähnungen von Inkubationen in byzantinischen Heiligenviten.

Die in unseren Beispielen durch Heilschlaf in der Kirche geheilten Krankheiten reichen von einer schweren, aber nicht näher definierten Krankheit, über einen Leisten- und einen Nabelbruch, chronische Kopfschmerzen, Migräne oder psychosomatische Stresssymptome, eine mit Blutungen zusammenhängende Erkrankung, Lähmung der unteren Extremitäten bis hin zu chronischem, unstillbaren Durst. Nimmt man andere Beispiele in den Blick, erweitert sich das Spektrum der durch Heilschlaf in der Kirche heilbaren Krankheiten zusehends. Wir finden Darstellungen 
von Heilungen von Blindheit, ${ }^{36}$ von Taub- bzw. Stummheit, ${ }^{37}$ von Lähmungen aller Art einschließlich durch Verletzung hervorgerufener Verrenkungen und Knochenbrüche, ${ }^{38}$ Heilungen von Kinderlosigkeit, ${ }^{39}$ von Fieber und Schüttelfrost (in modernen Erläuterungen häufig konkret als Malaria bezeichnet), wohl aber verschiedene Infektionskrankheiten mit umfassend, ${ }^{40}$ Heilungen von Lepra ${ }^{41}$ und verschiedenartigen Geschwüren, jeder Art von schwerer und lebensgefährlicher Krankheit einschließlich Tumoren und Karzinomen der verschiedensten Organe, von Epilepsie und Anfallsleiden, ${ }^{42}$ schließlich von psychischen Erkrankungen wie der sogenannten Besessenheit und Raserei. ${ }^{43}$ Nach der Darstellung der hagiographischen Literatur gibt es also nahezu keine bekannte Krankheit, die nicht durch Inkubation und Heilung im Schlaf geheilt werden könnte. Dabei werden die Krankheiten, wie wir gesehen haben, einmal mehr, einmal weniger konkret beschrieben. Es gibt zahlreiche Fälle, in denen nur pauschal von einer schweren Erkrankung unter Umständen in Verbindung mit Bettlägerigkeit die Rede ist. In anderen Fällen werden die Krankheiten dagegen genauer beschrieben und/oder auch benannt, so dass wir sie mit heute bekannten Krankheiten identifizieren können.

In den allermeisten Fällen erfolgt die Wunderheilung schnell. Es wird also kein länger andauernder Heilungsprozess angenommen, sondern ein plötzliches Ende der Krankheit. Die göttliche Allmacht entfernt einfach die Krankheit, deren Symptome damit auch auf einen Schlag verschwinden. Dieser Verlauf ist in Bezug auf einige Krankheiten vorstellbar, in Bezug auf andere Krankheiten jedoch weniger realistisch. Das relativ plötzliche Ende einer Nierenkolik etwa ist durch den natürlichen Abgang des Nierensteins über die Harnwege durchaus möglich, die Wiedererlangung der vollen Mobilität nach einer Lähmung ist dagegen ein eher langsamer und schrittweiser Prozess. Dieser Punkt wird jedoch in den hagiographischen Darstellungen oft übergangen, da eben auch und gerade die Plötzlichkeit der Heilung das Wunderbare des göttlichen Wirkens zum Ausdruck bringt.

Die Vorstellung von der Möglichkeit einer spontanen Heilung jeder Art von Krankheit mag auch auf der in Spätantike und Mittelalter verbreiteten christlichen Vorstellung von Krankheit beruhen. Nach der in den hagiographischen Texten kolportierten Vorstellung kommt nämlich jede Krankheit vom Teufel bzw. war eine Strafe Gottes. ${ }^{44}$ Entweder war die Krankheit eine Strafe für das sündhafte Verhalten der Menschen, oder weil der Diabolos den Menschen ein friedliches und gottgefälliges

36 Vgl. die Beispiele bei Pratsch, Topos 229-231; ArIANTZI, Kindheit 313.

37 Vgl. Pratsch, Topos 231f.; ARIANTZI, Kindheit $312 \mathrm{f}$.

38 Vgl. PRATSCH, Topos 232-234; ARIANTZI Kindheit 299-303.

39 Vgl. PRATSCH, Topos 234-236.

40 Vgl. Pratsch, Topos $237 \mathrm{f}$.

41 Vgl. Pratsch, Topos 238-240; ARIANTZI, Kindheit $310 \mathrm{f}$.

42 Vgl. ArIANTZI, Kindheit 306-310.

43 Vgl. Pratsch, Topos, 244-247; ARIANTZI, Kindheit 304-306.

44 Vgl. ODB 1, 638, s. v. „Disease“; LThK 6 (1997) 426-430, s. v. „Krankheit“. 
Leben nicht gönnt, fährt er entweder selbst in die Menschen und schlägt sie mit Krankheit, häufiger aber betraut er einen seiner zahlreichen Dämonen mit dieser Aufgabe. Diese Dämonen können nun aber durch göttliches Wirken wieder vertrieben werden. Sie verlassen dann den menschlichen Körper häufig in Gestalt eines Tieres, wie wir an dem Beispiel aus der Vita des Elias Spelaiotes gesehen haben. Vor dem Hintergrund dieser Vorstellung, dass nämlich Krankheiten von Dämonen verursacht werden, die durch göttliches Wirken plötzlich vertrieben werden können, erscheint die spontane Heilung einer Krankheit geradezu näherliegend als ein langsamer und gradueller Heilungsprozess.

Ob nun spontan oder nicht, ist Heilung im Schlaf durch Inkubation vom naturwissenschaftlichen Standpunkt aus gesehen denn überhaupt möglich? Diese Frage muß grundsätzlich mit ,ja“ beantwortet werden, wenn auch mit großen Einschränkungen. Zum einen gab und gibt es Erkrankungen, wie die oben erwähnten Nierenoder Blasensteine und andere Krankheiten, die in ihrem Verlauf auch ohne Behandlung abrupt enden können. Diese Heilung oder deren Beginn konnte mit dem Zeitraum der Inkubation zusammenfallen und im Nachhinein natürlich dem Heilschlaf zugeschrieben werden. Zum anderen gehörten zu der mit der Inkubation einhergehenden „Therapie“ nicht nur Gebete, sondern häufig auch die Verabreichung von Myron, einem wundertätigen, angeblichen Ausfluss des Leichnams des Heiligen, ${ }^{45}$ oder von heiligem Öl aus einer Lampe vom Grab des Heiligen. In beiden Fällen dürfte es sich um Pflanzenöle, am wahrscheinlichsten um Olivenöl, gehandelt haben, die innerlich und äußerlich angewendet wurden, also getrunken oder auf die Haut aufgetragen. Diese Behandlung konnte unter Umständen bei einer Reihe innerer und äußerer Erkrankungen, wie etwa Erkrankungen des Magen-Darm-Trakts oder Hautkrankheiten, eine Besserung oder gar Heilung herbeiführen. Ferner ist auch das breite Spektrum der psychischen Erkrankungen zu nennen, von denen einige, wie etwa bestimmte schizoide Zustände und Neurosen, durch Inkubation in einer Kirche, vielleicht durch eine zufällige Traumerscheinung, durch Festigung des Glaubens und Willens oder durch den Aufbau einer vertrauensvollen Beziehung zu einem der anwesenden Priester oder Mönche, durchaus Linderung oder Heilung erfahren konnten. Diese psychologische Komponente könnte unter Umständen auch im Hinblick auf andere organische Erkrankungen zum Tragen gekommen sein.

Die Inkubation und Heilung im Schlaf ist nur eine von mehreren Formen der Wunderheilung in Byzanz. Wunderheilungen ereigneten sich - jedenfalls nach Darstellung der Quellen - während oder nach dem Besuch einer heiligen Stätte (in den meisten Fällen ist dies eine Kirche, ein Kloster oder das Grab eines Heiligen u.ä.) oder eines heiligen Mannes (etwa eines Asketen in der Wüste, eines Eremiten oder eines heiligmäßig lebenden Bischofs). ${ }^{46}$ Sie erfolgten während oder nach einem intensiven Gebet zu einem bestimmten Heiligen, das oftmals an der heiligen Stätte abgehalten

45 Vgl. Pratsch, Topos 223-224; P. Menebisoglu, Meletemata peri hagiu myru. Athen 1999. $46 \mathrm{Zu}$ heiligen Männern vgl. PRATSCH, Topos 156-157. 
werden konnte. Die Wunder geschahen während oder nachdem Myron oder Öl vom Grab eines Heiligen innerlich oder äußerlich appliziert worden war, oder während oder nach einem Gespräch mit einer heiligmäßig lebenden Person, die den Patienten durch Handauflegen, Bekreuzigen, Segnen oder Gebete heilte.

Es ergibt sich also ein relativ komplexes Bild eines geistlichen Heilungswesens, ${ }^{47}$ das zwar auch von Fall zu Fall medizinisch wirksame Elemente enthielt, etwa die innerliche und äußerliche Anwendung von Pflanzenölen, Reinigung und Fasten, die psychotherapeutische Komponente der persönlichen Zuwendung sowie Ruhe und Konzentration des Patienten auf die Krankheit und den eigenen Körper und anderes mehr, das aber dennoch im Wesentlichen auf die Heilung durch Gott vertraute. Gott allein besaß nach dieser Vorstellung die Macht, die Menschen von einer jeden Krankheit zu erlösen, deshalb half auch nur ein fester Glaube und intensives Beten, am besten zu einem Heiligen, der dann Fürsprache bei Gott einlegte. Alles menschliche medizinische Bemühen erwies sich nach dieser Ansicht ohne Gottes Hilfe als nutzlos.

Es liegt auf der Hand, dass ein solches geistliches Heilungswesen mit der in Byzanz praktizierten ärztlichen Kunst und medizinischen Wissenschaft in einen deutlichen Gegensatz geraten konnte. ${ }^{48}$ Dieser Gegensatz wird häufig bereits in den Texten selbst artikuliert, wie wir auch schon an den angeführten Beispielen gesehen haben, wenn dort die Heilungserfolge von Ärzten mit denen des oder der Heiligen verglichen werden. Da heißt es dann beispielsweise sinngemäß: Nachdem er (sc. der Kranke) sämtliche Ärzte der Stadt konsultiert hatte, die ihm aber alle nicht hatten helfen können, wandte er sich an den Heiligen mit der Bitte um Heilung. ${ }^{49}$ Relativ häufig wird dabei auch der monetäre Aspekt miteinbezogen und thematisiert: Nachdem sie (sc. die Kranken oder die Verwandten der Kranken) viel Geld für Ärzte ausgegeben hatten, die aber nicht hatten helfen können, wandten sie sich an den unbezahlten Arzt, den Heiligen..$^{50}$ Hier wird, wie dies auch im Beinamen der Anargyroi Kosmas und Damian zum Ausdruck kommt, ${ }^{51}$ hervorgehoben, dass der Heilige, im Gegensatz zu den Ärzten, für seine Therapie kein Geld nimmt. Auf diesen Aspekt werden wir noch zurückkommen müssen. Den Ärzten wird dabei nicht nur der Heilungserfolg abge-

\footnotetext{
47 Der Begriff bezeichnet die Heilungen von Krankheiten durch einen Heiligen bzw. Gott nach einem Besuch in der Kirche und Bitte um Fürsprache bei dem Heiligen, hier speziell auch durch Inkubation in der Kirche. Er wird hier verwendet zur Unterscheidung vom menschlichen medizinischen Heilungswesen der Ärzte und Bader.

48 Vgl. $O D B$ 2, 1327-1328, s. v. „Medicine“ (mit weiterer Literatur).

49 Vgl. etwa Vita Phantini iun. (BHG 2366z) cap. 57 (ed. E. FolLIERI, La Vita di San Fantino il Giovane, introduzione, testo greco, traduzione, commentario e indici [Subsidia Hagiographica 77]. Brüssel 1993, 462-464).

50 Vgl. etwa den Fall der Frau des Patrikios und Magistros Stephanos: Vita Ioannic. (Sabas; $B H G$ 935) cap. 35 (Vita Ioannicii auctore Saba monacho, in: AASS Nov. II 1, 364C); Vita Ioannic. (Petros; BHG 936) cap. 63 (Vita Ioannicii auctore Petro monacho, in: AASS Nov. II 1, 427 A-B).

$51 O D B$ 1, 85, s. v. „Anargyroi“. „Anargyroi“ enthält die Aussage, dass diese Ärzte ihre Behandlungen „ohne Geld“, d.h. gratis ausführten.
} 
sprochen häufig wird ihnen gar eine Verschlimmerung der Krankheit durch Kunstfehler bei ihrer Behandlung vorgeworfen. Dies betrifft vor allem die gelegentlich erwähnten heidnischen oder jüdischen Ärzte, die zu konsultieren in der Darstellung der hagiographischen Texte natürlich besonders verwerflich ist. ${ }^{52}$

Die Selbstdarstellung des Heiligenkultes und der Wunderheilungen setzt sich also in einen deutlichen Kontrast $\mathrm{zu}$ den naturwissenschaftlichen Heilmethoden des ärztlichen Standes. Es sind zwar keine diesbezüglichen Zeugnisse erhalten, doch erscheint es keineswegs verfehlt anzunehmen, dass die Ärzteschaft im Gegenzug die Heilmethoden der Priester und Mönche, also Inkubation, Myronsalbung usw., mit einer gewissen Skepsis betrachtete. Diese Situation erinnert auffallend an die heutige Kluft zwischen den Verfechtern verschiedener heilpraktischer Methoden und der sogenannten Schulmedizin. Was aber war der Hintergrund dieser Konkurrenz? Es trifft zwar zu, dass die Urheber der Wunderheilungen, also die Heiligen mit Hilfe Gottes, für ihre Leistungen nicht bezahlt wurden, jedenfalls nicht persönlich. Es war aber doch Sitte und wurde erwartet, dass der Kranke bei Inanspruchnahme des geistlichen Heilungswesens freiwillig seine Dankbarkeit in Form von Geld oder anderen materiellen Schenkungen leistete, desgleichen noch einmal im Falle des Erfolgs der Heilung oder zumindest der Linderung des Leidens im Zusammenhang mit einer Danksagung an den Heiligen. Es musste jedoch nicht immer Bargeld sein: Wir wissen, dass in diesem Zusammenhang, insbesondere zur Erfüllung von Gelübden auch wertvolles Kirchengerät und andere Sachwerte gestiftet wurden. ${ }^{53}$ Der Wert der Schenkung war nicht feststehend und richtete sich in erster Linie nach den individuellen Möglichkeiten der Kranken, konnte aber bei einer wohlhabenden Person durchaus Größenordnungen annehmen, die den Neubau eines Klosters, die Renovierung einer Kirche, die Anschaffung wertvollen Kirchengeräts oder die Erweiterung des Landbesitzes ermöglichten. Von den größeren Stiftungen einmal abgesehen, addierten sich aber auch die kleineren Beträge letztendlich zu nicht $\mathrm{zu}$ vernachlässigenden Summen. Folglich hatte die Konkurrenz zwischen Wunderheilung und Medizin auch einen handfesten ökonomischen Hintergrund. Das Gesundheitswesen - sowohl das geistliche Heilungswesen einschließlich der Inkubation als auch die ärztliche Tätigkeit war also bereits im byzantinischen Mittelalter (auch) von wirtschaftlicher Bedeutung.

Die allgemeinen Rahmenbedingungen und Besonderheiten des christlichen Heilschlafs in der Kirche trafen nun selbstverständlich auch auf Jugendliche und junge Erwachsene in Byzanz zu. Ebenso wie Kinder oder ältere Erwachsene hatten auch Jugendliche und junge Erwachsene im Falle einer Erkrankung grundsätzlich die Wahl zwischen der traditionellen ärztlichen Kunst und einem geistlichen Heilungs-

52 Vgl. hierzu etwa das Beispiel eines jüdischen Arztes, der laut Aussage der Vita Symeons, des jüngeren Styliten, von Kaiser Iustinos II. konsultiert wurde, in: Vita Symeonis Stylitae iun. (BHG 1689) cap. 208 - 211 (ed. P.vAn DEN VEn, La vie ancienne de S. Syméon stylite le jeune [521- 592], I, II [Subsidia Hagiographica 32]. Brüssel 1962, 179-181); vgl. dazu auch PRATSCH, Topos 173f. mit Anm. 20.

53 Vgl. exemplarisch M. Mundell MANGo, Silver from Early Byzantium: The Kaper Koraon and Related Treasures (A Walters Art Gallery Publication in the History of Art). Baltimore 1986. 
wesen, die miteinander in Konkurrenz standen und beide auf eine gewisse, wenn auch aus heutiger Sicht begrenzte Wirksamkeit verweisen konnten. Die besondere Therapie des Heilschlafs in der Kirche wurde daher bisweilen auch von Jugendlichen und jungen Erwachsenen in Anspruch genommen. Sie konnten aber ebenso - und das zeigen einige Beispiele - nacheinander oder alternierend auf beide Methoden zurückgreifen. 



\section{Adolescentia in abendländischen Quellen des frühen Mittelalters zwischen Kindheit und Erwachsensein? Ein begriffsgeschichtlicher Zugang}

\section{Forschungsstand und Lebensalterlehre}

Wenn mir in diesem überwiegend byzantinischen Band die Aufgabe zufällt, über Verständnis und Praxis der Adoleszenz im abendländischen Westen zu referieren, kann ich mich bei diesem Thema, trotz einer Reihe von Vorarbeiten, nicht gerade auf einen hinreichenden Forschungsstand stützen, den man hier einfach zusammenfassen könnte, sondern wähle einen anderen Ansatz. Die einschlägigen Arbeiten beschränken sich weitgehend auf die (allgemeine) Lebensalterlehre, ${ }^{1}$ wie sie, mit antiker Vorgeschichte, ${ }^{2}$ vor allem von Augustin entwickelt ${ }^{3}$ und, daran angelehnt, aber auch

In diesem Beitrag werden folgende Abkürzungen verwandt (vor allem für die gängigen Quellensammlungen): CC SL = Corpus Christianorum. Series latina; CCCM = Corpus Christianorum. Continuatio mediaevalis; $\mathrm{CSEL}=$ Corpus scriptorum ecclesiasticorum latinorum; FSGA = Freiherr-vom-SteinGedächtnisausgabe (= Ausgewählte Quellen zur Geschichte des Mittelalters); MGH = Monumenta Germaniae Historica (Reihen: Capit. = Capitularia; Conc. = Concilia; Dt. MA = Deutsches Mittelalter; Epp. = Epistolae; Epp. sel. = Epistolae selectae; Ldl = Libelli de lite; QGG = Quellen zur Geistesgeschichte; SS = Scriptores, SS rer. Germ. = Scriptores rerum Germanicarum in usum scholarum [n.s. = nova series]; SS rer. Lang. = Scriptores rerum Langobardarum et Italicarum; SS rer. Merov. $=$ Scriptores rerum Merovingicarum); PL = Patrologia Latina; $\mathrm{SC}=$ Sources chrétiennes. Ferner ..$=$ anno; ep. $=$ epistola. Die Siglen für die Bibelbücher folgen den gängigen lateinischen Abkürzungen.

1 Kurze Überblicke darüber bieten: K. ARNoLD, Lebensalter (Mittelalter), in: Europäische Mentalitätsgeschichte, hrsg. von P. Dinzelbacher. Stuttgart 22008, ('1993), 216-222 und H.-H. KoRTüM, Menschen und Mentalitäten. Einführung in Vorstellungswelten des Mittelalters. Berlin 1996, 252-257. An ausführlichen Darstellungen seien genannt: J. A. BurRow, The Ages of Man. A Study in Medieval Writing and Thought. Oxford 1986; E. SEARS, The Ages of Man. Medieval Interpretations of the Life Cycle. Princeton 1986 (ist eher an den symbolischen Deutungen interessiert); Les âges de la vie au moyen âge. Actes du colloque du Département d'Études Médiévales de l’Université de Paris-Sorbonne et de l’Université Friedrich-Wilhelm de Bonn, Provins, 16-17 mars 1990, hrsg. von H. Dubois - M. Zink (Cultures et civilisations médiévales 7). Paris 1992; Medieval Life Cycles. Continuity and Changes, hrsg. von I. Cochelin - K. Smyth (International Medieval Research 18). Turnhout 2013. Zum Spätmittelalter: M. E. Goodich, From Birth to Old Age. The Human Life Cycle in Medieval Thought, 1250 - 1350. Lanham - New York - London; D. Youngs, The Life Cycle in Western Europe, c. 1300-c. 1500 (Manchester Medieval Studies). Manchester - New York 2006.

2 Vgl. K.-H. Schwarte, Die Vorgeschichte der augustinischen Weltalterlehre (Antiquitas. Reihe 1: Abhandlungen zur Alten Geschichte 12). Bonn 1966, besonders 43-52, zur römischen Geschichte im 
abgewandelt, über Isidor von Sevilla ${ }^{4}$ und Beda Venerabilis ${ }^{5}$ dem Mittelalter in verschiedenen Formen überliefert worden ist. ${ }^{6}$ Das ist bekannt und soll hier, als Folie, nur

Spiegel der Lebensalter (bei Cicero, Seneca, Florus, Ammian und anderen), der dem Lebensaltervergleich ansonsten aber kaum Beachtung schenkt; zu den antiken Anfängen der Lebensalterlehre: R. HäUSSLER, Vom Ursprung und Wandel des Lebensaltervergleichs. Hermes 92 (1964) 313-341. Zur Tradition der Lebensalterlehren vgl. auch SEARs, Ages (wie Anm. 1) 9-37.

3 Augustinus, De Genesi contra Manichaeos I 23,35- 41 (ed. D. WEBER [CSEL 91]. Wien 1998, 104-111): uideo enim per totum textum diuinarum scripturarum sex quasdam aetates operosas. [...] primordia enim generis humani, in quibus ista luce frui coepit, bene comparantur primo diei quo fecit deus lucem. haec aetas tanquam infantia deputanda est ipsius uniuersi saeculi, quod tanquam unum hominem proportione magnitudinis suae cogitare debemus: quia unusquisque homo cum primo nascitur, et exit ad lucem, primam aetatem agit infantiam. haec tenditur ab Adam usque ad Noe generationibus decem. quasi uespera huius diei fit diluuium; quia et infantia nostra tanquam obliuionis diluuio deletur. et incipit mane a temporibus Noe secunda aetas tanquam pueritia, et tenditur haec aetas usque ad Abraham aliis generationibus decem. et bene comparatur secundo diei quo factum est firmamentum inter aquam et aquam; quia et arca in qua erat noe cum suis, firmamentum erat inter aquas inferiores in quibus natabat, et superiores quibus compluebatur. haec aetas non diluuio deletur, quia et pueritia nostra non obliuione tergitur de memoria. meminimus enim nos fuisse pueros, infantes autem non meminimus. sed nec ista aetas secunda generauit populum dei, quia nec pueritia apta est ad generandum. mane ergo fit ab Abraham, et succedit aetas tertia similis adolescentiae. et bene comparatur diei tertio, quo ab aquis terra separata est. [...] haec enim aetas potuit iam generare populum deo, quia et tertia aetas, id est adolescentia filios habere iam potest. [...] et inde fit mane regnum Dauid. haec aetas similis iuuentutis est. et reuera inter omnes aetates regnat iuuentus, et ipsa est firmum ornamentum omnium aetatum: et ideo bene comparatur quarto diei, quo facta sunt sidera in firmamento coeli. [...] et porrigitur haec aetas usque ad aduentum domini nostri iesu christi, id est quinta aetas, scilicet declinatio a iuuentute ad senectutem, nondum senectus, sed iam non iuuentus: quae senioris aetas est [...]. et reuera sic ista aetas a regni robore inclinata et fracta est in populo iudaeorum, quemadmodum homo a iuuentute fit senior. [...] et finitur dies quintus: incipit sextus, in quo senectus ueteris hominis apparet. hac enim aetate illud carnale regnum uehementer attritum est, quando et templum deiectum est, et sacrificia ipsa cessauerunt; et nunc ea gens quantum ad regni sui uires attinet, quasi extremam uitam trahit. in ista tamen aetate tanquam in senectute ueteris hominis, homo nouus nascitur, qui iam spiritualiter uiuit. Vgl. auch Caesarius von Arles, Sermones. Sermo 169,1 (ed. D. G. MoRIN [CC SL 104]. Turnhout 1953, 692).

4 Isidor von Sevilla, Etymologiae sive Origines XI 2 (ed. W. M. LindSAY. Oxford 1911 [ND Oxford 2007], II, 21-26): Gradus aetatis sex sunt: infantia, pueritia, adolescentia, iuuentus, grauitas atque senectus. Prima aetas infantia est pueri nascentis ad lucem, quae porrigitur in septem annis. Secunda aetas pueritia, id est pura et necdum ad generandum apta, tendens usque ad quartumdecimum annum. Tertia adolescentia ad gignendum adulta, quae porrigitur usque ad uiginti octo annos. Quarta iuuentus firmissima aetatum omnium, finiens in quinquagesimo anno. Quinta aetas senioris, id est grauitas, quae est declinatio a iuuentute in senectutem; nondum senectus sed iam nondum iuuentus, quia senioris aetas est,

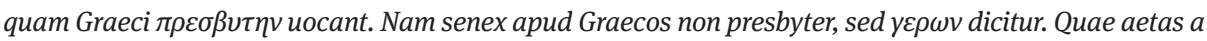
quinquagesimo anno incipiens septuagesimo terminatur. Sexta aetas senectus, quae nullo annorum tempore finitur; sed post quinque illas aetates quantumcumque uitae est, senectuti deputatur. Senium autem pars est ultima senectutis, dicta quod sit terminus sextae aetatis.

5 Beda Venerabilis, De temporum ratione 66 (ed. CH. W. Jones [CC SL 123B]. Turnhout 1977, 463f.): De sex huius mundi aetatibus ac septima uel octaua quietis uitae que caelestis et supra in conparatione primae ebdomadis, in qua mundus ornatus est, aliquanta perstrinximus, et nunc in conparatione aeui unius hominis, qui microcosmos Grecae a philosophis, hoc est, minor mundus solet nuncupari, de eisdem aliquanto latius exponemus. Prima est ergo mundi huius aetas ab Adam usque ad Noe, continens annos 
kurz in Erinnerung gerufen werden. Das Menschenalter unterteilt sich danach bekanntlich in verschiedene (vorrangig sechs) Lebensalter mit (scheinbar) festen Phasen (oder Altersstufen), die - das ist ausdrücklich zu betonen - nach Zahl (vier, fünf, sechs oder sieben), ${ }^{7}$ Phasen und zugeschriebenen Eigenschaften aber erheblich variieren können. Das wohl am meisten verbreitete, auf Augustin beruhende Schema bei Isidor von Sevilla umfasst

- die Kindheit (infantia) bis sieben Jahren,

- die Jugend (pueritia) bis 14 Jahren,

- die Adoleszenz (adolescentia) - ich übersetze bewusst erst einmal nicht - bis 28 Jahren (sie währt damit immerhin doppelt so lange wie die ersten beiden Menschenalter),

- die iuventus bis 50 Jahren,

- die gravitas bis 70 Jahren - begrifflich also das Alter der Würde wie auch bereits der Schwäche -,

- $\quad$ und schließlich das Greisenalter (senectus) bis zum Tod.

(Augustin lässt der iuventus hingegen den senectus als fünftes Lebensalter folgen und schließt dem als sechstes noch ein Dahinsiechen, aetas decrepita, an.)

iuxta hebraicam veritatem mille dclvi, iuxta lxx interpretes iimccxlii, generationes iuxta utramque editionem numero $x$. Quae uniuersali est deleta diluuio, sicut primam cuiusque hominis obliuio demergere consueuit aetatem; quotus enim quisque est, qui suam recordetur infantiam? secunda aetas a Noe usque ad Abraham generationes iuxta hebraicam auctoritatem conplexa $x$, annos autem ccxcii, porro iuxta lxx interpretes ann. cclxxii, generationes uero xi. Haec quasi pueritia fuit generis populi dei et ideo in lingua inuenta est, id est hebrea, a pueritia namque homo incipit nosse loqui post infantiam, quae hinc appellata est, quod fari non potest. Tertia ab Abraham usque ad Dauid generationes iuxta utramque auctoritatem xiiii, annos uero dccccxlii conplectens. Haec quaedam uelut adolescentia fuit populi dei, a qua aetate quia incipit homo posse generare, propterea Matheus euangelista generationum ex Abraham sumsit exordium, qui etiam pater gentium constitutus est, quando mutatum nomen accepit. Quarta a Dauid usque ad transmigrationem Babylonis, habens annos iuxta hebraicam veritatem cccclxxiii, iuxta lxx translationem xii amplius, generationes iuxta utrosque codices xvii; quas tamen euangelista Matheus certi mysterii gratia xiiii ponit. A qua uelut iuuenali aetate in populo dei regum tempora coeperunt, haec namque in hominibus aetas apta gubernando solet existere regno. Quinta quasi senilis aetas a transmigratione Babylonis usque in aduentum Domini saluatoris in carnem, generationibus et ipsa xiiii, porro annis dlxxxviiii extenta. In qua, ut graui senectute fessa, malis crebrioribus plebs hebrea quassatur. Sexta, que nunc agitur, aetas, nulla generationum uel temporum serie certa, sed ut aetas decrepita ipsa totius saeculi morte consumenda. Has erumnosas plenas que laboribus mundi aetates quique felici morte uicerunt; septima iam sabbati perennis aetate suscepti, octauam beatae resurrectionis aetatem, in qua semper cum domino regnent, exspectant.

6 Vgl. etwa Honorius Augustodunensis, Imago mundi II 59 (De homine microcosmo) (ed. V. I. J. FLINT. Archives d'histoire doctrinale et littéraires du Moyen Âge 49 [1982] 48-151) mit Zuweisung der vier Charaktere zu den Altersstufen, und vor allem ebd. II 78 (De aetate), ebd. 106 und 110.

7 SEARs, Ages (wie Anm. 1) gliedert ihre Kapitel geradezu nach der Anzahl der Lebensalter: Kapitel 1 (9-37): drei oder vier Alter, Kapitel 2 (38-53): sieben Alter, Kapitel 3 (54-79): sechs Alter, parallel den Weltaltern. 
In der Einleitung zu einem jüngst erschienenen Sammelband über „Medieval Life Cycles“ hat Isabelle Cochelin die ihr bekannten Lebensalterlehren vom 3. bis zum Ende des 12. Jahrhunderts zusammengestellt und graphisch umgesetzt. ${ }^{8}$ Diese Aufstellung lässt dreierlei erkennen: Bis auf wenige Ausnahmen fügen sich erstens alle Belege in ein relativ einheitliches System ein, das allerdings verschiedene Varianten aufweist. Cochelin geht von drei Hauptphasen, pueritia, iuventus und senectus aus, die jeweils oft wieder zweigeteilt sind. ${ }^{9}$ Man kann daraus zweitens auch umgekehrt schließen, dass immer wieder (meist alternativ) zwei der sechs Lebensalter (in aller Regel jeweils unter dem zweiten Namen) zusammengefasst werden, und zwar infantia und pueritia, adolescentia und iuventus sowie gravitas und senectus. Mit Ausnahme des Letzteren sind die Begriffe drittens erstaunlich ,stabil‘.

Kennzeichnend für die christlichen Lebensaltersysteme ist ferner, schon bei Augustin, Isidor und Beda, ihre Einbindung in übergreifende Zeitrhythmen: die sechs Schöpfungstage und die sechs Weltalter (aetates) bzw. die ,Lebensalter des Volkes Israel, aber auch die vier Jahreszeiten, die vier Elemente bzw. die vier davon bestimmten Körpersäfte (Blut, rote Galle, schwarze Galle, Schleim), Eigenschaften (warm, kalt, feucht, trocken) und Temperamente (Choleriker, Sanguiniker, Phlegmatiker und Melancholiker), ${ }^{10}$ und zwar unter Übergehung der pueritia sowie entweder der adolescentia (wie bei Honorius) oder der iuventus (wie bei Beda). Das Siebenerschema wird wiederum mit den sieben Planeten parallelisiert. Wichtiger in unserem Zusammenhang ist hingegen die inhaltliche Kennzeichnung (hier nach Augustin, Isidor und Beda): ${ }^{11}$

- Die infantia bleibt sprachlos (da das Kind noch keine richtigen Zähne hat) und vor allem ohne Erinnerung,

- die pueritia bedeutet Sprech- und Erinnerungsfähigkeit (so Augustin), doch ist der puer nach allen Autoren noch nicht zeugungsfähig,

- $\quad$ während die adolescentia sich vor allem mit Zeugungsfähigkeit verbindet;

- die iuventus ist Höhepunkt und stärkstes Alter. Danach beginnt der Abstieg.

Mittelalterlich greift die Adoleszenz also weit aus bis zur Erlangung der vollen Manneskraft. Bei dieser Spanne wird man kaum mehr von ,Jugend‘ sprechen können, wie der „Georges“ übersetzt, für den aber auch noch der bis zum 50. Lebensjahr währende iuventus (wortgetreu), Jugend‘ ist. Die Übersetzungsschwierigkeiten zeigen bereits an,

8 I. Cochelin, Introduction: Pre-Thirteenth-Century Definitions of the Life Cycle, in: Medieval Life Cycles (wie Anm. 1) 1-54, die Grafiken hier 3-5. Im Anhang (18-44) sind die Belege mit den Lebensaltern (und Altersangaben) aufgeschlüsselt, doch ist leider nicht der jeweilige Quellentext abgedruckt.

9 Vgl. dazu ebd. 6-11.

10 Vgl. dazu TH. BeIn, Lebensalter und Säfte. Aspekte der antik-mittelalterlichen Humoralpathologie und ihre Reflexe in Dichtung und Kunst, in: Âges de la vie (wie Anm. 1) 1- 54; SEARs, Ages (wie Anm. 1) 97-120: „Popular cosmology“.

$11 \mathrm{Vgl}$. Anm. 3 bis 5. 
dass die antik-mittelalterliche Lebensalterlehre von unseren Vorstellungen grundlegend abweicht und über die Phase des Erwachsenwerdens Jugendlicher hinausgeht. Entsprechend wird in der Forschung gern ihre Theoriehaftigkeit und Ferne von den tatsächlichen sozialen Verhältnissen betont. ${ }^{12}$ Schon Adolf Hofmeister hat auf die Relativität und Ungenauigkeit dieser Altersbezeichnungen im Vergleich zum tatsächlichen Lebensalter hingewiesen. ${ }^{13}$ Dabei war der Übergang von der adolescentia zur iuventus besonders fließend. ${ }^{14}$

Wenige der einschlägigen Studien (wie Burrow oder Sears) befassen sich zudem speziell mit der adolescentia (oder werden hier wenig konkret), wie sich die Forschung überhaupt weit mehr mit Kindheit ${ }^{15}$ und Alter $^{16}$ als mit Adoleszenz und Juventus be-

12 Vgl. Burrow, Ages (wie Anm. 1) 34; SEARS, Ages 6 (wie Anm. 1): ,the theme of the ages of man was a bookish one“.

13 A. Hofmeister, Puer, Iuvenis, Senex. Zum Verständnis der mittelalterlichen Altersbezeichnungen, in: Papsttum und Kaisertum. Forschungen zur politischen Geschichte und Geisteskultur des Mittelalters. Paul Kehr zum 65. Geburtstag dargebracht, hrsg. von A. Brackmann. München 1926, 287-316. 14 Ebd. 305. Insgesamt ist der adolescens mindestens 12-14 und höchstens 28 Jahre alt, doch gibt es davon auch Abweichungen (ebd. 316). Die Übergänge bei Gregor von Tours behandelt E. JAMES, Childhood and Youth in the Early Middle Ages, in: Youth in the Middle Ages, hrsg. von P. J. P. Goldberg F. Riddy. Woodbridge - Rochester 2004, 11-23.

15 Neben den inzwischen schon „klassischen“ Studien zur mittelalterlichen Kindheit von K. ARNoLD, Kind und Gesellschaft in Mittelalter und Renaissance. Beiträge und Texte zur Geschichte der Kindheit. Paderborn 1980; M. WinTER, Kindheit und Jugend im Mittelalter, Freiburg 1984 und S. SHAHAR, Childhood in the Middle Ages. London 1990 (nach dem initiierenden, aber einseitigen Essay von Philippe Ariès) seien genannt: The Church and Childhood. Papers Read at the Summer Meeting and the 1994 Winter Meeting of the Ecclesiastical History Society, hrsg. von D. Wood (Studies in Church History 31). Oxford 1994; Aufblühen und Verwelken. Mediävistische Forschungen zu Kindheit und Alter. 4. Tagung der Arbeitsgruppe „Marburger Mittelalterzentrum (MMZ)“, Marburg 17. November 2006, hrsg. von. I. Heiser - A. Meyer. Leipzig 2009; Als Aufriss versteht sich B. G BAILEY - M.E. BERNARD - G. CARrier - Ch. L. Elliott - J. LANGdon - N. Leishman - M. MlynARZ - O. Mykhed - L. C. Ridders, Coming of Age and the Family in Medieval England. Journal of Family History 33 (2008) 41-60.

16 Vgl. beispielsweise R. SPRANDEL, Altersschicksal und Altersmoral. Die Geschichte der Einstellungen zum Altern nach der Pariser Bibelexegese des 12.-16. Jahrhunderts (Monographien zur Geschichte des Mittelalters 22). Stuttgart 1981; Vieillesse et vieillissement au Moyen Âge (Senefiance 19). Aix-en-Provence 1987; Aging and Aged in Medieval Europe. Selected Papers from the Annual Conference of the Centre for Medieval Studies. University of Toronto, held 25-26 February and 11-12 November 1983, hrsg. von M. Sheehan (Papers in Mediaeval Studies 11). Toronto 1990; S. SHAHAR, Growing Old in the Middle Ages. ,Winter Clothes Us in Shadow and Pain‘. London - New York 1997; B.-U. HeRGEMÖLLER, Die Kindlein spotten meiner schier. Quellen und Reflexionen zu den Alten und zum Vergreisungsprozeß im Mittelalter (Hergemöllers Historiographische Libelli 4). Hamburg 2006; Youth and Age in the Medieval North, hrsg. von S. Lewis-Simpson (The Northern World. North Europe and the Baltic c. 400-1700 AD. Peoples, Economies and Cultures 42). Leiden - Boston 2008; Alterskulturen des Mittelalters und der frühen Neuzeit. Internationaler Kongress, Krems an der Donau, 16. bis 18. Oktober 2006, hrsg. von E. Vavra (Österreichische Akademie der Wissenschaften. Philosophisch-historische Klasse. Sitzungsberichte 780 = Veröffentlichungen des Instituts für Realienkunde des Mittelalters und der Frühen Neuzeit 21). Wien 2008; Aufblühen und Verwelken (wie Anm. 15); Generations in the Cloister, Generationen im Kloster. Youth and Age in Medieval Religious Life. Jugend und Alter in der mittelalterlichen vita religiosa, hrsg. von S. Heusinger - A. Kehnel (Vita regularis. Ordnungen und Deutungen 
fasst hat bzw. werden Kindheit und Jugend in der Regel zusammengefasst. Meist geht es um eine soziale Differenzierung (Adel), ${ }^{17}$ um Unterschiede zwischen Mönchtum und Laien ${ }^{18}$ oder um die Übergänge (rites de passage) von der Kindheit zur Jugend. ${ }^{19}$ Neben sehr speziellen Studien ${ }^{20}$ bieten lediglich die auf das späte Mittelalter bezogenen Werke von Michael Goodich und Deborah Youngs eigene Kapitel über die Adoleszenz, die beide allerdings wieder Adoleszenz und Jugend zusammenfassen. Youngs stellt hier als Kennzeichen des Lebensalters körperliche Merkmale (Stärke und Haartracht), Emotionen („lust“), aber auch Verstand, „misbehaviour“ und Training

religiösen Lebens im Mittelalter. Abhandlungen 36). Münster 2008; On Old Age: Approaching Death in Antiquity and the Middle Ages, hrsg. von Ch. Krötzl - K. Mustakallio (The History of Daily Life 2). Turnhout 2012.

17 Zur Adelserziehung im hohen Mittelalter (anhand der Biographie Guillaumes le Maréchal) vgl. M. PARMENTIER, Höfische Jugend um 1200. Die Karriere eines subkulturellen Stils im vormodernen Europa, in: Vormoderne Lebensläufe - erziehungshistorisch betrachtet, hrsg. von W. K. Rudolf - W. E. Wiersing (Beiträge zur historischen Bildungsforschung 12). Köln - Weimar - Wien 1994, 187-202. Zur Stellung in der Adelsgesellschaft: $\mathrm{CH}$. DETTE, Kinder und Jugendliche in der Adelsgesellschaft des frühen Mittelalters. Archiv für Kulturgeschichte 76 (1994) 1-34. Zur Rolle des Vaters bei der Vorbereitung, Unterweisung und Einweisung in die künftigen Pflichten und zur Einbeziehung in die Tätigkeiten des Adels: J. R. Lyon, Fathers and sons: preparing noble youths to be lords in twelfth-century Germany. Journal of Medieval History 34 (2008) 291-310, der sich damit zugleich gegen Dubys Thesen jugendlicher Neigung zur Rebellion wendet.

18 Vgl. J. A. Schultz, The Knowledge of Childhood in the German Middle Ages, 1100-1350 (Middle Ages Series). Philadelphia 1995. Aussagen der Klosterregeln zu adolescentes unter den (werdenden) Mönchen wertet aus: F. H. StoERTz, Adolescence and Authority in Medieval Monasticism, in: The Growth of Authority in the Medieval West. Selected Proceedings of the International Conference Groningen 6-9 November 1997, hrsg. von M. Gosman - A. Vanderjagt - J. Veenstra (Mediaevalia Groningana 25). Groningen 1999, 119-140. Das Verständnis der adolescentia in den Consuetudines der Cluniacenser vom 10.-12. Jahrhundert untersucht I. Cochelin, Adolescence Uncloistered (Cluny, Early Twelfth Century), in: Medieval Life Cycles (wie Anm. 1) 147-182; zum Verständnis der Kirchenreformer vgl. K. G. Cushing, Pueri, Iuvenes, and Viri: Age and Utility in the Gregorian Reform. The Catholic Historical Review 94 (2008) 435-449.

19 Vgl. Schultz, Knowledge (wie Anm. 18) Kap. 6, 148-162; James, Childhood (wie Anm. 14). Zum langfristigen Wandel des Adoleszenzverständnisses vom Mittelalter bis heute vgl. B. A. HANAWALT, Historical Descriptions and Prescriptions for Adolescence. Journal of Family History 17 (1992) 341-351. 20 So untersucht R. НомEт, Niños y adolescentes en fiestas y ceremonias. En la España Medieval 24 (2001) 145-169, die Funktion von Kindern und Jugendlichen bei Kirchenfesten; U. HeLfEnstein, Beiträge zur Problematik der Lebensalter in der mittleren Geschichte. Diss. Zürich 1952, fragt nach rechtlichen Altersstufen (Mündigkeit und kanonischem Alter); B. MCCREESH, The Birth, Childhood and Adolescence of the Early Icelandic Bishops, in: Youth and Age (wie Anm. 14) 87-102, geht Nachrichten zu ausgewählten Bischöfen nach. Nach Schwierigkeiten mit Heranwachsenden in der nordischen Literatur fragt C. LARRINGTON, Awkward Adolescents. Male Maturation in Norse Literature, in: ebd. 151166. Zur Bekehrung von Juden: W. Ch. Jordan, Adolescence and Conversion in the Middle Ages: A Research Agenda, in: Jews and Christians in Twelfth-Century Europe, hrsg. von M. A. Signer - J. Van Engen (Notre Dame Conferences in Medieval Studies 10). Notre Dame - Indiana 2001, 77-93; J. SHERwood, Rebellious Youth and Pliant Children: Jewish Conversion in Adolescentia, in: Medieval Life Cycles (wie Anm. 1) 183-209. 
heraus. ${ }^{21}$ In diesem Alter war man besonders gewalttätig, doch wurden die Taten auch am ehesten verziehen. ${ }^{22}$ Goodich stellt die altersgemäßen Tugenden (wie Großmut, Freigebigkeit, Hoffnung, Freundlichkeit, Mitgefühl und Bescheidenheit) und Laster (wie Unbeständigkeit, Vertrauensseligkeit, Sturheit, Verlogenheit und Maßlosigkeit) heraus, ${ }^{23}$ verweist auf (monastische oder laikale) Erziehung ${ }^{24}$ und betont vor allem den sexuellen Druck dieses Lebensalters. ${ }^{25}$ Man wird zu prüfen haben, ob Ähnliches schon für das frühere Mittelalter gilt.

Der grobe Überblick deckt darüber hinaus ein großes Forschungsproblem auf. Die bisherige Forschung subsumiert die Adoleszenz nicht nur unter ,Jugend', sondern fast alle Studien zur mittelalterlichen Jugend ${ }^{26}$ legen dabei den heutigen Begriff zugrunde, um dieses Lebensalter zu charakterisieren, sind zudem mehr an dem Lebensalter als an seinen Inhalten interessiert und gehen entsprechend kaum dem spezifisch mittelalterlichen Verständnis nach, auch wenn beides immer wieder gestreift wird ${ }^{27}$ (und sind damit eventuell nicht mehr mittelaltergemäß). Darauf hat bereits James Schultz verwiesen, ${ }^{28} \mathrm{zu}$ Recht eine Überprüfung an zeitgenössischen Quellen gefordert und darüber sämtliche Theorien über Adoleszenz als unhaltbar zurückgewiesen, ${ }^{29}$ weil sie in den Quellen keinerlei Stütze finden (wobei Schultz sich auf mittelhochdeutsche Quellen beschränkt). Mit gleicher Skepsis gegenüber den Quellen bestreitet er darüber hinaus, dass das Mittelalter überhaupt klare Vorstellungen von Adoleszenz hatte, da sämtliche Begriffe höchst schillernd sind, während unser Begriff von Adoleszenz keine mittelhochdeutsche Entsprechung hat ${ }^{30}:$,It is a clear sign that these texts do not

21 Youngs, Life Cycle (wie Anm. 1) 96-125.

22 Ebd. 120; ganz ähnlich DeTTE, Kinder (wie Anm. 17) 28.

23 Goodich, From Birth (wie Anm. 1) $105 \mathrm{f}$.

24 Ebd. 109-114.

25 Ebd. 121-141.

26 Zur Jugend vgl. etwa Youth in the Middle Ages (wie Anm. 14); zum Frühmittelalter hier lediglich: James, Childhood (wie Anm. 14), zu Gregor von Tours; Youth and Age (wie Anm. 16).

27 Das kann hier nicht resümierend dargelegt werden. Wichtige diesbezügliche Ergebnisse und Ansichten werden im Folgenden bei der jeweiligen Behandlung der Inhalte genannt werden.

28 J. A. Schultz, Medieval Adolescence: The Claims of History and the Silence of German Narrative. Speculum 66 (1991) 519-539.

29 Schultz unterscheidet den „psychologischen Ansatz“ (ebd. 520 - 526: „struggle with parents and the struggle for identity“), den „physiologischen Ansatz“, nämlich die implizite Annahme, dass das Mittelalter eine Vorstellung von Adoleszenz als dem Alter zwischen Pubertät und Erwachsensein (Reife) hatte (ebd. 526-530) - nicht einmal der Bartwuchs bei heranwachsenden Jungen sei in den Quellen ein eindeutiges Kennzeichen (ebd. 529) - sowie den „lexikalischen Ansatz“ (530 - 533), der nach adolescentia fragt, während die Verfechter tatsächlich über Adoleszenz (im modernen Sinn) schreiben.

30 Ebd. 533. Letztlich zeigt Schultz auf, dass die mittelhochdeutschen Texte keine Hinweise für Adoleszenz als Lebensphase in unserem Sinn bieten (so auch explizit ebd. 533). Da die mittelalterlichen Lebensalterlehren die Phasen anders organisieren, ist das allerdings wenig überraschend (und eben nur nötig gewesen, weil die bisherige Forschung zu wenig klar zwischen beiden Konzepten differenziert hat). 
recognize adolescence, and it is a strong indication that adolescence may simply not have occurred in the society in which they were written“31.

Das gilt für das Mittellateinische nun eindeutig nicht (wenngleich der Begriff auch hier nicht unserer ,Jugend“ entspricht) und geht in der Folgerung zu weit. Fragt man hier nach den Inhalten, so lassen sich, wie ich meine, durchaus mehr oder weniger konsistente Vorstellungen von Adoleszenz ermitteln. ${ }^{32}$ Ich wähle im Folgenden deshalb einen begriffsgeschichtlichen Ansatz, indem ich die Belege für adolescentia im früheren Mittelalter sichte und, nach verschiedenen Aspekten geordnet, aus dem Kontext herauszufinden suche, wie er in den Quellen überhaupt angewandt wird, was die Autoren damit verbinden und was er bedeutet. Mit der Betrachtung möglichst vieler Belege erweitere ich die (bislang im Mittelpunkt stehenden) Lebensalterschemata um eine erheblich breitere Quellenbasis für das Verständnis von ,Jugendlichen‘. Das Ergebnis sind erste Einblicke in die mittelalterlichen Vorstellungen von Adoleszenz. Aussagen zur damaligen sozialen Wirklichkeit, über ihre Stellung in der Gesellschaft, ergeben sich daraus allenfalls indirekt, vor allem aus Chroniken, die den Begriff scheinbar unreflektiert (aber mit ihrem Vorverständnis) auf historische Persönlichkeiten und Ereignisse anwenden. Ein farbiges Bild des Lebensstils mittelalterlicher Jugendlicher lässt sich aus den frühmittelalterlichen Quellen nicht nachzeichnen. Entgegen dem Wiener Projekt habe ich den Untersuchungszeitraum außerdem auf das 12. Jahrhundert ausgedehnt, weil es oft besonders aufschlussreiche Belege bietet (die natürlich aber nicht einfach zurückprojiziert werden dürfen).

\section{Häufigkeit}

Übersicht:

\section{Library of Latin Texts (LLT):}

$\begin{array}{lr}\text { 5. Jh.: } & 196 \\ \text { 6. Jh.: } & 98 \\ \text { 7. Jh.: } & 58 \\ \text { 8. Jh.: } & 49 \\ \text { 9. Jh.: } & 199 \\ \text { 10. Jh.: } & 50 \\ \text { 11. Jh.: } & 113 \\ \text { 12. Jh.: } & 1136\end{array}$

196

58 


\section{dMGH:}

\section{Scriptores:}

SS rer. Merov. $\quad 90$

SS rer. Lang. $\quad 8$

SS rer. Germ. ns 6

SS rer. Germ. $\quad 118$

$\mathrm{SS}^{33} \quad 744$

Ldl $\quad 32 \quad \sum$ SS 998

Leges (LL) 29

Diplomata (DD) 4

Epistolae (EE) 80

Antiquitates $\quad 13$

Necrologia 27

Andere $\quad 22$

Gesamt $\quad \sum$ gesamt 1173

Ein erster Überblick über die Häufigkeit des Begriffs zeigt dreierlei. Adolescentia ist erstens kein Ausnahmebegriff. Inhaltlich aussagekräftig sind allerdings nur wenige, im Folgenden ausgewertete Belege. Oft ist der Begriff nur ein Zusatz zum Namen ohne nähere Aussage, in erzählenden Texten ebenso wie etwa in Nekrologien. Die chronologische Ordnung nach der „Library of Latin Texts“ (LLT) zeigt zweitens gewisse Spitzen im 5. und 9. und einen absoluten Höhepunkt im 12. Jahrhundert (auch wenn das zum Teil auf die Gesamtproduktion und die Quellenbasis der LLT zurückgeht). Die Differenzierung nach den Großgattungen der MGH lässt drittens erkennen, dass der Begriff sich - neben den theologischen Texten der LLT - vor allem in der Historiographie sowie allenfalls noch in Briefen findet und ansonsten eher spärlich verwendet wird; das gilt auch für Rechtstexte: Rechtlich war die Altersstufe anscheinend nicht von besonderer Bedeutung. ${ }^{34}$

33 Soweit die Belege nicht bereits in den neueren Reihen berücksichtigt worden sind.

$34 \mathrm{Zu}$ einer bemerkenswerten Ausnahme vgl. unten Anm. 63. 


\section{Alter}

\section{a. Altersstufen}

Die ausgewerteten Belege bestätigen zunächst die Anwendung und Einbettung in die (meist) sechs Lebensalterstufen, in der Regel nach Isidor, wie bei Alkuin, ${ }^{35}$ oder nach Augustin, wie bei Sedulius Scotus oder in der Ars Laureshamensis (einem DonatusKommentar) aus dem 9. Jahrhundert, ${ }^{36}$ allerdings mit verschiedenen Varianten: ${ }^{37}$ So durchläuft ein Mann nach Aelred von Rievaulx drei Altersstufen: infantia, pueritia und adolescentia, bevor er zum Mann wird. ${ }^{38}$ Andere kennen nur fünf Altersstufen, wie Adso von Montier-en-Der (ohne pueritia), ${ }^{39}$ Rupert von Deutz (gemäß den Stunden des Tages, ohne infantia) ${ }^{40}$ oder Bernhard von Clairvaux (ohne gravitas)..$^{41}$ Wenn Agobard

35 Alkuin, Commentaria in s. Ioannis Euangelium (PL 100). Paris 1851, 733-1008, hier 792 C: Nam sexta aetas senectus est, quoniam prima est infantia, secunda pueritia, tertia adolescentia, quarta iuventus, quinta gravitas.

36 Sedulius Scotus, In Donati artem maiorem, pars 2 (ed. B. LöFSTEDT [CCCM 40B]. Turnhout 1977, 103): Sex namque sunt aetates hominum: infantia uidelicet et pueritia, adolescentia et iuuentus, senectus et senium. Quae omnes retrogradae sunt et hae sex pro tribus accipiuntur. Einen Superlativ hält Sedulius für falsch, weil er bereits auf das folgende Alter verweise: Superlatiuo autem ideo carent, quia si dicam iuuenissimus et adolescentissimus, desinit iam esse iuuenis et adolescens et incipit transire in antecedentem aetatem. Similiter senex senior: plus enim significat senex quam senior, id est spatium longioris temporis. Wörtlich übereinstimmend (nach Löfstedt aus gleicher Quelle): Ars Laureshamensis. Expositio in Donatum maiorem, pars 2, De nomine (ed. B. LöfSTEDT [CCCM 40 A]. Turnhout 1977, 26): Sex namque sunt aetates hominum: infantia uidelicet et pueritia, adolescentia et iuuentus, senectus et senium.

37 Abweichende Begriffe für die beiden letzten Lebensalter etwa bei Philipp von Harvengt, De obedientia clericorum 5 (PL 203). Paris 1855, 839-944, hier 849 B: Prima hominis aetas infantia est, secunda pueritia, tertia adolescentia, quarta iuuentus, quinta uirilis aetas, sexta senectus.

38 Aelred von Rievaulx, Sermones. Sermo 43,7 (coll. Claraevallensis secunda) (ed. G. RACITI, [CCCM 2 A]. Turnhout 1989, 243-370, hier 337): Istae aetates solent notari in homine: primo infantia, dein pueritia, postea adolescentia. Abhinc incipit homo esse uir.

39 Adso von Montier-en-Der, Vita sancti Basoli 7 (ed. M. Goullet [CCCM 198]. Turnhout 2003, 219269, hier 224): Igitur beatissimus Basolus, Aquitania prouintia inclyto parentum germine exortus, territorio urbis lemouicinę natu et genere fuit nobilissimus. Qui felici auspicio mętas infantię supergressus, cum tempora adolescentie sacri tirocinii incremento contigisset, ad diuine seruitutis opus totum se conuertit.

40 Rupert von Deutz, De sancta trinitate et operibus eius XXXVI 10 (ed. R. HAACKE [CCCM 24]. Turnhout 1972, 1914): Celebrato hoc mysterio, quotacumque sit hora diei siue mane siue hora tertia siue sexta siue nona siue uespera, id est quaecumque sit hominis aetas siue pueritia siue adolescentia siue iuuentus siue senectus siue aetas decrepita, nouus homo est et puer Domini noua creatura est et filius Dei. 41 Bernhard von Clairvaux, Homiliae in laudibus Virginis matris. Homilia super ,Missus est‘2,8 (ed. J. LECLERCQ - H. RochAIs, Sancti Bernardi Opera, IV [Sermones I]. Rom 1966, 13-58, hier 26): Hoc est enim a femina circumdari. Novimus viros, qui scilicet infantiam, pueritiam, adolescentiam atque iuventutem transeuntes, ad gradum usque senectuti proximum pervenerunt. 
von Lyon alle Eigenschaften und Funktionen Christi aufzählt, erwähnt er nur die ersten vier Lebensalter, weil Christus auf Erden bereits als iuvenis gestorben ist. ${ }^{42}$

Gleichermaßen bestätigt sich die symbolische Parallele der Lebensalter zu anderen ,Systemen': vorab den Weltaltern, die zugleich als Altersstufen eines Volkes (des Volkes Israel) gewertet werden, ${ }^{43}$ aber auch den Tagesstunden. ${ }^{44}$ Die Adoleszenz entspricht dabei nach Heiric von Auxerre der dritten Stunde, in der die Sonne aufsteigt, so wie in der Adoleszenz die Hitze zunimmt. ${ }^{45}$ Systematisch ist die Weltalterlehre bei Beda ${ }^{46}$ und danach, mit Abweichungen, zu Beginn der um 1000 entstandenen Quedlinburger Annalen verarbeitet, die jeweils aetas, deren Anfang und Ende, Zahl der Generationen, Dauer in Jahren sowie den inhaltlichen Bezug zum Lebensalter angeben. ${ }^{47}$ Schematisch umgesetzt, ergibt sich daraus folgendes Bild:

42 Agobard von Lyon, Aduersum dogma Felicis 28 (ed. L. VAN ACKER [CCCM 52]. Turnhout 1981, $73-$ 111, hier 96), über Christus: Accedentia uero illi sunt homo, infans, puer, adolescens, iuuenis, minister legis, seruus Patris, minor Patre, subiectus, frater electorum, particeps eorum, coheres, propinquus illorum, conformis, consimilis per omnia, excepto peccato, mediator Dei et hominum, propheta, euangelista, apostolus, sacerdos, pontifex. Hec omnia et his similia accedentia sunt illi secundum dispensationem carnis, non propria secundum potestatem deitatis.

43 Vgl. Hrabanus Maurus, De computo 96 (ed. W. M. STEVEns [CCCM 44]. Turnhout 1979, 199-321, hier 319f.), an Augustin angelehnt: Die erste aetas von Adam bis Noah entspricht der infantia, da mit der Sintflut alle Erinnerung ausgelöscht war, die zweite aetas von Noah bis Abraham der pueritia, wenn der Mensch sprechen kann, die dritte von David bis zur Babylonischen Gefangenschaft der Adoleszenz: Tertia ab ABRAHAM usque ad DAVID generationes iuxta utramque auctoritatem XIIII, annos uero DCCCCXLII complectens. Haec uelut quaedam adolescentia fuit populo DEI, a qua aetate quia incipit homo posse generare, propterea MATHEVS euangelista generationum ex ABRAHAM sumpsit exordium, qui etiam pater gentium constitutus est, quando mutatum nomen accepit. Die iuventus währte von David bis zur Babylonischen Gefangenschaft, die senilis aetas von da bis Christi Geburt, die aetas decrepita bis zur Gegenwart. Zu Altersstufen des Volkes Israel, die sich in Christi Gleichnis vom Jüngling widerspiegeln, vgl. auch Paschasius Radbertus, Expositio in Matheo IX (Mt 19,16) (ed. B. PAULus [CCCM 56B]. Turnhout 1984, 952): In seinem Gleichnis vom reichen und leichtfertigen Jüngling zeichnet Christus die ganze Geschichte Israels in seinen Altersstufen nach: Ac deinde ex hoc totam illam quae sequitur parabolam componit. Quapropter et hic primum proponit hunc adolescentem diuitem ac leuem gloriantem et amplius amantem bona sua carnalia quam celestia Christi promissa ut in persona huius adolescentis tota Iudaici populi forma exprimatur et passio, propter quam Christo credere noluerunt, id est propter sibi placentia propter iactantiam propter questus, quos capiebant ex lege. Propterea quaecumque dicuntur de adolescentulo isto sic sunt accipienda quasi de populo Iudaico dicta.

44 Vgl. Rupert von Deutz, De sancta trinitate et operibus eius XXXVI 10 (oben Anm. 40).

45 Heiric von Auxerre, Homiliae per circulum anni. I. Pars hiemalis, hom. 25 (ed. R. QUADRI [CCCM 116]. Turnhout 1992, 296): Possumus et has horas ad aetatis humanae momenta referre. Mane enim pueritiam significat; hora tertia, cum sol in altum proficit, adolescentiam, in qua calor aetatis crescit; sexta nichilominus iuuentutem, quia sicut sol sexta hora amplius utpote in medio caeli positus feruet, sic iuuentus ad omnia agenda fortior caeteris aetatibus exsistit; nona aeque senectus intellegitur. Vgl. auch unten Anm. 90.

46 Vgl. oben Anm. 5.

47 Annales Quedlinburgenses (ed. M. GIESE [MGH SS rer. Germ. 72]. Hannover 2004, 383f.): Prima aetas ab Adam usque ad Noe, continens generationes X, annos vero MDCLIII. Quae tota periit diluvio, sicut infantiam mergere solet oblivio. Secunda a Noe usque ad Abraham, similiter complexa generationes 


\begin{tabular}{|c|c|c|c|c|c|}
\hline Aetas & Zeit & $\begin{array}{l}\text { Gene- } \\
\text { ration }\end{array}$ & $\begin{array}{l}\text { Dauer } \\
\text { (Jahre) }\end{array}$ & Eigenschaft & Lebensalter \\
\hline 1. & Adam - Noah & 10 & 1653 & $\begin{array}{l}\text { Vernichtung durch } \\
\text { Sintflut // }\end{array}$ & $\begin{array}{l}\text { infantia: } \\
\text { Vergessen }\end{array}$ \\
\hline 2. & Noah - Abraham & 10 & 292 & Erfindung des Hebräischen & $\begin{array}{l}\text { pueritia: } \\
\text { Sprachvermögen }\end{array}$ \\
\hline 3. & Abraham - David & 14 & 942 & Zählung der Generationen & $\begin{array}{l}\text { adolescentia: } \\
\text { Zeugungsvermögen }\end{array}$ \\
\hline 4. & $\begin{array}{l}\text { David - Babylo- } \\
\text { nische Gefangenschaft }\end{array}$ & 14 & 484 & Zeit der Könige & $\begin{array}{l}\text { aetas iuvenilis: } \\
\text { zum Königtum fähig }\end{array}$ \\
\hline 5. & Bis Christi Geburt & 14 & 686 & Häufige Übel & $\begin{array}{l}\text { wie in der senectus: } \\
\text { starke Erschöpfung }\end{array}$ \\
\hline 6. & Jetztzeit & unb. & unb. & Ende der Zeiten & wie aetas decrepita: Tod \\
\hline
\end{tabular}

Wie schon mehrfach bemerkt worden ist, ${ }^{48}$ werden adolescentia und iuventus immer wieder zusammengesehen (oder auch zusammengefasst), in Wendungen wie adolescentia vel iuventus. ${ }^{49}$ Manche Autoren (wie Hinkmar von Reims) sprechen abwechselnd vom adolescens und vom iuvenis, ${ }^{50}$ und Heiric von Auxerre betont, dass die

$X$, annos autem CCXCII. In qua aetate lingua est Hebraea inventa. A puericia namque homo incipit loqui nosse post infantiam, quae inde nomen accepit, quia fari, id est loqui, non potest. Tertia aetas ab Abraham usque ad David, generationes XIIII, annos vero DCCCCXLII continens. Et quia ab adolescentia incipit homo posse generare, Matheus ab Abraham generationum sumpsit exordium. Qui etiam pater multarum gentium constitutus est, quoniam mutatum nomen accepit. Quarta aetas a David usque ad transmigrationem Babilonis, generationibus aeque iuxta Mattheum XIIII, annis autem CCCCLXXXIIII porrecta, a qua regum tempora expetunt. Aetas enim iuvenilis regno est habilis. Quinta deinde usque ad adventum salvatoris in carne, et ipsa generationibus XIIII porrecta, annis autem DLXXXVI extenta, in qua, ut senectute gravi fessa, malis crebrioribus plebs Hebraea quassatur. Sexta, quae nunc agitur, nulla generationum vel temporum serie certa, sed ut aetas decrepita ipsa totius seculi morte finienda. Secula generationibus constant, et inde dicuntur secula, quod se sequantur. Abeuntibus enim aliis alia succedunt, quorum decursus per generationes et regna ita inscribitur. Anschließend werden die Weltalter noch mit den Schöpfungstagen parallelisiert.

48 Vgl. etwa Hofmeister, Puer (wie Anm. 14) 305.

49 Vgl. etwa Gottfried (oder Irimbert?) von Admont, Homiliae dominicales 19 (De quinque spiritualibus aetatibus animae) (PL 174). Paris 1854, 21-386, hier 125: In quinque Dominicis, quas ab illo tempore ex quo, qui aeternus erat, pro nobis temporalis fieri dignatus est, celebravimus, quinque aetates, infantiam, pueritiam, adolescentiam vel iuventutem, senectutem, decrepitam aetatem satis congrue possumus accipere; Vita Landelini abbatis Lobbiensis et Crispiniensis prol. (ed. W. LEVISON [MGH SS rer. Merov. 6]. Hannover - Leipzig 1913, 433-444, hier 438): Benignitas nostri salvatoris semper adest parata in salutem eorum, quos praedestinavit ad capescenda futurae praemia vitae, et sicut ipse in euangelio spiritualiter docuit, quosdam mane, quosdam hora tertia, quosdam sexta et nona, quosdam undecima ad excolendam vineam suam convocat, quia quibusdam in infantia, quibusdam in iuventute vel adolescentia, aliquibus in senectute spiritualis assumere sationis concedit laborem.

50 Hinkmar von Reims, Vita Remigii 5 (unten Anm. 139). 
Begriffe auch in der Bibel manchmal identisch sind.$^{51}$ Bonifatius spricht in seinem Mahnbrief an König Aethelbald von Mercien gar von iuventus adolescentiae! ${ }^{52}$ Andererseits wird beides aber auch strikt unterschieden, wie in einer Canonessammlung aus dem 11. Jahrhundert, die geradezu Gegensätze zwischen infans und puer, iuvenis und adolescens andeutet, die denen zwischen reich und arm, frei und unfrei usw. entsprechen (ohne dass sie näher konkretisiert würden)..$^{53}$ Klarer ist demgegenüber der Beginn: Man wird adolescens, indem man das Knabenalter der pueritia hinter sich lässt. ${ }^{54}$

\section{b. Altersangaben}

Welches Alter die adolescentia konkret umfasst, ist hingegen weniger eindeutig. Durchblickt man die expliziten Äußerungen, ${ }^{55}$ dann ist der adolescens mindestens

51 Heiric von Auxerre, Homiliae per circulum anni. I. Pars hiemalis, hom. 18 (wie Anm. 45) 153: ,Nemo adolescentiam tuam contemnat" (1. Tim 4,11f.), sciendum est quod in sacro eloquio nonnumquam iuuentus adolescentia uocatur, ut illud: ,Laetare iuuenis in adolescentia tua (Eccle 11,9).

52 Bonifatius, Epistolae, ep. 73 (ed. M. TANGL [MGH Epp. sel. 1]. Berlin 1916, 150): praeterea, fili carissime, cum tu christianus et veri Dei cultor esse cognominaris, si in iuventute adolescentiae tuae putridine luxoriae inquinatus et foetore adulterii involutus et voragine libidinis quasi puteo inferni demersus fueras, iam tempus est, ut memor Domini tui a diaboli laqueis resipiscas et a foetore luxoriae sordidatam animam laves.

53 Collectio canonum in V libris (libri I-III) I 106 (ed. M. FoRNASARI [CCCM 6]. Turnhout 1970, 79f.): Non omnibus ergo una libra eademque pensanda sententia est poenitentiae, licet in uno constringatur uitio, sed discretio una sit cuique eorum, hoc est inter diuitem et pauperem, inter liberum et seruum, inter infantem et puerum, inter iuuenem et adolescentem, inter aetatem et senectutem, inter hebetes et aegrotos, inter gnaros et ignaros, inter laicos et cleros, inter monachos et continentes, inter episcopos et presbyteros, inter diacones et subdiacones, inter lectores et cantores, inter ingratos et gratos, siue inter innuptos seu copulatos coniugio, inter indigentes siue peregrinos, inter uirginem et meretricem, masculos et feminas, cuiusque aetatis ac sexus, uoluntarie uel casu, si in publico uel si in abscondito, si necessitate an uoluntate, tale commisit facinus.

54 Vgl. Vita Leonardi confessoris Nobiliacensis 2 (ed. B. KRUSCH [MGH SS rer. Merov. 3]. Hannover 1896, 396 - 399, hier 396): Puer autem Leonardus postquam evasit incrementa puerilia, adolescens factus (vgl. unten Anm. 220); Vita Betharii episcopi Carnoteni 3 (ed. B. KRUSCH [MGH SS rer. Merov. 3]. Hannover 1896, 613-619, hier 614): Cum autem, annis puerilibus transactis, adolescentiae metas transiret (vgl. unten Anm. 185); Formulae Sangallensis (Collectio Sangallensis Salomonis III. tempore conscripta) 46 (ed. K. ZeUMER [MGH Formulae]. Hannover 1886, 395-433, hier 429): quod omnibus amabiles et honorandi infantiam et pueritiam transcendentes, adolescentiae principia gratiosissimi captare videmini. Hingegen schließt JAMES, Childhood (wie Anm. 14) 22f., aus Gregor von Tours, dass adolescentia hier kein Lebensalter ist, sondern man von der Kindheit über die Pubertät gleich ins Erwachsenenalter gleitet.

55 In der bisherigen Literatur, etwa bei Hofmeister, Puer (wie Anm. 13), hat man das Alter von adolescentes genannten Männern oft aus anderen Quellenangaben erschlossen. Da das genaue Alter dem Autor oft nicht bekannt sein dürfte, beschränke ich mich hier auf explizite Altersangaben. 
12 Jahre alt (wie Otto III. beim Tod seines Vaters) ${ }^{56}$ - und Otto war immer noch adolescens, als er (mit 21 Jahren) verstarb. ${ }^{57}$ Dazwischen findet man Angaben mit 16, ${ }^{58}$ $18,{ }^{59} 19^{60}$ oder $20^{61}$ Jahren. Über dieses Alter hinaus habe ich, entgegen Isidors Einteilung, keine expliziten Werte mehr gefunden. ${ }^{62}$ Interessant ist vielleicht eine

56 So Rodulf Glaber, Historiae I 4,11, (ed. M. ARNoux, Turnhout 1996, 60; ed. J. FRANCE [Oxford Medieval Texts]. Oxford 1989, 24): Sequenti quoque post multa nobiliter gesta remque publicam decenter dispositam tempore, obiit Otto, relinquens filium Ottonem videlicet tertium, adolescentem tamen fere duodecim annorum, qui, ut erat iuvenculus, acer tamen viribus et ingenio suscepit iure paterno regimen imperii.

57 Vgl. Adalbert, Vita Heinrici II regis et imperatoris, Prolog II (ed. M. STUMPF [MGH SS rer. Germ. 69]. Hannover 1999, 333): Otto enim imperator, dum Rome obisset sine herede adhuc adolescens, eductus est et Aquisgrani sepultus; Alpert von Metz, De diversitate temporum II 4 (ed. H. van RIJ. Amsterdam 1980, 14): Post hęc vero imperator tercius Otto, bone indolis adolescens, in Italia moritur [...].

58 Vgl. Widukind von Corvey, Res gestae Saxonicae III 1 (ed. H.-E. LoHMANN - P. HIRSCH [MGH SS rer. Germ. 60]. Hannover $\left.{ }^{5} 1935,104\right)$, zu Liudolf: Ipse autem tener adhuc erat adolescens, aetatis non habens amplius quam sedecim annos.

59 Vgl. Sigebert von Gembloux, Chronica a. 465 (ed. L. Bethmann [MGH SS 6]. Hannover 1844, 300 374, hier 311): Theodericus adolescens annorum xuiii transito Danubio super Sarmatas irruit et regem eorum Babaz perimit et cum bellicis manubiis victor ad patrem redit.

60 Vgl. Hugeburg von Heidenheim, Vita Wynnebaldi abbatis Heidenheimensis 1 (ed. O. HolDER-EGGER [MGH SS 15/1]. Hannover 1887, 106-117, hier 107): qualiter, infantialis seu pueritialis transmeatis annorum curriculis, ad adolescentiae nondecem annorum aetate citatus celeps usque pervenit [...].

61 Vgl. Gesta Treverorum Cont. I, c. 19 (ed. G. WAITz [MGH SS 8]. Hannover 1848, 111-260 [Continuatio prima 175-200], hier 193), zu Heinrich V.: Heinricus videlicet nominis huius quintus rex, adhuc adolescens circiter annos 20, ei committitur, ut et regnum sua prudentia disponeret et heredem regni morum suorum honestate et disciplina, qua ipse prae omnibus pollebat, informaret, quousque in virum perfectum aetate et sapientia educatus succrevisset; Petrus Damiani, Epistolae, ep. 67 (ed. K. REINDEL [MGH Briefe der deutschen Kaiserzeit 4], I-IV, München 1983-1993, II, 1988, 288), an Herzog Gottfried von Tuszien, zu Otto III.: Hic itaque cum vicennalis adolescentiae vix adhuc excessisset etatem, inter insignia multa et praeclara quae gessit, tribus regni proceribus atque comitibus oculos eruit, Rodulfo videlicet, Raimundo et Arimundo.

$62 \mathrm{Zu}$ den (wenigen) Altersangaben in den Lebensaltersystemen vgl. Cochelin, Introduction (wie Anm. 8) 18-44. Die adolescentia reicht danach in zehn Fällen, bei Isidor von Sevilla und (danach) Hrabanus Maurus sowie im Benediktkommentar Hildemars des 9., in einer Fuldaer Handschrift aus dem 9. und einer Pariser Handschrift aus dem 10. Jahrhundert, einem apokryphen Lukasbrief und dem Juristen Papias Grammaticus aus dem 11. und bei Lambert von Saint-Omer, in einer Handschrift der Schule von Laon sowie bei Romuald von Salerno aus dem 12. Jahrhundert von 14 bis 27 Jahren und lediglich bei Honorius Augustodunensis und in einer Handschrift aus Chartres von 14 bis 20, im ,Tractatus quidam de Philosophia et partibus eius' aus dem 12. Jahrhundert von 15 bis 20 und in einer Ptolemaiosübersetzung von 1138 von 14 bis 21 Jahren. Nach Cushing, Pueri (wie Anm. 18) 439, gibt es keinen Konsens über das Ende der Adoleszenz, das bei 21, 25, 28, 30 oder sogar 35 Jahren angesetzt werde. Vgl. auch StoERTz, Adolescence (Anm. 18) 122: Die Adoleszenz beginnt mit ca. 14 Jahren und dauert bis in „late teens, twenties, or even the early thirties“. Gegen die herrschende Ansicht findet dagegen Cochelin, Adolescence (wie Anm. 18) 167-170, die Angaben in cluniazensischen Klosterregeln recht konsistent. 
Rechtsverfügung der Langobarden aus den 770er Jahren, das Jugendlichen unter 18 Jahren nicht erlaubt, (vor Gericht) für sich selbst zu sprechen. ${ }^{63}$

Interessanter noch ist die Tatsache, dass man innerhalb dieser Zeitspanne differenziert: So spricht die Vita Rimberti von der prima aetas adolescentiae, ${ }^{64}$ Bruno in seinem Buch vom Sachsenkrieg umgekehrt vom nahenden Ende der adolescentia Heinrichs IV., als er Adalbert von Bremen zu seinem Ratgeber erhob. ${ }^{65}$ Man kann (unter der Erziehung) „zum Adolescens heranwachsen“ (adolescere) ${ }^{66}$ oder sogar im Komparativ adolescentior sein als andere. ${ }^{67}$ Damit ist in der Regel allerdings nur gemeint, dass man jünger als andere, aber nicht unbedingt, dass man noch adolescens war. So war Bischof Gunther von Bamberg adolescentior als die anderen Bischöfe, die nach dem Bericht der Altaicher Annalen an der Jerusalemwallfahrt von 1064 teilnahmen, aber ihnen gleich an Weisheit und Tugend, ${ }^{68}$ doch gewiss kein ,Jüngling“ mehr.

63 Leges Langobardorum: Aregis principis capitula 7 (nach 774) (ed. F. BLUHME [MGH Fontes iuris Germanici antiqui 2]. Hannover 1869, 171): Ut adolescentem ante 10 et octo annos; ebd. 173: Et hoc constituimus, adolescentem ante 10 et octo anno etatis non posse solius ori sui manifestationem condempnari.

64 Vgl. Vita Rimberti 7 (ed. G. WAITZ [MGH SS rer. Germ. 55]. Hannover 1884, 81-100, hier 85): Accedit etiam ad praesagium virtutum beati viri visio quaedam de ipso revelata procuratori antistitis prima mox nocte adventus eius Hammaburg, cum ipse adhuc in prima aetate adolescentiae fuisset.

65 Brunos Buch vom Sachsenkrieg 16 (ed. H.-E. LohMANN [MGH Dt. MA 1]. Leipzig 1937, 22): Inter haec omnia iam abeunte adolescentia, mox ut Adalbertum Bremensem episcopum nactus est consiliarium, ipsius suasionibus coepit in desertis locis altos et natura munitos montes quaerere et in his huiusmodi castella fabricare, quae, si in locis competentibus starent, ingens regno firmamentum simul et ornamentum forent.

66 Vita Meinwerci episcopi Patherbrunnensis 160 (ed. F. TencKHOFF [MGH SS rer. Germ. 59]. Hannover 1921, 84): Adolescebant quoque secum in tirones milicię celestis Anno archiepiscopus Coloniensis, Frithericus Monasteriensis et perplures alii strennui postmodum in vinea Domini operarii. Zum Beginn des Zitats vgl. Anm. 142.

67 Vgl. Regino von Prüm, Chronicon a. 890 (ed. F. KURZE [MGH SS rer. Germ. 50]. Hannover 1890, 135), zu den beiden rivalisierenden Bretonenfürsten: Vidicheil war zwar jünger als Alanus, aber erfolgreicher in der Schlacht: Itaque Vidicheil, qui erat adolescentior, cupiens gloriam nominis sui exaltare, non expectato Alano cum sociis prelium conserit, multa milia hostium caedit, reliquos in quendam vicum fugere compulit.

68 Annales Altahenses maiores a. 1065 (ed. E. L. B. von OefELE [MGH SS rer. Germ. 4]. Hannover 1891, 66): Inter eos igitur, qui pergebant, principes isti erant: Sigifridus archiepiscopus Moguntiacensis, Wilelmus episcopus Traiectensis, Otto episcopus Ratisbonensis, Guntherius praesul Babenpergensis. Hic ergo, quamvis caeteris adolescentior esset aetate, reliquis tamen inferior non erat sapientia animique virtute, et quod iam post mortem eius sine dolore et gemitu non possumus recordari, tunc temporis decus et columna videbatur tocius regni. 


\section{Charakterisierung des Alters: Die Eigenschaften der Adoleszenz}

Wichtiger als das reale Alter sind den Autoren dessen Eigenschaften. Die Adoleszenz ist gewissermaßen ein Übergangsalter. ${ }^{69}$ Der Zisterzienser Aelred von Rievaulx hat das treffend zum Ausdruck gebracht: Der Adoleszent ist noch kein Mann, aber die Adoleszenz ist das Alter, in dem der Jüngling (durch das sanfte Joch des Glaubens) zum Mann heranwächst. ${ }^{70}$ Der Adoleszent ist demnach noch nicht vollkommen, er hat, wie Alkuin es ausdrückt, noch nicht die perfectior aetas erreicht, in der die Seele erstarkt. ${ }^{71}$ Adoleszenz ist folglich eine minor aetas (so Anselm von Canterbury), ${ }^{72}$ in der der Jüngling noch nicht genügend stark ist - auch Stärke ist Charakteristikum der iuventus - und noch nicht die volle Kraft besitzt. ${ }^{73}$ Sein Kennzeichen ist (wie schon in der pueritia) folglich (weiterhin) die Schwäche (imbecillitas), wie Sigebert von Gembloux betont. ${ }^{74}$ Entsprechend ist der Adoleszent nur an Jahren reif, aber ,aus Leich-

69 Den Übergang von der Kindheit zur Adoleszenz nahmen nach CocHELIN, Adolescence (wie Anm. 18) $160 \mathrm{f}$., auch die cluniazensischen Klosterregeln so wichtig, dass er fast überall erwähnt wird.

70 Aelred von Rievaulx, Sermo 43,20 (wie Anm. 38) 341: Si uero perseuerauerit homo sub isto iugo, crescet utique ab adolescentia in uirum perfectum, et ita suscipiet iugum illud suaue et onus leue, iugum caritatis, de quo Dominus dicit: ,Iugum meum suaue est et onus meum leue', mit Bezug auf Mt 11,29f. 71 Alkuin, Grammatica (PL 101). Paris 1863, 849-902, hier 854 A: Per has vero, filii charissimi, semitas vestra quotidie currat adolescentia, donec perfectior aetas et animus sensu robustior ad culmina sanctarum Scripturarum perveniat. Quatenus hinc inde armati verae fidei defensores et veritatis assertores omnimodis invincibiles efficiamini. Vgl. auch Gesta Treverorum Cont. I 19 (oben Anm. 61).

72 Anselm von Canterbury, ep. 117 (ed. F. S. Sснмiтт, S. Anselmi Cantuariensis archiepiscopi Opera omnia. III [epp. 1-147]. Edinburgh 1946 [ND Stuttgart - Bad Cannstatt 1968], 97-294, hier 254), an einen adolescens Wilhelm: Pudeat te deum minus thesaurario Belvacensi diligere, qui tuae aut minoris aetatis adolescens delicatus et pulcherrimus, valde dives et nobilissimus et immoderatus amator saeculi, nuper postquam ivi in Angliam, nescio qua causa veniens Beccum statim recessurus, subita sancti spiritus gratia mutatus, se monachum ibi permansurum, tonsura statim et habitu nostro sumpto, spopondit, se nunc laetiorem quam umquam in vita sua fuisset affirmans.

73 Vgl. Stephanus, Miracula s. Modoaldi III 1 (ed. G. H. PeRTz [MGH SS 8]. Hannover 1848, 224-246, hier 224), zur Barbarei der Normannen (iuvenes und adolescentes verteidigen sich nach ihren Kräften): Quae immanitate sui sceleris grassata, in cervices fidelium velut ursa raptis catulis efferata, nulli sexui, nulli aetati parcere, senes reverendos necare, iuvenes et adolescentes pro viribus vel tempore se defendentes confodere, parvulos matrum uberibus avulsos excerebrare, matronas castas, virgines pudicas dehonestare, urbes nobilissimas flammis edacibus concremare, sanctuaria Dei aut subvertere aut profanare, divina et humana pari contemptu pessumdare. Vgl. auch Vita Gangulfi martyris Varennensis 2 (ed. W. Levison [MGH SS rer. Merov. 7]. Hannover 1920, 155-174, hier 158): Dehinc, decursis adolescentiae metis, cum robur virilis evasisset aetatis, genere consimilem sortitur uxorem.

74 Sigebert von Gembloux (?), Vita altera Sigeberti (III.) regis Francorum 4,10 (PL 87). Paris 1851, $303-$ 314, hier 309 BC: Sequens annus luctum maximum intulit regi Sigeberto eiusque regno, Pipino maiore domus ex hac luce subtracto, qui ipsum Sigebertum paterne nutrierat a puero et imbecillitatem pueritie et adolescentiae eius valido sui auxilio sustentaverat brachio. 
tigkeit der Sitten unreif“ (inmaturus) - so Paschasius Radbertus,$-{ }^{75}$ noch nicht erwachsen, sondern unreif (impubes) - so Bernhard von Clairvaux. ${ }^{76}$ Das hat Folgen für die Einschätzung: Die Adoleszenz ist weder mehr die tenera aetas, das zarte Alter, noch das gefestigte (dura aetas), sondern das Alter, in dem noch alles offen und ungeformt ist wie Wachs, wie es Wilhelm von Conches, mit Berufung auf Plato, beschreibt, so dass es gerade den Anfängen der Lehre (oder Bildung) angemessen ist. ${ }^{77}$

Der Erzdiakon Arnulf von Sées, der spätere Bischof von Lisieux, schreibt in seiner Streitschrift jedem Alter seine spezifische (schlechte) Eigenschaft zu: der pueritia die Ungefälligkeit (illiberalitas), der iuventus den üblen Ruf (infamia), der virilis etas die Unanständigkeit (indecorus), der Adoleszenz aber den fluxus, das Zerfließen (und damit auch eine Ausgelassenheit).$^{78}$ Die Adoleszenz ist daher labilis aetas, ein gleitendes (oder auch: schlüpfriges) Alter, so die Aachener Synode von 862, ${ }^{79}$ und ein Alter der Veränderlichkeit (mobilitas), in dem der Mensch leicht verführt werden kann, ${ }^{80}$ wie die lothringische Synode von 865/867 zur Entschuldigung Lothars II.

75 Vgl. Paschasius Radbertus, Expositio in Matheo IX (ed. B. PAULus, [CCCM 56/56 A/56B]. Turnhout 1984, hier CCCM 56 B, 952): Iudaicus ergo populus erat adolescens non etate sed leuitate moribus inmaturus sed non annis. Sed et omnia carnalia uitia seu iunenilia gessit de quibus dicit apostolus: „Iunenilia desideria fuge“ (2. Tim 2,22). Superbissimum autem iuuenile uitium est gloriatio uana, qua plus uult uideri quam esse sicut populum Iudeorum fuisse cognoscimus. Unreife erwächst hier allerdings nicht aus dem Alter, sondern eben aus der Leichtigkeit der Sitten (leuitate moribus inmaturus).

76 Bernhard von Clairvaux, ep. 43 (Epistola de moribus et officio episcoporum) 25 (ed. J. LECLERCQ H. TALBOT - H. RochaIs, Bernardi Opera VII. Rom 1974, 121): Scholares pueri et impubes adolescentuli $o b$ sanguinis dignitatem promoventur ad ecclesiasticas dignitates, et de sub ferula transferuntur ad principandum presbyteris. Das kann hier auch einschränkend gemeint sein und sich nicht auf alle, sondern eben nur auf die noch unreifen Schüler beziehen. Die triefenden Augen eines adolescens bei Gregor von Tours, Historiae 5,48 (ed. B. KRUSCH - W. LEvison [MGH SS rer. Merov. 1,1]. Hannover 1951, 257) (Sed quia lippis erat in adolescentia oculis), wird man hingegen individuell und nicht als Kennzeichen des Lebensalters auffassen müssen.

77 Wilhelm von Conches, Dragmaticon Philosophiae VI 27,4 (ed. I. RonCA - A. BAdiA, [CCCM 152]. Turnhout 1997, 272): Principio uero doctrinae adolescentia est conueniens, quia, ut ait Plato, aetas hominis similis est cerae, quae, si nimis mollis sit, nec figuram recipit nec retinet; similiter si sit dura. Ergo nec nimis tenera aetas nec nimis dura conueniens est doctrinae. Terminus uero doctrinae est mors. Zur tenera aetas der Adoleszenz vgl. hingegen Gunther von Pairis, Ligurinus 6 (ed. E. Assmann [MGH SS rer. Germ. 63]. Hannover 1987, 351, v. 404-407) zu Geza und Stephan von Ungarn: Verba precesque ferens Gaize sub nomine regis. / Huic satis in teneris adolescens strenuus annis / Frater erat Stephanus, quem rex ad culmina regni / Aspirare dolo Belo suasore ferebat.

78 Arnulf Sagiensis, Invectiva 4 (ed. C. ZEY [MGH SS rer. Germ. 67]. Turnhout 1994, 96): At si vitae consideretur integritas et a primis annis sub examen omnia redigantur, nihil deformatum crimine, nihil infamia condempnatum; in eo enim nil illiberale pueritia, nil adolescentia fluxum, nil iuventus infame, nil virilis etas edidit indecorum. Alle diese Übel hatte Papst Innozenz II. danach nicht.

79 Synode von Aachen 862, Nr. 9. B. Libellus proclamationis Lothars II. (ed. W. HARTMANN [MGH Conc. 4]. Hannover 1998, 75): Et cum multipliciter ac generaliter nostram adolescentiam et labilem ętatem procurastis, specialiter quoque et diligenter circa fraudem nobis in nuncupata uxore illatam vigilastis. 80 Lothringische Synode von 865/67, ebd. Nr. 22, ebd. 200: Praesertim cum ipse rex, adolescentiae mobilitate et astutia hominum aliquando seductus, nunc autem ad meliora conversus, se totum domino praestante episcopalibus decretis ac salubribus monitis bonorumque consiliis aptare festinet. 
feststellt, der sich von seiner Gemahlin Theutberga zugunsten seiner Jugendgeliebten Waldrada trennen wollte, oder wie auch die Vita Heinrichs IV. betont: ut semper seductilis est adolescentia. ${ }^{81}$ (Dem Antiheinricianer Bernold von St. Blasien zufolge wurde Heinrich verführt, ${ }^{82}$ während er sich sogar nach dem königlichen Verteidiger Benzo von Alba immerhin durch Kriege und Unrecht der Böswilligen verwirren ließ). ${ }^{83}$ So wäre auch Ottos I. aufständischer Sohn Liudolf nach Folcwin von Lobbes ein ansehnlicher Jüngling gewesen, wenn er sich nicht hätte verführen lassen. ${ }^{84}$

Entsprechend unsicher sind die Sitten, wie Richer von Saint-Remi betont. ${ }^{85}$ Deshalb warnt Bernhard von Clairvaux davor, einem adolescens kirchliche Würden zu übertragen, ${ }^{86}$ und Benedikt von Aniane zitiert die Mönchsregel Isidors, nach der wegen mangelnder Würde kein adolescens zum Abt gewählt werden soll, weil ihm die Älteren sonst nicht gehorchen würden. ${ }^{87}$ Trotz solcher Äußerungen darf das Alter dennoch nicht einseitig als ausschließlich negativ betrachtet gelten, ${ }^{88}$ da den ado-

81 Vita Heinrici IV imperatoris 9 (ed. W. EBERHARD [MGH SS rer. Germ. 58]. Hannover - Leipzig 1899, 30), zum Aufstand des Königssohnes: Quid multa? Statim illectus et abstractus á concupiscentia, malignae suggestioni, ut semper seductilis est adolescentia, nec voto defuit nec facto.

82 Berthold von St. Blasien, Chronicon a. 1068 (ed. I. S. RoBinson, Die Chroniken Bertholds von Reichenau und Bernolds von Konstanz 1054-1100 [MGH SS rer. Germ. n.s. 14]. Hannover 2003, 163381, hier 206), zu Heinrich IV.: Huc rex adolescentie sue errore seductus, legitime coniugis adeo obliviscitur et tam nefandis criminibus involutus esse diffamatur, ut etiam principes eius eum regno privare molirentur.

83 Benzo von Alba, Ad Heinricum IV imperatorem libri VII (Sieben Bücher an Kaiser Heinrich IV.) V praef. (ed. H. SEYFFERT [MGH SS rer. Germ. 65]. Hannover 1996, 438): Sine intermissione quidem conturbatus est in sua adolescentia sive bellorum furiis seu malignantium iniuriis.

84 Folcwin, Gesta abbatum Lobiensium 25 (ed. G. WAITZ [MGH SS 4]. Hannover 1841, 65f.): Erat in ea coniuratione princeps ipsius regis filius Liudulphus, spectabilis adolescens, et unice clarus, si seductoribus minime credidisset, et heres esse, non proditor, maluisset [...].

85 So Richer von Saint-Remi, Historiae IV 27 (ed. H. HoffmanN [MGH SS 38]. Hannover 2000, 249f.) zu Erzbischof Arnulf von Reims: Sed quia eius utpote adolescentis mores affectusque incertos habemus, nostras solummodo rationes non sufficere ad hęc arbitramur.

86 Bernhard von Clairvaux, ep. 43 (oben Anm. 76).

87 Benedikt von Aniane, Concordia regularum 4,5,1-3 (ex regula s. Isidori 3) (ed. P. BonNERUE [CCCM 168 A]. Turnhout 1999, 61): Abbas interea eligendus est institutione sanctae uitae duratus atque inspectus, patientiae et humilitatis expertus, quique etiam et per exercitium uitam laboriosam tolerauit, ac transcendens aetatem adolescentiae iuuentutem seu senectutem tetigerit, cui et maiores non dedignentur parere oboedientes ei, tam pro aetate quam etiam pro morum probitate. Eine seltsam widersprüchliche Entwicklung nimmt CocHELIN, Adolescence (wie Anm. 18) 170 - 174, aus dem Befund cluniazensischer Consuetudines an: Danach begegnet Adoleszenz in unserem Sinn erst im 12. Jahrhundert und wird jetzt eher mit pueritia als mit iuventus verbunden. Bis zum frühen 12. Jahrhundert wurde von cluniazensischen Jungen und jungen Männern erwartet, dass sie gehorsame Söhne waren. Danach wurden sie nach außen hin ermutigt, gegen die weltlichen Gewalten zu revoltieren, im Innern aber wegen mangelnden Gehorsams kritisiert. Den Grund dafür sucht Cochelin in dem Umstand, dass immer weniger Kinder und immer mehr Jugendliche und Erwachsene dem Kloster übergeben wurden oder in ein Kloster eintraten.

88 Nach StoerTz, Adolescence (wie Anm. 18) 127, gibt es in den Klosterregeln insgesamt nur wenig negative Stereotypen. 
lescentes immer wieder auch bereits Reife ${ }^{89}$ und Tugenden zugeschrieben werden: Es ist das Alter, in dem der calor aetatis, die Hitze des Alters, wächst, wie Heiric von Auxerre es ausdrückt, ${ }^{90}$ oder, mit Sedulius Scottus (auf die Weltalter bezogen), die florida aetas, das blühende Alter. ${ }^{91}$

Bezüglich spezifischer Altersmerkmale lässt sich viererlei feststellen:

Erstens besitzt der adolescens bereits Stärke, es ist die fortior aetas. ${ }^{92}$ Das betrifft nach Hinkmar von Reims auch die Geistes- bzw. Willensstärke (robor mentis), die aber oft noch fehlt. ${ }^{93}$

Zweitens beginnt in diesem Alter der Bartwuchs. „Männlich (pubes) ist der erste Bart des Jugendlichen und männlich ist der adolescens, “ schreibt Sedulius Scotus. ${ }^{94}$ „In der Adoleszenz beginnt der Bart zu sprießen, die Natur der Männer (iuvenes) und Greise aber führt dazu, dass sie nicht ohne Bart sein können.“ bestätigt der Zisterzienserabt Burchard von Bellevaux, der um 1160 eine ausführliche Abhandlung über Bärte verfasst hat. „Wie es Kennzeichen von Kindern und Jungen ist, dass sie in diesem Alter

89 Vgl. Bernhard von Clairvaux, ep. 449 (wie Anm. 76) 426: Quaenam haec in rege dignatio, in adolescente maturitas!

90 Heiric von Auxerre, Homiliae per circulum anni. Pars hiemalis, hom. 18 (oben Anm. 45). Vgl. Miraculum s. Donatiani (ed. O. HoLDER-EGGER [MGH SS 14]. Hannover 1883, 178): Nam decursa, ut dictum est, minori aetate, iam grandiusculus eminebat, iam calentis adolescentiae terminos attigerat, cum acutissima quam Greci paralysin vocant passione dissolutus decubuit ac, membrorum destituta compage, a planta pedis usque ad verticem dimidius emarcuit.

91 Sedulius Scotus, In argumentum secundum Matthaeum expositiuncula 1-3 (PL 103). Paris 1851, 273-280, hier 276 A: Tertia aetas, quae est quasi florida mundi adolescentia, ab Abraham usque ad Dauid regem extenditur, quae quidem aetas habilis ad generandum habetur. Vgl. auch Chronicon Salernitanum 94 (ed. G. H. PERTz [MGH SS 3]. Hannover 1839, 469 - 561, hier 515) zu Ademar: eo quod esset adolescens florida aetate gerentes.

92 So das Konzil von Tribur 895 (ed. A. Boretius - V. Krause [MGH Capit. 2,2], Hannover 1897, Nr. 252, c. 24b, 227), zur Nonnenwerdung von Mädchen vor dem 12. Lebensjahr: At si annum et diem id dissimulando consenserint, ulterius nec illi nec ipsa mutare hoc poterunt. Porro si in fortiori etate adolescentula vel adolescens servire Deo elegerint, non est potestas parentibus hoc prohibendi.

93 Hinkmar von Reims, Opusculum LV capitulorum c. 43 (ed. R. SchIEFFER, Die Streitschriften Hinkmars von Reims und Hinkmars von Laon [MGH Conc. 4]. Suppl. 2. Hannover 2003, 315): Quod mente percipiebam et mihi admodum displicebat, sed, cum viderem te per iactantiam amore laudis flagrare et me non posse sentirem in ętatis adolescentia te ad tantum robur mentis accendere, ut ad discendi ac versificandi studium te provocarem et iactantiae a te pravitatem eraderem, ne acriori correptione fractus revocareris ab studio, permisi te interim, tantum ut studio litterali incumberes, de scientia iactitare, donec in maturiori aetate ab illo etiam vitio possem compescere, secutus pro modulo meo apostolum, qui, cum infirmum auditorem suum perpenderet aut prava adhuc velle agere aut de actione recta humanę laudis retributione gaudere [...].

94 Sedulius Scotus, In Donati artem maiorem, pars 2 (wie Anm. 36) 304: pubes est prima barba iuuenis et pubes est adolescens, quin etiam et plurium 〈numerus〉 eiusdem aetatis. 
noch keinen Bart haben, so (ändert sich das) in den folgenden Lebensaltern. “95 Aber nicht alle haben schon einen Bart. Burchard erzählt ein Gleichnis von zwei Jünglingen, von denen einem bereits ein Bart wuchs, dem anderen nicht - beides ist in diesem Alter also möglich -, und er deutet das allegorisch als Zeichen der Abwehrkräfte: Der bärtige Jüngling vermochte sich bereits selbst gegen die Dämonen zu wehren, der bartlose bedurfte dazu eines schützenden Engels. ${ }^{96}$

Drittens beginnt jetzt, wie schon in den Lebensalterlehren, die Zeugungsfähigkeit, die „Pubertät“. Anfangs noch impubis, ${ }^{97}$ also unbehaart, unerwachsen, unmannbar, ist die „Pubertät“ (im eigentlichen Sinn) das Alter, in dem die Zeugungsfähigkeit einsetzt. „Von diesem Alter an ist der Mensch zeugungsfähig,“ schreibt Hrabanus Maurus.9

95 Burchard von Bellevaux, Apologia de barbis. Sermo 3,2 (ed. R. B. C. HuYGEns [CCCM 62]. Turnhout 1985, 175): Secunda consideratio de barbae natura. Secunda consideratio est quod in eodem sexu, virili scilicet, quibusdam aetatibus ex natura proprium sit barbas omnino non habere, aliis, nisi ex ingenita vitiositate vel ex accidenti occasione contigerit, penitus barbis non carere. Sicut enim proprium est infantibus et pueris in his aetatibus sine barbis esse, ita reliquis aetatibus, in adolescentia barbis incipientibus pullulare, iuvenum videlicet et senum natura confert sine barbis esse non debere, nisi sicut iam dictum est aut vitiositas ingenita contradicat aut occasio ex tempore accidens auferat.

96 Ders., Sermo 3, 25, ebd. $201 \mathrm{f} .:$ De exemplo duorum adolescentium, unius barbam mittentis, alterius nundum mittentis barbam. Quibus adhuc in incremento sunt barbae, hoc est qui barbas incipiunt mittere vel qui nundum inter vos mittunt, sumant in exemplum duos illos adolescentes, horum altero barbam mittente, altero adhuc imberbi permanente, de quibus abbas Vindemius abbatem Macharium narrat fuisse locutum et narrasse, dicentem: Sedente me aliquando in Scithi, venerunt ad me duo adolescentes peregrini: unus quidem ipsorum incipiebat mittere barbam, alius vero adhuc non. Horum qualis postea fuit vita et conversatio ipse Macharius narrat, et qui vobis recitaverit, fratres, ista ipse narret, ut de perfectione illorum vestra proficiat imperfectio. Inde tamen modicum aliquid audite. Narrat Macharius quod super illum iuniorem, qui erat imberbis, viderit demones in similitudine muscarum venientes: Vidi, inquit, demones sicut muscas venientes super illum minorem, et alii quidem veniebant ut sederent super os eius, alii vero super oculos. Et vidi angelum Domini habentem gladium igneum et defendentem illum atque abigentem ab eo demones. Illi autem maiori non poterant appropinquare. Item iunior ille imberbis cum psalleret, per singulos versus exiebat lampada ignis de ore ipsius et ascendebat in caelum. Similiter autem et ille maior, qui mittebat barbam, cum aperiret os suum psallens, tanquam funiculus ignis egrediebatur de ore ipsius et pertingebat in caelum. Et cognovi, inquit Macharius, quia ille maior perfectus esset, illi autem minori adhuc repugnabat inimicus. Et conclusit: Venite, inquit, et videte martirium horum duorum peregrinorum. Ecce, fratres, audistis miraculum stuporis et admirationis, exultationis pariter et terroris, quod ad misterium imberbii et barbaturae pertinere videtur.

97 Vgl. Johannes von Salisbury, Policraticus VI 18 (ed. C. C. I. WebB. Oxford 1909, II 51f.), zu Theodosius dem Jüngeren: Nec mora; in primis adolescentiae annis impubes adhuc militiam aggressus est uultuque ferociori et fortiori manu diuini auxilii hostium corda concussit et fregit; Lampert von Hersfeld, Annales a. 1071 (ed. O. HolDER-EGGER [MGH SS rer. Germ. 38]. Hannover 1894, 124: Filius erat Balduwino, impubis adhuc adolescens et necdum armis militaribus maturus.

98 Hrabanus Maurus, De computo I 96 (wie Anm. 43) 320: Haec uelut quaedam adolescentia fuit populo Dei, a qua aetate quia incipit homo posse generare. Vgl. oben Anm. 43. Vgl. auch ähnlich Annales Quedlinburgenses (oben Anm. 47). Habilis ad generandum: Sedulius Scotus, In argumentum euangelii secundum Matthaeum (oben Anm. 91). 
Der Adoleszent ist daher entweder pubescens, ${ }^{99}$ „,heranreifend“, oder schon pubes, ${ }^{100}$ „reif“. Auch das kann negativ bewertet werden als Beginn wachsender Lust, wie in einem Ruthkommentar des 12. oder 13. Jahrhunderts, wonach der Adoleszent „zur Wollust der Begierde“ aufglüht. ${ }^{101}$ Es bedeutet aber auch, dass der adolescens schon verlobt sein (wie Giselbert von Lothringen), ${ }^{102}$ ja, bereits eine Konkubine haben kann (wie Lothar II. mit Waldrada). ${ }^{103}$ Mehr noch: Die adolescentia ist die Zeit der Heirat ${ }^{104}$ und der Kopulation. So kann Andreas von St. Viktor in seinem Salomonkommentar schreiben:

Dein sprudelnder Samen möge sich in der Zeugung von Nachkommen vervielfältigen. Die Frau deiner Adoleszenz, der du in deiner Adoleszenz beigewohnt hast, [...] sie sei dir [mit Prov 5,19] dein allerliebstes Reh und dein allerliebtes Maultier, das heißt so lieb wie ein Reh und Maultier ihren Ernährern ist. ${ }^{105}$

Mehrfach ist eine Heirat von adolescentes bezeugt (wie des byzantinischen Königssohnes Alexius mit der Schwester des Königs Philipp von Frankreich, ${ }^{106}$ der Tochter

99 Vgl. Petrus Damiani, ep. 117 (wie Anm. 61) III, 1989, 319): David vixdum primo florentis adolescenciae decore pubescens; ep. 110, ebd. 241: Scottigenarum rex primevo tunc adolescentiae flore pubescens, quia pater obiit, ei mox in regalis fastigii iura successit. Vgl. auch ep. 153, ebd. IV, 1993, 54: Vos autem, ephebi adolescentes, vos etiam pubescentes iuvenes, tanto propensioribus exhortationum auxiliis indigetis, quanto duriora carnalis incendii certamina toleratis.

100 Vgl. Sedulius Scotus, In Donati artem maiorem, pars 2 (oben Anm. 94).

101 Commentarium in Ruth (e codice Genouefensi 45) 2(b), 5 (ed. G. DE MANTEL [CCCM 81]. Turnhout 1990, 147 f.): Adolescens autem est, qui ad annos perueniens pubertatis in libidinum uoluptates incalescit et excrescit. Zum Beginn des Zitats vgl. unten Anm. 124.

102 Vgl. Widukind von Corvey, Res gestae Saxonicae I 30 (wie Anm. 58) 43 (zu Giselbert): Deinde videns adolescentem valde industrium, genere ac potestate, divitiis quoque clarum, liberaliter eum coepit habere, ac postremo desponsata sibi filia nomine Gerberga.

103 Vgl. Regino von Prüm, Chronicon a. 864 (wie Anm. 67) 80, zu Waldrada: quae eius fuerat concubina, cum adhuc adolescens esset in domo paterna. Mit dem adolescens ist hier sicher Lothar gemeint, obwohl sich der Begriff sowohl auf Lothar als auch auf Waldrada beziehen kann. Vgl. auch Gesta Treverorum 26 (wie Anm. 61) 164, zu Lothar II.: Hanc enim falsam infamiam Lotharius cum his episcopis composuerat, cupiens hoc modo reginam abicere et Waldradam, quam ipse adolescens concubinam habuerat, in regnum assumere.

104 Vgl. DetTE, Kinder (wie Anm. 17) 17: Mit der Zeugungsfähigkeit erhielt der adolescens „die Möglichkeit, eine eigene Familie zu gründen“.

105 Andreas von St. Viktor, Expositiones historicae in libros Salomonis. Expositio historica in Parabolis (ed. R. BERNDT [CCCM 53B]. Turnhout 1991, 33): Scatebra seminis tui per prolis procreationem multiplicetur. ,Mulierem adolescentiae tuae.' Quam in adolescentia tua tibi copulasti. ,Cerua gratissima et gratissimus hinnulus. 'Illa mulier adolescentiae tuae sit tibi ,cerua gratissima et gratissimus hinnulus (Prov 5,19), id est ita grata sicut cerua et hinnulus suis sunt nutritoribus.

106 Historia de expeditione Friderici imperatoris (ed. A. Chroust [MGH SS rer. Germ. n.s. 5]. Berlin 1928, 31): Quod autem pręscripti comites partem regni Grecię eo tempore tam confidenter invaserant, hęc eis fuit materia. Manuele imperatore Constantinopolitano defuncto filioque suo Alexio adolescentulo sceptra regni per tutores suos tenente, cui etiam pater non multo antea regis Francię Philippi germanam desponsaverat, surrexit quidam de sanguine eius nomine Andronicus, impius et scelestus tyrannus [...]. 
Herzog Hugos des Großen von Franzien mit dem hochadligen adolescens Richard ${ }^{107}$ oder Heinrichs VI. mit Konstanze von Sizilien). ${ }^{108}$ Ja, man kann, wie Heinrich IV., in diesem Alter sogar schon wieder geschieden sein. ${ }^{109}$

Viertens schließlich entscheidet sich in diesem Alter der Charakter. Bruno will in seinem Buch vom Sachsenkrieg deshalb einiges aus pueritia vel adolescentia Heinrichs IV. erzählen, um ihn zu diffamieren, damit der Leser bereits aus seinen frühen Taten erkennt, weshalb er als Mann einen Bürgerkrieg begonnen hat. ${ }^{110}$ Bruno beklagt, dass Heinrich in seiner Adolescenz den engen Pfad zur Rechten gemieden und lieber den breiten Platz zur Linken eingeschlagen hat, nämlich allen Tugenden entsagt und sich mit allen seinen Kräften den Begierden hingegeben habe. ${ }^{111}$ Hier wie noch häufig wird dem adolescens ein Hang zum Bösen nachgesagt: „Jedes Alter neigt von der adolescentia an zum Bösen, denn nichts ist unzuverlässiger als das Leben der adolescentes, “ so warnt das Konzil von Aachen von 816. ${ }^{112}$ Dabei neigt allerdings nicht der Adoleszent an sich zum Bösen - Laster gibt es vielmehr in jedem Alter - ${ }^{113}$ sondern der Mensch von diesem Alter an (ab adolescentia). ${ }^{114}$

107 Dudo, Historia Normannorum III 93 (ed. J. LAIR - F. LE Blanc-HARdEL, De moribus et actis primorum Normanniae ducum [Mémoires de la Société des Antiquaires de Normandie 23], Caen 1865, 251): Dedit itaque Hugo dux Magnus Richardo nobilissimo adolescenti filiam suam firmamento sacramenti, non tamen statuta lege Fescenninae coemptionis, verum denominato iuratoque termino conexionis conubialis.

108 Alberich von Troisfontaines, Chronicon a. 1185 (ed. P. SCHEFFER-BoICHORST [MGH SS 23]. Hannover 1874, 631-950, hier 858): zu Heinrich VI. und Konstanze, der Tochter Rogers von Sizilien: adhuc in adolescentia duxit in uxorem.

109 Berthold von St. Blasien, Chronicon a. 1068 (oben Anm. 82).

110 Brunos Buch vom Sachsenkrieg prol. (wie Anm. 65) 13: Sed priusquam incipiam ipsius belli seriem explicare, oportet me pauca de pueritia vel adolescentia eiusdem Heinrici praemittere, ut, dum qualem vitam puer vel adolescens agebat, lector agnoverit, minus miretur, quod intestinum bellum vir factus inceperit.

111 Ebd. 14: Verum postquam ille, puerili simplicitate transcursa, adolescentiam, quae communis est omnium campus flagitiorum, ingressus ad bivium Samiae litterae pervenit, derelicta sublimi semitae dexterioris angustia, declivem plateae sinistrae latitudinem, qua incederet, elegit, calleque virtutum penitus abdicato, totis viribus post concupiscentias suas ire disposuit.

112 Konzil von Aachen 816, c. 139 (unten Anm. 143).

113 Vgl. Epp. Variorum nr. 27 (Erzbischof Vulfad von Bourges an Kleriker und Laien, 866/76) (ed. E. DÜMMLER [MGH Epp. 6]. Berlin 1925, 128-206, hier 189): Et ideo, dilectissimi filii, obsecro, ut recurrat unusquisque ad conscientiam suam et diligenter requirat, qualis sit vel quae in pueritia, in adolescentia, in iuventute, et in omni aetate sua gessit; et si quis se homicidam, adulterum, sacrilegum, fornicatorem, periurum aut falsum testem viderit, ad Deum et matrem ecclesiam confugium faciat, et confessionem puram de omnibus iniquitatibus suis sacerdotibus donet, et penitentiam suscipiat et verissime eam adimpleat; et quod inique gessit elemosinis, ieiuniis, orationibus, abstinentia et ceteris bonis operibus delere et redimere satagat, quoniam nisi hic, dum tempus et locum habet, emendare studuerit, regnum Dei testante apostolo habere non poterit.

114 Bernhard von Clairvaux, Sermones de diuersis. Sermo 41,4 (wie Anm. 41) VI,1, 1972, 247): Primus autem gradus est oboedire libenter. Omnis aetas ab adolescentia sua prona est in malum, et unusquisque 
Die Sinne und Gedanken des menschlichen Herzens neigten von seiner Adoleszenz an zum Bösen. Man muß nämlich bemerken, dass die schlimmen Wünsche jeden Menschen von seiner Natur her und gleichsam von Beginn an anfechten.

schreibt Remigius von Auxerre. ${ }^{115}$ Genauso gut kann die Entscheidung aber auch in die umgekehrte Richtung gehen. (Auf beides ist noch zurückzukommen.) Erst der adolescens vermag nämlich zwischen Gut und Böse zu unterscheiden (und sich entsprechend zu entscheiden), wie Rupert von Deutz betont (der hier die Auslegung des Hieronymus aufgreift, aber gerade die für uns wichtige Stelle hinzufügt): „Denn ich bin seit meiner Jugend wie Adam, da die menschliche Natur seither die Unterscheidung von Gut und Böse hat. “116

\section{Verhalten}

Der Charakter des Lebensalters wirkt wiederum auf das Verhalten zurück. So erwächst aus der Pubertät, positiv gesehen, Scham, ${ }^{117}$ negativ hingegen Wollust: Seit Gregor dem Großen heißt es immer wieder (mit Prediger 11,10): „Adoleszenz und Lust sind eitel.“118 Bonifatius wirft dem König Aethelbald von Mercia vor, in seiner Jugend (in

voluntatem pravi cordis sui sectatur; Helmold von Bosau, Chronicon II 107 (ed. B. SCHMEIDLER [MGH SS rer. Germ. 31]. Hannover 1937, 210) zum Widerstand Wedekinds von Dasenburg: Hic enim ab adolescentia sua ad malum strennuus semper militiae usum in rapinas detorserat, sed ne malum posset, quod voluit, ducis refrenatione acrius tenebatur.

115 Remigius von Auxerre, Expositio super Genesim VIII 21 (ed. B. VAn NAME Edwards [CCCM 136]. Turnhout 1999, 94): Sensus enim et cogitatio humani cordis in malum prona sunt ab adolescentia sua. Notandum quia praua desideria naturaliter et quasi ab ortu unumquemque hominem impugnant.

116 Vgl. Rupert von Deutz, Commentaria in duodecim prophetas minores. In Zachariam prophetam 5 (PL 168). Paris 1893, 699-814, hier 801 B: Dicat ergo: non sum propheta, homo agricola sive, quod eodem respectu dicere potest, homo faber ego sum, quoniam Adam exemplum meum, id est homo peccator ego sum sicut Adam ab adolescentia mea, id est ex quo discretionem boni et mali habet natura humana et itidem talis ego sum arbitratu vestro. Nur der Beginn des Zitats folgt wörtlich Hieronymus, Commentarii in prophetas minores. In Zachariam III 13 (ed. M. ADRIAEN [CC SL 76 A]. Turnhout 1970, 873).

117 Vgl. Ekkehard von Aura, Chronicon a. 1105 (ed. G. WAITZ [MGH SS 6]. Hannover 1844, 33-267, hier 227; ed. F.-J. SCHMALE - I. SchMALE-OTT [FSGA 15]. Darmstadt 1972, 125 - 204 [erste Fassung], hier 192): zu Heinrich V.: si qua vero irrationabilia rogabantur, mira ac ultra suos annos prudenti responsione et avita magnanimitate confutavit, in omnibus his et sibimet miro modo servans adolescentiae verecundiam, et Christi sacerdotibus dignam exhibens reverentiam; Bernhard von Clairvaux, Sermones super Cantica Canticorum. Sermo 53,1,1 (wie Anm. 76) II, 1958, 95): Videns sponsa novam adolescentularum verecundiam, et verecundum timorem, quod scilicet de novo coepissent non audere se ingerere sancto otio ipsius, nec, sicut heri et nudiustertius, molestae fieri quiescenti in contemplatione praesumerent, agnoscit hoc sibi provenisse cura et opera sponsi.

118 Eccle (Ecclesiastes) 11,10: Aufer iram a corde tuo et amove malitiam a carne tua, adolescentia enim et voluptas vana sunt. Vgl. Gregor der Große, Moralia in Iob IV praef., (ed. M. AdRIAEN [CC SL 143, 143 A, 143 B]. Turnhout 1979/85, hier CC SL 143, 1979, 158); Alkuin, ep. 65 (ed. E. DüMMLER [MGH Epp. 4]. Berlin 1895, 18-481, hier 108); ep. 66, ebd. 110; Andreas von St. Viktor, Expositiones historicae in libros Salomonis. Expositio historica in Ecclesiasten 11,10 (wie Anm. 105) 135; Rupert von Deutz, De sancta 
iuventute adolescentiae tuae) „im Schmutz des Ehebruchs und im Abgrund der Wollust verstrickt und so gewissermaßen im Schlund der Hölle versenkt“ gewesen zu sein, und fordert ihn zur Buße auf. ${ }^{119}$ Von der „Eitelkeit der Geringfügigkeiten“ (levium rerum vanitatibus) des adolescens spricht der Geschichtsschreiber Richer von SaintRemi im Hinblick auf den westfränkischen König Ludwig V. ${ }^{120}$ Das „schwache Alter“ (infirma aetas) sei mit Fleischeslust verbunden, vor der man es bewahren müsse, warnt der Klosterreformer Benedikt von Aniane (mit Isidor von Sevilla), ${ }^{121}$ und Alkuin ermahnt in einem Brief die pueri des Martinsklosters in Tours zum gehorsamen Dienst an Gott, „weil die Nachstellungen teuflischen Betrugs zu fleischlicher Begierde und anderen Lastern der Adoleszenz gegenüber ,Jünglingchen‘ (adolescentulos) zahlreich sind““. ${ }^{122}$ Petrus Lombardus rät deshalb (mit 1. Tim 5,11) dazu, noch jugendliche Witwen (adolescentiores viduae) zu meiden! ${ }^{123}$ Ein Ruthkommentar des 12. (oder erst 13.) Jahrhunderts schreibt sogar: „Der adolescens aber ist derjenige, der in die Jahre der Geschlechtsreife (pubertas) gelangt und in der Begierde der Wollust erglüht und emporwächst.“ Danach kann erst der iuvenis solchem Verlangen aus vernünftigem Verständnis heraus widerstehen, während der Greis darüber bereits hinweg ist. ${ }^{124}$

„Diese Jugend stand bei den kirchlichen Autoren dieser Zeit in nicht eben hohem Ansehen,“ schreibt Christoph Dette. Sie war unerfahren, ungestüm und leicht verführbar, ${ }^{125}$ „gilt doch die Jugend als Synonym für Leichtsinn“. ${ }^{126}$ Die Neigung zu

trinitate et operibus eius XXV (In librum Psalmorum VI) (wie Anm. 40) 1407; Commentarium in Ruth (e codice Genouefensi 45), 2 (b),5 (unten Anm. 124); Petrus Cantor, Verbum adbreuiatum I 59 (ed. M. BOUTRY [CCCM 196]. Turnhout 2004, 402 und öfter).

119 Bonifatius, ep. 73 (oben Anm. 52).

120 Richer von Saint-Remi, Historiae 3,95 (wie Anm. 85) 222: Ludouicus vero quia morum informatorem non habebat, utpote adolescens levium rerum vanitatibus insistebat.

121 Benedikt von Aniane, Concordia regularum 72,22 (wie Anm. 87 ) 636 (aus der Regel Isidors von Sevilla 24,6): Adolescentuli autem uel nuper conuersi a tali ministerio remouendi, ne aut infirma aetas carnis desiderio polluatur aut rudis conuersio ad saeculi desideria reuertatur.

122 Alkuin, ep. 131 (wie Anm. 118) 194: Exhortamini illos sobrie, caste, pudice cum omni humilitate et oboedientia Deo servire in bonis moribus et sancta conversatione et relegiosa castitate, et maxime de confessione peccatorum suorum, quia multae sunt contra adolescentulos diabolicae fraudis insidiae in desiderio carnali et ceteris adolescentiae vitiis.

123 Petrus Lombardus, Collectanea in omnes Pauli apostoli Epistulas. In epistolam I ad Timotheum 5,11 (PL 192). Paris 1855, 325-384, hier 353: ,Adolescentiores autem viduas devita. ' Cum enim luxuriatae fuerint, in Christo nubere volunt, habentes damnationem, quia primam fidem irritam fecerunt.

124 Commentarium in Ruth (e codice Genouefensi 45) II (b) 5 (wie Anm. 101) 148: Adolescens autem est qui ad annos perueniens pubertatis in libidinum uoluptates incalescit et excrescit. Senex uero est, qui robur transgressus iuuentutis frequentibus querelis laborat et debilitatur. Quoniam igitur adolescentia et uoluptas uana sunt et inueteratus dierum malorum lapidatur, iuuentus autem medio tutissima pergens ualido uirtutis accessu roboratur, iuuenis praeesse messoribus non immerito perhibetur. Quis itaque per iuuenem, nisi rationalis exprimitur intellectus, qui petulantiae repugnans uoluptati, etiam uanitati morbosque uitiorum uexatum ex eis animum debilitantes, medentis rationis curationem propulsans, subtiliter et utiliter intelligit, quae sit uoluntas Dei in malorum expulsione bona, in bonorum exhibitione beneplacens et in instantia utriusque perfecta?

125 DetTe, Kinder (wie Anm. 17) 28. 
Lastern ist tatsächlich ein beständiger Vorwurf. Entsprechend groß sind die Klagen. Rahewin zufolge hörte der junge ungarische König Stephan auf die schlechten Ratschläge, „die das schlüpfrige Alter der Adoleszenz zu schlechtesten Neigungen verleiten“, ${ }^{127}$ und Hinkmar von Reims erinnert sich, in seiner Jugend, nämlich in der Adoleszenz und noch in der iuventus, sich vergangen $\mathrm{zu}$ haben (und muss darüber weinen). ${ }^{128}$ Florus von Lyon nennt das Lebensalter (mit Ambrosius) geradezu ein „besudeltes“ Leben, ${ }^{129}$ für Bruno ist es „ein Feld der Schandtaten“, nachdem „die kindliche Einfachheit durchlaufen ist““, ${ }^{130}$ Alpert von Metz hält es für „die Gewohnheit der adolescentes, sich allzu sehr in Versprechungen zu ergehen. ${ }^{131}$ Helmold von Bosau wirft dem jugendlichen, von den Normannen besiegten König Karl (dem Dicken) Trägheit (inertia) vor ${ }^{132}$ und Konzilscanones beklagen mangelnden Gehorsam. Eine Canonessammlung aus dem 11. Jahrhundert, die jeder Gruppe ein spezifisches Laster zuschreibt, vermisst gerade beim adolescens den Gehorsam. ${ }^{133}$ So erfüllt sich Heinrich IV. nach Bruno seine eigenen Wünsche und hält alle, die das nicht tun, für dumm. ${ }^{134}$ Bei dem weit objektiveren Frutolf von Michelsberg wird das, weniger anklagend, aber mit der gleichen Einstellung zur Adoleszenz, umgewertet: Heinrich habe die Freiheit

\section{Ebd. 30.}

127 Rahewin, Gesta Frederici III 13 (ed. G. WAITZ - B. von Simson [MGH SS rer. Germ. 46]. Hannover Leipzig 1912, 182; ed. F.-J. Schmale [FSGA 17].Darmstadt 1974, 392-714, Gesta III 15, hier 426): deinde pravorum consilia secutum, quae ad pessima studia lubricam in adolescente aetatem impellebant.

128 Hinkmar von Reims, De cavendis vitiis et virtutibus exercendis I 1 (ed. D. NACHTMANN [MGH QGG 16]. München 1998, 127): Quaeque nos in adolescentia, quęque in iuventute deliquisse meminimus, defleamus, morum operumque maculas lacrimis tergamus, amemus iam redemptoris nostri vestigia, quae peccando contempsimus.

129 Florus von Lyon, Collectio ex dictis XII patrum. Dicta Ambrosii Mediolanensis. Excerptum 235 (III) ad Ep. ad Galatas pertinens (ed. P. I. FrANSEN - B. COPPIETERS 'T WALLANT - R. DEMEULENAERE [CCCM 193 A]. Turnhout 2006, 163): Nam, si aetas senectutis uita immaculata, utique aetas adolescentiae est uita maculosa.

130 Brunos Buch vom Sachsenkrieg prol. (oben Anm. 110).

131 Alpert von Metz, De diversitate temporum II 15 (wie Anm. 57) 74, zu einem entflohenen Unfreien Balderichs: Ille autem, ut est consuetudo adolescentium, nimium in pollicitatione eius exultans, magna ei promittit munera et ut quam celeriter id efficiat hortatur. Ille vero fidem dat se ita facturum, et ad constitutum tempus reseratis portis summo mane dominum suum intromisit.

132 Helmold von Bosau, Chronicon I 7 (wie Anm. 114) 18: Karolus adolescens, filius Loduici ipso tempore Roma rediens cum grandi exercitu Northmannos iuxta Mosam fluvium apprehendit. [...] At illi regis adolescentis inertiam irridentes, ubi noxia libertate potiti sunt, rursum in unum conglobati tantas strages dederunt, ut crudelitas modum excesserit.

133 Collectio canonum in V libris (libri I-III), I 256 (wie Anm. 53) 155: De duodecim abusionibus ex quibus suffocatur iustitia: I sapiens sine operibus, II senex sine religione, III adolescens sine oboedientia, IIII diues sine helymosina, V femina sine pudicitia, VI dominus sine ueritate, VII christianus contentiosus, VIII pauper superbus, VIIII rex iniquus, X episcopus negligens, XI plebs indisciplinata, XII populus sine lege. Danach später wörtlich Liber quare, Appendix 2, additio 94 (ed. G. P. GöTz [CCCM 60]. Turnhout 1983, 233), zu den zwölf abusiua saeculi.

134 Brunos Buch vom Sachsenkrieg 8 (wie Anm. 65) 18: Stultum namque dixit esse, si non in omnibus satisfaceret suae desideriis adolescentiae. 
der Adoleszenz zu nutzen gewusst. ${ }^{135}$ Nach der Vita Ansberti ist der Adoleszent gar allem gegenüber widerwillig. ${ }^{136}$ Und er ist frech. In einem von Sedulius Scotus stilisierten Lehrgespräch zwischen einem Greis (senex) und einem adolescens gibt letzterer lauter freche Antworten. Dort heißt es im Wortlaut:

Der Greis sagte zum Jüngling: „Wenn du mein Schüler werden willst, werde ich dich darüber belehren, was du noch nicht weißt.“

Adolescens: „Noch nie hat man erlebt, daß ein Blinder einen Sehenden erleuchtet oder ein Ignorant den auf dem rechten Weg Wandelnden berichtigt.“

Der Greis sagte: „Ich weiß, dass deine Frechheit mit Schlägen und Tritten gezähmt werden muß.“ Der Jüngling sagte: „So schwer, wie dir die Worte fallen, so behindert erscheinst du auch an den Händen.“

Der Greis sagte: „Du machst mich bestürzt, aber noch fühle ich Mitleid mit deinem kindlichen Alter (infantia).“

Adolescens: „Ich erkenne weder, dass dein Herz an Verständnis überfließt, noch reicht deine Sprache zum Reden aus.“

Greis: „Je mehr du sagst, desto mehr offenbarst du deine Dummheit.“

Adolescens: „Wenn ein Weiser mit einem Dummkopf streitet, dann wird er entweder erzürnt oder lacht, aber er findet keine Ruhe.“

Greis: „Dein Maul spricht ständig Lügen und vermag nichts Wahres zu sagen. Leg daher deinen Finger an den Mund und schweig.“

Adolescens: „Es ist unmöglich, dass aus einer bitteren Quelle süßes Wasser entspringt oder dass an einem verdorrten Baum süße Früchte hängen."“137

135 Frutolf von Michelsberg, Chronicon a. 1068 (ed. G. WAITZ [MGH SS 6]. Hannover 1844, 33-231, hier 199): Heinricus rex adolescentiae usus libertate, Saxoniam solam ex omni Romano imperio coepit incolere, principes despicere, nobiles obprimere, inferiores sustollere, venatui, lusibus ceterisque huiusmodi exercitiis plus quam iusticiis faciendis, ut incusatus est, operam dare, filias illustrium quibuslibet obscure natis coniugare, privata presidia, nimirum potentibus regni non satis fidens, instituere.

136 Vita Ansberti 1 (ed. W. Levison [MGH SS rer. Merov. 5]. Hannover - Leipzig 1910, 618-641, hier 620) : Eruditur sine aliqua suorum instantia; fuerat namque in eo dulcis infantia, modesta pueritia, gravis adolescentia. Qui cum adultus pubertatis excederet annos, saecularis eius pater, ut unicum eum diligens, venatibus eum occupari et dulcedine saeculi armari cupiebat. Sed quo oblectabatur grandevus pater, adolescens filius per cuncta fastidiebat.

137 Sedulius Scotus, Collectaneum miscellaneum 3 (ex prouerbiis Graecorum) (ed. D. Simpson [CCCM 67]. Turnhout 1988, 12f.): Senex dixit contra adolescentem: Si uis fieri meus discipulus, ego instruam te quod nescis. / Adolescens: Nunquam uisum est, ut cecus illuminet uidentem aut ignorans corrigat recto itinere gradientem. / Senex dixit: Scio quia proteruia tua fustibus et calcibus debet dominari. / Adolescens dixit: Sicut uerbis difficilis es, ita et in manibus debilis apparebis. / Senex dixit: Multum me commoues, sed adhuc tue misereor infantiae. / Adolescens: Video quia nec cor tuum abundat ad intellegendum, nec lingua tua sufficit ad loquendum. / Senex: Quantum plus fueris locutus, tantum stulticiam tuam declarabis. / Adolescens: Vir sapiens si cum stulto contenderet, siue irascatur siue rideat, non inueniet requiem. / Senex: Os tuum semper mentitum est, et uerum dicere non ualet. Pone ergo digitum super os tuum et obmutesce. / Adolescens: Impossibile est ut ex amaro fonte dulcis egrediatur aqua, aut ex arbore arida dulcia pendeant poma. 
Der Jüngling präsentiert sich hier als Gegenteil dessen, was von ihm erwartet wird, dass er nämlich den Älteren ehren soll. ${ }^{138}$ Ähnlich frech geriert sich auch ein adolescens in der Remigiusvita Hinkmars von Reims, der sich darüber lustig macht, dass ein alter Mann wie ein Kind mit einem Rebhuhn spielt, und dafür vom Apostel Johannes mit einem Gleichnis zurechtgewiesen wird: Wenn er seinen Bogen zu lange spannt, verliert dieser an Spannkraft; so auch der Mensch, wenn er nicht ab und zu seiner menschlichen Schwäche nachgibt. ${ }^{139}$

Eine Folge solcher Einschätzungen sind daher zunächst einmal (häufige) Ermahnungen der adolescentes. Alkuin schreibt an einen jüngeren Schüler, er solle die adulescentuli, die jüngeren Jugendlichen, zur Keuschheit ihres Körpers, zur Bekennung ihrer Sünden, zu beständigem Lernen, zu nüchternem Umgang, zum Vermeiden von Genusssucht und der Eitelkeiten dieser Welt ermahnen (und selbst ein gutes Beispiel abgeben). ${ }^{140}$ Derselbe Alkuin fordert iuvenes, adolescentes und pueri aber auch dazu auf, sich vom Teufel zu befreien, indem sie Buße und Frömmigkeit üben und sich nicht durch fleischliche Gelüste die himmlischen Freuden verscherzen. ${ }^{141}$

138 Vgl. unten Anm. 146.

139 Hinkmar von Reims, Vita Remigii episcopi Remensis 5 (wie Anm. 50) 267 (nach Johannes Cassianus, Collationes 24,21, wo aber nicht von adolescentes die Rede ist): Fertur relatione maiorum, quia cum beato Iohanni apostolo avis quae perdix vocatur viva et sana a quodam oblata fuisset, cepit eam leni manu demulcendo tractare. Quod quidam adolescentium videns, ad coevos suos ridendo dixit: ,Videte;, quomodo ille senex cum avicula sicut et puer ludit. 'Beatus vero apostolus per spiritum ista cognoscens, vocavit ad se iuvenem, interrogans, quid manu teneret. Cui iuvenis: ,Arcum, 'inquid. Et beatus Ioannes: ,Quod habet officium illud quod manu tenes?' Et iuvenis: ,Sagittamus, ' inquid,' inde bestias sive aves vel alia queque.' Et beatus Iohannes: ,Quomodo,' inquit, ,vel quo ordine?‘ Et iuvenis, curvato arcu, tetendit illum et tensum in manu tenuit. Sed cum beatus Iohannes nihil ei subsecutus loquendo fuit, post aliquod spatium iuvenis arcum distendit. Cui beatus Iohannes: ,Cur, 'inquit, ,arcum distendisti?'Ad quem iuvenis: ,Quia si diutius tensus tenoretur, infirmius tela iactaret.' Et ad haec sanctus apostolus: ,Sic et humana fragilitas, si semper in rigore contemplationis persistat et suae fragilitati non condescendat, minus necessario valida contemplationis penna sublevatur. Quod pia provisione Conditoris et Salvatoris nostri agitur, ut per collatam gratiam crescamus in profectu virtutum et per infirmitatis nostrae recognitionem discamus humilitatis custodire virtutem. Nam et aquila cunctis avibus celsius volare, cunctis animantibus clarius solis radiis infigere consuevit obtutus; tamen naturae necessitate ad cadaver celsum deponere solet volatum. Sic et nostra fragilitas memor suae conditionis, quia illum, ad quem suspiramus in huius tempore vel loco peregrinationis, continue contemplari non possumus, quasi ab effectu contemplationis desiderato ad modicum retractus animus, crebra innovatione ardentius et fortius ad caelestia tendit. ' Quo facto et sermone audientibus magnam instructionis aedificationem et presentibus contulit et sequentibus se reliquit.

140 Alkuin, ep. 88, von 793/96 (wie Anm. 118) 132: Ammoneasque adulescentulos, qui tecum sunt, de castitate corporis sui, de confessione peccatorum suorum, de adsiduitate discendi, de conversatione sobria, de ebrietate cavenda, de luxoria fugienda, de vanitate huius saeculi non sequenda, sed discant in adolescentia, ut habeant, quid doceant in senectute. Deferant enim honores senioribus; ament vero ecclesiastica officia. Ergo praevide, ut bona videant in te exempla cum sanctorum exhortatione verborum. 141 Ders., ep. 131, ebd. 198: Agite nunc iuvenes adolescentes et pueri, liberate vosmetipsos de diabolica servitute. Concurrite per paenitentiam ad clementissimam omnipotentis Dei pietatem. Nolite per desideria carnis vestrae perdere gaudia caelestia et aeterni regni inter angelicos coetus beatitudinem, sed 
Nach der Vita des Bischofs Meinwerk von Paderborn ist daher eine strenge Erziehung und Abschirmung notwendig, wie sie Bischof Imad in seiner Kindheit erfahren hatte, der ,in solch strenger, klösterlicher Zucht erzogen worden war, dass es ihm niemals vergönnt war, seinen Vater außerhalb des Konvents zu sehen und zu sprechen“. ${ }^{142}$ Weil dieses Alter zum Bösen neigt, so das Aachener Reformkonzil von 816, sollten junge Kleriker in einer gemeinsamen Halle leben und dem erprobtesten Älteren zugeordnet werden, der ihr Lehrer hinsichtlich der Lehre wie des Lebens sein sollte, „damit sie ihre schlüpferigen Jahre nicht in Genusssucht, sondern in kirchlicher Unterweisung verbringen“. ${ }^{143}$ Adolescentes sollten, so die Benediktregel (und Benedikt von Aniane), nicht miteinander, sondern mit Älteren das Bett teilen ${ }^{144}$ und, so Augustin, Beda Venerabilis und andere (mit 1. Petr 5,5), der Aufsicht der Älteren unterworfen sein, ${ }^{145}$ ihnen, so Alkuin, wie den Eltern unterstehen, die Älteren aber sollten sie ihrerseits wie ihre Kinder behandeln. ${ }^{146}$ Adolescentes, so Rimbert, sollen die Älteren nachahmen. ${ }^{147}$

confortate vosmetipsos et viriliter pugnate cum adversario vestro, ut feliciter mereamini coronari cum sanctis Dei et perpetuam cum illis possidere gloriam.

142 Vita Meinwerci episcopi Patherbrunnensis 160 (wie Anm. 66) 84: Prefatus quoque Imadus episcopus tempore sue pueritię tanto disciplinę claustralis rigore ibidem est nutritus, ut numquam patrem suum videre extra conventum specialiter vel ei colloqui fuerit permissus.

143 Konzil von Aachen 816, c. 139 (ed. A. Werminghoff [MGH Conc. 2,1]. Hannover 1906, 413), als Zitat aus Toletanum IV, c. 24, von 633: Prona est omnis aetas ab adolescentia in malum, nihil incertius quam vita adolescentium. Ob hoc constituendum oportuit, ut, si quis in clero puer est aut adolescentes existunt, omnes in uno conclavi atrii commorentur, ut lubricae aetatis annos non in luxoria, sed in disciplinis ecclesiasticis agant, deputati probatissimo seniori, quem et magistrum doctrinae et testem vitae habeant, et caetera.

144 Regula Benedicti 22,7 (ed. A. DE Vogüé - J. Neufville 1-2 [SC 181/182]. Paris 1972, hier SC 181, 540/542): Adulescentiores fratres iuxta se non habeant lectos, sed permixti cum senioribus. Danach Benedikt von Aniane, Concordia regularum 29,7 (wie Anm. 87) 244.

145 Augustinus, Speculum 44 (ed. F. WeIHRICH [CSEL 12]. Wien 1887, 1-285, hier 267): similiter, adulescentes, subditi estote senioribus. Wörtlich auch Beda Venerabilis, In epistolas septem catholicas, ep. 2,5,5 (ed. D. HuRST [CCCM 121]. Turnhout 1983, 180 -342, hier 257); Hrabanus Maurus, ep. 15 (ed. E. DÜMMLER [MGH Epp. 5]. Berlin 1899, 381-516, hier 406); Aelred von Rievaulx, De speculo caritatis II 34 (ed. C. H. Talbot [CCCM 1]. Turnhout 1971, 82). Vgl. auch Cushing, Pueri (wie Anm. 18) 449. Zu Klosterregeln vgl. StоERTZ, Adolescence (wie Anm. 18) 128. Danach wurde die Aufsicht seit dem Ende des 11. Jahrhunderts (Lanfrank und Anselm von Bec) erheblich strikter betont (ebd. 134f.), so dass Stoertz eine Entwicklung zu zunehmendem Misstrauen gegenüber Jugendlichen sieht (so ebd. 139f.). Nach Cochelin, Adolescence (wie Anm. 18) 152, wurden adolescentes bereits in cluniazensischen Consuetudines als lästige Kinder angesehen, die unter strenge Aufsicht zu stellen waren.

146 Alkuin, ep. 51, von 782/96 (wie Anm. 118) 95, an die Mönche von St. Martin in Tours: Habete adolescentes quasi filios, quatenus illi vos habeant ut patres. Exemplis bonis illos instruite, ammonete ut carissimos, castigate ut conservos. Quia haec est fraterna caritas, ut omnes quasi vosmet ipsos ad coronam supernae beatitudinis perducere studeatis. Adolescentes vero senioribus quasi patribus subditi estote, sicut de deo Christo legitur in evangelio, quod subditus esset suis parentibus. Si vero Deus homo et dominus omnium subditus esse non dedignatur hominibus, quanto magis et vos pueri vel adolescentes subditi esse debetis magistris vestris, ut pro illorum bona doctrina proficere et florere et exaltari in ecclesia Christi et ad vitam aeternam cum illis Deo donante pervenire mereamini. 
Andererseits ist man jedoch immer wieder auch zur Nachsicht bereit, sind Fehltritte gerade bei adolescentes verzeihlich, die ihr „,abschüssiges Alter“ (aetas proclivior) entschuldigt, wie Bernhard von Clairvaux es ausdrückt, auch wenn es ihn schmerzt. ${ }^{148}$ Mit dem Hinweis auf die „kindliche Schwäche“ (puerilis fragilitas) des adolescens und auf frühere Beispiele für Nachsichtigkeit sucht ein bischöfliches Gutachten auf der Aachener Synode von 862 Lothar II. zu entschuldigen, weil „das, worin man sich in der Adoleszenz in kindlicher Zerbrechlichkeit vergangen hat, die Wiedergutmachung durch geeignete Besserung in ehelichem Bündnis reinigt““. ${ }^{49}$ Mord, Ehebruch, Sakrileg, Unzucht, Meineid oder falsches Zeugnis: jede Fehltat eines adolescens kann durch Almosen, Fasten, Gebete, Abstinenz und gute Werke gebüßt werden, meint Erzbischof Vulfad von Bourges. ${ }^{150}$

Und schließlich wird (nicht zuletzt natürlich bei Heiligen) herausgestellt, dass sie den Versuchungen dieses „ausschweifenden Lebensalters“ erfolgreich widerstanden haben, wie Abt Theoderich von Saint-Hubert in den Ardennen. ${ }^{151}$ Der Vita Wynnebaldi ist die lascivia überhaupt ein Kennzeichen des „infantilen“ und „puerilen“ Lebensalters, das der Adoleszent überwindet. ${ }^{152}$ Folglich gibt es in jedem Alter nicht nur Laster, sondern auch Tugenden, wie Willibald in seiner Bonifatiusvita betont. ${ }^{153}$ „Die

147 Rimbert, Vita Anskarii 35 (ed. G. WAITZ [MGH SS rer.Germ. 55]. Hannover 1884, 13-79, hier 66): Tamen, ut auditum habemus, ipse adolescens et iuvenis senibus et grandevis mirandus videbatur et imitandus.

148 Bernhard von Clairvaux, ep. 300 (wie Anm. 76) VIII 216: SI quando in vos filius vester visus est excessisse, doluimus, et dolemus non minus excessum filii quam matris iniuriam; quod tamen et ipsum excusabile est in adolescente filio. Nempe DELICTA IUVENTUTIS, ipsa, quae commisit, excusat aetas proclivior. An nescitis quia proni sunt sensus hominis et cogitationes in malum ab adolescentia? Confidendum mutatum iri in melius, patris meritis et eleemosynis. Die Bereitschaft zu verzeihen betonen auch Youngs und Dette (oben Anm. 22).

149 Synode von Aachen 862, Nr. 9 D: Gutachten einiger Bischöfe (wie Anm. 79) 85: vel quod delictum est in adolescentia puerili fragilitate, purgavit satisfactio emendatione congrua per maritalem confoederationem; siquidem in maiorum constitutis exempla nos docent adolescentię lapsibus quantum indulgeri oporteat siquidem adolescentem cum sorore lapsum pęnitentiae remedio dicunt esse sanandum, non a coniugio penitus removendum.

150 Epp. Variorum nr. 28 (oben Anm. 113).

151 Vita Theoderici abbatis Andaginensis 9 (ed. W. WATTENBACH [MGH SS 12]. Hannover 1856, 37-57, hier 42): Ad obediendum semper paratus, mirabilis erat omnibus; castitati vero luxuriam subdidit et incentiva vitiorum, et ipsos, qui non desunt in senibus naturales corporis motus, lectionum assiduitate, vigiliarum instantia et orationum frequentia in lascivientis adolescentiae annis viriliter compescuit et frenavit.

152 Hugeburg von Heidenheim, Vita Wynnebaldi abbatis Heidenheimensis 1 (wie Anm. 60) 107: qualiter, infantialis seu pueritialis transmeatis annorum curriculis, ad adolescentiae nondecem annorum aetate citatus celeps usque pervenit, et tunc ille strenuus. spretis atque dispectis pueritialis incentivorum lasciviis inlecebrosisque vitiorum petulantiis, magna dispensationis clementia arcessioneque episcopi nostri ad celsioris vitae aeternae meritorum profectionibus inhiando properabat.

153 Vgl. Willibald, Vita Bonifatii 9 (ed. W. Levison [MGH SS rer. Germ. 57]. Hannover - Leipzig 1905, 1-58, hier 56): Enumeratis igitur beati viri gestis, quibus in infantia et pueritia vel adolescentia et iuventute aut etiam in senectute floruerat [...]. 
Adoleszenz zählt sich nach Jahren, die Tugend des Geistes aber wird in den Gliedern beherrscht,“ schreibt der Autor der Vita des heiligen Vincentianus, der in solchem Verständnis schon als zehnjähriger Knabe ein adolescens war. ${ }^{154}$ Das biologische Alter wird dadurch relativiert, die Reife nicht nach Jahren, sondern nach Tugenden bemessen, wie auch ein Brief des 11. Jahrhunderts betont. ${ }^{155}$ Adolescens ist man nicht dem Alter nach, sondern durch Verdienst, meint ähnlich Bernhard von Clairvaux. ${ }^{156}$ Daher kann man schon als adolescens dem Geist nach ein Greis sein (was hier, anders als wir es heute verstehen würden, natürlich positiv gemeint ist). ${ }^{157}$

Der adolescens kennt folglich nicht nur Laster, sondern auch Tugenden und gute Sitten, auch wenn sie vor allem wieder in Heiligenviten betont werden und den Heiligen damit gegenüber der ,Normalität“ auszeichnen wollen: „Die heilige Adoleszenz“ des heiligen Basolus war nach der Basolusvita Adsos von Montier-en-Der „nicht den Lastern unterworfen, von denen dieses Lebensalter (sonst) allzusehr umschlungen wird“. ${ }^{158}$ So berichtet Radbod von Utrecht in seiner (der zweiten) Vita des Bonifatius über dessen „,würdige Sitten, die er (schon) in der Adoleszenz hatte, und über seine rechtmäßigen Werke, mit denen er in seiner iuventus gefestigt überzeugte“. ${ }^{159}$ Abt

154 Vita Vincentiani confessoris Avolcensis 4 (ed. W. LEvison [MGH SS rer. Merov. 5]. Hannover Leipzig 1910, 116 -128, hier 117): In puericia vero positus, cepit Domino militare cum apicibus. Erat autem decem annorum, sed iuventutem corporis vincebat rigore mentis. Adolescencia computabatur in annis, sed virtus mentis regebatur in membris.

155 Briefe der Zeit Heinrichs IV. Hannoversche Sammlung. Hildesheimer Briefe, ep. 56 (R. an Hezilo von Hildesheim) (ed. C. ERDMANn [MGH Briefe der deutschen Kaiserzeit 5]. Weimar 1950, 15-106, hier 102): Tu enim senex es, ego vero puer vel adolescens - non de annis loquor, pater, sed de moribus. 156 Bernhard von Clairvaux, ep. 243,1 (wie Anm. 76) VIII 131: Ita nunc quoque, etsi ,adolescentulus sum ego et contemptus' (Ps 118,141), - adolescentulus dico, non annorum paucitate, sed meritorum -, ,potens est' tamen ,Deus' (Mt 3,9) dare etiam voci meae vocem virtutis, per quam fiat ut et is populus, quem nihilominus constat esse seductum, ad iudicium revertatur.

157 So die Vita Athanasii episcopi Neapolitani 3 (ed. G. WAITZ [MGH SS rer. Lang. 7]. Hannover 1878, 439-449, hier 442): Futurae namque humilitatis specimen, quod in pontificatu de culmine exhibuit, in adolescentia senili iam mente ostendebat.

158 Adso von Montier-en-Der, Vita sancti Basoli 7 (wie Anm. 39) 224: Qui licet communiter in seculo aliquandiu conuersatus, non tamen sacram adolescentiam uitiis subdiderat, quibus illa ętas nimium implicari solet. Iam tum quidem aestuans amore cęlestium semperque tendens in aucmenta uirtutum, et quamuis secularis rei superducta specie teneretur, disciplinis tamen cęlestibus animum inserebat, euangelicam perfectionem in illa etiam infirmitate sibi prę oculis inferens, patrem matremque minus dilexerat, ut Deo se approbante fieri dignior potuisset.

159 Radbod von Utrecht, Vita altera Bonifatii 12 (ed. W. LEvison [MGH SS rer. Germ. 57]. Hannover Leipzig 1905, 62-78, hier 70 f.): Sed iam nunc tempus ammonet ut decursis hiis, que dignis eius moribus, quos in adolescentia habuit, legitimisque laboribus, quibus in iuuentute certavit, necnon et sancte conversationi, qua in sacerdocio tamquam stella lucidissima fulsit, conueniunt non tam secundum gestorum dignitatem, quam secundum uires meas ad martirii eius reuelationem hystoriuncula nostra perueniat. 
Pardulf von Guéret erschien in seinem Knabenalter „vor den übrigen Jünglingen“ (adolescentuli) als „der Ruhigste und Besonnene, mit höchster Ehrerbietung“. ${ }^{160}$

Tugenden werden aber auch an weltlichen Jünglingen gelobt. Nach Widukind von Corvey schmückte König Heinrich I., der Sohn Ottos des Erlauchten, „sein Leben schon in frühem Alter mit jeder Art von Tugend" (und als der Vater die Klugheit dieses Jünglings (adolescens) sah, überließ er ihm ein Heer). ${ }^{161}$ Die Klugheit (prudentia) und alle Tugend Ludwigs V. lobt auch Richer von Saint-Remi. ${ }^{162}$ Widukind von Corvey stellt die Energie (industria) Herzog Giselberts heraus, den Heinrich I. mit seiner Tochter Gerberga verlobte und dem er die Herrschaft über Lothringen übertrug, ${ }^{163}$ während es Heinrich IV. nach Wido von Ferrara schon in annis adolescentiae an Sitten und Königstugenden mangelte: an Standhaftigkeit, Tapferkeit, Strenge, Großmut, Gefälligkeit und Freigebigkeit. ${ }^{164}$ Der „Jungfrauenspiegel“ (Speculum virginum) aus dem 12. Jahrhundert kennt drei altersspezifische Tugenden des adolescens: Keuschheit, Gehorsam und Respekt vor dem Alter. ${ }^{165}$

160 Vita Pardulfi abbatis Waractensis 1 (ed. W. Levison [MGH SS rer. Merov. 7]. Hannover- Leipzig 1920, 24-40, hier 25): Qui dum puerili degeret vita, mitissimus ac modestus cum summa reverencia prae ceteris adolescentulis videbatur.

161 Widukind von Corvey, Res gestae Saxonicae I 17 (wie Anm. 58) 27: Natus est ei filius toto mundo necessarius, regum maxime optimus, Heinricus, qui primus libera potestate regnavit in Saxonia. Qui cum in primaeva aetate omni genere virtutum vitam suam ornaret, de die in diem proficiebat; nam maximum ei ab adolescentia studium erat in glorificando gentem suam et pacem confirmando in omni potestate sua. Pater autem videns prudentiam adolescentis et consilii magnitudinem, reliquit ei exercitum et militiam adversus Dalmantiam, contra quam diu ipse militavit. Danach Frutolf von Michelsberg, Chronicon a. 912 (wie Anm. 135) 179.

162 Richer von Saint-Remi, Historiae IV 1 (wie Anm. 85) 232: Alii quoque cum duce ei immorandum [commorandum] asserebant, eo quod adolescens tanti principis prudentia simul et virtute informari indigeret.

163 Widukind von Corvey, Res gestae Saxonicae I 30 (wie Anm. 58) 43, zu Giselbert von Lothringen: Erat autem Isilberhtus nobili genere ac familia antiqua natus. Quem rex satis laetus suscepit, quia per ipsum solum totum Lotharii regnum se habiturum arbitratus est. Deinde videns adolescentem valde industrium, genere ac potestate, divitiis quoque clarum, liberaliter eum coepit habere, ac postremo desponsata sibi filia sua nomine Gerberga, affinitate pariter cum amicitia iunxit eum sibi, sublegato ei omni Lotharii regno. Danach Frutolf von Michelsberg, Chronicon a. 920 (wie Anm. 135) 181.

164 Wido von Ferrara, De scismate Hildebrandi 3 (ed. R. WiLMANS - E. DÜMmLER [MGH Ldl 1]. Hannover 1891, 532-567, hier 536): Tum vero rex Heinricus in annis adolescentiae constitutus et eiusdem aetatis consiliariis assuetus, nobilium et maiorum contra regiam consuetudinem familiaritates horrebat, et cum morum gravitas plurimum habeat laudis in rege - quia decet esse regem constantem, fortem, severum, magnanimum, beneficum, liberalem -, relictis senibus gravibusque personis, levibus delectabatur et pueris tam sensu quam annis; hinc actum est, ut ad vitia propensior haberetur, quia difficile quis, quod diligit, aspernatur.

165 Speculum uirginum 9 (ed. J. SEYFARTH [CCCM 5]. Turnhout 1990, 1-377, hier 285): Tres enim principales gradus sunt adolescentię, pulchritudo castitatis, obedientię deuotio, maioribus reuerentię exhibitio. In primo gradu angelica dignitas in terris uidetur, in secundo fructus ęternitatis est, in tercio ordo naturę seruatur. Keuschheit benennt auch Alkuin, ep. 168 (wie Anm. 118) 276: Quapropter cum gratiarum actione accipiat, quod ei pastoralis cura sufficere praevideat. Sit enim sanctae pacis concordia 
Bei solcher Sachlage ist es wenig verwunderlich, wenn eine Wertung gegenüber der adolescentia durchaus gegensätzlich ausfallen konnte: Erlaubten die Gefahren der noch unabgeschlossenen Persönlichkeit auf der einen Seite negative Urteile, so konnte mancher Heranwachsende, der in diesem Alter die richtige Entscheidung getroffen hatte oder schon große Taten vollbrachte, konnte aber auch die adolescentia als solche positiv bewertet werden. ${ }^{166}$ Jedenfalls ist, trotz mancher Schmähungen, die Meinung, adolescentes würden durchweg negativ beurteilt, ${ }^{167}$ zurückzuweisen. Zahlreiche Autoren wie Augustin, Hinkmar von Reims, Hrabanus Maurus und andere warnen daher (mit 1. Tim 4,12): „Niemand soll deine Adoleszenz verachten“ (Nullus contempnat adolescentiam tuam)! ${ }^{168}$ Sie nutzen das allerdings zugleich wieder zur Ermahnung, sich entsprechend zu verhalten. Ein ganz anderes Charakteristikum wird in der Vita Heinrichs IV. angesprochen, nämlich die Neigung gerade von adolescentes, Verbrüderungen oder Busenfreundschaften (sodalitates) zu schließen. ${ }^{169}$

\section{Aufgaben und Tätigkeiten}

Mit der adolescentia beginnt gewissermaßen die Verantwortung, werden Aufgaben und Tätigkeiten übernommen. (Radbod von Utrecht spricht von „legitimen Arbeiten“ (legitimi labores). ${ }^{170}$ Von seiner Adoleszenz an, so lehrte Aelred von Rievaulx, mit angeblicher Berufung auf Jeremias, könne (und solle) jeder sein Joch tragen. ${ }^{171}$ Es ist

inter cunctos; vitae castitas in adolescentibus; morum gravitas in senibus; fervor operis in iuvenibus: nec quisquam se canonicis horis vel regularibus psalmodiis subtrahat.

166 Vgl. auch Adalard von Gent, Vita Dunstani Cantuarensis 55 (ed. W. STUBвs, Memorials of Saint Dunstan [Rerum Britannicarum medii aeui scriptores 63]. London 1874, 55 f.).

167 Vgl. Dette, Kinder (wie Anm. 17) 28. StoerTz, Adolescence (wie Anm. 18) 140, sieht im „distrust“ gegenüber Adoleszenten in Klosterregeln erst eine spätere Entwicklung.

168 Vgl. Augustinus, De doctrina christiana IV 27,60 (ed. J. MARTIN [CC SL 32]. Turnhout 1962, 1-167, hier 164): Denique apostolus scribens ad Timotheum, cum dixisset: ,Nemo adolescentiam tuam contemnat', subiecit, unde non contemneretur, atque ait: ,Sed forma esto fidelium in sermone, in conuersatione, in dilectione, in fide, in castitate ' (1. Tim 4,12); Hinkmar von Reims, Opusculum LV capitulorum 42 (wie Anm. 93) 306: Nemo adolescentiam tuam contemnat, id est: talem te exhibe, ut propter aetatis adolescentiam nemo te contemptibilem habeat; Hrabanus Maurus, ep. 12 (wie Anm. 145) 399: Nemo adolescentiam tuam contempnat, sed exemplum esto fidelium, in verbo, in conversatione, in caritate, in fide, in castitate. Dum venio, adtende lectioni et exhortationi [et] doctrine; Speculum virginum 9 (oben Anm. 165).

169 Vgl. Vita Heinrici IV imperatoris 9 (wie Anm. 81) 29: Denique, ut fit inter adolescentes, quodam sodalitatis glutino iuncti sunt, ut etiam fidem dextramque mutuis secretis darent.

170 Vgl. Radbod von Utrecht, Vita Bonifatii (oben Anm. 159).

171 Aelred von Rievaulx, Sermo 43,7 (wie Anm. 38) 337: Istorum perfectionem et ipsius perfectionis uiam describit sanctus Ieremias, dicens: ,Bonum est uiro, cum portauerit iugum ab adolescentia sua; sedebit solitarius et silebit, quia leuauit se supra se.' Perfectio ergo illorum, qui uere solitarii sunt, est leuare se supra se. Via, ab adolescentia portare iugum, et sic sedere solus et tacere. Bonum est, inquit, uiro, cum portauerit iugum ab adolescentia. Vgl. ebd. 43,20 (oben Anm. 70). Der zitierte Vers findet sich nicht bei Jeremias, sondern Lam 3,27 f. 
bezeichnend, dass die möglichen biblischen Vorlagen - hier ist ja häufig vom Joch (iugum) die Rede - nirgends von Adoleszenz sprechen, Aelred das Alter also aus seinen mittelalterlichen Vorstellungen heraus eingeflochten hat.

\section{a. Ausbildung, Erwerb von Bildung, geistliche Erleuchtung}

$\mathrm{Zu}$ den typischen Tätigkeitsfeldern gehören zunächst die Ausbildung zum Kleriker und der Bildungserwerb. „Sie (die Schüler) sollen in der Jugend (adolescentia) lernen, damit sie jetzt erwerben, was sie im Greisenalter lehren sollen,“ schreibt Alkuin. ${ }^{172}$ Kinder und Jugendliche (pueri adolescentesque) sollen sorgfältig in die „Buchwissenschaft“ (scientia librorum) eingeführt werden, so ermahnt Alkuin den angelsächsischen Bischof Aedilberct von Hexham, damit dieser würdige Nachfolger finden könne. ${ }^{173}$ Die adolescentia ist daher, wie vielfach bezeugt ist, die Zeit der Ausbildung und der Studien, ${ }^{174}$ die Zeit, die zur scientia aufbaut (so Aelred von Rievaulx), ${ }^{175}$ weil sich dieses Alter dazu am besten eignet; es ist (so Wilhelm von Conches) am besten dafür „temperiert“, um das Verstandene zu behalten (während Kinder noch keine und Greise keine Lehre mehr aufnehmen). ${ }^{176}$ Dem Jungen (so Andreas von St. Viktor) wird Wissen über Gleichnisse vermittelt, dem adolescens hingegen Wissen und Verständnis. ${ }^{177}$ Adolescentes, aber auch pueri, werden daher häufig als „Schüler“ (scholares) vorgestellt. ${ }^{178} \mathrm{Im}$ Glauben sind das für Jonas von Bobbio allerdings erst „die allerersten Grundlagen“ (primordia rudimenta). ${ }^{179}$

172 Alkuin, ep. 88 (oben Anm. 140).

173 Alkuin, ep. 31, ebd. 73, von 793/95: Pueros adolescentesque diligenter librorum scientiam ad viam Dei docete, ut digni vestri honoris fiant successores, etiam et intercessores pro vobis.

174 Vgl. Petrus Damiani, ep. 44 (wie Anm. 61) II 30: Adolescentem me in Faventina urbe propter litterarum studia constitutum audire contigit, quod enarro; Sedulius Scotus, Collectaneum miscellaneum, diuisio 3 (oben Anm. 137) Vgl. DETTE, Kinder (wie Anm. 17) 25 (Zugang zur Bildung bei kirchlicher Laufbahn).

175 Aelred von Rievaulx, Sermo 42,12 (wie Anm. 38) 334: In adolescentia erexit ad scientiam.

176 Wilhelm von Conches, Glosae super Boetium In Consolationem 1. In prosam 3 (ed. L. NAUTA [CCCM 158]. Turnhout 1999, 69): Similiter est aetas hominis. Quae, si nimis est tenera, nullam recipit doctrinam ut infantes. Similiter si nimis est dura ut in senibus. Sed in adolescentibus ita est temperata aetas, ut cito intelligat et intellecta conseruet memoria.

177 Andreas von St. Viktor, Expositiones historicae in libros Salomonis. Expositio historica in Parabolis, zu Prov 1,4 (wie Anm. 105) 11: Et adolescenti scientia et intellectus [...]. Paruulo tanquam minoriper parabolam scientia datur contra malorum insidias cautela, adolescenti uero, qui maioris est aetatis, scientia et intellectus.

$178 \mathrm{Vgl}$. Bernhard von Clairvaux, Epistola de moribus et officio episcoporum (oben Anm. 76). Notger von Lüttich hatte daher immer adolescentes scholares um sich: Anselm, Gesta episcoporum Leodiensium, Rez. II 27 (ed. G. WAITZ [MGH SS 14]. Hannover 1883, 106-120, hier 109): Et quanta fuerit illi in educandis pueris scolaribusque disciplinis instruendis sollicitudo, hinc maxime probatur, quod semper, dum in via pergeret, longe seu prope, scolares adolescentes secum ducebat, qui uni ex capellanis suis sub 
Der adolescens kann aber auch bereits ein erfahrener Schreiber, ein Schriftgelehrter, ein fruchtbarer Weiser, ein vorausblickender Ratgeber, ein wahrer Prediger und ein vorsichtiger Redner mit vollem Wissen sein, wie Agnellus von Ravenna es im 9. Jahrhundert von dem Notar Johannicius berichtet. ${ }^{180}$ Auch Abt Theoderich von Angers wurde seiner Vita zufolge

binnen kurzem ebenso im Schmuck der Sitten wie in der Bildung in den sieben Künsten, die wir die freien nennen, zu einem Philosophen, der, obgleich noch adolescentulus, bereits von den gelehrtesten Greisen bewundert wurde ${ }^{181}$

(auch wenn so etwas hier sicherlich wieder als Ausnahme geschildert wird). Der spätere Bischof Salomon von Konstanz war Ekkehards „Casus s. Galli“ zufolge immerhin bereits als adulescens gut gebildet (bene educatus), ${ }^{182}$ und Ratpert von St. Gallen war seit seiner Adoleszenz sogar selbst bereits Lehrer der Klosterschule (magister scholarum). ${ }^{183}$

In diesem Alter (der Unterscheidung von Gut und Böse) ereignet sich daher oft auch die „geistliche Erleuchtung“, erfolgt mit dem Eintritt in den „göttlichen Dienst“, wie es bei Adso von Montier-en-Der heißt, ${ }^{184}$ die Entscheidung zum religiösen Leben, ${ }^{185}$ wächst die Gottes- und Nächstenliebe (amor Dei et proximi) ${ }^{186}$ oder der Ent-

artissima scolarum parerent disciplina. Vgl. auch Hinkmar von Reims, Opusculum LV capitulorum c. 43 (oben Anm. 93).

179 Jonas von Bobbio, Vita Iohannis abbatis Reomaensis 1 (ed. B. KRUSCH [MGH SS rer. Germ. 37]. Hannover - Leipzig 1905, 326-344, hier 329): quique etiam inter ipsa primordie rudimenta tam in puericia quam in adolescentia sub christiani vigoris cultu atque religionis a parentibus alitus est atque nutritus.

180 Agnellus de Ravenna, Liber pontificalis ecclesiae Rauennatis 120 (ed. O. HoLDER-EGgER [MGH SS rer. Lang.]. Hannover 1878, 275-391, hier 356; ed. D. Mauskopf DeliYANnis [CCCM 199]. Turnhout 2006, 291): Est hic adolescens unus Iohanicius nomine, scriba peritissimus, in scripturis doctus, in sapientia fecundus, in consilio providus, in sermone verax, cautus eloquio omnique scientia plenus, nobilissimis ortus natalibus.

181 Vita Theoderici abbatis Andaginensis 8 (wie Anm. 151) 42: In brevi tam ornatu morum quam eruditione septem artium, quas liberales dicimus, factus philosophus, magnae admirationi adhuc adolescentulus eruditissimis senibus fuit.

182 Ekkehard von St. Gallen, Casus s. Galli 3 (ed. G. MEYER von KnonAu [Mitteilungen zur vaterländischen Geschichte 15/16. St. Gallische Geschichtsquellen 3]. St. Gallen 1877, 3): Salomon, iam adolescens bene educatus.

183 Ebd. 34, ebd, 130: scolarum ab adolescentia magister

184 Adso von Montier-en-Der, Vita Basoli 7 (oben Anm. 39).

185 Vgl. Vita Betharii episcopi Carnoteni 3 (wie Anm. 54) 614: Cum autem, annis puerilibus transactis, adolescentiae metas transiret et die noctuque sancto insisteret proposito atque oboedientiam sibi commissam totis viribus exornaret vitamque solitariam toto nisu arripere festinaret, humili cum devotione bonae memoriae Pabolo episcopo studuit indicare.

186 Gottfried von Admont, Homiliae dominicales 19 (PL 174). Paris 1854, 21-386, hier 125 D: Tertiam aetatem, quae dicitur adolescentia vel iuventus, verbi Dei praedicationem appellamus, quam parit bonum illud, quod est amor Dei et proximi. Gottfried spricht hier allerdings von adolescentia vel iuventus. 
schluss zur „Arbeit im Weinberg“, ${ }^{187}$ wird das Leben Gott geweiht, wie es Gregor von Tours an Gallus lobt, ${ }^{188}$ wurde man Mönch ${ }^{189}$ oder Nonne. ${ }^{190}$ (Dazu ist, wie Anselm von Canterbury betont, zuvor allerdings von Abt und Konvent der Lebenswandel genauestens zu prüfen. ${ }^{191}$ ) Ansgar wurde als Mönch adolescentior und ließ die menschliche Zerbrechlichkeit hinter sich. ${ }^{192}$ Natürlich wird so etwas wieder vorzugsweise von Heiligen berichtet und somit vom ,Normalfall' abgehoben. So lobt die Vita des adligen Abtes Leutfrid des Klosters Merey (das nach ihm später La Croix-Saint Leufroy benannt wurde) den Heiligen:

In seiner Adoleszenz, wo er doch im eitlen Ruhm dieser Welt hätte emporwachsen können, weihte er seinen ganzen Kriegsdienst (militia) dem wahren König, Gott, und wollte lieber arm und nackt Christus folgen als, mit weltlichen Reichtümern bekleidet, wie eine Blume nur zeitweilig blühen. ${ }^{193}$

Armenfürsorge und Nächstenliebe waren bei dem heiligen Pardulf „die ersten Proben seiner Adoleszenz und das Spiel seiner pueritia."194 Wieder kann man auch von der Jugend (pueritia) an auf Studien bedacht sein, wie der Kleriker Odo in Orléans zur Zeit

\footnotetext{
Vgl. ebd. hom. 84, ebd. 587 D: Non puerum, non senem, sed adolescentem esse decernit, cuius animum ardens dilectionis ignis in interiorem quietis intimae securitatem, orationis ascensu, vehementer accendit. 187 So (aber wieder auf jedes Alter bezogen) die Vita Landelini prol. (oben Anm. 49).

188 Gregor von Tours, Liber vitae patrum 6,1 (ed. B. KRUSCH [MGH SS rer. Merov. 1/2]. Hannover 1885, 211-294, hier 230): Sanctus denique Gallus ab adolescentia sua devotus Deo esse coepit, diligensque ex tota anima Dominum, et ea, quae Deo dilecta esse noverat, dilegebat.
}

189 Vgl. Hermann von Tournai, Liber de restauratione monasterii sancti Martini Tornacensis 38 (ed. G. WAITz [MGH SS 14]. Hannover 1883, 274-317, hier 290), zu Alulf, dem Sohn des Kanonikers Sigulf: videns adolescentem filium suum monachus factum. In dieser Schrift ist sechsmal von einem adolescens die Rede. Nach Cochelin, Adolescence (wie Anm. 18) 154, wurde man in Cluny mit 15 Jahren, also „mitten in der Adoleszenz“, in die Klausur aufgenommen.

190 Vgl. das Konzil von Tribur 895, c. 24b (oben Anm. 92).

191 Anselm von Canterbury, ep. 38 (wie Anm. 72) 148: De fratre adolescente quod petitis nec domnus abbas nec nostra congregatio concederet, nisi prius eius vitam diuturnae conversationis experimento cognosceret.

192 So Rimbert, Vita Anskarii 3 (wie Anm. 147) 21: Cumque post haec apud vos tonsoratus atque in monasticis disciplinis positus adolescentior fuisset factus, humana subripiente fragilitate, aliquantulum coepit a propositi prioris rigore frigescere.

193 Vita Leutfredi abbatis Madriacensis 1 (ed. W. LEvison [MGH SS rer. Merov. 7]. Hannover - Leipzig 1920, 7-16, hier 9): Beatus vir Leutfredus infra Gallias pago Ebrocensi exortus est, sanguinis nobilitate conspicuus, parentibus christianissimis natus. Hic in adolescentia sua, cum in vana mundi huius gloria excrescere posset, totam militiam suam Domino vero regi devovit, magis cupiens pauper Christum nudus ipse sequi, quam, divitiis saeculi vestitus, ad tempus sicut flos feni florere. Vgl. Gesta archiepiscoporum Salisburgensium. Vita Chunradi archiepiscopi Salisburgensis 3 (ed. W. WATTENBACH [MGH SS 11]. Hannover 1854, 64): Non quia adolescens et iuvenis secularem gloriam et iactantiam fugere adhuc didicerit, cuius vanitatem studio vestium in tantum secutus est, ut palliatus appellaretur Chuonradus. 194 Vita Pardulfi 3 (wie Anm. 160) 26: Haec sunt adolescencie suae prima rudimenta, hic est ludus puericie suae. 
König Philipps I. von Frankreich. ${ }^{195}$ Erneut sind adolescentes allerdings noch nicht hinreichend vollkommen, um den Sinn etwa der Heiligen Schrift voll zu verstehen, wie Alkuin mahnt, ${ }^{196}$ oder um in die geistigen Höhen $\mathrm{zu}$ gelangen, wie Bernhard von Clairvaux betont. ${ }^{197}$ Teils fliehen sie sogar die Bildung ${ }^{198}$ und müssen wegen ihrer Keckheit und ihres Übermutes deshalb hart erzogen und gezüchtigt werden. ${ }^{199}$

\section{b. Dienste und Ämter}

In diesem Alter tritt der Jugendliche entsprechend häufig seinen Dienst bei einem Herrn an, wie Pippins Vater Waltchisus, der am Hof König Dagoberts „hochedel im Kriegswesen und in höfischer Zucht“ erzogen wurde, ${ }^{200}$ oder wie es häufig in Viten und Chroniken in Bezug auf bestimmte Personen betont wird (meist im Hinblick auf Dienst und Treue seit der Adoleszenz). Der spätere Bischof Desiderius von Cahors stand zuvor ab adolescentiae suae tempore als Schatzmeister (thesaurarius) in treuen Diensten König Dagoberts. ${ }^{201}$ Der Autor der Vita des Bischofs Caesarius von Arles wiederum kann sich auf mehrere Priester und Diakone als Gewährsleute stützen, die

195 Hermann von Tournai, Liber de restauratione monasterii sancti Martini Tornacensis 1 (wie Anm. 189) 274: Hic a puericia studiis litterarum instanter intentus, intra tempus adolescentie tantum scientie est adeptus, ut nemini sui temporis Francigenarum in ea iudicaretur secundus.

196 Alkuin, Grammatica (oben Anm. 71).

197 Bernhard von Clairvaux, Sermones super Cantica Canticorum 19,7 (wie Anm. 117) I 112: Hi ergo omnes, prout capiunt, diligunt. Sed enim adolescentulae, quoniam minus sapiunt, minus et capiunt, nec omnino sufficiunt ad tam sublimia: parvulae quippe in Christo sunt, lacte et oleo nutriendae.

$198 \mathrm{Vgl}$. Anselm, Gesta episcoporum Leodiensium II (recensione altera) 28 (wie Anm. 178) 109: Quid de Huboldo dicam, qui dum adolescentulus a scolari disciplina hinc inde aufugisset, Parisius veniens, sanctae Genovefae canonicus adhesit, in brevi multorum scolarium instructor fuit?

199 Vgl. das Konzil von Aachen 816, c. 135 (oben Anm. 143); ebd. c. 145, ebd. 420: Delinquentes zelo rectitudinis pariter arguant et castigent, pueros et adolescentes neglegentes verberum disciplinis corripiant. Vgl. auch Vita Meinwerci episcopi Patherbrunnensis 160 (wie Anm. 66) 84: dicente episcopo pueros et adolescentes cum districtione debere erudiri et non nocivis blandimentis deliniri, quoniam audacie et ferocię nutrimenta eis ministrarent blandimenta.

200 Gesta abbatum Fontanellensium 1 (ed. S. LöWENFELD [MGH SS rer. Germ. 28]. Hannover 1886, 11f.): Huius genitor Waltchisus nuncupatus nomine, ut veracium didicimus traditione seniorum, patruus gloriosissimi Pippini ducis Francorum, filii Anschisi, extitit. Denique idem vir Domini, adolescentiae dum polleret aetatis in annis atque in aula gloriosissimi regis Dagoberti nobilissime militaribus negociis ac aulicis disciplinis educaretur, ab eodem rege comes palatii constitutus, ac deinde ducatus offitio mancipatur.

201 So Vita Desiderii Cadurcae urbis episcopi 14 (ed. B. KRUSCH [MGH SS rer. Merov. 4]. Hannover Leipzig 1902, 563 - 602, hier 572 [CC SL 117]. Turnhout 1957, 345-401, hier 358f.): Dum et vestri sanctitati bene credimus esse conpertum, qualiter fidelis noster, uir illustris Desiderius, tesaurarius noster nobis ab adolescentiae suae tempore fideliter deservivit [...], in einer inserierten Urkunde König Dagoberts; vgl. Die Urkunden der Merowinger Nr. 37 von 630 (ed. TH. KöLzER [MGH Diplomata regum Francorum e stirpe Merovingica]. Hannover 2001, I 100. 
dem Bischof „von Jugend an“ gedient haben und seine Schüler waren. ${ }^{202}$ Der gleichnamige, adoleszente Sohn Landulfs von Capua stand bis zur Nachfolge im Hofdienst; ${ }^{203}$ Dunstan von Canterbury trat in diesem Alter zunächst in den Dienst eines verwandten Erzbischofs, mit dem er auch in einem Haus wohnte, und dann König Aethelstans. ${ }^{204}$ Otto der Große rief seinen Bruder Brun, den späteren Erzbischof von Köln, noch als adolescentulus von der Schule an seinen Hof, ${ }^{205}$ und, immer noch adolescens, führte Brun als erste Anordnung (prima dispensatio) bereits, teils unter Zwang, Klosterreformen durch. ${ }^{206}$ Der Adoleszent ist schon Kleriker - so bildete Remigius von Reims an der Domschule adolescentes clericos aus ${ }^{207}$ - oder Kaplan und zu Missionen herangezogen, ${ }^{208}$ aber noch zu jung für ein Bistum, wie Willibald in seiner

202 Vita Caesarii episcopi Arelatensis I 1 (ed. B. KRUSCH [MGH SS rer. Merov. 3]. Hannover 1896, 457501, hier 457): Et multa quidem ipsius beatissimi domni nobis narratione comperta, multa a nobis ipsis visa, nonnulla etiam venerabilium presbyterorum sive diaconorum, discipulorum suorum relatione prolata didicimus, praecipue tamen venerabilis Messiani presbyteri et fidelissimi viri Stephani diaconi, qui ei ab adolescentia servierunt: ea dumtaxat quae minus onerent et sint a prolixitate submota. Vgl. ebd. 1,63, ebd. 483: Rogamus tamen vos, sancti fratres Messiane presbyter et Stephane diacone, quibus de illo multa comperta sunt, pro eo quod ab adolescentia in obsequio ipsius fuistis, ut huic opusculo vestram quoque collationem iungatis.

203 Erchempert, Historia Langobardorum Beneventanorum 21 (ed. G. WAITZ [MGH SS rer. Lang.]. Hannover 1878, 234-264, hier 242): Subtracto vero ex hac luce Landulfo Capuano comite, ut post tergum redeam, quatuor reliquid liberos, Landonem videlicet iam fatum virum, Pandonem, Landonolfum et Landolfum, futurum pontificem, viros singularis prudentia virtutisque efficatia valde compotes; ex quibus Lando Capuam, Pando marepahissatum, Landonolfus Teanum regebat, Landulfus vero adhuc adolescens palatinis excubabat obsequiis.

204 Adalard von Gent, Vita Dunstani Cantuarensis. Epistola Adelardi ad Elfegum archiepiscopum (ed. W. StuввS, Memorials of Saint Dunstan [Rerum Britannicarum medii aeui scriptores 63]. London 1874, 55f.) (11. Jh.): Cumque iam flos adolescentiae in annis adolesceret de Glaestonia egressus archiepiscopo Dorobernensi Athelmo patruo scilicet suo se iunxit et cohabitare coepit. In quo idem pontifex gratiam dei admirans et futurorum honorum indicia praevidens in palatio eum praesentavit et regi Aethelstano, quem sacra unctione livit magno affectu commendavit, ubi quotidie crescens virtutibus magnus coram rege factus est et principibus.

205 Ruotger, Vita Brunonis 5 (ed. I. Отт [MGH SS rer. Germ. n.s. 10]. Weimar 1951, 6): Hic germanum suum Brunonem Deo dicatum, adhuc adolescentulum, honorabiliter, ut par fuit, e scolis in palatium evocavit, locum videlicet tam lucidissimo aptum speculo, ubi, quicquid deforme per totum pene mundum esset, in studiis liquidius appareret.

206 Ebd. 10, ebd. 10: Prima dispensatio credita est illi adhuc adolescenti in quibusdam monasteriis, in quibus degentes cum idoneo ecclesiae testimonio partim voluntate, partim vi ad regularem vitam constrinxit, sciens quod et invitis bona prestantur.

207 Vgl. Flodoard von Reims, Historia Remensis ecclesiae IV 9 (ed. M. StRatmann [MGH SS 36]. Hannover 1998, 401f.): Praefatus denique presul honorabilis Folco sollicitus circa Dei cultum et ordinem ecclesiasticum, amore quoque sapientie fervens duas scolas Remis, canonicorum scilicet loci atque ruralium clericorum, iam pene delapsas restituit et evocato Remigio Autissiodorense magistro liberalium artium studiis adolescentes clericos exerceri fecit.

208 Vgl. Chronicon s. Michaelis in pago Virdunensi 11 (ed. G. WaItz [MGH SS 4]. Hannover 1841, 79-86, hier 82): Hic igitur adhuc adolescentulus ab abbate suo in discipulatu capellani constitutus, frequenter cum eo ad palatium et perplura locorum habuit comitatum, et in tantum prudentiae in brevi accrevit, ut abbas, velut fastidiens mundialis pompae negotia, cum iam quasi consecretalem primorum 
Bonifatiusvita den Heiligen selbst beteuern lässt, ${ }^{209}$ auch wenn es selbst dazu Ausnahmen gab. 210

Adoleszente Königssöhne erhielten amtliche Aufgaben und Missionen. ${ }^{211} \mathrm{Im}$ weltlichen Bereich sind das vor allem die Jagd, die nach den Gesta Dagoberti für adolescentes geradezu zum mos Francorum zählt, ${ }^{212}$ und Waffen- und Kriegsdienste, wie es vielfach bezeugt wird, ${ }^{213}$ auch wenn das gegenüber der „süßen Kindheit“ und der „mäßigen Jugend“ (pueritia), so die Vita Ansberti, als hart empfunden werde. ${ }^{214}$ So begleitete Ludwig der Fromme seinen Vater gleich nach der Schwertleite auf dessen Awarenfeldzug, musste allerdings am Wienerwald umkehren. ${ }^{215}$ Lothar I. aber schickte seinen Sohn Ludwig, bonae adolescentiae iuvenem, gegen die Sarazenen in Apulien, die er besiegte, ${ }^{216}$ und Heinrich IV. legte gegen Ende seiner Adoleszenz Burgen in Sachsen an, die später zu den Sachsenkriegen führen sollten. ${ }^{217}$ Schon in

existimaret, et cuius fidei possent committi, quae sunt tacenda praeter quibus diriguntur, secreta legationum et consiliorum.

209 Willibald, Vita Bonifatii 24 (wie Anm. 153) 24f.: Qui etiam, humiliter repente respuens, minime se dignum esse episcopatus gradu referebat et, ne sibi tantae dignitatis fastigium adolescentiae adhuc in annis constituto inponeret, deprecatus est et, quoniam quinquagesimi anni iuxta canonicae rectitudinis normam necdum plene reciperet aetatem, testatus est, ut omni se penitus excusationis tergiversatione ab huius gradus a celsitudine declinaret.

210 Vgl. Ekkehard von St. Gallen, Casus s. Galli 103 (wie Anm. 182) 366: Thietericus vero Metensis episcopus adolescens; Annalista Saxo a. 1090 (ed. K. NAß [MGH SS 37]. Hannover 2006, 483): et Gebehardus adolescens Ratisponensis episcopus constituitur.

211 Vgl. Ademar von Chabannes, Chronicon (recensiones beta et gamma) III 57 (ed. P. BouRgain, unter Mitarbeit von R. LANDES und G. Pon [CCCM 129]. Turnhout 1999, 178), zu Herzog Wilhelm von Aquitanien (a. 1024): Quadragesima superveniente dux Romam abiens, filio suo prudentissimo adolescenti Willelmo imperavit ut revertens ordinatum reperiret episcopum.

212 Gesta Dagoberti I. regis Francorum 2 (ed. B. KRUSCH [MGH SS rer. Merov. 2]. Hannover 1888, 399 425, hier 401): Cum autem adolescentiae aetatem, ut genti Francorum moris est, venationibus exerceret, agere cervum quadam die instituit. Vgl. auch Vita Ansberti (oben Anm. 136).

213 Vgl. etwa Adso von Montier-en-Der, Vita Walberti 4 (ed. M. GoulLEt [CCCM 198]. Turnhout 2003, 81): Qui uidelicet uir in uita sua gloriosissimus, licet fuerit posterior ordine, non tamen inferior exstitit meritorum qualitate. Cuius quoque annos adolescentię in armis tradunt primum excellentissime floruisse. Vgl. DetTE, Kinder (wie Anm. 17) $17 \mathrm{f} .:$ Als „nobilis“ erhielt der adolescens das Schwert und später den Ritterschlag.

214 Vita Ansberti episcopi Rotomagensis 1 (oben Anm. 136).

215 Astronomus, Vita Hludowici imperatoris 6 (ed. E. TREMP [MGH SS rer. Germ. 64]. Hannover 1995, 280 -555, hier 300): (Ludwig trifft seinen Vater bei Ingelheim und geht mit ihm nach Regensburg): Ibique ense, iam appellens adolescentię tempora, accinctus est ac deinde patrem in Auares exercitum ducentem usque ad Chuneberg comitatus, iussus est reverti et usque ad reversionem pater nam cum Fastrada manere regina.

216 Johannes, Gesta episcoporum Neapolitanorum 61 (ed. G. WAITZ [MGH SS rer. Lang.]. Hannover 1878, 402-436, hier 433): Eodem quoque anno supplicatione huius Sergii principumque Langobardorum direxit Lhotharius imperator filium suum Lhodoguicum, bonae adolescentiae iuvenem, propter catervas Saracenorum Apuliae sub rege commanentes et omnium fines depopulantes.

217 Brunos Buch vom Sachsenkrieg 16 (oben Anm. 65). Vgl. auch Regino von Prüm, Chronicon a. 890 (wie Anm. 67). 
primis adolescentiae annis, so schreibt Johannes von Salisbury über König Heinrich I. von England, „trat dieser gegen Heere an und erschütterte und zerbrach die Herzen der Feinde““.218 Nach der Herkunftssage der Thüringer bei Widukind von Corvey überlistete ein Thüringer bereits in diesem Alter die Sachsen. ${ }^{219}$ Wenn der heilige Leonardus als adolescens den Kriegsdienst verweigerte, so wurde dieser folglich normalerweise in diesem Alter „gemäß der Gewohnheit der königlichen Familie“ geleistet. ${ }^{220}$ Viele weitere Belege ließen sich anführen. So besiegte und vertrieb der adolescens Konrad, der Sohn des einstigen Herzogs Konrad von Kärnten, den an seiner Stelle eingesetzten Herzog Adalbero; ${ }^{221}$ Friedrich Barbarossa arbeitete sich a prima adolescentia bellicis [...] officiis $\mathrm{ab}^{222}$ und Lothar III. war schon vor seiner Königswahl $a b$ adolescentia in Kriegen hocherfahren. ${ }^{223}$ Adolescentes erringen daher Siege (wie der Westfrankenkönig Ludwig über die Normannen, ${ }^{224}$ Ottos I. Sohn Liudolf, bevor er zum Verräter wurde, ${ }^{225}$ oder der junge Barbarossa ${ }^{226}$ ). Andererseits tötete Ekbert den Sohn des Grafen Bernhard, einen egregium adolescentem, weil dieser noch kaum zum

218 Johannes von Salisbury, Policraticus VI 18 (wie Anm. 97) 51: militiam aggressus est [...] hostium corda concussit et fregit.

219 Widukind von Corvey, Res gestae Saxonicae I 5 (wie Anm. 58) 6: Ea igitur tempestate contigit adolescentem quendam egredi de navibus oneratum multo auro torque aurea simulque armillis aureis. 220 Vita Leonardi confessoris Nobiliacensis 2 (wie Anm. 54) 396: Puer autem Leonardus postquam evasit incrementa puerilia, adolescens factus, iuxta consuetudinem parentelae regali noluit implicari militia, sed Spiritu divino tactus, potius voluit sequi archipontificis Remigii vestigia. Vgl. aber auch Lampert von Hersfeld, Annales a. 1071 (oben Anm. 97), wonach der adoleszente Sohn Balduins noch nicht reif zum Kriegsdienst war.

221 Hermann von Reichenau, Chronicon a. 1019 (ed. G. H. PERTZ [MGH SS 5]. Hannover 1844, 74-133, hier 119): Counradus adolescens, filius Counradi quondam ducis Carentani, auxiliante patruele suo Counrado postea imperatore, Adalberonem, tunc ducem Carentani, apud Ulmam pugna victum fugavit. 222 Otto von Freising, Gesta Frederici I prol. (ed. G. WAITZ - B. von Simson [MGH SS rer. Germ. 46]. Hannover - Leipzig 1912, 162-346, hier 11; ed. F.-J. SchmaLe [FSGA 17]. Darmstadt 1974, 392-714, hier 118): Inter omnes enim Romanorum principes tibi pene soli hoc reservatum est privilegium, ut, quamvis a prima adolescentia bellicis desudasse cognoscaris officiis, obscenum tibi nondum vultum fortuna verterit. 223 Annalista Saxo a. 1125 (wie Anm. 210) 585, zum Bartholomäustag in Mainz: Ibi episcopi, duces, marchiones, comites omnes unanimiter ducem Saxonie Liuderum sive Lotharium in regem eligunt, virum iam inde ab adolescentia in bellis experientissimum et in victoriis frequentissimum.

224 Annales Vedastini a. 881 (ed. B. von SimSon [MGH SS rer. Germ. 12]. Hannover - Leipzig 1909, 40 - 82, hier 50 f.), zum Normannensieg des Westfrankenkönigs Ludwig: Hac vero patrata victoria, quia multos contigit ibi ruere Nortmannos, rex ovans repedavit trans Hisam, perpauci vero Dani, qui evasere, interitum suorum nuntiavere in castra, indeque Nortmanni Hludowicum regem adolescentem timere coeperunt.

225 Ruotger, Vita Brunonis 18 (wie Anm. 205) 16, zu Ottos I. Sohn Liudolf: Erat in ea coniuratione princeps imperatoris filius Liudolfus, unice clarus et spectabilis adolescens, qui non solum ad conservanda, verum etiam ad exornanda patrię monimenta victoriae sufficeret, si seductoribus minime credidisset et heres esse, non proditor, voluisset.

226 Otto von Freising, Gesta Frederici I 26 (wie Anm. 222) 44 (ed. WAITz) bzw. 180 (ed. Schmale [Gesta 1,27]): sicque adolescens prefatum ducens comitem ad propria cum victoria revertitur. 
Kriegsdienst gereift war (sed vixdum militiae maturum). ${ }^{227}$ Auch hier schimmert folglich dieselbe Ambivalenz durch, die sich schon bei Tugenden und im Verhalten beobachten ließ. Viele adolescentes aber zeichneten sich bereits im Krieg aus.

So verwundert es nicht, dass schon adolescentes (erbliche) Ämter innehaben konnten. Sie waren Grafen (wie Landulf von Capua) ${ }^{228}$ oder Markgrafen (wie Liutbald von Tuszien), ${ }^{229}$ Fürsten ${ }^{230}$ und Herzöge (wie Konrad von Lothringen), ${ }^{231}$ Könige $^{232}$ (wie Otto III., ${ }^{233}$ Heinrich III., ${ }^{234}$ Heinrich IV. ${ }^{235}$ oder Karl der Einfältige ${ }^{236}$ ) oder sogar Kaiser

227 Lampert von Hersfeld, Annales a. 1057 (wie Anm. 97) 159: Sed Egberdus quamquam graviter saucius, dolore tamen interempti fratris efferatus, rapido cursu in confertissimos hostes praecipitem se mittit, Bernhardi comitis filium, egregium adolescentem sed vixdum militiae maturum, interficit.Vgl. auch Arnulf von Mailand, Liber gestorum recentium I 9 (ed. C. ZEY [MGH SS rer. Germ. 67]. Hannover 1994, 130): Cumque foret permissum viros adolescentes muliebriter superindutos, subtus autem accinctos mucronibus, cautissime venire mandavit. Ubi vero ingressi sunt navem, ilico irruentes in hostes evaginatis ensibus indifferenter quosque trucidant.

228 Vgl. Erchempert, Historia Langobardorum Beneventanorum 21 (oben Anm. 203). Vgl. auch Wilhelm von Tyrus, Chronicon XIV 25 (ed. R. B. C. HuYgens - H. E. MAYER - G. RösCH, Chronicon [CCCM 63/63 A], hier CCCM 63 A. Turnhout 1986, 663f.) zu dem Grafen Raymund von Tripoli.

229 Vgl. Hermann von Reichenau, Chronicon a. 1043 (wie Anm. 221) 124: Ubi etiam Liutpaldus, Adalberti marchionis filius, magnae virtutis et pietatis adolescens, ab ipso rege marchio promotus, et post paucos dies defunctus, Treveri a patruo suo Poppone archiepiscopo sepultus est. Vgl. auch Vita Bertulfi Renticensis 9 (Acta Sanctorum 1. Feb. I, 639): cum Balduino filio adulescentulo marchione advenit. 230 Vgl. Folcwin, Gesta abbatum Lobiensium 25 (oben Anm. 84).

231 Vgl. Widukind von Corvey, Res gestae Saxonicae II 33 (wie Anm. 58) 94: cui et unicam filiam suam rex desponsavit; qui erat adolescens acer et fortis, domi militiaque optimus, commilitonibus suis carus; ebd. 3,33, ebd. 119 f.: Pontifex cum duce Cuonrado, cum adolescentem non possent inclinare, quatinus patri subderetur eiusque sententiam secutus sustineret, discesserunt ab eo, Deo regique sese iungentes. Vgl. auch Wilhelm von Tyrus, Chronicon XVI 23 (wie Anm. 228) 748, zum Herzog Friedrich von Schwaben: Fredericus Suevorum dux, adolescens admirande indolis.

232 So mehrfach, zur biblischen und römischen Geschichte, in der Chronik Frechulfs von Lisieux, zum Beispiel I 7,9 (Ptolemaios) (ed. M. I. Allen [CCCM 169 A]. Turnhout 2002, 388); ebd. I 7,10 (Herodes), ebd. 394: Herodem quoque, minorem natu atque admodum adolescentem, pari honore Galileae praefecit; ebd. I 7,11 (Caesar Augustus), ebd. 394. Vgl. auch Petrus Damiani, ep. 117 (wie Anm. 61) III 319, zu David: David vixdum primo florentis adolescenciae decore pubescens, de post foetantes paternae subieccionis eligitur, et ad crispandum spiritalis allegoriae psalterium, atque ad optinendum regalis sceptri fastigium promovetur. Unhaltbar ist die These von DETTE, Kinder (wie Anm. 17) 11: „Ein Kind als König wäre in der Kriegergesellschaft des frühen Mittelalters undenkbar gewesen [...].“

233 Vgl. Rodulf Glaber, Historiae I 4,11 (oben Anm. 56).

234 Vgl. Arnulf von Mailand, Liber gestorum recentium II 17 (wie Anm. 227) 162: Defuncto autem Chuonrado Heinricus adolescens eius filius rex vivente patre creatus augustus ab omnibus appellatur, vir rei publice gerende satis ydoneus.

235 Vgl. Brunos Buch vom Sachsenkrieg 42 (wie Anm. 65) 41, in einem Brief des Erzbischofs Werner von Magdeburg an den Erzbischof Siegfried von Mainz: Dominus noster rex, postquam factus adolescens abiecto principum suorum consilio sui iuris esse coepit et eorum, qui nec aequum nec bonum considerant, magisterio se subdidit, semper nos insolito more quaerebat opprimere.

236 Vgl. Hermann von Reichenau, Chronicon a. 892 (wie Anm. 221) 110: Apud Gallias Karolus adolescens, Ludowici Balbi postumus filius, a quibusdum primatibus rex effectus, multa deinceps Odoni mala fecit et ab eo vicissim sustinuit. 
(wie Heinrich III.), ${ }^{237}$ auch schon in frühen Jahren der Adoleszenz (primevo tunc adolescentiae flore pubescens), wie ein schottischer König, der die Würde jedoch niederlegen und Mönch werden wollte, ${ }^{238}$ oder sie stellten Ansprüche auf das Königtum (wie Ottos I. Bruder Heinrich). ${ }^{239}$ Auch hier mochte es vorkommen, dass ein Amtsträger dem noch nicht wirklich gewachsen war, wie, aus der Sicht Lamperts von Hersfeld, Herzog Gozelo von Lothringen, der „zwar ein Jüngling von ausgezeichneter Gesinnung, doch buckelig“ und, so ergänzt der Annalista Saxo, von kleiner Statur war. ${ }^{240}$ Das Hindernis lag in diesem Fall also nicht am (mangelnden) Alter, sondern in der unfürstlichen Gestalt. Schließlich konnten adoleszente Amtsträger auch schon wieder versterben ${ }^{241}$ und adoleszente Frauen bereits Witwen sein. ${ }^{242}$

\section{Fazit}

Jugend, so stellte schon Georges Duby fest, ist mehr als ein Lebensalter, es ist eine sozioökonomische Bedingung. ${ }^{243}$ Die vielbehandelten Lebensalterlehren sind nur ein - sehr schematischer - Informationsherd. Betrachtet man die - oft beiläufigen adolescentia-Belege in verschiedenen Schriften des frühen und hohen Mittelalters, dann erweitert sich das Bild zu einem Panorama der zeitgenössischen Vorstellungen von der Adoleszenz. Genaue Altersangaben und Altersgrenzen - sie betreffen eher den Abschnitt zwischen 12 und 20 als traditionell (oder theoretisch in Lebensalter-

$237 \mathrm{Vgl}$. Arnulf von Mailand, Liber gestorum recentium II 17 (oben Anm. 234). Vgl. auch Historia de expeditione Friderici (oben Anm. 106).

238 Vgl. Petrus Damiani, ep. 110 (wie Anm. 61) III 241: Scottigenarum rex primevo tunc adolescentiae flore pubescens, quia pater obiit, ei mox in regalis fastigii iura successit. Sed cum sollicite consideraret, quam fragile sit, quicquid in hoc saeculo tumet, quam labile, quod supereminet, quam velociter extinguibile sit omne, quod splendet, cum coniugatus iam esset, decrevit regales infulas regni caelestis amore deponere, coronam capitio, purpuram cuculla mutare.

239 Vgl. Widukind von Corvey, Res gestae Saxonicae II 12 (wie Anm. 58) 78: Heinricus autem erat eo tempore nimis adolescens, fervens animo, et nimia regnandi cupiditate illectus eo pacto crimine solvit eum, quo coniuratione secum facta contra regem dominum suum et fratrem sibi regni diadema, si possibile foret, inponeret. Vgl. auch Galbert von Brügge, De multro, traditione. et occisione gloriosi Karoli comitis Flandriarum 1 (ed. J. RYDER [CCCM 131]. Turnhout 1994, 5): zu Balduin von Flandern: Igitur comes Balduinus, adolescens fortissimus, moriendo, nepoti suo Karolo regnum simul cum principibus contradidit et sub fidei securitate commendavit.

240 Vgl. Lampert von Hersfeld, Annales a. 1070 (wie Anm. 97) 112, zum Tod Herzog Gottrieds von Niederlothringen: cui filius eius Gozelo successit, prestantis quidem animi adolescens, sed gibbosus. Der Annalista Saxo a. 1070 (wie Anm. 210) 416, ergänzt: et statura pusillus.

241 Vgl. Hermann von Reichenau, Chronicon a. 911 (wie Anm. 221) 416, zu Ludwig dem Kind: Ludowicus rex adolescens moritur.

242 Vgl. Flodoard von Reims, Historiae Remensis ecclesiae I 6 (wie Anm. 207) 77: Hieronymus schreibt ad quandam adulescentulam viduam Aggeruchiam nobilem.

243 So Cushing, Pueri (wie Anm. 18) 441, mit Berufung auf G. Duby, Youth and Aristocratic Society. London 1977, 113f., wo allerdings nur von der Zugehörigkeit zu einer bestimmten Gruppe die Rede ist. 
lehren) zwischen 14 und 28 Lebensjahren - spielen dabei eine untergeordnete Rolle gegenüber dem Charakter und dem Verhalten, so dass dem Alter nicht nur bestimmte Eigenschaften und Verhaltensweisen zugeschrieben werden, sondern sogar umgekehrt das Alter nach dem Verhalten bemessen werden kann. Adoleszenz wird als ein labiles und schwieriges, aber entscheidendes Übergangsalter des Erwachsenwerdens zwischen unbekümmerter Kindheit und verantwortungsvoller iuventus wahrgenommen, das sich körperlich in Bartwuchs (beim Mann) und Zeugungsfähigkeit äußert. Der Adoleszent ist kein Kind mehr, aber auch noch kein Mann, noch nicht zu voller Stärke herangewachsen, noch ungeformt und unvollkommen und daher manchmal, jedenfalls als Klischee, störrisch oder rebellisch und leicht verführbar, doch sind die Fehler in diesem Alter noch verzeihlich. Entsprechend ambivalent ist auch die Wertung: Adolescentes bedürfen (noch) der Aufsicht, aber auch der Nachsicht; aus der Zeugungsfähigkeit erwachsen sowohl Wollust als auch Scham, dem „Hang zum Bösen“ stehen Tugenden gegenüber.

Wichtiger aber erscheint den Autoren etwas anderes: Adoleszenz ist zugleich das Alter der Formung: im Bildungserwerb ebenso wie in der Moral und in der Lebensentscheidung. Mit (oder seit) der Adoleszenz entscheidet sich der Charakter wie auch der weitere Lebensweg, der Eintritt in ein Kloster oder eine kirchliche ,Laufbahn‘, denn erst jetzt vermag der Mensch zwischen gut und böse zu unterscheiden. Entgegen Warnungen, adolescentes bereits Kirchen- und Klosterleitungen anzuvertrauen, scheint der weltliche, zumindest der hochadlige Adoleszent durch Heirat, Hofdienst, Kriegsdienst und Amtsführung als Graf, Herzog, König oder Kaiser bereits ganz in das soziale Leben integriert gewesen zu sein.

Wenn die Vorstellungen von Adoleszenz hier relativ kohärent erscheinen, dann liegt das sicher auch daran, dass es sich vielfach um Ermahnungen handelt, die sich grundsätzlich an das Alter selbst und an beide Geschlechter richten. Eine geschlechterspezifische Differenzierung tritt damit ebenso wenig in das Blickfeld der Autoren wie große Entwicklungslinien der Adoleszenzvorstellungen zwischen dem 7. und dem 12. Jahrhundert. Für eine feinere zeitliche Differenzierung reicht die Zahl der Belege insgesamt, obwohl sie größer ist, als zunächst vermutet, letztlich nicht aus, doch ist vieles anscheinend über die Jahrhunderte hinweg erhalten geblieben. Auch zwischen Geistlichen und Laien verblassen vielfach die - in der Lebensform ja zweifellos vorhandenen - Unterschiede, zumal die Verfasser sämtlich demselben Milieu entstammen. Anders verhält es sich dort, wo die Lebenspraxis reflektiert wird. Vor allem in der Zuschreibung von Aktivitäten scheinen lebensweltliche Unterschiede durch. Dabei erweitert sich der hier gewählte, vorstellungsgeschichtliche Ansatz, der zunächst nur Zugang zum frühmittelalterlichen Konzept der Adoleszenz verschafft, von einem Spiegelbild der Vorstellungen und Erwartungen (das vielfach sicherlich stereotyp und klischeehaft wirkt) unversehens zugleich zu einem Spiegelbild der sozialen Wirklichkeit des frühen und hohen Mittelalters, auf die es zurückwirkt.

Abschließend sei zumindest angedeutet, dass die okzidentalen Adoleszenzvorstellungen eine Reihe von Ähnlichkeiten mit den Verhältnissen in Byzanz aufweisen, wo allerdings terminologisch weit stärker differenziert wird und auch die rechtliche 
Bestimmung des Lebensalters präziser erfolgt. ${ }^{244}$ Hier wie dort ist Adoleszenz jedoch ein Übergangsalter, das in das Erwachsenwerden hineinreicht, spielen sexuelle Versuchungen und Heirat, aber auch Freundschaften eine Rolle, werden die Leidenschaften des Alters angeprangert, wird in dieser Zeitspanne aber auch der Charakter ausgebildet. Im Westen wie im Osten gibt es Spannungen zwischen der Unbeständigkeit des Alters und den Anforderungen an die Adoleszenten, so dass es zu entsprechenden Mahnungen kommt. Stereotypen Negativbildern stehen „Zeichen und Signale für sozialen Rollenwechsel in der Familie und im Berufsleben“ gegenüber. ${ }^{245}$ Ob solche Übereinstimmungen auf die gleichen (antiken) Grundlagen zurückzuführen sind oder eine Art anthropologischer „Konstanten“ bilden, bliebe noch zu untersuchen.

244 Vgl. dazu D. ARIANTzI, Terminologische und sozialhistorische Untersuchungen zur Adoleszenz in Byzanz (6.-11. Jahrhundert). Teil 1. Theorien, Konzepte, narrative Quellen. JÖB 63 (2013) 1-31.

245 Vgl. ebd. 29. 



\section{List of Contributors}

Despoina Ariantzi is a post-doctoral researcher in the Department of Byzantine and Modern Greek Studies, University of Vienna. She works on Byzantine social history, especially history of childhood and adolescence, social marginalisation and subculture groups, Byzantine pilgrimage, and hagiography. Her publications include Kindheit in Byzanz. Emotionale, geistige und materielle Entwicklung im familiären Umfeld vom 6. bis zum 11. Jahrhundert (2012).

Leslie Brubaker is Professor of Byzantine Art and Director of the Centre for Byzantine, Ottoman and Modern Greek Studies, University of Birmingham. She has published widely on gender, iconoclasm and Byzantine manuscripts. Her books include Gender in the Early Medieval World (with Julia Smith, 2004); The Cult of the Mother of God in Byzantium (with Mary Cunningham, 2011); Byzantium in the Era of Iconoclasm (with John Haldon, 2011); Inventing Byzantine Iconoclasm (2012); and Vision and Meaning in Ninth - Century Byzantium (1999).

Béatrice Chevallier Caseau is Professor in Byzantine History at the University of Paris -Sorbonne and a member of the Centre d'Histoire et Civilisation de Byzance. Her publications explore religious anthropology and sacred spaces of the late antique and Byzantine periods and include Euodia: The Use and Meaning of Fragrances in the Ancient World and Their Christianization (100900AD) (1994); She is coeditor of Pèlerinages et lieux saints dans l'Antiquité et le Moyen âge (2006) and of Pratiques de l'eucharistie dans les Églises d'Orient et d'Occident (Antiquité et le Moyen Âge) (2009); Les Réseaux Familiaux. Antiquité tardive et Moyen Âge in memoriam A. Laiou et É. Patlagean (Paris 2012).

Catia Galatariotou is a cultural historian and a practising psychoanalyst. Her research has been mostly inter-disciplinary and focused on aspects of Byzantine culture, the Cyprus conflict, and Psychoanalysis. Her publications include The Making of a Saint. The life, times and sanctification of Neophytos the Recluse (2004).

Cecily Hennessy is senior lecturer at Christie's Education, London. Her research has focused on children and young people in Byzantine art as well as on Constantinopolitan manuscripts, paintings and topography. Her publications include Image of Children in Byzantium (2008).

Hans-Werner Goetz is Professor Emeritus of Medieval History at the University of Hamburg, and former president of the Mediävistenverband (2001-2007). His main fields of research, all concerning the Earlier Middle Ages (5th-12th century) are: history of concepts, medieval thinking and mentality; historiography; political, social, constitutional and gender history; state and perspectives of medieval studies. His major publications include: Vorstellungsgeschichte. Gesammelte Schriften zu Wahrnehmungen, Deutungen und Vorstellungen im Mittelalter (2007); Gott und die Welt. Religiöse Vorstellungen des frühen und hohen Mittelalters, 3 vols. (2011-2016); Die Wahrnehmung anderer Religionen und christlich-abendländisches Selbstverständnis im frühen und hohen Mittelalter (5.-12. Jahrhundert), 2 vols. (2013).

Tonia Kiousopoulou is Professor of Byzantine History in the Department of History and Archaeology at the University of Rethymon. Her research has focused on family, childhood, political ideology

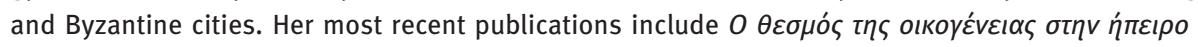

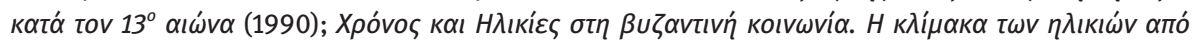

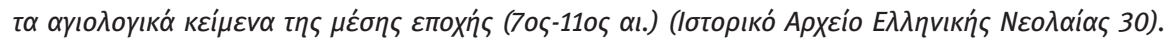

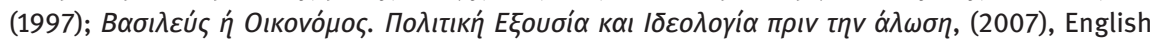


Translation by P. Magdalino, Power and Manager. Power and Political Ideology in Byzantium be-

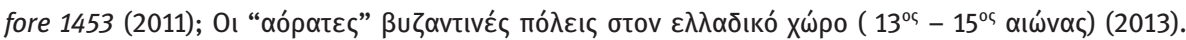

Petra Melichar is the editor-in-chief of the journal Byzantinoslavica - revue international des études byzantines and post-doctoral researcher at the Slavonic Institute of the Czech Academy of Sciences in Prague. Her research has focused on female piety in Byzantium and late Byzantine empresses.

Thomas Pratsch researches and teaches at the Johannes Gutenberg-Universität Mainz. His main fields of research are church history, prosopography and hagiography of the middle Byzantine period. He has written books on Theodore of Studios (1998), the use of hagiographical topoi in Saint's Lives (2005) and the Empress Theodora (2011).

Günter Prinzing is Professor Emeritus of Byzantine Studies at the Johannes Gutenberg - University Mainz. His wide-ranging research covers such topics as relations between Byzantium and southeast and east-central Europe and, most recently, slavery and slaves in the Byzantine Empire. His is editor of the works of Archbishop Demetrios Chomatinos of Ohrid (CFHB 38, 2002).

Ulrike Sirsch is Assistant Professor at the Faculty of Psychology, University of Vienna, Austria. Her research focus is in the field of developmental psychology, specifically in becoming adult and the transition from adolescence to young adulthood.

Alice-Mary Talbot is a former Director of Byzantine Studies at Dumbarton Oaks Research Library and Collection. Her research has focused on Byzantine monasticism and hagiography, and women's studies. Her most recent books are: Women and religious life in Byzantium (2001); The History of Leo the Deacon (with Dennis Sullivan, 2005); The life of Saint Basil the Younger (with Dennis Sullivan and Stamatina Mcgrath, 2014); Holy Men of Mount Athos (with Richard P. H. Greenfield, 2016). 


\section{Index}

Abbot $84,88-90,92-94,96,241,268,279$, 280, 284, 285,

abuse 45,94

Achris (Achrida/Ohrid) 13, 32-34, 40, 42, 49 f., 57, 59, 66, 68-70, 72f., 102, 296

Adalbero, duke of Carinthia 289

Adalbert, archbishop of Hamburg and bishop of Bremen 265

Adso of Montier-en-Der, abbot at the monastery Montier-en-Der Abbey in France 260, 284 Aelred of Rievaulx, St. $\quad 260,278,282,283$

Aethelbald of Mercia, king of Mercia 273

Aethelstan, king of the Anglo-Saxons and king of the English 287

Agnellus of Ravenna, bishop of Ravenna 284 Agobard of Lyon, archbishop of Lyon $260 \mathrm{f}$. agriculture 7,118,122, 139

Alcuin, scholar $260,266,274,277,278,281$, 283, 286

Alexander, co-emperor, elder brother of Leon VI 117, 145f., 151, 155, 159

Alexandria 24, 112, 142

Alexios I. Komnenos, emperor 35, 180

Alexios II. Komnenos, emperor 188

Alexios Komnenos, son of sebastokrator Andronikos I 126

Ambrose, bishop of Milan, St. 19, 23

Amorion 124

amusements $120,129,133,138 \mathrm{f}$.

- actress 132

- brothel(s) 5,128, 134, 138

- dancing and dancers 129

- drinking (wine) 126, $130 \mathrm{f}$.

- festival/feasts 129

- fighting ability 125

- games of dice 129

- jesting 129

- playing 123

- prostitutes visit 128,133

- singing $102 \mathrm{f}$.

- stage/spectacle 129

- tavern(s) visit $\quad 5,130$

- theater visit 5, 129,134, 137

Andreas Salos, St. 130-135

Andronikos, sebastokrator 126

Andronikos Trichas 80

animals 92, 123f., 127, 132

- birds 124, 132, 244
- deer 124, 132

- fish $87,93,95$

- fox 132, 204

- horses 9, 125-127

Anna Komnene 7,126

Anna, wife of Constantinos Papaioannopulos 49,65

Anselm of Canterbury, Benedictine monk, abbot 266, 285

Anastasios (in the life of Theodore of Sykeon), illoustrios 125

Antony Kauleas, St. $\quad 86,97$

Antony Mauros 241

Anthony the Younger, St. 84

Apostolina, widow from Ennakosia/Thrakien 75

Apulia 288

Armenopoulos, teacher at the Vatopedi monastery on Athos 91

Arnulf of Sées, bishop of Lisieux 267

Arta 4, 45f., 64, 120

Artemios, St. 238-240, 244

asceticism 14, 24, 28, 90, 94, 101, 103

Asklepios 237

Athanasios of Athos, St. $87,90 \mathrm{f}$.

Athanasios of Meteora, St. 84, 94, $99 \mathrm{f}$.

Athanasios I. Patriarch, St. 99

Athanasios, young man in the life of Niketas of Medikion $9-11,68,90,99 \mathrm{f}$.

athletic activities: $123,125,128,140$

- archery 126

- ball game 126

- battle 8

- boxing contest 123,125

- horse-riding 123f., 125-127, 213

- hunt $8,123-128,132,212,288$

- hunting $8 \mathrm{f} ., 17 \mathrm{f} ., 123,125,205,212 \mathrm{f}$.

- jumping 9, 123, 125

- polo 128

- running 9, $14 \mathrm{f} ., 101 \mathrm{f} ., 105,111,123-125$, 145

- struggle $136 \mathrm{f}$.

- wrestling 9,123,125

Augustin, St. 19, 26, 251, 253f., 260 f., 278, 282

Augustus, emperor $148-150,290$

Auxentios, St. $91 \mathrm{f}$. 
Bakchos the Younger, St. 86 f., 97

bakery 93

Balaneidiotis, stratopedarchis 110

Barnabas, priest-monk 50, 72

Basileios Kaballarios, nobleman 110

Basileios Kaliges from Leukas 45, 64

Basileios Pediadites, metropolitan in Corfu 46

Basil the Great 11

Basolus, St. 260, 280

Bayezid I., Sultan 58

Bede the Venerable, St. 252, 278

behaviour 22, 24, 43, 100, 103, 118-120, 122 f., 127, 130 f., 133, 135-137, 139, 140, 144, 204 f., 209 f., 216, 228, 234

- behavioural norms 121, 134

- behavioural patterns 118

- violence 4, 131, $135 \mathrm{f}$.

- violent behaviour 4, 131

Benedict of Aniane, Benedictine monk and monastic reformer 268, 274, 278

Benzo of Alba, bishop 268

Bernhard of Clairvaux, abbot and reformer 267 f., 269, 272f., 279, 280, 283, 286

Berroia, in Macedonia $40,56 \mathrm{f}$., 66-71

betrothal $7 \mathrm{f} .$, 32, $34 \mathrm{f} .$, 44, 154, 162, 239

biological development (characteristics) or psychical development of adolescents $1,4,6$, 14, 100, 103, 131, 204

- beard 6f., 16, 19, 22, 83-86, 90 f., 97, $142-150,152,155,178,194,196,201$, 212, 257, 269 f., 292

- beardless $14,83-87,89,92,95,97,108$, 142, 144 f., $147-151,179-181,190,192-196$, 201f., 270

- beauty of adolescence 73

- bodily changes 218, 222

- body 7, 88 f., 103, 109, 123, 180-182, 184, 200 f., 206, 208 f. 227, 234, 240 f., 246 f., 297

- carnal and sexual desire(s) 133, 277

- desire(s) 8f., 11, 14, 85, 87 f., 95, 101, 103, 106, 108, 112, 114, 128, 133, 205, 209, 212, 231, 271f., 274

- facial hair $6,85-87,100,144,149,179$, 192, 257, 269, 292

- fertility 17, 254, 270 f., 292

- hormonal changes $7,117,131 \mathrm{f}$

- menarche 203, 218

- physical activity $127 \mathrm{f}$., 131

- physical strength $6,142,123$

Blasios of Amorion, St. 85, 94
Boniface, St. $\quad 263,273$ f., 279 f., 288

boy(s) (pais) 52, 54, 67, 124, 147, 212, 235 , 255

bride $10,15,26 \mathrm{f}$., $44 \mathrm{f}$., $47 \mathrm{f}$., 52, 58, 64, $74 \mathrm{f}$., $76,105 \mathrm{f} ., 110,145,152,156,187 \mathrm{f}$.

Brun, archbishop of Cologne, brother of Otto the Great 287

Burchard of Bellevaux, abbot 269

burial 141f., 157

Caesarius of Arles, archbishop of Arles 286

Callistus I, patriarch of Constantinople 36, 76 canonical penalties (epitimia) 34

Cappadocia 89, 111, 184f., 192

career $6-9,11,13,18,23,29,36,38 \mathrm{f} ., 40 \mathrm{f}$. 48, 51, 54 f., 60, 86 f., 93, 102, 106, 121, 256

- court service $8,15,105,287,292$

- ecclesiastical profession $9,29,36,48,51$, $54 \mathrm{f} ., 60$

- military service $\quad 285,288-290,292$

- public offices 8, 121, 200, $286 \mathrm{f}$., 290

Carl III, king 275

Charikles, hero in the novel Drossila and Charikles 16, $211 \mathrm{f}$.

Charles the Simple, king 290

Christodoulos, abbot of Lavra monastery of Stylos $83,89,181$

church(es)

- Church of Archangel in Chonai 135

- Elmali Church in Cappadocia 185

- Hagia Euphemia in Constantinople $146 \mathrm{f}$.

- Church of St. Ioannes in Constantinople 240

- Karanlik Church in Cappadocia 185

- Church of St. Nikolaos in Corfu 70

- Church of St. Panteleimon in Nerezi 180

- Church of the Resurrection in Constantinople 82

- Church of Our Saviour in the Chora 193195, 200

- Hagia Sophia in Constantinople 54, 144 146, $185 \mathrm{f}$

- Church of Theotokos 125

- New Church of Tokali 184, 192

coin/coinage 144, 147-151, 155, 199

Coloneia, thema, in the diocese Diabolis/

Devol 45, 65, 72

concubine $40,58,66,271$

Conrad I, duke of Carinthia 289

Conrad of Lorraine, duke 290 
Constance, queen of Sicily, wife of Henry VI 272

Constans II, emperor, grandson of Herakleios 147-149, 164-166

Constantine Argyros, archōn tēs ekklēsias $\quad 48$, 75

Constantine, co-emperor, son of Theophilos 150

Constantine IV, son of Justinian II 148 f., 166

Constantine Manasses 211

Constantine Palates, imperial pageboy 44,74

Constantine Papaioannopoulos 49

Constantine Pegonites, son of Demetrios Pegonites 79

Constantine Rhamatas 78

Constantine, son of Basileios Kaliges from Leukas 45,64

Constantine V, emperor 149, 166 f., 176

Constantine VI, emperor 144, 146-151, 168

Constantinople $6 \mathrm{f} ., 9,13,16,32 \mathrm{f} ., 84-87$, $89-91,97,100,103,138,148,152,169$, 176, 178, 193, 195, 211

Cosmas und Damianos, physicians, St. 237, 247

Crete 11, 84f., 90, 108

Cyprus 91, 211, 295

Cyril of Philea, St. 138

Cyril of Skythopolis, monk, priest and hagiographer 112, 210

Cyzikus 136

Daniel (Old Testament) 160, 182

David of Mytilene, St. 11

Demetrianos of Chytroi, St. 97

Demetrios Asomatianos 51, 53

Demetrios Chomatenos, Archbishop of Achris $13,33,40,50,59,63,65$

Demetrios Eskammatismenos, priest from Thessalonike $52 \mathrm{f} ., 76$

Demetrios Kydones 79

Demetrios Pegonites 79

demons $89,111,129,243$ f., 246, 270

Desiderius of Cahors, bishop of Cahors, St. 286

developmental psychology $1,2,6,106,114$, 117 f., 121,123, 131,139, 215-217, 220-222, 224-226, 232, 235, 296,

Digenes Akrites 16, 204, 211

Dionysius the Areopagite, judge at the court Areopagus 145 diseases and illnesses 12, 17, 102, 208, 237, $243 \mathrm{f} ., 246 \mathrm{f}$.

Dosikles, hero in the novel Rhodanthe and Dosikles of Theodoros Prodromos $211 \mathrm{f}$.

dowry $10 \mathrm{f} ., 34 \mathrm{f} ., 42,44,48 \mathrm{f} ., 61,70,72$, $74 \mathrm{f} ., 78-82$

Dragoste, concubine of loannis $\quad 50,72$

Drama 43, 74

Drosilla, hero in the novel Drossila and Charikles 16, $211 \mathrm{f}$.

Dunstan of Canterbury, archbishop 282, 287

education $\quad 3,6-9,14$ f., 18,23 f., 87,90 f., 99 , 101-103, 113, 117, 121f., 129, 134, $226-$ 228, 256 f., 265, 278, 295

- arithmetic 91, 122, 227

- astronomy 91

- classmates 102

- educational facilities (paideuteria) 91

- geometry 91

- military arts $8,15,103$

- military training of emperors or other adolescents $8,18,125 \mathrm{f}$.

- professional training 121,139

- rhetoric 2, 91

- school education 15, 103, 118, 122

- school 17, 27, 87, 90 f., 102 f., 122, 219, 224, 230, 234, 264

- secondary education (enkyklios paideia) 91

- students $90 \mathrm{f} ., 102,220,232$

- tachygraphy 91

- teacher(s) 91, 102, 122, 127, 278, 284

Eirene, daughter of Michael Kaludes 43, 64

Eirene, mother of Constantine VI 144, $147 \mathrm{f} ., 150 \mathrm{f}$.

Eirene of Chrysobalanton, St. $\quad 89,111,114$

Eirene Raoulaina, aunt of Theodora Palaiologina 110

Eirene, widow of loannes Lakapenos 66

Eirene, wife of loannes II Komnenos 146

Eirene, wife of John, third son of Andronikos II 108

Eirene, wife of Theodore Chloropodes, craftsmen 47,73

Elias of Heliopolis, St. 86

Elias Spelaiotes, St. 132, 243 f., 246

engagement $6,10,22,110,227$

Epiphanios, friend of Andreas Salos 97, $132 \mathrm{f}$.

Epirus 4, 41, 211

epithalamion $145,152,155,187 \mathrm{f}$. 
Euarestos, St. 138

euchologion $6 \mathrm{f}$.

Eudocia Nestongonissa, Megale Papiaina 42 , 57,74

Eudokia Doukaina Komnene Synadene Palaiologina, wife of Theodore Komnenos Doukas $152 \mathrm{f.,} 170,172$

Eulogia Choumnaina, daughter of the famous Nikephoros Choumnos $86,108 \mathrm{f}$.

Eunuch $85,100,108,144,156,178$

Euphrosyne, daughter of Theodoule, founder of monastery of the Virgin of Certain Hope 146, $152 \mathrm{f} .$, 163, 171, $186 \mathrm{f}$.

Euphrosyne Doukaina Palaiologina, granddaughter of John Synadenos, wife of the Constantine Komnenos Palaiologos Raoul 152

Euphrosyne Petraleiphina, nun 75

Euphrosynos, young kitchen worker at the monastery, in the vita of Blasios of Amorion 94

Eusebios, bishop 136

Eustratios of Agauros, St. 97

Euthymios, St. 11, 32, 132

Euthymios the Younger, St. 10, 92, 97

family $1,6-16,19,23-26,28,31 \mathrm{f} ., 35,48$, $50,57-59,81,88,94,100-102,105-110$, $113 \mathrm{f} ., 118 \mathrm{f} ., 121 \mathrm{f} ., 124 \mathrm{f} ., 128 \mathrm{f} ., 136,139$, $141-143,145,152,175,177,185$ f., $188 f$., 202, 208-210, 222f., 224, 228 f., 230, 255f., 271, 289, 293, 295

- aristocratic families 7, 136

- family relations 3

- father authority 121

- guardians $6,10,57,71,78,80$

- Hypexousia 210

- parental authority 25, 206

- parental care 2, 6, 9, 11f., 14

- parents 1, 6-11,13,15, 23, 25, 27f., 42, 44 f., 47 f., 64, 70 f., 73f., 88, 100 f., 106 f., $109,112,114,117,121,129$ f., 134, 139, 143 , 146, 154, 184, 195, 197 f., 200, 204, 206-211, $222-225,227,278$

- rural families 122,139

fasting $23,88,247,279$

father Zosimas, spiritual father of St. Mary of Egypt 112

Febronia, St. 183, 237, 239

feeling(s) 15, 25, 108f., 206, 232
Filopappous, young man in the Epic of Digenis Akrites 210

Florus of Lyon, diacon of Lyon and ecclesiastical writer 275

Folcwin of Lobbes, abbot of Lobbes 268

Frederick I Barbarossa, emperor 289

friendship(s) 117, 121, 227, 282, 293

Frutolf of Michelsberg, monk $275 \mathrm{f}$., 281

funeral oration 144

George Akropolites 91

George of Cyprus 91

George of Mytilene, St. 97

George Pachymeres 110

Georgios Bardanes, metropolitan in Corfu 45 , 64

Georgios Komnenos, duke from Firom 53, 73

Georgios Euschemanos, anagnost 55

Georgios Kallistos, priest 55, 77

Georgios Kuritzes from Achris 70

Georgios Serbopoulos 49, 72

George Tarchaniotes 44, 74

Germanos Maroules, monk 84, 93

Germanos of Athos, St. 99

Gilbert, duke of Lorraine 271

girl(s) (kore) 35f., 38, 47f., 52, 54, 59, 67, 118, $121,130,134,147,235,239,255,269$

Glaukeias, inhabitant of Moros 243

Göreme (city in Cappadocia) 184 f., 192

Gozelo of Lorraine, duke 291

Gregory I pope 273

Gregory of Dekapolis, St. $\quad 86,97,131 \mathrm{f} ., 240$, 244

Gregory of Nazianzos, St. $\quad 142,144,157$

Gregory of Sinai, monk, St. 93

Gregory Pakourianos, founder of the monastery of the Mother of God Petritzonitissa in Bachkovo and author of its typikon 90

Gunther of Bamberg, bishop 265

hair $16,111,132,142-144,149,152 \mathrm{f} ., 156$, 178, $180-184,187,192 \mathrm{f} ., 201 \mathrm{f}$

- hairstyle 5, 7

harbor cities 134, 237-243, 245-249

healing 17

health $112 \mathrm{f} ., 150$

- health care 17

Heiric of Auxerre, Benedictine theologian and writer 261-263, 269

Helena, wife of Manuel II Palaiologos 145, 160 
Helmold of Bosau, historian 273, 275

Henry I, king 281, 289

Henry III, emperor $290 \mathrm{f}$.

Henry IV, king $265,268,272,275,280,282$, 288, 290

Henry V, king 273

Henry VI. king 272

Herakleios Constantine, son of Herakleios 148

Herakleios, emperor $147 \mathrm{f}$.

Heraklonas, son of Herakleios' second wife, Martina 144,148

Hilarion, monk, spiritual father 241

Hilarion of Dalmatos, St. 97

Hinkmar of Reims, archbishop of Reims 262, 269, 275, 277, 282, 284

homosexuality 84,102

household $8,10,87,114$

- domestic work 7

- servants $24,26,46,87,91,93,109,144 \mathrm{f}$., 156, 191, 193

Hrabanus Maurus, bishop of Mainz 261, 264, 270, 278, 282

Hugh the Great, duke of Franks 272

lakobos, monk 78, 84, 189, 192

lakobos Tarchaniotes 78

Ignatios Patriarch, St. $\quad 97,144$

Ignatios the Deacon, cleric and writer of the life of Gregorios Dekapolites 122, 132, 240

incubation 17

inheritance (see also property) 12, 13, 21, 24, 34, 67-68, 109

initiation rites (see also rites de passage) 6 , 16, 117, 205, 212

Ioannakios Achyraites from Berroia 69

loannina 43, 64

loannis Argyropoulos 14, $102 \mathrm{f}$.

Ioannis, bishop of Polyboton, St. $138 \mathrm{f}$.

Ioannis Chrysaphes 80

Ioannis, Illustrios from Amorion 100 f., 123, 126, 260

Ioannis of Herakleia, St. 99

Ioannis Plytos, sebastos 40

loannes Teichotitzes 68

Ioseph, metropolitan and Katholikos Kritēs of Apros 42, 54f., 76, 190

Isidore of Seville, archbishop of Seville 19

Issac I Komnenos, emperor 178
Jairos (figure of the New Testament) 143

Jerome, St. 273

Jerusalem $87,89,112,146,184,188-190$, 195, 197, 201

Johannicius, notary 284

John Apokaukos, Archbishop of Naupaktos 4, 13, 33-38, 43-46, 49, 51, 53f., 59, $63-65,102$

John Klimax, abbot, St. 88

John of Athos, St. 84

John of Kathara, St. 97

John II Komnenos, emperor 146

John Palaiologos, first son of Manuel II. Palaiologos 145

John Psichaites, St. 12

John III Vatatzes, emperor 91

Joseph, husband of Maria, the Mother of God 143, 146, 154, 162, 174, 189 f., 192-198, 200

Joseph the Hymnographer, St. $\quad 86,97$

Justinian I, emperor 42

Justinianic legislation $210 \mathrm{f}$.

Kalligone, Kleadros's beloved in the Byzantine romance Drossila and Charikles 212

Kastoria 41, 69

Katablattas, judge from Thessalonike 14, $102 \mathrm{f}$.

kinship (biological and spiritual) 12, 42, 189, 203

- adopted daughter 42, 57, 74

- adoption 42, 55 f., 77

- brothers 11f., 16, 40, 42, 48, 52, 55, 61, 66, 70 f., $73,93,148,150,188-190,194-196$, 199, 202, 243, 287, 291

- daughter 11, 15, 23f., 26, 43-47, 51f., 61, $64,66,71-75,78,108,110,113,143,146$, 151, 177, 186 f., 189 f., 196, 239, 271f., 281

- esōgambros 44, 48

- father 9-11, 23-25, 27, 40-42, 44-45, $48,52,55,66-68,70$ f., 75, 79, 85, 106-108, $113,124,126-127,135,139,144,148-151$, 187, 194, 196, 212, 223, 241, 256, 264, 278, 281, 286, 288

- grandfather $40,67,148 \mathrm{f}$.

- grandmother 47

- grandparents $10,70,121,210$

- grandson 70,147, 180

- husband or spouse $10,24,43,45,47 \mathrm{f} ., 67$, $75,107,109,154,189,210$ 
- mother 8, $10 f ., 16,24,26,40-42,44,50$, 57, 61, 66, 68-71, 74 f., $79-81,106 f ., 110$, $112 \mathrm{f} ., 122,146-148,150 \mathrm{f} ., 153 \mathrm{f} ., 177,186 \mathrm{f}$. , 189 f., 193, 196, 200, 202, 204, 206, 223, 239. 295

- nephew 12, 52, 79, 80, 94

- relatives $1,6,8-11,13,43,52 \mathrm{f} ., 55,57$, $60 \mathrm{f.,} \mathrm{69,} \mathrm{73,} \mathrm{87,} \mathrm{119,} \mathrm{244,} \mathrm{247,} 287$

- son 3, 10, 24, 26f., 45, 50, 57, 64, 67f., 71f., 75, 77, 79, 81, 100, 108, 113, 122, 124, 126f., 144, 148-152, 166, 176 f., 185, $187-$ $191,193-200,212,238,256,268,271,281$, 285, 287- 289

- spiritual father $54,87,108,135,243,247 f$, 293

- stepparents 13, 60

- uncles 9, 11f., 42, 57, 67-68, 70, 80, 126, 212

- wife (wives) and spouse $8,38,45,47,48$, $64,67,73,79,81 \mathrm{f} ., 108,121,145,148,152 \mathrm{f}$., 160, 189 f., 204

kitchen (monastery kitchen) 92-94, 244

Kleandros, the hero in the byzantine romance Drosilla and Charikles 212

Landulf of Capua, count 287, 290

lavra of Stylos on Latros 83

Lazaros of Galesion, St. $100,133,135 \mathrm{f}$.

Leo III, emperor 137, 149, $166 \mathrm{f}$., 176

Leo IV, emperor 149f., 168

Leo VI, emperor $11,28,52,55,146,151,155$, 160

Leonardus, St. 263, 289

Leon Kontos from Bodina 57, 71

Leontios of Jerusalem, Patriarch 86, 97

Lesbos 51, 78, 97

Leusista/Lapsista 43, 64

life cycle $16,143 \mathrm{f} ., 203,251,254,256 \mathrm{f}$.

- adulthood 1, 4, 6, 8, 12f., 16f., 19 f., 24, $26 \mathrm{f} ., 115,142,148,175 \mathrm{f} ., 188,202,210 \mathrm{f}$., $215,217,219,220-224,226-230,232$, 234f., 251, 255, 257, 263, 268, 292, 293, 296

- adult life $6-8,25 \mathrm{f} ., 80,102,118,177,219$, 221-224, 226-230, 263,

- childhood 1, 3f., 7f., 10-14, 16, 19-21, 24, 26 f., 35, 83, $100 f ., 103,105$ f., 113, 118, $120-$ 122, 125 f., 133, 137 f., 142 f., 175 f., 178, 188, 196, 202, $210-212,223,224,226,251,253$, $255-257,263,266,278,288,292,295$
- child (paidion) 4, 6, $10 \mathrm{f} ., 13,16 \mathrm{f} ., 22-26$, $28,39-40,42,47-48,50-51,54-55$, $65-66,68,70-72,78,80,87,90,93,100 \mathrm{f}$., $105,107,114,121-122,127,129,130,134$, 139, $143-145,149-154,175,177,181,186 \mathrm{f}$. , 193, 195-197, 199, 202, 204, 206f., 209212, 216-217, 223, 228, 230, 234, 248, 254, 269, 277-278, 283, 292

- geron or elder 93

- hebe 126f., 203, 212

- infancy $6,115,141,151,184,189$ f., 192 , 196, 199-201, 206

- infans 261, 263

- majority and age of majority $1,19,20,177$, 184

- manhood 17, 20f., 108, 181, 185, 204, 212

- older generation 3, 5, 121

- old men 143f., 155f., 184, 212

- old women $16,143 \mathrm{f}$.

- teenage (teenage years) 13, 19f., 87f., 105110, 112, 142

- transitional phase or period 4, 6, 12, 201 and 8, 19

- transitional state 204

- younger generation 3,117

- young man (neaniskos) 19, 45, 64, 67, 73, 124-126, 132

- young woman (neãnis) 7, 35 f., 47, 52, 59, 105, 107, 111f., 118, 121, 134, 153f., 239

Lincoln College typikon 146, 152 f., 186

Liudolf, son of Otto I $\quad 264,268,289$

Liutbald of Tuscia, margrave 290

Lombards 265

Lothar II, king 267, 271, 279

Louis V of France, king 274, 281

love $5,15 \mathrm{f}$., $89,108-112,126,180,205-207$, 210, 212, 232

Luke of Steiris, St. $\quad 86,93,97$

Makarios Makres, monk at the Vatopedi monastery on Athos 91, 94, 97

Mamas, St. 92

Manuel Buzenos 81

Manuel I, emperor, son of John II. Komnenos 1122-1142 127, 199, 211

Manuel II Palaiologos, emperor 145, 160

Manuel Sbenilos 68

Manuel Trichas, uncle of Andronikos Trichas 80 
manuscript(s) 13, 24, 93, 143-146, 152-155, 178, 179, 182, $184-186,188$ f., 193, 195 f., 198

Maria Branaina, daughter of Euphrosyne Petraleiphina 75

marriage $6-8,10,12,14-18,20,25-28,32$, $35,42,50,100-103,106,108-110,113$, 142, 153 f., 176, 189, 203f., 210 f., 228, 231

Martha Palaiologina, mother of Theodora, the wife of Basileios Kaballarios 110

Mary-Marinos, St. $107 \mathrm{f} ., 114$

Mary of Egypt, St. $112,114,142 \mathrm{f}$.

Masai 204

matrimonies $32,42,44,51-53,67,74 \mathrm{f} ., 77$

Matrona of Chios. St. $106 \mathrm{f} ., 112,114$

Matthaios I Patriarch 86, 88, 94, 97

Maximos of Kausokalyvia or Kausokalybites, monk St. $86,88,94,97,99 \mathrm{f}$.

Maximos Planoudes 91

Mazaris 14, 103

Meinwerk, bishop of Paderborn 278

Melias, a young, half-orphaned from Beroia 40, 56, $66 \mathrm{f}$.

Methymna (in Lesbos) 51, 78

metochion 84, 90

Michael Bodaniates, craftsman 47

Michael III, emperor 126, 151

Michael II the Amorian, emperor 150

Michael Kabasilas 54, 76

Michael Kaludes, priest $\quad 43,64$

Michael Magistros Pothos 78

Michael Maleinos, St. 28, 90, 97

Michael VIII Palaiologos, emperor 110, 152, $185 \mathrm{f}$.

Michael Palaiologos (citizen of Constantinople) $78 \mathrm{f} . ., 82$

Michael Phortos 52

Michael Psellos 113, 122

monasteries $11,14,22,25,28,78,80 \mathrm{f}$., $83-85,88,90,93,105,107,114,122,141$, 179, 181f., 185, 193, 241f., 243f., 246 f., 248, 255 f., 258, 264, 266, 268, 274, 284 f., 287, 292

- Epiphanios of Maximina monastery in Constantinople 97

- Lavra monastery of Stylos 83

- monastery of Akataleptos 91

- monastery of Bačkovo 90

- monastery of Charsianeites 85

- monastery of Chryse Petra in Pontos
- monastery of Eleousa in Macedonia $83 \mathrm{f}$.

- monastery of Emathia near Ephesus 85

- monastery of Esphigmenou 94, 100, 193

- monastery of Gregory Thaumatourgos in Ephesus 91

- monastery of Hodegetria at Turnovo 100

- monastery of Kosmosoteira in Thrace 83

- monastery of Lazaros in the Galesion 100 ,

- monastery of Lips Constantinople 85, 89, 92

- monastery of Makarios Choumnos 95

- monastery of Mar Saba in Palestine 83

- monastery of Myrelaion 78

- monastery of Phoberou in Bithynia 83, 92

- monastery of Pissadinoi on Bithynian Mt. Olympos 92

- monastery of Philanthropenos

81

- monastery of Prodromos on Mt. Menoikeion 83

- monastery of St. John on Patmos 83

- monastery of St. Nicholas 90

- monastery of Stoudios 84, 87-89, 91f., 97, 178, 193, 242 f., 296

- Vatopedi on Athos 91,93

monastic hierarchy 12

monastic rules $11,108,256,258,264,266$ f., 282

monastic vows $23,27 \mathrm{f} ., 86,88-90,111 \mathrm{f}$.

monk $11 \mathrm{f} ., 14,23 \mathrm{f} ., 50,55,61,73,80 \mathrm{f}$., 83-96, 100-102, 108, 132f., 146, 153, 181, 195 f., 240-244, 246, 248, 278, 285, 291

Mount Athos 22, 179 f., $182-185,193,241-$ 244, 246, 248, 278

Mount Kyminas 90

Mount Olympos 92

Myron $246 \mathrm{f}$.

Mytilene 11, 97

Naupaktos 4, 13, 33, 45, 59, 102

Neilos Damilas 85,90

Neilos the Younger, St. 10

Neophytos, bishop 43, 64, 97

Neophytos the Recluse, St. 211, 295

Nicholaos Bambakas $51 \mathrm{f} ., 78$

Nicholaos Exotrochos 81

Nicholas of Stoudios / Nicholas the Stoudite, St. $\quad 91,97$

Nicholaos Tzachenos, archon 66

Nikephoros Blemmydes 85,91 
Nikephoros Choumnos 108

Nikephoros Gregoras 101

Nikephoros I, emperor 150

Nikephoros II, emperor 151

Nikephoros of Chios, writer the life of Matrona 106

Nikephoros Syropulos from Berroia 71

Nikephoros und loseph, sons of Theorodos Syropoulos 42

Nikephoros von Melit 122

Niketas of Medikion, St. 9-11

Niketas Stethatos, writer of the life of Symeon New Theologian, St. 94

Nikon „ho Metanoeite“, St. 92

Nostongonissa, halfsister of Theodora Palaiologina 110

novices $14,83,85 \mathrm{f} ., 88-92,94 \mathrm{f} ., 112$

nun $7 \mathrm{f} ., 14 \mathrm{f} ., 23 \mathrm{f} ., 37 \mathrm{f} ., 40,42-44,46,50 \mathrm{f}$., 53, 55, $66-71,73 f ., 75 f ., 78-81,86,89 f .$, $92,106,108-112,114,133,135,152 \mathrm{f} ., 187$, 237, 239-241, 243, 246, 248, 258, 269, 285

orphans $13,22,24,29,39-41,52,55-56$, $60,66-68,69 f ., 70,75,77,130$

Otto I, emperor 268, 289, 291

Otto III, king 264, 290

Palaiologos Synadenos $\quad$ 152, 170

Pankalo 45, 64

Paphlagonia 87, 124

Pardulphus, Benedictine abbot, St. 281, 285

Paschasius Radbertus, abbot of Corbie 261, 267

Patmos $83,87,89,181 \mathrm{f}$.

Paul of Latros, St. $\quad 11,89,94$

Paul of Monembasia, St. 10

peers 7, 15, 114, 123, 134, 139 f., 228

- peer groups 7,15

Perrios Lampadenos 79

Peter Lombard, scholastic theologian and bishop of Paris 274

Peter of Argos, St. $11 \mathrm{f}$.

Peter of Atroa, St. 11, 97

Petros from Resine/Resen (in Prespa-Sea) 44

Phantinos the Younger, St. 11, 97

Philip of France, king 271, 286

Philippoi 43

Philotheos Kokkinos, Patriarch of Constantinople 93
Phocas, priest 80

Phrygia 124

pilgrimage centre 134

Plato, a young man in the miracula of Artimius 12, $129 \mathrm{f} ., 138,215,240,267$

Plato 215, 267

Plato of Sakkudion, St. $\quad 129,130,138$

priest $34,36,38,45-47,51-53,55,61,64 \mathrm{f}$., $69,75-77,80,90,142,154,199,246$, 248, 286

property (see also inheritance) $12 \mathrm{f} ., 13,20 \mathrm{f}$., 21, $67 \mathrm{f} ., 109$

protosebastos 152

Prousa 102

psychoanalysis 16, 203, 205, 209, 295

- emotional independence or autonomy 117 , 139, 219, 227

- emotional maturity 13,25

- identity (personal) and social identity 15 , 112, 120, 128, 138, 193, 205, 208, 210, 216, 221, 223-226, 232, 257

- immaturity $4 \mathrm{f} ., 51,84,135,176$

- intimate emotional relationships 117, 228

- maturity 2, 4, 20, 23, 112, 141-143, $145 \mathrm{f}$, 149 -152, 175 f., 179 f., 183f., 188, 196, 201, 204

- self-actualization 3

- self-assurance 9

- self-confidence 107

- self-control 27, 124

- self-image 107

- self-responsibility $3,7,118,219$

- separation of parents 9-11, 101

psychosexual development 5, 205

puer senex $120,144,184,196$

Radbod of Utrecht, bishop of Utrecht 280 , 282

Ratpert of St. Gallen, chronicler 284

relationship (to God); romantic (erotic or sexual), mother-daughter 10,14f., 102, 105, $107-110,112 \mathrm{f} ., 117,135,147,151,182$, 208, 223

Remigius of Auxerre, Benedictine monk 273 Rimberti, St. 265

rites de passage $\quad 6,117,204,209,256$

Romanos, neaniskos from Choropanion 67

Romylos of Vidin, St. 93, 95, $99 \mathrm{f}$.

Rules of Makarios Choumnos 95 
Rupert of Deutz, Benedictine theologian 260 f., 273

Sabas, St. $\quad 11,83,95,247$

Sabas the Younger, St. $\quad 84,86,94$ f., 97

Samoa 118, 210

saracens 288

sarcophagus 142, 201

Saxony $288 \mathrm{f}$.

Sbina, wife of Georgios Serbopoulos $\quad 49,72$

Sedulius Scotus (or Scottus), Irish teacher, Latin grammarian and scriptural commentator 260, 269-271, 276, 283

sexuality $131,131-133,181,205,222$

- sexual identity 133, 138, 209

- sexual life 206

- sexual maturity 22,196

- sexual passion $120,124,131-133$

- sexual relationship 7,117, 135

- sexual temptation(s) 85, 89, 131, 133, 293

shepherds 118, 122, 139

Sicily 131,272

Sigebert of Gembloux, writer and Benedictine monk 264, 266

Slaba, wife of soldier Rados 48,73

social anthropology 203

social behavior 5

social class $20,123,125,203,235$

- aristocracy $8,13,26,125,127 \mathrm{f}$.

- elite $7 f ., 26,141,143,152$

- lower social strata 7, $9 \mathrm{f}$.

- slaves 21f., 27, 45, 86, 296

- upper class $9,26,40,59-60,87,125$

social integration $7,12,15,118,139$,

socialisation $12,103,118 \mathrm{f} ., 142$

Solomon 146, 182

Sophronios, author the life of St. Mary of Egypt 112

sport $9,15,126,128,139,213$

Staurakios (son of Nikephoros I.) $150 \mathrm{f}$.

Stephan the Younger, St. 11

Symeon, brother of David of Mytilene or Lesbos, St. $11,84,87,89$ f., 92 f., 97, 130, 133, 182, 248

Symeon Eulabes, spiritual father of Symeon the New Theologian 87-90, 92

Symeon Salos, St. 130, 133

Symeon the New Theologian, St. 9, 11, 84, $88-90,92,94$

Sympolos (Cembalo/Crimea) 79 synagogue 146,181

Syracuse 131

Tarasios, Patriarch, St. 122

Thekla, sister of Michael III 151

Theoderich, abbot 279, 284

Theodora Buzene Philanthropene, wife of Manuel Buzenos 81

Theodora, mother of Michael III $\quad 110,151$

Theodora of Kaisaris, St. 11

Theodora of Thessaloniki, St. 11

Theodora Palaiologina, niece of Michael Palaiologos (later Michael VIII) 110

Theodora Raoulaina, niece of Theodora Palaiologina 110

Theodora Tarchaneiotissa 114

Theodore Branas 75

Theodore Chloropodes, craftsman $\quad 47,73$

Theodore Diakonites 75

Theodore II Laskaris, emperor (1254-1258)

110

Theodore of Edessa, St. $\quad 83,97,181$

Theodore of Stoudios, St. 97

Theodore of Sykeon, St. 11, 24, 124-125

Theodore Prodromos 126, 211

Theodore Syropulos, grammatikos 42

Theodosia of Constantinople, St. 11

Theodosios Basilikos 40

Theodote, mother of Michael Psellos 113

Theodoule, founder of the monastery of the Virgin of Certain Hope 146, 163

Theodoulos, novice at the monastery of Makarios Choumnos 95

Theoktistos, abbot 89, 195

Theoleptos, bishop of Philadelphia $108 \mathrm{f}$.

Theophanes, monk at the Stoudios monastery; uncle of Nikolaos Stoudios 86-88, 91, 94, $123 \mathrm{f}$.

Theophanes the Confessor $86,97,123-124$

Theophilos, emperor (829-842), son of Michael II $123,126,150$

Theophylact, co-emperor, son of the emperor Michael I $150 \mathrm{f}$.

Theophylaktos of Nikomedeia, St. 97

therapy (see also healing) 17, 246, 247, 249

Thessaloniki/Thessalonike 11, 15, 46, 52 f., 76, $84,91,95,102 \mathrm{f} ., 127,143,184$

Theutberga, wife of Lothar II., king of Lotharingia 268

Tiberios, co-emperor, son of Constans II $148 \mathrm{f}$. 
Turnovo 100

typikon for the Phoberou monastery 84

typikon of Evergetis 88

typikon of the Bebaia Elpis 90

typikon of the Lips 92

typikon of the Machairas monastery on Cyprus 91

Union of Lyon 110

upbringing $8,121-122,139,257,265,278$

urban centres 9,139

virginity 15, 23f., 49, 106, 204

Virgin Mary $144,146,153$

Vlachs 46

Waldrada, mistress of Lothar II, king of Lotharingia 268,271 warfare 9,18

weapons $8 \mathrm{f} .124 \mathrm{f} ., 127,132,288$

- spear 124

Wido of Ferrara, historian 281

widow(s) 291

Widukind of Corvey, chronicler $\quad 271,281,289$, $290 \mathrm{f}$.

Wilhelm of Conches, philosopher 267, 283

wine $87,94,99,129-131,243$

women/females $7 \mathrm{f} ., 10,15 \mathrm{f} ., 21,24,26,35$, $37,50,59,105-108,110,112-115,128,132$, $135,141-145,152,154-156,177,181,183$, 187, 203f., 211, 231, 291, 296

work of children and adolescents 27, 118, $121-123,139,140,177$

youth gang 123,135 